\title{
IntechOpen
}

\section{Advanced Electromagnetic Waves}

Edited by Saad Osman Bashir

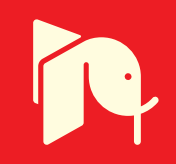





\section{ADVANCED ELECTROMAGNETIC WAVES}

Edited by Saad Osman Bashir 
Advanced Electromagnetic Waves

http://dx.doi.org/10.5772/59382

Edited by Saad Osman Bashir

\section{Contributors}

Mikhail Nesterenko, Irismar Da Paz, Heurison Silva, Paulo Miranda, Raul Valenzuela, Chunchuan Xu, Chengyin Liu, Jorge Avella Castiblanco, Divitha Seetharamdoo, Marion Berbineau, Michel Ney, Francois Gallee, Ibrahim Massy Sanchez, Adam Kusiek, Paul Denis Smith, Audrey Markowskei, Galyna Safonova, Elena Vinogradova, Jan Olof Jonson

\section{(c) The Editor(s) and the Author(s) 2015}

The moral rights of the and the author(s) have been asserted.

All rights to the book as a whole are reserved by INTECH. The book as a whole (compilation) cannot be reproduced, distributed or used for commercial or non-commercial purposes without INTECH's written permission.

Enquiries concerning the use of the book should be directed to INTECH rights and permissions department (permissions@intechopen.com).

Violations are liable to prosecution under the governing Copyright Law.

\section{(cc) BY}

Individual chapters of this publication are distributed under the terms of the Creative Commons Attribution 3.0 Unported License which permits commercial use, distribution and reproduction of the individual chapters, provided the original author(s) and source publication are appropriately acknowledged. If so indicated, certain images may not be included under the Creative Commons license. In such cases users will need to obtain permission from the license holder to reproduce the material. More details and guidelines concerning content reuse and adaptation can be foundat http://www.intechopen.com/copyright-policy.html.

\section{Notice}

Statements and opinions expressed in the chapters are these of the individual contributors and not necessarily those of the editors or publisher. No responsibility is accepted for the accuracy of information contained in the published chapters. The publisher assumes no responsibility for any damage or injury to persons or property arising out of the use of any materials, instructions, methods or ideas contained in the book.

First published in Croatia, 2015 by INTECH d.o.o.

eBook (PDF) Published by IN TECH d.o.o.

Place and year of publication of eBook (PDF): Rijeka, 2019.

IntechOpen is the global imprint of IN TECH d.o.o.

Printed in Croatia

Legal deposit, Croatia: National and University Library in Zagreb

Additional hard and PDF copies can be obtained from orders@intechopen.com

Advanced Electromagnetic Waves

Edited by Saad Osman Bashir

p. $\mathrm{cm}$.

ISBN 978-953-51-2205-0

eBook (PDF) ISBN 978-953-51-6398-5 


\section{We are IntechOpen, \\ the world's leading publisher of Open Access books}

Built by scientists, for scientists

\section{$3,800+$}

Open access books available

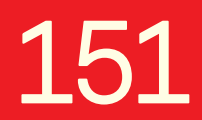

Countries delivered to

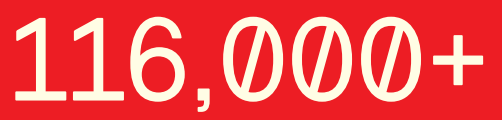

International authors and editors
$120 \mathrm{M}+$

Downloads

Our authors are among the

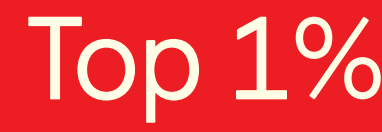

most cited scientists

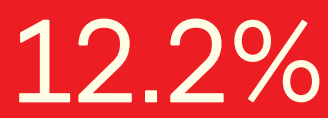

Contributors from top 500 universities

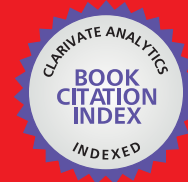

WEB OF SCIENCE ${ }^{\mathrm{TM}}$

Selection of our books indexed in the Book Citation Index in Web of Science ${ }^{\mathrm{TM}}$ Core Collection (BKCI)

Interested in publishing with us?

Contact book.department@intechopen.com

Numbers displayed above are based on latest data collected.

For more information visit www.intechopen.com

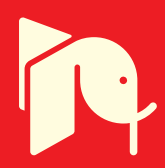





\section{Meet the editor}

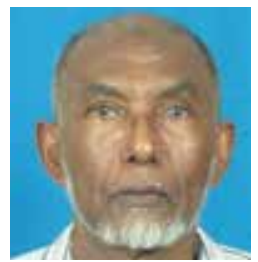

Prof. Dr. Bashir received his PhD in Electronics and Telecommunications Engineering, University of Bradford, West Yorkshire, in 1980/84. His research interests include antennas and radio wave propagation, optical communication systems, microwave and satellite systems, EM theory, and EM scattering computation methods; there is a significant amount of original publications in these fields. An InTech book, Electromagnetic Radiation, edited by Prof. Bashir was first published in June 2012 (p. cm. ISBN 978-953-51-0639-5). 



\section{Contents}

Preface XI

Chapter 1 What Effect does Rounding the Corners have on Diffraction from Structures with Corners? 1

Paul D. Smith and Audrey J. Markowskei

Chapter 2 Probing the Molecular Ordering in Azopolymer Thin Films by Second-Order Nonlinear Optics 29

Heurison S. Silva, Irismar G. Paz and Paulo B. Miranda

Chapter 3 The Electromagnetic Force between Two Parallel Current Conductors Explained Using Coulomb's Law 61 Jan Olof Jonson

Chapter 4 Electromagnetic Waves Propagation and Detection in Shielded Dielectric Power Cables 93

Chunchuan Charles Xu and Chengyin Liu

Chapter 5 Nonreciprocal Devices Utilizing Longitudinally Magnetized Ferrite Coupled Lines 117

Adam Kusiek, Wojciech Marynowski, Rafal Lech and Jerzy Mazur

Chapter 6 Electromagnetic Waves Excitation by Thin Impedance Vibrators and Narrow Slots in Electrodynamic Volumes 147

Mikhail V. Nesterenko, Sergey L. Berdnik, Victor A. Katrich and Yuriy M. Penkin

Chapter 7 Rigorous Approach to Analysis of Two-Dimensional Potential Problems, Wave Propagation and Scattering for Multi-conductor Systems 177

Galyna Safonova and Elena Vinogradova 
Chapter 8 Characterization of Magnetic Phases in Nanostructured Ferrites by Electron Spin Resonance 209

Rebeca Díaz-Pardo and Raúl Valenzuela

Chapter 9 Time-Domain Electromagnetic Wave Propagation in Confined Environments 239

Jorge Avella-Castiblanco, Divitha Seetharamdoo, Marion Berbineau, Michel Ney, Ibrahim Massy and Franois Gallée 


\section{Preface}

This book endeavors to give the reader a strong base in the advanced theory of electromagnetic waves and its applications, while keeping pace with research in various other disciplines that apply electrostatics/electrodynamics theory. The treatment is highly mathematical, which tends to obscure the principles involved. Hence, the book is designed for distinguished academics in this field and is by no means easy for the standard student to assimilate.

An earlier book, Electromagnetic Radiation, was originally published in 2012 by InTech. The subject of that book was radiation of EM waves or EMC. The present book retains essentially the same subject matter, in an expanded and modernized change which has taken place in the past decade.

Special thanks to Ms. Iva Simcic, Publishing Process Manager, and to InTech publishers for choosing me to be the editor of this book.

Prof. Saad Osman Bashir

International Islamic University

Malaysia 



\title{
Chapter 1
}

\section{What Effect does Rounding the Corners have on Diffraction from Structures with Corners?}

\author{
Paul D. Smith and Audrey J. Markowskei \\ Additional information is available at the end of the chapter
}

http://dx.doi.org/10.5772/61152

\begin{abstract}
In studying electromagnetic wave diffraction, the choice of an appropriate canonical structure is significant in elucidating the dominant features of a scattering scenario. This study was originally motivated by the influence that the corners of buildings and their surface cladding might have on the wave propagation. When an integral equation approach is employed as the basis of numerical studies of the scattering of plane waves by an obstacle, a common technique for dealing with domains with corners is to round the corners. In order to clarify the effect of such corner rounding, this work examines the diffraction from cylindrical scatterers which possess corners, that is, points at which the normal changes discontinuously. Specifically we develop a numerical method for the scattering of an E- polarised plane wave by such cylindrical structures. We examine three different boundary conditions: soft, hard and an impedance loaded boundary condition, each enforced at all points on the cross-sectional boundary of the cylinder. We quantify the difference between test structures with corners and similar structures where the corners have been rounded to assess the impact on near- and far-field scattering, as a function of the radius of curvature in the vicinity of the rounded corner points.
\end{abstract}

Keywords: Scattering and diffraction, two-dimensional structures, impedance boundary condition, integral equations, geometrical theory of diffraction

\section{Introduction}

Diffraction of electromagnetic waves by canonical shapes and structures of more general and arbitrary shape is of enduring interest. The choice of an appropriate canonical structure to model the dominant features of a scattering scenario can be very illuminating. The study in this paper was originally motivated by the influence that the corners of buildings and their surface cladding have on electromagnetic wave propagation. A recent publication by Rawlins 
[1] considered an approximate model relevant to the understanding of signal strength for phones in this environment. It studied the diffraction of an E-polarised wave by an absorbing rectangular cylinder, based upon Keller's method of GTD and its extensions to deal with multiple diffraction. It utilized the diffraction coefficient derived for the canonical problem of diffraction by an impedance corner to obtain relatively simple high frequency approximate expressions for the scattered far-field resulting from a plane wave obliquely incident on an imperfectly conducting rectangle.

In order to validate the results of [1], Smith and Rawlins [2] undertook a numerical study of the scattering of an E-polarised plane wave by an infinite cylindrical structure in which an impedance boundary condition is enforced at all points on the cross-sectional boundary of the cylinder. It employed the integral equation formulation of Colton and Kress [3] for the unknown surface distribution comprising a single-layer potential and the adjoint of the double-layer potential. A Nyström method similar to that expounded by Colton and Kress [4] for the soft boundary condition was developed to obtain numerical solutions of this integral equation. The computed scattered far-fields were compared with the results of Rawlins [1] in order to validate his solutions over the range of impedances and wavenumbers examined. The study concluded that the approximations developed in [1] provide reasonably accurate patterns for rectangular structures for the range of wavenumbers and dimensions examined, but some divergences appear at smaller wavenumbers. There was a limitation to the study [2]: the method was applicable only to cylindrical cross-sections that are smooth (having a continuously varying normal vector at each point), and so the exactly rectangular structures investigated in [1] were treated by a replacing them by an appropriate "super-ellipse" that approximates the rectangle with rounded corners.

In order to clarify the effect of corner rounding this paper examines the diffraction from cylindrical scatterers which possess corners, that is, points at which the normal changes discontinuously. Specifically we develop a numerical method for the scattering of an E-polarised plane wave by such cylindrical structures. The work in [5] is significantly extended. We examine three different boundary conditions: soft, hard and an impedance loaded boundary condition. In each case the boundary condition is enforced at all points on the cross-sectional boundary of the cylinder. We implement the Nyström method expounded by Colton and Kress [4] for the soft boundary condition to obtain numerical solutions of this integral equation. We then develop other Nyström methods similar to [4] for the hard and impedance boundary conditions to obtain numerical solutions of the respective integral equations.

We use these numerical methods to examine the difference between a test structure with a corner and a rounded corner to assess the impact on near and far field scattering, as a function of the radius of curvature in the vicinity of the rounded corner point. We then extend the numerical methods developed thus far to examine a test structure with two corners. We conclude by examining the effect on the scattered field of rounding these corners as a function of the radius of curvature in the vicinity of the rounded corner points.

\section{Formulation}

\subsection{The Scatterer}

We consider an infinitely long cylinder with uniform cross section. Without loss of generality we may assume that the axis of the cylinder is parallel to the $z$-axis. The cylinder is 
illuminated by an incident plane wave propagating with direction parallel to the $x-y$ plane. We will assume that the cross-section $D$ lying in the $x-y$ plane has a closed boundary $\partial D$ that can be parameterised by

$$
\boldsymbol{x}(t)=\left(x_{1}(t), x_{2}(t)\right), \quad t \in[0,2 \pi]
$$

\subsection{The incident and scattered fields}

The incident field illuminating the scatterer induces a scattered field. We assume that the incident and scattered fields are time harmonic with a temporal factor $e^{-i \omega t}$. The spatial component $u^{i n c}(x, y)$ of the incident wave travelling in the direction of the unit vector $\boldsymbol{d}=$ $\left(\cos \theta_{0}, \sin \theta_{0}\right)$ takes the form

$$
u^{i n c}(x, y)=e^{i k \boldsymbol{x} \cdot \boldsymbol{d}}
$$

and satisfies the Helmholtz equation

$$
\Delta u^{i n c}(x, y)+k^{2} u^{i n c}(x, y)=0, \quad(x, y) \in \mathbb{R}^{2} .
$$

The spatial component $u^{s c}(x, y)$ of the scattered field obeys the Helmholtz equation

$$
\Delta u^{s c}(x, y)+k^{2} u^{s c}(x, y)=0, \quad(x, y) \in \mathbb{R}^{2},
$$

at all points $(x, y)$ exterior to the body, where $k=\omega / c$ is the wavenumber and $c$ the speed of light in free space; moreover it obeys the two-dimensional form of the Sommerfeld radiation condition [4]

$$
\lim _{|\boldsymbol{x}| \rightarrow \infty} \sqrt{|\boldsymbol{x}|}\left(\frac{\partial u^{s \mathcal{C}}(\boldsymbol{x})}{\partial \boldsymbol{x}}-i k u^{s \mathcal{C}}(\boldsymbol{x})\right)=0, \quad \boldsymbol{x} \in \mathbb{R}^{2} \backslash D
$$

\subsection{The boundary conditions}

The nature of the scatterer imposes certain conditions that must be satisfied by the total field

$$
u^{t o t}=u^{i n c}+u^{s c}
$$

on the boundary of the scatterer $\partial D$.

This work considers sound soft scatterers, sound hard scatterers, and impedance loaded scatterers. All the scatterers induce a scattered acoustic potential. We define the boundary conditions for the different scatterers below. 


\subsubsection{Sound soft scatterers}

The total field $u^{\text {tot }}$ vanishes on the boundary of a sound soft scatterer $\partial D$. Thus

$$
u^{t o t}(\boldsymbol{x})=0, \quad \boldsymbol{x} \in \partial D
$$

and from (6) we determine

$$
u^{s c}(\boldsymbol{x})=-u^{i n c}(\boldsymbol{x}), \quad \boldsymbol{x} \in \partial D
$$

This sound soft boundary condition is a Dirichlet boundary condition.

\subsubsection{Sound hard scatterers}

The normal derivative of the total field with respect to the unit outward normal $\boldsymbol{n}$ to $\partial D$, vanishes on the boundary of a sound hard scatterer $\partial D$. Thus

$$
\frac{\partial u^{t o t}}{\partial n}(\boldsymbol{x})=0, \quad \boldsymbol{x} \in \partial D
$$

and from (6) we determine

$$
\frac{\partial u^{s c}}{\partial \boldsymbol{n}}(\boldsymbol{x})=-\frac{\partial u^{i n c}}{\partial \boldsymbol{n}}(\boldsymbol{x}), \quad \boldsymbol{x} \in \partial D
$$

This sound hard boundary condition is a Neumann boundary condition.

\subsubsection{Impedance loaded scatterers}

The impedance boundary value problem is prescribed by the boundary condition

$$
\frac{\partial u^{t o t}}{\partial \boldsymbol{n}}(\boldsymbol{x})+i k \lambda u^{t o t}(\boldsymbol{x})=0, \quad \boldsymbol{x} \in \partial D
$$

where $\boldsymbol{n}(x)$ is the unit outward normal to the boundary at the point $\boldsymbol{x}$ and $\lambda=\lambda(\boldsymbol{x})$ is a continuous function of position. From (6) we determine

$$
\frac{\partial u^{s c}}{\partial \boldsymbol{n}}(\boldsymbol{x})+i k \lambda u^{s c}(\boldsymbol{x})=-\frac{\partial u^{i n c}}{\partial \boldsymbol{n}}(\boldsymbol{x})-i k \lambda u^{i n c}(\boldsymbol{x}), \quad \boldsymbol{x} \in \partial D
$$

The scattered field is uniquely determined by the boundary and radiation conditions, provided $\operatorname{Re}(\lambda)$ is positive on the boundary $\partial D$. In this work, $\lambda$ will be restricted to be a (complex) constant. 


\subsection{Green's function}

As shown in [3], the problem of determining the scattered field may be solved by employing the single- and double-layer potentials associated with the two dimensional free-space Green's function

$$
G(\boldsymbol{x}, \boldsymbol{y})=\frac{i}{4} H_{0}^{(1)} k(|\boldsymbol{x}-\boldsymbol{y}|),
$$

where $H_{0}^{(1)}$ denotes the Hankel function of first kind and order zero. The Green's function satisfies the Helmholtz equation

$$
\Delta_{\boldsymbol{x}} G(\boldsymbol{x}, \boldsymbol{y})+k^{2} G(\boldsymbol{x}, \boldsymbol{y})=0,
$$

everywhere except at $\boldsymbol{x}=\boldsymbol{y}$, and satisfies the Sommerfeld radiation condition (5).

For a fixed point $\boldsymbol{y} \in \partial D$, the normal derivative of the Green's function with respect to the outward unit normal at $\boldsymbol{y}$ is

$$
\frac{\partial G(\boldsymbol{x}, \boldsymbol{y})}{\partial \boldsymbol{n}(\boldsymbol{y})}=\nabla_{\boldsymbol{y}} G(\boldsymbol{x}, \boldsymbol{y}) \cdot \boldsymbol{n}(\boldsymbol{y})
$$

It satisfies the Helmholtz equation (14) except at $\boldsymbol{x}=\boldsymbol{y}$, and satisfies the Sommerfeld radiation condition (5).

\subsection{Integral operators}

We define two operators associated with the single- and double-layer potentials of a continuous density $\phi(\boldsymbol{y})$ defined on the boundary $\partial D$,

$$
\begin{aligned}
& (\mathcal{S} \phi)(\boldsymbol{x})=2 \int_{\partial D} G(\boldsymbol{x}, \boldsymbol{y}) \phi(\boldsymbol{y}) d s(\boldsymbol{y}), \\
& (\mathcal{K} \phi)(\boldsymbol{x})=2 \int_{\partial D} \frac{\partial G(\boldsymbol{x}, \boldsymbol{y})}{\partial \boldsymbol{n}(\boldsymbol{y})} \phi(\boldsymbol{y}) d s(\boldsymbol{y})
\end{aligned}
$$

their normal derivatives are, respectively

$$
\begin{aligned}
& \left(\mathcal{K}^{\prime} \phi\right)(\boldsymbol{x})=2 \int_{\partial D} \frac{\partial G(\boldsymbol{x}, \boldsymbol{y})}{\partial \boldsymbol{n}(\boldsymbol{x})} \phi(\boldsymbol{y}) d s(\boldsymbol{y}) \\
& (\mathcal{T} \phi)(\boldsymbol{x})=2 \frac{\partial}{\partial \boldsymbol{n}(\boldsymbol{x})} \int_{\partial D} \frac{\partial G(\boldsymbol{x}, \boldsymbol{y})}{\partial \boldsymbol{n}(\boldsymbol{y})} \phi(\boldsymbol{y}) d s(\boldsymbol{y}) .
\end{aligned}
$$


The integral operators (16), (17), (18) and (19) are compact [3].

The acoustic single-layer potential $u$ with integrable density $\phi$ is

$$
u(\boldsymbol{x})=\frac{1}{2} \mathcal{S} \phi(\boldsymbol{x})
$$

and is continuous and bounded throughout $\mathbb{R}^{2} \backslash \partial D$ and at all points on the boundary $\partial D$ [4]. The double-layer potential $v$ with integrable density $\phi$ is

$$
v(\boldsymbol{x})=\frac{1}{2} \mathcal{K} \phi(\boldsymbol{x})
$$

and is continuous and bounded throughout $\mathbb{R}^{2} \backslash \partial D$. It is discontinuous at all points on the boundary $\partial D$, but can be continuously extended form $D$ to $\bar{D}$ and from $\mathbb{R}^{2} \backslash \partial \bar{D}$ to $\mathbb{R}^{2} \backslash \partial D$ with limiting values [4]

$$
v_{ \pm}(\boldsymbol{x})=\int_{\partial D} \frac{\partial G(\boldsymbol{x}, \boldsymbol{y})}{\partial \boldsymbol{n}(\boldsymbol{y})} \phi(\boldsymbol{y}) d s(\boldsymbol{y}) \pm \frac{\phi(\boldsymbol{x})}{2}, \quad \boldsymbol{x} \in \boldsymbol{\partial} D
$$

where

$$
v_{ \pm}(\boldsymbol{x})=\lim _{h \rightarrow+0} v(\boldsymbol{x} \pm h \boldsymbol{n}(\boldsymbol{x}))
$$

\subsection{Integral representations}

The solution to the exterior Dirichlet problem for all $\boldsymbol{x} \in \mathbb{R}^{2} \backslash \bar{D}$, is based on representing the scattered field as a combination of the single (20) and double-layer (21) potentials

$$
u^{s c}(\boldsymbol{x})=\int_{\partial D}\left\{\frac{\partial G(\boldsymbol{x}, \boldsymbol{y})}{\partial \boldsymbol{n}(\boldsymbol{y})}-i \eta G(\boldsymbol{x}, \boldsymbol{y})\right\} \phi(\boldsymbol{y}) d s(\boldsymbol{y}), \quad \boldsymbol{x} \in \mathbb{R}^{2} \backslash \bar{D}
$$

where $\eta$ is a coupling parameter, provided the continuous density $\phi(\boldsymbol{x})$ is a solution to the following integral equation on $\partial D$ :

$$
I \phi+\mathcal{K} \phi-i \eta \mathcal{S} \phi=2 g
$$

where $g=-2 u^{i n c}$. This integral equation is uniquely solvable for all wave numbers satisfying $\operatorname{Im} k \geq 0$ [3]. 
The single-layer potential (20)

$$
u^{s \mathcal{C}}(\boldsymbol{x})=\int_{\partial D} G(\boldsymbol{x}, \boldsymbol{y}) \phi(\boldsymbol{y}) d s(\boldsymbol{y}), \quad \boldsymbol{x} \in \mathbb{R}^{2} \backslash \bar{D},
$$

is a solution to the exterior Neumann problem for all $\boldsymbol{x} \in \mathbb{R}^{2} \backslash \bar{D}$, provided that the continuous density $\phi(\boldsymbol{x})$ is a solution of the following integral equation on $\partial D$ [6]:

$$
\phi-\mathcal{K}^{\prime} \phi=-2 h
$$

where

$$
h(\boldsymbol{x})=-\frac{\partial u^{i n c}}{\partial \boldsymbol{n}}(\boldsymbol{x}), \quad \boldsymbol{x} \in \partial D
$$

and $\phi(\boldsymbol{x})$ satisfies

$$
\int_{\partial D} \phi d s=0
$$

Further, in $\mathbb{R}^{2}$, the exterior Neumann problem is uniquely solvable if and only if

$$
\int_{\partial D} h d s=0
$$

is satisfied [6].

The solution to the exterior impedance problem for all $\boldsymbol{x} \in \mathbb{R}^{2} \backslash \bar{D}$, is

$$
u^{s \mathcal{C}}(\boldsymbol{x})=\int_{\partial D} G(\boldsymbol{x}, \boldsymbol{y}) \phi(\boldsymbol{y}) d s(\boldsymbol{y}), \quad \boldsymbol{x} \in \mathbb{R}^{2} \backslash \bar{D},
$$

provided $\phi(\boldsymbol{x})$ is a solution to

$$
\phi-\mathcal{K}^{\prime} \phi-i k \lambda \mathcal{S} \phi=-2 m
$$

where

$$
m(\boldsymbol{x})=-\frac{\partial u^{i n c}}{\partial \boldsymbol{n}}(\boldsymbol{x})-i k \lambda u^{i n c}(\boldsymbol{x}), \quad \boldsymbol{x} \in \partial D
$$


This solution is unique provided that $k$ is not an interior Dirichlet eigenvalue [3]. Uniqueness is guaranteed by considering a suitable combination of single- and double-layer potentials, ie the combined potential

$$
u^{s c}(\boldsymbol{x})=\int_{\partial D}\left\{\frac{\partial G(\boldsymbol{x}, \boldsymbol{y})}{\partial \boldsymbol{n}(\boldsymbol{y})}-i \eta G(\boldsymbol{x}, \boldsymbol{y})\right\} \phi(\boldsymbol{y}) d s(\boldsymbol{y}), \quad \boldsymbol{x} \in \mathbb{R}^{2} \backslash \bar{D},
$$

where $\eta \neq 0$ such that $\eta \operatorname{Re} k \geq 0$, solves the exterior impedance problem uniquely provided that the density $\phi(\boldsymbol{x}) \in \partial D$ is a solution of the integral equation [3]

$$
(I-i \eta \lambda) \phi-\left(\mathcal{K}^{\prime}+i \eta \mathcal{T}+i \eta \lambda \mathcal{K}+\lambda \mathcal{S}\right) \phi=-2 m .
$$

\section{Numerical solution}

We use the Nyström method based on weighted trigonometric interpolation quadratures as the numerical method used to approximate the solution to the integral equations (24), (26) and (31) with a mesh of $2 n$ points. We parameterize the boundary $\partial D$ as

$$
\boldsymbol{x}(t)=\left(x_{1}(t), x_{2}(t)\right), \quad t \in[0,2 \pi] .
$$

So for $\boldsymbol{x}, \boldsymbol{y} \in \partial D$, we let

$$
\begin{array}{ll}
\boldsymbol{x}=\boldsymbol{x}(t)=\left(x_{1}(t), x_{2}(t)\right), \quad t \in[0,2 \pi], \\
\boldsymbol{y}=\boldsymbol{x}(\tau)=\left(x_{1}(\tau), x_{2}(\tau)\right), \quad \tau \in[0,2 \pi] .
\end{array}
$$

The outward pointing unit normal at $\boldsymbol{x}(\tau)$ is

$$
\boldsymbol{n}(\boldsymbol{x}(\tau))=\frac{\left(x_{2}^{\prime}(\tau),-x_{1}^{\prime}(\tau)\right)}{J(\tau)}
$$

where $J(\tau)$ is the Jacobian factor

$$
J(\tau)=\sqrt{\left(x_{1}^{\prime}(\tau)\right)^{2}+\left(x_{2}^{\prime}(\tau)\right)^{2}} .
$$

The operators (25), (27) and (32) may then be expressed as

$$
\begin{aligned}
(\mathcal{K} \phi)(\boldsymbol{x}(t)) & =\int_{0}^{2 \pi} K_{0}(t, \tau) \phi(\tau) d \tau, \\
(\mathcal{S} \phi)(\boldsymbol{x}(t)) & =\int_{0}^{2 \pi} S_{0}(t, \tau) \phi(\tau) d \tau, \\
\left(\mathcal{K}^{\prime} \phi\right)(\boldsymbol{x}(t)) & =\int_{0}^{2 \pi} K_{0}^{\prime}(t, \tau) \phi(\tau) d \tau,
\end{aligned}
$$


where $\phi(\tau)=\phi(x(\tau))$, and the associated kernels

$$
\begin{aligned}
& S_{0}(t, \tau)=2 G(\boldsymbol{x}(t), \boldsymbol{x}(\tau)) J(\tau), \\
& K_{0}(t, \tau)=2 \frac{\partial G(\boldsymbol{x}(t), \boldsymbol{x}(\tau))}{\partial \boldsymbol{n}(\tau)} J(\tau), \\
& K_{0}^{\prime}(t, \tau)=2 \frac{\partial G(\boldsymbol{x}(t), \boldsymbol{x}(\tau))}{\partial \boldsymbol{n}(t)} J(\tau),
\end{aligned}
$$

each have a logarithmic singularity at $t=\tau$. Thus we transform the integral operator formulation (25) of the exterior Dirichlet problem into the parametric form

$$
\phi(t)+\int_{0}^{2 \pi}\left\{K_{0}(t, \tau)-i \eta S_{0}(t, \tau)\right\} \phi(\tau) d \tau=g(t), \quad 0 \leq t \leq 2 \pi,
$$

the integral operator formulation (27) of the exterior Neumann problem into the parametric form

$$
-\phi(t)+\int_{0}^{2 \pi} K_{0}^{\prime}(t, \tau) \phi(\tau) d \tau=h(t), \quad 0 \leq t \leq 2 \pi,
$$

and the integral operator formulation (32) of the exterior impedance problem into the parametric form

$$
-\phi(t)+\int_{0}^{2 \pi}\left\{K_{0}^{\prime}(t, \tau)+i k \lambda S_{0}(t, \tau)\right\} \phi(\tau) d \tau=m(t), \quad 0 \leq t \leq 2 \pi .
$$

A method developed by Martensen and Kussmaul [4] for the logarithmic singularities arising in (41), (42) and (43) was employed. The singular parts of the kernels (44), (45) and (46) are isolated in the following manner so that

$$
\begin{aligned}
& K_{0}(t, \tau)=K_{1}(t, \tau) \ln \left(4 \sin ^{2} \frac{t-\tau}{2}\right)+K_{2}(t, \tau), \\
& S_{0}(t, \tau)=S_{1}(t, \tau) \ln \left(4 \sin ^{2} \frac{t-\tau}{2}\right)+S_{2}(t, \tau), \\
& K_{0}^{\prime}(t, \tau)=K_{1}^{\prime}(t, \tau) \ln \left(4 \sin ^{2} \frac{t-\tau}{2}\right)+K_{2}^{\prime}(t, \tau),
\end{aligned}
$$

where $K_{1}, K_{2}, S_{1}, S_{2}, K_{1}^{\prime}, K_{2}^{\prime}$ are analytic. 
The smooth components of the kernel $K_{0}(t, \tau)$ are evaluated using the trapezoidal rule to approximate

$$
\int_{0}^{2 \pi} K_{2}(t, \tau) \phi(\tau) d \tau \approx \frac{\pi}{n} \sum_{j=0}^{2 n-1} K_{2}\left(t, t_{j}\right) \phi\left(\tau_{j}\right) d t
$$

An identical rule was applied for

$$
\int_{0}^{2 \pi} S_{2}(t, \tau) \phi(\tau) d \tau
$$

and

$$
\int_{0}^{2 \pi} K_{2}^{\prime}(t, \tau) \phi(\tau) d \tau
$$

A different quadrature rule is used to estimate the singular part of the kernel $K_{0}(t, \tau)$ which replaces the integrand by its trigonometric interpolation polynomial and integrates this interpolant exactly. We apply the following quadrature rule

$$
\int_{0}^{2 \pi} \ln \left(4 \sin ^{2} \frac{t-\tau}{2}\right) K_{1}(t, \tau) \phi(\tau) d \tau \approx \sum_{j=0}^{2 n-1} R_{j}^{(n)}(t) K_{1}\left(t, t_{j}\right) \phi\left(t_{j}\right), \quad \text { for } 0 \leq t \leq 2 \pi
$$

to approximate the integral of the logarithmic part of the kernel $K_{0}(t, \tau)$. The quadrature weights $R_{j}^{(n)}$ are given by

$$
R_{j}^{(n)}(t)=-\frac{2 \pi}{n} \sum_{m=1}^{n-1} \frac{1}{m} \cos m\left(t-t_{j}\right)-\frac{\pi}{n^{2}} \cos n\left(t-t_{j}\right), \quad \text { for } j=0, \ldots, 2 n-1 .
$$

An identical rule was applied for

$$
\int_{0}^{2 \pi} \ln \left(4 \sin ^{2} \frac{t-\tau}{2}\right) S_{1}(t, \tau) \phi(\tau) d \tau
$$

and

$$
\int_{0}^{2 \pi} \ln \left(4 \sin ^{2} \frac{t-\tau}{2}\right) K_{1}^{\prime}(t, \tau) \phi(\tau) d \tau
$$


Three different spacings of the $2 n$ mesh points were used. For smooth scatterers we used a mesh of $2 n$ uniformly spaced points $t_{j}=\frac{\pi j}{n}$, for $j=0,1, \ldots, 2 n-1$, in the parameterisation (36). However, for domains with corners, the solutions to (25), (27) and (32) have singularities in the derivatives in the corners. To deal with these singularities, the uniform mesh is replaced by a non-uniform graded mesh. This is achieved by substituting a new variable such that the derivatives of the transformed integrand vanish up to a certain order at the corners [4]. The previous quadrature rules (Martensen-Kussmaul and trapezoidal) are then modified as follows. For any function $f(t)$, its definite integral over $[0,2 \pi]$ is evaluated by the trapezoidal quadrature rule after the substitution $t=w(s)$ by an appropriately chosen function $w(s)$ :

$$
\int_{0}^{2 \pi} f(t) d t=\int_{0}^{2 \pi} f(w(s)) w^{\prime}(s) d s \approx \frac{\pi}{n} \sum_{j=1}^{2 n-1} a_{j} f\left(s_{j}\right),
$$

with weights $a_{j}=w^{\prime}\left(t_{j}\right)$ and mesh points $s_{j}=w\left(t_{j}\right)$.

For a domain with a single corner, the scatterer boundary $\partial D$ is defined as having one corner at the point $\boldsymbol{x}_{0}$ and $\partial D \backslash\left\{\boldsymbol{x}_{0}\right\}$ is assumed to be $C^{2}$ and piecewise analytic. The angle $\gamma$ at the corner is assumed to be $0<\gamma<2 \pi$. A suitable choice of the function $w(s)$ is [4]

$$
w(s)=2 \pi \frac{[v(s)]^{p}}{[v(s)]^{p}+[v(2 \pi-s)]^{p}}, \quad 0 \leq s \leq 2 \pi,
$$

where

$$
v(s)=\left(\frac{1}{p}-\frac{1}{2}\right)\left(\frac{\pi-s}{\pi}\right)^{3}+\frac{1}{p} \frac{s-\pi}{\pi}+\frac{1}{2}
$$

and the integer $p$ is chosen to be at least 2 . The function $w(s)$ is strictly monotonically increasing and the derivatives at the end points $s=0$ and $2 \pi$ vanish up to order $p$. This choice of substitution ensures that approximately half of the quadrature points are uniformly distributed around the surface of the scatterer and that the other half are concentrated at the corner end points $s=0$ and $2 \pi$. In this study we set $p=8$. Use of this particular function $w(s)$ (61) requires that the parameterisation of the surface (36) is such that the corner $\boldsymbol{x}_{0}$ occurs at $t=0$.

The required substitution is applied to the discretization of (41) by setting $t=w(s)$ and $\tau=w(\sigma)$ to obtain

$$
\int_{0}^{2 \pi} K_{0}(t, \tau) \phi(\tau) d \tau=\int_{0}^{2 \pi} K_{0}(w(s), w(\sigma)) \phi(w(\sigma)) w^{\prime}(\sigma) d \sigma,
$$


and decomposing

$$
K_{0}(w(s), w(\sigma))=K_{1}(s, \sigma) \ln \left(4 \sin ^{2} \frac{s-\sigma}{2}\right)+K_{2}(s, \sigma)
$$

where

$$
K_{1}(s, \sigma)=K_{1}(w(s), w(\sigma))
$$

and

$$
K_{2}(s, \sigma)=K_{0}(w(s), w(\sigma))-K_{1}(s, \sigma) \ln \left(4 \sin ^{2} \frac{s-\sigma}{2}\right), \quad s \neq \sigma .
$$

The kernels $K_{1}(s, \sigma)$ and $K_{2}(s, \sigma)$ are analytic. The operator is now discretized using the points $s_{j}=w\left(t_{j}\right)$ and weights $a_{j}=w^{\prime}\left(t_{j}\right)$. Fuller details are in [4]. The same discretization procedure is applied to discretize (42) and (43).

For a domain with two corners, the scatterer boundary $\partial D$ is defined as having a corner at the point $\boldsymbol{x}_{0}$ and a second at the point $\boldsymbol{x}_{\pi}$ and $\partial D \backslash\left\{\boldsymbol{x}_{0} \cup \boldsymbol{x}_{\pi}\right\}$ is assumed to be $C^{2}$ and piecewise analytic. The angle $\gamma$ at the corners is assumed to satisfy $0<\gamma<2 \pi$. Our choice of the function $w(s)$ is

$$
w(s)=s-\frac{3}{4} \sin 2 s+\frac{3}{20} \sin 4 s-\frac{1}{60} \sin 6 s, \quad 0 \leq s \leq 2 \pi .
$$

The function $w(s)$ is strictly monotonically increasing between the corners and the derivatives at the corner points $s=0, \pi$ and $2 \pi$ vanish. This choice (67) of substitution ensures that approximately half of the quadrature points are uniformly distributed around the surface of the scatterer between the two corners and that the other half is concentrated at the corner end points $s=0, \pi$ and $2 \pi$. Use of this particular function $w(s)(67)$ requires that the parameterisation of the surface (36) is such that the corner $\boldsymbol{x}_{0}$ occurs at $t=0$ and that the corner $\boldsymbol{x}_{\pi}$ occurs at $t=\pi$.

With any of the above quadrature rules evaluated at the $2 n$ points $t_{j}$ we have obtained a system of $2 n$ linear equations for the boundary values $\phi\left(t_{j}\right)$ for $j=0,1, \ldots, 2 n-1$ that is a discretization of the integral equations (25), (27) and (32). The solutions are obtained by the usual Gaussian elimination procedure.

Implementation of the graded mesh ensures an exponentially fast convergence rate (as a function of $n$ ) for scatterers with one or two corners with the Neumann and impedance boundary conditions. In the case where these scatterers have a Dirichlet boundary condition further modifications are necessary to achieve comparable convergence rates. For these domains the kernel of (24) is no longer weakly singular at the corner. 
The modification for domains with a single corner at $\boldsymbol{x}_{0}$ and the Dirichlet boundary condition [4], uses the fundamental solution

$$
G_{0}(\boldsymbol{x}, \boldsymbol{y})=\frac{1}{2 \pi} \ln \frac{1}{|\boldsymbol{x}-\boldsymbol{y}|}, \quad \boldsymbol{x} \neq \boldsymbol{y},
$$

to the Laplace equation in $\mathbb{R}^{2}$ to subtract a vanishing term. This transforms (24) into

$$
u^{s c}(\boldsymbol{x})=\int_{\partial D}\left\{\left\{\frac{\partial G(\boldsymbol{x}, \boldsymbol{y})}{\partial \boldsymbol{n}(\boldsymbol{y})}-i \eta G(\boldsymbol{x}, \boldsymbol{y})\right\} \phi(\boldsymbol{y})-\frac{\partial G_{0}(\boldsymbol{x}, \boldsymbol{y})}{\partial \boldsymbol{n}(\boldsymbol{y})} \phi\left(\boldsymbol{x}_{0}\right)\right\} d s(\boldsymbol{y}), \quad \boldsymbol{x} \in \mathbb{R}^{2} \backslash \bar{D},
$$

and the associated boundary equation (25) is reformulated as

$$
\begin{aligned}
\phi(\boldsymbol{x})-\phi\left(\boldsymbol{x}_{0}\right)+2 \int_{\partial D}\left\{\frac{\partial G(\boldsymbol{x}, \boldsymbol{y})}{\partial \boldsymbol{n}(\boldsymbol{y})}-i \eta G(\boldsymbol{x}, \boldsymbol{y})\right\} \phi(\boldsymbol{y}) d s(\boldsymbol{y}) \\
-2 \int_{\partial D} \frac{\partial G_{0}(\boldsymbol{x}, \boldsymbol{y})}{\partial \boldsymbol{n}(\boldsymbol{y})} \phi\left(\boldsymbol{x}_{0}\right) d s(\boldsymbol{y})=-2 u^{i n c}(\boldsymbol{x}), \quad \boldsymbol{x} \in \partial D .
\end{aligned}
$$

An analysis showing the existence of a solution to (70) is provided in [4]. The integral equation (70) is rewritten in parameterised form

$$
\begin{aligned}
\phi(t)-\phi(0) & -\int_{0}^{2 \pi} \hat{K}(t, \tau) \phi(\tau) d \tau \\
& -\int_{0}^{2 \pi} H(t, \tau) \phi(0) d \tau=g(t), \quad 0 \leq t \leq 2 \pi,
\end{aligned}
$$

where

$$
H(t)=\left\{\begin{array}{cl}
\frac{1}{\pi} \frac{x_{2}^{\prime}(\tau)\left[x_{1}(t)-x_{1}(\tau)\right]-x_{1}^{\prime}(\tau)\left[x_{2}(t)-x_{2}(\tau)\right]}{|\boldsymbol{x}(t)-\boldsymbol{x}(\tau)|^{2}}, & t \neq \tau, \\
\frac{1}{2 \pi} \frac{x_{2}^{\prime}(t) x_{1}^{\prime \prime}(t)-x_{1}^{\prime}(t) x_{2}^{\prime \prime}(t)}{\left|\boldsymbol{x}^{\prime}(t)\right|^{2}}, & t=\tau, t \neq 0,2 \pi
\end{array}\right.
$$

and

$$
\hat{K}(t, \tau)=K(t, \tau)-i \eta S(t, \tau), \quad 0 \leq t \leq 2 \pi .
$$


We now apply the substitution (60) to (71) and obtain

$$
\begin{aligned}
\int_{0}^{2 \pi} \hat{K}(t, \tau) \phi(\tau) d \tau & -\int_{0}^{2 \pi} H(t, \tau) \phi(0) d \tau \\
= & \int_{0}^{2 \pi} \hat{K}(w(s), w(\sigma)) w^{\prime}(\sigma) \phi(w(\sigma)) d \sigma \\
& -\int_{0}^{2 \pi} H(w(s), w(\sigma)) w^{\prime}(\sigma) \phi(0) d \sigma .
\end{aligned}
$$

The logarithmic singularity present in the kernel $\hat{K}(t, \tau)$ remains to be accounted for. This is done in the same manner as (64). Using the quadrature rules (53) and (56) to discretize the kernel, and the trapezoidal rule to discretize the kernel $H(t, \tau)$ and $\phi_{0}=\phi(0)$ at the corner $s_{0}=0$ gives

$$
\begin{array}{rlrl}
\phi_{i}-\phi_{0} & +\sum_{j=1}^{2 n-1}\left[R_{|i-j|}(t)\left\{K_{1}\left(w\left(s_{i}\right), w\left(s_{j}\right)\right)-i \eta S_{1}\left(w\left(s_{i}\right), w\left(s_{j}\right)\right)\right\}\right. & & \\
& \left.+\frac{\pi}{n}\left\{K_{2}\left(w\left(s_{i}\right), w\left(s_{j}\right)\right)-i \eta S_{2}\left(w\left(s_{i}\right), w\left(s_{j}\right)\right)\right\}\right] a_{j} \phi_{j} & \\
& -\sum_{j=1}^{2 n-1} \frac{\pi}{n} H\left(w\left(s_{i}\right), w\left(s_{j}\right)\right) a_{j} \phi_{0}=g\left(w\left(s_{i}\right)\right), & \text { for } i=0, \ldots, 2 n-1 .
\end{array}
$$

We have obtained a system of $2 n-1$ linear equations for the boundary values $\phi\left(t_{j}\right)$, for $j=1,2, \ldots, 2 n-1$, that is a discretization of the integral equation (70). The solution is obtained by the usual Gaussian elimination procedure.

The described modification (70) applied to (25) ensures that exponentially fast convergence is achieved for scatterers with the Dirichlet boundary condition and a single corner on $\partial D$.

This modification needs to be extended when the scatterer has two corners on $\partial D$. There are now two points in the domain with singularities in the derivatives: at $t=0$ and $t=\pi$. Each of these singularities have a contributing effect that needs to be accounted for. We use the fundamental solution to the Laplace equation in $\mathbb{R}^{2}(68)$ to subtract vanishing terms. To reflect these combined contributions (69) is reformulated as

$$
\begin{aligned}
& u^{s c}(\boldsymbol{x})=\int_{\partial D}\left\{\left\{\frac{\partial G(\boldsymbol{x}, \boldsymbol{y})}{\partial \boldsymbol{n}(\boldsymbol{y})}-i \eta G(\boldsymbol{x}, \boldsymbol{y})\right\} \phi(\boldsymbol{y})\right. \\
& \left.-\cos ^{2} \frac{t(\boldsymbol{x})}{2} \frac{\partial G_{0}(\boldsymbol{x}, \boldsymbol{y})}{\partial \boldsymbol{n}(\boldsymbol{y})} \phi\left(\boldsymbol{x}_{0}\right)-\sin ^{2} \frac{t(\boldsymbol{x})}{2} \frac{\partial G_{0}(\boldsymbol{x}, \boldsymbol{y})}{\partial \boldsymbol{n}(\boldsymbol{y})} \phi\left(\boldsymbol{x}_{\pi}\right)\right\} d s(\boldsymbol{y}), \quad \boldsymbol{x} \in \mathbb{R}^{2} \backslash \bar{D},
\end{aligned}
$$


where $\boldsymbol{x}_{0}$ and $\boldsymbol{x}_{\pi}$ are the two corner points and $t(\boldsymbol{x})$ denotes the parameter value of point $\boldsymbol{x}$. The associated boundary equation (70) is now

$$
\begin{aligned}
& \phi(\boldsymbol{x})-\left(\cos ^{2} \frac{t(\boldsymbol{x})}{2} \phi\left(\boldsymbol{x}_{0}\right)\right.\left.+\sin ^{2} \frac{t(\boldsymbol{x})}{2} \phi\left(\boldsymbol{x}_{\pi}\right)\right) \\
&+2 \int_{\partial D}\left\{\frac{\partial G(\boldsymbol{x}, \boldsymbol{y})}{\partial \boldsymbol{n}(\boldsymbol{y})}-i \eta G(\boldsymbol{x}, \boldsymbol{y})\right\} \phi(\boldsymbol{y}) d s(\boldsymbol{y}) \\
&- 2 \int_{\partial D}\left(\cos ^{2} \frac{t(\boldsymbol{x})}{2} \frac{\partial G_{0}(\boldsymbol{x}, \boldsymbol{y})}{\partial \boldsymbol{n}(\boldsymbol{y})} \phi\left(\boldsymbol{x}_{0}\right)+\sin ^{2} \frac{t(\boldsymbol{x})}{2} \frac{\partial G_{0}(\boldsymbol{x}, \boldsymbol{y})}{\partial \boldsymbol{n}(\boldsymbol{y})} \phi\left(\boldsymbol{x}_{\pi}\right)\right) d s(\boldsymbol{y}) \\
&=-2 u^{i n c}(\boldsymbol{x}), \quad \boldsymbol{x} \in \partial D,
\end{aligned}
$$

which in parameterised form is

$$
\begin{aligned}
\phi(t) & -\left(\cos ^{2} \frac{t}{2} \phi(0)+\sin ^{2} \frac{t}{2} \phi(\pi)\right)-\int_{0}^{2 \pi} \hat{K}(t, \tau) \phi(\tau) d \tau \\
& -\cos ^{2} \frac{t}{2} \int_{0}^{2 \pi} H(t, \tau) \phi(0) d \tau-\sin ^{2} \frac{t}{2} \int_{0}^{2 \pi} H(t, \tau) \phi(\pi) d \tau=g(t), \quad 0 \leq t \leq 2 \pi,
\end{aligned}
$$

where $H(t)$ is as (72) except that for $t=\tau, t \neq 0, \pi, 2 \pi$ and $\hat{K}(t, \tau)$ is as in (73). We then apply the substitution (60) as in the case for the single corner domain with graded mesh (67) and discretize in the same fashion.

\section{Verification of numerical results}

The numerical results discussed in the results section were obtained after implementation of the above schemes in a MATLAB code. A number of tests were applied to verify its correctness, including those applied in [5]. Analytical solutions were derived for a circular scatterer for the three boundary conditions and the Mie series method was used to compute an actual solution. This enabled comparison of the scattered field computed by the methods described in this section for a circular scatterer. For all three boundary conditions the error was in the order of $10^{-15}$ which was considered a suitable tolerance. Also, the condition number of the systems was checked to ensure that uniqueness problems arising for wavenumbers $k$ near an interior Dirichlet eigenvalue of the scatterer were avoided.

However, there is no analytical expression for the scattered field from a non-circular scatterer and as such, there is no true solution to which we can compare results. For this study, we use a significant digit measurement to determine the convergence of the solution.

We choose a point $\boldsymbol{x}$ in the domain external to the scatterer and compute the field. As the number of quadrature points increases, if the solution is convergent, the number of significant digits in agreement increases. Thus we measure the number of unchanging digits 
in the approximate solution as the number of quadrature points $N$ increases, and terminate the calculation when the truncation of the computed value to a prespecified number of significant digits does not change as $N$ increases.

Two measures were used determine the convergence of the solutions. Firstly, a near field measure of the real and imaginary parts of the scattered field $u^{s c}$. This measurement was taken at a radius $r=10$ from the origin in the direction $\boldsymbol{x}=(-1,1)$.

The second measure employs the far-field. It is measured in a specified direction $\hat{\boldsymbol{x}}$. For the Dirichlet boundary condition, the far field pattern is calculated as

$$
u_{\infty}(\hat{\boldsymbol{x}})=\frac{e^{-i \frac{\pi}{4}}}{\sqrt{8 \pi k}} \int_{\partial D}\{k \boldsymbol{n}(y) \cdot \hat{\boldsymbol{x}}+\eta\} e^{-i k \hat{\boldsymbol{x}} \cdot \boldsymbol{y}} \phi(\boldsymbol{y}) d s(\boldsymbol{y}), \quad|\hat{\boldsymbol{x}}|=1
$$

and for Neumann and impedance boundary conditions the calculation is

$$
u_{\infty}(\hat{\boldsymbol{x}})=\frac{e^{-i \frac{\pi}{4}}}{\sqrt{8 \pi k}} \int_{\partial D} e^{-i k \hat{\boldsymbol{x}} \cdot \boldsymbol{y}} \phi(\boldsymbol{y}) d s(\boldsymbol{y}), \quad|\hat{\boldsymbol{x}}|=1
$$

\section{Results and discussion}

\subsection{Effect of corner rounding on a domain with a single corner}

Consider the curve given by the parametric representation (it is half of the so-called lemniscate of Gerono):

$$
\boldsymbol{x}=\boldsymbol{x}(t)=a\left(2 \sin \frac{t}{2},-\sin t\right), \quad t \in[0,2 \pi]
$$

where $a$ is a parameter. It has the corner at $t=0$ and with an interior right angle. Henceforth the parameter $a$ is set equal to 1 length unit.

We will also consider a family of curves in which the corner has been rounded; the family is parameterized by the quantity $\varepsilon(0 \leq \varepsilon \leq 1)$ :

$$
\boldsymbol{x}=\boldsymbol{x}(t)=a\left(2 \sqrt{\varepsilon^{2}+\left(1-\varepsilon^{2}\right) \sin ^{2} \frac{t}{2}},-\sin t\right), \quad t \in[0,2 \pi] .
$$

Figure 1 illustrates the two shapes, with $a=1$. The radius of curvature $\rho$ at the corner point $t=0$ is $2 \varepsilon /\left(1-\varepsilon^{2}\right)$. 


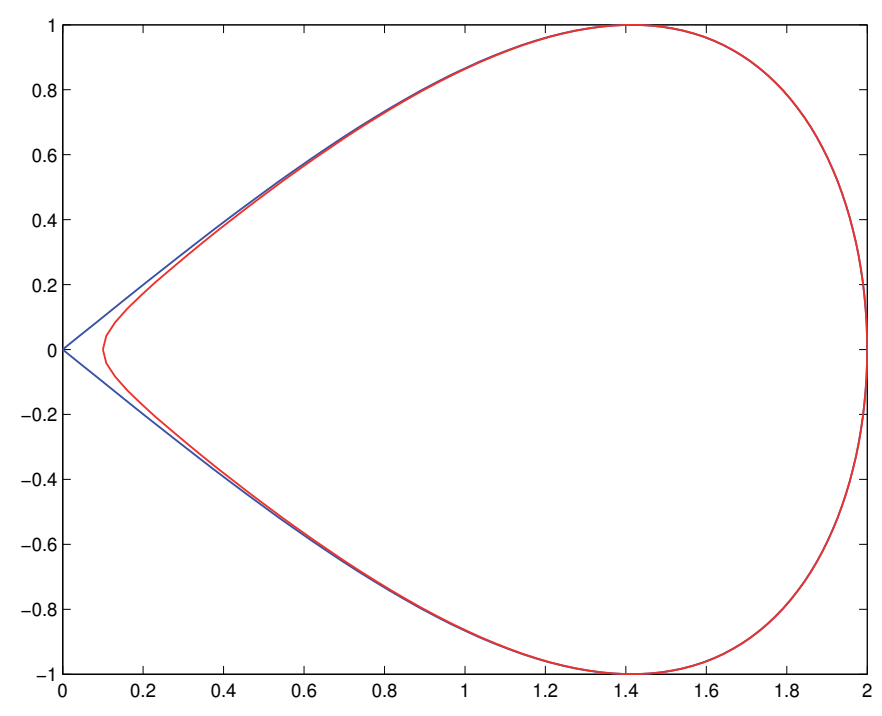

Figure 1. Leminscate (blue). The interior (red) curve with rounded corner has parameter $\varepsilon=0.05(\rho=0.1)$.

The near- and far-fields were computed for each of the boundary conditions using the graded mesh (61) for the lemniscate (81). A variety of angles of incidence were tested and in the case of the impedance loaded lemniscate a number of impedance parameters were tried. All tests were performed for $k a=1,5,10$ and $2 \pi$. Colton and Kress [4] have published results for the Dirichlet boundary condition. We were able to reproduce these results. In all cases an examination of the convergence rate as a function of $N$ was observed to be exponentially fast (super-algebraic). Some typical results are as follows. Table 1 shows the scattered near- and far-field patterns for the lemniscate illuminated by a plane wave incident at angle $\theta_{0}=0$ with $k a=2 \pi$. For the impedance boundary condition, the impedance parameter shown is $\lambda=1+i$.

We then examined the effect of rounding the corner of the lemniscate. The near- and far-fields were computed for each of the boundary conditions using a uniform mesh $t_{j}=\frac{\pi j}{n}$, for $j=$ $0,1, \ldots, 2 n-1$, in the parameterisation (36) of the rounded lemniscate (82) and the lemniscate (81).

A variety of angles of incidence were tested and in the case of the impedance loaded scatterers a number of impedance parameters were tried. All tests were performed for $k a=1,5,10$ and $2 \pi$, and radii of curvature $\rho=0.1,0.08, \ldots, 0.02,0.01$. The results were similar in all cases. As expected a decrease in the radius of curvature shows a decrease in the rate of convergence. For radius of convergence $\rho=0.1$ use of a uniform mesh achieves 10 significant digits of agreement and eventually for small radii $(\rho<0.04)$ the solution fails to converge (agreement of less than 6 significant digits). The results for the lemniscate, as expected, exhibit non-convergence.

The same series of experiments were then re-run using the graded mesh (61). In all cases this discretization method exhibits superior results. Super-algebraic convergence was 


\begin{tabular}{lllll}
\hline \multicolumn{5}{c}{ Lemniscate using graded mesh } \\
\hline$N$ & $\operatorname{Re} u^{S C}(\boldsymbol{x})$ & $\operatorname{Im} u^{S \mathcal{C}}(\boldsymbol{x})$ & $\operatorname{Re} u_{\infty}(\boldsymbol{d})$ & $\operatorname{Im} u_{\infty}(\boldsymbol{d})$ \\
\hline Dirichlet & & & & \\
32 & -0.07494830903628 & -0.07116098685594 & -1.87242780404153 & 1.24490326848555 \\
64 & -0.07494835562512 & -0.07116093299795 & -1.87243588474719 & 1.24489457829233 \\
128 & -0.07494835564211 & -0.07116093293816 & -1.87243588474320 & 1.24489457829267 \\
256 & -0.07494835564212 & -0.07116093293813 & -1.87243588474320 & 1.24489457829268 \\
Neumann & & & & \\
32 & 0.04164120404373 & 0.03521714231575 & 1.59458645194898 & 0.92713146438117 \\
64 & 0.04164071915392 & 0.03521722967811 & 1.59457738453702 & 0.92713314620758 \\
128 & 0.04164071916034 & 0.03521722965359 & 1.59457738456520 & 0.92713314620969 \\
256 & 0.04164071916034 & 0.03521722965358 & 1.59457738456522 & 0.92713314620969 \\
Impedance & $\lambda=1+i$ & & & \\
32 & 0.00222570467763 & -0.04334130654021 & 1.26634214415129 & 1.65780088985777 \\
64 & 0.00222588468293 & -0.04334146584422 & 1.26633733538116 & 1.65780860014239 \\
128 & 0.00222588466664 & -0.04334146583637 & 1.26633733538197 & 1.65780860013947 \\
256 & 0.00222588466664 & -0.04334146583637 & 1.26633733538197 & 1.65780860013947 \\
\hline \hline
\end{tabular}

Table 1. Direction of incident plane wave $\theta_{0}=0$ with $k a=2 \pi, \boldsymbol{d}=(1,0)$ and $u^{s c}(\boldsymbol{x})$ for $\boldsymbol{x}=(-1,1)$ with $k r=20 \pi$.

observed in all cases when examining the convergence rate as a function of $N$, demonstrating the advantage of using the graded mesh. In all cases 15 significant digit convergence was achieved. Of interest is the observation that even though the rounded lemniscate (82) has a smooth boundary $\partial D$, as the radius of curvature decreases use of the uniform mesh for discretization fails to produce a convergent solution for small radii of curvature. This suggests that the type of discretization method chosen should be decided on a more sophisticated approach rather than a simplistic smooth versus not smooth criterion.

A set of typical results is provided in Table 2 which shows the values for the near-field using uniform mesh and then using graded mesh for a scatterer with the impedance boundary condition with impedance parameter $\lambda=1+i$, illuminated by a plane wave incident at angle $\theta_{0}=0$ with $k a=2 \pi$. Table 3 shows the results of the far-field for the same experiments.

Having established that the graded mesh gives superior results for the rounded lemniscate, we attempt to answer the concern that rounding the corner will produce significant deviation from the solution where corners are not rounded. The difference between the actual solution, $u_{\infty}^{L}(\hat{\boldsymbol{x}})$ for $\hat{\boldsymbol{x}} \in[0,2 \pi]$, and that produced by rounding, $u_{\infty}^{R}(\hat{\boldsymbol{x}})$, is measured using the $L^{2}$ norm

$$
\left\|u_{\infty}^{L}-u_{\infty}^{R}\right\|_{2}=\left(\int_{0}^{2 \pi}\left|u_{\infty}^{L}(\hat{\boldsymbol{x}})-u_{\infty}^{R}(\hat{\boldsymbol{x}})\right|^{2} d \hat{\boldsymbol{x}}\right)^{\frac{1}{2}}
$$

and $L^{\infty}$ norm

$$
\left\|u_{\infty}^{L}-u_{\infty}^{R}\right\|_{\infty}=\max _{\hat{x} \in[0,2 \pi]}\left|u_{\infty}^{L}(\hat{\boldsymbol{x}})-u_{\infty}^{R}(\hat{\boldsymbol{x}})\right|
$$




\begin{tabular}{|c|c|c|c|c|}
\hline \multicolumn{5}{|c|}{ Rounded Lemniscate with impedance boundary condition: near field } \\
\hline & \multicolumn{2}{|l|}{ Uniform Mesh } & \multicolumn{2}{|l|}{ Graded Mesh } \\
\hline$N$ & $\operatorname{Re} u^{S C}(\boldsymbol{x})$ & $\operatorname{Im} u^{S C}(\boldsymbol{x})$ & $\operatorname{Re} u^{S C}(\boldsymbol{x})$ & $\operatorname{Im} u^{S C}(\boldsymbol{x})$ \\
\hline \multicolumn{5}{|c|}{$\rho=0.1$} \\
\hline 16 & 0.04109806757344 & -0.04113428021951 & 0.03720901114453 & -0.04700162395186 \\
\hline 32 & 0.03739551348295 & -0.04213065825702 & 0.03720928577876 & -0.04213508567513 \\
\hline 64 & 0.03720998623363 & -0.04213533443707 & 0.03720928577876 & -0.04213527920590 \\
\hline 128 & 0.03720928580093 & -0.04213527920719 & 0.03720928577876 & -0.04213527920590 \\
\hline 256 & 0.03720928577876 & -0.04213527920590 & 0.03720928577876 & -0.04213527920590 \\
\hline \multicolumn{5}{|c|}{$\rho=0.08$} \\
\hline 16 & 0.03440987434478 & -0.04437085759214 & 0.02784748818262 & -0.05030877964670 \\
\hline 32 & 0.02919063786326 & -0.04546211186317 & 0.02875233753249 & -0.04543747633458 \\
\hline 64 & 0.02875717614250 & -0.04543850947697 & 0.02875258961627 & -0.04543766502331 \\
\hline 128 & 0.02875259055193 & -0.04543766521258 & 0.02875258961627 & -0.04543766502331 \\
\hline 256 & 0.02875258961627 & -0.04543766502331 & 0.02875258961627 & -0.04543766502331 \\
\hline \multicolumn{5}{|c|}{$\rho=0.06$} \\
\hline 16 & 0.02888124025951 & -0.04585721669170 & 0.01971124100506 & -0.05179809647240 \\
\hline 32 & 0.02153837811679 & -0.047 & 137736 & -0.0469241 \\
\hline 64 & 0.02057080115803 & -0.04693227871137 & 0.02054161007537 & -0.04692430220810 \\
\hline 128 & 0.02054165076197 & -0.04692431 & 0.02054161007 & -0.046924 \\
\hline 256 & 0.02054161007559 & -0.04692430220817 & 0.02054161007537 & -0.04692430220810 \\
\hline \multicolumn{5}{|c|}{$\rho=0.04$} \\
\hline 16 & 0.02589808135523 & -0.04581451169315 & 7156670 & -0.05 \\
\hline 32 & 5268719371 & 395117 & 532463556 & 38096770 \\
\hline 64 & 0.013 & 090670 & 4495255 & -0.04680156017172 \\
\hline 128 & 0.01304736974804 & -0.04680236337734 & 0.01304554495255 & -0.04680156017172 \\
\hline 256 & 0.01304554533578 & -0.04680156034492 & 0.01304554495255 & -0.04680156017172 \\
\hline \multicolumn{5}{|c|}{$\rho=0.02$} \\
\hline 16 & 0.02925003757755 & -0.04467314700387 & 0.00598950684038 & -0.05028232677963 \\
\hline 32 & 0.01176640513049 & -0.04644167438759 & 0.00673781449261 & -0.04541082809958 \\
\hline 64 & 0.00766782061888 & -0.04575605837829 & 0.00673802661846 & -0.04541100344294 \\
\hline 128 & 0.00681329578610 & -0.04544795488922 & 0.00673802661846 & -0.04541100344294 \\
\hline 256 & 0.00673882182373 & -0.04541144923285 & 0.00673802661846 & -0.04541100344294 \\
\hline \multicolumn{5}{|c|}{$\rho=0.01$} \\
\hline 16 & 0.03794586127165 & -0.04413616075373 & 0.00345639312266 & -0.04927243930309 \\
\hline 32 & 0.01249317081358 & -0.04622574470432 & 0.00419970408560 & -0.04440251423153 \\
\hline 64 & 0.00629912969982 & -0.04516736933935 & 0.00419991683968 & -0.04440269063368 \\
\hline 128 & 0.00461128601429 & & 91683968 & -0.04440269063368 \\
\hline 256 & 0.00423419335659 & -0.04442201458794 & 0.00419991683968 & -0.04440269063368 \\
\hline \multicolumn{5}{|c|}{ Lemnis } \\
\hline 16 & -0.01163732525088 & -0.03 & 7105834 & -0.04820 \\
\hline 32 & -0.00397500132190 & -0.04021321442543 & 0.00222570467763 & -0.04334130654021 \\
\hline 64 & -0.00040933936032 & -0.04209018109850 & 0.00222588468293 & -0.04334146584422 \\
\hline 128 & 0.00114252253408 & -0.04283149822654 & 0.00222588466664 & -0.04334146583637 \\
\hline 256 & 0.00178850801274 & -0.04313421526217 & 0.002225884666664 & -0.04334146583637 \\
\hline
\end{tabular}

Table 2. Direction of incident plane wave $\theta_{0}=0$ with $k a=2 \pi$ and $u^{s c}(\boldsymbol{x})$ for $\boldsymbol{x}=(-1,1)$ with $k r=20 \pi$. Impedance paramater $\lambda=1+i$. 


\begin{tabular}{|c|c|c|c|c|}
\hline \multicolumn{5}{|c|}{ Rounded Lemniscate with Impedance boundary condition: far field } \\
\hline & \multicolumn{2}{|l|}{ Uniform Mesh } & \multicolumn{2}{|l|}{ Graded Mesh } \\
\hline$N$ & $\operatorname{Re} u_{\infty}(\boldsymbol{d})$ & $\operatorname{Im} u_{\infty}(\boldsymbol{d})$ & $\operatorname{Re} u_{\infty}(\boldsymbol{d})$ & $\operatorname{Im} u_{\infty}(\boldsymbol{d})$ \\
\hline \multicolumn{5}{|c|}{$\rho=0.1$} \\
\hline 16 & 1.26738976138034 & 1.66105526777388 & 1.25530278020801 & 1.64198030138557 \\
\hline 32 & 1.26778462660553 & 1.66045261136046 & 1.26781160112455 & 1.66041662811791 \\
\hline 64 & 1.26780743084847 & 1.66042387986373 & 1.26780750283287 & 1.66042376203697 \\
\hline 128 & 1.26780750283088 & 1.66042376204068 & 1.26780750283287 & 1.66042376203697 \\
\hline 256 & 1.26780750283287 & 1.66042376203697 & 1.26780750283287 & 1.66042376203697 \\
\hline \multicolumn{5}{|c|}{$\rho=0.08$} \\
\hline 16 & 1.26679319682521 & 1.66100369319241 & 1.25514611227298 & 1.64136408633317 \\
\hline 32 & 1.26723239948607 & 1.66019266418943 & 1.26728244518451 & 1.66011880476277 \\
\hline 64 & 1.26727771729194 & 1.66012685149464 & 1.26727812591656 & 1.66012608299574 \\
\hline 128 & 1.26727812584623 & 1.66012608315554 & 1.26727812591656 & 1.66012608299574 \\
\hline 256 & 1.26727812591656 & 1.66012608299574 & 1.26727812591656 & 1.66012608299574 \\
\hline \multicolumn{5}{|c|}{$\rho=0.06$} \\
\hline 16 & 1.26628254876688 & 1.660942666686662 & 1.25496277020654 & 1.64065624538918 \\
\hline 32 & 1.26675739510909 & 1.65984471852085 & 1.26684907150266 & 1.65968759452492 \\
\hline 64 & 1.26684233823722 & 1.65969990319333 & 1.26684456821701 & 1.65969501037167 \\
\hline 128 & 1.26684456565199 & 1.65969501752357 & 1.26684456821701 & 1.65969501037167 \\
\hline 256 & 1.26684456821700 & 1.65969501037171 & 1.26684456821701 & 1.65969501037167 \\
\hline \multicolumn{5}{|c|}{$\rho=0.04$} \\
\hline 16 & 1.26581970905186 & 1.66101371994892 & 1.25479198100542 & 1.63987507228103 \\
\hline 32 & 1.26636387623415 & 1.65947078536106 & 1.26652994925162 & 1.65913395755207 \\
\hline 64 & 1.26651395959820 & 1.65917079145125 & 1.26652530266827 & 1.65914149765931 \\
\hline 128 & 1.26652520551250 & 1.65914182632024 & 1.26652530266827 & 1.65914149765931 \\
\hline 256 & 1.26652530265039 & 1.65914149772878 & 1.26652530266827 & 1.65914149765931 \\
\hline \multicolumn{5}{|c|}{$\rho=0.02$} \\
\hline 16 & 1.26516216215893 & 1.66171371564432 & 1.25467501349357 & 1.63904489275113 \\
\hline 32 & 1.26602051309766 & 1.65923831994890 & 1.26634907227985 & 1.65848212898845 \\
\hline 64 & 1.26629109761250 & 1.65864493833610 & 1.26634432336604 & 1.65848977294699 \\
\hline 128 & 1.26634078340330 & 1.65850339846154 & 1.26634432336604 & 1.65848977294699 \\
\hline 256 & 1.26634429273403 & 1.65848992335866 & 1.26634432336604 & 1.65848977294699 \\
\hline \multicolumn{5}{|c|}{$\rho=0.01$} \\
\hline 16 & 1.26439990092700 & 1.662961180 & 1.25465229232071 & 1.63863275250060 \\
\hline 32 & 1.26579068367998 & 1.65938609457059 & 1.26632066601268 & 1.65813602895562 \\
\hline 64 & 1.26619618222096 & 1.65849076674951 & 1.26631588055722 & 1.65814371438451 \\
\hline 128 & 1.26629637930118 & 1.65821726963456 & 1.26631588055722 & 1.65814371438451 \\
\hline 256 & 1.26631455164602 & 1.65815016944201 & 1.26631588055722 & 1.65814371438451 \\
\hline \multicolumn{5}{|c|}{ Lemniscate } \\
\hline 16 & 1.26753738579244 & 926109144 & 1.25466535533964 & 1.63824863702138 \\
\hline 32 & 1.26675455678284 & 1.65668969319373 & 1.26634214415129 & 1.65780088985777 \\
\hline 64 & 1.26649349543064 & 1.65733861326598 & 1.26633733538116 & 1.65780860014239 \\
\hline 128 & 1.26639670753949 & 1.65761574700699 & 1.26633733538197 & 1.65780860013947 \\
\hline 256 & 1.26636023315794 & 1.65773061188777 & 1.26633733538197 & 1.65780860013947 \\
\hline
\end{tabular}

Table 3. Direction of incident plane wave $\theta_{0}=0$ with $k a=2 \pi$ and $\boldsymbol{d}=(1,0)$. Impedance parameter $\lambda=1+i$. 
These tests were run for all three boundary conditions for $k a=1,5,10$, and $2 \pi$, and radii of curvature $\rho=0.1,0.08, \ldots, 0.02,0.01$ and, in the case of the impedance loaded scatterers, a number of impedance parameters. The results were similar for all wave numbers and Table 4 presents the results for $k a=2 \pi$. The smaller the radius of curvature used for the rounding, the smaller the measured error. Both the absolute and relative errors were measured. The relative error is expressed as a percentage of the same norm of the lemniscate far-field. Using a radius of curvature of $\rho=0.02$, using the $L^{2}$ norm measures an error of $2.4 \%$ in the Dirichlet case, $0.9 \%$ in the Neumann case and $1.7 \%$ for the impedance boundary condition. Similarly, the $L^{\infty}$ norm measures an error of $1.4 \%$ in the Dirichlet case, $0.4 \%$ in the Neumann case and $0.8 \%$ for the impedance boundary condition. Using a radius of curvature of $\rho=0.01$, using the $L^{2}$ norm measures an error of $0.9 \%$ in the Dirichlet case, $0.03 \%$ in the Neumann case and $0.8 \%$ for the impedance boundary condition. Similarly, the $L^{\infty}$ norm measures an error of $0.6 \%$ in the Dirichlet case, $0.1 \%$ in the Neumann case and $0.4 \%$ for the impedance boundary condition.

\begin{tabular}{ccccc}
\hline \multicolumn{5}{c}{ Comparison of rounding effect to actual lemniscate } \\
\hline $\boldsymbol{\rho}$ & $L^{2}$ Norm & \% Difference & $L^{\infty}$ Norm & \% Difference \\
\hline Dirichlet & & & & \\
0.1 & 0.0550 & 20 & 0.2537 & 11 \\
0.08 & 0.0413 & 15 & 0.1929 & 8.6 \\
0.06 & 0.0284 & 10 & 0.1343 & 6.0 \\
0.05 & 0.0224 & 8.1 & 0.1064 & 4.7 \\
0.04 & 0.0167 & 6.0 & 0.0799 & 3.5 \\
0.03 & 0.0114 & 4.1 & 0.0550 & 2.4 \\
0.02 & 0.0067 & 2.4 & 0.0325 & 1.4 \\
0.01 & 0.0026 & 0.9 & 0.0130 & 0.6 \\
Neumann & & & & \\
0.1 & 0.0315 & 13 & 0.1278 & 6.9 \\
0.08 & 0.0220 & 8.8 & 0.0885 & 4.8 \\
0.06 & 0.0137 & 5.5 & 0.0547 & 3.0 \\
0.05 & 0.0101 & 4.1 & 0.0401 & 2.2 \\
0.04 & 0.0070 & 2.8 & 0.0274 & 1.5 \\
0.03 & 0.0042 & 1.7 & 0.0167 & 0.9 \\
0.02 & 0.0021 & 0.9 & 0.0082 & 0.4 \\
0.01 & 0.0006 & 0.3 & 0.0024 & 0.1 \\
Impedance & $\lambda=1+i$ & & & \\
0.1 & 0.0267 & 12 & 0.1121 & 5.4 \\
0.08 & 0.0203 & 9.3 & 0.0863 & 4.1 \\
0.06 & 0.0142 & 6.5 & 0.0612 & 2.9 \\
0.05 & 0.0113 & 5.2 & 0.0491 & 2.4 \\
0.04 & 0.0087 & 4.0 & 0.0376 & 1.8 \\
0.03 & 0.0061 & 2.8 & 0.0267 & 1.3 \\
0.02 & 0.0038 & 1.7 & 0.0166 & 0.8 \\
0.01 & 0.0017 & 0.8 & 0.0076 & 0.4 \\
\hline \hline
\end{tabular}

Table 4. Direction of incident plane wave $\theta_{0}=0$ with $k a=2 \pi$. 


\subsection{Effect of Corner Rounding on a Domain with Two Corners}

Consider the curve given by the parametric representation:

$$
\boldsymbol{x}=\boldsymbol{x}(t)=a\left(\frac{\cos t}{1+|\sin t|}, \frac{\sin t}{1+|\sin t|}\right), \quad t \in[0,2 \pi]
$$

where $a$ is a parameter. It has the corners at $t=0$ and $t=\pi$ respectively, with interior right angles. Henceforth the parameter $a$ is set equal to 1 length unit.

We will also consider a family of curves in which the corner has been rounded; the family is parameterized by the quantity $\varepsilon(0 \leq \varepsilon \leq 1)$ :

$$
\boldsymbol{x}=\boldsymbol{x}(t)=a\left(\frac{\cos t}{1+\sqrt{\varepsilon^{2}+\sin ^{2} t}}, \frac{\sin t}{1+\sqrt{\varepsilon^{2}+\sin ^{2} t}}\right), \quad t \in[0,2 \pi] .
$$

Figure 2 illustrates the two shapes, with $a=1$. The radius of curvature $\rho$ at the corner points $t=0$ and $\pi$ is

$$
\rho(\boldsymbol{x})=\left|\frac{\left(x_{1}^{\prime}(t)^{2}+x_{2}^{\prime}(t)^{2}\right)^{3 / 2}}{x_{1}^{\prime}(t) x_{2}^{\prime \prime}(t)-x_{2}^{\prime}(t) x_{1}^{\prime \prime}(t)}\right|, \quad t \in[0,2 \pi]
$$

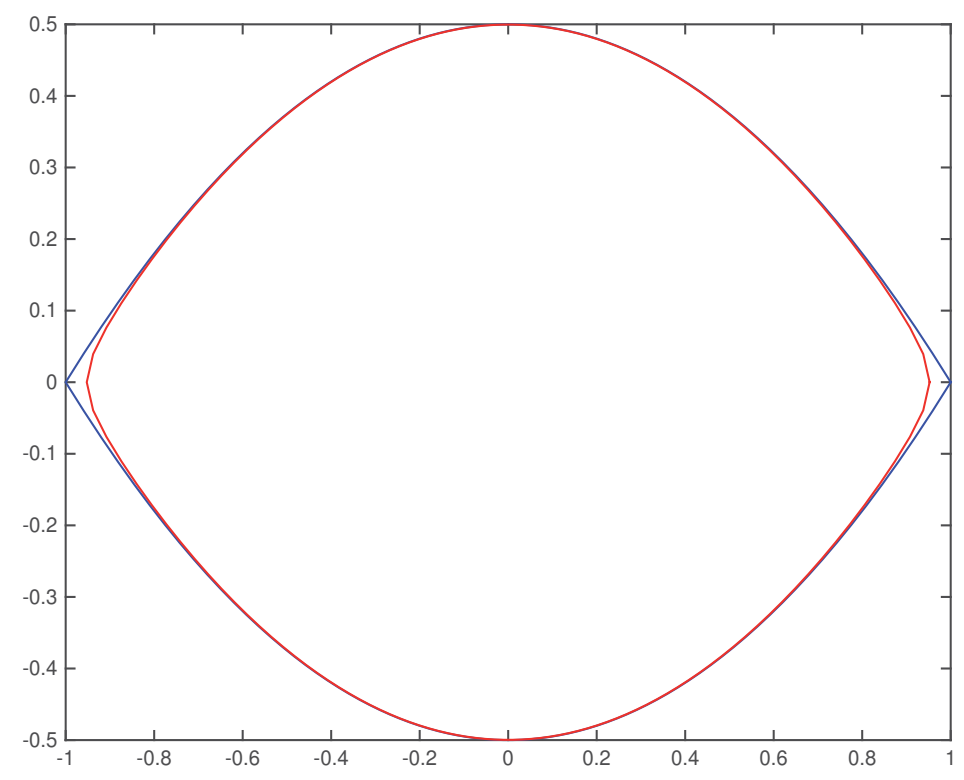

Figure 2. Two-corner scatterer (blue). The interior (red) curve with rounded corners has parameter $\varepsilon=0.05(\rho \approx 0.05)$. 
The near- and far-fields were computed for each of the boundary conditions using the graded mesh (67) for the two-corner scatterer (85). A variety of angles of incidence were tested and in the case of the impedance loaded two-corner scatterer a number of impedance parameters were tried. All tests were performed for $k a=1,5,10$ and $2 \pi$. In all cases an examination of the convergence rate as a function of $N$ was observed to be exponentially fast (super-algebraic). We note that unlike the case of the lemniscate (81) we obtained 12 significant digit convergence rather than 15 . This is attributed to the choice of function (67) used for the graded mesh for the two-corner scatterer: the derivatives at the points $s=0, \pi, 2 \pi$ vanish up to order 6 whereas the function (61) used for the lemniscate vanish up to order 8 . Some typical results are as follows. Table 5 shows the scattered near- and far-field patterns for the two-corner scatterer illuminated by a plane wave incident at angle $\theta_{0}=0$ with $k a=2 \pi$. For the impedance boundary condition, the impedance parameter shown is $\lambda=1+i$.

\begin{tabular}{lllll}
\hline \multicolumn{5}{c}{ Two-corner scatterer using graded mesh } \\
\hline$N$ & $\operatorname{Re} u^{s c}(\boldsymbol{x})$ & $\operatorname{Im} u^{s c}(\boldsymbol{x})$ & $\operatorname{Re} u_{\infty}(\boldsymbol{d})$ & $\operatorname{Im} u_{\infty}(\boldsymbol{d})$ \\
\hline Dirichlet & & & & \\
32 & 0.09714066817949 & -0.04206954201768 & -1.30520131280366 & 0.52677041231075 \\
64 & 0.09713891064627 & -0.04207166784754 & -1.30520131989700 & 0.52676949835567 \\
128 & 0.09713890337649 & -0.04207167577468 & -1.30520131965813 & 0.52676949545313 \\
256 & 0.09713890336079 & -0.04207167579114 & -1.30520131965776 & 0.52676949544743 \\
Neumann & & & & \\
32 & -0.04208998732029 & 0.03926860763238 & 0.61684378748988 & 0.11166701105301 \\
64 & -0.04208918412475 & 0.03926998017758 & 0.61684312041463 & 0.11166735322848 \\
128 & -0.04208918124931 & 0.03926998510688 & 0.61684311922066 & 0.11166735401338 \\
256 & -0.04208918124342 & 0.03926998511698 & 0.61684311921824 & 0.11166735401498 \\
Impedance & & & & \\
32 & 0.04240217311338 & 0.01943563484368 & 0.53623358174525 & 1.08920867424572 \\
64 & 0.04240224749500 & 0.01943484871137 & 0.53623292759500 & 1.08920881290892 \\
128 & 0.04240224762591 & 0.01943484562635 & 0.53623292505883 & 1.08920881334528 \\
256 & 0.04240224762614 & 0.01943484561999 & 0.53623292505360 & 1.08920881334620 \\
\hline \hline
\end{tabular}

Table 5. Direction of incident plane wave $\theta_{0}=0$ with $k a=2 \pi, \boldsymbol{d}=(1,0)$ and $u^{s c}(\boldsymbol{x})$ for $\boldsymbol{x}=(-1,1)$ at $k r=20 \pi$.

As in the case of the lemniscate (81), we then examined the effect of rounding the two corners of the scatterer. The near- and far-fields were computed for each of the boundary conditions using a uniform mesh $t_{j}=\frac{\pi j}{n}$, for $j=0,1, \ldots, 2 n-1$, in the parameterisation (36) of the rounded scatterer (86) and the two-corner scatterer (85).

A variety of angles of incidence were tested and in the case of the impedance loaded scatterers a number of impedance parameters were tried. All tests were performed for $k a=1,5,10$ and $2 \pi$, and radii of curvature $\rho=0.1,0.05,0.04, \ldots, 0.01$. The results were similar in all cases. As expected a decrease in the radius of curvature shows a decrease in the rate of convergence and eventually for small radii $(\rho \leq 0.05)$ the solution fails to converge. The results for the two-corner scatterer, as expected, exhibit non-convergence.

The same series of experiments were then re-run using the graded mesh (67). In all cases this discretization method exhibits superior results. Super-algebraic convergence was observed in all cases when examining the convergence rate as a function of $N$, demonstrating the 


\begin{tabular}{|c|c|c|c|c|}
\hline \multicolumn{5}{|c|}{ Rounded two-corner scatterer with impedance boundary condition: near field } \\
\hline & Uniform Mesh & & Graded Mesh & \\
\hline$N$ & $\operatorname{Re} u^{S C}(\boldsymbol{x})$ & $\operatorname{Im} u^{S C}(\boldsymbol{x})$ & $\operatorname{Re} u^{s c}(\boldsymbol{x})$ & $\operatorname{Im} u^{s C}(\boldsymbol{x})$ \\
\hline \multicolumn{5}{|c|}{$\rho=0.05$} \\
\hline 16 & 0.03470677457659 & 0.03798379196971 & 0.03843618042815 & 0.03338842573544 \\
\hline 32 & 0.03897149015734 & 0.03486730759441 & 0.03940928962261 & 0.03387147655152 \\
\hline 64 & 0.03939900905631 & 0.03392160276388 & 0.03940929911450 & 0.03387163276154 \\
\hline 128 & 0.03940928308095 & 0.03387177435745 & 0.03940929911442 & 0.03387163276137 \\
\hline 256 & 0.03940929911413 & 0.03387163276369 & 0.03940929911442 & 0.03387163276137 \\
\hline \multicolumn{5}{|c|}{$\rho=0.04$} \\
\hline 16 & 0.03481359170705 & 0.03603686016428 & 0.03973252285591 & 0.03005487424932 \\
\hline 32 & 0.04004351467396 & 0.03216490387031 & 0.04069913302070 & 0.03060510102866 \\
\hline 64 & 0.04067397485734 & 0.03074186288893 & 0.04069909427737 & 0.03060516627501 \\
\hline 128 & 0.04069899786205 & 0.03060639554837 & 0.04069909427756 & 0.03060516627559 \\
\hline 256 & 0.04069909426695 & 0.03060516643891 & 0.04069909427756 & 0.03060516627559 \\
\hline \multicolumn{5}{|c|}{$\rho=0.03$} \\
\hline 16 & 0.03403888671802 & 0.03482751799820 & 0.04067458127216 & 0.02679979723427 \\
\hline 32 & 0.04063667872250 & 0.02981632754250 & 0.04162851228396 & 0.02737654283524 \\
\hline 64 & 0.04156807903825 & 0.02773425361138 & 0.04162851666947 & 0.02737622783093 \\
\hline 128 & 0.04162795338930 & 0.02738656204370 & 0.04162851666923 & 0.02737622782875 \\
\hline 256 & 0.04162851649254 & 0.02737623967346 & 0.04162851666923 & 0.02737622782875 \\
\hline \multicolumn{5}{|c|}{$\rho=0.02$} \\
\hline 16 & 0.03217155484158 & 0.03507379516897 & 0.04121333829419 & 0.02405994803154 \\
\hline 32 & 0.04067690299734 & 0.02828422652111 & 0.04216254273554 & 0.02454661770912 \\
\hline 64 & 0.04202756957945 & 0.02536776882377 & 0.04216259142752 & 0.02454704861633 \\
\hline 128 & 0.04215961390872 & 0.02461376091346 & 0.04216259142616 & 0.02454704861049 \\
\hline 256 & 0.04216259955285 & 0.02454764108611 & 0.04216259142616 & 0.02454704861049 \\
\hline \multicolumn{5}{|c|}{$\rho=0.01$} \\
\hline 16 & 0.02764745305810 & 0.03908271244059 & 0.04145153747908 & 0.02129232793218 \\
\hline 32 & 0.03994042453183 & 0.02818804933763 & 0.04242372188117 & 0.02166516985259 \\
\hline 64 & 0.04207481477062 & 0.02365888639225 & 0.04242374291713 & 0.02166462301523 \\
\hline 128 & 0.04239986579142 & 0.02210111122351 & 0.04242374292179 & 0.02166462302828 \\
\hline 256 & 0.04242431719588 & 0.02170326321857 & 0.04242374292179 & 0.02166462302828 \\
\hline \multicolumn{5}{|c|}{2 Corners } \\
\hline 16 & 0.04613478369077 & 0.02381635331120 & 0.04141767031255 & 0.01902504141843 \\
\hline 32 & 0.04354116342657 & 0.02025415654551 & 0.04240217311338 & 0.01943563484368 \\
\hline 64 & 0.04274414603582 & 0.01951718789786 & 0.04240224749500 & 0.01943484871137 \\
\hline 128 & 0.04250241810608 & 0.01939520893768 & 0.04240224762591 & 0.01943484562635 \\
\hline 256 & 0.04243092698390 & 0.01939826345460 & 0.04240224762614 & 0.01943484561999 \\
\hline
\end{tabular}

Table 6. Direction of incident plane wave $\theta_{0}=0$ with $k a=2 \pi$ and $u^{s c}(\boldsymbol{x})$ for $\boldsymbol{x}=(-1,1)$ with $k r=20 \pi$. Impedance parameter $\lambda=1+i$.

advantage of using the graded mesh. In all cases 15 significant digit convergence was achieved for the rounded scatterer. As in the case of the rounded lemniscate, we observe that even though the rounded two-corner scatterer (86) has a smooth boundary $\partial D$, as the radius of curvature decreases use of the uniform mesh for discretization fails to produce a convergent solution for small radii of curvature. It demonstrates the need to consider an appropriate distribution of quadrature points. 


\begin{tabular}{|c|c|c|c|c|}
\hline \multicolumn{5}{|c|}{ Rounded two-corner scatterer with impedance boundary condition: far field } \\
\hline & Uniform Mesh & & Graded Mesh & \\
\hline$N$ & $\operatorname{Re} u_{\infty}(\boldsymbol{d})$ & $\operatorname{Im} u_{\infty}(\boldsymbol{d})$ & $\operatorname{Re} u_{\infty}(\boldsymbol{d})$ & $\operatorname{Im} u_{\infty}(\boldsymbol{d})$ \\
\hline \multicolumn{5}{|c|}{$\rho=0.05$} \\
\hline 16 & 0.54786382850918 & 1.09142671531078 & 0.53499654458393 & 1.08180757916455 \\
\hline 32 & 0.54407696878866 & 1.08791439414992 & 0.54339168688582 & 1.08744061118358 \\
\hline 64 & 0.54342318785131 & 1.08745614894034 & 0.54339178610716 & 1.08744068563751 \\
\hline 128 & 0.54339187292392 & 1.08744071591287 & 0.54339178610706 & 1.08744068563756 \\
\hline 256 & 0.54339178610846 & 1.08744068563798 & 0.54339178610706 & 1.08744068563756 \\
\hline \multicolumn{5}{|c|}{$\rho=0.04$} \\
\hline 16 & 0.54802405041036 & 1.09282346754333 & 0.53343196943322 & 1.08255766004404 \\
\hline 32 & 0.54302476711729 & 1.08885799431672 & 0.54191178786334 & 1.08818730305528 \\
\hline 64 & 0.54199881644045 & 1.08822602834406 & 0.54191183753492 & 1.08818739837731 \\
\hline 128 & 0.54191259166592 & 1.08818763880005 & 0.54191183753526 & 1.08818739837727 \\
\hline 256 & 0.54191183763337 & 1.08818739840296 & 0.54191183753526 & 1.08818739837727 \\
\hline \multicolumn{5}{|c|}{$\rho=0.03$} \\
\hline 16 & 0.54904031395079 & 1.09419728294031 & 0.53185218383499 & 1.08314160094841 \\
\hline 32 & 0.54222295015728 & 1.08967328649225 & 0.54039372823952 & 1.08875566456820 \\
\hline 64 & 0.54062723594874 & 1.08884581254326 & 0.54039354075047 & 1.08875566295939 \\
\hline 128 & 0.54039993768851 & 1.08875750430892 & 0.54039354074910 & 1.08875566295913 \\
\hline 256 & 0.54039354788384 & 1.08875566458283 & 0.54039354074910 & 1.08875566295913 \\
\hline \multicolumn{5}{|c|}{$\rho=0.02$} \\
\hline 16 & 0.55147905440958 & 1.09551480873753 & 0.53047388953404 & 1.08352661580456 \\
\hline 32 & 0.54197193671198 & 1.09026975943922 & 0.53900018451662 & 1.08910173339606 \\
\hline 64 & 0.53955928159085 & 1.08927803170276 & 0.53900044710649 & 1.08910181964229 \\
\hline 128 & 0.53904260169118 & 1.08911245582527 & 0.53900044710318 & 1.08910181964229 \\
\hline 256 & 0.53900080793236 & 1.08910189288763 & 0.53900044710318 & 1.08910181964229 \\
\hline \multicolumn{5}{|c|}{$\rho=0.01$} \\
\hline 16 & 0.55834834543519 & 1.09730788959491 & 0.52900328898032 & 1.08374759482932 \\
\hline 32 & 0.54310925871843 & 1.09061324880737 & 0.53749456980150 & 1.08927299148915 \\
\hline 64 & 0.53897642362642 & 1.08955599900788 & 0.53749420294315 & 1.08927299186681 \\
\hline 128 & 0.53778718513610 & 1.08932494624636 & 0.53749420294888 & 1.08927299186695 \\
\hline 256 & 0.53751853798100 & 1.08927708680932 & 0.53749420294888 & 1.08927299186695 \\
\hline \multicolumn{5}{|c|}{2 Corners } \\
\hline 16 & 0.53760520970805 & 1.08630428265680 & 0.52768053495681 & 1.08369527189265 \\
\hline 32 & 0.53651537005059 & 1.08836129033128 & 0.53623358174525 & 1.08920867424572 \\
\hline 64 & 0.53621106278279 & 1.08896400236454 & 0.53623292759500 & 1.08920881290892 \\
\hline 128 & 0.53617782659103 & 1.08914505371977 & 0.53623292505883 & 1.08920881334528 \\
\hline 256 & 0.53619704154265 & 1.08919444740499 & 0.53623292505360 & 1.08920881334620 \\
\hline
\end{tabular}

Table 7. Direction of incident plane wave $\theta_{0}=0$ with $k a=2 \pi$ and $\boldsymbol{d}=(1,0)$. Impedance parameter $\lambda=1+i$.

A set of typical results is provided in Table 6 which shows the values for the near-field using uniform mesh and then using graded mesh for a scatterer with the impedance boundary condition with impedance parameter $\lambda=1+i$, illuminated by a plane wave incident at angle $\theta_{0}=0$ with $k a=2 \pi$. Table 3 shows the results of the far-field for the same experiments.

Having established that use of the graded mesh gives excellent results for the two-corner scatterer, we may now examine the effect of rounding the corners and determine the relationship between the radius of curvature of the rounding and the deviation from the 
solution produced by the two-corner scatterer. The difference between the actual solution and that produced by rounding is measured using the $L^{2}$ norm (83) and $L^{\infty}$ norm (84).

The tests were run for all three boundary conditions for $k a=1,5,10$, and $2 \pi$, and radii of curvature $\rho=0.05,0.04, \ldots, 0.01$ and, in the case of the impedance loaded scatterers, a number of impedance parameters. The results were similar for all wave numbers and Table 8 presents the results for $k a=2 \pi$. The smaller the radius of curvature used for the rounding, the smaller the measured error. Both the absolute and relative errors were measured. The relative error is expressed as percentage of the same norm of the far-field of the two-corner scatterer.

Using a radius of curvature of $\rho=0.02$, using the $L^{2}$ norm produces an error of $3.8 \%$ in the Dirichlet case, $1.6 \%$ in the Neumann case and $2.4 \%$ for the impedance boundary condition. Similarly, the $L^{\infty}$ norm measures an error of $2.4 \%$ in the Dirichlet case, $1.5 \%$ in the Neumann case and $1.4 \%$ for the impedance boundary condition. Using a radius of curvature of $\rho=0.01$, using the $L^{2}$ norm produces an error of $1.2 \%$ in the Dirichlet case, $0.4 \%$ in the Neumann case and $1 \%$ for the impedance boundary condition. Similarly, the $L^{\infty}$ norm measures an error of $0.9 \%$ in the Dirichlet case, $0.4 \%$ in the Neumann case and $0.6 \%$ for the impedance boundary condition.

\begin{tabular}{|c|c|c|c|c|}
\hline \multicolumn{5}{|c|}{ Comparison of rounding effect to actual two-corner scatterer } \\
\hline$\rho$ & $L^{2}$ Norm & $\%$ Difference & $L^{\infty}$ Norm & \% Difference \\
\hline \multicolumn{5}{|l|}{ Dirichlet } \\
\hline 0.05 & 0.0219 & 10 & 0.1052 & 7.5 \\
\hline 0.04 & 0.0164 & 7.7 & 0.0792 & 5.6 \\
\hline 0.03 & 0.0112 & 5.3 & 0.0543 & 3.9 \\
\hline 0.02 & 0.0068 & 3.8 & 0.0332 & 2.4 \\
\hline 0.01 & 0.0026 & 1.2 & 0.0128 & 0.9 \\
\hline \multicolumn{5}{|l|}{ Neumann } \\
\hline 0.05 & 0.0094 & 7 & 0.0433 & 6.6 \\
\hline 0.04 & 0.0065 & 4.9 & 0.0300 & 4.6 \\
\hline 0.03 & 0.0040 & 3.0 & 0.0184 & 2.8 \\
\hline 0.02 & 0.0021 & 1.6 & 0.0097 & 1.5 \\
\hline 0.01 & 0.0006 & 0.4 & 0.0027 & 0.4 \\
\hline Impedance & $\lambda=1+i$ & & & \\
\hline 0.05 & 0.0113 & 6.9 & 0.0487 & 4.0 \\
\hline 0.04 & 0.0086 & 5.3 & 0.0374 & 3.1 \\
\hline 0.03 & 0.0061 & 3.7 & 0.0264 & 2.1 \\
\hline 0.02 & 0.0039 & 2.4 & 0.0167 & 1.4 \\
\hline 0.01 & 0.0017 & 1.0 & 0.0074 & 0.6 \\
\hline
\end{tabular}

Table 8. Direction of incident plane wave $\theta_{0}=0$ with $k a=2 \pi$.

\section{Conclusion}

In this paper we have described numerical schemes and their implementation for the solution of scattering of a plane wave by two different cylindrical structures: a single-cornered structure and a second structure with two corners, each with three different boundary conditions imposed on their surfaces - soft, hard and an impedance boundary condition. We 
have numerically demonstrated that the field scattered by the rounded structure converges, in both the $L^{2}$ and $L^{\infty}$ norm, to that scattered by the corresponding sharp cornered object as the radius of curvature in the vicinity of the corner tends to zero.

It is important to use an appropriate quadrature scheme - a graded mesh - in order to obtain numerical results efficiently, for both the scatterer with sharp corners and for the scatterer with rounded corners possessing small radii of curvature. We anticipate that improvements to the graded mesh employed for the two-cornered object will match the rate of convergence demonstrated for the single-cornered lemniscate.

Our results show that for the soft boundary condition, the $L^{\infty}$ norm difference between the near or far scattered field of the single-cornered scatterer and that of the rounded scatterer is less than $4 \%$ when the radius of curvature is restricted so that $k \rho \leq 3 \pi / 50$. This percentage reduces to $3 \%$ or $2 \%$ respectively, when the boundary condition is replaced by the Neumann boundary condition or the impedance boundary condition (with $\lambda=1+i$ ), respectively. More precise measures of the difference are given in Table 4 . Similar results were obtained for the the two-cornered object, and are displayed in Table 8.

Our approach provides a relatively simple yet efficient and accurate method for computing near and far-fields scattered by sharp cornered objects of diameter $D$ up to a few wavelengths in extent. Accuracy was of paramount importance in this study in assessing the effects of rounding a corner. Our calculations rigorously examined the regime $1 \leq k a \leq 10$ corresponding to $0.318 \leq D / \lambda_{0} \leq 3.18$, where $\lambda_{0}$ is wavelength.

A more sophisticated approach to the scattering from soft cylindrical structures with sharp corners is given in [7]. It employs the so-called recursively compressed inverse preconditioning method, and as the authors note in their survey of the two dimensional scattering literature, it alone addresses the problem of accurate near-field evaluation in scatterers with corners.

In conclusion, this paper provides some precise quantification and assessment of the impact that the rounding of the corner of a sharp cornered object has on the scattering of acoustic waves. The method would seem to be extendible considerably beyond the wavelength range examined, constrained mainly by computer resources.

\section{Author details}

Paul D. Smith* and Audrey J. Markowskei

*Address all correspodence to: paul.smith@mq.edu.au

Department of Mathematics, Macquarie University, Sydney, Australia

\section{References}

[1] Rawlins AD. High Frequency diffraction of a electromagnetic plane wave by an imperfectly conducting rectangular cylinder. J Eng Math 2012; 76(1): 157-180. 
[2] Smith PD, Rawlins AD. Diffraction from structures with an impedance boundary. In: Electromagnetics in Advanced Applications (ICEAA), 2013 International Conference on; 9-13 Sept. 2013, Torino, Italy. 2013. pp. 1297-1300.

[3] Colton DL, Kress R. Integral equation methods in scattering theory. New York: Wiley; 1983. $271 \mathrm{p}$.

[4] Colton DL, Kress R. Inverse acoustic and electromagnetic scattering theory. 3rd ed. New York: Springer; 2013. 405 p.

[5] Smith PD, Markowskei A, Rawlins AD. Two-dimensional diffraction by impedance loaded structures with corners. In: Electromagnetics in Advanced Applications (ICEAA), 2014 International Conference on; 3-8 Aug. 2014, Aruba. 2014. pp. 644-647. 412 p.

[6] Kress R. Linear Integral Equations. 3rd ed. New York: Springer; 2014.

[7] Helsing J, Karlsson, A. An accurate boundary value problem solver applied to scattering from cylinders with corners. IEEE Transactions on Antennas and Propagation 2013; 61(7): 3693-3700. 
Chapter 2

\title{
Probing the Molecular Ordering in Azopolymer Thin Films by Second-Order Nonlinear Optics
}

\author{
Heurison S. Silva, Irismar G. Paz and Paulo B. Miranda \\ Additional information is available at the end of the chapter
}

http://dx.doi.org/10.5772/61180

\begin{abstract}
Second-harmonic generation (SHG), a second-order nonlinear optical technique, was used to investigate the molecular ordering of self-assembled layer-by-layer films of $\mathrm{PAH}$, a cationic polyelectrolyte, and PS-119, an anionic polyelectrolyte containing photoisomerizable azo groups. Possible phase transitions in these multilayer films and their thermal stability were investigated by probing the SHG signal as a function of temperature and comparing the molecular order before and after thermal treatment. These studies were also performed with different $\mathrm{pH}$ values for the assembling solutions, a relevant parameter for polyelectrolyte adsorption. The results have shown that the films are not thermally stable, with the SHG signal nearly vanishing at a temperature of $150^{\circ} \mathrm{C}$, in contrast to what is reported in the literature. SHG measurements have also confirmed that the films are isotropic in the plane of the samples, independent of their number of layers or the $\mathrm{pH}$ of assembling solutions. SHG signal before and after heating indicates that the SHG signal was considerably reduced at high temperatures, but after slow cooling it was recovered to almost the same value as before heating, showing that the thermal disorder is reversible. No phase transition was observed, since the SHG signal reduction was slow and gradual, without any sudden change that would characterize a glass transition. We demonstrate that the SHG technique provides information on the film arrangement at the microscopic level which could be difficult to get with traditional techniques.
\end{abstract}

Keywords: Nonlinear optics, second-harmonic generation, second-order susceptibility, polyelectrolytes, layer-by-layer self-assembled films

\section{Introduction}

The molecular organization and thermal stability of self-assembled thin films containing azopolymers are important to their applications, such as organic diodes [1, 2], optical storage 
[3], and biosensors [4, 5], to name a few. Here we are interested in probing the molecular organization of azopolymer thin films fabricated by the layer-by-layer technique resulting from their intermolecular and interlayer interactions. The thermal stability of these films will also be studied by measuring the nonlinear optical signal from second-harmonic generation (SHG) while the sample is heated.

Polymeric thin films may be very different from thick films. Moreover, the surface properties of thick films can be very different from those of their bulk [6]. For a full characterization of polymeric thin films, it is necessary to probe their thermal behavior, including the glass transition temperature, $T_{g}$, that is, the temperature where molecules acquire a more mobile state, which leads to lower film viscosity. Particularly, the $T_{g}$ of polymeric materials gives us information about the intermolecular interactions. Furthermore, glass transition temperatures have practical importance for optical storage devices because near $\mathrm{T}_{\mathrm{g}}$ the information recorded in the molecular arrangement is lost due to increased motion, which leads to molecular disorder.

In this sense, several techniques have been used to characterize these polymeric materials, and the most common are differential scanning calorimetric (DSC), thermal gravimetric analysis (TGA), and dynamic mechanical analysis (DMA). However, these thermal techniques characterize bulk samples but are ineffective for studying films with thickness on the order of a few nanometers. Some authors have applied these techniques to probe free-standing films of polystyrene (PS) [7], but for films adsorbed on solid substrates, such as layer-by-layer (LbL) and Langmuir-Blodgett (LB) films, or on liquid interfaces, such as Langmuir films, those techniques cannot be applied.

Recently, Lutkenhaus et al. have reported an alternative methodology for using traditional techniques (DSC and TGA) to study LbL films fabricated by secondary interactions [8], like $\mathrm{H}$ bonding for PEO/PAA films, and PAH/PAA films fabricated by electrostatic interaction [9]. The method is based on using films with many (around 100 or 200) layers, and average thickness per bilayer about $80 \mathrm{~nm}$. These films were removed from the inert substrate (Teflon) to be investigated by conventional techniques. Curiously, for PEO/PAA films (assembled by secondary interactions) it was possible to find $\mathrm{T}_{\mathrm{g}}$, but not for PAH/PAA (strongly bound by electrostatic interaction). However, those films are not exactly what we could call ultrathin LbL films, because both films are very thick $(2-8 \mu \mathrm{m})$, and the methodology removes the influence of the substrate. This influence is retained only in the conformation of the initial layers, but their contribution is negligible in the thermal analysis. It is therefore quite challenging to investigate the $\mathrm{T}_{\mathrm{g}}$ of ultrathin polymeric films, and in particular of LbL films with only a few bilayers.

Optical techniques such as ellipsometry and Raman spectroscopy [10] have been used to probe the $\mathrm{T}_{\mathrm{g}}$ of thin films, but they do not allow to investigate how the molecular arrangement is changed during the glass transition, although their results show excellent agreement with the values of $\mathrm{T}_{\mathrm{g}}$ obtained by other techniques. Because $\mathrm{T}_{\mathrm{g}}$ is associated with a transition to a state of molecular disorder, it is natural to apply second-order nonlinear optical techniques, such as second-harmonic generation (SHG), which are quite sensitive to molecular orientation [1113]. With increasing temperature, the molecules become increasingly mobile (more random 
and dynamic conformation), resulting in increased disorder and reduced SHG signal, which is dependent on orientational ordering. Therefore, for a system consisting of only one material, we would expect a sudden variation of the signal when the $T_{g}$ of the material is reached [14].

Another issue that is interesting is the thermal stability of the films. Here, we do not consider thermal degradation of the film, but the film's ability to maintain its molecular arrangement while temperature varies. Lvov et al. showed that the SHG signal from of PDDA/PAZO LbL films was substantially reduced when heated to about $120^{\circ} \mathrm{C}$ [15], showing that the ordering of the films was destroyed and they were not thermally stable. It is worth mentioning that, unlike the work of Han et al. [14], ref. [15] does not directly aim at measuring the $\mathrm{T}_{\mathrm{g}}$ of LbL films, but intends only to check their thermal stability. However, some authors have described thermally stable layer-by-layer films, showing stability around 20\% [16].

In this chapter, we will describe the basic theory for SHG in thin films, showing how its polarization dependence can be used to determine the orientation of chromophores in azopolymer LbL films, and how phase measurements of the SHG signal can probe the molecular reorientation after azopolymer adsorption. SHG measurements as a function of number of layers, of sample azimuthal angle, and of $\mathrm{pH}$ of the self-assembly solution reveal how the molecular organization depends on sample fabrication conditions. We also probe the thermal stability and effect of heat treatment on molecular ordering.

\section{Basic theory}

\subsection{Fundamentals of nonlinear optics}

Radiation-matter interaction processes lead to numerous effects due to light-induced polarization, which acts as a source of new electromagnetic waves. The polarization induced by the electric field of the electromagnetic wave, $\vec{E}(\vec{r}, t)$, is responsible for several optical processes. If this electric field is relatively weak in comparison to the electrostatic field acting on electrons within an atom or molecule, the polarization has a linear dependence on the electric field:

$$
\vec{P}=\chi \vec{E}
$$

where $\chi$ is the linear susceptibility and $\vec{P}$ is the linear polarization. This is the linear optics regime.

However, if the electric field of the electromagnetic wave is strong, new contributions turn out to be significant. Then, an approximate relation between electric field (cause) and polarization (effect) is a power series in $\vec{E}(\vec{r}, t)$ :

$$
\vec{P}=\vec{\chi}^{(1)} \cdot \vec{E}+\vec{\chi}^{(2)}: \vec{E} \vec{E}+\vec{\chi}^{(3)}: \vec{E} \vec{E} \vec{E}+\ldots \equiv \vec{P}^{(1)}+\vec{P}^{(2)}+\vec{P}^{(3)}+\ldots
$$


The terms $\chi^{(\mathrm{n})}$ and $\mathrm{P}^{(\mathrm{n})}$ are the susceptibilities and polarizations of $\mathrm{n}^{\text {th }}$-order, respectively. Figure 1 shows the linear polarization of Equation (1) and a typical example of the nonlinear response, Equation (2).
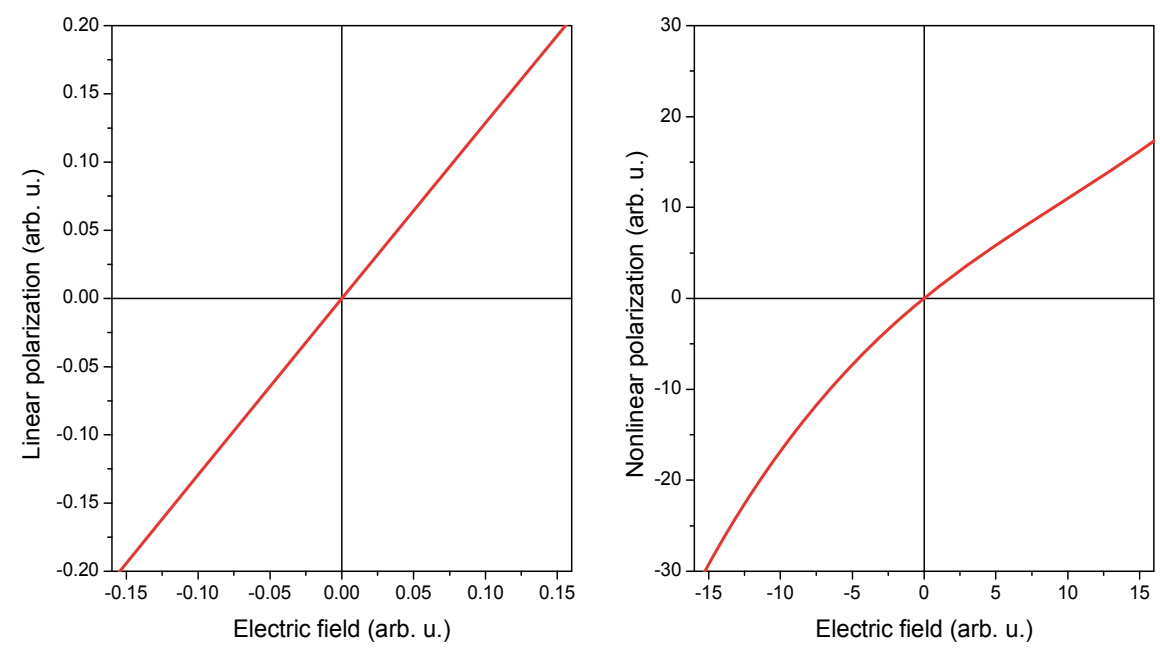

Figure 1. Typical linear (left) and nonlinear (right) polarization as a function of applied electric field.

Here, we are interested in second-order effects, which are related to the second-order nonlinear susceptibility, $\chi^{(2)}$. For the moment we will neglect the vector character of the electric field and polarization, and consider initially the local and instantaneous relation (neglecting spatial and frequency dispersion) between the field and the oscillating induced polarization. With the applied electric field $E(t)=E_{0} \cos \omega t$, the polarization up to third order is

$$
\begin{aligned}
& P=\chi^{(1)} E_{0} \cos \omega t+\chi^{(2)}\left(E_{0} \cos \omega t\right)^{2}+\chi^{(3)}\left(E_{0} \cos \omega t\right)^{3}+\ldots \\
& =\chi^{(1)} E_{0} \cos \omega t+\chi^{(2)} E_{0}{ }^{2}(\cos \omega t)^{2}+\chi^{(3)} E_{0}^{3}(\cos \omega t)^{3}+\ldots
\end{aligned}
$$

Using the trigonometric relations $(\cos \omega t)^{2}=\frac{1}{2}(1+\cos 2 \omega t)$ and $(\cos \omega t)^{3}=\frac{1}{4}(3 \cos \omega t+\cos 3 \omega t)$ we get

$$
\begin{gathered}
P=\chi^{(1)} E_{0} \cos \omega t+\chi^{(2)} \frac{1}{2} E_{0}{ }^{2}(1+\cos 2 \omega t)+\chi^{(3)} \frac{1}{4} E_{0}{ }^{3}(3 \cos \omega t+\cos 3 \omega t)+\ldots \\
\Rightarrow P=\chi^{(1)} E_{0} \cos \omega t+\chi^{(2)}\left[\frac{1}{2} E_{0}{ }^{2}+\frac{1}{2} E_{0}{ }^{2} \cos 2 \omega t\right]+ \\
\chi^{(3)}\left[\frac{3}{4} E_{0}{ }^{3} \cos \omega t+\frac{1}{4} E_{0}{ }^{3} \cos 3 \omega t\right]+\ldots
\end{gathered}
$$


We can see that polarization generated by electric field $E(t)=E_{0} \cos \omega t$ produces light with the same, double and triple of incident light frequency (named second- and third-harmonic, respectively), and a static component (optical rectification).

In general, if we have an incident electric field such as $E(t)=E_{1} \cos \omega_{1} t+E_{2} \cos \omega_{2} t$, representing two waves of frequencies $\omega_{1}$ and $\omega_{2}$ travelling through the material, it is easy to show that

$$
\begin{gathered}
P^{(2)}=\chi^{(2)}\left(E_{1} \cos \omega_{1} t+E_{2} \cos \omega_{2} t\right)^{2}= \\
=\chi^{(2)}\left[E_{1}^{2}+E_{2}^{2}+E_{1}^{2} \cos 2 \omega_{1} t+E_{2}^{2} \cos 2 \omega_{2} t+\frac{1}{2} E_{1} E_{2} \cos \left(\omega_{1}-\omega_{2}\right) t+\frac{1}{2} E_{1} E_{2} \cos \left(\omega_{1}+\omega_{2}\right) t\right] .
\end{gathered}
$$

From Equation (5) we can expect the generation of static fields, second-harmonic generation, a term that oscillates with the difference of incident frequencies (difference-frequency generation - DFG), and a term that oscillates with the sum of incident frequencies (sum-frequency generation - SFG). Therefore, it is possible to generate light with new frequencies through wave mixing nonlinear processes.

In the preceding equations, the susceptibility relates the electric field with the respective polarization. If both $\vec{E}(t)$ and $\vec{P}(t)$ are vector quantities, the nonlinear $\mathrm{n}^{\text {th }}$-order susceptibility is an $(n+1)^{\text {th }}$ rank tensor. A convenient form to express the term in Equation (5) responsible for SFG is:

$$
P_{i}\left(\omega_{3}=\omega_{1}+\omega_{2}\right)=\sum_{j, k} \chi_{i j k}^{(2)}\left(\omega_{3}=\omega_{1}+\omega_{2} ; \omega_{1}, \omega_{2}\right) E_{j}\left(\omega_{1}\right) E_{k}\left(\omega_{2}\right)
$$

The ijk indices run through the xyz Cartesian coordinates of electric field and polarization.

\subsection{Origin of nonlinear response of molecules}

The correct interpretation of nonlinear phenomena such as sum-frequency generation (SFG) or second-harmonic generation (SHG) is related to the understanding of nonlinear secondorder susceptibility, $\chi^{(2)}$. Here, we are going to discuss only the main characteristics of $\chi^{(2)}$, but many publications [17-23] present a complete theory of $\chi^{(2)}$ effects. However, we will try to be succinct while addressing relevant issues. The classical treatment is well-known, providing satisfactory results with a phenomenological theory. However, the quantum interpretation is more comprehensive and generally allows calculating the nonlinear susceptibilities and their dispersion.

The origin of nonlinear optical activity of organic molecules can be understood by a Taylor series expansion of the induced dipole moment of the molecule [21]:

$$
p_{l}=\alpha_{l m} E_{m}+\beta_{l m n} E_{m} E_{n}+\gamma_{l m n o} E_{m} E_{n} E_{o}+\ldots
$$


In Equation (7), $\alpha_{1 \mathrm{~m}}$ is the (first order) linear polarizability, $\beta_{\mathrm{lmn}}$ and $\gamma_{\mathrm{lmno}}$ are the second- and third-order hyperpolarizabilities, respectively.

Through second-order time-dependent perturbation theory [17], we can derive an expression for $\beta_{\operatorname{lmn}}$ that is quite complicated. But a simplification happens when we consider the case of SHG with the fundamental frequency $\omega$ away from electronic resonances, but with the secondharmonic $2 \omega$ resonant with an electronic transition of the molecule, $\omega_{\mathrm{vg}}$ [22]:

$$
\beta_{l m n}=\frac{1}{2 \hbar} \frac{Q_{l} P_{m n}}{2 \omega-\omega_{v g}+i \Gamma}
$$

where $\Gamma$ is the width of the electronic transition. Terms $P_{m n}$ and $Q_{1}$ are due to off-resonance and resonant transition moments, respectively. They are given by

$$
\begin{gathered}
P_{m n}=\sum_{s}\left[\frac{\left\langle v\left|\hat{\mu}_{m}\right| s\right\rangle\left\langle s\left|\hat{\mu}_{n}\right| g\right\rangle}{\left(\omega-\omega_{v s}\right)\left(\omega-\omega_{s g}\right)}-\frac{\left\langle v\left|\hat{\mu}_{n}\right| s\right\rangle\left\langle s\left|\hat{\mu}_{m}\right| g\right\rangle}{\left(\omega+\omega_{v s}\right)\left(\omega+\omega_{s g}\right)}\right], \\
Q_{l}=\left\langle g\left|\widehat{\mu}_{l}\right| v\right\rangle .
\end{gathered}
$$

In Equations (9) and (10), $\hat{\mu}$ is the electric dipole operator, $|g\rangle$ is the fundamental state, $|v\rangle$ is the excited state resonant with the second-harmonic, and $|s\rangle$ is any other state.

\subsection{Second-order susceptibility}

The nonlinear second-order susceptibility is a macroscopic average of second-order nonlinear polarizability $\beta$. It is defined as the term that describes the interaction between the material medium and the optical electric field [23]. The relation between $\chi^{(2)}$ and $\beta$ can be described by a coordinate transformation from the molecular reference frame to the laboratory frame, as shown in the following equation:

$$
\chi_{i j k}^{(2)}=N \sum\langle R(\psi) R(\theta) R(\varphi)\rangle \beta_{l m n} .
$$

where $\mathrm{N}$ is the number of molecules by volume, and $\langle R(\psi) R(\theta) R(\varphi)\rangle$ is the product of three rotation matrices that relate the molecular coordinate system $(\mathrm{l}, \mathrm{m}, \mathrm{n})$ to the laboratory coordinate system. The symbol $\diamond$ represents the orientational average. If we know the $\beta$ tensor for molecules, measurements of $\chi^{(2)}$ elements can give us information about their molecular orientation, as will be described in Section 2.4. 
Second-harmonic generation (SHG) arises from a term of Equation (5) proportional to $\cos (2 \omega t)$, when just one electric field at frequency $\omega$ is applied. In that case, Equation (6) becomes:

$$
\vec{P}^{(2)}(2 \omega=\omega+\omega)=\vec{\chi}^{(2)}: \vec{E}(\omega) \vec{E}(\omega)
$$

SHG is intrinsically sensitive to surfaces and interfaces due to its selection rule. As we will see later [see Equation (13)], the SHG intensity is proportional to the square of the second-order susceptibility $\chi^{(2)}$. As a polar third-rank tensor, $\chi^{(2)}$ changes sign under the inversion operation (under the electric dipole approximation): $\chi_{i j k}^{(2)}=-\chi_{-i-j-k}^{(2)}$. However, in centrosymmetric media $\chi^{(2)}$ remains unchanged upon inversion of coordinates: $\chi_{i j k}^{(2)}=\chi_{-i-j-k}^{(2)}$. Therefore, the only possible solution for the two earlier equations is $\chi^{(2)}=0$. We can conclude that, under the electric dipole approximation, for media with inversion symmetry, no second-order optical process is possible, including SHG. Most bulk molecular materials do have inversion symmetry. This is because the functional groups in the bulk of these systems are, in general, randomly or oppositely oriented [24]. However, because inversion symmetry is usually broken at the surface/interface, SHG is not forbidden in those cases. Figure 2 illustrates SHG at an interface between two centrosymmetric media. The incidence angles obey the momentum conservation along the interface plane: for refraction of incident laser: $k_{\omega}^{(2)} \sin \alpha_{\omega}^{(2)}=k_{\omega}^{(1)} \sin \alpha_{\omega}^{(1)}$ (Snell's law); for reflective/refractive SHG generation: $k_{2 \omega}^{(1)} \sin \alpha_{2 \omega}^{(1)}=2 k_{\omega}^{(1)} \sin \alpha_{\omega}^{(1)} ;$ and $k_{2 \omega}^{(2)} \sin \alpha_{2 \omega}^{(2)}=2 k_{\omega}^{(1)} \sin \alpha_{\omega}^{(1)}$. Index (i), $\mathrm{i}=1,2$, refer to media 1 and 2 in Figure 2 . For the specific case of thin polymeric films adsorbed on solid substrate such layer-by-layer films, if asymmetric molecules (or functional groups) adsorb with random orientations, the net SHG signal is canceled out. Conversely, if there is a substantial SHG signal, we can conclude that molecules have a net average orientation at the interface. More detailed considerations about the importance of symmetry on the interpretation of SHG (and other second-order processes, such as SFG) can be found elsewhere $[13,17,18,22]$.

For second-harmonic generation at interfaces between two different media, as shown in Figure 2 , Y. R. Shen demonstrated $[13,18]$ that the intensity of the second-harmonic signal is given by

$$
I(2 \omega)=\frac{8 \pi^{3}(2 \omega) \sec ^{2} \alpha}{\mathrm{c}^{3} \hbar\left[\varepsilon_{1}(2 \omega)\right]^{1 / 2} \varepsilon_{1}(\omega)}\left|\hat{\mathrm{e}}(2 \omega) \cdot \vec{\chi}_{s}^{(2)}: \hat{\mathrm{e}}(\omega) \hat{\mathrm{e}}(\omega)\right|^{2} I^{2}(\omega),
$$

where the SHG signal is expressed in terms of the net second-order susceptibility of the surface $\chi_{s}^{(2)}$. The term $\chi_{e f f}^{(2)}=\hat{e}(2 \omega) \cdot \vec{\chi}_{s}^{(2)}: \hat{e}(\omega) \hat{e}(\omega)$ is the effective susceptibility, which also depends on the polarizations of the input and output beams, $\hat{e}\left(\omega_{i}\right)$ and the Fresnel factors $L_{n n}\left(\omega_{i}\right)$, since $\hat{e}\left(\omega_{i}\right)=\hat{\epsilon}\left(\omega_{i}\right) \cdot \overleftrightarrow{L}\left(\omega_{i}\right)$. The Fresnel factors are given by the following expressions: 


$$
\begin{gathered}
L_{x x}\left(\omega_{i}\right)=\frac{2 \varepsilon_{1}\left(\omega_{i}\right) k_{2 z}\left(\omega_{i}\right)}{\varepsilon_{2}\left(\omega_{i}\right) k_{1 z}\left(\omega_{i}\right)+\varepsilon_{1}\left(\omega_{i}\right) k_{2 z}\left(\omega_{i}\right)}, \\
L_{y y}\left(\omega_{i}\right)=\frac{2 k_{1 z}\left(\omega_{i}\right)}{k_{1 z}\left(\omega_{i}\right)+k_{2 z}\left(\omega_{i}\right)}, \\
L_{z z}\left(\omega_{i}\right)=\frac{2 \varepsilon_{1}\left(\omega_{i}\right) k_{2 z}\left(\omega_{i}\right)\left(\frac{\varepsilon_{2}}{\varepsilon_{s}}\right)}{\varepsilon_{2}\left(\omega_{i}\right) k_{1 z}\left(\omega_{i}\right)+\varepsilon_{1}\left(\omega_{i}\right) k_{2 z}\left(\omega_{i}\right)} .
\end{gathered}
$$

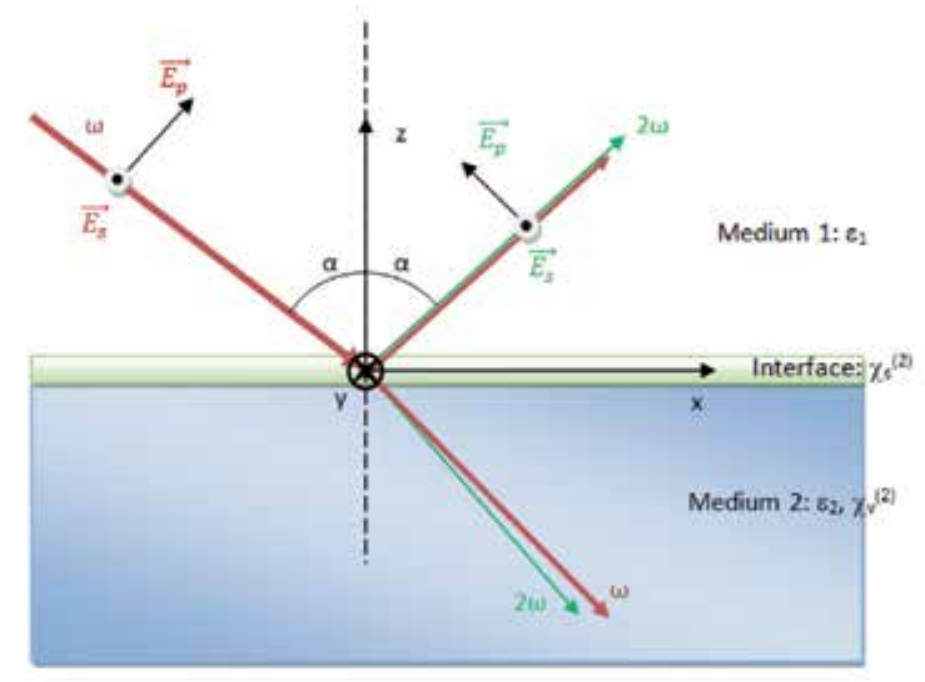

Figure 2. SHG geometry from the interface between two different media, showing the SHG beams generated in reflection and transmission. $\chi_{\mathrm{s}}^{(2)}$ in usually non-vanishing, while in general $\chi_{\mathrm{v}}{ }^{(2)}=0$.

From Equation (13), we can see that the SHG signal $I(2 \omega)$ is proportional to $\left|\chi_{\text {eff }}^{(2)}\right|{ }^{2}$, the effective second-order susceptibility of surface. From Equations (8) and (11), we can write $\chi_{\text {eff }}^{(2)}$ as a complex number:

$$
\chi_{\text {eff }}^{(2)}=\frac{A}{2 \omega-\omega_{v g}+i \Gamma}=\left|\chi_{e f f}^{(2)}\right| e^{i \phi},
$$

where $\left|\chi_{\text {eff }}^{(2)}\right|$ is its modulus and $\phi$ is the phase. Frequently, it is necessary to experimentally measure the phase of $\chi_{\text {eff }}^{(2)}$, because it is related to the relative orientation (up or down) of molecules at the interface. This can be accomplished by interference between SHG signals from sample (polymer films) and a nonlinear reference, like crystalline quartz or zinc sulfide, $\mathrm{ZnS}$ $[25,26]$. In practice, we measure the effective $\chi_{\text {eff }}^{(2)}$ that is the sum of the reference and the film signals: 


$$
\chi_{e f f}^{(2)}=\left|\chi_{r e f}^{(2)}\right| e^{i \phi_{r e f}}+\left|\chi_{f i l m}^{(2)}\right| e^{i \phi_{f i m}}
$$

As the SHG signal is proportional to square of $\chi_{e f f}^{(2)}$, we have

$$
\begin{gathered}
\left|\chi_{e f f}^{(2)}\right|^{2}=|| \chi_{\text {ref }}^{(2)}\left|e^{i \phi_{\text {ref }}}+\right| \chi_{\text {film }}^{(2)}\left|e^{i \phi_{\text {fllm }}}\right|^{2}= \\
=\left|\chi_{\text {ref }}^{(2)}\right|^{2}+\left|\chi_{\text {film }}^{(2)}\right|^{2}+2\left|\chi_{\text {ref }}^{(2)}\right|\left|\chi_{\text {film }}^{(2)}\right| \cos (\Delta \phi),
\end{gathered}
$$

where $\Delta \phi=\left(\phi_{\text {film }}-\phi_{\text {ref }}\right)=\frac{2 \pi}{\lambda} \Delta \mathrm{l}$ is the phase difference between two signals, and $\Delta \mathrm{l}$ is the difference of optical length due to a compensator inserted in the detection beam path (amorphous quartz window). Figure 3 shows the experimental setup. The angle $\theta$ of the compensator determines the additional optical path $\Delta \mathrm{l}=\frac{\Delta n d}{\cos \theta}$ traveled by the SHG and pump beams from the sample to the detector, where $\Delta \mathrm{n}$ is the difference in refractive indices of the compensator for the fundamental and SHG beams. Therefore, the phase difference between the two signals is given by

$$
\Delta \phi=\frac{2 \pi}{\lambda} \frac{\Delta n d}{\cos \theta}
$$

Figure 3 illustrates the (normalized) interference pattern intensity as a function of compensator angle $\theta$.

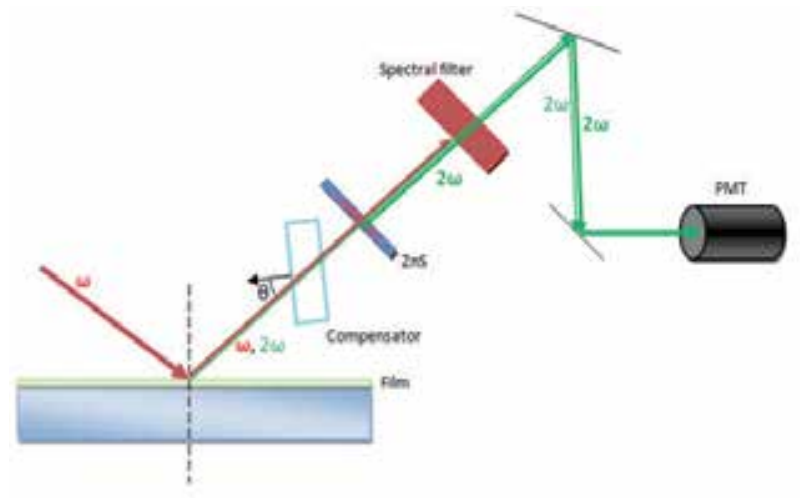

(a)

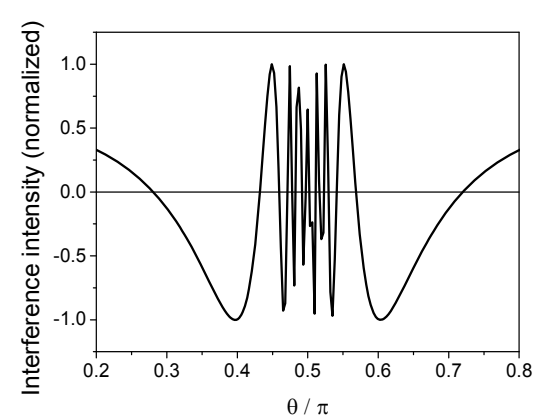

(b)

Figure 3. a) Experimental setup for SHG phase measurements. (b) Detected intensity (normalized) as a function of compensator angle $\theta$. 


\subsection{Molecular orientation from SHG measurements}

As seen earlier, Equation (11), the macroscopic quantity $\chi_{i j k}^{(2)}$ is related to microscopic quantity $\beta_{l m n}$ through an orientational average of a coordinate transformation, where $\beta_{l m n}$ is a tensor that relates the components of the second-order contribution to the dipole moment $\vec{p}$ of the molecule to local electric field components $\vec{E}_{\text {local }}$.

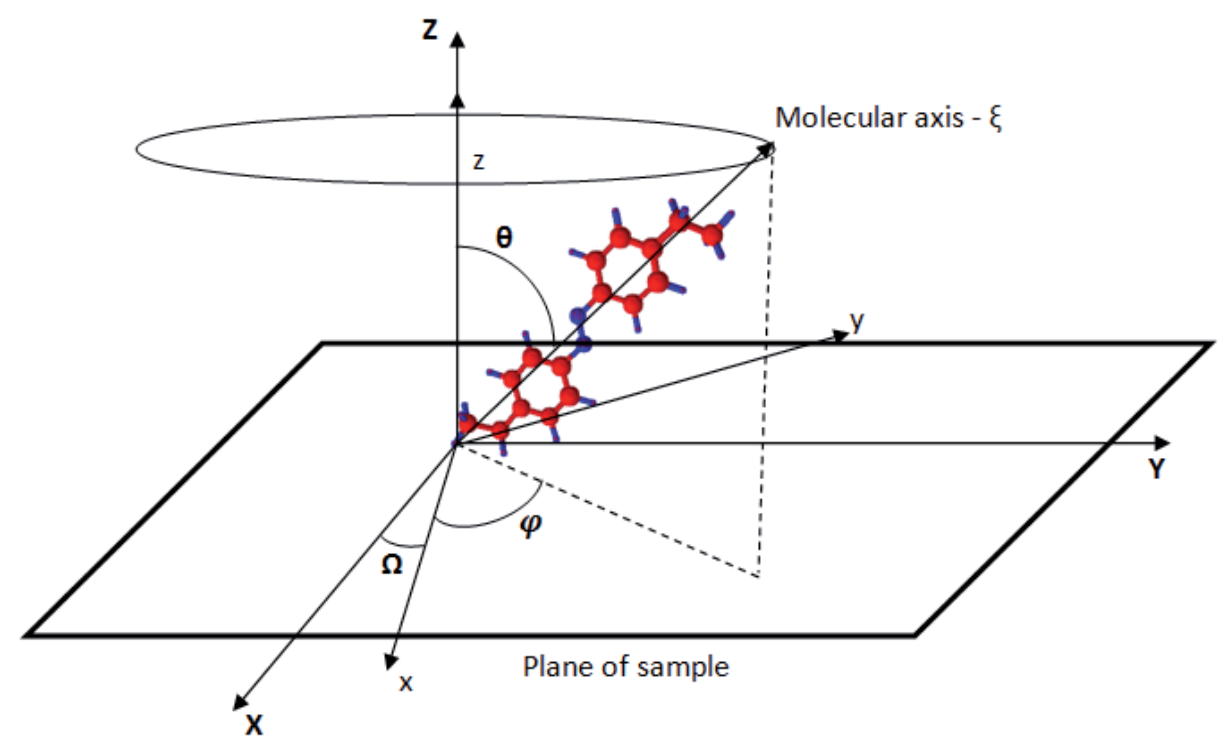

Figure 4. Molecular geometry with the azobenzene group $(\mathrm{Ph}-\mathrm{N}=\mathrm{N}-\mathrm{Ph})$ along the $\xi$ axis. The frame $(\mathrm{x}, \mathrm{y}, \mathrm{z})$ is the sample reference frame, with $\mathrm{xZ}$ as a mirror plane. The $(X, Y, Z)$ frame is the laboratory coordinate system, with $X Z$ as the incidence plane. The molecule is tilted by the polar angle $\theta$ with respect to the surface normal, and $\varphi$ is the azimuthal angle with respect to the sample symmetry direction, which in turn is rotated by $\Omega$ with respect to the incidence plane ( $\mathrm{X}$ direction).

For molecules with electrons delocalized mainly along a single direction, the hyperpolarizability $\beta_{l m n}$ will have only one element, that is, $\beta_{\xi \xi \xi}$, along the molecular axis $\xi$, as shown in Figure 4 for an azobenzene group. Then, for the case of a molecular monolayer adsorbed on the surface, Equation (11) can be written as

$$
\chi_{i j k}^{(2)}=N(\hat{i} \cdot \hat{\xi})(\hat{j} \cdot \hat{\xi})(\hat{k} \cdot \hat{\xi}) \beta_{\xi \xi \xi} \cdot
$$

The transformation of coordinates from the molecular frame $\hat{l}, \hat{m}$, and $\hat{n}$ to the sample frame $\hat{i}, \hat{j}$, and $\hat{k}$ is given by

$$
\hat{i} \cdot \hat{\xi}=\sin \theta \cos \varphi
$$




$$
\begin{gathered}
\hat{j} \cdot \hat{\xi}=\sin \theta \sin \varphi, \\
\hat{k} \cdot \hat{\xi}=\cos \theta .
\end{gathered}
$$

Considering a medium with a $\mathrm{C}_{1 \mathrm{v}}$ symmetric distribution of molecules on the plane $\mathrm{xy}$ ( $\mathrm{xz}$ is the sample plane of symmetry), we obtain six independent elements of the tensor $\chi_{i j k}^{(2)}$ [27]:

$$
\begin{gathered}
\chi_{1}=\chi_{z z z}^{(2)}=N \cos ^{3} \theta \beta_{\xi \xi \xi}, \\
\chi_{2}=\chi_{x x x}^{(2)}=N \sin ^{3} \theta \cos ^{3} \varphi \beta_{\xi \xi \xi}, \\
\chi_{3}=\chi_{y y z}^{(2)}=\chi_{z y y}^{(2)}=\chi_{y z y}^{(2)}=N\left(\cos \theta-\cos ^{3} \theta\right)\left(1-\cos ^{2} \varphi\right) \beta_{\xi \xi \xi}, \\
\chi_{4}=\chi_{x x z}^{(2)}=\chi_{z x x}^{(2)}=\chi_{x z x}^{(2)}=N\left(\cos \theta-\cos ^{3} \theta\right) \cos ^{2} \varphi \beta_{\xi \xi \xi}, \\
\chi_{5}=\chi_{x z z}^{(2)}=\chi_{z x z}^{(2)}=\chi_{z z x}^{(2)}=N\left(\sin \theta-\sin ^{3} \theta\right) \cos \beta_{\xi \xi \xi}, \\
\chi_{6}=\chi_{x y y}^{(2)}=\chi_{y x y}^{(2)}=\chi_{y y x}^{(2)}=N\left(\cos \varphi-\cos ^{3} \varphi\right) \sin ^{3} \theta \beta_{\xi \xi \xi},
\end{gathered}
$$

Therefore, measuring these six elements in the preceding equations allows determining up to five parameters of the orientation distribution function of the adsorbed monolayer (since usually the product $\mathrm{N} \beta_{\xi \xi \xi \xi}$ is unknown). This can be performed by SHG measurements with several combinations of polarization, such as $\mathrm{S}_{\text {in }} \mathrm{S}_{\text {out }}, \mathrm{S}_{\text {in }} \mathrm{P}_{\text {out }}, \mathrm{P}_{\text {in }} \mathrm{S}_{\text {out }}, \mathrm{P}_{\text {in }} \mathrm{P}_{\text {out }}, \mathrm{M}_{\text {in }} \mathrm{S}_{\text {out }}, \mathrm{M}_{\text {in }} \mathrm{P}_{\text {out }}$, where the first polarization is for the pump beam at $\omega$ and the other is for the generated beam at $2 \omega$. $\mathrm{S}$ indicates the polarization with the electric field perpendicular to the incidence plane, and $\mathrm{P}$ is with the electric field parallel to the incidence plane. M polarization is that where the electric field has equal components perpendicular and parallel to the incidence plane (mixed polarization). Figure 2 shows both $\mathrm{S}$ and $\mathrm{P}$ polarization.

We should note, however, that in general the laboratory coordinate system (XYZ), defined by incidence plane $X Z$ and the sample plane $X Y$, is not coincident with the sample coordinate system (xyz), defined by the sample plane xy and the plane of mirror symmetry xz. We define the angle $\Omega$ describing the relation between the two coordinate systems, as shown in Figure 4 .

Therefore, to fully determine $\chi_{\text {eff }}^{(2)}$ in laboratory frame, we need to do an additional coordinate transformation from the sample frame (xyz) to the laboratory frame (XYZ). Thus, we obtain $\chi_{\text {eff }}^{(2)}$ for six possible polarization combinations as a function of independent components $\chi_{1}$ to $\chi_{6}$ (Equations (22) to (27)), which characterizes the distribution of orientations for the molecules in study: 


$$
\begin{aligned}
& \chi_{e f f, S S}^{(2)}=L_{y y}(2 \omega) L_{y y}^{2}(\omega)\left[\sin ^{3}(\Omega) \chi_{2}+3 \sin (\Omega) \cos ^{2}(\Omega) \chi_{6}\right], \\
& \chi_{e f f, S P}^{(2)}=\sin (\alpha) L_{z z}(2 \omega) L_{y y}^{2}(\omega)\left[\cos ^{2}(\Omega) \chi_{3}+\sin ^{2}(\Omega) \chi_{4}\right]- \\
& \cos (\alpha) L_{x x}(2 \omega) L_{y y}^{2}(\omega)\left[\cos (\Omega) \sin ^{2}(\Omega) \chi_{2}+\left(\cos ^{3}(\Omega)-2 \sin ^{2}(\Omega) \cos (\Omega)\right) \chi_{6}\right], \\
& \chi_{\text {eff }, P S}^{(2)}=\cos ^{2}(\alpha) L_{y y}(2 \omega) L_{x x}^{2}(\omega)\left[\sin (\Omega) \cos ^{2}(\Omega) \chi_{2}+\left(\sin ^{3}(\Omega)-2 \sin (\Omega) \cos ^{2}(\Omega)\right) \chi_{6}\right] \\
& +2 \cos (\alpha) \sin (\alpha) L_{y y}(2 \omega) L_{z z}(\omega) L_{x x}(\omega)\left[\sin (\Omega) \cos (\Omega)\left(\chi_{4}-\chi_{3}\right)\right] \\
& +\sin ^{2}(\alpha) L_{y y}(2 \omega) L_{z z}^{2}(\omega) \sin (\Omega) \chi_{5} \text {, } \\
& \chi_{e f f, P P}^{(2)}=-\cos ^{3}(\alpha) L_{x x}(2 \omega) L_{x x}^{2}(\omega)\left[\cos ^{3}(\Omega) \chi_{2}+\left(3 \sin ^{2}(\Omega) \cos (\Omega)\right) \chi_{6}\right] \\
& +\left[\sin (\alpha) \cos ^{2}(\alpha) L_{z z}(2 \omega) L_{x x}^{2}(\omega)-2 \cos ^{2}(\alpha) \sin (\alpha) L_{x x}(2 \omega) L_{x x}(\omega) L_{z z}(\omega)\right] \\
& {\left[\sin ^{2}(\Omega) \chi_{3}-\cos ^{2}(\Omega) \chi_{4}\right]+\left[2 \sin ^{2}(\alpha) \cos (\alpha) L_{z z}(2 \omega) L_{x x}(\omega) L_{z z}(\omega)-\cos (\alpha) \sin ^{2}(\alpha) L_{x x}(2 \omega) L_{z z}^{2}(\omega)\right]} \\
& {\left[\cos \Omega \chi_{5}\right]+\sin ^{3}(\alpha) L_{z z}(2 \omega) L_{z z}^{2}(\omega) \sin (\Omega) \chi_{1} \text {, }} \\
& \chi_{e f f, M S}^{(2)}=\frac{1}{2} L_{y y}(2 \omega) L_{y y}^{2}(\omega)\left[\sin ^{3} \Omega \chi_{2}+\left(3 \sin \Omega \cos ^{2} \Omega\right) \chi_{6}\right] \\
& +\frac{1}{2} \cos ^{2} \alpha L_{y y}(2 \omega) L_{x x}^{2}(\omega)\left[\sin (\Omega) \cos ^{2}(\Omega)+\left(\sin ^{3}(\Omega)-2 \sin (\Omega) \cos ^{2} \Omega\right) \chi_{6}\right] \\
& +\frac{1}{2} \sin ^{2}(\alpha) L_{y y}(2 \omega) L_{z z}^{2}(\omega)[\sin (\Omega)] \chi_{5} \\
& +\cos (\alpha) L_{y y}(2 \omega) L_{y y}(\omega) L_{x x}(\omega)\left[\cos \Omega \sin ^{2}(\Omega) \chi_{2}+\left(\cos ^{3} \Omega-2 \sin ^{2}(\Omega) \cos (\Omega)\right) \chi_{6}\right] \\
& +\sin (\alpha) L_{y y}(2 \omega) L_{y y}(\omega) L_{z z}(\omega)\left[\cos ^{2} \Omega \chi_{3}+\sin ^{2}(\Omega) \chi_{4}\right] \\
& +\sin (\alpha) \cos (\alpha) L_{y y}(2 \omega) L_{x x}(\omega) L_{z z}(\omega)\left[\sin (\Omega) \cos (\Omega)\left(\chi_{4}-\chi_{3}\right)\right] \text {, } \\
& \chi_{e f f, M P}^{(2)}=-\frac{1}{2} \cos (\alpha) L_{x x}(2 \omega) L_{y y}^{2}(\omega)\left[\sin ^{2}(\Omega) \cos (\Omega) \chi_{2}+\left(\cos ^{3} \Omega-2 \sin ^{2}(\Omega) \cos (\Omega)\right) \chi_{6}\right]- \\
& \frac{1}{2} \cos ^{3}(\alpha) L_{x x}(2 \omega) L_{x x}^{2}(\omega)\left[\cos ^{3} \Omega \chi_{2}+3 \sin ^{2}(\Omega) \cos (\Omega) \chi_{6}\right]-\cos ^{2} \alpha L_{x x}(2 \omega) L_{y y}(\omega) L_{x x}(\omega) \\
& {\left[\sin \Omega \cos ^{2} \Omega \chi_{2}+\left(\sin ^{3} \Omega-2 \sin \Omega \cos ^{2} \Omega\right) \chi_{6}\right]} \\
& +\frac{1}{2} \sin \alpha L_{z z}(2 \omega) L_{y y}^{2}(\omega)\left[\cos ^{2} \Omega \chi_{3}+\sin ^{2}(\Omega) \chi_{4}\right]+\frac{1}{2} \sin ^{3} \alpha L_{z z}(2 \omega) L_{z z}^{2}(\omega) \chi_{1}+ \\
& \sin ^{2} \alpha L_{z z}(2 \omega) L_{y y}(\omega) L_{z z}(\omega)\left[\sin \Omega \chi_{5}\right]+\sin ^{2} \alpha L_{z z}(2 \omega) L_{y y}(\omega) L_{z z}(\omega)\left[\sin (\Omega) \chi_{5}\right]+ \\
& {\left[\sin ^{2} \alpha \cos \alpha L_{z z}(2 \omega) L_{x x}(\omega) L_{z z}(\omega)-\frac{1}{2} \sin ^{2} \alpha \cos \alpha L_{x x}(2 \omega) L_{z z}{ }^{2}(\omega)\right] \cos \Omega \chi_{5}+} \\
& {\left[\frac{1}{2} \cos ^{2} \alpha \sin \alpha L_{z z}(2 \omega) L_{x x}{ }^{2}(\omega)-\sin \alpha \cos ^{2} \alpha L_{x x}(2 \omega) L_{x x}(\omega) L_{z z}(\omega)\right]\left[\sin ^{2}(\Omega) \chi_{3}+\cos ^{2} \Omega \chi_{4}\right]+} \\
& {\left[\begin{array}{c}
\cos \alpha \sin \alpha L_{z z}(2 \omega) L_{y y}(\omega) L_{x x}(\omega)- \\
\cos \alpha \sin \alpha L_{x x}(2 \omega) L_{y y}(\omega) L_{z z}(\omega)
\end{array}\right]\left[\sin \Omega \cos \Omega\left(\chi_{4}-\chi_{3}\right)\right] .}
\end{aligned}
$$


In practice, $\mathrm{SHG}$ measurements consist in recording the $\mathrm{SH}$ intensity as a function of the sample azimuthal angle $\Omega$ for several polarization combinations. From them, we can determine the independent components, from $\chi_{1}$ to $\chi_{6}$, that are related to the orientational distribution of molecules on the sample. For example, for the case of an isotropic sample on its xy plane, only $\chi_{1}$ and $\chi_{3}=\chi_{4}$ will be nonvanishing, so that all $\chi_{e f f}^{(2)}$ are either null or independent of azimuthal angle $\Omega$, as expected. In this case, the ratio $\frac{\chi_{1}}{\chi_{3}}=\frac{\cos ^{3} \theta}{\cos \theta-\cos ^{3} \theta}$ depends only on the average molecular tilt with respect to the normal direction (polar angle $\theta$ ). Therefore, measurements can immediately be qualitatively interpreted to determine if samples are isotropic or not about the surface, and in that case, if polar orientation changes significantly.

In order to fully determine the orientation of adsorbed molecules at an interface (film), we assume that this orientation is described by a distribution function such as

$$
F(\theta, \varphi)=A e^{-\frac{\left(\theta-\theta_{0}\right)^{2}}{2 \sigma^{2}}}\left[d_{0}+d_{1} \cos (\varphi)+d_{2} \cos (2 \varphi)+d_{3} \cos (3 \varphi)\right] .
$$

The first term of $F(\theta, \varphi)$ is a Gaussian distribution function of the polar angle $\theta$, where $\theta_{0}$ is the average molecular tilt and $\sigma$ is the polar distribution width. $A$ is a normalization constant, given by

$$
A=\frac{1}{(2 \pi)^{1 / 2} \sin \left(\theta_{0}\right) \sigma\left(1-\frac{\sigma^{2}}{2}\right)} .
$$

This distribution describes the orientation angle of adsorbed molecules with respect to the zaxis, which is perpendicular to plane of sample (see Figure 4).

The second factor of Equation (34) is a Fourier series on the azimuthal angle $\varphi$, truncated at the third term. There, $d_{0}$ is the normalization constant equal to $1 / 2 \pi$. This distribution describes the anisotropy of adsorbed molecules along to plane of sample, with respect to the mirror plane $\mathrm{xz}$.

In order to obtain the parameters in the orientational distribution function $F(\theta, \varphi)$, we need to experimentally measure $\chi_{\text {eff }}^{(2)}$ in the six polarization combinations as the sample is rotated (varying the sample azimuthal angle $\Omega$ ). We then adjust the data (simultaneous fitting) to Equations (28) to (33) in order to find the parameters $\theta_{0}, \sigma, d_{1}, d_{2}$, and $d_{3}$, as well as the initial sample azimuth $\Omega_{0}$ (initial angle between sample symmetry direction and laboratory frames, see Figure 4).

In this chapter, we are interested in probing the molecular orientation of layer-by-layer films of azopolymers and their thermal stability using the SHG technique. In the next section, we will briefly describe the most important characteristics of these ultrathin films that are relevant to the physical interpretation of SHG results. 


\section{Layer-by-layer thin films}

G. Decher and co-workers [28, 29] were the first to propose this simple and efficient method of physical deposition. Specifically, layer-by-layer (LbL) deposition is a fast and practical deposition process based on the electrostatic interaction between polyelectrolytes and opposite charges on solid substrates, such as glass, silica, or mica [29-33]. It can be used to fabricate thin films from a few nanometers to hundreds of nanometers. In most cases the mechanism responsible for adsorption is mainly the electrostatic interaction, but secondary interactions such as hydrophobicity, Van der Waals, or H-bonding are also relevant [7].

Some advantages of electrostatic $\mathrm{LbL}$ with respect to other thin film fabrication techniques, for example, Langmuir-Blodgett (LB) depositions, are the use of water-soluble molecules involving a large variety of materials, its independence of size and topology of substrate [31, 32], and the applicability to almost any hydrophobic or hydrophilic solid, such as glass, quartz, mica, and gold [33].

In the LbL self-assembly process, spontaneous sequential adsorption of oppositely charged polyelectrolytes (polyions) is carried out from most often dilute aqueous solutions on charged surfaces. Figure 5 illustrates the experimental procedures for adsorbing LbL films. Typically, a charged substrate (frequently glass or quartz) is immersed in an oppositely charged polyion solution. Electrostatic attraction occurs between the charged surface and the oppositely charged molecules in solution. It is expected that adsorption occurs until overall charge neutrality or charge reversal is reached at the substrate surface, implying that the adsorption process is self-limited. After washing the substrate with an aqueous solution (usually of the same $\mathrm{pH}$ as the adsorption solution) in order to remove excess adsorbed material and to ensure that only one strongly adsorbed monolayer remains, the substrate is dried by $\mathrm{N}_{2}$ flow. We have shown that this drying step promotes the inhomogeneity of the film in the micrometer scale [34], making it unfit for certain applications. The next step is immersing the substrate with the first adsorbed layer in an oppositely charged polyion solution. The oppositely charged polyelectrolytes will complex at the film/solution interface, leading to adsorption of the second layer and overall charge reversal again. Now the signal of net surface charge (substrate plus adsorbed film) is restored to that of the original substrate. Other rinsing and drying steps complete the fabrications of the first bilayer. The whole procedure can be repeated as many times as necessary, with the same or a different pair of materials, which may also include nanoparticles, dendrimers, enzymes, etc. Therefore, in addition to allowing precise control of film thickness, the LbL method allows making films with their compositions controlled at the nanometer scale up to several hundreds of nanometers in thickness, simply by properly choosing the materials used for fabricating each layer.

Since there are no restrictions in the selection of polyelectrolytes, there are many materials that may be employed in the manufacture of LbL films. Thus, some of the most used are the PEI (poly(ethylene imine)), PAH (poly(allylamine chloride)), and PDAC (poly(dimethyldiallylammonium chloride)) as polycations; and PVS (poly(vinyl sulfonic acid)), PSS (poly(sodium styrene sulfonate)), Ma-co-DR13 (a side-chain-substituted azobenzene copolymer derived from azodye Disperse Red 13), PS-119 (Poly(vinylamine) backbone azo chromophore), and 
A
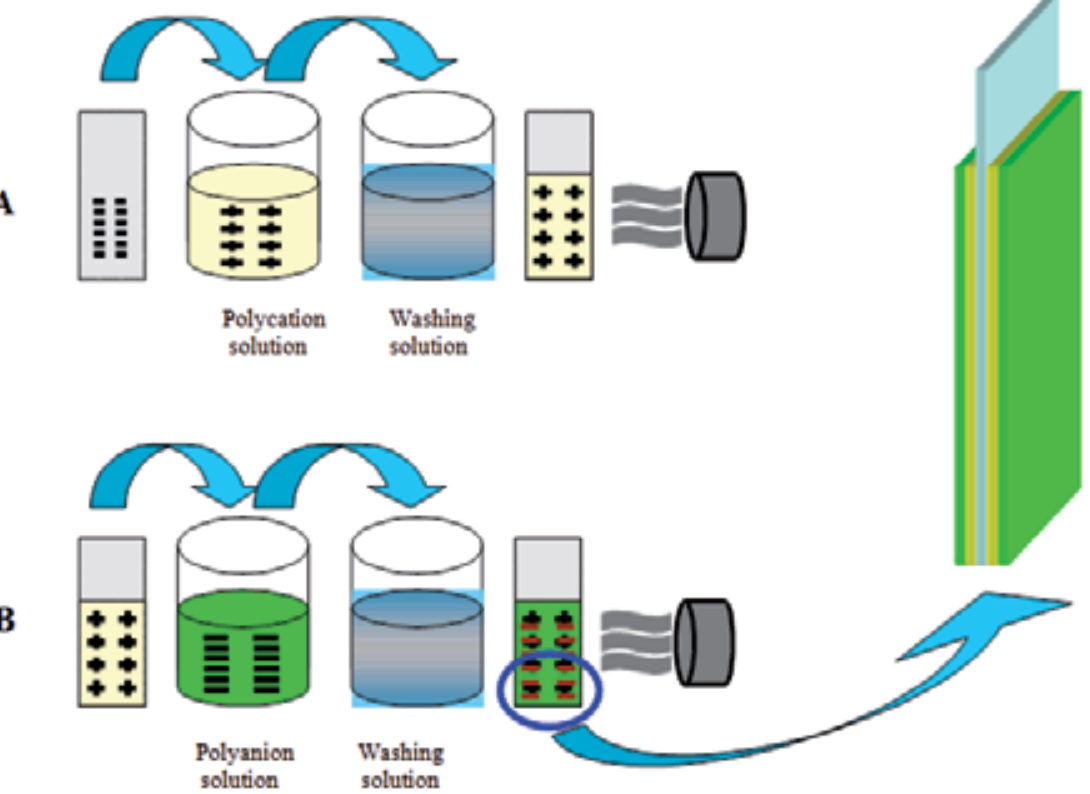

Figure 5. Self-assembly process. Part A: adsorption, rinsing, and drying of the first layer polyelectrolyte (polycation). Part B: adsorption, rinsing, and drying of the second layer (polyanion). Repetition of this process determines the desired number of bilayers.

PAA (poly(acrylic acid)) as polyanions. Figure 6 displays the structural formulas of some of these polyelectrolytes.
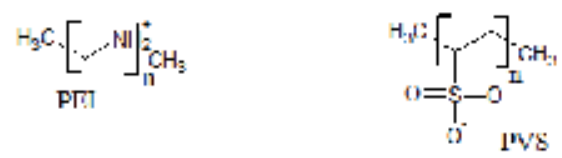<smiles>[CH][Te](C)(CCC)CC(C)(C)CC</smiles>
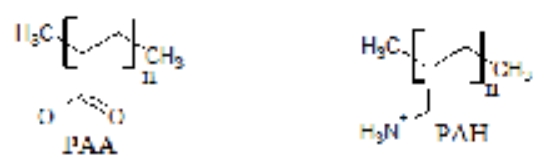

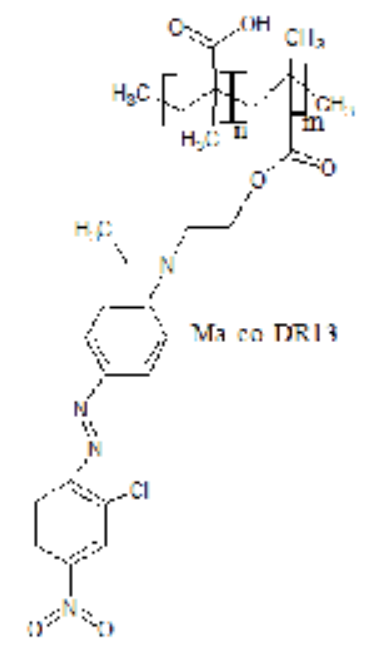

Figure 6. Structural formula for some polyelectrolytes used in LbL assembly. 
In this chapter, we discuss the molecular orientation of self-assembled LbL films fabricated with polyelectrolytes containing the azo-group. PAH $\left(\mathrm{M}_{\mathrm{w}}=15000\right)$ and Poly S-119 $\left(\mathrm{M}_{\mathrm{w}}=\right.$ unknown) were obtained from Aldrich and used as received. LbL films on BK7 glass substrates (area $10 \times 30 \mathrm{~mm}^{2}$, thickness $4 \mathrm{~mm}$ ) were prepared from aqueous solutions of PAH and Poly S-119 with $1.0 \mathrm{mg} / \mathrm{ml}$ concentration and $\mathrm{pH} 3.5,7.0$, and 10.0. For a given choice of $\mathrm{pH}$, both polyelectrolyte solutions and the rinsing solution had the same $\mathrm{pH}$ value, which was adjusted by addition of $\mathrm{HCl}$ (from Qhemis, 37\%, analytical grade) and $\mathrm{NaOH}$ (from Aldrich, electronic grade, purity $99.99 \%)$. Substrates were cleaned by piranha solutions $\left(\mathrm{H}_{2} \mathrm{SO}_{4} / \mathrm{H}_{2} \mathrm{O}_{2}\right.$ at $3: 1$ proportions by volume) for $20 \mathrm{~min}$, extensively rinsed with Milli-Q water (resistivity 18.3 $\mathrm{M} \Omega \bullet \mathrm{cm}$ ) and dried by nitrogen-flow right before use.

The LbL films were prepared by alternate adsorption of cationic (PAH) and anionic (Poly S-119) polyelectrolytes on the BK7 glass substrates, as described in literature $[29,33]$. In this work, we used just one final drying process: drying by slow water evaporation, that is, the films were prepared without any drying after adsorption or rinsing stages. In order to dry the samples after the self-assembly is complete, the substrates were loosely covered by a Petri dish to avoid contamination and stored for a period of 48 hours at room temperature $\left(\sim 23^{\circ} \mathrm{C}\right)$ and air humidity around $40 \%$. Only after this period, the second-harmonic signals were recorded.

Figure 7 shows absorbance at $445 \mathrm{~nm}$ (due to the azodye sidechain of PS-119) as a function of number of bilayers for films fabricated with three different $\mathrm{pH}$ values. As we can see, the film content of PS-119 increases linearly with the number of bilayers, demonstrating that the same amount of azopolymer is adsorbed at each bilayer. Figure 7 also shows that the adsorbed amount per layer is larger for $\mathrm{pH} 10$, suggesting the formation of thicker films under such conditions [35].

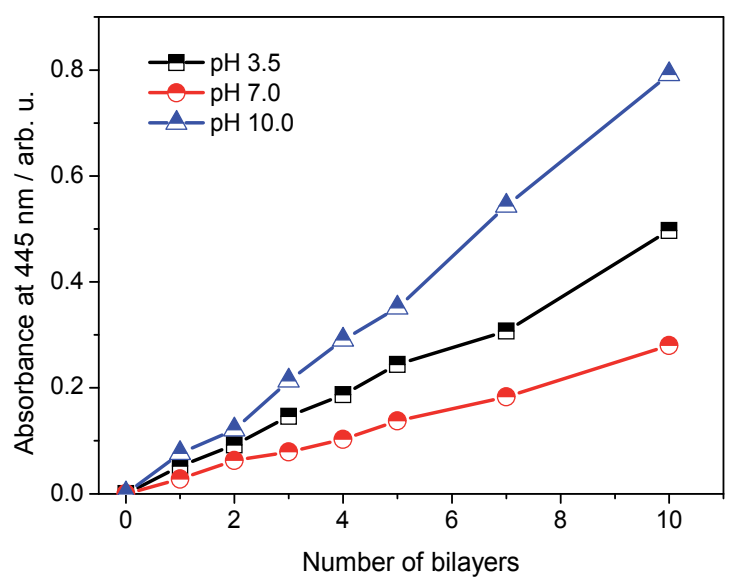

Figure 7. UV-vis absorbance at $445 \mathrm{~nm}$ for PAH/PS-119 LbL films fabricated at different $\mathrm{pH}$ values. 


\section{Second-harmonic generation from LbL films}

\subsection{SHG instrumentation}

Due to electronic resonance at $532 \mathrm{~nm}$, PS-119 polyelectrolyte is strongly active in secondharmonic generation if excited by a $1064 \mathrm{~nm}$ laser beam [see Eq. (8)], while PAH is optically inactive. Therefore, using this pump wavelength we are probing only one polyelectrolyte (PS-119), while the other is used only to assemble the film. This facilitates the interpretation of experimental results.

Our SHG instrumentation is shown in Figure 8 for SHG measurements as a function of azimuthal angle $\Omega$, where we can see a double-functional rotation/translation stage, allowing rotation of sample around the $\mathrm{z}$ axis (azimuthal angle $\Omega$ ), and its translation on the horizontal plane $x y$. A pulsed $\mathrm{Nd}^{3+}$ :YAG laser is used to excite the samples. The repetition rate, pulse duration, and the pump energy of the IR beam at $1064 \mathrm{~nm}$ were $20 \mathrm{~Hz}, 30 \mathrm{ps}$, and $2.0 \mathrm{~mJ}$, respectively. The area of beam on the sample surface was approximately $2 \mathrm{~mm}^{2}$, and the angles of incidence/reflection were $60^{\circ}$, since the phase matching condition along the surface plane leads to $k_{S H G} \sin \alpha_{S H G}=2 k_{I R} \sin \alpha_{I R}$, which gives $\alpha_{S H G}=\alpha_{I R}$ in the reflection direction (air side). Polarizers are used to set the polarization combination.

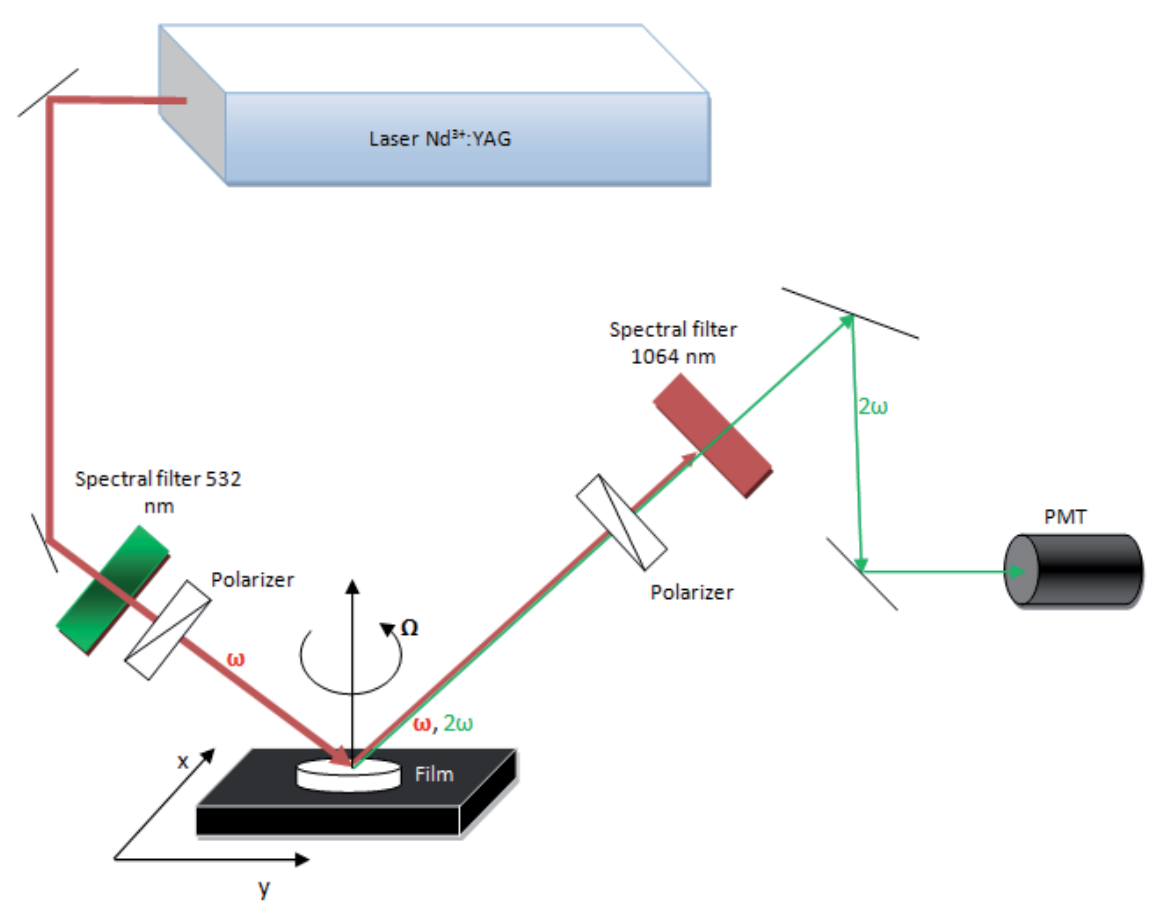

Figure 8. Layout for SHG measurements, consisting of a mode-locked $\mathrm{Nd}^{+3}$ :YAG $(1064 \mathrm{~nm})$ laser pumping the sample, which is positioned at a rotation/translation stage, and a detection system based on a photomultiplier tube (PMT). 
For studying the effect of heating, we used a temperature-controlled sample cell and the $S_{\text {in }} P_{\text {out }}$ polarization combination. Figure 9 shows the sample cell for SHG measurements as a function of temperature, which is aligned by a mirror mount positioned on top of a computerized xy translation and rotation stage. A commercial temperature controller was used to vary the temperature with $\sim 0.34^{\circ} \mathrm{C} / \mathrm{min}$ heating rate from room temperature (around $25^{\circ} \mathrm{C}$ ) to $190^{\circ} \mathrm{C}$. Figure 9 also shows in more detail the heating cell used in this experiment, with a sample inside.

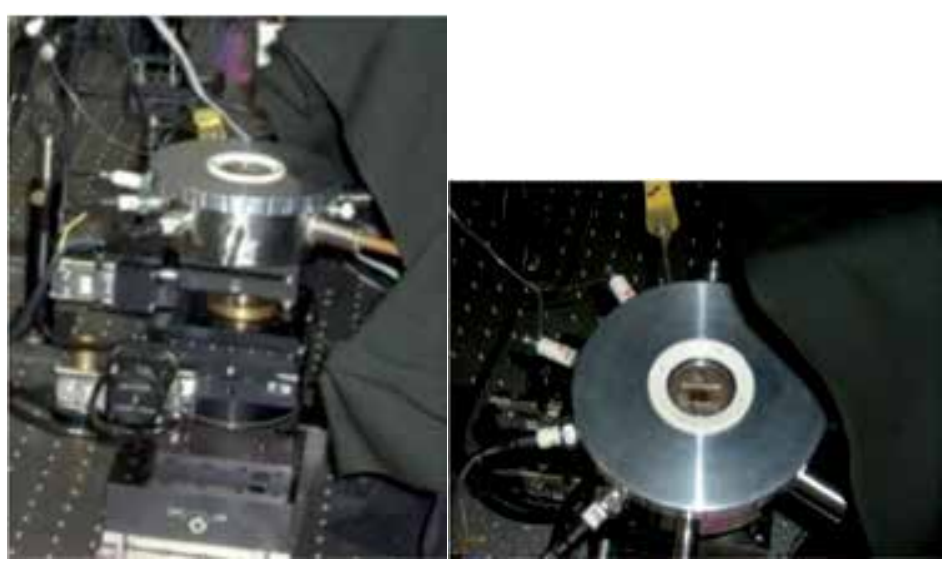

Figure 9. Experimental sample cell used to probe SHG signal as a function of temperature. It allows measurements either in air or vacuum, and for alignment purposes it is positioned on a mirror mount attached to the rotation/translation stage.

\subsection{Molecular ordering as a function of number of layers}

Measurements of SHG as a function of azimuthal angle of rotation $\Omega$ were performed for the SP (S pump and P SHG signal, or $\mathrm{S}_{\text {in }} \mathrm{P}_{\text {out }}$ ) and SS (S pump and S SHG signal, or $\mathrm{S}_{\text {in }} \mathrm{S}_{\text {out }}$ ) polarization combinations for LbL films of PAH/PS-119, varying the number of monolayers and the $\mathrm{pH}$ of the adsorption/rinsing solutions. For samples with isotropic ordering, the signal in the SP polarization combination should be intense, while for the SS polarization combination the SHG signal is only allowed if the sample is anisotropic. However, if there are orientational domains much smaller than the area of the pump beam in the sample, but larger than the wavelength of the beam, there should be an intense and isotropic SHG signal for both SP and SS polarization, confirming the microscopic anisotropy of the sample. Therefore, the absence of signal in the SS polarization is indicative of an isotropic molecular arrangement at the scale of the pump beam wavelength.

As a control measurement, an SHG rotational scan was obtained for a sample of z-cut quartz crystal and also for a gold surface prepared by thermal evaporation on glass. As can be seen in Figure 10, the measurement for quartz presents six directions where the signal is maximum, reflecting the $C_{3 v}$ symmetry of this quartz crystal surface. For the gold surface, it is isotropic and the electronic resonance at $532 \mathrm{~nm}$ yields a high second-order susceptibility [22] charac- 

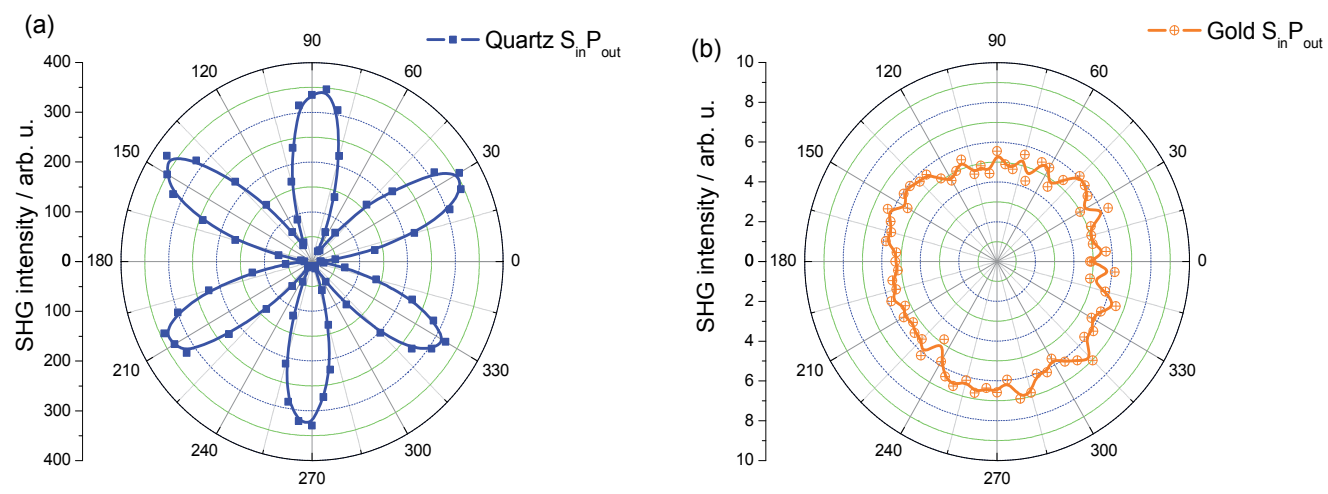

Figure 10. SHG measurements as a function of sample orientation $(\Omega)$ for (a) z-cut $\alpha$-quartz and (b) thermally evaporated gold film, with the $\mathrm{S}_{\text {in }} \mathrm{P}_{\text {out }}$ polarization combination.

teristic of this metal. Thus, the SHG signal from gold is independent of orientation and is fairly strong.

As an example of determining the orientational distribution function for layer-by-layer films, we consider the results displayed in Figure 11, where we can see the SHG signal as a function of azimuthal angle $\Omega$ for a 10 bilayer film of PAH/Ma-co-DR13 [36]. As described in Section 2.4, the results were adjusted to Equations (28) - (33) to determine $\chi_{1}$ through $\chi_{6}$, and the best fit lines are shown in Figure 11. Using their definitions (Equations (22) - (27)) and the orientation distribution function given by Equation (34), the six equations were solved to find the orientation parameters in $\mathrm{F}(\theta, \varphi)$. For these films, the following values were determined: $\theta_{0}=$ $37.41 \pm 0.24, \sigma=12.85 \pm 0.53, \mathrm{~d}_{1}=0.009 \pm 0.001, \mathrm{~d}_{2}=0.014 \pm 0.002, \mathrm{~d}_{3}=-0.001 \pm 0.003$, and $\Omega_{0}=$ $-11.87 \pm 4.78$, which fully characterize the orientational distribution of azo-groups in the sample.
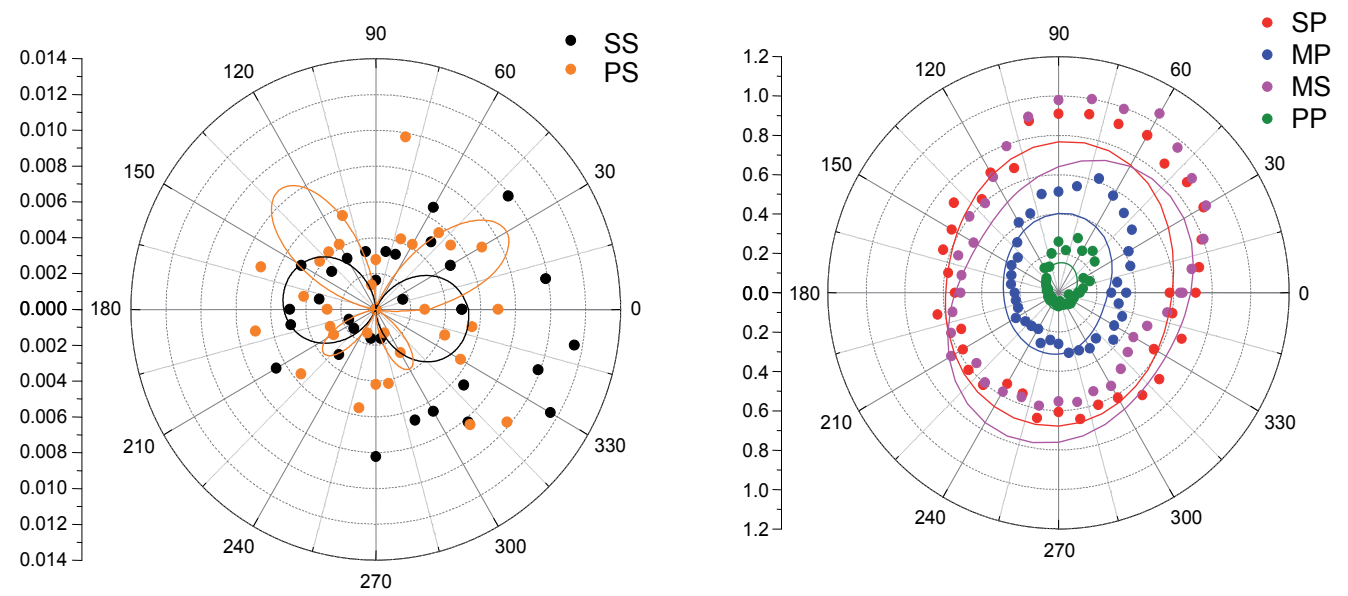

Figure 11. Azimuthal dependence of SHG signal for azopolimer (PAH/Ma-co-DR13) ${ }_{10}$ layer-by-layer films. (From Reference [36]) 
The marked effect of drying on the molecular arrangement of azopolymer LbL films can also be investigated by SHG. Figure 12(a) shows SHG measurements as a function of azimuthal angle $\Omega$ for a one bilayer film of (PAH/PS-119) obtained at three different points on each sample, for two samples fabricated with solutions of different $\mathrm{pH}$ values and dried by slow evaporation. They show that this fabrication procedure leads to isotropic and homogeneous LbL films, in agreement with the results already found for films of PAH/PSS [34]. The opposite was observed for self-assembled films of PAH/Ma-co-DR13, shown in Figure 12(b), which were fabricated with dry nitrogen flow drying. Indeed BAM (Brewster Angle Microscopy) measurements revealed the presence of orientational domains and inhomogeneity [36]. However, we have found that thicker films tend to became globally (not locally) more isotropic, since the SHG signals show smaller variations as a function of rotation, but the SS polarization combination does not vanish.

(a)

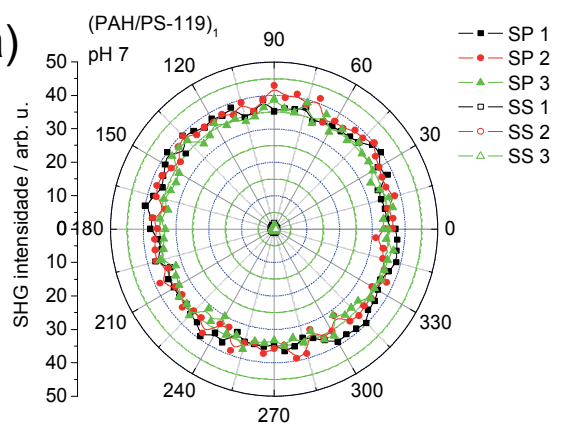

(b)

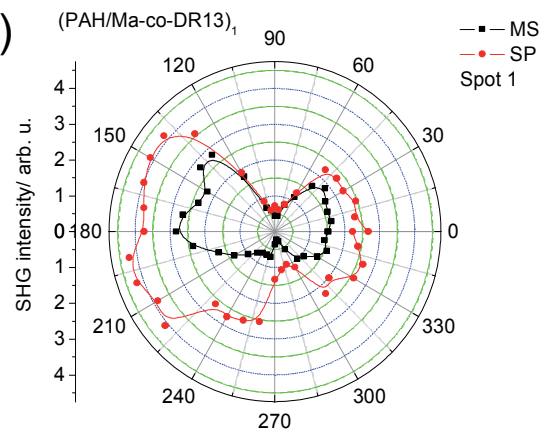

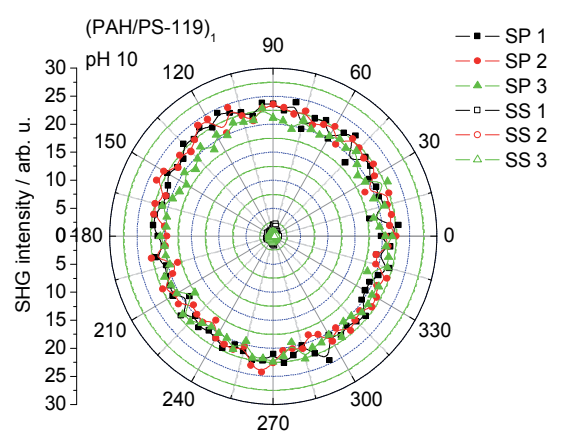

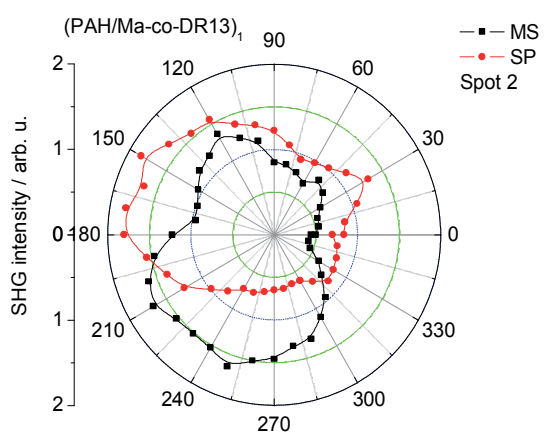

Figure 12. a) SHG measurements in three different spots for two different (PAH/PS-119) ${ }_{1}$ films fabricated with spontaneous drying (pH 7.0 and pH 10.0 solutions). (b) SHG measurements in two different spots of the same (PAH/Ma-coDR13) $)_{1}$ film fabricated with nitrogen flow drying.

SHG measurements as a function of the azimuthal angle $\Omega$ for films of PAH/PS-119 of different thicknesses prepared at $\mathrm{pH} 7$ showed that the films are always isotropic in the sample plane, since they have strong signal with SP polarization combination that is independent of the sample orientation. Furthermore, the SS polarization signal is practically zero whatever the number of layers, indicating that the samples are also microscopically isotropic. However, we 
note that the SP signal undergoes a change with the number of layers, which is related to the relative orientation of the azo-groups in each layer.

Similarly to the study of PAH/PSS films [24], for PAH/PS-119 films fabricated at pH 7.0 there is an alternation of the SP intensities as the last layer of the film is PAH or PS-119. Since the samples are always isotropic, Figure 13 shows the average intensities of the azimuthal SHG measurements with SP polarization as a function of the number of layers for the three $\mathrm{pH}$ values studied. Some groups have reported a linear increase of $\chi^{(2)}$ with the number of bilayers, especially above 10 bilayers [37-41], implying that average chromosphere orientations in each bilayer is the same (e.g., all pointing up, on average, in every bilayer). As noted in Figure 13, the square root of the SHG signal (which is related to the effective value of $\chi^{(2)}$ ) does not grow linearly with the number of bilayers for these PAH/PS-119 films. For pH 10.0, the signal rapidly decreases with the number of layers, up to 20 layers, but remains approximately constant for $\mathrm{pH}$ 7. In the case of $\mathrm{pH} 3.5$, there is a slight increase of signal with thickness up to 10 bilayers, but the signal is significantly reduced for thicker films, around 30 bilayers (not shown). Moreover, we always noted alternating SHG intensity after adsorption of each polyelectrolyte (integer vs. half-integer number of bilayers), at least for the first few bilayers. Specifically, for the films of PAH/PS-119 at pH 3.5 the authors of references [38] and [39] report a linear growth of $\chi^{(2)}$ for films up to 100 bilayers. However, we observe that for the same $\mathrm{pH}$ value the signal initially grows with the number of bilayers, but also alternating as the last layer is PAH or PS-119, and decreases from $~ 10$ bilayers, in disagreement with references [37-41]. Even for other films fabricated at other $\mathrm{pH}$ values, the increase was not linear. This behavior was reproduced in another set of samples manufactured with other solutions. Similar effect was observed by Lvov and co-workers for PDDA/PAZO LbL films where they reported an increase of $\chi^{(2)}$ up to 5 bilayers, but a reduction for thicker films [15]. At the moment we have no explanation for this discrepancy between our experimental results and those of references [3741]. However, we have reported the changes in molecular conformation including the molecular ordering after adsorption of the subsequent layer [24], and therefore a linear increasing of $\chi^{(2)}$ would be quite surprising, because that would mean that each and every PS-119 layer has an identical average orientation, and in the same direction. This would be especially unexpected considering effects such as interpenetration of layers and increasing film roughness with the number of layers, as has been observed for films of POMA/PVS [43] and PAH/Ma-co-DR13 [42].

In summary, comparing the intensities in Figure 13, we see that the signal initially grows with the number of layers for low $\mathrm{pH}$ (3.5), and it only decreases for high $\mathrm{pH}$ (10.0) and remains nearly unchanged for almost neutral $\mathrm{pH}$ (7.0). When the number of bilayers is high, we observed that the SHG signal always decreases considerably.

From the absorbance measurements in Figure 7, the amount of PS-119 per bilayer is constant in each sample for all three $\mathrm{pH}$ values. Therefore, we may conclude that the azopolymer chains do not remain with the same degree of ordering as the film grows, otherwise we should have observed a linear increase of $\chi^{(2)}$ with thickness. Specifically, since there is a decrease in signal with increasing number of layers, it is necessary that the adsorption of the last layers is affected by the average ordering of the previous layers, pointing on average in the opposite direction 


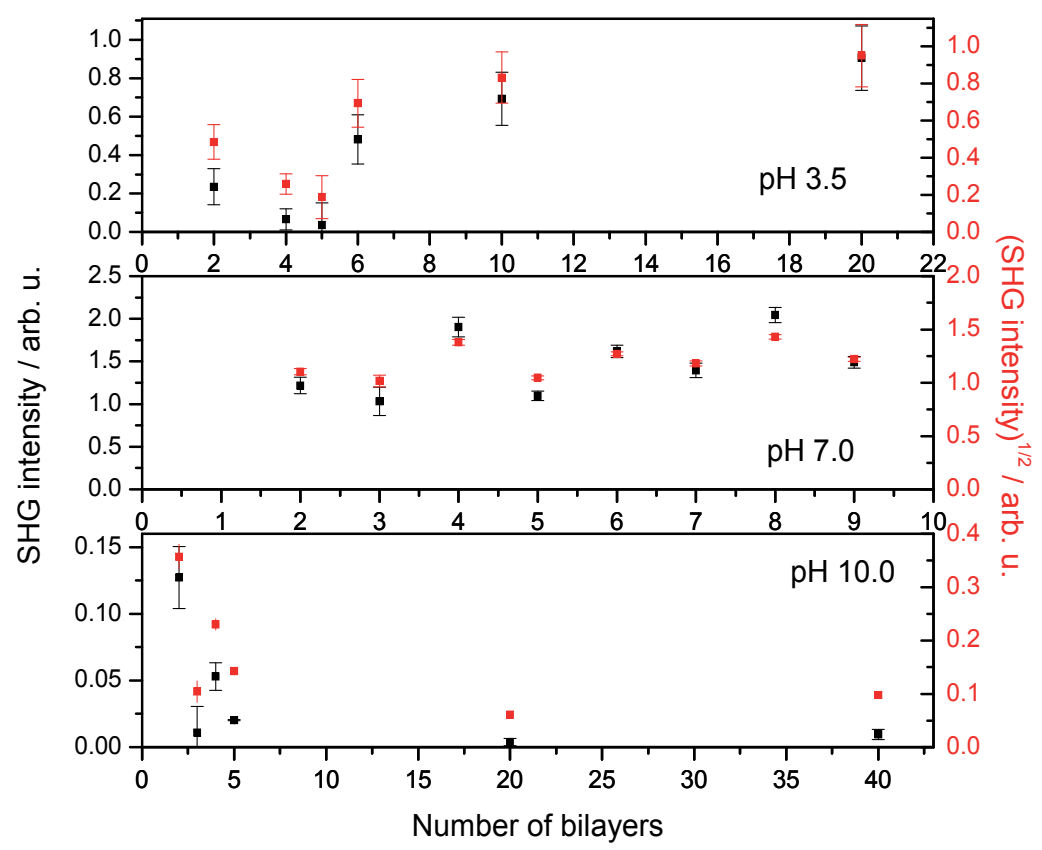

Figure 13. SHG signal and square root of SHG signal as a function of number of bilayers for PAH/PS-119 films fabricated at $\mathrm{pH} 3.5,7.0$, and 10.0 .

of the first ones. If the first few layers were ordered and the following ones had an isotropic arrangement, the SHG signal should initially grow and then saturate at a constant value for thick films.

At $\mathrm{pH} 3.5$, PAH has an ionization degree of about $100 \%$, while the glass is hardly ionized (only about $9 \%$ of the surface Si-OH groups, according to references $[43,44])$ and the first layer of $\mathrm{PAH}$ would be expected to be very thin due to the high charge density in the chains and low substrate charge. However, due to a high ionic strength, the electrostatic shield makes the PAH chain a little more coiled. Therefore, these films at $\mathrm{pH} 3.5$ are slightly thicker and rougher due to the reduction of electrostatic interactions by an increased ionic strength. This means that the SHG signal initially increases with thickness, but the net ordering of each additional bilayer is reduced for thick films, leading to a saturation (and eventual reduction) of SHG signal, as shown in Figure 13. For $\mathrm{pH} 10$, the glass is highly charged, but the $\mathrm{PAH}$ is only about $30 \%$ ionized, thus forming more folded layers than at $\mathrm{pH}$ 3.5. Therefore, the adsorbed amount is large but there are few sites in the PAH layer available for complexation with the PS-119, thus decreasing the drive for orientational ordering of PS-119 and leading to films that rapidly become disordered with increasing thickness, reducing the SHG signal. For $\mathrm{pH} 7$, both the substrate and PAH are quite charged, with a low ionic strength in the solutions, favoring electrostatic interactions and allowing the film growth with a relative stability of the SHG signal. 
Another interesting feature is the alternation of the SHG signal for films of a few bilayers (data not shown). For example, for the film of 3 layers (or 1.5 bilayers), (PAH/PS-119)/PAH, typically the signal is canceled out or significantly reduced, except for $\mathrm{pH} 7$ where the alternating signals are all large. Here, we will consider only the films formed at $\mathrm{pH} 3.5$ and 10, which show a different behavior from that observed in our previous report [24]. Compared to the first bilayer, PAH/PS-119, which presents considerable SHG signal, the (PAH/PS-119)/PAH film has a new ordering of PS-119 azo-groups, with random or symmetric configuration in the z-direction of film growth, which yields a vanishing SHG signal. This because in the 1 bilayer film, the negatively charged azo-groups of PS-119 are oriented on average toward the cationic PAH layer. In the three-layer film, the signal is greatly reduced, since the third PAH layer modifies the orientation of the previously adsorbed PS-119 layer, thereby reducing the SHG signal. In particular, for $\mathrm{pH} 3.5$ the signal is almost completely canceled, indicating an almost perfectly symmetrical configuration of the azopolymer active groups. This is reasonable because the two PAH layers of the PAH/PS-119/PAH film are highly and equally charged, exerting nearly the same influence on the central PS-119 layer.

To investigate in more detail the orientation of azo-groups of PS-119 in very thin films, in the anomalous region of ordering as a function of thickness for $\mathrm{pH} 3.5$ films (see Figure 13), we performed a direct measurement of the phase of $\chi^{(2)}$ with the SP polarization combination, using as a reference a thin film of zinc sulfide $(\mathrm{ZnS})$, as described in Section 2.3.

Figure 14(a) shows that the phase of the SHG signal from the sample, which is related to the average direction of orientation of the azopolymer is always the same for films formed at $\mathrm{pH}$ 3.5 with an integer number of bilayers where the last layer is the azopolymer, covering a highly charged cationic layer of PAH. For the first bilayer, it is expected that the preferred arrangement of the azo-groups of PS-119 is toward the highly charged layer of PAH, that is, toward the substrate side. Thus, this behavior is preserved for all films whose last layer is PS-119. Since the signal for the film with 3 layers, PAH/PS-119/PAH, is null, we can conclude that occurs a rearrangement of the chromophores in direction to both layers of $\mathrm{PAH}$, such as observed for PAH/PSS films [24]. Therefore, the results in Figure 14(a) confirm our assumptions about the orientation of the azopolymer groups.
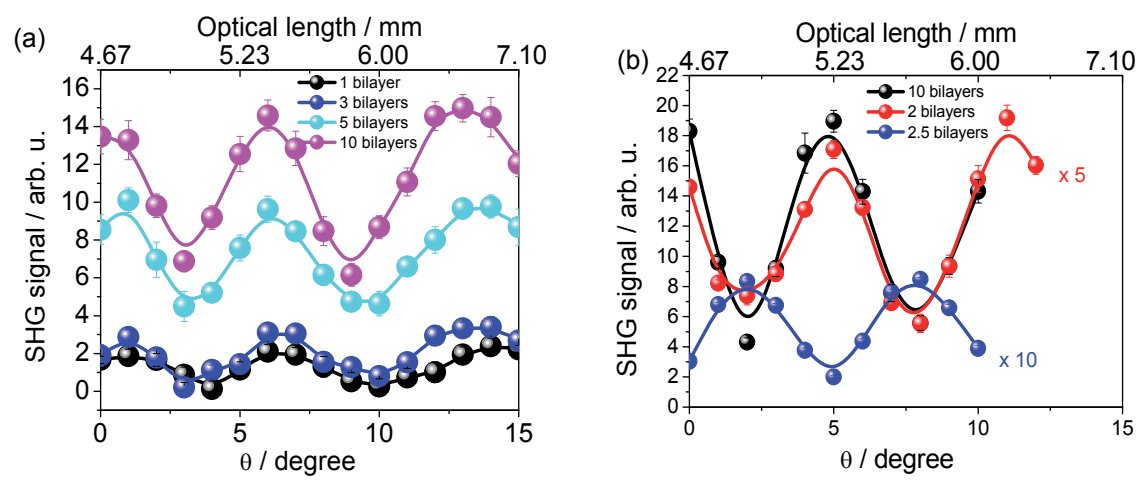

Figure 14. SHG interference pattern for PAH/PS-119 films fabricated at $\mathrm{pH} 3.5$ with various numbers of bilayers. $\theta$ is the angle of the silica compensator plate. 
For films with 2 or 2.5 bilayers, whose signals are less intense, the phase measurement, while difficult to be performed, confirms that the reordering of the azo-groups also occurs, as shown in Figure 14(b). When the film is finished with a layer of PAH, PS-119 chromophores undergo reorientation and then acquire a small average ordering in the direction opposite to the substrate, causing a reversal of the phase of the SHG signal. Therefore, films with an even number of layers have the same phase, with azo-groups pointing toward the substrate, while the 2.5 layer film had the opposite net orientation.

\subsection{Effect of temperature on the ordering of azopolymer films}

Now, we shall discuss the effect of heating on the ordering of azopolymer films. Figure 15 shows results for PAH/PS-119 films fabricated from solutions at $\mathrm{pH} 3.5$, which are illustrative of the general behavior of molecular ordering as a function of heating. We begin at room temperature $\left(\sim 20^{\circ} \mathrm{C}\right)$ and ramp the temperature up to $190^{\circ} \mathrm{C}$. As can be seen, there is no abrupt variation of SHG signal, but a gradual and significant decrease of intensity, even for thicker films with 5 or 10 bilayers. Similar behavior was observed for PDDA/PAZO films [15].

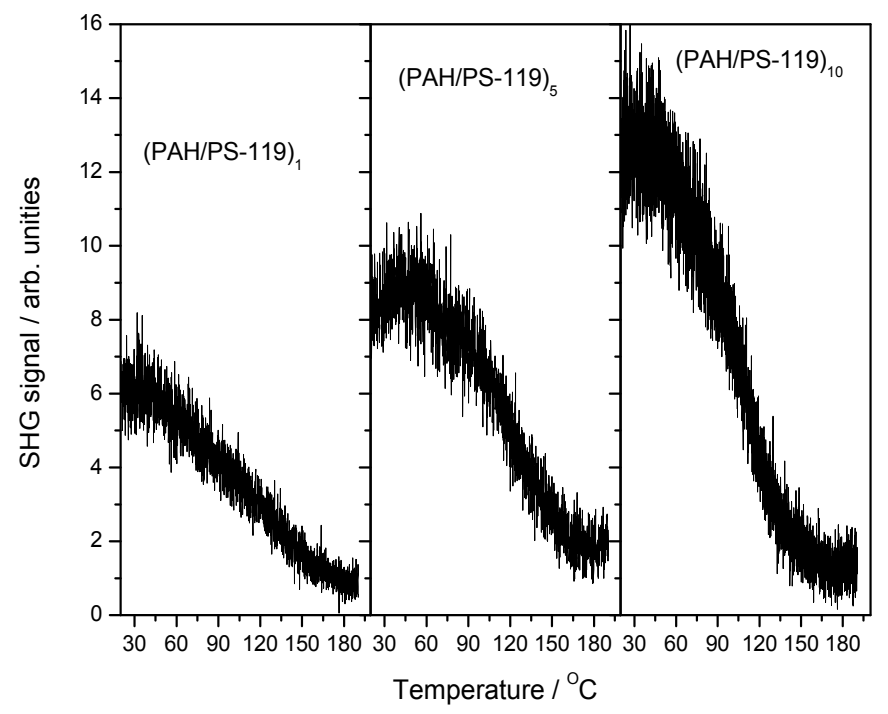

Figure 15. SHG intensity as a function of temperature for PAH/PS-119 films ( $\mathrm{pH}$ 3.5).

It is interesting to note that the literature reports that these films are quite thermally stable. They state that the SHG signal decreases about $20 \%$ from the initial value at room temperature, for temperatures above $\mathrm{T}_{\mathrm{g}}\left(\sim 140^{\circ} \mathrm{C}\right)$. Clearly, Figure 15 shows that thermal stability was not confirmed. The SHG intensity goes to almost zero in some cases, such as for the (PAH/PS-119)/ PAH film (result not shown). The SHG signal for the 1 bilayer film at $150^{\circ} \mathrm{C}$ is only $\sim 25 \%$ of initial signal at $20^{\circ} \mathrm{C}$ : a reduction of $75 \%$, instead of only $20 \%$ as previously reported $[16,38$, 40]. However, these authors do not mention how this $T_{g}$ was measured. It is not clear if it is for the LbL film, including the substrate effect, or for complexed molecules in the bulk. 
The fact that we do not observe an abrupt decrease of the SHG signal (indicating a glass transition temperature) can be due interactions in the film that are different from those in the bulk materials, like lateral (intralayer) or interlayer interactions. Furthermore, our films are formed by two different molecules and we should also consider the substrate effect because the films studied here have only a few layers and the substrate/polymer electrostatic interaction is considerable. Our results on films fabricated at $\mathrm{pH} 7.0$ show this influence on the film adsorption (data not shown [45]). For these films, it was observed that the SHG signal vanishes at high temperatures for a 2-bilayer film, but not for thicker films. This suggests that these thicker films, with a more efficient complexation between layers, have an improved thermal stability because it is more difficult to thermally induce disorder.

Figure 16 shows the ratio of $\chi^{(2)}$ at $180^{\circ} \mathrm{C}$ and $30^{\circ} \mathrm{C}$ as a function of the number of bilayers, for films fabricated at both $\mathrm{pH} 7$ and 3.5. Thicker films are more stable at $\mathrm{pH} 7$, but for films at $\mathrm{pH}$ 3.5 , that ratio was quite independent of thickness. However, there is an important difference in the temperature at which the SHG signal reaches the lowest value for $\mathrm{pH} 3.5$ films. For a 1bilayer film, this temperature is around $180^{\circ} \mathrm{C}$, but for films with 5 and 10 bilayers, this temperature is near $160^{\circ} \mathrm{C}$. This behavior suggests a significant influence of substrate charge density on the first layers, increasing the complexation effect between layers and the thermal stability for these thin films. For thicker films, the complexation/interpenetration of layers is not as disturbed as for thin films and the thermal stability increases. However, at $\mathrm{pH} 3.5$ the silica substrate is less charged and has less influence on the complexation of layers, resulting in a thermal stability which is independent of film thickness, with only a slight reduction in the stabilization temperature for thicker films. For films fabricated at $\mathrm{pH}$ 7, the substrate charge is higher, which promotes more efficient complexation between the polyelectrolytes and yields more thermally stable films (except for a 2-bilayer film that presents an anomalous behavior).

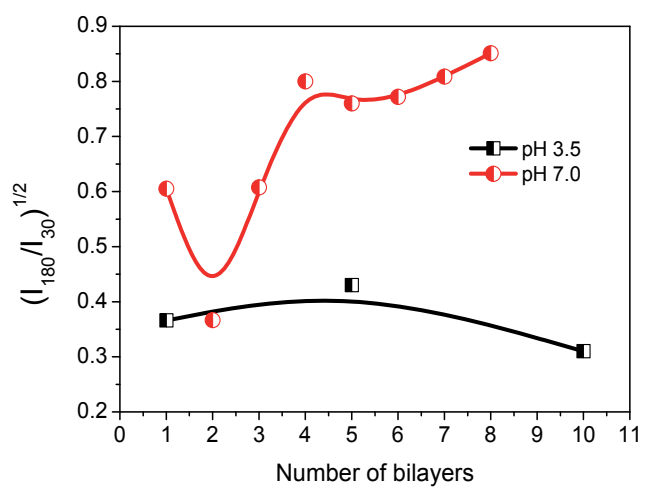

Figure 16. Reduction of $\chi^{(2)}$ due to heating as a function of number of layers, for films fabricated at $\mathrm{pH} 7.0$ and 3.5. The points are the ratio of $\chi^{(2)}$ measured at $180^{\circ} \mathrm{C}$ to that at $30^{\circ} \mathrm{C}$, and the lines are guides to the eye.

In order to verify the effect of heating on the structure of films, we compare the SHG signal for films at $\mathrm{pH} 3.5$ before heating, and after slow cooling to room temperature. Results indicate that thermally induced disordering is not permanent because the SHG signal is restored after 
slow cooling. This behavior is similar to what happens in the spontaneous drying assembly, since as the film cools the chains are losing mobility, but slowly enough for them to recover the best configuration induced by electrostatic interaction, thus recovering the order and restoring the SHG signal. For the films fabricated at $\mathrm{pH} 3.5$, the SHG signal as a function of the azimuthal angle has the same isotropic profile before and after heating. On the other hand, the same was not verified for films fabricated at $\mathrm{pH} 10$, where we can observe that after heating the ordering is no longer isotropic, as shown in Figure 17 for a 1-bilayer PAH/PS-119 film. This suggests that the films fabricated at this $\mathrm{pH}$ value have larger mobility than those at $\mathrm{pH} 3.5$ or 7 , which allows the rearrangement of chains to form macroscopic domains ( hundreds of micrometers) with preferential orientation along the substrate plane.
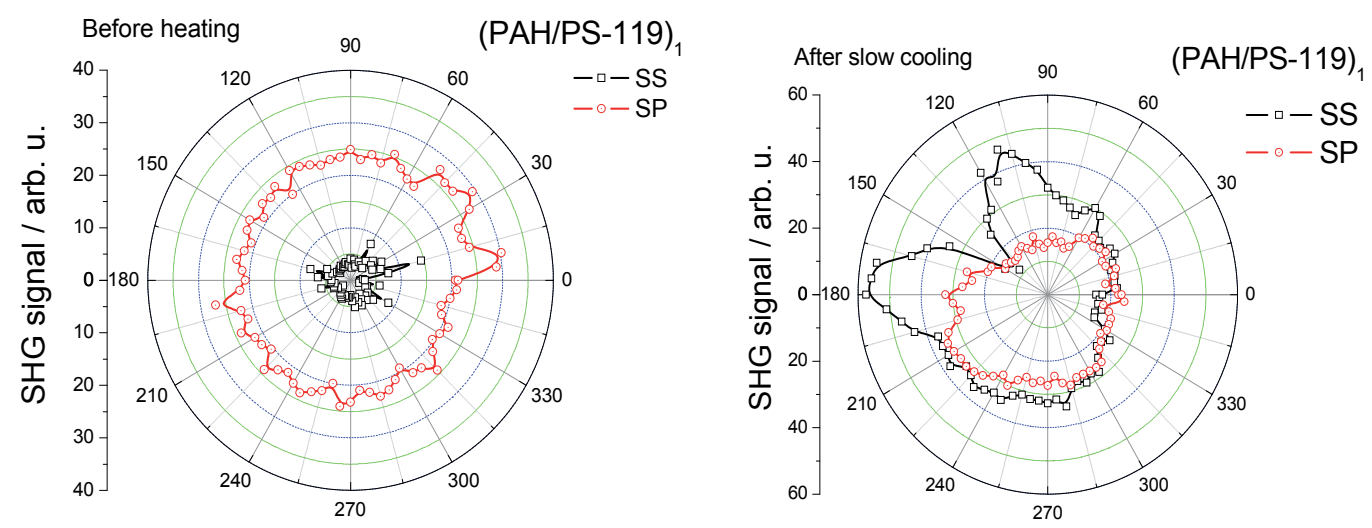

Figure 17. SHG signal in SS and SP polarization combination for a one-bilayer PAH/PS-119 film fabricated at $\mathrm{pH}$ 10, before and after heating to $190^{\circ} \mathrm{C}$.

\section{Conclusions}

In this chapter we have discussed how nonlinear optical methods, and in particular secondharmonic generation (SHG), can be used to investigate the molecular order in polyelectrolyte layer-by-layer films containing azopolymers. After a brief outline of the basic theory of SHG for interface studies, we have shown how its polarization dependence can be used to obtain quantitative information about the orientational distribution function of azo-groups in these thin films. However, even a qualitative analysis of the SHG signal can give important information about the film structure. For example, the SHG dependence on the azimuthal rotation of the sample has shown that the way the films are dried has a marked influence of their molecular arrangement, which is isotropic for slow (spontaneous) drying, while it becomes anisotropic and inhomogeneous with nitrogen-flow drying.

We have also investigated how the molecular ordering depends on the film thickness and fabrication conditions, especially the $\mathrm{pH}$ of the assembling/rinsing solutions. In contrast to previous reports in the literature, we did not find that all layers keep the same relative 
orientation, leading to a linear increase of the optical nonlinearity with thickness. Instead, we find that for films fabricated at low or high $\mathrm{pH}$, the nonlinearity tends to decrease for thick films ( 10 bilayers). Films fabricated at neutral $\mathrm{pH}$ generate an SHG signal that does not vary significantly with thickness, except for a slight alternation in intensity for films with odd or even number of layers. These results are due to the influence of adjacent polyelectrolyte layers on the order of an adsorbed layer, that is, the order of the last adsorbed layer is different than that for layers within the film. Phase measurements of the SHG signal confirm the reorientation of polymer groups in the last layer after adsorption of an additional polyelectrolyte layer. Finally, we have also studied the thermal stability of the molecular arrangement by SHG measurements as a function of sample temperature. We found that the nonlinear response presents a gradual and significant reduction upon heating, so that a clear glass transition temperature cannot be defined for these ultrathin layer-by-layer films. Again, the thermal stability of the samples depends on their fabrication conditions ( $\mathrm{pH}$ and thickness), with higher charge density in the polyelectrolytes and substrate promoting better complexation and improving their thermal stability. We also noted that the disordering effect of heating is reversible, and the SHG signal is recovered upon cooling. However, a few samples had their molecular arrangement becoming anisotropic after a heating/cooling cycle, as a result of aggregation and formation of molecular domains at the scale of tens of micrometers. We hope that these examples of SHG applied to the study of thin nonlinear optical polymer films have shown how powerful the technique can be to obtain information about the film structure at the molecular level, with also important consequences for their applications in optical devices.

\section{Author details}

Heurison S. Silva ${ }^{1 *}$, Irismar G. Paz ${ }^{1}$ and Paulo B. Miranda ${ }^{2}$

*Address all correspondence to: heurison@ufpi.edu.br

1 Universidade Federal do Piauí, Departamento de Física, Campus Universitário Ministro Petrônio Portella, Teresina, PI, Brazil

2 Universidade de São Paulo, Instituto de Física de São Carlos, Departamento de Física e Ciência dos Materiais, São Carlos, SP, Brazil

\section{References}

[1] Delongchamp, D., Hammond, P. T., Advanced Materials, v. 13, p. 1455, 2001.

[2] Roy, S., Kundu, S., Roy, S. K., Pal, A. J., Materials Chemistry and Physics, v. 77, p. 784, 2002. 
[3] Geest, B. G., Déjugnat, C., Verhoeven, E., Sukhorukov, G. B., Jonas, A. M., Plain, J., Demeester, J., Smedt, S. C., Journal of Controlled Release, v. 116, p. 159, 2006.

[4] Hoshi, T., Sagae, N., Daikuhara, K., Anzai, J.-I., Talanta, v. 71, p. 644, 2007.

[5] Shirsat, M. D., Too, C. O., Wallace, G. G., Electroanalysis, v. 20, p. 150, 2008.

[6] Chen, X., Clarke, M. L., Wang, J., Chen, Z. Sum frequency generation vibrational spectroscopy studies on molecular conformation and orientation of biological molecules at interfaces. International Journal of Modern Physics B, v. 19, n. 4, p. 691-713, Feb. 2005.

[7] Forrest, J. A., Dalnoki-Veres, K., The glass transition in thin polymer films, Advanced in Colloid and Interface Science, v. 94, p. 167-196, 2001.

[8] Lutkenhaus, J. L., Hrabak, K. D., McEnnis, K., Hammond, P. T., Elastomeric flexible free-standing hydrogen-bonded nanoscale assemblies, Journal of American Chemical Society, v. 127, p. 17228-17234, 2005.

[9] Shao, L., Lutkenhaus, J. L., Thermochemical properties of free-standing electrostatic layer-by-layer assemblies containing poly(allylamine hydrochloride) and poly(acrylic acid). Soft Matter, v. 6, p. 3363-3369, 2010.

[10] Liem, H., Cabanillas-Gonzalez, J., Etchegoin, P., Bradley, D. D. C., Glass transition temperatures of polymer thin films monitored by Raman scattering, Journal of Physics: Condensed Matter, v. 16, p. 721-728, 2004.

[11] Shen, Y. R., Hunt, J. H., Guyot-Sionnest, P., Observation of C-H stretch vibrational of monolayers of molecules optical sum-frequency generation, Chemical Physics Letters, v. 133, 189-192, 1987.

[12] Ficher, P., Buckingham, A. D., Surface second-order nonlinear optical activity. Journal of Optical Society of America B, v. 15, n. 12, p. 2951-2957, Dec. 1998.

[13] Shen, Y. R. Surf. Sci., v. 299/300, p. 551-562, 1994,.

[14] Han, S. H., Belkin, M. A., Shen, Y. R. Optically active second-harmonic generation from a uniaxial fluid medium. Optics Letters, v. 29, p. 1527-1529, 2004.

[15] Lvov, Y., Yamada, S., Kunitake, T., Non-linear optical effects in layer-by-layer alternate films of publications and an azobenzene-containing polyanion, Thin Solid Films, v. 300, p. 107-112, 1997.

[16] Figura, C., Neyman, P. J., Marciu, D., Brands, C., Murray, M. A., Hair, S., Davis, R. M., Miller, M. B., Heflin, J. R., Thermal stability and immersion solutions dependence of second order nonlinear optical ionically self-assembled films. In: Bradley, Donald D. C., Kippelen, Bernard (Ed.) Organic Photonic Materials and Devices II. San Jose, CA: SPIE, 2000. Proceedings of SPIE, v. 3939, p. 214-222, 2000. doi:10.1117/12.386378.

[17] Boyd, R. W., Nonlinear Optics, Academic Press, New York, 2004. 
[18] Shen, Y.-R., Fundaments of Nonlinear Optics, Academic Press: San Diego, CA, 1988, Chapter 25.

[19] Bloemberg, N., Nonlinear Optics; Addison-Wesley, New York, 1992.

[20] Sutherland, R. L., Handbook of Nonlinear Optics, Marcel Dekker, Inc., New York, 1996.

[21] Nalwa, H. S., Watanabe, T., Miyata, S., Organic materials for second-order nonlinear optics, in: H. S. Nalwa, S. Miyata (Ed.) Nonlinear Optics of Organic Molecules and Polymers, CRC Press, New York, 1997.

[22] Lambert, A. G., Davis, P. B., Neivandt, D. J., Implementing the Theory of Sum Frequency Generation Vibrational Spectroscopy: A Tutorial Review, Applied Spectroscopy Reviews, v. 40, p. 103-145, 2005.

[23] Prasad, P. N., Williams, D. J., Introduction to Nonlinear Optical Effects in Molecules and Polymers, John Wiley \& Sons, New York, 1991.

[24] Silva, H. S., Miranda, P. B., Molecular ordering of layer-by-layer polyelectrolyte films studied by sum-frequency vibrational spectroscopy. Journal of Physical Chemistry. B, v. 113, p. 10068-10071, 2009.

[25] Superfine, R., Huang, J. Y., Shen, Y.-R., Phase measurement for surface infrared-visible sum-frequency generation, Optics Letters, v. 15, n. 22, p. 1276-1278, 1990.

[26] Ji, N., Ostroverkhov, V., Chen C. Y., Shen Y.-R., Phase-sensitive sum-frequency vibrational spectroscopy and its application to studies of interfacial alkyl chains, Journal of American Chemistry Society, v. 129, p. 10056-10057, 2007.

[27] Shen, Y.-R. Optical second harmonic generation at interfaces. Annual Review of Physical Chemistry, v. 40, p. 327-350, Oct. 1989.

[28] Lvov, Y., Decher, G., Mohwald, H., Assembly, Structural Characterization, and Thermal Behavior of Layer-by-Layer Deposited Ultrathin Films of Poly(vinyl sulfate) and Poly(allylamine), Langmuir, v. 9, p. 481, 1993.

[29] Decher, G., Hong, J. D., Schimtt, J., Buildup of ultrathin multilayer films by a self-assembly process: III. Consecutively alternating adsorption of anionic and cationic polyelectrolytes on charged surfaces, Thin Solids Films, v. 210/211, 831, 1992

[30] Lvov, Y., Ariga, K., Ichinose, I., Kunitake, T., Molecular film assembly via layer-bylayer adsorption of oppositely charged macromolecules (Linear Polymer, Protein and Clay) and concanavalin A and glycogen, Thin Solid Films, Thin Solid Films, v. 284, p. 797, 1996

[31] Lvov, Y. M., Handbook of Surfaces and Materials, edited by H. S. Nalwa, Volume 3: Nanostructured Materials, Micelles, and Colloids, 2001. 
[32] Oliveira, Jr., Raposo, D., Handbook of Surfaces and Interfaces of Materials, edited by H. S. Nalwa, Volume 4: LB and Self-assembly Polymeric Films, 2001.

[33] Oliveira, Jr., O. N., He, J.-A., Zucoloto, V., Balasurbramaniam, S., Li, L., Nalwa, H. S. Kumar, J. and Tripathy, S. K., Handbook of Polyelectrolytes and Their Applications, edited by S. K. Tripathy, J. Kumar and H. S. Nalwa, Volume 1: Polyelectrolyte-Based Multilayers, Self-Assemblies and Nanoestructures, 2001.

[34] Silva, H. S., Uehara, T. M., Bergamaski, K., Miranda, P. B., Molecular ordering in layer-by-layer polyelectrolyte films studied by sum-frequency vibrational spectroscopy: The effects of drying procedures, Journal of Nanoscience and Nanotechnology, p. 3399-3405, 2008.

[35] Perinoto, A. C., Study of photoinduced birefringence in azopolymer and azodye selfassembled films. [thesis] Master Program in Physics, Physics Institute of São Carlos, University of São Paulo, São Carlos, 2005.

[36] Lopes, F. J. S., Study of molecular orientation in azopolimer self-assembled films using the Second-Harmonic generation (SHG) technique. [thesis] Master Program in Science and Engineering of Materials, Physics Institute of São Carlos, Chemistry Institute of São Carlos, Engineering School of São Carlos, University of São Paulo, São Carlos, 2006. (http://www.teses.usp.br/teses/disponiveis/88/88131/ tde-18122006-161256/pt-br.phpv)

[37] Figura, C. C., Second order nonlinear optics in ionically self-assembled thin films. [thesis] Faculty of Virginia Polytechnic, Institute and State University, Virginia, 1999.

[38] Neyman, P. J., Second-order nonlinear optical characteristics of nanoscale self-assembled multilayer organic films. [thesis] Faculty of Virginia Polytechnic, Institute and State University, Virginia, 2004.

[39] Heflin, J. R., Guzy, M. T., Neyman, P. J., Gaskins, K. J., Brands, C., Wang, Z., Gibson, H. W., Davis, R. M., Van Cott, K. E., Efficient, Thermally Stable, Second Order Nonlinear Optical Response in Organic Hybrid Covalent/Ionic Self-Assembled Films. Langmuir, v. 22, n. 13, p. 5723-5727, 2006.

[40] Heflin, J. R., Figura, C., Marciu, D., Liu, Y., Claus, R. O., Thickness dependence of second-harmonic generation in thin films fabricated from ionically self-assembled monolayers, Applied Physics Letters, v. 74, n. 4, p. 495-97, 1999.

[41] Lobo, R. F. M., Pereira-da-Silva, M. A., Raposo, M., Faria, R. M., Oliveira Jr., O. N., The morphology of layer-by-layer films of polymer polyelectrolyte studies by atomic force microscopy. Nanotechnology, v. 14, n. 1, p. 101-108, 2003.

[42] Souza, N. C., ; Zucolotto, V., Silva, J. R., Santos, F. R., Santos Junior, D. S., Balogh, D. T., Oliveira Junior., O. N., Giacometti, J. A., Morphology characterization of layer-bylayer films from PAH/MA-co-DR13: the role of film thickness. Journal of Colloid and Interface Science, v. 285, p. 544-550, 2005. 
[43] Choi, J., Rubner, M. R., Influence of the degree of ionization on weak polyelectrolyte multilayer assembly. Macromolecules, v. 38, n. 1, p. 116-124, 2005.

[44] Eisenthal, K. B., Liquid interfaces probed by second-harmonic and sum-frequency spectroscopy. Chemical Reviews, v. 96, p. 1343-1360, 1996.

[45] Silva, H. S., Miranda, P. B., Manuscript in preparation. 

Chapter 3

\title{
The Electromagnetic Force between Two Parallel Current Conductors Explained Using Coulomb's Law
}

\author{
Jan Olof Jonson \\ Additional information is available at the end of the chapter \\ http://dx.doi.org/10.5772/61221
}

\begin{abstract}
In this book chapter the electromagnetic force between two parallel electric conductors has been derived, applying thereby the effects of propagation delay and the Special Relativity theory, taking thereby also into count the thus far neglected effects introduced by the voltage sources of both circuits. This has been done for a specific case consisting of two rectangular circuits, aligned to each other along one of the long sides, at a distance that is short compared to the long sides. The intention in doing so is to make a meaningful application of the concept of "two parallel conductors of infinite length", so that it is possible to make a complete calculation of the force between the two circuits, avoiding thus making a vague claim as for example Maxwell, saying that the other parts of the conductors do not contribute to the force. What is radically new in this interpretation is that it is Coulomb's law that is responsible for the force.

Keywords: Ampère's Bridge, Ampère's Law, Coulomb's Law, electromagnetic force, Lorentz force, Lorentz transformation, parallel conductors, propagation delay, retarded action, Special Relativity theory, Sagnac effect, time dilatation
\end{abstract}

\section{Introduction}

In several papers evidence has been presented that is able to refute the widely recognized electromagnetic theory of today [1-5]. One such fundamental law is Lorentz' force law. Already 1997 a paper presented mathematical proofs showing that this law is unable to explain the repulsive force between collinear currents, demonstrated in the case of Ampère's bridge [1]. Even Graneau's exploding wires and Hering's pump cause difficulties, when trying to use Lorentz' force law in order to explain the effects that have been registered [6-11]. Therefore it is most exciting to explain one of the most frequent applications of Lorentz force law, the attractive force exerted between two parallel conductors carrying a DC current. Confessedly, 
others have made efforts in this respect already near 200 years ago, most famous of them Ampère [12]. Successors, like Grassmann, have made serious efforts to make the Lorentz force (in his early pre-Lorentz formulation) appear to be in accordance with Ampère's results [13]. In a more recent paper, this claim has been discarded through a mathematical analysis of Grassmann's derivation [14]. Additionally, in order to introduce a new theory, it must be able not only to explain experiments that a recognized theory cannot, but also to explain the experiments that it apparently is successful in explaining. One crucial phenomenon is that of light, or electromagnetic radiation. In fact, it has been possible to explain this, too, using basically Coulomb's law [15-18]. One may mention also electromagnetic induction [3-5].

The traditional methods have the benefit of being able to predict certain experiments, but not all. A new method must therefore in order to be better both done the first thing, but also be able to explain more evidence. By going back to the most basic well-corroborated law, Coulomb's law, one would expect a possible solution, provided one is very careful and applies mathematical method in a very strict fashion.

\section{Method}

\subsection{Description of a physical circuit describing two parallel conductors}

A geometry is defined, with two parallel conductors aligned to the $\mathrm{x}$ axis in a Cartesian coordinate system. They are assumed to be of a length that is very long compared to the rectilinear distance. Already Maxwell complained that this kind of analysis is incomplete, if not taking into account the track along which the respective currents returns to its origin and that the apparent conflict between the theory of Ampère and that of Grassmann is related to this [19]. This is done here, too.

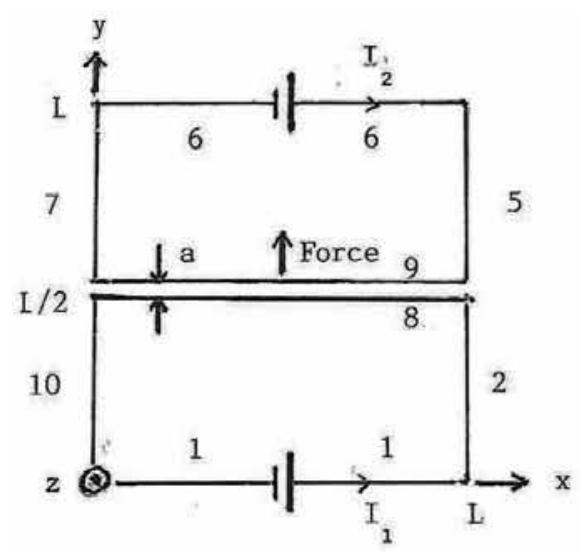

Figure 1. The configuration according to Ampère's bridge used in order to realize a "two-infinite-parallel-conductor" circuit 


\subsection{Mathematical treatment of 'infinite length' and other approximations}

A geometry is defined, with two parallel conductors aligned to the $\mathrm{x}$ axis in a Cartesian coordinate system. They are assumed to be of a length that is very long compared to the rectilinear distance between themselves. Already Maxwell complained that this kind of analysis is incomplete, if not taking into account the track along which the respective currents returns to its origin and that the apparent conflict between the theory of Ampère and that of Grassmann is related to this [20].This has been done here, too. What Maxwell did not specify closer, was the mathematical treatment of the concept 'infinite length'. It must of course be infinite with respect to a smaller entity in the circuit. Choosing quadrangle circuits, with side $L$ and the distance between the two sides of respective quadrangle circuit that are aligned along each other are situated at a distance $a$. Treating the length of respective side as infinite will be mathematically expressed through

$$
\mathbf{L}>\mathbf{a}
$$

where $L[\mathrm{~m}]$ denotes the length of the long side of respective circuit and $a[\mathrm{~m}]$ the distance between the two parallel branches that are close to each other. Generally, what concerns the calculations, the integration results do not display higher order terms that are negligible with respect to the dominating terms. Therefore, $\mathrm{a} \cong$ sign will often be used when accounting for the integration result.

\subsection{The electromagnetic force between two currents}

The two respective currents are being analyzed, using Coulomb's law, taking into account the effects of propagation delay and the Special Relativity Theory. The effects of the propagation delay were derived by this author in a paper 1997 [1], using thereby a different interpretation, than Feynman [21] and Jackson [22]. This author has been successful in showing what the fallacies are [2]. In the 1997 paper [1] it was crucial to the success in using Coulomb's law that the propagation delay was correctly being derived, both due to the "sending charges" of the "first conductor" and to the "receiving charges" of the "second conductor". Having done that analysis, it remains to take into account to the effects of the Special Relativity theory, especially the Lorentz transformation of lengths. Since that effect is related only to the relative movements of the two coordinate axes, and has nothing to do with the propagation delay an observer faces, it may be multiplied straightforwardly to the effect of propagation delay.

An electric current carried by a conductor implies that both the immobile lattice ions and the moving electrons contribute to the force exerted on other charges. In the case those are embedded in a neighboring electric conductor that is also carrying an electric current, they interact with both positive lattice ions and moving conductor electrons. This implies that altogether four kinds of interaction will take place, each demanding their own mathematical treatment respectively: from the positive ions of the first conductor to both kinds of charges of the second conductor and from the electrons of the first conductor to both kinds of charges 
of the second conductor. In the case of quadrangle circuits, the two respective currents appear both parallel and perpendicular to each other.

\subsection{Coulomb's law, basic formulation}

In order to integrate the contribution to the total force between two currents, carried by conductors, it is most suitable to use the differential force that an incremental segment gives rise to. If the immobile positive ions of both conductors are taken into account, one may write

$$
\Delta \overrightarrow{\mathbf{F}}=\frac{\rho_{1} \rho_{2} \Delta \mathbf{x}_{1} \Delta \mathbf{x}_{2} \overrightarrow{\mathbf{u}}_{\mathbf{r}}}{4 \pi \varepsilon_{0} \mathbf{r}^{2}}
$$

where $\Delta \vec{F}[\mathrm{~N}]$ denotes the incremental the electric force vector between two points of the two circuits, $\rho_{1}$ denotes the line charge density of the first circuit $[\mathrm{C} / \mathrm{m}], \rho_{2}$ the line charge density of the second circuit $[\mathrm{C} / \mathrm{m}] \Delta x_{1}[\mathrm{~m}]$ an infinitesimal length element along the $x$ direction of the first conductor, $\Delta x_{2}[\mathrm{~m})$ an infinitesimal length element along the $x$ direction of the second conductor, $\vec{r}[\mathrm{~m}]$ the distant vector from a point of the first conductor to a point of the second conductor $\vec{u}_{r}[\mathrm{~m}]$ a unit vector along the distant vector from a point of the first conductor to a point of the second conductor and $\varepsilon_{0}[\mathrm{~F} / \mathrm{m}]$ denotes the permittivity of vacuum, assuming thus the currents being aligned along the $x$ axis, but this will change throughout the chapter, dependent on which sections of the circuits are being treated, where the distant vector $r$ has been dissolved into its three Cartesian components.

$$
\overrightarrow{\mathbf{r}}=\left(\mathbf{x}_{2}-\mathbf{x}_{1}, \mathbf{y}_{2}-\mathbf{y}_{1}, 0\right)
$$

For simplicity the z-coordinate has been chosen to zero, based on the model with the circuits situated in the $x-y$-plane as defined in Fig. 1.

The force between the two currents will appear as the $y$ component of the total force, according to the following expression:

$$
\Delta \overrightarrow{\mathbf{F}} \bullet \overrightarrow{\mathbf{u}}_{\mathbf{y}}=\frac{\rho_{1} \rho_{2} \Delta \mathbf{x}_{1} \Delta \mathbf{x}_{2} \cdot \overrightarrow{\mathbf{u}}_{\mathbf{r}} \bullet \overrightarrow{\mathbf{u}}_{\mathbf{y}}}{4 \pi \varepsilon_{0} \mathbf{r}^{2}}
$$

A new variable has here been introduced, $\vec{u}_{y}[\mathrm{~m}]$ a unit vector along the positive $y$ axis

The case when both conductors are parallel to each other, especially along the $x$ axis.as for example between line 8 and 9 , in the figure above, the attractive or repulsive forces between them may be described as the $y$-component of the force in Eq. (2), as expressed in Eq. (4) above.

In this case, where all charges are stationary, there will be neither a propagation delay effect nor a relativistic effect due to the Lorentz contraction of one or both coordinates. 


\subsection{The method deriving propagation delay}

As was mentioned previously, the effects of propagation delay becomes relevant, when the charges are moving, so that the electric field due to a sending charge must be evaluated at an earlier time event than at the time, when the field was activated at a distant point. Going farther away, the travel time becomes longer. This is described in the following figure, which is based on the analysis first being done in another paper [1]:

The expression for the charge density that is being felt, or observed, at a point at a distance, when the charge density $\rho_{1}$ is due to individual charges moving with the velocity $\vec{v}_{1}$, was derived in the cited paper and is

$$
\rho_{1, \text { ret }}=\rho_{1}\left(1-\frac{\overrightarrow{\mathbf{v}}_{1} \bullet \overrightarrow{\mathbf{r}}}{\mathbf{r c}}\right)
$$

where $\rho_{1 \text { ret }}[\mathrm{C} / \mathrm{m}]$ denotes the retarded charge density of the first circuit, $\vec{v}_{1}[\mathrm{~m} / \mathrm{s}]$ the velocity of a charge element (electrons) of the first conductor and $c[\mathrm{~m} / \mathrm{s}]$ the speed of light, which may also be written:

$$
\rho_{1, \text { ret }}=\rho_{1}\left(1-\frac{\mathbf{v}_{1}}{\mathbf{c}} \cdot \cos \theta\right)
$$

where $\theta$ denotes the angle between the direction of the first current and the distant vector $\vec{r}$ $[\mathrm{m}]$.

This expression for the charge density will be used when the electrons are being studied at the first conductor.

In this connection it has to be mentioned that the traditional interpretation of propagation delay, as by Feynman [21] in his derivation of the Liénard-Wiechert potentials is fallacious [2]. However, additionally, there will appear a propagation delay effect also with respect to the charges receiving the action, since the farther away these charges are situated from the sending charges, the longer the way to travel, and hence, the charge density will appear to be smaller to the sender than what is the simultaneous charge density. Correspondingly, the expression for the charge density that is being felt by the sending charges, is also derived in the cited paper and is

$$
\rho_{2^{\prime}, \text { ret }}=\rho_{2}\left(1-\frac{\overrightarrow{\mathbf{v}}_{2} \bullet \overrightarrow{\mathbf{r}}}{\mathbf{r c}}\right)
$$

where $\rho_{\text {2ret }}[\mathrm{C} / \mathrm{m}]$ denotes the retarded charge density of the second circuit, $\vec{v}_{2}[\mathrm{~m} / \mathrm{s}]$ the velocity of a line charge element (electrons) of the second conductor, which may also be written 


$$
\rho_{2, \text { ret }}=\rho_{2}\left(1-\frac{\mathbf{v}_{2}}{\mathbf{c}} \cdot \cos \psi\right)
$$

where $\psi$ denotes the angle between the distant vector $\vec{r}[\mathrm{~m}]$ and the direction of the second current. This expression for the charge density will be used when the electrons are being studied at the second conductor.

\subsection{Coulomb's law, taking into account the effects of propagation delay}

The total force between two parts of respective circuit consists of the sum of the forces due to the four combinations of positive lattice ions and conduction electrons. Using the results of the preceding section, they are:

The first case is when the electric force due to positive charges of both conductors is being studied. The expression for the force will in this case be

$$
\Delta \overline{\mathbf{F}}_{+\rightarrow+} \bullet \overrightarrow{\mathbf{u}}_{\mathbf{y}}=\frac{\rho_{1} \rho_{2} \cdot \overrightarrow{\mathbf{u}}_{\mathbf{r}} \bullet \overrightarrow{\mathbf{u}}_{\mathbf{y}} \cdot \Delta \mathbf{x}_{1} \Delta \mathbf{x}_{2}}{4 \pi \varepsilon_{0} \mathbf{r}^{2}}
$$

where $\Delta \vec{F}_{+\rightarrow+}[\mathrm{N}]$ denotes the incremental force from the positive charges of the first conductor to the positive charges of the second conductor, still sticking to the case with both sections aligned along the $\mathrm{x}$ axis.

The second case, applying to conduction electrons of the first conductor affecting the positive immobile ions of the second conductor will be

$$
\Delta \overline{\mathbf{F}}_{-\rightarrow+} \bullet \overrightarrow{\mathbf{u}}_{\mathbf{y}}=\frac{-\rho_{1, \text { ret }} \cdot \rho_{2} \cdot \overrightarrow{\mathbf{u}}_{\mathbf{r}} \bullet \overrightarrow{\mathbf{u}}_{\mathbf{y}} \cdot \Delta \mathbf{x}_{1} \Delta \mathbf{x}_{2}}{4 \pi \varepsilon_{0} \mathbf{r}^{2}}
$$

where $\Delta \vec{F}_{-\rightarrow+}[\mathrm{N}]$ denotes the incremental force from the negative charges of the first conductor to the positive charges of the second conductor, thereby using Eq. (5) for the electrons, applying then the minus sign.

The third case, applying to the positive immobile ions of the first conductor exerting a force on the conduction electrons of the second conductor, will correspondingly be

$$
\Delta \overline{\mathbf{F}}_{+\rightarrow-} \bullet \overrightarrow{\mathbf{u}}_{\mathbf{y}}=\frac{\rho_{1}\left(-\rho_{2, \text { ret }}\right) \cdot \overrightarrow{\mathbf{u}}_{\mathbf{r}} \bullet \overrightarrow{\mathbf{u}}_{\mathbf{y}} \cdot \Delta \mathbf{x}_{1} \Delta \mathbf{x}_{2}}{4 \pi \varepsilon_{0} \mathbf{r}^{2}}
$$

where $\Delta \vec{F}_{+-}[\mathrm{N}]$ denotes the incremental force from the positive charges of the first conductor to the negative charges of the second conductor. 
Finally, the fourth case, applying to conduction electrons of the first conductor exerting a force on the conduction electrons of the second conductor will be:

$$
\Delta \overline{\mathbf{F}}_{-\rightarrow-} \bullet \overrightarrow{\mathbf{u}}_{\mathbf{y}}=\frac{\rho_{1, \text { ret }} \cdot \rho_{2, \text { ret }} \cdot \overrightarrow{\mathbf{u}}_{\mathbf{r}} \bullet \overrightarrow{\mathbf{u}}_{\mathbf{y}} \cdot \Delta \mathbf{x}_{1} \Delta \mathbf{x}_{2}}{4 \pi \varepsilon_{0} \mathbf{r}^{2}}
$$

where $\Delta \vec{F}_{-\rightarrow-}[\mathrm{N}]$ denotes the incremental force from the negative charges of the first conductor to the negative charges of the second conductor.

Adding these four contributions, keeping in mind also that

$$
\mathbf{I}_{1}=\rho_{1} \mathbf{v}_{1}
$$

where $I_{1}[\mathrm{~A}]$ denotes the current of the first conductor and

$$
\mathbf{I}_{2}=\rho_{2} \mathbf{v}_{2}
$$

where $I_{2}[\mathrm{~A}\}$ denotes the current of the second conductor and

$$
\frac{1}{\varepsilon_{0} \mu_{0}}=\mathbf{c}^{2}
$$

where $\mu_{0}$ [N / A2] denotes the permeability of vacuum, gives rise to the following expression for the total electric force between two electric currents, carried by conductors, and has been earlier derived [1], :

$$
\Delta \overrightarrow{\mathbf{F}}=\frac{\mu_{0} \mathbf{I}_{1} \mathbf{I}_{2} \cos \theta \cos \psi \cdot \Delta \mathbf{x}_{1} \Delta \mathbf{x}_{2}}{4 \pi \cdot \mathbf{r}^{2}} \cdot \overrightarrow{\mathbf{u}}_{\mathbf{r}}
$$

valid for the more general case, when the angles between two conductors may be chosen arbitrarily. In the case of two parallel conductors

$$
\theta=\psi
$$

This expression was also successful in predicting the repulsive force between the two parts of Ampère's bridge, whereas the Lorentz force wasn't [23]. 


\subsection{Coulomb's law, taking into account also the effects of the Special relativity theory}

The Special relativity theory implies that relative movement makes the extension of the moving things become smaller, as viewed from point of view of the laboratory system, thereby using the so-called standard configuration [24]. Hence, the vectors between moving and not-moving charges must necessarily be adjusted accordingly.

Hence, in order to derive more exact expressions for the electric force due to moving charges, all terms containing the distance vector between charges in the expressions above, even implicitly, must be modified by using the Lorentz contraction of space, more precisely the point of the vector that connects to a moving charge element [25]:

$$
\mathbf{x}^{\prime}=\frac{\mathbf{x}-\mathbf{v t}}{\sqrt{1-\mathbf{v}^{2} / \mathbf{c}^{2}}}
$$

where $x^{\prime}[\mathrm{m}]$ denotes the Lorentz transformation of the $x$ variable of the first circuit, movement assun'med to take place along the $x$ axis, i.e. Standard Configuration.where some authors prefer to use the term Lorentz factor [26] :

$$
\gamma(\mathbf{v})=\frac{1}{\sqrt{1-\mathbf{v}^{2} / \mathbf{c}^{2}}}
$$

where the Lorentz factor $\gamma(v)$ is dimensionless. One thing that must be taken into account, when the Lorentz transformation is concerned, is that every single incremental charge element that is moving must be denoted its own specific Lorentz transformation, since the Lorentz transformation is in fact dealing with single points moving with a velocity $v$. This becomes evident, when realizing that it is one event that is observed from two different coordinate systems, i.e. inertial systems [27]. This way of using the Lorentz transformation was furthermore successful in explaining the Sagnac effect [28].

When performing the calculations, a simplification will be introduced that the electrons carrying the both currents are propagating with the same velocity, i.e.

$$
\mathbf{v}_{1}=\mathbf{v}_{2}=\mathbf{v}
$$

For convenience it is here repeated that $\vec{v}_{1}[\mathrm{~m} / \mathrm{s}]$ denotes the velocity of a charge element (electrons) of the first conductor and $\vec{v}_{2}[\mathrm{~m} / \mathrm{s}]$ the velocity of a charge element (electrons) of the second conductor.This assumption in turn leads to

$$
\gamma\left(\mathbf{v}_{1}\right)=\gamma\left(\mathbf{v}_{2}\right)=\gamma(\mathbf{v})
$$


Some necessary preparations will also be needed before it is possible to perform the integrations, since the denominators of the terms that have to be integrated are on a form that makes integration on a closed form unfeasible, except for Eq. (9). Serial expansion of the denominators in the shape of binomial series [29] will make it possible to move terms of higher order of $\left(\frac{v}{c}\right)$ embedded in the $\gamma(v)$ terms of the denominator up to the numerator.

The Lorentz transformation according to the Special Relativity theory will be applied. By practical reasons the calculations have been separated into two categories: the parts of the conductors being interacting with each other being parallel respectively perpendicular to each other. It may be remarked that the Lorentz transformation will affect the infinitesimal incremental length element $d x$ in the denominator, so we will have

$$
\rho^{\mathbf{R}}=\gamma(\mathbf{v}) \cdot \frac{\mathbf{d Q}}{\mathbf{d} \mathbf{x}}
$$

where $\rho^{R}[\mathrm{C} / \mathrm{m}]$ denotes the Lorentz transformed line charge density.

Along a specified distance (according to the reference system $\mathrm{K}$ ) along the positive direction of movement, there will apparently be more charges and if this would be the case for all directions of movement, as when the charges are turning back to their origin, hence in the opposite direction, charges would seem to have been "created". However, a second effect, the 'time dilation', will make the opposite thing with the incremental length elements in that direction, and, hence, the sum of charges will remain unchanged, independently of from which coordinate system one prefers to observe the events.This is described in the following.

Additionally, there is also an effect, time dilatation that has to be taken into account. This effect causes the observer of $\mathrm{K}$ to register different time proceeding dependent of the direction of movement. The basic formula describing time dilatation is [28], [30]:

$$
\frac{\mathbf{d t}^{\prime}}{\mathrm{dt}}=\gamma(\mathbf{v})\left(1-\frac{\mathbf{v}}{\mathbf{c}^{2}} \frac{\mathbf{d x}}{\mathbf{d t}}\right)
$$

Assuming the velocity of the moving electrons being $v$, makes Eq.(23) transform to:

$$
\frac{\mathbf{d t ^ { \prime }}}{\mathbf{d t}}=\gamma(\mathbf{v})\left(1-\frac{\mathbf{v}^{2}}{\mathbf{c}^{2}}\right)
$$

where $t\left[\mathrm{~s}\right.$ ] denotes the time according to the $\mathrm{K}$ inertial system, $t^{\prime}[\mathrm{s}]$ the Lorentz transformed time being observed in $\mathrm{K}$ to take place in the $\mathrm{K}^{\prime}$ system.

Eq. (24) may in turn be re-written to 


$$
\frac{\mathbf{d t}^{\prime}}{\mathbf{d t}}=\frac{1}{\gamma(\mathbf{v})}
$$

which implies that $\gamma(v)$ times more charge will flow through a cross section given a certain time.

but logically, if assuming that the movement takes place in the opposite direction, applying this on Eq. (23) leads to approximately

$$
\frac{\mathbf{d t}^{\prime}}{\mathbf{d t}}=\gamma(\mathbf{v})
$$

in that case,

since

$$
\left(1+\frac{\mathbf{v}^{2}}{\mathbf{c}^{2}}\right) \cong 1-\frac{\mathbf{v}^{2}}{\mathbf{c}^{2}}
$$

if neglecting higher order terms of $\frac{v}{c}$.

Eq. (24) may accordingly be re-written to

$$
\frac{\mathbf{d t}^{\prime}}{\mathbf{d t}}=(\gamma(\mathbf{v}))^{3}
$$

This implies that $(\gamma(v))^{3}$ times less charge will flow through a cross section given a certain time, i.e. one will have to divide the force by $(\gamma(v))^{3}$.

In the case of a rotating disc, the Sagnac effect describes, how light being propagated along the direction of rotation will travel a longer distance than a light beam sent counterclockwise along the same disc [28]. If for example two electron currents flow along the same positive axis, there will not be any time dilatation when comparing them, but if the currents are directed in opposite direction to each other, the effect will be doubled. However, in the case of perpendicular currents, there will be no such difference, since all movement is perpendicular to each other and, hence, there will not be any difference in propagation time in either direction. This all will become more evident, when defining the incremental force contributions due to every incremental displacement. 


\subsection{The parts of the two conductors being parallel, aligned along the $x$ axis}

The expressions for the electric force due to the four combinations of charges (9), (10), (11) and (12) will be modified, using the Lorentz transformation. In the first case, described by Eq. (9), there will be no change, since all the charges are being at rest and, accordingly, no relativistic effects will be observed:

$$
\Delta \overrightarrow{\mathbf{F}}_{+\rightarrow+}^{\mathbf{R}} \bullet \overrightarrow{\mathbf{u}}_{\mathbf{y}}=\Delta \overline{\mathbf{F}}_{+\rightarrow+} \bullet \overrightarrow{\mathbf{u}}_{\mathbf{y}}=\frac{\rho_{1} \rho_{2} \cdot \overrightarrow{\mathbf{u}}_{\mathbf{r}} \bullet \overrightarrow{\mathbf{u}}_{\mathbf{y}} \cdot \Delta \mathbf{x}_{1} \Delta \mathbf{x}_{2}}{4 \pi \varepsilon_{0} \mathbf{r}^{2}}
$$

where

$$
\overrightarrow{\mathbf{r}}=\left(\mathbf{x}_{2}-\mathbf{x}_{1}, \mathbf{y}_{2}-\mathbf{y}_{1}, 0\right)
$$

where $\vec{r}[\mathrm{~m}]$ denotes the distant vector from a point of the first conductor to a point of the second conductor.

The case when conduction electrons of the first conductor are affecting the immobile, positive ions of the second conductor, as they are moving along the positive $\mathrm{x}$ axis, will be more complicated. Applying Eq. (22) and (25) leads implies that

$$
\Delta \overrightarrow{\mathbf{F}}_{-\rightarrow^{\prime}+}^{\mathbf{R}} \bullet \overrightarrow{\mathbf{u}}_{\mathbf{y}}=(\gamma(\mathbf{v}))^{2} \frac{-\rho_{1}\left(1-\frac{\mathbf{v}_{1}}{\mathbf{c}} \cos \theta^{\prime}\right) \rho_{2} \cdot \overrightarrow{\mathbf{u}}_{\mathbf{r}^{\prime}} \bullet \overrightarrow{\mathbf{u}}_{\mathbf{y}} \Delta \mathbf{x}_{1} \Delta \mathbf{x}_{2}}{4 \pi \varepsilon_{0}\left(\mathbf{r}^{\prime}\right)^{2}}
$$

where $\theta^{\prime}$ denotes the Lorentz transformed angle for this case, $r^{\prime}[\mathrm{m}]$ the Lorentz transformed absolute value of the Lorentz transformed distance vector $\vec{r}^{\prime}[\mathrm{m}]$ and $\vec{u}_{r^{\prime}}[\mathrm{m}]$ a unit vector along the Lorentz transformed distant vector $\dot{r}^{\prime}$ from a point of the first conductor to a point of the second conductor, where

$$
\overrightarrow{\mathbf{r}}^{\prime}=\left(\mathbf{x}_{2}-\frac{\mathbf{x}_{1}}{\gamma\left(\mathbf{v}_{1}\right)}, \mathbf{y}_{2}-\mathbf{y}_{1}, 0\right)
$$

and

$$
\cos \theta^{\prime}=\frac{\mathbf{x}_{2}-\frac{\mathbf{x}_{1}}{\gamma\left(\mathbf{v}_{1}\right)}}{\mathbf{r}^{\prime}}
$$

The case when the positive, immobile ions of the first conductors are is exerting a force on the conduction electrons of the second conductors, as they are moving along the positive $x$ axis, will be also be more complicated. Applying Eq. (22) and (25) leads to: 


$$
\Delta^{2} \overrightarrow{\mathbf{F}}_{+\rightarrow^{\prime}-}^{\mathbf{R}} \bullet \overrightarrow{\mathbf{u}}_{\mathbf{y}}=\left(\gamma(\mathbf{v})^{2}\right) \cdot \frac{-\rho_{1} \rho_{2}\left(1-\frac{\mathbf{v}_{2}}{\mathbf{c}} \cos \theta^{\prime \prime}\right) \cdot \overrightarrow{\mathbf{u}}_{\mathbf{r}^{\prime \prime}} \bullet \overrightarrow{\mathbf{u}}_{\mathbf{y}} \Delta \mathbf{x}_{1} \Delta \mathbf{x}_{2}}{4 \pi \varepsilon_{0}\left(\mathbf{r}^{\prime \prime}\right)^{2}}
$$

where $\theta^{\prime \prime}$ denotes the Lorentz transformed angle for this case, $r^{\prime \prime}[\mathrm{m}]$ the Lorentz transformed absolute value of the Lorentz transformed distance vector $\vec{r}^{\prime \prime}[\mathrm{m}]$ and $\vec{u}_{r^{\prime \prime}}[\mathrm{m}]$ a unit vector along the Lorentz transformed distant vector ${ }^{\prime}$ " from a point of the first conductor to a point of the second conductor, where

$$
\begin{gathered}
\overrightarrow{\mathbf{r}}^{\prime \prime}=\left(\frac{\mathbf{x}_{2}}{\gamma\left(\mathbf{v}_{2}\right)}-\mathbf{x}_{1}, \mathbf{y}_{2}-\mathbf{y}_{1}, 0\right) \\
\cos \theta^{\prime \prime}=\frac{\frac{\mathbf{x}_{2}}{\gamma\left(\mathbf{v}_{2}\right)}-\mathbf{x}_{1}}{\mathbf{r}^{\prime \prime}}
\end{gathered}
$$

In the case either of the currents flows in the opposite direction, instead of Eq. (25) should be applied Eq. (28) together with Eq. (22) on the equation dealing with that current (i.e. either Eq. (31) or Eq. (34).

The last case, the conduction electrons of the first conductor affecting the conduction electrons of the second conductor, there will be no time dilation effect, but still a Lorentz contraction., This will give rise to the following expression:

$$
\Delta \overrightarrow{\mathbf{F}}_{-\rightarrow^{\prime}-}^{\mathbf{R}} \bullet \overrightarrow{\mathbf{u}}_{\mathbf{y}}=\left(\gamma(\mathbf{v})^{2}\right) \cdot \frac{\rho_{1}\left(1-\frac{\mathbf{v}_{1}}{\mathbf{c}} \cdot \cos \theta^{\prime \prime \prime}\right) \rho_{2}\left(1-\frac{\mathbf{v}_{2}}{\mathbf{c}} \cdot \cos \theta^{\prime \prime \prime}\right) \cdot \overrightarrow{\mathbf{u}}_{\mathbf{r}^{\prime \prime \prime}} \bullet \overrightarrow{\mathbf{u}}_{\mathbf{y}} \cdot \Delta \mathbf{x}_{1} \Delta \mathbf{x}_{2}}{4 \pi \varepsilon_{0}\left(\mathbf{r}^{\prime \prime \prime}\right)^{2}}
$$

where

$$
\overrightarrow{\mathbf{r}}^{\prime \prime}=\left(\frac{\mathbf{x}_{2}}{\gamma\left(\mathbf{v}_{2}\right)}-\frac{\mathbf{x}_{1}}{\gamma\left(\mathbf{v}_{1}\right)}, \mathbf{y}_{2}-\mathbf{y}_{1}, 0\right)
$$

and

$$
\cos \theta^{\prime \prime}=\frac{\frac{\mathbf{x}_{2}}{\gamma\left(\mathbf{v}_{2}\right)}-\frac{\mathbf{x}_{1}}{\gamma\left(\mathbf{v}_{1}\right)}}{\mathbf{r}^{\prime \prime}}
$$


where $\theta$ " ' denotes the Lorentz transformed angle for this case, $r$ "' $[\mathrm{m}]$ the Lorentz transformed absolute value of the Lorentz transformed distance vector $\vec{r}^{\prime \prime \prime}[\mathrm{m}]$ and $\vec{u}_{r^{\prime \prime}}[\mathrm{m}]$ a unit vector along the Lorentz transformed distant vector ${ }^{\prime}$ '" from a point of the first conductor to a point of the second conductor. Both the conduction electrons of the first circuit and the conduction electrons of the second circuit are moving, thereby both implying the need for a Lorentz transformation. Hence,

$$
\Delta \overrightarrow{\mathbf{F}}_{-\rightarrow-}^{\mathbf{R}} \bullet \overrightarrow{\mathbf{u}}_{\mathbf{y}}=\frac{\rho_{1}\left(1-\frac{\mathbf{v}_{1}}{\mathbf{c}} \cdot \cos \theta^{\prime \prime \prime}\right) \cdot \rho_{2}\left(1-\frac{\mathbf{v}_{2}}{\mathbf{c}} \cdot \cos \theta^{\prime \prime \prime}\right) \cdot\left(\mathbf{y}_{2}-\mathbf{y}_{1}\right) \cdot \Delta \mathbf{x}_{1} \Delta \mathbf{x}_{2}}{4 \pi \varepsilon_{0} \cdot\left(\frac{1}{\left((\gamma(\mathbf{v}))^{2}\right)} \cdot\left(\mathbf{x}_{2}-\mathbf{x}_{1}\right)^{2}+\left(\mathbf{y}_{2}-\mathbf{y}_{1}\right)^{2}\right)^{\frac{3}{2}}} .
$$

Adding the four contributions to the force, assuming the velocities being equal, expressed through Eq. (29), Eq. (31), Eq. (34) and Eq. (37) gives, thereby using Eq. (34) (39), the result for the case the currents are being directed along the same direction:

$$
\Delta \mathbf{F}_{\text {total }}^{\mathbf{R}} \bullet \overrightarrow{\mathbf{u}}_{\mathbf{y}} \cong \frac{\rho_{1} \Delta \mathbf{x}_{1} \cdot \rho_{2} \Delta \mathbf{x}_{2} \cdot\left(\mathbf{y}_{2}-\mathbf{y}_{1}\right)}{4 \pi \varepsilon_{0} \mathbf{r}^{5}} \cdot\left(\left(\frac{\mathbf{v}}{\mathbf{c}}\right)^{2}\left(-1+\cos ^{2} \theta\right)\right)
$$

where $\vec{F}_{\text {total }}^{R}[\mathrm{~N}]$ denotes the total force due to Lorentz transformed entities, due to the sum of the contributions from all participating charges, for two specified sections of respective conductor, or, using Eq. (13), Eq. (14) and Eq. (15) one may as well write

$$
\Delta \overrightarrow{\mathbf{F}}_{\text {total }}^{\mathbf{R}} \bullet \overrightarrow{\mathbf{u}}_{\mathbf{y}}=\frac{\mu_{0} \mathbf{I}_{1} \mathbf{I}_{2} \Delta \mathbf{x}_{1} \Delta \mathbf{x}_{2} \cdot\left(\mathbf{y}_{2}-\mathbf{y}_{1}\right)}{4 \pi \cdot \mathbf{r}^{3}} \cdot\left(-1+\frac{\left(\mathbf{x}_{2}-\mathbf{x}_{1}\right)^{2}}{\mathbf{r}^{2}}\right)
$$

One may already now observe the leading term -1 in Eq. (42), pointing to the attractive force between parallel electric currents.

When the direction is the opposite of either current, in the case either or both of the currents flows along the negative $x$ axis, instead of Eq. (25) Eq. (28) would have to be applied together with Eq. (22) on the equation dealing with that current (i.e. either Eq. (31), Eq. (34) or Eq. (37).

Eq. (29) will remain unchanged, since in that case both charges are at rest, implying thus no relativistic effects.

The result is the following modified versions of these equations:

$$
\Delta \overrightarrow{\mathbf{F}}_{-\rightarrow^{\prime}+}^{\mathbf{R}} \bullet \overrightarrow{\mathbf{u}}_{\mathbf{y}}=(\gamma(\mathbf{v}))^{4} \frac{-\rho_{1}\left(1-\frac{\mathbf{v}_{1}}{\mathbf{c}} \cos \theta^{\prime}\right) \rho_{2} \cdot \overrightarrow{\mathbf{u}}_{\mathbf{r}^{\prime}} \bullet \overrightarrow{\mathbf{u}}_{\mathbf{y}} \Delta \mathbf{x}_{1} \Delta \mathbf{x}_{2}}{4 \pi \varepsilon_{0}\left(\mathbf{r}^{\prime}\right)^{2}}
$$




$$
\begin{gathered}
\Delta^{2} \overrightarrow{\mathbf{F}}_{+\rightarrow^{\prime}-}^{\mathbf{R}} \bullet \overrightarrow{\mathbf{u}}_{\mathbf{y}}=\cdot \frac{-\rho_{1} \rho_{2}\left(1-\frac{\mathbf{v}_{2}}{\mathbf{c}} \cos \theta^{\prime \prime}\right) \cdot \overrightarrow{\mathbf{u}}_{\mathbf{r}} \bullet \overrightarrow{\mathbf{u}}_{\mathbf{y}} \Delta \mathbf{x}_{1} \Delta \mathbf{x}_{2}}{4 \pi \varepsilon_{0}\left(\mathbf{r}^{\prime \prime}\right)^{2}} \\
\Delta \overrightarrow{\mathbf{F}}_{-\rightarrow^{\prime}-}^{\mathbf{R}} \bullet \overrightarrow{\mathbf{u}}_{\mathbf{y}}=\left(\gamma(\mathbf{v})^{2}\right) \cdot\left(1+2 \cdot\left(\frac{\mathbf{v}}{\mathbf{c}}\right)^{2}\right) \cdot \frac{\rho_{1}\left(1-\frac{\mathbf{v}_{1}}{\mathbf{c}} \cdot \cos \theta^{\prime \prime \prime}\right) \rho_{2}\left(1-\frac{\mathbf{v}_{2}}{\mathbf{c}} \cdot \cos \theta^{\prime \prime \prime}\right) \cdot \overrightarrow{\mathbf{u}}_{\mathbf{r}^{\prime \prime \prime}} \bullet \overrightarrow{\mathbf{u}}_{\mathbf{y}} \cdot \Delta \mathbf{x}_{1} \Delta \mathbf{x}_{2}}{4 \pi \varepsilon_{0}\left(\mathbf{r}^{\prime \prime \prime}\right)^{2}}
\end{gathered}
$$

The multiplicative term is the sum of the effect of both the forwards moving electrons of the first conductor and the backwards moving electrons of the second conductor.

Adding the four contributions to the force, setting the velocities equal, expressed through Eq. (29), Eq. (43), Eq. (44) and Eq. (45) gives, thereby using Eq. (39), the result for the case the currents are flowing opposite to each other, along the $x$ axis:

$$
\Delta \mathbf{F}_{\text {total }}^{\mathbf{R}} \bullet \overrightarrow{\mathbf{u}}_{\mathbf{y}} \cong \frac{\rho_{1} \Delta \mathbf{x}_{1} \cdot \rho_{2} \Delta \mathbf{x}_{2} \cdot\left(\mathbf{y}_{2}-\mathbf{y}_{1}\right)}{4 \pi \varepsilon_{0} \mathbf{r}^{5}} \cdot\left(\left(\frac{\mathbf{v}}{\mathbf{c}}\right)^{2}\left(1-\cos ^{2} \theta\right)\right)
$$

which apparently implies that the force will be of equal strength, but with opposite sign. In that case. Using Eq. (13), Eq. (14) and Eq. (15) one may as well write

$$
\Delta \overrightarrow{\mathbf{F}}_{\text {total }}^{\mathbf{R}} \bullet \overrightarrow{\mathbf{u}}_{\mathbf{y}}=\frac{\mu_{0} \mathbf{I}_{1} \mathbf{I}_{2} \Delta \mathbf{x}_{1} \Delta \mathbf{x}_{2} \cdot\left(\mathbf{y}_{2}-\mathbf{y}_{1}\right)}{4 \pi \cdot \mathbf{r}^{3}} \cdot\left(1-\frac{\left(\mathbf{x}_{2}-\mathbf{x}_{1}\right)^{2}}{\mathbf{r}^{2}}\right)
$$

\subsubsection{Branch 8 to 9}

In order to calculate the force between the two branches 8 and 9, one has to insert

$$
\mathbf{y}_{2}-\mathbf{y}_{1}=\mathbf{a}
$$

where $a[\mathrm{~m}]$ indicates the distance between the two parallel branches that are close to each other, in Eq. (42), or

$$
\int \mathbf{d} \overrightarrow{\mathbf{F}}_{\text {total }}^{\mathbf{R}} \bullet \overrightarrow{\mathbf{u}}_{\mathbf{y}} \cong \int_{\mathbf{x}_{2}=0}^{\mathbf{L}} \int_{\mathbf{x}_{1}=0}^{\mathbf{L}} \frac{\mu_{0} \mathbf{I}_{1} \mathbf{I}_{2} \mathbf{d} \mathbf{x}_{1} \mathbf{d} \mathbf{x}_{2} \cdot \mathbf{a}}{4 \pi \cdot \mathbf{r}^{3}} \cdot\left(-1+\frac{\left(\mathbf{x}_{2}-\mathbf{x}_{1}\right)^{2}}{\mathbf{r}^{2}}\right)
$$

Solving the integral gives the result 


$$
\mathbf{F}_{\text {total }}^{\mathbf{R}}(8 \rightarrow 9)=\int \mathrm{d} \overrightarrow{\mathbf{F}}_{\text {total }}^{\mathbf{R}} \bullet \overrightarrow{\mathbf{u}}_{\mathbf{y}} \cong-\frac{4}{3} \frac{\mu_{0} \mathbf{I}_{1} \mathbf{I}_{2} \mathbf{L}}{4 \pi \cdot \mathbf{a}}
$$

where $F_{\text {total }}^{R}(n \rightarrow n)[\mathrm{N}]$ indicates the total force due to Lorentz transformed entities, due to the sum of the contributions from all participating charges, for two specified sections of respective conductor,

The negative sign implies that it is a question of an attractive force.

\subsubsection{Branch 8 to 6}

In this case the currents are of opposite direction and hence, instead of applying Eq. (25) on the electrons of branch 6, one will have to apply Eq. (28).

Here

$$
\mathbf{y}_{2}-\mathbf{y}_{1}=\frac{\mathbf{L}}{2}
$$

The summation of the contributions from all the charges will in this case be

$$
\int \mathbf{d} \overrightarrow{\mathbf{F}}_{\text {total }}^{\mathbf{R}} \bullet \overrightarrow{\mathbf{u}}_{\mathbf{y}} \cong \int_{\mathbf{x}_{2}=0}^{\mathbf{L}} \int_{\mathbf{x}_{1}=0}^{\mathbf{L}} \frac{\mu_{0} \mathbf{I}_{1} \mathbf{I}_{2} \mathbf{d x} \mathbf{x}_{1} \mathbf{d x} \mathbf{x}_{2} \cdot\left(\frac{\mathbf{L}}{2}\right)}{4 \pi \cdot \mathbf{r}^{3}} \cdot\left(1-\frac{\left(\mathbf{x}_{2}-\mathbf{x}_{1}\right)^{2}}{\mathbf{r}^{2}}\right)
$$

Solving the integral gives the result:

$$
\mathbf{F}_{\text {total }}^{\mathbf{R}}(8 \rightarrow 6)=\int \mathrm{d} \overrightarrow{\mathbf{F}}_{\text {total }}^{\mathbf{R}} \bullet \overrightarrow{\mathbf{u}}_{\mathbf{y}} \cong \frac{\mu_{0} \mathbf{I}_{1} \mathbf{I}_{2}}{4 \pi} \cdot \frac{2}{3} \cdot\left(\frac{9}{\sqrt{5}}-1\right)
$$

\subsubsection{Branch 8 to the voltage source of branch 6}

The claim that the voltage source is playing a role in the balance of forces between electric currents was shown already in an earlier paper [31] and accordingly, Eq. (49) would have to be replaced by an integral applying an impulse current instead of $I_{2}$, namely .$I_{2} \cdot 3 L \cdot \delta\left(x_{2}-L / 2,\right)$

It has to be observed that the direction of that current is opposite to $I_{2}$, implying thereby the need for using Eq. (42), i.e. the current $I_{1}$ has the same direction as $I_{2} \cdot L \cdot \delta\left(x_{2}-L / 2\right.$, ).

In this case $y_{2}-y_{1}=\frac{L}{2}$ 
The total contribution to the force here will accordingly be:

$$
\int \mathbf{d} \overrightarrow{\mathbf{F}}_{\text {total }}^{\mathbf{R}} \bullet \overrightarrow{\mathbf{u}}_{\mathbf{y}} \cong \int_{\mathbf{x}_{2}=0}^{\mathbf{L}} \int_{\mathbf{x}_{1}=0}^{\mathbf{L}} \frac{\mu_{0} \mathbf{I}_{1} \cdot\left(3 \mathbf{L} \cdot \mathbf{I}_{2} \delta\left(\mathbf{x}_{2}-\frac{\mathbf{L}}{2}\right) \cdot \mathbf{d} \mathbf{x}_{1} \mathbf{d} \mathbf{x}_{2} \cdot\left(\frac{\mathbf{L}}{2}+\mathbf{a}\right)\right.}{4 \pi \cdot \mathbf{r}^{3}} \cdot\left(-1+\frac{\left(\mathbf{x}_{2}-\mathbf{x}_{1}\right)^{2}}{\mathbf{r}^{2}}\right)
$$

Solving the integral gives the result:

$$
\mathbf{F}_{\text {total }}^{\mathbf{R}}(8 \rightarrow \mathbf{V S} 6)=\int \mathbf{d} \overrightarrow{\mathbf{F}}_{\text {total }}^{\mathbf{R}} \bullet \overrightarrow{\mathbf{u}}_{\mathbf{y}} \cong \frac{\mu_{0} \mathbf{I}_{1} \mathbf{I}_{2}}{4 \pi} \cdot(-5 \sqrt{2})
$$

where $V S n$ indicates a voltage source with applied branch number.

\subsubsection{Branch 1 to 9}

Since the geometry is exactly the same as in the case, when branch 8 is affecting branch 6 , the integral equation will be almost the same, even though here

$$
\mathbf{y}_{2}-\mathbf{y}_{1}=\frac{\mathbf{L}}{2}
$$

The summation of the contributions from all the charges will in this case be

$$
\int \mathbf{d} \overrightarrow{\mathbf{F}}_{\text {total }}^{\mathbf{R}} \bullet \overrightarrow{\mathbf{u}}_{\mathbf{y}} \cong \int_{\mathbf{x}_{2}=0}^{\mathbf{L}} \int_{\mathbf{x}_{1}=0}^{\mathbf{L}} \frac{\mu_{0} \mathbf{I}_{1} \mathbf{I}_{2} \mathbf{d} \mathbf{x}_{1} \mathbf{d} \mathbf{x}_{2} \cdot\left(\frac{\mathbf{L}}{2}\right)}{4 \pi \cdot \mathbf{r}^{3}} \cdot\left(1-\frac{\left(\mathbf{x}_{2}-\mathbf{x}_{1}\right)^{2}}{\mathbf{r}^{2}}\right)
$$

The integration result according to Eq. (53) can be used straightforwardly, since the small difference in $y_{2}-y_{1}$ compared to $\mathrm{Eq}(51)$ will be negligible.

$$
\mathbf{F}_{\text {tottal }}^{\mathbf{R}}(1 \rightarrow 9)=\int_{\mathbf{x}_{2}=0}^{\mathbf{L}} \mathbf{d} \mathbf{x}_{2} \int_{\mathbf{x}_{1}=0}^{\mathbf{L}} \mathbf{d} \mathbf{x}_{1} \mathbf{F}_{\text {total }}^{\mathbf{R}} \bullet \overrightarrow{\mathbf{u}}_{\mathbf{y}} \cong \frac{\mathbf{I}_{1} \mathbf{I}_{2}}{4 \pi \varepsilon_{0}} \cdot \frac{2}{3} \cdot\left(\frac{9}{\sqrt{5}}-1\right)
$$

\subsubsection{Branch 1 to 6}

In order to calculate the force between the two branches 1 and 6 , one will have to set

$$
\mathbf{y}_{2}-\mathbf{y}_{1}=\mathbf{L}
$$


in Eq. (42), or

$$
\int \mathbf{d} \overrightarrow{\mathbf{F}}_{\text {total }}^{\mathbf{R}} \bullet \overrightarrow{\mathbf{u}}_{\mathbf{y}} \cong \int_{\mathbf{x}_{2}=0}^{\mathbf{L}} \int_{\mathbf{x}_{1}=0}^{\mathbf{L}} \frac{\mu_{0} \mathbf{I}_{1} \mathbf{I}_{2} \mathbf{d} \mathbf{x}_{1} \mathbf{d} \mathbf{x}_{2} \cdot \mathbf{L}}{4 \pi \cdot \mathbf{r}^{3}} \cdot\left(-1+\frac{\left(\mathbf{x}_{2}-\mathbf{x}_{1}\right)^{2}}{\mathbf{r}^{2}}\right)
$$

Solving the integral gives the result

$$
\mathbf{F}_{\text {total }}^{\mathbf{R}}(1 \rightarrow 6)=\int_{\mathbf{x}_{2}=0}^{\mathbf{L}} \mathbf{d} \mathbf{x}_{2} \int_{\mathbf{x}_{1}=0}^{\mathbf{L}} \mathbf{d} \mathbf{x}_{1} \mathbf{F}_{\text {total }}^{\mathbf{R}} \bullet \overrightarrow{\mathbf{u}}_{\mathbf{y}} \cong \frac{\mu_{0} \mathbf{I}_{1} \mathbf{I}_{2}}{4 \pi} \cdot \frac{2}{3} \cdot\left(1-\frac{3}{\sqrt{2}}\right)
$$

\subsubsection{Branch 1 to the voltage source of branch 6}

In this case involving a voltage source, one will have to apply an impulse current instead of $I_{2}$, namely $.3 I_{2} \cdot L \cdot \delta\left(x_{2}-L / 2,\right)$

It has to be observed that the direction of that current is opposite to $I_{2}$, implying thus the need for using Eq. (42), i.e. the current $I_{1}$ has the same direction as $I_{2} \cdot L \cdot \delta\left(x_{2}-L / 2\right)$.

In this case $y_{2}-y_{1}=L$

The total contribution to the force here will be:

$$
\int \mathrm{d} \overrightarrow{\mathrm{F}}_{\text {total }}^{\mathbf{R}} \bullet \overrightarrow{\mathbf{u}}_{\mathbf{y}} \cong \int_{\mathbf{x}_{2}=0}^{\mathbf{L}} \int_{\mathbf{x}_{1}=0}^{\mathbf{L}} \frac{\mu_{0} \cdot \mathbf{I}_{1} \cdot 3 \mathbf{L} \cdot \mathbf{I}_{2} \cdot \delta\left(\mathbf{x}_{2}-\frac{\mathbf{L}}{2}\right) \cdot \mathbf{d} \mathbf{x}_{1} \mathbf{d} \mathbf{x}_{2} \cdot \mathbf{L}}{4 \pi \cdot \mathbf{r}^{3}} \cdot\left(-1+\frac{\left(\mathbf{x}_{2}-\mathbf{x}_{1}\right)^{2}}{\mathbf{r}^{2}}\right)
$$

Solving the integral gives the result:

$$
\mathbf{F}_{\text {total }}^{\mathbf{R}}(\mathbf{V S 1} \rightarrow 6)=\int \mathrm{d} \overrightarrow{\mathbf{F}}_{\text {total }}^{\mathbf{R}} \bullet \overrightarrow{\mathbf{u}}_{\mathbf{y}} \cong \frac{\mu_{0} \mathbf{I}_{1} \mathbf{I}_{2}}{4 \pi} \cdot\left(\frac{28}{5 \sqrt{5}}\right)
$$

\subsubsection{Voltage source of branch 1 to branch 9}

In this case involving a voltage source, one will have to apply an impulse current instead of $I_{1}$, namely.$I_{1} \cdot 3 L \cdot \delta\left(x_{1}-L / 2,\right)$

It has to be observed that the direction of that current is opposite to $I_{2}$, implying thus the need for using Eq. (42), i.e. the current $I_{2}$ has the same direction as $I_{1} \cdot 3 L \cdot \delta\left(x_{1}-L / 2,\right)$.

In this case $y_{2}-y_{1}=\frac{L}{2}$ 
The total contribution to the force here will be:

$$
\int \mathbf{d} \overrightarrow{\mathbf{F}}_{\text {total }}^{\mathbf{R}} \bullet \overrightarrow{\mathbf{u}}_{\mathbf{y}} \cong \int_{\mathbf{x}_{2}=0}^{\mathbf{L}} \int_{\mathbf{x}_{1}=0}^{\mathbf{L}} \frac{\mu_{0} \cdot 3 \mathbf{L} \cdot \mathbf{I}_{1} \delta\left(\mathbf{x}_{1}-\frac{\mathbf{L}}{2}\right) \cdot \mathbf{I}_{2} \cdot \mathbf{d} \mathbf{x}_{1} \mathbf{d} \mathbf{x}_{2} \cdot\left(\frac{\mathbf{L}}{2}\right)}{4 \pi \cdot \mathbf{r}^{3}} \cdot\left(-1+\frac{\left(\mathbf{x}_{2}-\mathbf{x}_{1}\right)^{2}}{\mathbf{r}^{2}}\right)
$$

Solving the integral gives the result:

$$
\mathbf{F}_{\text {total }}^{\mathbf{R}}(\mathbf{V S 1} \rightarrow 9)=\int \mathrm{d}_{\mathbf{F}_{\text {total }}^{\mathbf{R}}}^{\mathbf{R}} \bullet \overrightarrow{\mathbf{u}}_{\mathbf{y}} \cong \frac{\mu_{0} \mathbf{I}_{1} \mathbf{I}_{2}}{4 \pi} \cdot(-5 \sqrt{2})
$$

\subsubsection{Voltage source of branch 1 to branch 6}

In this case involving a voltage source, one will necessarily have to apply an impulse current instead of $I_{1}$, namely.$_{1} \cdot 3 L \cdot \delta\left(x_{1}-L / 2\right.$, )

It has to be observed that the direction of that current is opposite to $I_{2}$, implying thus the need for using Eq. (42), i.e. the current $I_{2}$ has the same direction as $I_{1} \cdot 3 L \cdot \delta\left(x_{1}-L / 2\right.$, ).

In this case $y_{2}-y_{1}=L$

The total contribution to the force here will be:

$$
\int \mathbf{d} \overrightarrow{\mathbf{F}}_{\text {total }}^{\mathbf{R}} \bullet \overrightarrow{\mathbf{u}}_{\mathbf{y}} \cong \int_{\mathbf{x}_{2}=0}^{\mathbf{L}} \int_{\mathbf{x}_{1}=0}^{\mathbf{L}} \frac{\mu_{0} \cdot 3 \mathbf{L} \cdot \mathbf{I}_{1} \delta\left(\mathbf{x}_{1}-\frac{\mathbf{L}}{2}\right) \cdot \mathbf{I}_{2} \cdot \mathbf{d} \mathbf{x}_{1} \mathbf{d} \mathbf{x}_{2} \cdot \mathbf{L}}{4 \pi \cdot \mathbf{r}^{3}} \cdot\left(-1+\frac{\left(\mathbf{x}_{2}-\mathbf{x}_{1}\right)^{2}}{\mathbf{r}^{2}}\right)
$$

Solving the integral gives the result:

$$
\mathbf{F}_{\text {total }}^{\mathbf{R}}(\mathbf{V S 1} \rightarrow 6)=\int \mathrm{d} \overrightarrow{\mathbf{F}}_{\text {total }}^{\mathbf{R}} \bullet \overrightarrow{\mathbf{u}}_{\mathbf{y}} \cong \frac{\mu_{0} \mathbf{I}_{1} \mathbf{I}_{2}}{4 \pi} \cdot\left(\frac{28}{5 \sqrt{5}}\right)
$$

\subsubsection{Voltage source of branch 1 to the voltage source of branch 6}

In this case Eq. (37) will again be used, but in this case replacing both the currents $I_{1}$ and $I_{2}$ in Eq. (42) with impulse currents, namely $I_{1} \cdot 3 L \cdot \delta\left(x_{2}-L / 2\right.$, ) and $I_{2} \cdot 3 L \cdot \delta\left(x_{2}-L / 2\right.$, ).

In this case $y_{2}-y_{1}=L$

The total contribution to the force will in this case be: 
$\int \mathbf{d} \overrightarrow{\mathbf{F}}_{\text {total }}^{\mathbf{R}} \bullet \overrightarrow{\mathbf{u}}_{\mathbf{y}} \cong \int_{\mathbf{x}_{2}=0}^{\mathrm{L}} \int_{\mathbf{x}_{1}=0}^{\mathrm{L}} \frac{\mu_{0} \cdot 3 \mathbf{L} \cdot \mathbf{I}_{1} \delta\left(\mathbf{x}_{1}-\frac{\mathbf{L}}{2}\right) \cdot 3 \mathbf{L} \cdot \mathbf{I}_{2} \cdot \delta\left(\mathbf{x}_{2}-\frac{\mathbf{L}}{2}\right) \cdot \mathbf{d} \mathbf{x}_{1} \mathbf{d x} \mathbf{x}_{2} \cdot \mathbf{L}}{4 \pi \cdot \mathbf{r}^{3}} \cdot\left(-1+\frac{\left(\mathbf{x}_{2}-\mathbf{x}_{1}\right)^{2}}{\mathbf{r}^{2}}\right)$

Solving the integral gives the result:

$$
\mathbf{F}_{\text {total }}^{\mathbf{R}}(\mathbf{V S} 1 \rightarrow \mathbf{V S} 6)=\int \mathrm{d} \overline{\mathbf{F}}_{\text {total }}^{\mathbf{R}} \bullet \overrightarrow{\mathbf{u}}_{\mathbf{y}} \cong \frac{\mu_{0} \mathbf{I}_{1} \mathbf{I}_{2}}{4 \pi} \cdot(-9)
$$

\subsection{The parts of the two conductors being parallel, aligned along the y axis}

In this case both branches are situated along the y axis, necessary changes in the equation for the force between parallel currents will be needed. The earlier results concerning currents aligned to the $x$ axis, expressed in Eq. (42) and Eq. (47) must necessarily be modified in order to fit with these facts. This means that the expression dealing with currents moving both in the same y direction will obey the following equation

$$
\Delta \overrightarrow{\mathbf{F}}_{\text {total }}^{\mathbf{R}} \bullet \overrightarrow{\mathbf{u}}_{\mathbf{y}}=\frac{\mu_{0} \mathbf{I}_{1} \mathbf{I}_{2} \Delta \mathbf{y}_{1} \Delta \mathbf{y}_{2} \cdot\left(\mathbf{y}_{2}-\mathbf{y}_{1}\right)}{4 \pi \cdot \mathbf{r}^{3}} \cdot\left(-1+\frac{\left(\mathbf{y}_{2}-\mathbf{y}_{1}\right)^{2}}{\mathbf{r}^{2}}\right)
$$

Further, in the case the currents flow opposite to each other, the following equation will have to be chosen:

$$
\Delta \overrightarrow{\mathbf{F}}_{\text {total }}^{\mathbf{R}} \bullet \overrightarrow{\mathbf{u}}_{\mathbf{y}}=\frac{\mu_{0} \mathbf{I}_{1} \mathbf{I}_{2} \Delta \mathbf{y}_{1} \Delta \mathbf{y}_{2} \cdot\left(\mathbf{y}_{2}-\mathbf{y}_{1}\right)}{4 \pi \cdot \mathbf{r}^{3}} \cdot\left(1-\frac{\left(\mathbf{y}_{2}-\mathbf{y}_{1}\right)^{2}}{\mathbf{r}^{2}}\right)
$$

\subsubsection{Branch 10 to 7}

In this case the currents flow opposite to each other and one should therefore be tempted to use Eq. (71). However, since the two currents come close to each other at one point, thereby giving rise to a singularity that is impossible to treat straightforwardly, it is necessary to extend the definition of the 'thin conductors' carrying the respective currents, to let them have extension in the $\mathrm{x}$ direction, though small, so that $x_{1}: 0 \rightarrow w$ and $x_{2}: 0 \rightarrow w$. In that case it is furthermore more suitable to apply Eq. (71). This all will be done the following way:

$$
\int \mathrm{d} \overrightarrow{\mathbf{F}}_{\text {total }}^{\mathbf{R}} \bullet \overrightarrow{\mathbf{u}}_{\mathbf{y}}=\frac{1}{\mathbf{w}^{2}} \cdot \int_{\mathbf{x}_{1}=0}^{\mathbf{w}} \mathbf{d} \mathbf{x}_{1} \int_{\mathbf{x}_{2}=0}^{\mathbf{w}} \mathbf{d} \mathbf{x}_{2} \int_{\mathbf{y}_{1}=0}^{\frac{\mathrm{L}}{2}-\mathbf{a}} \mathrm{d} \mathbf{y}_{1} \int_{\frac{\mathbf{L}}{2}}^{\mathbf{L}} \mathbf{d y _ { 2 }} \frac{\mu_{0} \mathbf{I}_{1} \mathbf{I}_{2} \cdot\left(\mathbf{y}_{2}-\mathbf{y}_{1}\right)}{4 \pi \cdot \mathbf{r}^{3}} \cdot\left(1-\frac{\left(\mathbf{y}_{2}-\mathbf{y}_{1}\right)^{2}}{\mathbf{r}^{2}}\right)
$$


The approximation that has to be done, when both the conductors are to be regarded as 'thin', i.e. $w \rightarrow 0$ and the distance between their meeting points are small, $a<<L$, is to decide, which one is the very smallest. If choosing $w<<a<<L$

when solving the integral the following result will arise:

$$
\mathbf{F}_{\text {total }}^{\mathbf{R}}(10 \rightarrow 7)=\int \mathbf{d} \overrightarrow{\mathbf{F}}_{\text {total }}^{\mathbf{R}} \bullet \overrightarrow{\mathbf{u}}_{\mathbf{y}} \cong \frac{\mu_{0} \mathbf{I}_{1} \mathbf{I}_{2}}{4 \pi} \cdot\left(-\ln \frac{\mathbf{L}}{\mathbf{a}}+\ln 4\right)
$$

\subsubsection{Branch 10 to5}

In order to calculate the force between branch 10 and 5, one will have to insert $x_{2}-x_{1}=L$ in Eq. (70). Since the currents of the two branches flow in the same direction, Eq (70) will be applied.

The summation of the contributions from all the charges will in this case be

$$
\int \mathrm{d} \overrightarrow{\mathbf{F}}_{\text {total }}^{\mathbf{R}} \bullet \overrightarrow{\mathbf{u}}_{\mathbf{y}}=\int_{\mathbf{y}=0_{1}}^{\frac{\mathbf{L}}{2}-\mathbf{a}} \mathbf{d} \mathbf{y}_{1} \int_{\mathbf{y}_{2}=\frac{\mathbf{L}}{2}}^{\mathbf{L}} \mathbf{d y} \mathbf{y}_{2} \frac{\mu_{0} \mathbf{I}_{1} \mathbf{I}_{2} \cdot\left(\mathbf{y}_{2}-\mathbf{y}_{1}\right)}{4 \pi \cdot \mathbf{r}^{3}} \cdot\left(-1+\frac{\left(\mathbf{y}_{2}-\mathbf{y}_{1}\right)^{2}}{\mathbf{r}^{2}}\right)
$$

Solving the integral gives the result:

$$
\mathbf{F}_{\text {total }}^{\mathbf{R}}(10 \rightarrow 5)=\int \mathrm{d}_{\mathbf{F}_{\text {total }}^{\mathbf{R}}} \bullet \overrightarrow{\mathbf{u}}_{\mathbf{y}} \cong \frac{\mu_{0} \mathbf{I}_{1} \mathbf{I}_{2}}{4 \pi} \cdot \frac{1}{3}\left(\frac{1}{\sqrt{2}}-\frac{2}{\sqrt{5}}+\frac{\mathbf{a}}{\mathbf{L}}\right)
$$

\subsubsection{Branch 2 to 7}

In order to calculate the force between branch 2 and 7, one will have to insert $x_{2}-x_{1}=-L$ in Eq (70). Since the currents of the two branches flow in the same direction, Eq. (70) will be applied. Here the summation of the contributions from all the charges will in this case be

$$
\int \mathrm{d} \overrightarrow{\mathbf{F}}_{\text {total }}^{\mathbf{R}} \bullet \overrightarrow{\mathbf{u}}_{\mathbf{y}}=\int_{\mathbf{y}=0_{1}}^{\frac{\mathrm{L}}{2}-\mathbf{a}} \mathbf{d} \mathbf{y}_{1} \int_{\mathbf{y}_{2}=\frac{\mathbf{L}}{2}}^{\mathbf{L}} \mathbf{d y _ { 2 }} \frac{\mu_{0} \mathbf{I}_{1} \mathbf{I}_{2} \cdot\left(\mathbf{y}_{2}-\mathbf{y}_{1}\right)}{4 \pi \cdot \mathbf{r}^{3}} \cdot\left(-1+\frac{\left(\mathbf{y}_{2}-\mathbf{y}_{1}\right)^{2}}{\mathbf{r}^{2}}\right)
$$

Solving the integral gives the result:

$$
\mathbf{F}_{\text {total }}^{\mathbf{R}}(2 \rightarrow 7)=\int \mathrm{d} \overrightarrow{\mathbf{F}}_{\text {total }}^{\mathbf{R}} \bullet \overrightarrow{\mathbf{u}}_{\mathbf{y}} \cong \frac{\mu_{0} \mathbf{I}_{1} \mathbf{I}_{2}}{4 \pi} \cdot \frac{1}{3}\left(\frac{1}{\sqrt{2}}-\frac{2}{\sqrt{5}}+\frac{\mathbf{a}}{\mathbf{L}}\right)
$$




\subsubsection{Branch 2 to 5}

In this case the currents flow opposite to each other and one should therefore be tempted to use Eq. (71). However, since the two currents come close to each other at one point, thereby giving rise to a singularity that is impossible to treat straightforwardly, it will be necessary to extend the definition of the 'thin conductors' carrying the respective currents, to let them have extension in the $x$ direction, though small, so that $x_{1}: L-w \rightarrow L$ and $x_{2}: L-w \rightarrow L$. In this case it is furthermore more suitable to apply Eq. (71). This all will be done the following way:

$$
\int \mathrm{d}_{\mathbf{F}_{\text {total }}}^{\mathbf{R}} \bullet \overrightarrow{\mathbf{u}}_{\mathbf{y}}=\frac{1}{\mathbf{w}^{2}} \cdot \int_{\mathbf{x}_{1}=\mathbf{L}-\mathbf{w}}^{\mathrm{L}} \mathbf{d} \mathbf{x}_{1} \int_{\mathbf{x}_{2}=\mathbf{L}-\mathbf{w}}^{\mathrm{L}} \mathbf{d} \mathbf{x}_{2} \int_{\mathbf{y}_{1}=0}^{\frac{\mathrm{L}}{2}-\mathbf{a}} \mathbf{d} \mathbf{y}_{1} \int_{\mathbf{y}_{2}=\frac{\mathbf{L}}{2}}^{\mathrm{L}} \mathbf{d y _ { 2 }} \frac{\mu_{0} \mathbf{I}_{1} \mathbf{I}_{2} \cdot\left(\mathbf{y}_{2}-\mathbf{y}_{1}\right)}{4 \pi \cdot \mathbf{r}^{3}} \cdot\left(1-\frac{\left(\mathbf{y}_{2}-\mathbf{y}_{1}\right)^{2}}{\mathbf{r}^{2}}\right)
$$

where $w[\mathrm{~m}]$ indicates the width of the conductor. The approximation that has to be done, when both the conductors are to be regarded as 'thin', i.e. $w \rightarrow 0$, and the distance between their meeting points are small, so that $a<<L$, is to decide, which one is the very smallest. If choosing $w<<a<<L$ when solving the integral will give rise to the following result:

$$
\mathbf{F}_{\text {total }}^{\mathbf{R}}(2 \rightarrow 5)=\int \mathbf{d}_{\mathbf{F}_{\text {total }}}^{\mathbf{R}} \bullet \overrightarrow{\mathbf{u}}_{\mathbf{y}} \cong \frac{\mu_{0} \mathbf{I}_{1} \mathbf{I}_{2}}{4 \pi} \cdot\left(-\ln \frac{\mathbf{L}}{\mathbf{a}}+\ln 4\right)
$$

\subsection{The parts of the two conductors being perpendicular to each other, from $y$ axis to $x$ axis}

In the case one branch is situated along the $y$ axis and the other along they $x$ axis, necessary changes in the equation for the force between parallel currents have to be undertaken. The equations describing the four different contributions to the force, due to the four combinations of charges, Eq. (29), Eq. (31), Eq. (34) and Eq. (37), will be used, but modified with respect to the new directions. As mentioned earlier, in Ch. 2.6, there will be no time dilation effect. This leads to the following equation for the total incremental force between the two branches, in the case both currents flow along the respective positive axis:

$$
\Delta \overrightarrow{\mathbf{F}}_{\text {total }}^{\mathbf{R}} \bullet \overrightarrow{\mathbf{u}}_{\mathbf{y}}=\frac{\mu_{0} \mathbf{I}_{1} \mathbf{I}_{2} \Delta \mathbf{y}_{1} \Delta \mathbf{x}_{2} \cdot\left(\mathbf{y}_{2}-\mathbf{y}_{1}\right)^{2} \cdot\left(\mathbf{x}_{2}-\mathbf{x}_{1}\right)}{4 \pi \cdot \mathbf{r}^{5}}
$$

If one of the currents flows along a negative axis, the equation will change sign, so that:

$$
\Delta \overrightarrow{\mathbf{F}}_{\text {total }}^{\mathbf{R}} \bullet \overrightarrow{\mathbf{u}}_{\mathbf{y}}=-\frac{\mu_{0} \mathbf{I}_{1} \mathbf{I}_{2} \Delta \mathbf{y}_{1} \Delta \mathbf{x}_{2} \cdot\left(\mathbf{y}_{2}-\mathbf{y}_{1}\right)^{2} \cdot\left(\mathbf{x}_{2}-\mathbf{x}_{1}\right)}{4 \pi \cdot \mathbf{r}^{5}}
$$




\subsubsection{Branch 10 to 9}

In order to calculate the force between the two branches 10 and 9 , one will have to insert $x_{1}=0$ and $y_{2}=\frac{L}{2}$ in Eq. (80), or

$$
\int \mathbf{d F}_{\text {total }}^{\mathbf{R}} \cdot \overrightarrow{\mathbf{u}}_{\mathbf{y}} \cong \int_{\mathbf{x}_{2}=0}^{\mathrm{L}} \int_{\mathbf{y}_{2}=0}^{\frac{\mathbf{L}}{2}-\mathbf{a}} \frac{\mu_{0} \mathbf{I}_{1} \mathbf{I}_{2} \mathbf{d} \mathbf{x}_{2} \mathbf{d} \mathbf{y}_{1} \cdot\left(\frac{\mathbf{L}}{2}-\mathbf{y}_{1}\right)^{2} \cdot \mathbf{x}_{2}}{4 \pi \cdot \mathbf{r}^{5}}
$$

Solving the integral gives the result

$$
\mathbf{F}_{\text {total }}^{\mathbf{R}}(10 \rightarrow 9) \cong \frac{\mu_{0} \mathbf{I}_{1} \mathbf{I}_{2}}{4 \pi} \cdot\left(\frac{1}{3}\right) \cdot\left(-\frac{\mathbf{a}}{\mathbf{L}}+\ln \frac{\mathbf{L}}{\mathbf{a}}+\frac{1}{\sqrt{5}}-2 \cdot \ln 2+\ln (\sqrt{5}+1)\right)
$$

\subsubsection{Branch 2 to 9}

In order to calculate the force between the two branches 2 and 9 , one will have to assume $x_{1}=L$ and $y_{2}=\frac{L}{2}$ in Eq. (80), or

$$
\int \mathbf{d F}_{\text {total }}^{\mathbf{R}} \bullet \overrightarrow{\mathbf{u}}_{\mathbf{y}} \cong \int_{\mathbf{x}_{2}=0}^{\mathbf{L}} \int_{\mathbf{y}_{2}=0}^{\frac{\mathbf{L}}{2}-\mathbf{a}} \frac{\mu_{0} \mathbf{I}_{1} \mathbf{I}_{2} \mathbf{d} \mathbf{x}_{2} \mathbf{d} \mathbf{y}_{1} \cdot\left(\frac{\mathbf{L}}{2}-\mathbf{y}_{1}\right)^{2} \cdot\left(\mathbf{x}_{2}-\mathbf{L}\right)}{4 \pi \cdot \mathbf{r}^{5}}
$$

Solving the integral gives the result

$$
\mathbf{F}_{\text {total }}^{\mathbf{R}}(2 \rightarrow 9) \cong \frac{\mu_{0} \mathbf{I}_{1} \mathbf{I}_{2}}{4 \pi} \cdot\left(\frac{1}{3}\right) \cdot\left(-\frac{\mathbf{a}}{\mathbf{L}}+\ln \frac{\mathbf{L}}{\mathbf{a}}+\frac{1}{\sqrt{5}}-2 \cdot \ln 2+\ln (\sqrt{5}+1)\right)
$$

\subsubsection{Branch 10 to 6}

In order to calculate the force between the two branches 10 and 6, one has to insert $x_{1}=0$ and $y_{2}=L$ in Eq. (81), thus keeping in mind the change of direction of the second current with respect to the two preceding sections, in this case:

$$
\int \mathbf{d F}_{\text {total }}^{\mathbf{R}} \bullet \overrightarrow{\mathbf{u}}_{\mathbf{y}} \cong \int_{\mathbf{x}_{2}=0}^{\mathbf{L}} \int_{\mathbf{y}_{1}=0}^{\frac{\mathbf{L}}{2}-\mathbf{a}} \frac{\mu_{0} \mathbf{I}_{1} \mathbf{I}_{2} \mathbf{d} \mathbf{x}_{2} \mathbf{d} \mathbf{y}_{1} \cdot\left(-\left(\mathbf{L}-\mathbf{y}_{1}\right)^{2}\right) \cdot \mathbf{x}_{2}}{4 \pi \cdot \mathbf{r}^{5}}
$$


Solving the integral gives the result

$$
\mathbf{F}_{\text {total }}^{\mathbf{R}}(10 \rightarrow 6) \cong \frac{\mu_{0} \mathbf{I}_{1} \mathbf{I}_{2}}{4 \pi} \cdot\left(\frac{1}{3}\right) \cdot\left(\frac{1}{\sqrt{5}}-\frac{1}{\sqrt{2}}-\ln 2-\ln \frac{-1+\sqrt{5}}{-1+\sqrt{2}}\right)
$$

\subsubsection{Branch 2 to 6}

In order to calculate the force between the two branches 10 and 6 , one has to set $x_{1}=L$ and $y_{2}=L$ in Eq. (81), or:

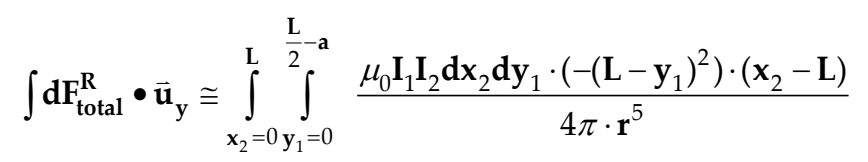

Solving the integral gives the result

$$
\mathbf{F}_{\text {total }}^{\mathbf{R}}(2 \rightarrow 6) \cong \frac{\mu_{0} \mathbf{I}_{1} \mathbf{I}_{2}}{4 \pi} \cdot\left(\frac{1}{3}\right) \cdot\left(\frac{1}{\sqrt{5}}-\frac{1}{\sqrt{2}}-\ln 2-\ln \frac{-1+\sqrt{5}}{-1+\sqrt{2}}\right)
$$

\subsubsection{Branch 10 to Voltage source 6}

In this case involving a voltage source, one will have to apply an impulse current instead of $I_{2}$, namely $I_{2} \cdot 3 L \cdot \delta\left(x_{2}-L / 2,\right)$

In this case $x_{1}=0$ and $y_{2}=L$

It has to be observed that the direction of that current is opposite to $I_{2}$, implying thus the need for changing sign compared to the integral equation for section 2.9.3 dealing with the forces between branch 10 and branch 6, so that instead Eq. (80) should be used, thereby expressing the total: contribution to the force here:

$$
\int \mathbf{d F}_{\text {total }}^{\mathbf{R}} \bullet \overrightarrow{\mathbf{u}}_{\mathbf{y}} \cong \int_{\mathbf{x}_{2}=0}^{\mathbf{L}} \int_{\mathbf{y}_{1}=0}^{\frac{\mathbf{L}}{2}-\mathbf{a}} \frac{\mu_{0} \mathbf{I}_{1} \mathbf{I}_{2} \cdot 3 \mathbf{L} \cdot \delta\left(\mathbf{x}_{2}-\frac{\mathbf{L}}{2}\right) \cdot \mathbf{d x}_{2} \mathbf{d} \mathbf{y}_{1} \cdot\left(\left(\mathbf{L}-\mathbf{y}_{1}\right)^{2}\right) \cdot \mathbf{x}_{2}}{4 \pi \cdot \mathbf{r}^{5}}
$$

Solving the integral gives the result:

$$
\mathbf{F}_{\text {total }}^{\mathbf{R}}(10 \rightarrow \mathbf{V S} 6)=\int \mathrm{d}_{\mathbf{F}_{\text {total }}^{\mathbf{R}}}^{\mathbf{R}} \cdot \overrightarrow{\mathbf{u}}_{\mathbf{y}} \cong \frac{\mu_{0} \mathbf{I}_{1} \mathbf{I}_{2}}{4 \pi} \cdot\left(-\frac{5}{\sqrt{2}}+\frac{56}{5 \sqrt{5}}\right)
$$




\subsubsection{Branch 2 to Voltage source 6}

In this case involving a voltage source, one will have to apply an impulse current instead of $I_{2}$, namely $I_{2} \cdot 3 L \cdot \delta\left(x_{2}-L / 2,\right)$

In this case $x_{1}=L$ and $y_{2}=L$

It has to be observed that the direction of that current is opposite to $I_{2}$, implying thus the need for changing sign compared to the integral equation for section 2.9.3 dealing with the forces between branch 10 and branch 6, so that instead Eq. (80) should be used, thereby expressing the total: contribution to the force here:

$$
\int \mathbf{d F}_{\text {total }}^{\mathbf{R}} \bullet \overrightarrow{\mathbf{u}}_{\mathbf{y}} \cong \int_{\mathbf{x}_{2}=0}^{\mathbf{L}} \int_{\mathbf{y}_{1}=0}^{\frac{\mathbf{L}}{2}-\mathbf{a}} \frac{\mu_{0} \mathbf{I}_{1} \mathbf{I}_{2} \cdot 3 \mathbf{L} \cdot \delta\left(\mathbf{x}_{2}-\frac{\mathbf{L}}{2}\right) \cdot \mathbf{d} \mathbf{x}_{2} \mathbf{d} \mathbf{y}_{1} \cdot\left(\left(\mathbf{L}-\mathbf{y}_{1}\right)^{2}\right) \cdot\left(\mathbf{x}_{2}-\mathbf{L}\right)}{4 \pi \cdot \mathbf{r}^{5}}
$$

Solving the integral gives the result:

$$
\mathbf{F}_{\text {total }}^{\mathbf{R}}(2 \rightarrow \mathbf{V S} 6)=\int \mathrm{d} \overrightarrow{\mathbf{F}}_{\text {total }}^{\mathbf{R}} \bullet \overrightarrow{\mathbf{u}}_{\mathbf{y}} \cong \frac{\mu_{0} \mathbf{I}_{1} \mathbf{I}_{2}}{4 \pi} \cdot\left(-\frac{5}{\sqrt{2}}+\frac{56}{5 \sqrt{5}}\right)
$$

\subsection{The parts of the two conductors being perpendicular to each other, from the $x$ axis to} the $\mathrm{y}$ axis

In the case one branch is situated along the $x$ axis and the other along they $y$ axis, necessary changes in the equation for the force between parallel currents have to be undertaken. The earlier results concerning currents aligned to the $x$ axis, expressed in Eq. (42) and Eq. (47) must necessarily be modified in order to fit with these facts. This will lead to the following equation:

$$
\Delta \overrightarrow{\mathbf{F}}_{\text {total }}^{\mathbf{R}} \bullet \overrightarrow{\mathbf{u}}_{\mathbf{y}}=\frac{\mu_{0} \mathbf{I}_{1} \mathbf{I}_{2} \Delta \mathbf{x}_{1} \Delta \mathbf{y}_{2} \cdot\left(\mathbf{y}_{2}-\mathbf{y}_{1}\right)^{2} \cdot\left(\mathbf{x}_{2}-\mathbf{x}_{1}\right)}{4 \pi \cdot \mathbf{r}^{5}}
$$

If one of the currents flows along a negative axis, the equation will change sign, so that:

$$
\Delta \overrightarrow{\mathbf{F}}_{\text {total }}^{\mathbf{R}} \cdot \overrightarrow{\mathbf{u}}_{\mathbf{y}}=-\frac{\mu_{0} \mathbf{I}_{1} \mathbf{I}_{2} \Delta \mathbf{x}_{1} \Delta \mathbf{y}_{2} \cdot\left(\mathbf{y}_{2}-\mathbf{y}_{1}\right)^{2} \cdot\left(\mathbf{x}_{2}-\mathbf{x}_{1}\right)}{4 \pi \cdot \mathbf{r}^{5}}
$$

\subsubsection{Branch 1 to 7}

In order to calculate the force between the two branches 1 and 7 , one has to insert $y_{1}=0$ and $x_{2}=0$ in Eq. (94), and integrating, or 


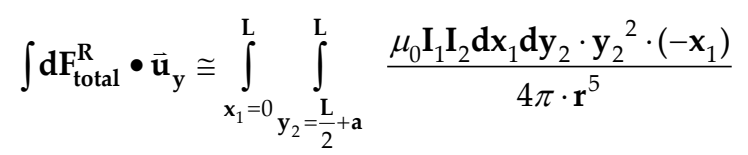

Solving the integral gives the result:

$$
\mathbf{F}_{\text {tottal }}^{\mathbf{R}}(1 \rightarrow 7) \cong \frac{\mathbf{I}_{1} \mathbf{I}_{2}}{4 \pi \varepsilon_{0}} \cdot\left(\frac{1}{3}\right) \cdot\left(-\frac{1}{\sqrt{2}}+\frac{1}{\sqrt{5}}-\ln \frac{1+\sqrt{5}}{1+\sqrt{2}}\right)
$$

\subsubsection{Branch 1 to 5}

In order to calculate the force between the two branches 1 and 7 , one has to insert $y_{1}=0$ and $x_{2}=0$ in Eq. (95), and integrating, or

$$
\int \mathrm{dF}_{\text {total }}^{\mathrm{R}} \bullet \overrightarrow{\mathbf{u}}_{\mathbf{y}} \cong \int_{\mathbf{x}_{1}=0}^{\mathrm{L}} \int_{\mathbf{y}_{2}=\frac{\mathbf{L}}{2}}^{\mathrm{L}} \frac{-\mu_{0} \mathbf{I}_{1} \mathbf{I}_{2} \mathbf{d} \mathbf{x}_{1} \mathbf{d y} \mathbf{y}_{2} \cdot \mathbf{y}_{2}{ }^{2} \cdot\left(\mathbf{L}-\mathbf{x}_{1}\right)}{4 \pi \cdot \mathbf{r}^{5}}
$$

Solving the integral gives the result:

$$
\mathbf{F}_{\text {tottal }}^{\mathbf{R}}(1 \rightarrow 5) \cong \frac{\mathbf{I}_{1} \mathbf{I}_{2}}{4 \pi \varepsilon_{0}} \cdot\left(\frac{1}{3}\right) \cdot\left(-\frac{1}{\sqrt{2}}+\frac{1}{\sqrt{5}}-\ln \frac{1+\sqrt{5}}{1+\sqrt{2}}\right)
$$

\subsubsection{Branch 8 to 7}

In order to calculate the force between the two branches 8 and 7 , one has to insert $y_{1}=\frac{L}{2}-a$ and $x_{2}=0$ in Eq. (52d'), or

$$
\int \mathbf{d F}_{\text {total }}^{\mathbf{R}} \bullet \overrightarrow{\mathbf{u}}_{\mathbf{y}} \cong \int_{\mathbf{x}_{1}=0}^{\mathrm{L}} \int_{\mathbf{y}_{2}=\frac{\mathbf{L}}{2}}^{\mathrm{L}} \frac{-\mu_{0} \mathbf{I}_{1} \mathbf{I}_{2} \mathbf{d} \mathbf{x}_{1} \mathbf{d} \mathbf{y}_{2} \cdot\left(\mathbf{y}_{2}-\frac{\mathbf{L}}{2}+\mathbf{a}\right)^{2} \mathbf{x}_{1}}{4 \pi \cdot \mathbf{r}^{5}}
$$

Solving the integral gives the result:

$$
\mathbf{F}_{\text {tottal }}^{\mathbf{R}}(8 \rightarrow 7) \cong \frac{\mathbf{I}_{1} \mathbf{I}_{2}}{4 \pi \varepsilon_{0}} \cdot\left(\frac{1}{3}\right) \cdot\left(-\frac{\mathbf{a}}{\mathbf{L}}+\ln \frac{\mathbf{L}}{\mathbf{a}}-\frac{1}{\sqrt{5}}+\ln (1+\sqrt{5})\right)
$$




\subsubsection{Branch 8 to 5}

In order to calculate the force between the two branches 8 and 7 , one has to insert $y_{1}=\frac{L}{2}-a$ and $x_{2}=L$ in Eq. $\left(52 c^{\prime}\right)$, or

$$
\int \mathbf{d F}_{\text {total }}^{\mathbf{R}} \cdot \overrightarrow{\mathbf{u}}_{\mathbf{y}} \cong \int_{\mathbf{x}_{1}=0}^{\mathbf{L}} \int_{\mathbf{y}_{2}=\frac{\mathbf{L}}{2}}^{\mathbf{L}} \frac{\mu_{0} \mathbf{I}_{1} \mathbf{I}_{2} \mathbf{d} \mathbf{x}_{1} \mathbf{d} \mathbf{y}_{2} \cdot\left(\mathbf{y}_{2}-\frac{\mathbf{L}}{2}+\mathbf{a}\right)^{2} \cdot\left(\mathbf{L}-\mathbf{x}_{1}\right)}{4 \pi \cdot \mathbf{r}^{5}}
$$

Solving the integral gives the result:

$$
\mathbf{F}_{\text {tottal }}^{\mathbf{R}}(8 \rightarrow 5) \cong \frac{\mathbf{I}_{1} \mathbf{I}_{2}}{4 \pi \varepsilon_{0}} \cdot\left(\frac{1}{3}\right) \cdot\left(-\frac{\mathbf{a}}{\mathbf{L}}+\ln \frac{\mathbf{L}}{\mathbf{a}}-\frac{1}{\sqrt{5}}+\ln (1+\sqrt{5})\right)
$$

\subsubsection{The Voltage source of branch 1 to branch 7}

In this case involving a voltage source, one will have to apply an impulse current instead of $I_{1}$, namely.$I_{1} \cdot 3 L \cdot \delta\left(x_{1}-L / 2\right.$, )

In this case $x_{2}=0$ and $y_{1}=0$

It has to be observed that the direction of that current is opposite to $I_{1}$, implying thus the need for changing sign compared to the integral equation in Sec. 2.10.1 dealing with the forces between branch 1 and branch 7. Hence, instead Eq. (95) would have to be used, thereby expressing the total: contribution to the force here:

$$
\int \mathbf{d F}_{\text {total }}^{\mathbf{R}} \bullet \overrightarrow{\mathbf{u}}_{\mathbf{y}} \cong \int_{\mathbf{x}_{1}=0}^{\mathbf{L}} \int_{\mathbf{y}_{2}=\frac{\mathbf{L}}{2}+\mathbf{a}}^{\mathbf{L}} \frac{-\mu_{0} \cdot \mathbf{I}_{1} \cdot 3 \mathbf{L} \cdot \delta\left(\mathbf{x}_{1}-\frac{\mathbf{L}}{2}\right) \cdot \mathbf{I}_{2} \mathbf{d x}_{1} \mathbf{d y _ { 2 }} \cdot \mathbf{y}_{2}{ }^{2} \cdot\left(-\mathbf{x}_{1}\right)}{4 \pi \cdot \mathbf{r}^{5}}
$$

Solving the integral gives the result:

$$
\mathbf{F}_{\text {tottal }}^{\mathbf{R}}(\mathbf{V S} 1 \rightarrow 7) \cong \frac{\mathbf{I}_{1} \mathbf{I}_{2}}{4 \pi \varepsilon_{0}} \cdot\left(\frac{1}{3}\right) \cdot\left(-\frac{1}{\sqrt{2}}+\frac{16}{5 \sqrt{5}}\right)
$$

\subsubsection{The Voltage source of branch 1 to branch 5}

In this case involving a voltage source, one will have to apply an impulse current instead of $I_{1}$, namely.$I_{1} \cdot 3 L \cdot \delta\left(x_{1}-L / 2\right.$, $)$ 
In this case $x_{2}=L$ and $y_{1}=0$

It has to be observed that the direction of that current is opposite to $I_{1}$, implying thus the need for changing sign compared to the integral equation in Sec. 2.10.2 dealing with the forces between branch 1 and branch 5. Instead, Eq. (94) should be used, thereby expressing the total: contribution to the force here:

$$
\int \mathbf{d F}_{\text {total }}^{\mathbf{R}} \bullet \overrightarrow{\mathbf{u}}_{\mathbf{y}} \cong \int_{\mathbf{x}_{1}=0}^{\mathbf{L}} \int_{\mathbf{y}_{2}=\frac{\mathbf{L}}{2}}^{\mathbf{L}} \frac{\mu_{0} \cdot \mathbf{I}_{1} \cdot 3 \mathbf{L} \cdot \delta\left(\mathbf{x}_{1}-\frac{\mathbf{L}}{2}\right) \cdot \mathbf{I}_{2} \mathbf{d} \mathbf{x}_{1} \mathbf{d y _ { 2 }} \cdot \mathbf{y}_{2}{ }^{2} \cdot\left(\mathbf{L}-\mathbf{x}_{1}\right)}{4 \pi \cdot \mathbf{r}^{5}}
$$

Solving the integral gives the result:

$$
\mathbf{F}_{\text {tottal }}^{\mathbf{R}}(\mathbf{V S} 1 \rightarrow 5) \cong \frac{\mathbf{I}_{1} \mathbf{I}_{2}}{4 \pi \varepsilon_{0}} \cdot\left(\frac{1}{3}\right) \cdot\left(-\frac{1}{\sqrt{2}}+\frac{16}{5 \sqrt{5}}\right)
$$

\section{Judgment (assessment) of the calculations}

If assessing all the calculations for the different parts of the circuits, it becomes evident that it is only one that dominates over all the others, namely the force between the parts of the two circuits aligned along each other at a distance $a$ a that is small compared to all the other dimensions of the circuits that are of order $L$, or $\frac{a}{L} \rightarrow 0$. The result is given through Eq. (50), implying an attractive force between these parts of the conductors. The force is furthermore proportional to the length of the conductors and inverse proportional to their mutual distance.

\section{Conclusion}

The two respective currents have been thoroughly analyzed, using Coulomb's law, taking into account the effects of propagation delay and the Special Relativity Theory. The way the effects of the propagation delay have been derived is that of this author in a paper 1997 [1], which differs fundamentally from the traditional interpretation, as that of Feynman [21] and Jackson [22]. This author has been successful in showing what the fallacies are [2]. Basically, Feynman committed a mathematical fault with respect to the calculation of the propagation delay, when deriving the Liénard-Wiechert potentials [21]. In the 1997 paper by this author [1] it was crucial to the success of Coulomb's law that the effects of propagation delay had been correctly derived, both with respect to the "sending charges" of the "first conductor" and to the "receiving charges" of the "second conductor". The first effect gives account for the dependence of the first current in the expression for the electromagnetic force, the second one for the 
second current in that same expression, but of course, it is arbitrary, which one is treated as sending or receiving current. This treatment makes it possible to see a product between two currents in an application of Coulomb's law, and, hence, there will be no need for the Lorentz force. When that analysis has been done, it remains to take into account to the effects of the Special relativity theory, especially the Lorentz transformation of lengths. Since this effect is related only to the relative movements of the two coordinate axes, and has nothing to do with the propagation delay an observer faces, it may be multiplied straightforwardly to the effect of propagation delay. The expression for the force between the two currents are compared to the expression that Ampère arrive at and to Lorentz' force law. Thereafter follows a discussion of the pro et contra of respective model. The result in this article is based on two wellcorroborated natural laws: Coulomb's law [23] and the Special relativity theory. Ampére in turn, derives his law in a strictly empirical sense [32], searching for similarities with Coulomb's law. However, since in his time, the individual electron had not yet been discovered and, secondly, the Special relativity theory had not been defined. Hence, Ampère had no other choice than to establish a fairly good empirical law. Lorentz (or first: Grassmann) faced the same problem, but his formula was derived through evident mathematical faults [15]. $\mathrm{Nb}$. This term 'Ampère's force law' is not the same law as that Jackson denotes Ampère's law. Please cf. the original paper by Ampère [13] and Jackson [24]. This would make it possible to create a continuous, logical chain, from the findings by Ampère to the established Maxwell electrodynamics. Assis has made an effort to prove that both Ampère's law and Grassmann's law produce the same result, when the forces within Ampère's bridge are being derived [15]. Admittedly, he concedes that they are not equal at every point, but in the integral sense, when a complete, closed electric circuit is taken into account. From a strictly mathematical pint of view, however, if two functions are not equal at every point, they don't express equal functions. This is taught in the most basic undergraduate courses. Anyhow, stating that all electric circuits are necessarily closed, he arrives at the conclusion that both laws are equally applicable on electric circuits.

To conclude, all three of them: Coulomb's law, Lorentz' force law and Ampère's force law can account for the attractive force exerted between two parallel electric conductors, carrying a current in the same direction. On the mere basis of the shape of the functions, it is not possible to decide, which one is best expressing physical reality, since the very measurements of currents involves a theory for the force between currents in the context of traditional measurement instruments. Hence, for every choice of model, there will necessarily appear a coupling constant that makes the measurements fit with the theory. Therefore, it remains to make a qualitative analysis of the three models. Above it has already been explored that Coulomb's has been used in a very strict manner, applying only the effects of propagation delay and the Special Relativity theory, whereas Ampère's force law is only expressing an empirical estimation of the force and the Lorentz force has been fallaciously derived, using Ampère's force law.

Hence, the conclusion to be drawn is that Coulomb's law gives the most comprehensive explanation to the force. 


\section{Author details}

Jan Olof Jonson ${ }^{1,2,3}$

Address all correspondence to: jajo8088@bahnhof.se

1 Royal Institute of Technology, Stockholm, Sweden

2 Stockholm University, Stockholm, Sweden

3 European Physical Society and the John Chappell Natural Philosophy Society, USA

\section{References}

[1] J. O. Jonson, 'The Magnetic Force between Two Currents Explained Using Only Coulomb's Law', Chinese Journal of Physics, Vol. 35, No. 2, 1997, p. 139-149

[2] J. O. Jonson, 'Refutation of Feynman's Derivation of the Lienard-Wiechert Potentials', Proc. 10th Natural Philosophy Alliance Conference, Storrs, CT, United States,2003, Journal of New Energy, Volume 7, No. 3, p. 42-44

[3] J. O. Jonson, 'The Law of Electromagnetic Induction Proved to be False Using Classical Electrostatics', Journal o Theoretics, Volume 5, No. 3, 2003. Available from: http:// www.journaloftheoretics.com/Articles/aArchive.htm

[4] J. O. Jonson, 'The Use of Finite Differences on Electric Currents Gives Credit to Coulomb's Law as Causing Electromagnetic Forces, thereby Explaining Electromagnetic Induction', IJMO, Vol. 3, No. 4, p. 373-376, 2013. DOI: 10.7763/IJMO.2013.V3.301

[5] J. O. Jonson, 'The Claim that Neumann's Induction Is Consistent with Ampère's Law Rejected' IJMO, Vol. 4, No. 4, p. 326-331, 2014. DOI: 10.7763/IJMO.2014.V4.394

[6] J. P. Wesley, 'Ampere Repulsion and Graneau's Exploding Wire', Progress in SpaceTime Physics, Benjamin Wesley-Publisher, 1987, p. 181-186

[7] P. Graneau, 'Longitudinal magnet forces', J. Appl. Phys. 55, 2598 (1984). DOI: $10.1063 / 1.333247$

[8] J. P. Wesley, 'Ampere Repulsion Drives the Graneau-Hering Submarine and Hering's Pump',, Progress in Space-Time Physics, Benjamin Wesley-Publisher, 1987, p. 181. ISBN: 3980094227 OCLC: 16693905

[9] P. Graneau, 'Ampere Tension in Electric Conductors', IEEE Transactions on Magnetics, Vol. Mag-20, No. 2, March 1984, p. 452-455. DOI: 10.1109/TMAG.1984.1063069

[10] J. P. Wesley, 'Ampere Repulsion and Graneau's Exploding Wires', Progress in SpaceTime Physics, Benjamin Wesley-Publisher, 1987, p. 182 
[11] P. Graneau, 'Ampere Tension in Electric Conductors', IEEE Transactions on Magnetics, Vol. Mag-20, No. 2, March 1984, p. 444.DOI: 10.1109/TMAG.1984.1063069

[12] A. M. Ampère, "Mémoire. Sur la théorie mathématique des phénomènes électrodynamiques uniquement déduite de l'expérience, dans lequel se trouvent réunis les Mémoires que M. Ampère a communiqués à l'Académie royale des Sciences, dans les séances des 4 et 26 décembre 1820, 10 juin 1822, 22 décembre 1823, 12 septembre et 21 novembre 1825", 'Mémoires de l'Académie Royale des Sciences de l'Institut de France Année 1823, Tome VI, Paris, chez Firmin Didot, Père et fils, libraires, Rue Jacob, No 24, p $204 \mathrm{ff}$.

[13] H. G. Grassmann, Poggendorffs Ann. Phys. Chemie 64 (1845), p. 1

[14] J. O. Jonson, 'Ampère's Law Proved Not to Be Compatible with Grassmann's Force Law', Engineering » Electrical and Electronic Engineering » "Electromagnetic Radiation", book edited by Saad Osman Bashir, ISBN 978-953-51-0639-5, DOI: $10.5772 / 37978$

[15] J. O. Jonson, 'Towards a Classical Explanation to the Stable Electron Paths around Nuclei and to Radiation in Connection with the De-Excitation of Excited Electrons', Proc. 2004, International Congress 2004: Fundamental Problems of Natural Sciences and Engineering, St. Petersburg, Russia, p. 111-118

[16] J. O. Jonson, 'Towards a Classical Explanation for the Stable Electron Paths Around Nuclei and to Radiation in Connection with the De-Excitation of Excited Electrons', Proceedings of the NPA, Volume 4, No. 1, p. 92-96, 2007, 14th Natural Philosophy Alliance Conference, Storrs, CT, United States

[17] J. O. Jonson, 'Photon as a Classical Wave Packet from Classically Stabilized Electron Orbits', Proc. The Nature of Light, Volume 6664, The Nature of Light: What Are Photons?, San Diego, CA, United States. DOI: 10.1117/12.747574

[18] J. O. Jonson, 'Coulomb's Law is the Basis for Radiation Energy', Proceedings of the NPA, Volume 8, p. 303-305, 2011, 18th Natural Philosophy Alliance Conference, College Park, MD, United States

[19] J. C. Maxwell, 'A Treatise on Electricity and Magnetism', London, UK, Oxford University Press, 1873, Vol. 2, p. 319. ARK:13960/t2s47ss48

[20] J. O. Jonson, 'The Electromagnetic Force between Two Parallel Electric Currents of 'Infinite' Length Attained Using Respectively Ampère's Law and Coulomb's Law Including a Relativistic Analysis.',2010 Available from: http://www.worldsci.org/php/ index.php?tab0=Abstracts\&tab1=Display\&id=5860\&tab=2

[21] R.P. Feynman, 'The Feynman Lectures on Physics', (mainly Electromagnetism in Matter), GB, Addison-Wesley, 1989, Vol. 2, p. 21-9-11. ISBN 10: 020102117X / ISBN 13: 9780201021172 
[22] J. D. Jackson, 'Classical Electrodynamics', Second Edition, John Wiley \& Sons, 1975, ARK:13960/t38065f08,p. 654f

[23] J. O. Jonson, 'The Magnetic Force between Two Currents Explained Using Only Coulomb's Law', Chinese Journal of Physics, Vol. 35, No. 2, 1997, p. 144

[24] A. Ramgard, 'Relativitetsteori’, Teoretisk Fysik, KTH, 1977, p. 2, 11

[25] R. Resnick, 'Introduction to Special Relativity', John Wiley \& Sons, USA, 1968, p. 60

[26] A. Ramgard, 'Relativitetsteori', Teoretisk Fysik, KTH, Stockholm, 1977, p. 14

[27] R. Resnick, 'Introduction to Special Relativity', John Wiley \& Sons, USA, 1968, p. 56

[28] J. O. Jonson, 'The Sagnac Effect Explained Using the Special Relativity Theory', 2009, 16th Natural Philosophy Alliance Conference, Storrs, CT, United States. Available on: http://www.worldsci.org/php/index.php?tab0=Abstracts\&tab1=Display\&id=1214\&tab=2 (available 20150127)

[29] M. Abramowitz and I. A. Stegun, 'Handbook of Mathematical Functions', Dover Publications, New York, 1972, Eq. (3.6.9) p. 15

[30] A. Ramgard, 'Relativitetsteori', Teoretisk Fysik, KTH, Stockholm, 1977, p. 18

[31] J. O. Jonson, 'The Magnetic Force between Two Currents Explained Using Only Coulomb's Law', Chinese Journal of Physics, Vol. 35, No. 2, 1997, p. 144

[32] A. M. Ampère, "Mémoire. Sur la théorie mathématique des phénomènes électrodynamiques uniquement déduite de l'expérience, dans lequel se trouvent réunis les Mémoires que M. Ampère a communiqués à l'Académie royale des Sciences, dans les séances des 4 et 26 décembre 1820, 10 juin 1822, 22 décembre 1823, 12 septembre et 21 novembre 1825", 'Mémoires de l'Académie Royale des Sciences de l'Institut de France Année 1823, Tome VI, Paris, chez Firmin Didot, Père et fils, libraires, Rue Jacob, No 24, pp. 175-387. Copy belonging to the Stockholm University Library (www.sub.su.se). 



\title{
Chapter 4
}

\section{Electromagnetic Waves Propagation and Detection in Shielded Dielectric Power Cables}

\author{
Chunchuan Charles Xu and Chengyin Liu \\ Additional information is available at the end of the chapter \\ http://dx.doi.org/10.5772/61055
}

\begin{abstract}
Partial discharge occurs a lot in shielded dielectric power cables. Partial discharge pulses are high-frequency electromagnetic waves that propagate in the shielded dielectric power cables. This chapter will study partial discharge propagation and detection in shielded dielectric power cables.
\end{abstract}

Keywords: Propagation, Detection, Shielded Power Cables

\section{Introduction}

\subsection{Shielded dielectric power cables}

Since the 1970s, shielded dielectric cables have been used overseas, and since the mid-1980s, they have been extensively used in North America as well. Today, because of environmental concerns, shielded solid dielectric cables are used more and more and most new power cables installed are solid dielectric cables. Figure 1 [1] shows the structure of a typical high-voltage shielded dielectric power cable. The conductor core $\mathrm{F}$ is the part that delivers power. The conductor shields $\mathrm{C}$ and $\mathrm{E}$ help smooth the electric field between the conductor and the insulation materials. The dielectric or insulation $\mathrm{D}$ is used to insulate high-voltage core $\mathrm{F}$ from the ground, i.e. neutral wires B. Encapsulating jacket A prevents water and dust from getting into the cable. 


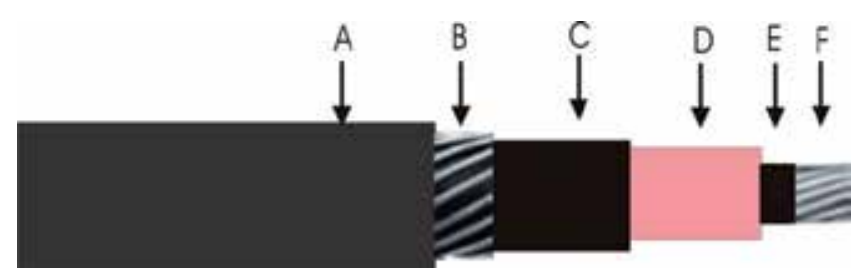

Figure 1. A typical high-voltage extruded solid dielectric cable. A, encapsulating jacket; B, neutral wires; $\mathrm{C}$, ground shield; D, dielectric; E, conductor shield; F, conductor; the conductor shield and ground shield are semiconductive. Usually, they are made by adding carbon black into a polymer and the particle size of the carbon black ranges from 15 to $50 \mathrm{~nm}$. The basic function of this configuration is to confine the electric field within the cable and obtain a symmetrical radial distribution of the electric within the dielectric [1].

\subsection{Partial discharge in shielded dielectric power cables}

Most failures occurring in the shielded dielectric cables are related to partial discharge. Partial discharges are localized breakdowns in a small portion of the insulations, which can be solid or fluid electrical insulation. When high voltage is applied to high-voltage equipment, defects introduced during the manufacturing process such as contained insulation cracks, contained insulation surfaces, or voids can all lead to partial discharges. In some cases, even without any defects, aging can cause the degradation of the insulator leading to partial discharges. PD makes damage to the equipment, and equipment with PD occurring within will eventually fail after a certain time depending on the strength of the PD if proper treatments are not applied. It's important to monitor partial discharges in high-voltage systems and if PD is detected, appropriate actions should be taken to prevent sudden failures, which can cause big blackouts.

Figure 2 gives us some basic ideas on how partial discharges occur. The cavity in the insulation normal contains gas which could be ionized when the electrical field exceeds the cutoff strength. When this happens, electromagnetic waves in the radiofrequencies are generated along with light, heat, noise, and possibly gas. With appropriate technologies and by detecting the HF radio signals, the magnitude as well as the location of partial discharges can be identified and used for assessing the health status of the shielded dielectric cable.

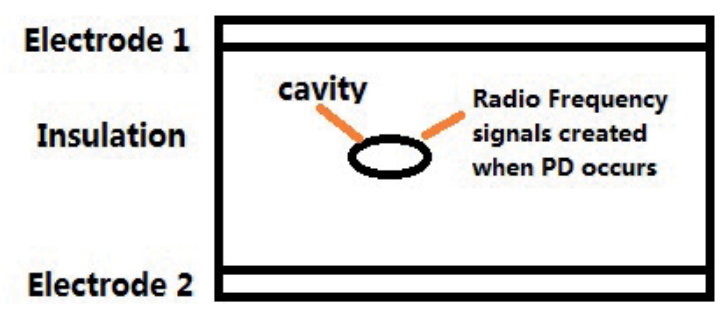

Figure 2. A "typical" partial discharge mechanism. The cavity in the insulation normally contains gas, which could be ionized when the electrical field exceeds the cutoff strength. 


\section{Electromagnetic wave propagation in shielded dielectric cables}

\subsection{Partial discharge pulse properties}

As discussed above, PD can create high-frequency electromagnetic waves in the radiofrequencies, heat, light, gas, etc. A lot of research has been conducted to study for detecting PD using sensors for heat, light, gas, etc. In this chapter, we focus on radiofrequency highfrequency PD signal detection as it provides a way to detect PD over long distances, which makes it useful in many cases. To effectively detect and analyze PD, first we have to understand the PD pulse. The authors will analyze the PD pulse spectrums, frequency properties, etc. Note that PD occurring in different high-voltage apparatus varies significantly due to the insulation properties and electric stress needed for triggering PD. Since this chapter focuses on PD in shielded dielectric cables, we will focus on the PD pulse properties in shielded dielectric cables.

Because the formation of electron avalanches is within the nanosecond range, the PD event is associated with a very fast current pulse. As of today, there is no direct way to measure the PD current pulse. A lot of theories have been proposed to simulate and study the current pulse and different measuring techniques were developed to measure PD current pulses. The PD is caused by the flow of the electrons and ions. The moving speed of the electrons is much faster than the ions. Some early theoretical simulations [2-5] predicted that the pulse current in the voids of the shielded dielectric cables has a rising time and pulse widths in the nanosecond range followed by a long-duration, low-magnitude pulse. Figure 3 shows one theoretically predicted PD current pulse. With the high-speed oscilloscope available, some PD experiments were conducted by Fujimoto and Boggs (1981) and Boggs and Stone (1982) [4] and the experimental results agree with the theoretical simulation results.

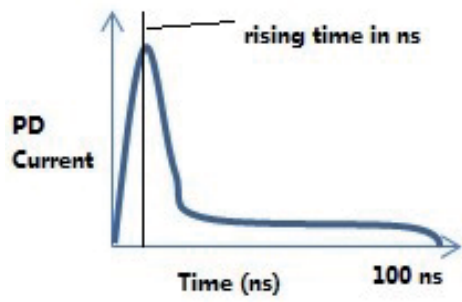

Figure 3. One theoretically predicted PD current pulse [2-5].

There is no standard spectrum chart for PD pulses in the power cables because under different situations, the PD pulse can be in different shapes leading to different energy spectrums. Some experimental results [6] show that the partial discharge spectrum has a peak point at about $100 \mathrm{MHz}$ when measuring PD at a distance very close to the PD source. When the partial discharge pulse propagates in the high-voltage cable, its high-frequency components are attenuated by the losses majorly caused by the semiconducting materials and, after a 100-meter trip, the peak frequency of the spectrum can be reduced to $20-30 \mathrm{MHz}$. 


\subsection{Shielded dielectric cable HF attenuation properties}

When the electromagnetic PD wave propagates in the cable, its HF components are significantly attenuated by the shielded cables. How far the pulse can travel and be detected by the PD detection devices heavily relies on the attenuation properties of the cable. A lot of research [7-17] has been conducted to study different aspects of the HF attenuation features of the shielded dielectric cables. From Figure 1, the HF attenuation is caused by various components. One measurement result [9] shown in Figure 4 gives HF attenuation losses for different components for different frequencies. From Figure 4, it can be seen that for this measurement, conductor and neutral wire skin effect losses dominate for low frequencies up to $5 \mathrm{MHz}$. After $5 \mathrm{MHz}$, grounding shielding losses as well as the dielectric losses start to play a more important role. This can be explained by the fact that at low frequencies, the capacitive current is low thus the current passing through the dielectric, conductor and ground shielding is low leading to relatively low losses. When frequencies are higher, the larger capacitive current flowing through the resistive component of the conductor and ground shielding makes big losses.

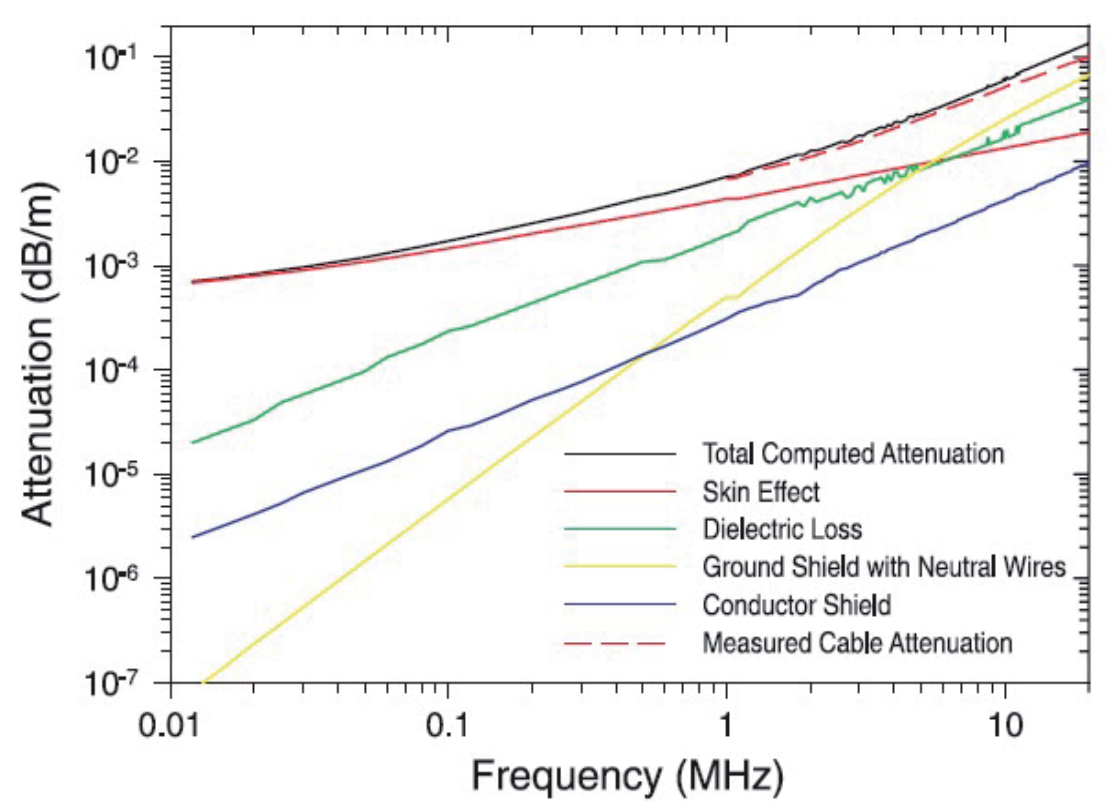

Figure 4. Measurement results for one shielded cable [9].

\subsubsection{Shield HF property measurements}

From the above analysis, it can be seen that the dielectric properties of the shielding are critical to HF losses of the shielding dielectric cables. The dielectric properties of the cable semiconducting shielding can be measured by a HF impedance analyzer. Figure 5 [10 and 17] shows a typical measurement configuration for HF dielectric properties (dielectric constant and 
conductivity) of the cable shielding. Conducting electrodes with small surface resistivity, often metal paint, are applied to the two surfaces of the shield material. Normally, contact with the electrodes is made along one edge of the sample. There is a voltage drop across the "conducting" electrodes, which can cause errors in the dielectric properties measurement when the current flowing through the sample and the nonperfect electrode resulting in possible measurement errors.
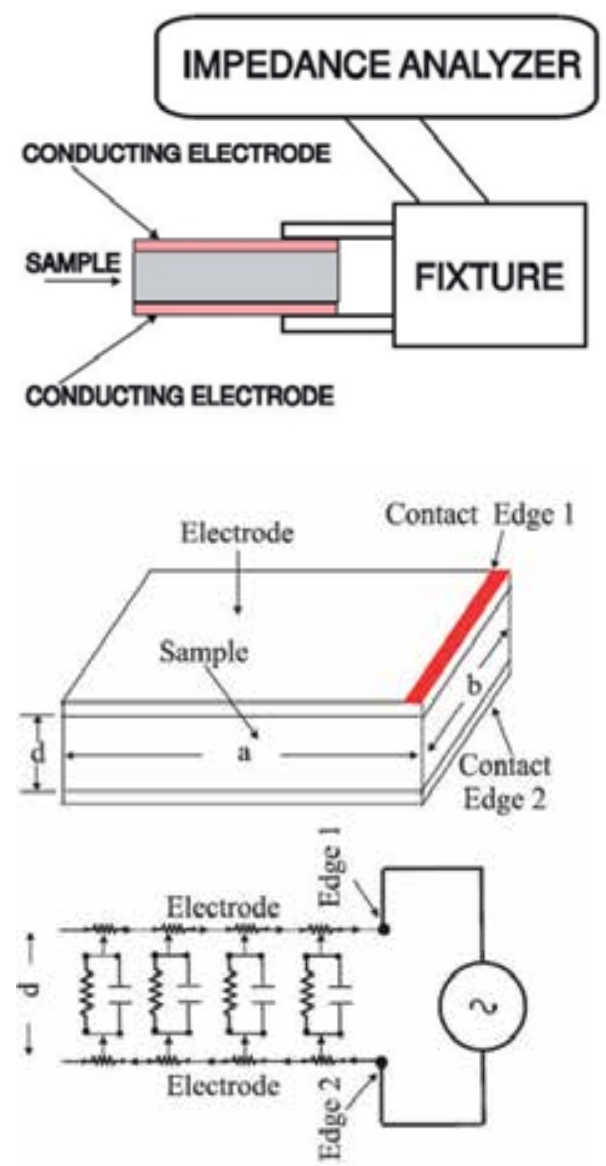

Figure 5. Typical measurement method for HF dielectric properties of the cable shielding and a simplified sample geometry and lumped element representation [10 and 17].

The measured sample impedance is normally interpreted as resulting from the dielectric constant and conductivity of the sample if we assume that the losses and voltage drop in the electrodes are negligible [7 and 10]. The applied electrode has a finite conductivity (normal silver paint) resulting in changes in the measured loss leading to errors in predicting the shield properties. These errors increase with frequency because the current through the electrodes increase significantly with frequency as a result of the large dielectric constant of the shield material. If we assume that voltage is applied along one edge of the upper and lower surface 
of a rectangular sample, as shown in Figure 5, and define $I 1(x)$ is the current as a function of distance from that edge on one surface (electrode), then the magnitude of the current in the other surface is also $I 1(x)$ with an opposite direction. Likewise, if the voltage on energized surface as a function of distance from that edge is $U 1(x)$, then the voltage on the grounded surface is $U_{0}-U 1(x)$. Uo is the applied voltage. From the geometry shown in Figure 5, the following equations can be derived

$$
\begin{aligned}
& l_{1}(x)=-\frac{U_{0} \sqrt{\frac{A b}{2 \rho}\left(\exp \left(x \sqrt{\frac{2 \rho A}{b}}\right)-\exp \left[(2 a-x) \sqrt{\frac{2 \rho A}{b}}\right]\right)}}{\left[1+\exp \left(2 a \sqrt{\frac{2 \rho A}{b}}\right)\right]} \\
& U_{1}(x)=\frac{U_{0}}{2}\left(\frac{\exp \left(x \sqrt{\frac{2 \rho A}{b}}\right)+\exp \left[(2 a-x) \sqrt{\frac{2 \rho A}{b}}\right]+\exp \left(2 a \sqrt{\frac{2 \rho A}{b}}\right)+1}{1+\exp \left(2 a \sqrt{\frac{2 \rho A}{b}}\right)}\right) \\
& A=\frac{b\left(\sigma+j \omega \varepsilon \varepsilon_{0}\right)}{d}
\end{aligned}
$$

where $d$ is the sample thickness, $\rho$ is the electrode surface resistivity in $\Omega /$ sq, $a$ is the sample length, $b$ is the sample width, $\omega$ is the angular frequency, and $\sigma$ and $\varepsilon$ are the conductivity and dielectric constant of the sample, respectively. Furthermore, we can derive the measured conductivity and relative dielectric constant as [17]

$$
\sigma_{P}=\frac{\left(2 P_{\text {electrode }}+P_{\text {shield }}\right) d^{2}}{V U_{0}^{2}}
$$

and

$$
\varepsilon_{P}=\frac{d I_{c}}{U_{0} \omega \varepsilon_{0} a b}
$$

From Eq. 2, it can be seen that the power losses $P_{\text {electrode }}$ caused by the electrodes cause errors as we are measuring losses by the shield $P_{\text {shield }}$, not losses by the electrodes. On the other hand, there is a voltage drop along the electrode due to the larger current and finite conductivity of the electrode resulting in some areas where there is no current flowing through. Thus, the measured losses can be larger or smaller than the actual shield losses. The shield conductivity property measurements affect cable high-frequency attenuation calculations a lot, thus it's very important that we minimize the errors caused by the electrode resistance. A first-order estimate of the maximum frequency to which accurate measurements are likely by using the DC conductivity and low frequency shield dielectric constant to roughly estimate the maximum 
frequency at which the impedance of the shield is comparable to that of the sample electrodes resulting in Equation 4 for a rectangle sample and Equation 5 for a circular sample [10 and 17]:

$$
\begin{aligned}
& f_{\max }=\frac{\sqrt{-4 \sigma^{2} \rho^{2} a^{2}+\left(\frac{d}{a}\right)^{2}}}{4 \pi a \rho \varepsilon \varepsilon_{0}}
\end{aligned}
$$

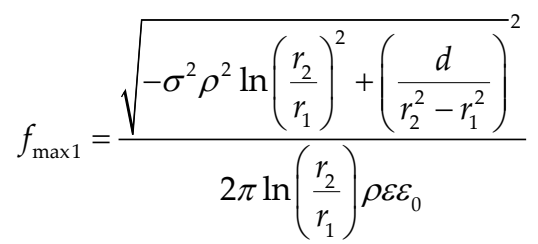

where $r_{2}$ is the "conducting" electrode radius, $r_{1}$ is the contact radius at the center of the electrode, and $d$ is the sample thickness. Figure 6 shows the measured ground shield relative dielectric constant and conductivity for a shield power cable as well as the calculated and measured HF attenuation for the shielded cables.

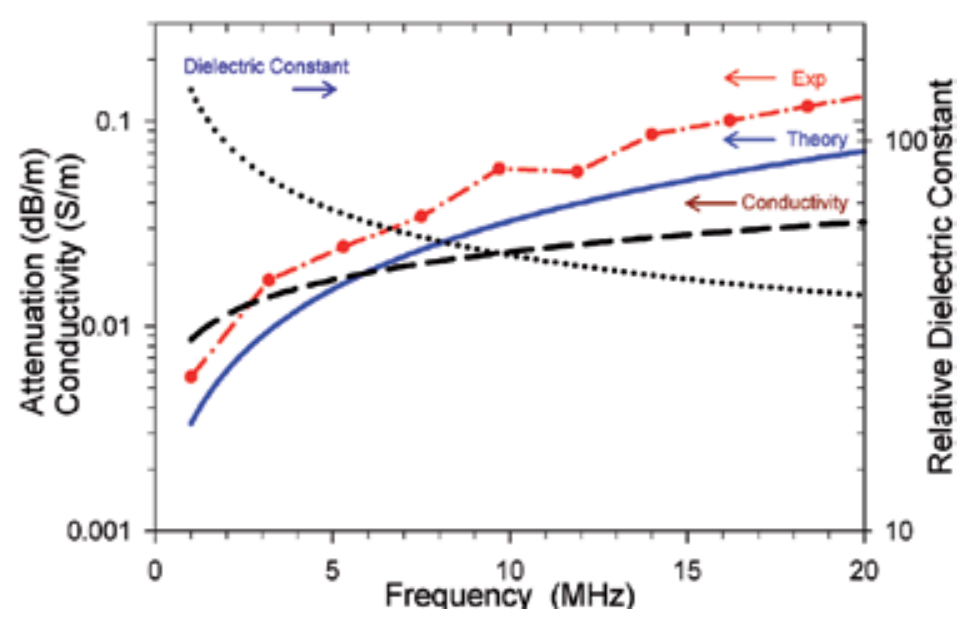

Figure 6. The measured ground shield relative dielectric constant and conductivity for a shielded power cable.

\subsubsection{Shielded cable HF attenuation calculations}

From Figure 4, there are losses from different components of the shielded power cable. The losses come from insulation, skin effect, conduct shield, and ground shield. For high frequencies $(5-20 \mathrm{MHz})$, the loss in the ground shield could dominate the shield cable loss [11,12]. This chapter discusses the loss calculations in the ground shield as it dominates losses at high frequency, which is critical for HF PD pulse propagation, detection and analysis. The shielded 
power cable can have different geometries, configurations, and number of neutral wires. The chapter won't cover all cable configurations. Two typical shielded cables will be studied.

\subsubsection{Normal jacketed shield power cable HF loss calculations}

The conductor and ground shield layers of shielded distribution cables help provide a smooth interface between conductors and insulation. Since the losses caused by the conductor shield are pretty small compared to the losses in other components, these losses are sometimes ignored in analyses. Figure 7 shows a typical jacketed shielded cable geometry and its simplified computation model [11 and 17]. The current flows from the conductor through the insulation (dielectric) passing through the ground semiconducting shield before it reaches the neutral, resulting in big losses in the shield at high frequency. The current passing through the ground shield is determined by the conductor voltage level and dielectric impedance, and can be considered as a "current source."

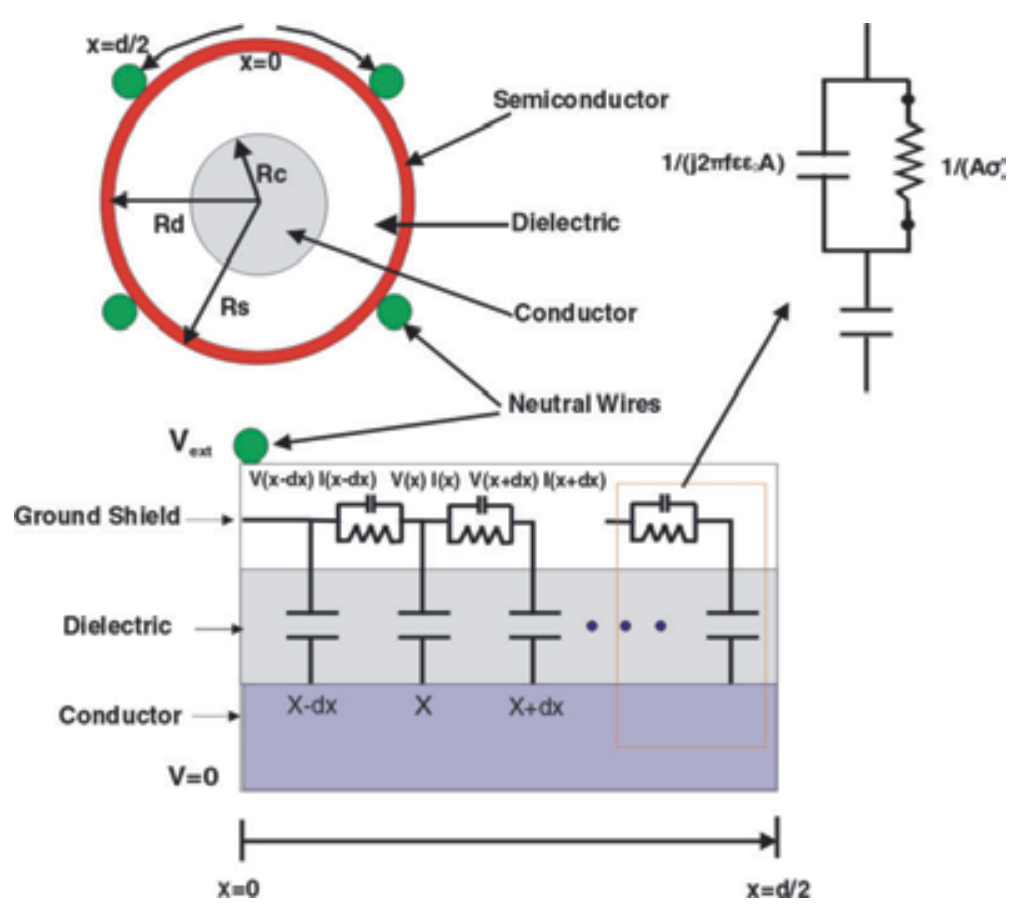

Figure 7. A typical jacketed shielded cable geometry and its simplified computation model [11 and 17]. The current flows from the conductor through the insulation (dielectric) passing the ground semiconducting shield before it reaches the neutral resulting in big losses in the shield at high frequency.

The loss caused by the current in the ground shield, including the component caused by the propagation of the current in a circumferential direction to reach a neutral wire, can be calculated with known cable parameters. 
With this simplified model, the ground shield current and voltage distributions of such a system can be derived [11 and 17]:

$$
\begin{aligned}
& V(x)=-\frac{V_{0}\left\{\exp \left(x \sqrt{\frac{K_{1}}{K_{2}}}\right)+\exp \left[(d-x) \sqrt{\frac{K_{1}}{K_{2}}}\right]\right\}}{\left[1+\exp \left(d \sqrt{\left.\frac{K_{1}}{K_{2}}\right) \sqrt{K_{2}}}\right]\right.} \\
& I(x)=\frac{V_{0}\left\{\exp \left[(d-x) \sqrt{\frac{K_{1}}{K_{2}}}\right]-\exp \left(x \sqrt{\frac{K_{1}}{K_{2}}}\right)\right\}}{\left[1+\exp \left(d \sqrt{\frac{K_{1}}{K_{2}}}\right)\right] \sqrt{K_{1} K_{2}}} \\
& K_{1}=\frac{1}{\left(\sigma_{3}+j \omega \varepsilon_{0} \varepsilon_{3}\right) T} \\
& K_{2}=\frac{T_{2}}{\sigma_{4}+j \omega \varepsilon_{0} \varepsilon_{4}}-\frac{2 j \pi R_{s}}{\omega C}
\end{aligned}
$$

where $d$ is the circumferential distance between two neutral wires; $V_{0}$ is the applied external voltage; $x$ is the position along the circumference of the ground shield; $\sigma_{3}$ is the conductivity of the ground shield; $\varepsilon_{3}$ is the dielectric constant of the ground shield; $\sigma_{4}$ is the conductivity of the conductor shield; $\varepsilon_{4}$ is the dielectric constant of the conductor shield; $\omega$ is the angular frequency of the applied voltage; $C$ is the capacitance of the insulation per meter; $R_{\mathrm{s}}$ is the radius of the ground shield; $T$ is the thickness of the ground shield; and $T_{2}$ is the thickness of the conductor shield. From Figure 7, it is the current that passes through the resistive component of the grounding shield that causes losses. To find the loss, the current through the resistive component needs to be derived, which is given by [11 and 17]

$$
I_{r 1}=\frac{j \sigma_{3} I(x)}{j \sigma_{3}-\omega \varepsilon_{0} \varepsilon_{3}}
$$

and similarly, the current passing through the resistive component of the conductor shield can be found as

$$
I_{r 2}=\frac{j \sigma_{4} I_{c}(x)}{j \sigma_{4}-\omega \varepsilon_{0} \varepsilon_{4}}
$$

With these two currents known, the losses in the conductor and ground shields can be found by calculating the power dissipation, i.e. the product of $I_{\mathrm{r} 1}$ and $I_{\mathrm{r} 2}$ and the resistance of the ground and conductor shields. With some math software such as Maple program and integrating the product of current and the resistive components, the amount of power dissipated can be found. To make it easy for the reader to perform such calculations, the Maple program is attached in the Appendix. 
The losses in the ground shield with some typical parameters (neutral wire conductor radius of $4 \mathrm{~mm}$, dielectric thickness of $7 \mathrm{~mm}$, conductor shield thickness of $0.2 \mathrm{~mm}$, and ground shield thickness of $0.5 \mathrm{~mm}$, the relative dielectric constant of 200, and conductivity of $0.1 \mathrm{~S} / \mathrm{m}$ ) can be seen in Figure 8 for a different number of neutral wires. Finite analysis element calculations are also conducted and the results of which are compared with the analytic results. They agree with each other well.

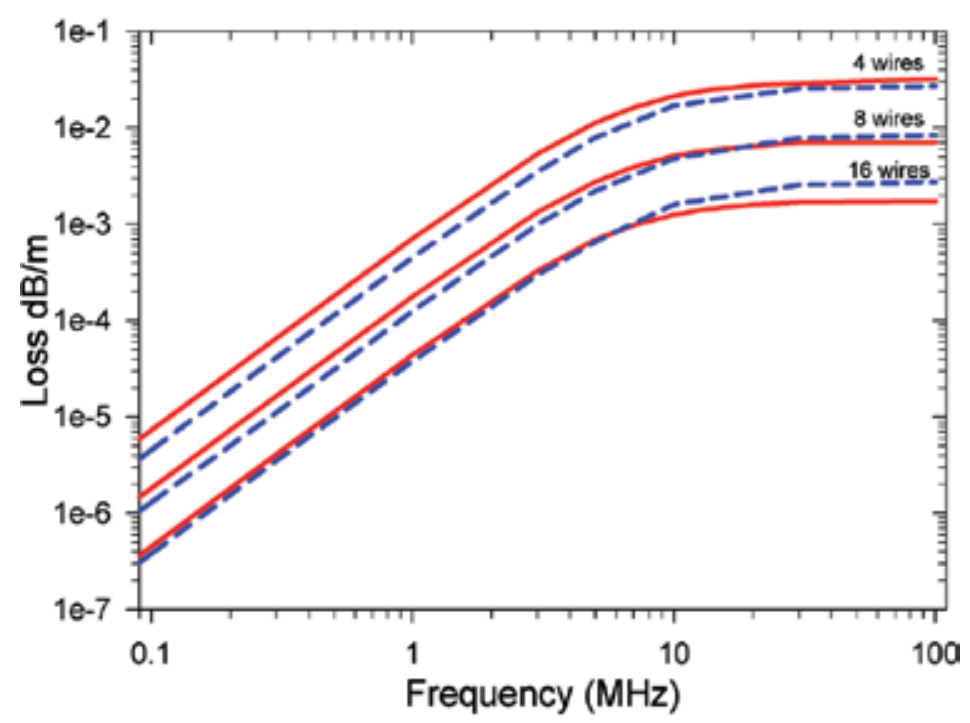

Figure 8. Losses in the ground shield for different numbers of neutral wires [11 and 17]. Finite analysis element calculations are also conducted and the results of which are compared with the analytic results. They agree with each other well.

To give the reader a better idea on the total HF losses in the shielded power cable, one more plot is given in Figure 9 for a cable with six neutral wires [11 and 17]. From Figure 9, it can be seen that for high frequencies (from 5 to $20 \mathrm{MHz}$ ), the ground shield loss, including the losses caused by the interaction of the ground shield with the neutral wires, dominates the HF loss for this six neutral-wire cable. The interaction of the neutral wires with the ground shield plays a critical role for the total loss, as can be seen by comparison with the line showing the ground shield loss with and without the effect of the circumferential current.

\subsubsection{Unjacketed shield power cable HF loss calculations}

To save costs, sometimes, unjacketed cables are installed. A cable jacket ensures intimate contact between ground shield and neutral wires. For unjacketed cables, this contact is not ensured resulting in the loss caused by the ground shield as a function of the separation between the neutral wires and the ground shield and the distance between points of contact of the neutral wires with the ground shield [13]. This section will address the loss as a function of neutral wire separation from the ground shield, contact interval between the neutral wires, 


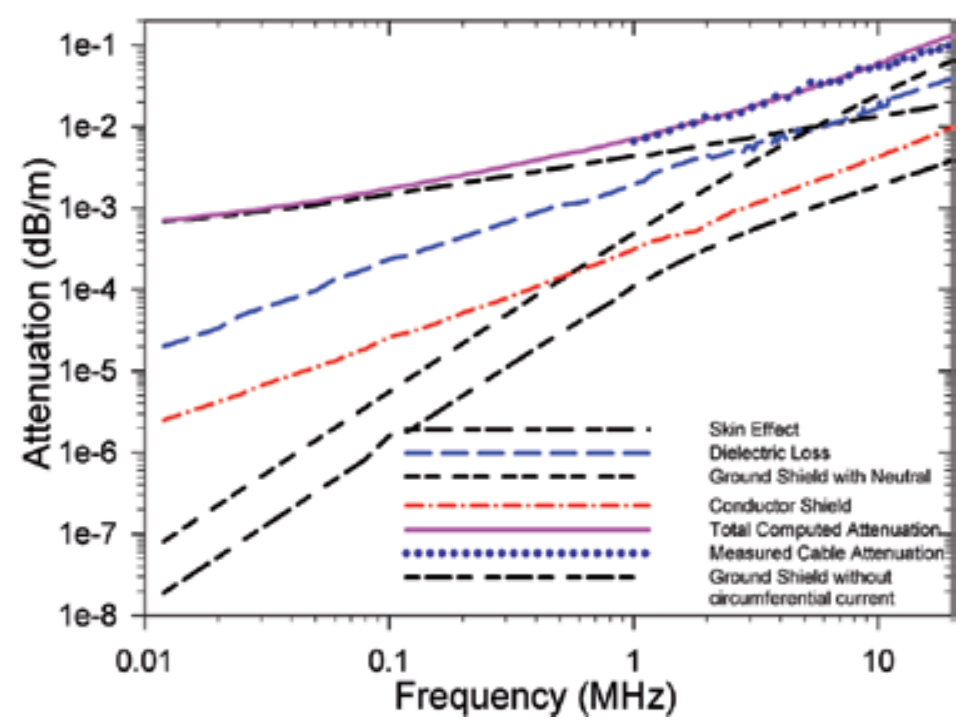

Figure 9. The total losses in a cable with six neutral wires. The losses from the current flowing through the ground losses contribute significantly to the total loss.

ground shield dielectric properties, frequency, etc. Figure 10 [13] gives a simplified model for the unjacketed cable.

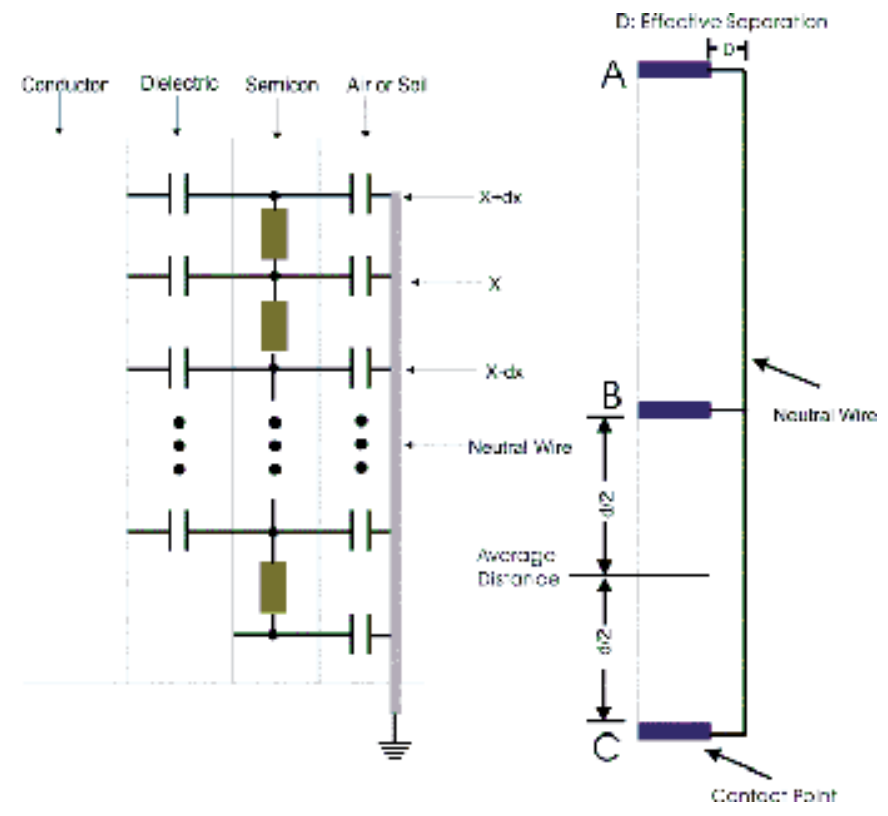

Figure 10. A simplified model for the unjacketed cable. Due to the nature of unjacketed cables, the neutral wires only have contacts with certain points, A, B and C. 
Due to the nature of unjacketed cables, the neutral wires only have contacts with certain points, A, B, and C. Similar to a normal jacketed cable, the current flowing through resistive component of the ground shield leads to loss that dominates the loss for high frequencies. The voltage and current distributions as well as the current passing through the resistive component of such a system can be found as [13]

$$
\begin{aligned}
& V(x)=-\frac{V_{0}\left(Z_{c}+Z_{c} \exp (-d \sqrt{K 1})+Z_{c 1}\{\exp (-x \sqrt{K 1})+\exp [(x-d) \sqrt{K 1}]\}\right)}{\left(Z_{c 1}+Z_{c}\right)[1+\exp (-d \sqrt{K 1})]} \\
& I(x)=\frac{V_{0} \sqrt{Z_{c 1}\left(Z_{c}+Z_{c 1}\right)}\{\exp (-x \sqrt{K 1})-\exp [(x-d) \sqrt{K 1}]\}}{\left[\left(Z_{c 1}+Z_{c}\right)(1+\exp (-d \sqrt{K 1}))\right] \sqrt{Z_{s} Z_{c}}}, \\
& K 1=\frac{Z_{s}\left(Z_{c 1}+Z_{c}\right)}{Z_{c 1} Z_{c}} \\
& Z_{c}=\frac{-j \ln \left(\frac{R_{d}}{R_{c}}\right)}{2 \omega \pi \varepsilon \varepsilon_{0}} \\
& Z_{c 1}=\frac{-j}{\omega C_{t l}} \\
& Z_{s}=\frac{\sigma-j \omega \varepsilon_{1} \varepsilon_{0}}{8 \pi R_{d} T\left[\sigma^{2}+\left(\omega \varepsilon_{1} \varepsilon_{0}\right)^{2}\right]} \\
& I_{r 1}=\frac{\left(\sigma^{2}-j \sigma \omega \varepsilon_{0} \varepsilon_{1}\right) I(x)}{\sigma^{2}+\left(\omega \varepsilon_{0} \varepsilon_{1}\right)^{2}}
\end{aligned}
$$

Similarly to a normal jacketed cable, the losses can be found by integrating the product of the resistive current and the resistance. The Maple program is similar to the normal jacketed shield cable and can be found in paper [13], and thus is not given in this chapter. The ground shield losses in the unjacketed cable, which dominates the HF loss, can vary a lot for different shield dielectric constants, conductivity, and thickness of the ground shield. The thickness of the ground shield plays an important role because the displacement current from the conductor to the ground shield flows longitudinally through the resistance of the ground shield before it reaches a neutral wire-ground shield contact. This loss is maximized under the condition that the resistive impedance of this path is comparable to the capacitive impedance of the dielectric, which is significantly affected by the thickness of the ground shield. For a typical ground shield thickness of $1 \mathrm{~mm}$, and conductor radius, $3.62 \mathrm{~mm}$; insulation thickness, 4.83 $\mathrm{mm}$; capacitance between ground shield and neutral wires, $150 \mathrm{pF} / \mathrm{m}$; insulation dielectric constant, 2.2; distance between two neutral wire-ground shield contacts, $3 \mathrm{~cm}$; frequency, 20 $\mathrm{MHz}$, the loss of such a jacketed cable vs. dielectric constant and conductivity is shown in 
Figure 11 [13]. From Figure 11, it can be seen that the losses can be pretty large due to the long distance needed for the displacement current to flow before it reaches the neutral wires, which means that the HF electromagnetic PD wave can be attenuated significantly in the unjacketed cable.

As stated at the beginning of this section, shield cables have different geometries and configurations. The authors won't address too many configurations and only two typical shielded cables, i.e. jacketed normal shielded cable and unjacketed shielded cables, are studied in this section. The reader should keep in mind that when designing and analyzing the HF electromagnetic PD wave signals, it's very important to know the shield dielectric properties and cable actual geometry and configurations to get a rough idea of the cable's HF attenuation properties. The readers should also know that it's not easy to do the HF dielectric property measurements and use caution when conducting such measurements. Furthermore, the reader needs to know that the shields in the cables are under pressure whereas the shields under test have no pressure. Some compensation might be necessary to offset the pressure difference.

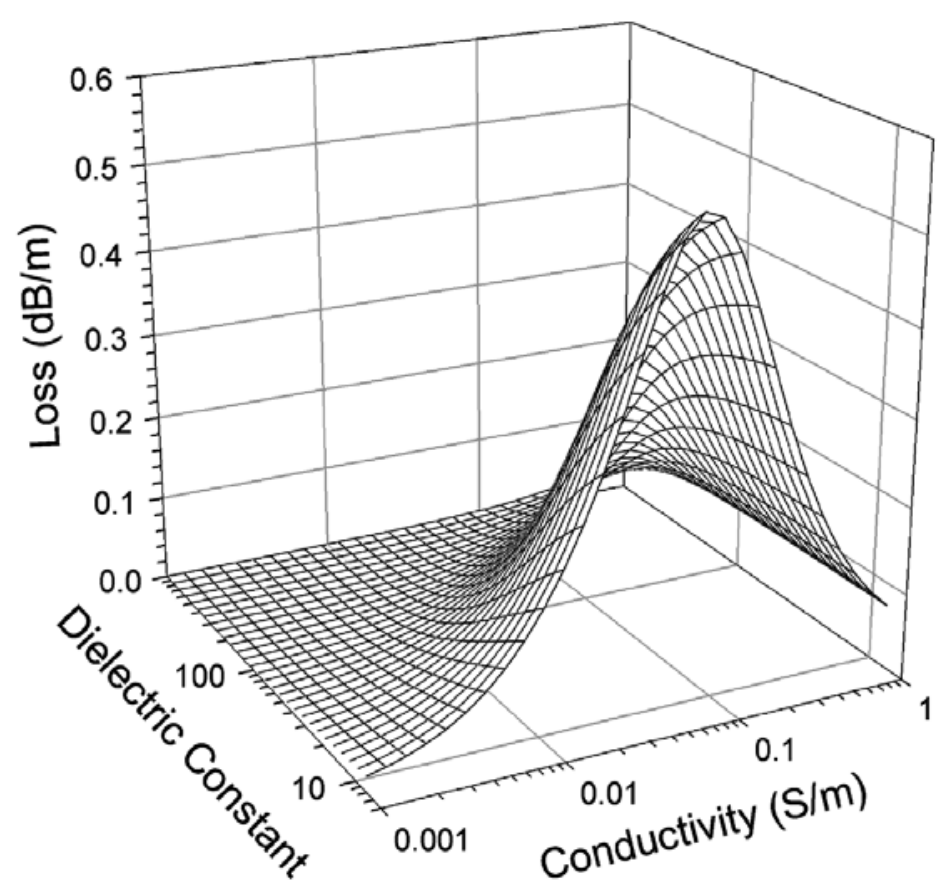

Figure 11. Losses vs. dielectric constant and conductivity for typical parameters.

\subsection{HF electromagnetic PD wave propagation}

The HF electromagnetic PD wave propagates in the shield cable and the wave is attenuated while it travels. How significant the wave is is mainly determined by the HF loss properties of the cable, which has been discussed extensively in Section 2.2. Besides the losses of the cable, 
the cable splices and termination can also affect the wave propagation and attenuation. Figure 12 shows one typical PD wave propagation. The PD wave propagates in both directions passing through the cable splices before it reaches the cable termination and reflects back if the waves are not attenuated completely. When it travels along the cable, HF components are most attenuated due to the facts discussed in Section 2.2, because the displacement current increased with frequency resulting in higher losses for higher frequencies. This provides the electromagnetic wave pulse a big pulse width as shown in Figure 12. If the pulse is not attenuated completely, it will reach the cable termination and reflect backward toward the PD source as shown in Figure 12. To analyze the effect of the cable losses, the electromagnetic wave pulse can be treated as a Gaussian pulse. If a Gaussian pulse in time domain is applied at the left end of the cable to simulate the PD source signal, it will propagate through the splices before it can reach the termination. For simplicity, splices are thought in parallel with loads having characteristic impedance. Since we apply a Gaussian pulse in time domain, we need to transform it to the frequency domain, multiply it with attenuation factor caused by splices in frequency domain, then transform it back to time domain. The output voltage is attenuated by a factor of Vatt which is

$$
\text { Vatt }=\frac{2 Z}{Z+Z_{c}}, Z=\frac{Z_{s} Z_{c}}{Z_{s}+Z_{c}}
$$

where $Z_{\mathrm{s}}$ is splice effective impedance, $Z_{\mathrm{c}}$ is characteristic impedance. By doing so, we get loss vs. number of splices as shown in Figure 13. Parameters used in computation are given in the caption of Figure 13. We also can treat the problem by calculating the energy dissipated by the cable. Current flows through semicon will result in loss. By calculating the loss, we can get a voltage attenuation ratio in frequency domain, Vatt1, which is:

$$
\operatorname{Vat} 1=\sqrt{1-\frac{P_{L}}{P_{I}}}
$$

where $P_{\mathrm{L}}$ is the power dissipated by splice whereas $P_{\mathrm{I}}$ is the input power. A Gaussian pulse multiplied by this ratio results in an attenuated Gaussian pulse as seen in Figure 14.

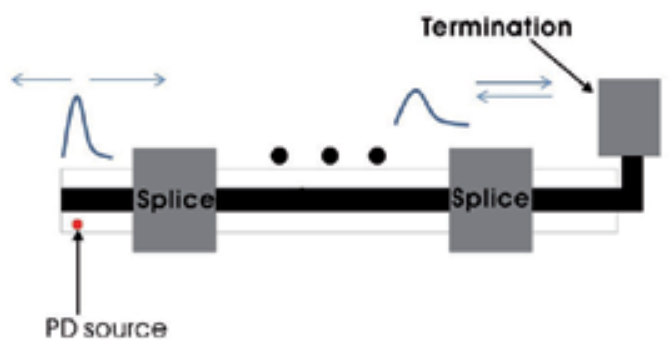

Figure 12. One typical PD wave propagation pattern. Note that the wave propagates in both directions although only the right section is shown here. 


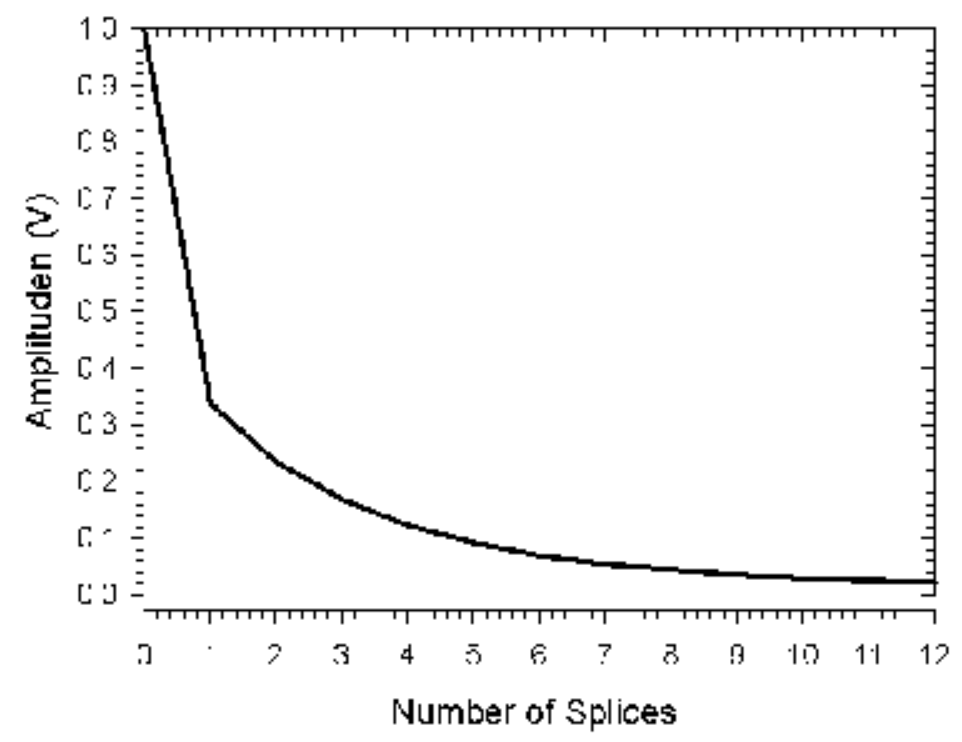

Figure 13. Amplitude as a function of number of splice number. Splices have the following parameters: length, $60 \mathrm{~cm}$; semicon conductivity, $5 \mathrm{~s} / \mathrm{m}$; conductor radius, $4 \mathrm{~mm}$; thickness of the insulation, $4 \mathrm{~mm}$; thickness of semicon, $6 \mathrm{~mm}$; dielectric constant of semicon, 300; dielectric constant of insulation, 2.2.

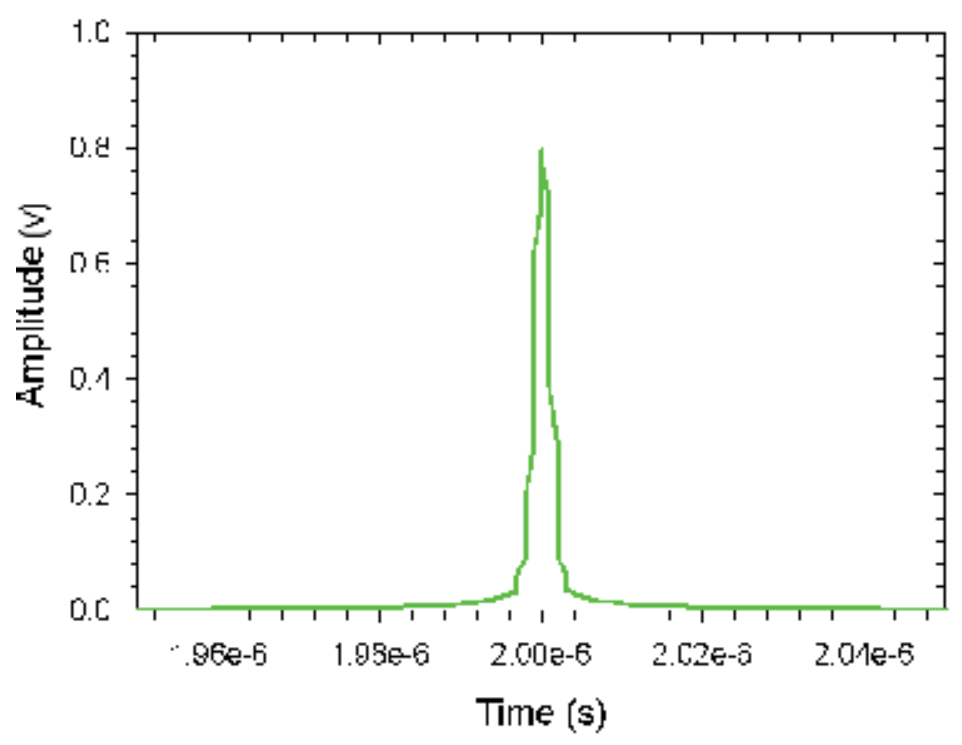

Figure 14. Attenuated Gaussian pulse. The splice has the following parameters: radius of conductor, $1 \mathrm{~cm}$; thickness of insulation, $0.5 \mathrm{~cm}$; thickness of semicon, $0.3 \mathrm{~cm}$; conductivity of semicon, $10 \mathrm{~S} / \mathrm{m}$; dielectric constant of semicon, 300; dielectric constant of insulation, 3.3; distance of splice, $40 \mathrm{~cm}$. 
The reader should keep in mind that the studied case is a simulation result based on some assumed shield dielectric properties. To assess the losses caused by the splices, some field measurements as well as further computations are necessary. Normally, the splices have smaller losses compared with the long cable.

\section{Electromagnetic wave detection in shielded dielectric cables}

PD detection has been extensively used for high-voltage apparatus diagnostic and status assessment. This section focuses on electromagnetic PD wave detection as, due to its relatively smaller losses, the HF PD signal can propagate for a long distance and can be detected by the PD sensors located either at the termination or other appropriate locations of the power circuit for the shielded power cables.

\subsection{PD pulse sensor}

Different sensors (optical, acoustic, electrical, etc.) are used for detecting the PD. For detecting the electromagnetic waves propagating in shielded dielectric cables, normally a high-frequency current transformer (HFCT) and ultra high-frequency (UHF) sensor are used [18-21]. The HFCT is normally placed around the shielded cable or sometimes around the ground strip to collect the PD signals. The UHF sensor captures the UFH electromagnetic waves propagating in the shielded cable, which can be attenuated quickly depending on the loss characteristic of the cable. Figure 15 shows some typical configurations for using HFCT in the PD measurements. There are a lot of different other sensors such as coupling capacitor, inductively coupled probes, integrated partial discharge sensor, etc. that are used in PD electromagnetic pulse detections. Among them, the HFCTs are one of the most widely used sensors due to their high bandwidth and ease of use.

For processing the measured PD signals, different theories and algorithms have been proposed for optimizing PD testing results [18-21]. For partial discharge measurements, as stated in the above sections, the PD signals cannot be detected directly due to its nature as the PD is inside the insulation. The electromagnetic wave pulse signals we capture are indirect measurements of the PD and analysis needs to be done to extract useful information such as PD locations, PD magnitude, etc. Some PD detection technologies require an excitation voltage to produce a partial discharge signal pulse to find the PD location. For locating the PD location, two sensors are placed at two different locations along the cable and if the attenuation along the cable is the same, then when the two sensors reads the same level of PD, the PD source is in the middle of the two sensors assuming that the cable splices contribute little or few losses for the PD pulse propagation. Theoretically, the technology should work but an excitation voltage could damage the cable and the cable needs to be taken out of service. More and more PD detection systems have been developed for online real-time measurements as they don't require dangerous excitation voltages and can be used to assess the HV apparatus under more realistic conditions. However, the highly noisy environment caused by the high voltage and high current of the power cable makes extracting useful PD information difficult. To improve the 
signal-to-noise ratio for optimized measurement results, different technologies, such as noise filtering, digital signal processing optimization, signal amplifying, etc., are used.

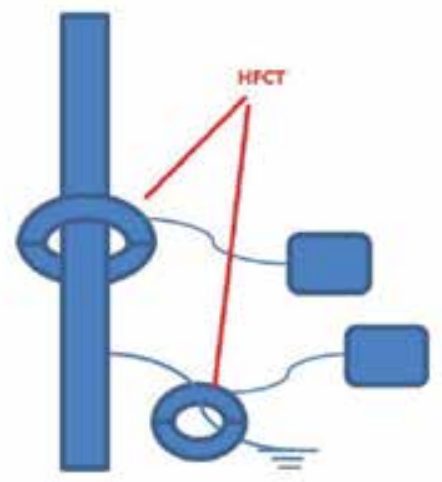

Figure 15. HFCT used in PD detection.

\subsection{PD pulse detection data processing and transmitting}

Some PD detection systems detect PD at the hot spots identified by the utility companies and can't be used to monitor the system continuously due to the complicated data processing and communication. Due to the fact that the PD signals are normally HF signals which can attenuate quickly if a normal coaxial cable is used for connecting the PD detection sensor and the PD signal analyzer and also for safety concerns, sometimes, optical coupling [22] is used for long-distance data transmitting between the sensor and the PD data analyzer. After the field signals are received by data processors, which can be a wide-band oscilloscope or a spectrum analyzer, the PD data are processed for waveform, phase, span, etc. [28]. For better results, field signals can also be filtered and amplified and processed with specialized processing computers for trend analysis and alarm generation, etc.

With the advancement of modern wireless communication and digital signal processing technologies, real-time continuously online PD detection becomes more realistic [23-28]. Different wireless technologies have been used for transmitting measured processed data back to the controlling center. The processed output from the oscilloscope or other digital instruments can be fed into data transmitters using Ethernet, wireless LTE modem, Wifi, Zigbee network, etc. Ethernet is reliable and easy to use but sometimes it can be unavailable. Wireless LTE is flexible but can be expensive due to its common monthly data fee. Wifi is also a good option if it's available. Zigbee is a low-cost and low-maintenance method compared with cellular data service as it does not have a monthly fee. The Zigbee node is small and, if set correctly, it can run for a long time without maintenance. Figure 16 shows one customized Zigbee node [29]. However, its data rate is relatively low compared with Ethernet and Wifi. To monitor the PD in real time, the captured PD signals can be processed with a wideband local oscilloscope or other devices such as a spectrum analyzer and the output can be fed into 
one of the data transmitting routers. The PD measurements will lead to a lot of raw data and normally they can't be sent directly to the remote controlling center due to its high data volume. After processing, the data can be sent back to the controlling and data concentrator for appropriate actions such as cable maintenance, alarm generation, etc.

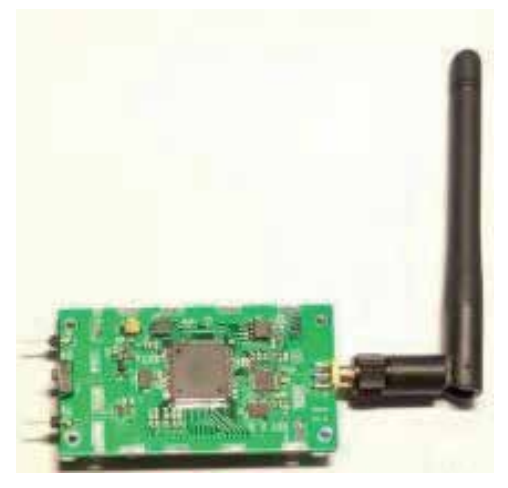

Figure 16. One Zigbee node.

\section{Summary and discussion}

Because the shielded power cables are widely used in modern HV power systems and the fact that the partial discharge is a powerful tool for assessing the HV power system status, HF PD electromagnetic wave propagation, detection, and analysis have been extensively studied. In this chapter, the authors look into the details of the shield power cable structure, their HF attenuation properties, PD electromagnetic wave propagation, and address the detection and analysis of the HF electromagnetic wave in the shielded power cable.

\section{Appendix: Maple program for loss calculations in a typical jacketed shielded cable}

>restart;

$>\mathrm{Rc}:=4 \mathrm{e}-3$ :\# Rc is the conductor radius.

>epsilon:=2.2:\#Epsilon is the dielectric constant of the insulation.

>epsilon0:=8.85e-12:

$>$ mu1:=1*4* ${ }^{*}{ }^{*} 1 \mathrm{e}-7$ :

$>$ Rs:=11.7e-3: \#Rs is the ground shield radius (m).

$>\mathrm{Rd}:=11.2 \mathrm{E}-3$ : $\mathrm{Rd}$ is the insulation radius (m). 
$>\mathrm{V} 0:=1$ :

$>\mathrm{N}:=4$ :\# $\mathrm{N}$ is the number of neutral wires.

>omega:=2*Pi*f:

>epsilon $0:=8.85 \mathrm{e}-12$ :

$>1:=1$ :\# let the length of the cable be $1 \mathrm{~m}$;

$>\mathrm{T}:=0.5 \mathrm{e}-3$ : $\# \mathrm{~T}$ is the thickness of the ground shield.

$>\mathrm{T} 2:=0.2-3: \# \mathrm{~T} 2$ is the thickness of the conductor shield.

$>\mathrm{d}:=\operatorname{evalf}\left(2^{*} \mathrm{Pi}^{*} \mathrm{Rs} / \mathrm{N}\right)$ :\# $\mathrm{d}$ is the length between two neutral wires.

$>\mathrm{C}:={ }^{*} \mathrm{Pi}^{*}$ epsilon${ }^{*}$ epsilon $0 * 1 /(\ln (\mathrm{Rs} / \mathrm{Rc}))$ :\# Capacitance of the insulation per meter.

$>C 1:={ }^{*} \mathrm{Pi}^{*}$ epsilon*epsilon0/( $\left.\ln (\mathrm{Rd} / \mathrm{Rc})\right)$ :

$>\mathrm{L} 1:=\mathrm{mu} 1 * \ln (\mathrm{Rs} / \mathrm{Rc}) /(2 * \mathrm{Pi})$ :

$>$ Zc:=evalf(sqrt(L1/C1)):\#Characteristic impedance.

$>\mathrm{K} 1:=1 /\left(\left(\text { sigma3+omega*epsilon } 0^{*} \text { epsilon } 3^{*} \mathrm{I}\right)^{*} \mathrm{1}^{*} \mathrm{~T}\right)$ :

$>\mathrm{K} 2:=-2 * \mathrm{I} /$ omega/C/1*Pi*Rs+T2/(sigma4+omega*epsilon4*epsilon0*I)/1:

$>\mathrm{I} 1:=$

$-\mathrm{V} 0^{*}\left(\exp \left(1 / \mathrm{K} 2^{\wedge}(1 / 2)^{*} \mathrm{~K} 1^{\wedge}(1 / 2)^{*} \mathrm{x}\right)-\exp \left(-\mathrm{K} 1^{\wedge}(1 / 2)^{*}(-\mathrm{d}+\mathrm{x}) / \mathrm{K} 2^{\wedge}(1 / 2)\right)\right) / \mathrm{K} 1^{\wedge}(1 /$

$2) / \mathrm{K} 2^{\wedge}(1 / 2) /\left(1+\exp \left(1 / \mathrm{K} 2^{\wedge}(1 / 2)^{*} \mathrm{~K} 1^{\wedge}(1 / 2)^{*} \mathrm{~d}\right)\right): \#$

Current distribution along the ground shield.

$>\mathrm{V} 1:=-\mathrm{V} 0^{*}\left(\exp \left(1 / \mathrm{K} 2^{\wedge}(1 / 2)^{*} \mathrm{~K} 1^{\wedge}(1 / 2)^{*} \mathrm{x}\right)+\exp \left(-\mathrm{K} 1^{\wedge}(1 / 2)^{*}(-\mathrm{d}+\mathrm{x}) / \mathrm{K} 2^{\wedge}(1 / 2)\right)\right) / \mathrm{K} 2^{\wedge}($

$1 / 2) /\left(1+\exp \left(1 / \mathrm{K} 2 \wedge(1 / 2)^{*} \mathrm{~K} 1^{\wedge}(1 / 2)^{*} \mathrm{~d}\right)\right): \#$

90

Voltage distribution along the ground shield.

$>\mathrm{I} 2:=(-\mathrm{V} 1) / \mathrm{K} 2$ :\# Current flowing through the conductor shield.

$>\mathrm{dZr} 4:=\mathrm{T} 2 /\left(\operatorname{sigma} 4^{*} \mathrm{l}^{*} \mathrm{dx}\right): \# \mathrm{dZr} 4$ is the elemental resistive impedance of the conductor shield.

$>\mathrm{dZc} 4:=1 /\left(\mathrm{I}^{*}\right.$ omega* $\left(\right.$ epsilon0*epsilon $\left.\left.4^{*} \mathrm{l}^{*} \mathrm{dx} / \mathrm{T} 2\right)\right)$ :\# $\mathrm{dZc} 4$ is the elemental capacitive impedance of the conductor shield.

>Ratio2:=simplify $(\mathrm{dZc} 4 /(\mathrm{dZc} 4+\mathrm{dZr} 4))$ :\# To get the current flowing through the resistive component of the conductor shield, we calculate Ratio2.

>Irtotal2:=I2* Ratio2:\# Irtotal2 is the current flowing through resistive component 
of the conductor shield.

$>\mathrm{dZr} 3:=\left(1^{*} \mathrm{dx}\right) /\left(\operatorname{sigma}^{*}{ }^{*} \mathrm{~T}\right): \#$ Same as above.

$>\mathrm{dZc} 3:=1 /\left(\mathrm{I}^{*}\right.$ omega ${ }^{*}($ epsilon0*epsilon3*1*T/dx)):\# Same as above.

>Ratio1:=simplify(dZc3/(dZc3+dZr3)):\# Same as above.

>Irtotal:=I1*Ratio1:\# Irtotal1 is the current flowing through resistive component

of the ground shield.

$>\operatorname{Ii}:=\operatorname{Im}(\operatorname{Irtotal})$ :

$>\operatorname{Ir}:=\operatorname{Re}($ Irtotal):

$>$ Ii2:=Im(Irtotal2):

$>\operatorname{Ir} 2:=\operatorname{Re}(\operatorname{Irtotal} 2)$ :

$>\operatorname{Pd} 4:=\operatorname{int}\left(\left((\operatorname{Ir} 2)^{\wedge} 2+(\operatorname{Ii} 2)^{\wedge} 2\right)^{*}(\mathrm{~T} 2 /(\operatorname{sigma} 4)), \mathrm{x}=0 . . \mathrm{d} / 2\right)$ :\# Conductor shield power

dissipation

$>$ Pd3:=int $\left(\left((\operatorname{Ir})^{\wedge} 2+(\mathrm{Ii})^{\wedge} 2\right)^{*}\left(1 /\left(\operatorname{sigma3}{ }^{*} \mathrm{~T}\right)\right), \mathrm{x}=0 . . \mathrm{d} / 2\right)$ :\# Ground shield power dissipation

$>$ Pin:=(V0)^2/Zc:\# Input power.

$>\mathrm{dB} 3:=10 * \log 10\left(1-\left(\mathrm{Pd}^{*}{ }^{*} \mathrm{~N} / \mathrm{Pin}\right)\right)$ :\# Ground shield attenuation.

$>\mathrm{dB} 4:=10^{*} \log 10\left(1-\left(\mathrm{Pd} 4{ }^{*}{ }^{*} \mathrm{~N} / \mathrm{Pin}\right)\right)$ :\# Conductor shield attenuation.

\section{Author details}

Chunchuan Charles $\mathrm{Xu}^{1^{*}}$ and Chengyin $\mathrm{Liu}^{2}$

*Address all correspondence to: xuchunchuan@gmail.com

1 Underground Systems, Inc, Armonk, NY, U.S.A.

2 Harbin Institute of Technology Shenzhen Graduate School, Shenzhen, China

\section{References}

[1] Chunchuan $\mathrm{Xu}$, "Recent Developments in Power System Diagnostics and Protection: Partial Discharge Detection and Analysis." Recent Patents on Electrical \& Electronic Engineering (Formerly Recent Patents on Electrical Engineering), Volume 3, Number 1, January 2010, pp. 51-54(4). 
[2] Wolfgang Hauschild, et al. "High-Voltage Test and Measuring Techniques", Springer Heidelberg New York Dordrecht London. 2014.

[3] P. Degn, "Partial Discharges in Solid Dielectrics", Ph.D. Thesis, Electric Power Engineering Department, Technical Uni of Denmark, Lyngby, 1971.

[4] S. Boggs, et al. "Fundamental limitations in the measurement of corona and partial discharge", IEEE Transactions on Electrical Insulation, Volume EI-17, Issue 2, pp. 143-150, 1982.

[5] C.A. Bailey, "A study of internal discharge in cable insulation", IEEE Paper No. 31, pp. 66-363, 1966.

[6] Z. Liu, et al. "The Propagation of Partial Discharge Pulses in a High Voltage Cable", Proc. of AUPEC/EECON eds, 1999. Citeseer.

[7] G.C. Stone, et al. "Propagation of partial discharge pulses in shielded power cable," in 1982 Annu. Rep. Conf. Elect. Insul. Dielect. Phenomena. Washington, DC: Natl. Acad. Sci., pp. 275-280.

[8] W. L. Weeks, et al. "Wave propagation characteristics in underground power cable," IEEE Trans. PAS-103, (Oct) pp. 2816-2825, 1984.

[9] Chunchuan $\mathrm{Xu}$, et al. "High Frequency Properties of Shielded Power Cable Part 1: Overview of Mechanisms", IEEE Electrical Insulation Magazine, Volume 21, Issue 6, pp. 24-28, 2005.

[10] Chunchuan Xu, et al. "High Frequency Properties of Shielded Power Cable Part 2: Sources of Error in Measuring Shield Dielectric Properties", IEEE Electrical Insulation Magazine, Volume 22, Issue 1, pp. 7-13, 2006.

[11] Chunchuan Xu, et al. "High Frequency Properties of Shielded Power Cable. Part 3: Loss from Neutral Wire-Shield Interaction", IEEE Electrical Insulation Magazine 04/2007; 23(2):12-16.

[12] Chunchuan $\mathrm{Xu}$, et al. "High Frequency Loss from Neutral Wire-Shield Interaction of Shielded Power Cable", Power Delivery, IEEE Transactions on, Volume 23, Issue 2, 2008, pp. 531-536.

[13] Chunchuan Xu, et al. "High-Frequency Loss in Unjacketed Distribution Cable and Its Effect on PD Measurement", Power Delivery, IEEE Transactions on, Volume 24, Issue 2, 2009, pp. 495-500.

[14] Jim Jun Guo, et al. "High Frequency Attenuation in Transmission Class Solid Dielectric Cable", Power Delivery, IEEE Transactions on, Volume 23, Issue 4, 2008, pp. 1713-1719.

[15] M. Vakilian, et al. "Investigation of PD Signal Propagation Characteristics in XLPE Cables”, International Conf. Power Syste. Techn., pp. 1863-1868, 2004. 
[16] G. Mugala, et al. "High frequency characteristics of a shielded medium voltage XLPE cable," in 2001 Annu. Rep. IEEE Conf. Elect. Insul. Dielect. Phenomena, 2001, pp. 132-136.

[17] Chunchuan $\mathrm{Xu}$, “High Field Phenomena in Polymer Films and High Frequency Attenuation in Shielded Power Cable", Ph.D thesis, Uconn 2007.

[18] M. Wild et al. "Power Cable Modeling for PD Pulse Propagation and Sensitivity", 2013 IEEE Electrical Insulation Conference (EIC), June 2-5, pp. 57-60.

[19] Khushboo Arora, et al. "Wavelet Analysis Used for Partial Discharge Pattern Recognition", International Journal of Emerging Technology and Advanced Engineering. Website: www.ijetae.com (ISSN 2250-2459 (Online), Volume 4, Special Issue 1, February 2014) pp. 125-130.

[20] Naima Oussalah, et al. "Analytic Solutions for Pulse Propagation in Shielded Power Cable for Symmetric and Asymmetric PD Pulses", IEEE Transactions on Dielectrics and Electrical Insulation Vol. 14, No. 5, October 2007, pp. 1264-1270.

[21] Xiaoli Zhou, et al. "The Application of Partial Discharge Detection for the condition assessment of XLPE Power Cables" Przegląd Elektrotechniczny (Electrical Review), ISSN 0033-2097, R. 88 NR 6/2012, pp. 313-316.

[22] Y. Tian, et al. "Continuous on-line monitoring of partial discharges in high voltage cables", Conference Record of the 2004 IEEE International Symposium on Electrical Insulation, 2004. 19-22 Sept. 2004 pp. 454-457.

[23] Marco Tozzi, "Partial Discharges in Power Distribution Electrical Systems: Pulse Propagation Models and Detection Optimization", PhD thesis, Alma Mater Studiorum-University of Bologna, 2010.

[24] Ghadir Madi, et al. "Impacts of impulsive noise from partial discharges on wireless systems performance: application to MIMO precoders", Madi et al. EURASIP Journal on Wireless Communications and Networking 2011, 2011:186.

[25] Shan, Qingshan, et al. "Performance of Zigbee in Electricity Supply Substations", International Conference on Wireless Communications, Networking and Mobile Computing, 2007. WiCom 2007. 21-25 Sept. 2007, pp. 3871-3874.

[26] S.A. Bhatti, et al "Vulnerability of Zigbee to Impulsive Noise in Electricity Substations", General Assembly and Scientific Symposium, 2011 XXXth URSI, 13-20 Aug. 2011, pp. 1-4.

[27] P.C. Baker, et al. "Data Management of On-Line Partial Discharge Monitoring Using Wireless Sensor Nodes Integrated with a Multi-Agent System", International Conference on Intelligent Systems Applications to Power Systems, 2007. ISAP 2007. pp. 1-6. 
[28] Chen Rui-Long, et al. "The Design of Partial Discharge On-Line Monitoring System for XLPE Power Cable", Research Journal of Applied Sciences, Engineering and Technology 6(21): 4063-4069, 2013.

[29] Chengyin Liu, et al. "Development and validation of wireless strain sensor network", Conference Record of the 13th International Symposium on Structural Engineering, 2014, Oct. 24-27, Hefei, China. 

Chapter 5

\title{
Nonreciprocal Devices Utilizing Longitudinally Magnetized Ferrite Coupled Lines
}

\author{
Adam Kusiek, Wojciech Marynowski, Rafal Lech and \\ Jerzy Mazur
}

Additional information is available at the end of the chapter

\begin{abstract}
This chapter presents authors' recent research on the nonreciprocal devices utilizing longitudinally magnetized ferrite coupled line (FCL) junction. The principle of operation of FCL junction is explained and the hybrid techniques of analysis are shown. Numerical and experimental results concerning the nonreciprocal devices utilizing the different configurations of FCL junctions are presented and discussed.
\end{abstract}

Keywords: ferrite, nonreciprcoal, Faraday rotation

\section{Introduction}

Nonreciprocal devices have been extensively used in modern microwave and millimeter systems [2-4, 14, 25, 27, 28]. In order to obtain the nonreciprocal effects, one needs to utilize the magnetized ferrite materials $[2,4,14,25,27,28]$ or active elements such as amplifiers [3]. Recently, the longitudinally magnetized ferrite coupled striplines or slotlines [2, 9, 14, 27] are being developed and employed to realize integrated nonreciprocal devices. Significant interest in these devices results from their advantages which are weak biasing magnetic field and wide operation bandwidth.

The basic part of ferrite coupled line (FCL) devices is longitudinally magnetized FCL section composed of two coupled lines placed on ferrite substrate [6, 21]. This structure was first proposed and experimentally verified by [6]. Next, Next, Mazur \& Mrozowski in [21] using the coupled-mode method (CMM) developed the model of FCL section which explains the operation of this structure and gives basis steps in their design procedure. According to this model, in the ferrite section, a gyromagnetic coupling occurs, resulting in Faraday rotation effect. The wide operation bandwidth and high isolation are obtained, when the Faraday 
rotation phenomenon is optimal. This optimal effect is achieved when the ferrite material is placed in the region where the wave is linearly polarized and occurs in cylindrical waveguide with coaxially located ferrite rod or suspended stripline. In order to construct devices such as circulators [9, 23], gyrators [19, 22], or isolators [13], the FCL junction has to be cascaded with reciprocal sections providing input signals to the FCL which are either in phase or out of phase [20].

So far, studies concerning FCL devices have been focused mainly on structures realized in a planar line technology $[1,4,9]$. Such structures allow one to obtain fully integrated FCL devices. However, due to the significant length of the ferrite section, the main drawbacks are high insertion losses occurring in ferrite material and large dimensions of the structure.

There were several attempts to improve performance and to reduce total dimensions of planar FCL devices. Promising results concerning low insertion losses and high isolation were obtained for the nonreciprocal devices employing a ferrite coupled slotline [9] and stripline junctions $[23,24]$. For the fabricated devices, obtained insertion losses were not lower than $3 \mathrm{~dB}$ and isolation was better than $12 \mathrm{~dB}[9,18,23]$. Moreover, in order to reduce the dimensions of the planar FCL devices in [4], the circulator with appropriate matching networks at the ports ensuring multiple reflections was proposed. For presented device, the FCL junction length reduction by a factor of two was obtained. The drawback of this structure was high value of insertion losses caused by multiple transmission of signal through the lossy ferrite junction. Also similar length reduction of FCL junction was achieved with the use of periodic left-handed/ferrite coupled line (LH-FCL) structures [1]. However, for the simulated circulator utilizing LH-FCL section, the insertion losses were not lower than $4 \mathrm{~dB}$.

The better performance in comparison to currently proposed planar configurations was obtained for nonreciprocal devices utilizing cylindrical ferrite coupled line (CFCL) junction [10]. Due to the similar geometry to the circular waveguide with coaxially located ferrite rod, such structure allows to obtain close-to-optimal Faraday rotation effect. Moreover, in such configuration, stronger gyromagnetic coupling occurs which is a result of high magnetic field concentration in the ferrite medium. These make possible to design shorter ferrite junctions ensuring lower insertion losses in comparison to planar ones. This junction was successfully applied to realization of nonreciprocal devices such as isolators and circulators [11, 12].

This chapter presents the authors' recent research on the nonreciprocal devices utilizing longitudinally magnetized FCL junction. The operation principle of FCL junction is explained, and the hybrid techniques of analysis are shown. Numerical and experimental results concerning the nonreciprocal devices using different configurations of FCL junctions are presented and discussed.

\section{Formulation of the problem}

The general view of FCL junction is presented in Fig. 1. This junction is a four-port structure that contains a ferrite section realized as two coupled lines placed on ferrite substrate. The ferrite section is fed from dielectric coupled lines with the same cross section where instead of ferrite, the dielectric materials are used. When the ferrite junction is longitudinally magnetized, the Faraday rotation phenomenon occurs, resulting in nonreciprocal properties of the FCL junction. For better understanding of the nonreciprocal effect, an example of a junction ensuring $45^{\circ}$ Faraday rotation has been considered (see Fig. 1(b) and (c)). When 


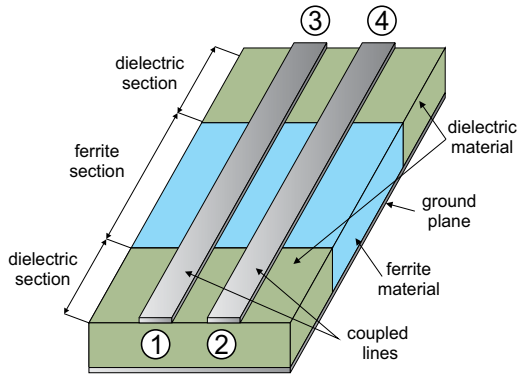

(a)

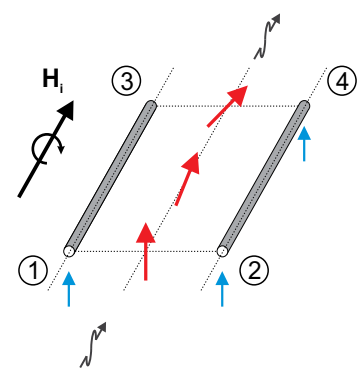

(b)

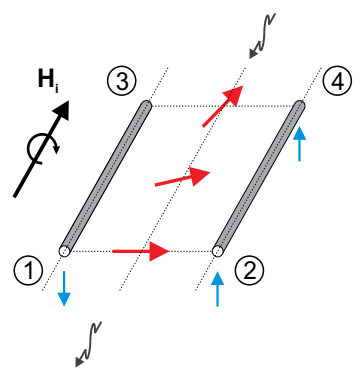

(c)

Figure 1. Ferrite coupled line junction: (a) general view of the structure and transmission through the junction for (b) even-mode excitation and (c) single-port excitation

ports (1) and (2) of the structure are excited with in phase signals of equal amplitude (see Fig. 1(b)), due to the Faraday rotation, the output signal is observed only at port (4), while port (3) is isolated. On the other hand, when only port (4) of the structure is excited, the out of phase signals of equal amplitudes appear in ports (1) and (2) (see Fig. 1(c)). These phenomena occurring in the FCL section directly indicate the presence of the nonreciprocal effect. Based on the abovementioned phenomena, it was noted in [20] that in order to obtain nonreciprocal transmission, the FCL section should be cascaded with the structures that allow for the evenor odd-mode signal excitation. This makes possible to realize FCL circulators, which can then be used to design a variety of other nonreciprocal circuits such as isolators or phase shifters.

One of the basic nonreciprocal circuits built based on the FCL section is a three-port circulator shown in Fig 2(a). This structure consists of a cascade connection of $T^{e}$ - or $T^{o}$-junction with four-port FCL junction. Note that the direction of circulation of the circulator structure depends on the choice of T-junction type. Taking into account the circulator with $T^{e}$-junction, when port (1) of the structure is excited the output signal appears in port (2). Excitation of port (2) of the circulator results in the signal transmission to port (3). Finally, if port (3) is excited, the signal is transmitted to port (1). Therefore, such circuit provides transmission sequence between ports $(1) \rightarrow(3) \rightarrow(2) \rightarrow(1)$. The circulation direction will reverse when the FCL section is cascaded with $T^{0}$-junction. In addition, the circulation direction can be reversed by changing into opposite the direction of biasing magnetic field which results in reversed direction of Faraday rotation. The described circulator can be used for the realization of isolator, by introducing a matched load in one of the circulator ports.

The four-port circulators can be obtained by replacing in three-port circulator from Fig. 2(a) the normal T-junction with magic T-junction (see Fig. 2(b)) or just by cascading two three-port FCL circulators (see Fig. 2(c)). The advantage of structure from Fig. 2(b) is that the signal for each transmission passes through the ferrite section only once, and as a result, this structure can be characterized by lower insertion losses. However, in the case of integrated circuits, the realization of this circulator requires the design of a complex and technologically demanding magic T-junction. In the case of nonreciprocal devices utilizing two FCL sections, it is possible to obtain better isolation in comparison to structure from Fig. 2(b). However, due to the double signal passing through the ferrite section, the losses of the structure are two times 


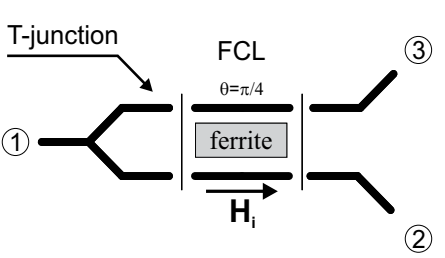

(a)

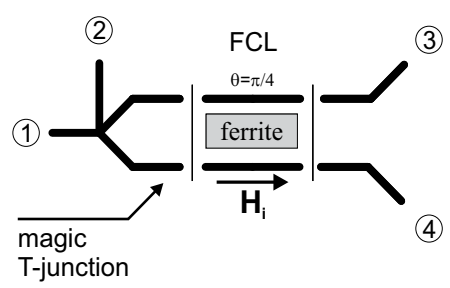

(b)

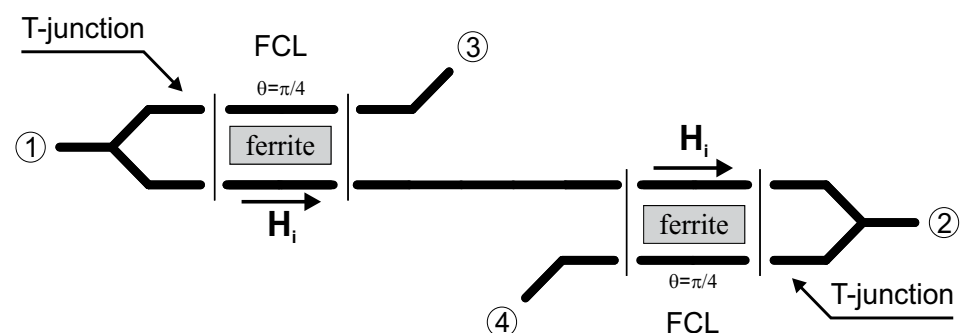

(c)

Figure 2. Nonreciprocal devices utilizing ferrite coupled line junction: (a) three-port circulator, (b) four-port circulator with single FCL section, and (c) four-port circulator with double FCL section

higher. It should be noted that presented nonreciprocal devices from Fig. 2 are analogous to cylindrical waveguide nonreciprocal devices with Faraday rotation ([7]).

In order to determine the scattering parameters of the FCL junction, two different hybrid techniques have been proposed and developed ([12, 16-18]) (see Fig. 3). The first approach

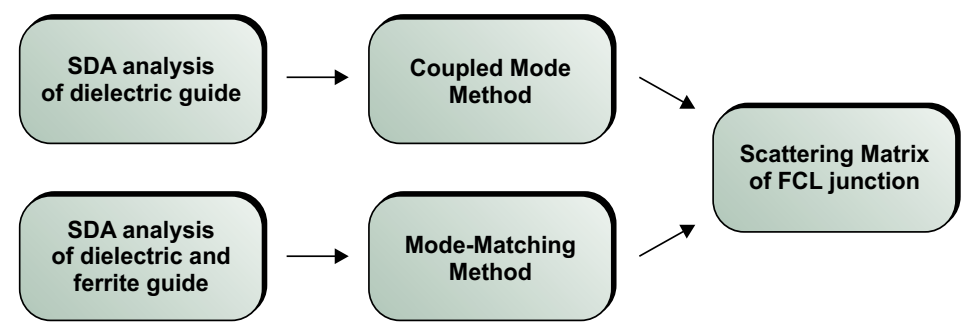

Figure 3. Proposed analysis methods of investigated configurations of FCL junctions

is based on the combination of spectral-domain approach (SDA) and coupled-mode method $(\mathrm{CMM})([16,17])$. The analysis using SDA/CMM involves introducing an isotropic basic guide. This guide is complementary to the ferrite one; however, instead of ferrite, the dielectric characterized by the same permittivity as ferrite and relative permeability $\mu_{r}=1$ is utilized. In this approach, the basic guide modes obtained from SDA are used to determine the wave parameters of ferrite modes. As a result of the analysis, the dispersion characteristics of the ferrite line, the gyromagnetic coupling coefficient, and the scattering parameters of the ferrite junction are obtained. The second method is based on a combination of SDA with mode-matching (MM) technique $([12,18])$. In this approach, the SDA is utilized to determine the propagation coefficients and field distribution of two fundamental modes in 
a dielectric and ferrite section. Then by applying the continuity conditions for the tangential field components at both the interfaces between dielectric and ferrite sections, we formulate the scattering matrix of four-port FCL junction.

The formulation of SDA for dielectric/ferrite guides with different cross sections has been presented in section 2.1. The details of scattering matrix calculation utilizing CMM and MM method have been presented in sections 2.2 .1 and 2.2.2, respectively.

\subsection{Analysis of dielectric/ferrite guide using SDA}

In Fig. 4, the cross sections of the investigated lines are presented. In order to determine the propagation coefficients and field distribution in the structure, the spectral domain approach is utilized. Depending on the type of the considered line, the method is formulated in rectangular, cylindrical, or elliptic coordinate system.

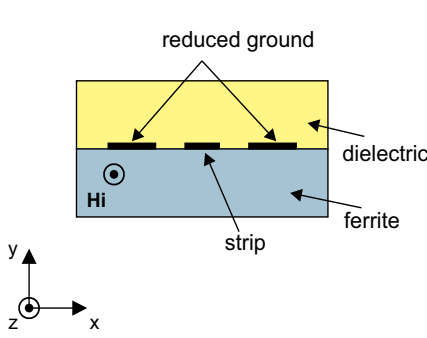

(a)

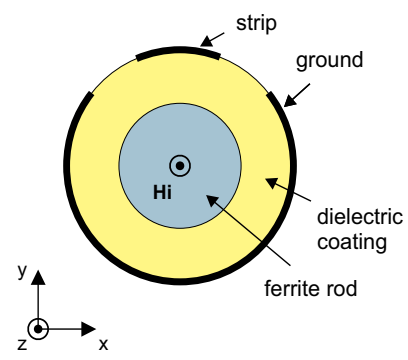

(b)

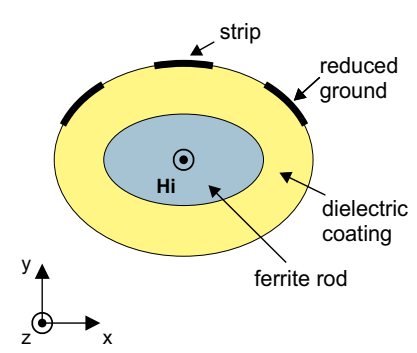

(c)

Figure 4. Investigated types of coupled lines: (a) planar three-strip line, (b) cylindrical coupled slotline and (c) elliptical three-strip line

The Fourier transform in each coordinate system takes the following form:

- Rectangular coordinates $(x, y, z)$

$$
\widetilde{f}(p)=\int_{\mathbb{R}} f(y) e^{-\mathrm{j} p y} \mathrm{~d} y, \quad f(y)=\frac{1}{2 \pi} \int_{\mathbb{R}} \widetilde{f}(p) e^{\mathrm{j} p y} \mathrm{~d} p,
$$

- Cylindrical coordinates $(\rho, \varphi, z)$

$$
\widetilde{f}(p)=\frac{1}{2 \pi} \int_{0}^{2 \pi} f(\varphi) e^{-\mathrm{j} p \varphi} \mathrm{d} \varphi, \quad f(\varphi)=\sum_{p=-\infty}^{\infty} \widetilde{f}(p) e^{\mathrm{j} p \varphi},
$$

- Elliptic coordinates $(u, v, z)$ 


$$
\begin{aligned}
& \widetilde{f}^{e(o)}(p)=\frac{1}{2 \pi} \int_{0}^{2 \pi} f(v)\left\{\begin{array}{l}
c e_{p}(q, v) \\
s e_{p}(q, v)
\end{array}\right\} \mathrm{d} v, \\
& f(v)=\sum_{p=0}^{\infty} \widetilde{f}^{e}(p) c e_{p}(q, v)+\sum_{p=1}^{\infty} \widetilde{f}^{o}(p) s e_{p}(q, v),
\end{aligned}
$$

where $\tilde{f}(\cdot)$ is the image of the function $f(\cdot), c e_{p}(\cdot, \cdot)$ and $s e_{p}(\cdot, \cdot)$ are even and odd angular Mathieu functions of $p$ th order, $q=k_{0}^{2} d^{2} / 4, k_{0}=\omega \sqrt{\mu_{0} \varepsilon_{0}}$, and $d$ is the focal length. The electromagnetic field in the structure can be described by Maxwell's equations defined in the spectral domain, as follows:

$$
\begin{aligned}
& \nabla \times \widetilde{\mathbf{E}}=-k_{0} \boldsymbol{\mu}_{r} \eta \widetilde{\mathbf{H}} \\
& \nabla \times \eta \widetilde{\mathbf{H}}=k_{0} \varepsilon_{r} \widetilde{\mathbf{E}}
\end{aligned}
$$

where $\widetilde{\mathbf{E}}$ and $\widetilde{\mathbf{H}}$ are Fourier transforms of the electric and magnetic fields, respectively, $\eta=$ $-\mathrm{j} \eta_{0}, \eta_{0}=\sqrt{\mu_{0} / \varepsilon_{0}}$, and $\boldsymbol{\mu}_{r}$ denotes the permeability tensor of ferrite material. For the longitudinally magnetized ferrite material along $z$-axis, $\boldsymbol{\mu}_{r}=\mathbf{T} \boldsymbol{\mu}_{r}^{\prime} \mathbf{T}^{-1}$, where $\boldsymbol{\mu}_{r}^{\prime}=\mu\left(\mathbf{i}_{x} \mathbf{i}_{x}+\right.$ $\left.\mathbf{i}_{y} \mathbf{i}_{y}\right)+\mathbf{j} \mu_{a}\left(\mathbf{i}_{x} \mathbf{i}_{y}-\mathbf{i}_{y} \mathbf{i}_{x}\right)+\mathbf{i}_{z} \mathbf{i}_{z}$ is a permeability tensor in a dyadic form defined in rectangular coordinates, $\mathbf{T}$ is transformation matrix from rectangular to cylindrical or elliptic coordinates, and $\mu$ and $\mu_{a}$ are defined according to [7].

By applying the boundary and continuity conditions to the relations (5), (6) and assuming the fields and currents variation along $z$-axis as $e^{-\mathrm{j} \beta z}$, one obtains a set of equations combining tangential electric field $\left(\widetilde{E}_{z}\right.$ and $\left.\widetilde{E}_{\xi}\right)$ and current densities $\left(\widetilde{J}_{z}\right.$ and $\left.\widetilde{J}_{\xi}\right)$ at the strips:

$$
\left[\begin{array}{l}
\widetilde{E}_{z}\left(\varsigma=\varsigma_{0}, p\right) \\
\widetilde{E}_{\mathcal{\zeta}}\left(\varsigma=\varsigma_{0}, p\right)
\end{array}\right]=[\mathbf{G}(p, \beta)]\left[\begin{array}{l}
\widetilde{J}_{\mathcal{J}}\left(\varsigma=\varsigma_{0}, p\right) \\
\widetilde{J}_{z}\left(\varsigma=\varsigma_{0}, p\right)
\end{array}\right]
$$

where $(\zeta, \xi)=\{(x, y),(\rho, \varphi),(u, v)\}$ and $\mathbf{G}(p, \beta)$ is a dyadic Green's function ([8]). In order to solve (7) the current on the patch is expanded in terms of basis functions:

$$
\begin{aligned}
& J_{\xi}(\xi)=\left\{\begin{array}{cc}
\sum_{n=1}^{N} a_{n} \sin \left(\frac{n \pi(2 \xi+w)}{2 w}\right), & |\xi| \leq \frac{w}{2} \\
0, & \text { otherwise, }
\end{array}\right. \\
& J_{z}(\xi)=\left\{\begin{array}{cc}
\sum_{n=0}^{N} b_{n} \frac{\cos \left(\frac{n \pi(2 \xi+w)}{2 w}\right)}{\sqrt{1-\left(\frac{2 \xi}{w}\right)^{2}}}, & |\xi| \leq \frac{w}{2} \\
0, & \text { otherwise, }
\end{array}\right.
\end{aligned}
$$


where $a_{n}$ and $b_{n}$ are unknown current expansion coefficients. Next, using MoM with current basis functions (8) and (9) chosen as a testing functions (Galerkin method) a homogeneous set of equations is obtained ([26]). The nontrival solutions of the problem provide us with a phase coefficients $\beta$ and corresponding current coefficients allowing us to determine the current density distributions on the strips as well as the electric and magnetic fields in the cross section of the structure.

\subsection{Scattering matrix of ferrite coupled junction}

\subsubsection{Coupled-mode method}

Using the coupled-mode method, a gyromagnetic coupling coefficient, propagation coefficients of ferrite modes, and scattering matrix of FCL junction can be determined $([5,21])$. In this method, the transverse electric and magnetic fields in the investigated ferrite guide are expressed in terms of basic guide field eigenfunctions. Assuming two fundamental modes propagated in the basic guide, the Maxwell's equations for basic and ferrite guides are combined together, and after some mathematical manipulation, the following set of coupled-mode equations is obtained:

$$
\begin{array}{r}
\frac{\partial}{\partial z} \widehat{U}_{e}(z)+\mathrm{j} \beta_{e} Z_{e} \widehat{I}_{e}(z)=C_{e o} \widehat{I}_{o}(z), \\
\frac{\partial}{\partial z} \widehat{U}_{o}(z)+\mathrm{j} \beta_{o} Z_{o} \widehat{I}_{o}(z)=C_{o} \widehat{I}_{e}(z), \\
\frac{\partial}{\partial z} \widehat{I}_{e}(z)+\mathrm{j} \beta_{e} Y_{e} \widehat{U}_{e}(z)=0, \\
\frac{\partial}{\partial z} \widehat{I}_{o}(z)+\mathrm{j} \beta_{o} Y_{o} \widehat{U}_{o}(z)=0,
\end{array}
$$

where

$$
C_{e o}=-C_{e o}^{*}=k_{0} \eta_{0} \mu_{a} \int_{\Omega_{f}}\left(\mathbf{h}_{t, e} \times \mathbf{h}_{t, o}^{*}\right) \cdot \mathbf{i}_{z} \mathrm{~d} \Omega_{f}
$$

define the coupling between two fundamental modes in the basic guide, $\widehat{U}_{e(o)}(z)$ and $\widehat{I}_{e(o)}(z)$ are $z$-dependent voltage and current functions in the ferrite guide, $Z_{e(o)}=1 / Y_{e(o)}$ are wave impedances of fundamental modes, $\mathbf{h}_{t, e(o)}$ are the eigenfunctions of magnetic fields of fundamental modes, and $\Omega_{f}$ is a ferrite area in the cross section. Next, taking into consideration field distributions $\mathbf{H}_{t, e(o)}$ of basic modes instead of their eigenfunction $\mathbf{h}_{t, e(o)}$, the gyromagnetic coupling coefficient can be written as follows:

$$
C_{e o}=k_{0} \eta_{0} \mu_{a} \frac{\sqrt{Z_{e} Z_{o}}}{\sqrt{P_{e} P_{o}}} \int_{\Omega_{f}}\left(\mathbf{H}_{t, e} \times \mathbf{H}_{t, o}^{*}\right) \cdot \mathbf{i}_{z} \mathrm{~d} \Omega_{f},
$$

where $P_{e(o)}$ denotes powers of two fundamental basis modes. As one can see, the gyromagnetic coupling occurs in the guide when the ferrite is placed in the area where 
magnetic field vectors of two fundamental modes are orthogonal to each other and linearly polarized.

The above-defined transmission line model of the ferrite guide can be used to determine scattering matrix of FCL junction. At first, utilizing symmetry properties of the investigated structure, the modal even/odd voltage and current can be related to voltage and current defined for each of two coupled lines (see Fig. 5)

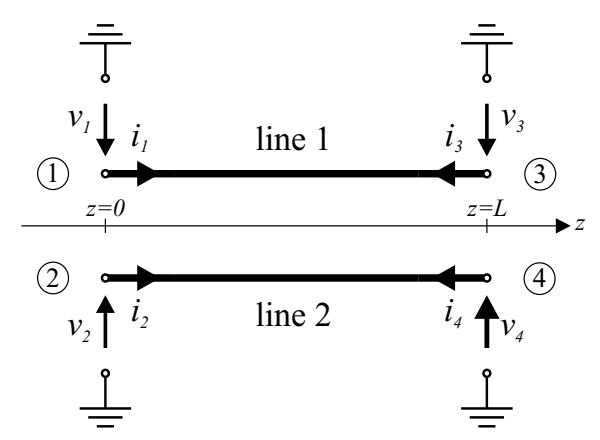

(a)

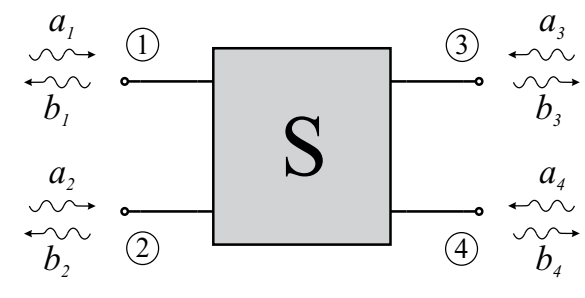

(b)

Figure 5. Four-port FCL junction: (a) circuit model, (b) network representation

as follows:

$$
\begin{array}{ll}
\widehat{U}_{\mathcal{e}}(z)=\widehat{U}_{1}(z)+\widehat{U}_{2}(z), & \widehat{I}_{\mathcal{e}}(z)=\widehat{I}_{1}(z)+\widehat{I}_{2}(z), \\
\widehat{U}_{o}(z)=\widehat{U}_{1}(z)-\widehat{U}_{2}(z), & \widehat{I}_{o}(z)=\widehat{I}_{1}(z)-\widehat{I}_{2}(z) .
\end{array}
$$

Next, applying the above relations to (10), the following eigenproblem is obtained:

$$
\mathbf{Q K}=\mathbf{K k}
$$

where matrix $\mathbf{Q}$, diagonal matrix of eigenvalues $\mathbf{k}$, and matrix of eigenvectors $\mathbf{K}$ are defined in [10]. The solutions of (14) are the eigenvalues $k_{1}$ and $k_{2}$, defining propagation coefficients of two fundamental modes in ferrite guide which take the following form:

$$
k_{1,2}= \pm \sqrt{\frac{\beta_{e}^{2}+\beta_{o}^{2}}{2} \pm \sqrt{\left(\frac{\beta_{e}^{2}-\beta_{o}^{2}}{2}\right)^{2}+\frac{C_{e o}^{2}}{Z_{e} Z_{o}} \beta_{e} \beta_{o}}}
$$

As one can see, the increase of the coupling results in the increase of the difference between $k_{1}$ and $k_{2}$. Finally, the length of the ferrite section ensuring $45^{\circ}$ Faraday rotation decreases when the coupling $C_{e o}$ increases. 
Using the solution of (15), the voltage and current in each of the two coupled lines can be defined at considered $z$ cross section as follows:

$$
\left[\begin{array}{c}
\widehat{U}_{1}(z) \\
\widehat{U}_{2}(z) \\
\widehat{I}_{1}(z) \\
\widehat{I}_{2}(z)
\end{array}\right]=\mathbf{K}\left[\begin{array}{cccc}
e^{-\mathrm{j} k_{1} z} & 0 & 0 & 0 \\
0 & e^{\mathrm{j} k_{1} z} & 0 & 0 \\
0 & 0 & e^{-\mathrm{j} k_{2} z} & 0 \\
0 & 0 & 0 & e^{\mathrm{j} k_{2} z}
\end{array}\right]\left[\begin{array}{c}
A_{1}^{+} \\
A_{1}^{-} \\
A_{2}^{+} \\
A_{2}^{-}
\end{array}\right]
$$

where $A_{1}^{+(-)}$and $A_{2}^{+(-)}$are unknown amplitudes of the forward and backward partial waves in the equivalent transmission line. Assuming notation from Fig. 5(a) and utilizing (16), we can write the relations between voltages and currents in the ports of the considered junction defined at interfaces $z=0$ and $z=L$ :

$$
\left[\begin{array}{l}
\widehat{U}_{1}(z) \\
\widehat{U}_{2}(z) \\
\widehat{I}_{1}(z) \\
\widehat{I}_{2}(z)
\end{array}\right]_{z=0}=\left[\begin{array}{l}
v_{1} \\
v_{2} \\
i_{1} \\
i_{2}
\end{array}\right] \quad \text { and } \quad\left[\begin{array}{l}
\widehat{U}_{1}(z) \\
\widehat{U}_{2}(z) \\
\widehat{I}_{1}(z) \\
\widehat{I}_{2}(z)
\end{array}\right]_{z=L}=\left[\begin{array}{c}
v_{3} \\
v_{4} \\
-i_{3} \\
-i_{4}
\end{array}\right]
$$

Combining equations (16) and (17), we obtain the following relation:

$$
\left[\begin{array}{c}
v_{3} \\
v_{4} \\
-i_{3} \\
-i_{4}
\end{array}\right]=\mathbf{K}\left[\begin{array}{cccc}
e^{-j k_{1} L} & 0 & 0 & 0 \\
0 & e^{\mathrm{j} k_{1} L} & 0 & 0 \\
0 & 0 & e^{-\mathrm{j} k_{2} L} & 0 \\
0 & 0 & 0 & e^{\mathrm{j} k_{2} L}
\end{array}\right] \mathbf{K}^{-1}\left[\begin{array}{c}
v_{1} \\
v_{2} \\
i_{1} \\
i_{2}
\end{array}\right]
$$

Finally, we define incident and reflected waves in each $i$ th port of the structure as follows:

$$
a_{i}=\frac{v_{i}}{\sqrt{Z_{0}}}+i_{i} \sqrt{Z_{0}} \quad \text { and } \quad b_{i}=\frac{v_{i}}{\sqrt{Z_{0}}}-i_{i} \sqrt{Z_{0}}
$$

where $Z_{0}$ is a wave impedance of the port. Applying above relations to (18), we obtain the scattering matrix of four-port FCL junction which is defined as follows:

$$
\left[\begin{array}{l}
b_{1} \\
b_{2} \\
b_{3} \\
b_{4}
\end{array}\right]=\left[\begin{array}{llll}
S_{11} & S_{12} & S_{13} & S_{14} \\
S_{21} & S_{22} & S_{23} & S_{24} \\
S_{31} & S_{32} & S_{33} & S_{34} \\
S_{41} & S_{42} & S_{43} & S_{44}
\end{array}\right]\left[\begin{array}{l}
a_{1} \\
a_{2} \\
a_{3} \\
a_{4}
\end{array}\right]
$$

Assuming instead of the voltage and current waves the real voltage and current distribution in the proposed transmission line model of FCL junction, the wave impedances $Z_{e(o)}$ and $Z_{0}$ can be treated as characteristic impedances. 


\subsubsection{Mode-matching method}

In order to determine the S-matrix of the proposed FCL junction, the mode-matching method is utilized. At first, the junction is analyzed as a two-port structure composed of dielectric section followed by ferrite section and another dielectric section as presented in Fig. 6. Since

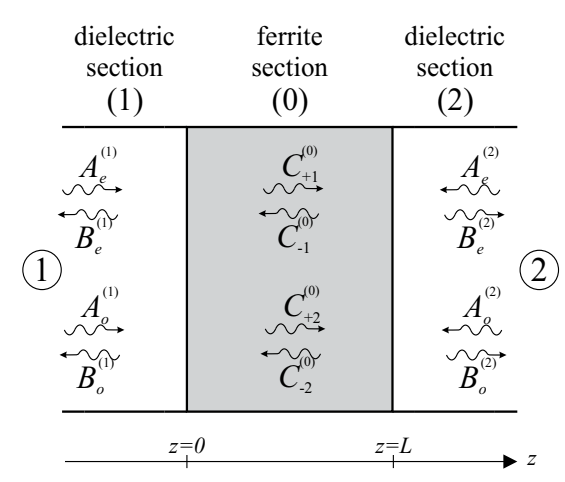

Figure 6. Two port FCL junction composed of ferrite and dielectric sections.

the widths of the strips in the ferrite and dielectric sections are the same, the higher modes are not excited, and therefore, they are neglected in the analysis. In this case, only two fundamental modes are taken into consideration. The modes propagated in the dielectric and ferrite sections are called dielectric and ferrite waves, respectively. Due to the symmetry of the structure, we can distinguish even- and odd-mode waves in the dielectric section. Despite the fact that at port (1) or (2) only one of the dielectric waves can appear, both ferrite waves are excited. The wave parameters and field distributions of modes in the ferrite and dielectric sections are determined using SDA ([15]). The total field in each section is determined as a superposition of both modes propagating in forward $(+)$ and backward (-) directions. Using the notation from Fig. 6, the total field in dielectric sections $i=1,2$ can be written in the following form:

$$
\mathbf{F}_{t}^{(i)}=\left[\mathbf{F}_{+e}^{(i)}, \mathbf{F}_{+o}^{(i)}, \mathbf{F}_{-e^{\prime}}^{(i)}, \mathbf{F}_{-o}^{(i)}\right]\left[\begin{array}{c}
A_{e}^{(i)} \\
A_{o}^{(i)} \\
B_{e}^{(i)} \\
B_{o}^{(i)}
\end{array}\right]
$$

where $\mathbf{F}=(\mathbf{E}, \mathbf{H})$ represents tangential electric or magnetic field and $A$ and $B$ are the unknown expansion coefficients describing forward and backward waves, respectively, of even $(e)$ and odd $(o)$ modes. The total field in ferrite section can be written as follows:

$$
\mathbf{F}_{t}^{(0)}=\left[\mathbf{F}_{+1}^{(0)}, \mathbf{F}_{+2}^{(0)}, \mathbf{F}_{-1}^{(0)}, \mathbf{F}_{-2}^{(0)}\right] \mathbf{D}(z)\left[\begin{array}{c}
C_{+1}^{(0)} \\
C_{+2}^{(0)} \\
C_{-1}^{(0)} \\
C_{-2}^{(0)}
\end{array}\right]
$$


where $\mathbf{D}(z)=\operatorname{diag}\left(\left[e^{j k_{+1}^{(v)} z}, e^{j k_{+2}^{(v)} z}, e^{j k_{-1}^{(v)} z}, e^{j k_{-2}^{(v)} z}\right]\right), k_{ \pm 1(2)}^{(0)}$ are the propagation coefficients of both fundamental modes in ferrite section and $\mathbf{F}=(\mathbf{E}, \mathbf{H})$ and $C_{ \pm 1(2)}^{(0)}$ are the unknown expansion coefficients describing forward $(+)$ and backward (-) waves. Using relations (20) and (21), the continuity conditions for the tangential components of electric and magnetic fields at two interfaces $z=0$ and $z=L$ can be written as follows:

$$
\begin{aligned}
\left.\mathbf{E}_{t}^{(1)}\right|_{z=0}=\left.\mathbf{E}_{t}^{(0)}\right|_{z=0^{\prime}} & \left.\mathbf{H}_{t}^{(1)}\right|_{z=0}=\left.\mathbf{H}_{t}^{(0)}\right|_{z=0^{\prime}} \\
\left.\mathbf{E}_{t}^{(2)}\right|_{z=L}=\left.\mathbf{E}_{t}^{(0)}\right|_{z=L^{\prime}} & \left.\mathbf{H}_{t}^{(2)}\right|_{z=L}=\left.\mathbf{H}_{t}^{(0)}\right|_{z=L^{\prime}}
\end{aligned}
$$

This set of equations can be solved using the orthogonality expansion method. As a result, the relation between forward and backward waves in dielectric sections can be derived in the following form:

$$
\mathbf{B}^{\prime}=\mathbf{S}^{\prime} \mathbf{A}^{\prime}
$$

where

$$
\mathbf{S}^{\prime}=\left[\begin{array}{llll}
S_{e e}^{11} & S_{e o}^{11} & S_{e e}^{12} & S_{e o}^{12} \\
S_{o e}^{11} & S_{o o}^{11} & S_{o e}^{12} & S_{o o}^{12} \\
S_{e e}^{21} & S_{e 0}^{21} & S_{e e}^{22} & S_{e 0}^{22} \\
S_{o e}^{21} & S_{o o}^{21} & S_{o e}^{22} & S_{o o}^{22}
\end{array}\right]
$$

and $\mathbf{A}^{\prime}$ and $\mathbf{B}^{\prime}$ are the vectors of unknown expansion coefficients for the fields in each section $\mathbf{A}^{\prime}=\left[A_{e}^{(1)}, A_{o}^{(1)}, A_{e}^{(2)}, A_{o}^{(2)}\right]^{T}, \mathbf{B}^{\prime}=\left[B_{e}^{(1)}, B_{o}^{(1)}, B_{e}^{(2)}, B_{o}^{(2)}\right]^{T}$. The $\mathbf{S}^{\prime}$ matrix defines the two-mode scattering matrix of two-port FCL junction. The element $S_{n m}^{j i}$ defines the relation between $m$ incident wave in the $i$ th port and $n$ reflected wave in the $j$ th port, where $m, n=\{e, o\}$ and $i, j=\{1,2\}$.

In the designing procedure of the integrated nonreciprocal devices, more useful is the S-matrix defined from the point of view of the incident and reflected waves at four ports of the FCL junction. The scheme of such junction is presented in Fig. 7.

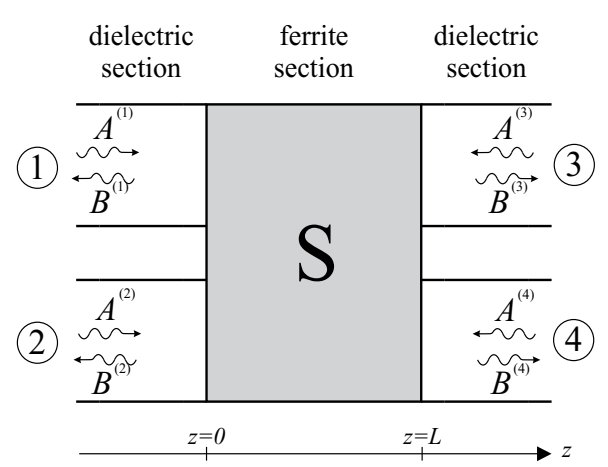

Figure 7. Four-port FCL junction 
Using the symmetry properties of the even and odd modes propagated in the dielectric sections, the matrix $\mathbf{S}^{\prime}$ can be rearranged in terms of port waves, and finally, the scattering matrix of four-port FCL junction is obtained. The incident and reflected waves at $i$ th port are denoted by $A^{(i)}$ and $B^{(i)}$, respectively. Due to symmetry of the waves in the dielectric sections, they can be written as superpositions of waves in each port of four-port FCL junction as follows:

$$
\begin{array}{ll}
A_{e}^{(1)}=\left(A^{(1)}+A^{(2)}\right) / \sqrt{2}, & A_{o}^{(1)}=\left(A^{(1)}-A^{(2)}\right) / \sqrt{2}, \\
B_{e}^{(1)}=\left(B^{(1)}+B^{(2)}\right) / \sqrt{2}, & B_{o}^{(1)}=\left(B^{(1)}-B^{(2)}\right) / \sqrt{2}, \\
A_{e}^{(2)}=\left(A^{(3)}+A^{(4)}\right) / \sqrt{2}, & A_{o}^{(2)}=\left(A^{(3)}-A^{(4)}\right) / \sqrt{2}, \\
B_{e}^{(2)}=\left(B^{(3)}+B^{(4)}\right) / \sqrt{2}, & B_{o}^{(2)}=\left(B^{(3)}-B^{(4)}\right) / \sqrt{2} .
\end{array}
$$

which can be expressed in the matrix form

$$
\mathbf{A}^{\prime}=\mathbf{T A} \quad \text { and } \quad \mathbf{B}^{\prime}=\mathbf{T B},
$$

where $\mathbf{A}=\left[A^{(1)}, A^{(2)}, A^{(3)}, A^{(4)}\right]^{T}, \mathbf{B}=\left[B^{(1)}, B^{(2)}, B^{(3)}, B^{(4)}\right]^{T}$, and

$$
\mathbf{T}=\left[\begin{array}{cc}
\mathbf{T}_{1} & \mathbf{0} \\
\mathbf{0} & \mathbf{T}_{1}
\end{array}\right], \quad \mathbf{T}_{1}=\frac{1}{\sqrt{2}}\left[\begin{array}{cc}
1 & 1 \\
1 & -1
\end{array}\right]
$$

Finally, using the two-mode S-matrix (24) and relations (27), the scattering matrix of the four-port FCL junction is obtained.

$$
\mathbf{B}=\mathbf{S ~ A}, \quad \text { where } \quad \mathbf{S}=\mathbf{T}^{-1} \mathbf{S}^{\prime} \mathbf{T} .
$$

Such S-matrix can be used in the analysis of the transmission properties of FCL junction with the assumed excitation.

\section{Numerical results}

\subsection{Microstrip ferrite coupled line junction}

The first investigated structure is a planar microstrip ferrite coupled line (MFCL) junction presented in Fig. 8(a). The cross section of the junction is shown in Fig. 8(b). It is a multilayer structure in which two conductive strips are placed at $h_{2}$ interface while the ground is placed at $h_{1}$ interface. A ferrite material with a relative permittivity $\varepsilon_{r 2}=13.3$, saturation magnetization $M_{s}=239 \mathrm{kA} / \mathrm{m}$, internal bias $H_{i}=0$, and thickness $d_{2}=0.5 \mathrm{~mm}$ is placed in layer (2) located above the conductive strips. The dielectric sections have the same cross section as ferrite section, although instead of ferrite, a dielectric material is used with relative permeability $u_{r}=1$ and with the same relative permittivity as the ferrite material. The investigated structure has a plane of symmetry $A A^{\prime}$ passing through the center of the gap 


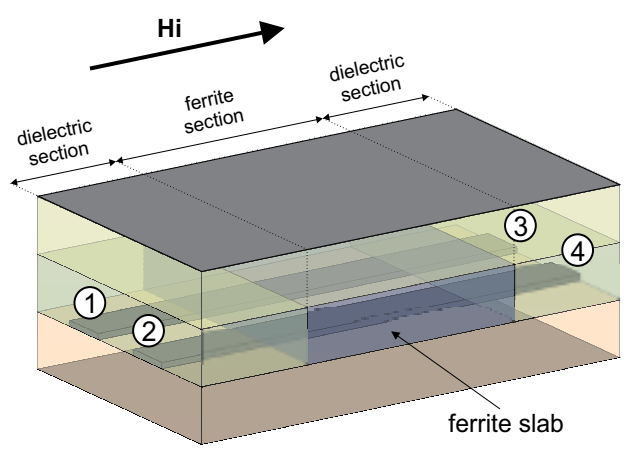

(a)

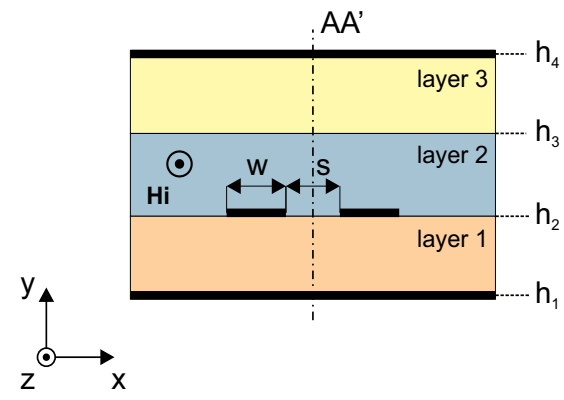

(b)

Figure 8. Planar junction of microstrip ferrite coupled lines: (a) 3D view and (b) cross section of ferrite guide

between the strips. In the dielectric section, this symmetry plane is an electric or magnetic wall for odd or even mode, respectively.

Utilizing the developed method of analysis, described in section 2, the dispersion characteristics of the investigated structure are first calculated. The calculations were performed in the frequency range from 9 to $18 \mathrm{GHz}$. The characteristics of the propagation coefficients of the two basic modes propagating in dielectric and ferrite sections are shown in Fig. 9(a). The ferrite modes have a cutoff frequency near $f_{M}=\gamma\left(H_{i}+M_{s}\right)=8.4 \mathrm{GHz}$ where gyromagnetic coefficient $\gamma=35.2 \mathrm{MHz} / \mathrm{kA}$. This means that the ferrite modes propagate in this structure, when the $\mu_{\text {eff }}=\left(\mu^{2}-\mu_{a}^{2}\right) / \mu>0$, where $\mu$ and $\mu_{a}$ are the elements of relative permeability tensor defined in section 2.1. It can be noted that with the increasing frequency, the propagation coefficients of the modes in the ferrite line converge to propagation coefficients of the modes in dielectric line. This effect is due to the fact that with

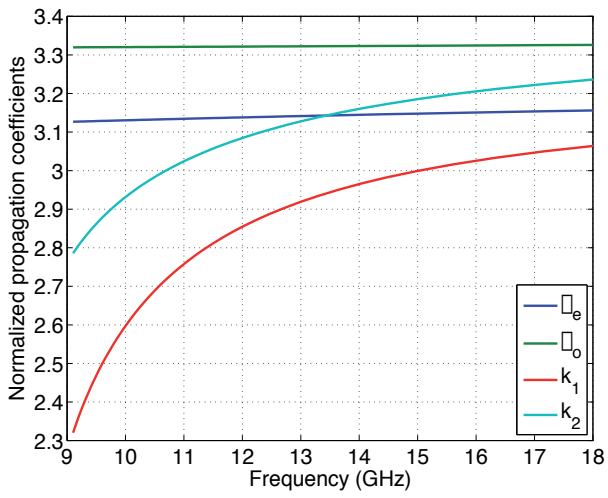

(a)

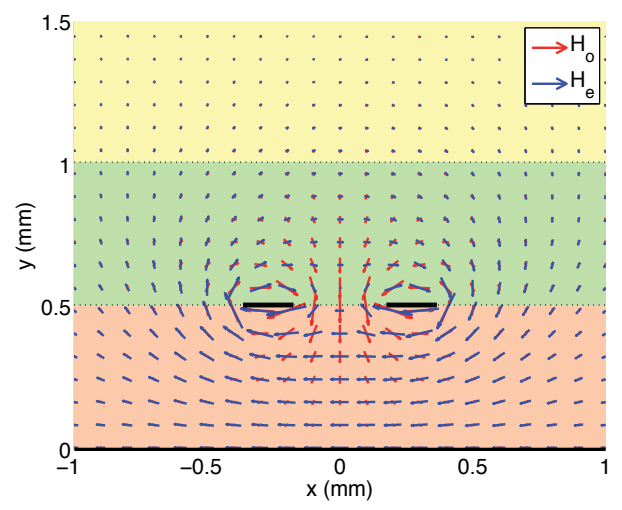

(b)

Figure 9. Simulation results of MFCL junction: (a) dispersion characteristics and (b) magnetic field distribution of even and odd mode in dielectric section of MFCL junction at $f_{0}=12.4 \mathrm{GHz}$ 
the increase of frequency, the value of $\mu_{a}$ element of the permeability tensor (responsible for gyromagnetic property of the ferrite) decreases.

Figure 9(b) shows the distributions of the transverse components of magnetic fields for the even and odd modes in the dielectric section. Calculations are performed at frequency $f_{0}=12.4 \mathrm{GHz}$. As can be seen, the magnetic field vectors of the dielectric modes are orthogonal to each other in the areas above and below the strips in the symmetry plane $A A^{\prime}$ of the structure. According to the definition of coupling coefficient (12), if instead of one of these layers the ferrite material is introduced, the optimal gyrotropic coupling effect will be obtained.

In the next step, utilizing the MM method described in section 2.2.2, the scattering parameters of the investigated MFCL junction are determined. The scattering parameters of the junction in a function of ferrite section length at $f_{0}=12.4 \mathrm{GHz}$ are shown in Fig. 10. The optimal

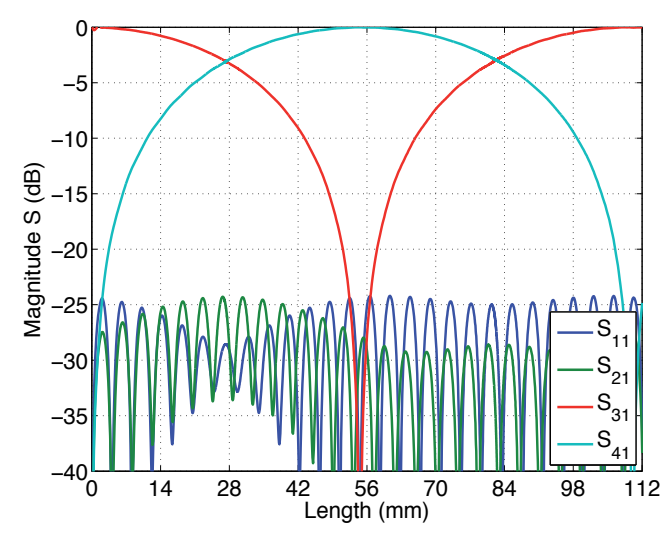

(a)

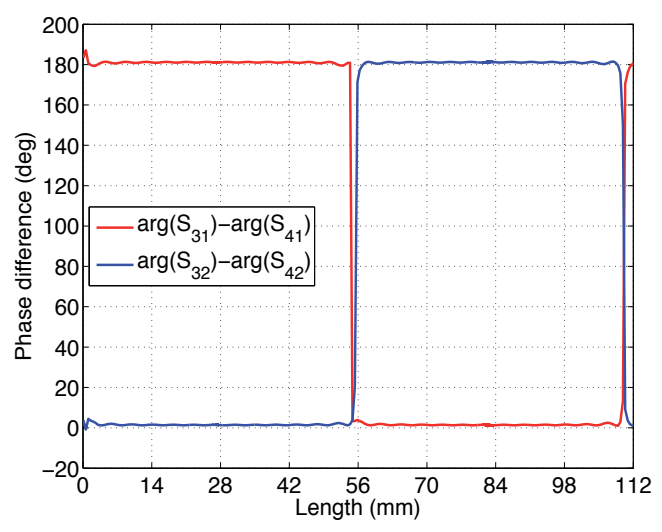

(b)

Figure 10. Simulated scattering parameters of MFCL junction versus length of ferrite section at $f_{0}=12.4$ GHz: (a) magnitude and (b) phase difference

length $L$ of the ferrite section providing $45^{\circ}$ Faraday rotation is determined by amplitude and phase conditions ([21]). According to these conditions, when port (1) of the structure is excited, the signal should be equally divided between ports (3) and (4) of the structure, while port (2) should be isolated. Moreover, the phase difference between signals in ports (3) and (4) for ports (1) or (2) excitation should be equal to 0 or $\pm 180^{\circ}$. From the obtained results, it can be seen that optimal length of the section for which the amplitude and phase conditions are fulfilled is $L=28 \mathrm{~mm}$.

In order to illustrate the nonreciprocal properties occurring in the investigated FCL, the change of power concentration along the structure has been calculated and presented in Fig. 11.

Based on the obtained results, one can see the periodic effect of signal exchange between coupled lines in the ferrite section due to the Faraday rotation phenomenon. Furthermore, the distributions of the electric and magnetic fields in the input and output ports of FCL junction providing $45^{\circ}$ Faraday rotation angle $(L=28 \mathrm{~mm})$ have been calculated and 


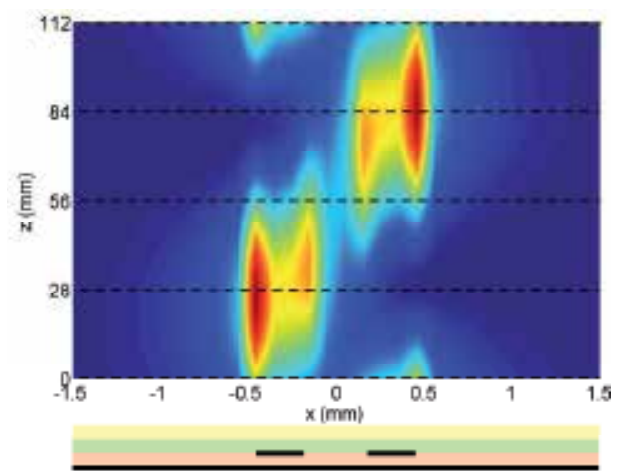

Figure 11. Power density distribution along the investigated MFCL section
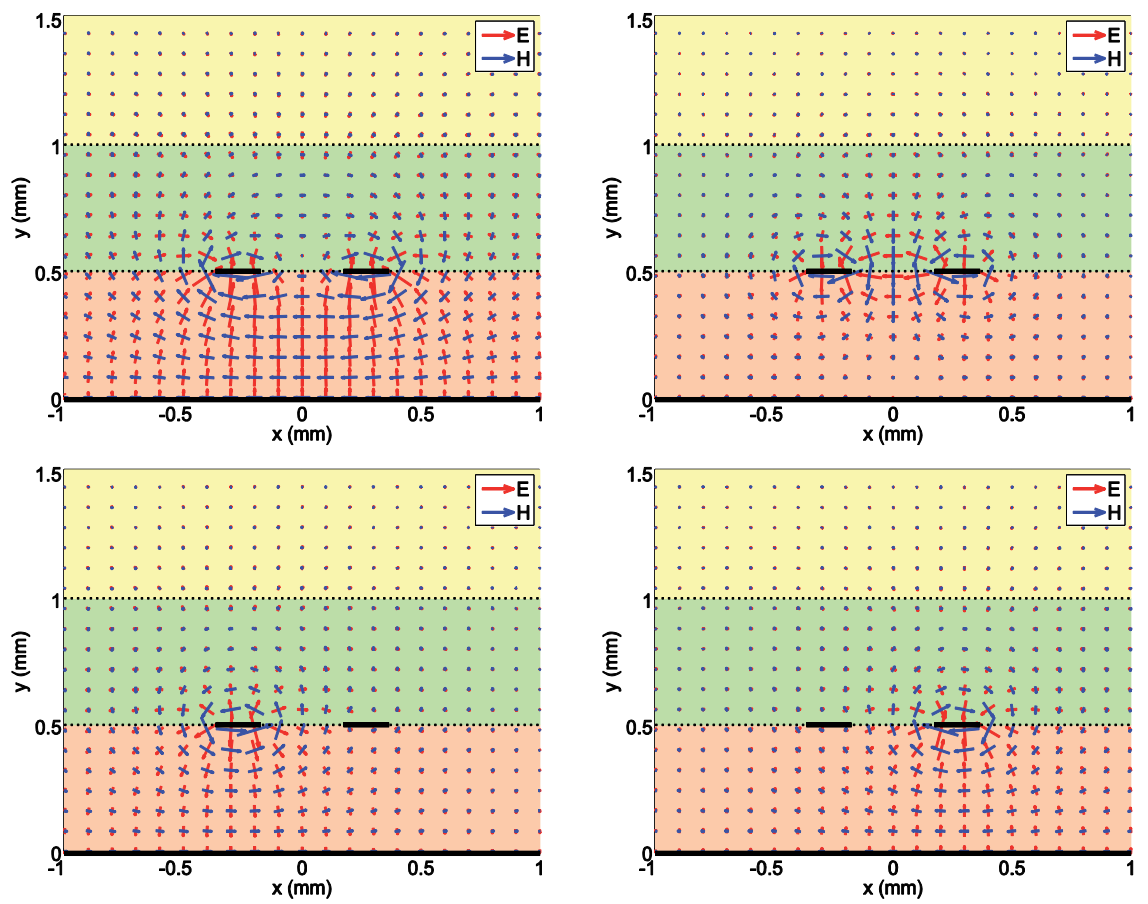

(a)

(b)

Figure 12. Field distribution in dielectric section of MFCL junction at $z=0$ (upper row) and $z=28 \mathrm{~mm}$ (bottom row) mm for: (a) even-mode excitation and (b) odd-mode excitation

presented in Fig. 12. In the analysis, the even- and odd-mode excitations of the junction were assumed. From the presented results, it can be observed that when such junction is excited with the even or odd mode, the signal concentrates around the left or right line at the output of the structure. If the direction of the magnetization will be reversed, the signal will concentrate on the opposite strips. 
For the FCL junction with the optimal length $L=28 \mathrm{~mm}$ of the ferrite section, the scattering parameters were calculated in a function of frequency (see Fig. 13).

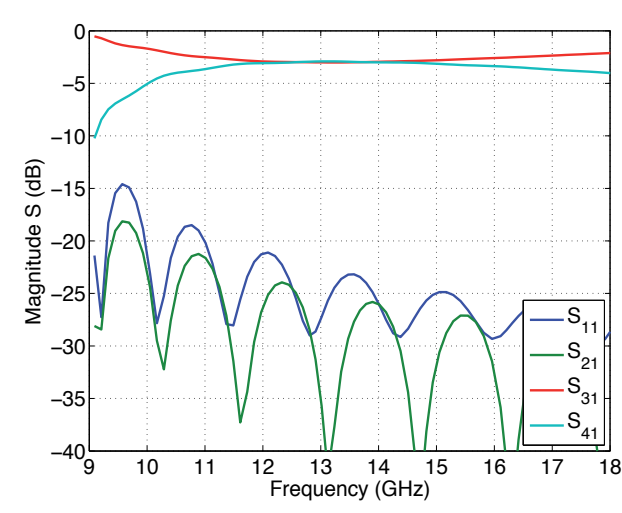

(a)

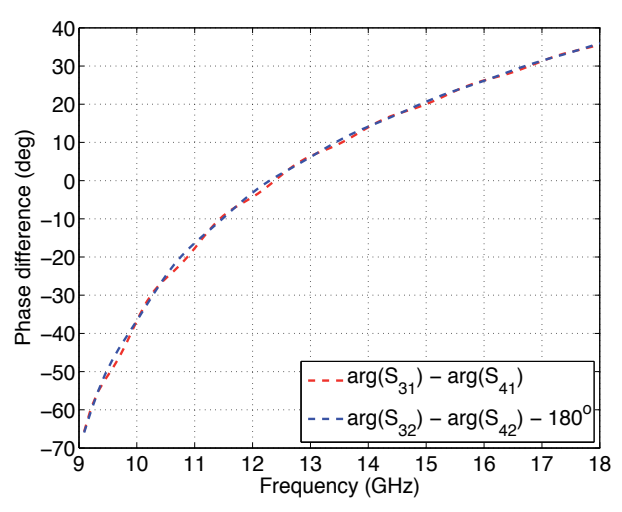

(b)

Figure 13. Simulated frequency-dependent scattering parameters of MFCL junction: (a) magnitude and (b) phase difference

From the obtained results, it can be seen that the transmission coefficients $S_{31}$ and $S_{41}$ are equal to $-3 \pm 0.5 \mathrm{~dB}$ in the frequency range from 11 to $16 \mathrm{GHz}$, with isolation $S_{21}$ and reflection losses $S_{11}$ better than $-20 \mathrm{~dB}$. In the considered frequency range, the phase difference between the output signals in ports (3) and (4) for ports (1) or (2) excitation varies in the range from -18 to $27^{\circ}$ (see Fig. 13(b)). The optimal $45^{\circ}$ Faraday rotation angle is obtained for $f_{0}=12.4 \mathrm{GHz}$.

\subsection{Cylindrical ferrite coupled line junction}

Another investigated structure is a cylindrical ferrite coupled line (CFCL) junction. The cross section of ferrite coupled lines is shown in Fig. 14.

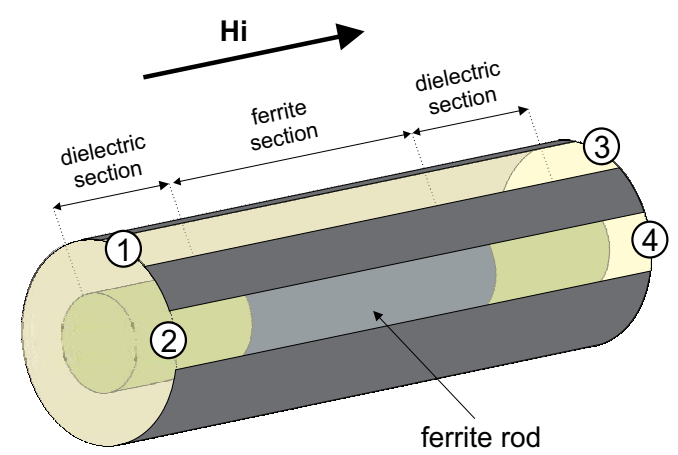

(a)

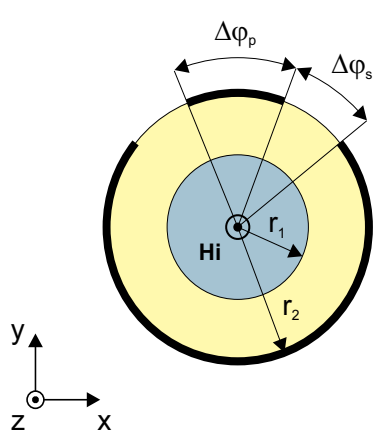

(b)

Figure 14. Cylindrical ferrite coupled line junction: (a) 3D view and (b) cross section of ferrite guide 
This structure consists of a cylindrical ferrite rod of radius $r_{1}$ covered with dielectric layer of thickness $d=r_{2}-r_{1}$ on which the conductive strips are etched. The dielectric section has the same cross section as ferrite section; however, instead of a ferrite rod, a dielectric rod is used with relative permeability $\mu_{r}=1$ and the same relative permittivity as the ferrite material.

Utilizing the developed method of analysis, described in section 2.1, the dispersion characteristics of the investigated structure are first calculated. In the analysis, the following dimensions and material parameters of the junction were assumed: $r_{1}=2.2 \mathrm{~mm}, \varepsilon_{r f}=15$, saturation magnetization $M_{s}=131 \mathrm{kA} / \mathrm{m}$, internal bias $H_{i}=0$, and dielectric coating: $d=0.127 \mathrm{~mm}, \varepsilon_{r d}=2.2$. The angular slot/strip widths were $\Delta \varphi_{p}=25^{\circ}, \Delta \varphi_{s}=15^{\circ}$. The characteristics of the propagation coefficients of the dielectric and the ferrite lines are shown in Fig. 15(a).

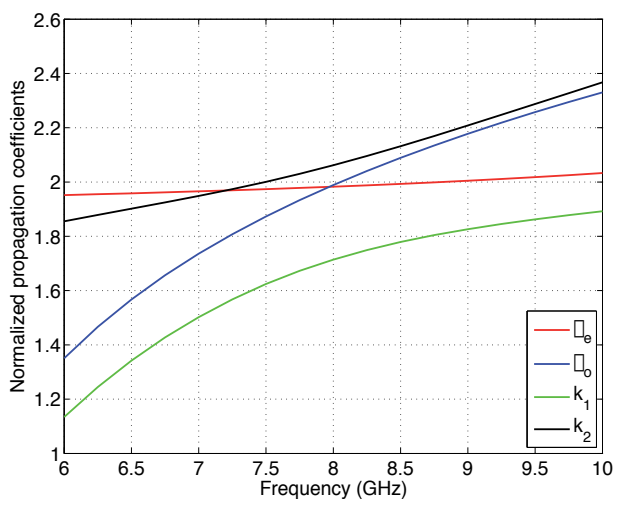

(a)

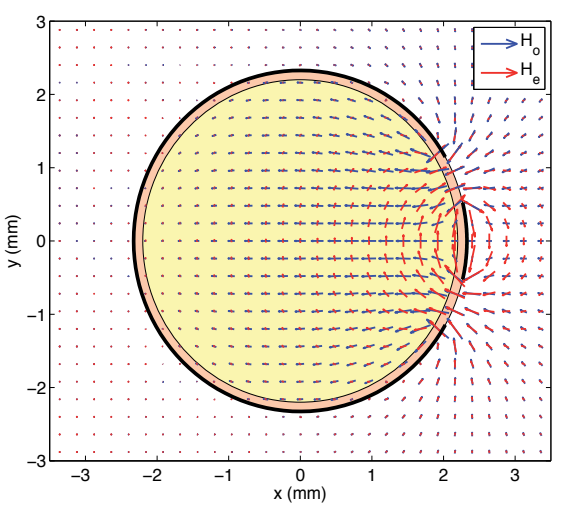

(b)

Figure 15. Simulation results of CFCL junction: (a) dispersion characteristics and (b) magnetic field distributions of even and odd modes in dielectric section of CFCL junction at $f_{0}=8.2 \mathrm{GHz}$

Based on the obtained characteristics for the investigated dielectric lines, it can be noted that for a specific frequency $f_{0}=8 \mathrm{GHz}$, the phase velocities of the even and odd modes are equal. It means that in such a line, the isotropic coupling vanishes and only gyromagnetic coupling occurs. This allows to obtain the optimal conditions for the Faraday rotation.

Figure 15(b) shows the magnetic field distributions of the even and odd modes in dielectric line specified for $f_{0}=8.2 \mathrm{GHz}$. From these distributions, it can be seen that there are areas in the line where the magnetic fields of both modes are orthogonal; hence, placing the ferrite material in this area of a line will produce the gyromagnetic coupling between the basic field modes.

In the next step, the scattering parameters of CFCL junction are calculated in a function of ferrite section length at $f_{0}=8.2 \mathrm{GHz}$ and shown in Fig 16 .

From the presented results, it can be noticed that the optimal length of the ferrite section providing $45^{\circ}$ Faraday rotation is $L=26 \mathrm{~mm}$. For such length of section $S_{31}=S_{41}=-3 \mathrm{~dB}$ and the phase difference between signals in ports (3) and (4) for ports (1) and (2), excitation is then 0 or $\pm 180^{\circ}$. 


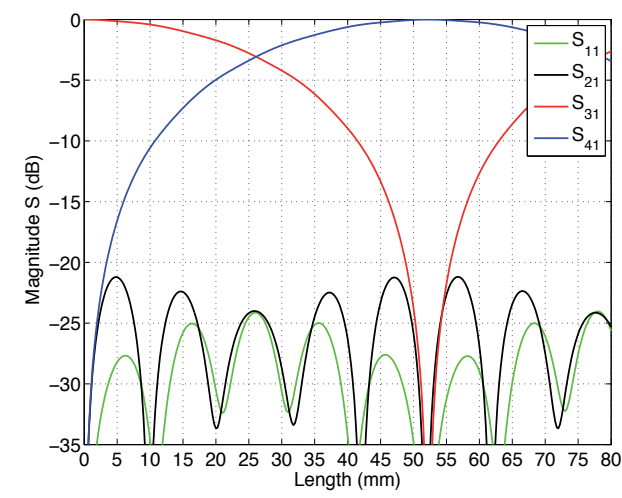

(a)

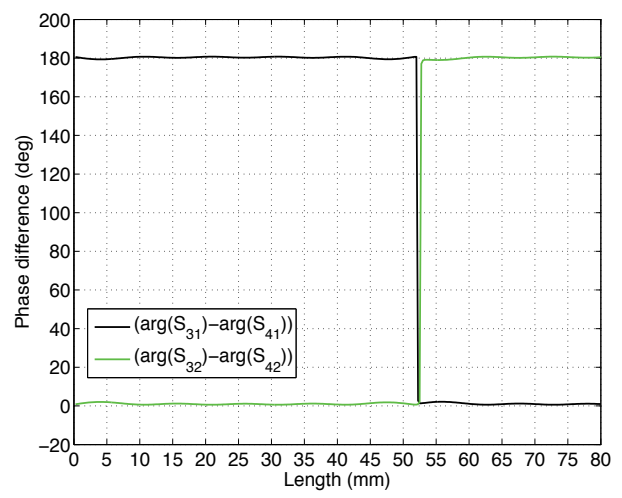

(b)

Figure 16. Scattering parameters of CFCL junction versus length of ferrite section: (a) magnitude and (b) phase difference

Similarly to the planar case presented in section 3.1, in order to illustrate the nonreciprocal properties occurring in the investigated CFCL junction, the power concentration along the structure has been determined (see Fig. 17).
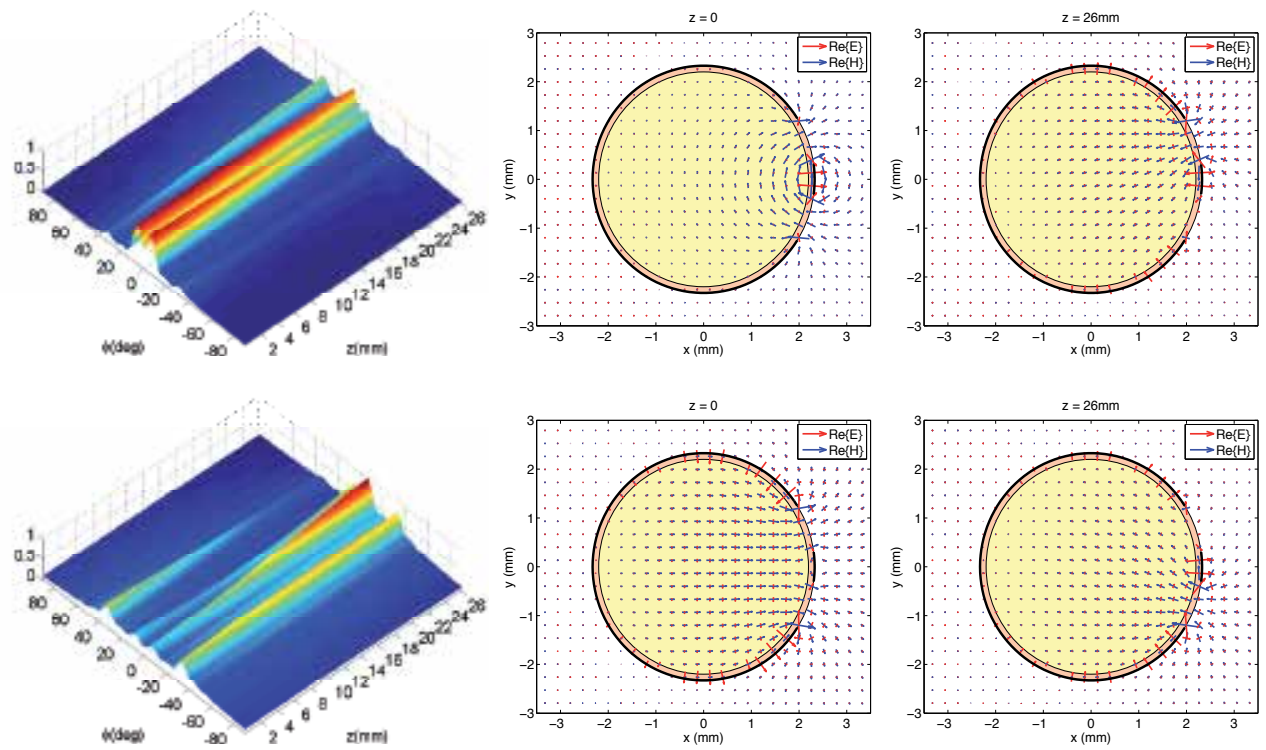

Figure 17. Power density and transversal field distribution in the CFCL section for even- (upper row) and odd-mode (bottom row) excitation calculated at $f_{0}=8.2 \mathrm{GHz}$

Furthermore, the distributions of the electric and magnetic fields in the input $(z=0)$ and output ports $(z=26 \mathrm{~mm})$ of FCL junction providing $45^{\circ}$ Faraday rotation angle have been calculated. In the analysis, the even- and odd-mode excitations of the junction were assumed. The obtained results confirm the nonreciprocal behavior of the designed CFCL junction. 
For the CFCL junction with the ferrite section of length $L=26 \mathrm{~mm}$, the scattering parameters were calculated in a function of frequency and presented in Fig. 18.

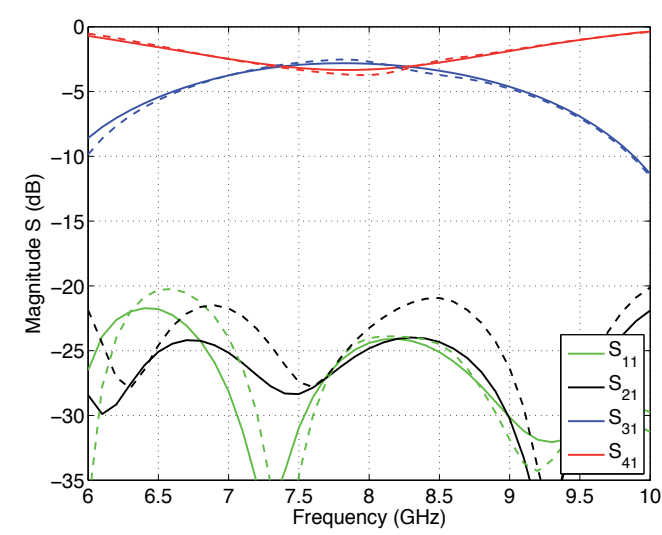

(a)

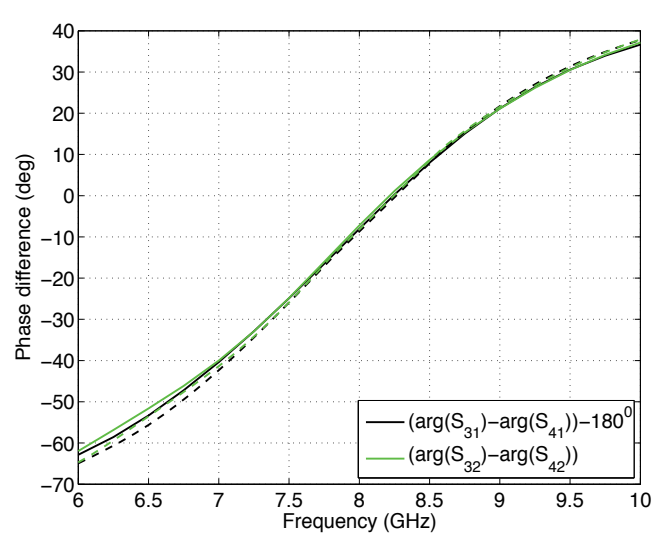

(b)

Figure 18. Frequency-dependent scattering parameters of CFCL junction for $L=26 \mathrm{~mm}$ : (a) magnitude and (b) phase difference (solid line, our method; dashed line, HFSS)

From the obtained results, it can be seen that the equal power division defined by $S_{31}=$ $S_{41}=-3 \pm 0.5 \mathrm{~dB}$ is obtained in the frequency range from 7 to $8.6 \mathrm{GHz}$ (see Fig. 18(a)). In this frequency range, the phase difference between the signals in ports (3) and (4) for ports (1) or (2) excitation varies in the range from $-40^{\circ}$ to $10^{\circ}$ (see Fig. 18(b)). In addition, it can be seen that the optimal amplitude and phase conditions required for $45^{\circ}$ Faraday rotation are fulfilled at the frequency $f_{0}=8.25 \mathrm{GHz}$. The result of the proposed approach is compared with those obtained from commercial software HFSS, and a good agreement can be observed.

\section{Nonreciprocal devices}

In this section, the results of investigation of nonreciprocal devices made in both the planar and conformal line technology are presented. Section 4.1 presents the results of the three-port circulator realized in slotline technology. Section 4.2 presents the results of a double isolator realized in microstrip coupled lines technology. In Section 4.3, the results of the four-port circulator realized utilizing cylindrical ferrite coupled line section are discussed.

\subsection{Three-port circulator utilizing ferrite coupled slotline junction}

The first investigated circuit is three-port circulator realized in coupled slotline technology, which is shown in Fig 19(a).

The device is realized as a cascade connection of a T-junction, ferrite coupled slotline (FCSL) junction, and the output section being the transformer from the coupled slotlines to the microstrip lines. The cross section of the FCSL junction is shown in Fig. 19(b). The FCSL junction is a four-layer structure, where the coupled slotlines are realized on a thin laminate situated above the ferrite material. The T-junction and the output section have the same cross section as ferrite section, although instead of ferrite, a dielectric material with relative 


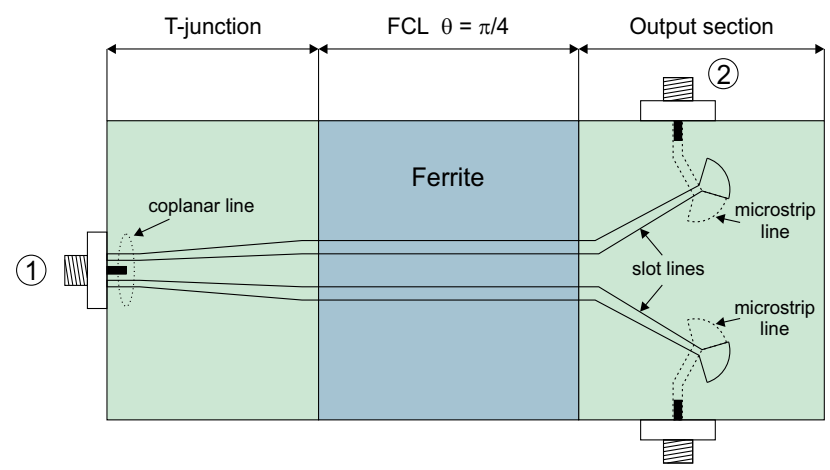

(3)

(a)

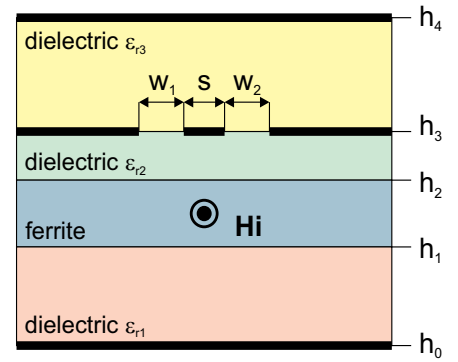

(b)

Figure 19. FCSL circulator: (a) schematic view of the device and (b) cross section of FCSL junction

permittivity $\varepsilon_{r}=9.6$ is used. T-junction is realized as a transformer from coplanar line into coupled slotlines. Due to the fact that the coplanar line is fed from the coaxial connector, the junction provides even excitation of FCSL section. On the other hand, the odd mode, which is transmitted from the ferrite section, is totally reflected in the plane of the coaxial connector. The output structure allows for the transmission of the signal from the specific slot of ferrite junction to the corresponding output microstrip port, while the remaining ports of the circulator are isolated.

In order to determine the scattering parameters of the circulator shown in Fig. 19(a), the scattering matrices of the ferrite junction, T-junction, and output section were first calculated. The scattering parameters of the FCSL junction were simulated with the use of our own software based on the methods described in section 2. The feeding circuits (T-junction and output section) were designed with the use of commercial software. The circulator scattering parameters were obtained by cascade connection of the calculated scattering matrices of individual sections. The simulated frequency characteristics of the scattering parameters are shown in Fig 20.

From the obtained results, it can be seen that the investigated configuration has return losses and isolation better than $10 \mathrm{~dB}$ over a wide frequency range from 13 to $20.5 \mathrm{GHz}$. In the considered frequency band, the average transmission losses are about $1 \mathrm{~dB}$ in the case of a single pass of the signal through the ferrite section and about $2 \mathrm{~dB}$ in the case of double pass of the signal through the ferrite section. In the analysis, the material losses were not taken into consideration, and the resulting level of losses is due to return losses and the lack of a perfect isolation between the ports of circulator.

A photograph of the manufactured prototype of the circulator is shown in Fig. 21. The measured characteristics of its scattering parameters are depicted in Fig. 22.

Measurements were performed in the frequency range from 10 to $21 \mathrm{GHz}$. The results show that the investigated device operates in a wide frequency band. In the frequency range from 12 to $19 \mathrm{GHz}$, the average transmission losses are about $2.5 \mathrm{~dB}$, and the level of isolation and return losses is about $10 \mathrm{~dB}$ for a single pass of the signal through the ferrite 


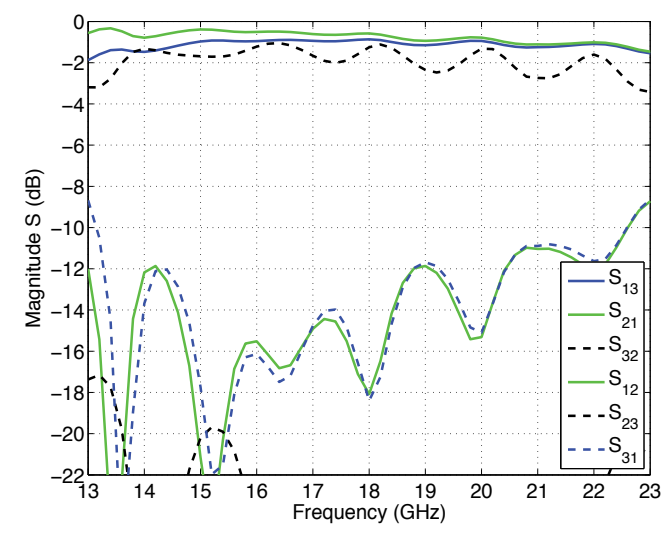

(a)

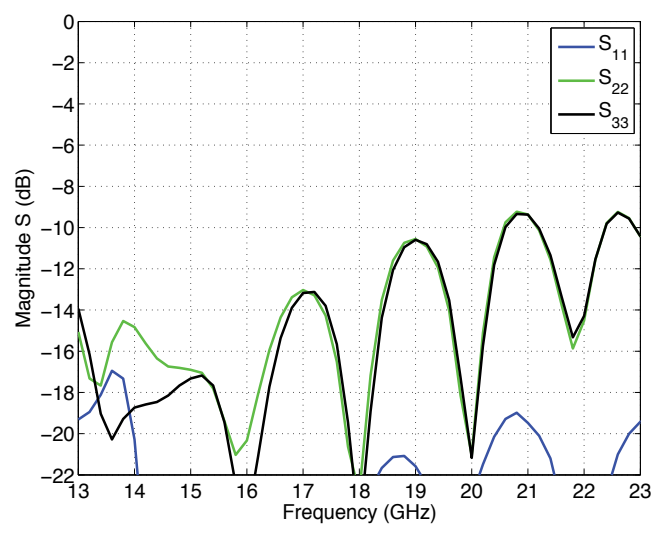

(b)

Figure 20. Simulated scattering parameters of FCSL circulator: (a) transmission with isolation and (b) reflection

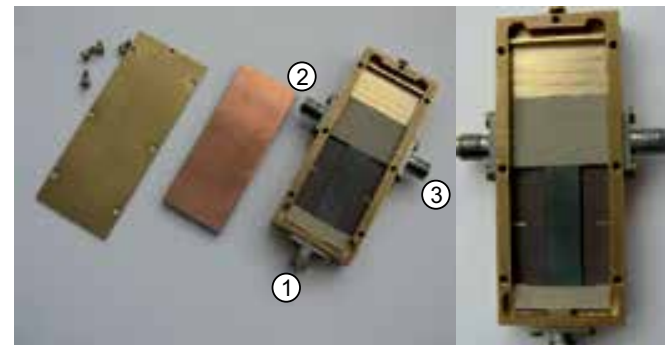

(a)

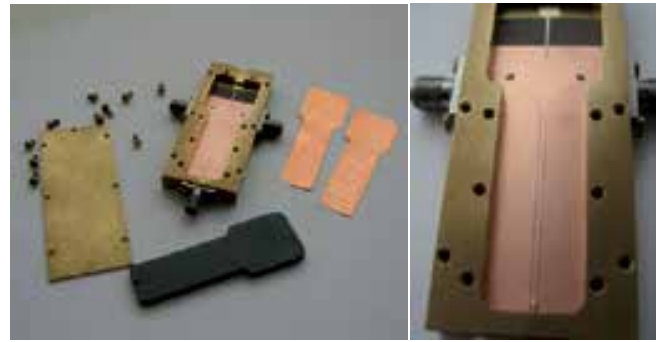

(b)

Figure 21. Photograph of the fabricated FCSL circulator: (a) top view and (b) bottom view

section. The best isolation is observed in the frequency range from 12 to $15 \mathrm{GHz}$ and is better than $16 \mathrm{~dB}$. In the case of a double pass of the signal through the ferrite section, the transmission losses are two times higher and are about $5.5 \mathrm{~dB}$ in the frequency range from 12 to $19 \mathrm{GHz}$. The isolation is better than $14 \mathrm{~dB}$ in the entire frequency range. In the measured transmission characteristics, small periodically repeating resonances occur, which result from the inaccuracies in the manufacturing process.

\subsection{Double isolator utilizing microstrip coupled line section}

Another investigated configuration is a double isolator shown in Fig. 23. This arrangement is composed of two interconnected and magnetized in the same direction three-port circulators (see Fig. 2(c)), in which the appropriate ports are terminated by matched loads. The advantage of this configuration is the ability to achieve high isolation. Unfortunately, due to the fact that the device uses two ferrite sections, the losses in the system are twice as high as in the case of isolator with a single section of FCL. 


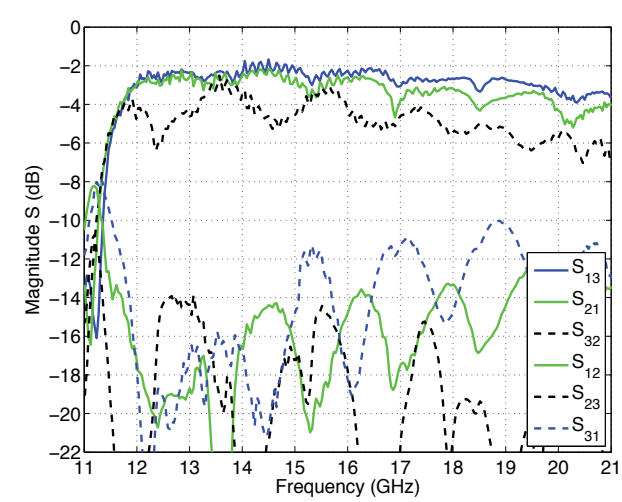

(a)

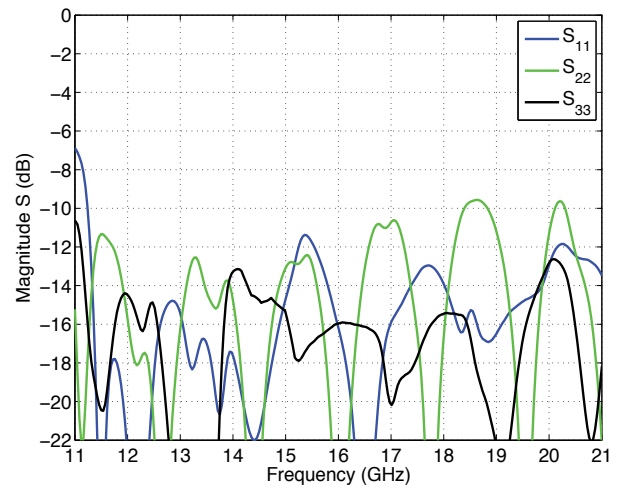

(b)

Figure 22. Measured scattering parameters of FCSL circulator: (a) transmission with isolation and (b) reflection

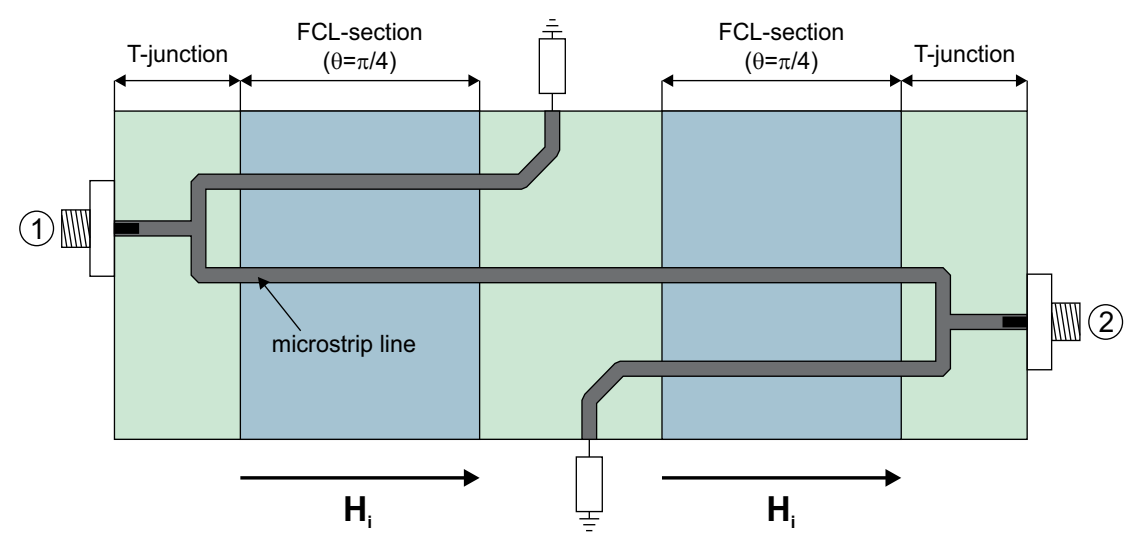

Figure 23. Double isolator utilizing microstrip ferrite coupled line junction

Utilizing own software, as well as commercial simulator, the double isolator utilizing ferrite junction from Fig. 8 was designed. The simulation results are shown in Fig. 24.

From the obtained results, it can be seen that in the frequency range from 10 to $16 \mathrm{GHz}$, the isolation is better than $20 \mathrm{~dB}$ with return losses better than $10 \mathrm{~dB}$.

The designed structure was manufactured. A photograph of the prototype is shown in Fig. 25. To obtain the double isolator, ports (3) and (4) of the structure were terminated with the matched SMA connectors. The obtained experimental results are presented in Fig. 26.

It can be seen that the device works in the frequency band from 9 to $16 \mathrm{GHz}$. In the given frequency range the isolation and the average return losses are better than $15 \mathrm{~dB}$. Furthermore, the average transmission losses are $3.5 \mathrm{~dB}$ and they change from $3 \mathrm{~dB}$ at $9 \mathrm{GHz}$ to $4 \mathrm{~dB}$ at $16 \mathrm{GHz}$. Based on these results, it can be estimated, that the losses for a single pass of the signal through the investigated microstrip ferrite section are about $1.8 \mathrm{~dB}$. The losses are lower by about $1.5 \mathrm{~dB}$ in comparison to the results published for a single section configuration in [4]. 


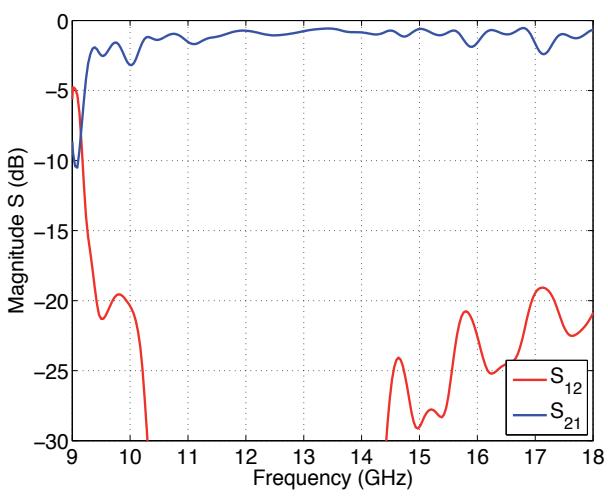

(a)

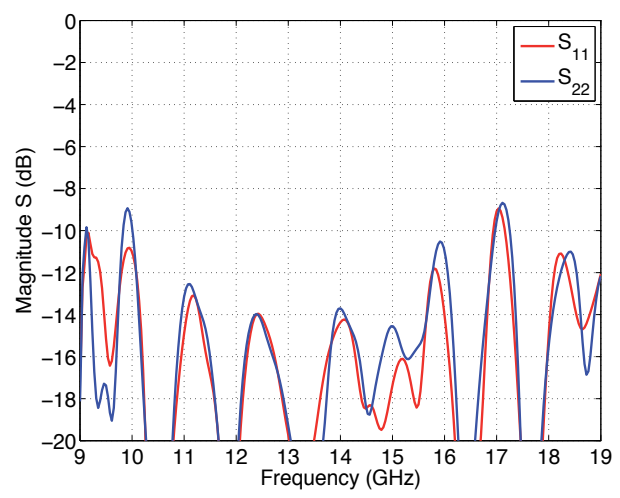

(b)

Figure 24. Simulated scattering parameters of double MFCL isolator: (a) transmission with isolation and (b) reflection

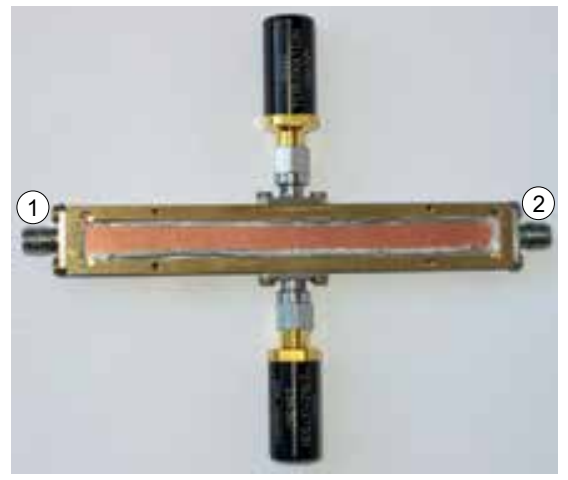

(a)

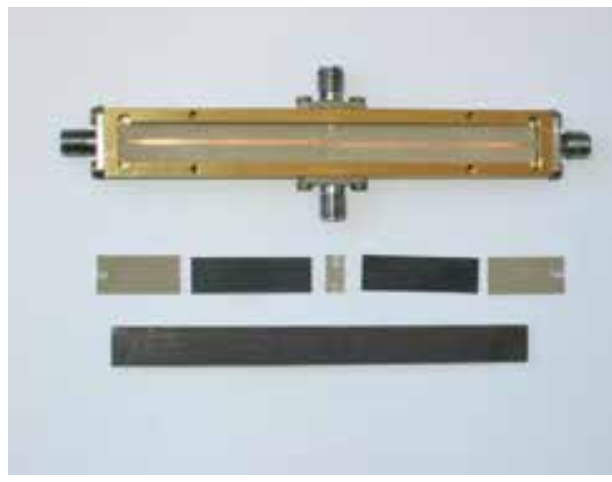

(b)

Figure 25. Photograph of the manufactured double MFCL isolator

\subsection{Four-port circulator utilizing cylindrical ferrite coupled line junction}

The last investigated device is a four-port circulator shown in Fig. 27. The advantage of this circulator is that the signal transmission in the circulation direction requires only one pass through the ferrite coupled line section. As a result, this allows for reduction of losses in the device in comparison to the alternative configuration of the four-port circulator realized with the use of double ferrite coupled line section.

The investigated device is realized as a cascade connection of the magic- $T$ structure, the CFCL junction, and the output section being the transformer from the cylindrical coupled lines to the uncoupled microstrip lines. The magic-T structure (shown in Fig. 28(a) and (b)) allows to excite ferrite section with odd or even mode.

In the case of port (1) excitation, the signal is transmitted directly through the structure ensuring even mode excitation of cylindrical coupled lines. On the other hand, when port (2) is excited, the signal is coupled to slotline, which results in the odd mode signal at ports (3) and (4). Output section, shown in Fig. 28(c), is a multilayer structure, which transforms 


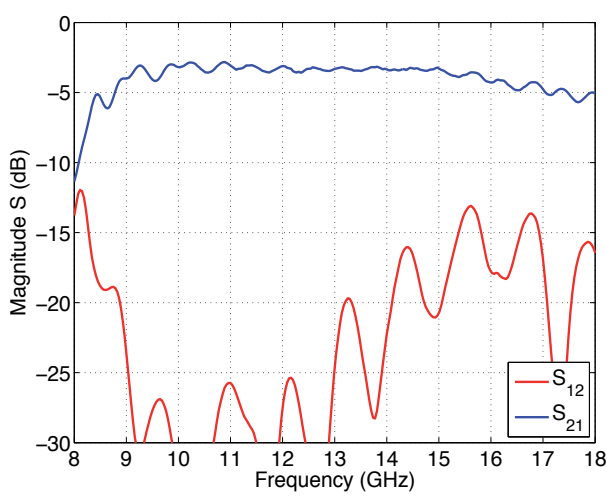

(a)

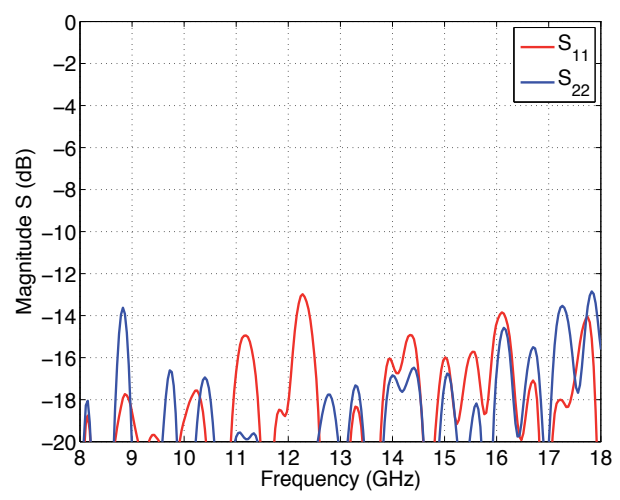

(b)

Figure 26. Measured scattering parameters of double MFCL isolator: (a) transmission with isolation and (b) reflection

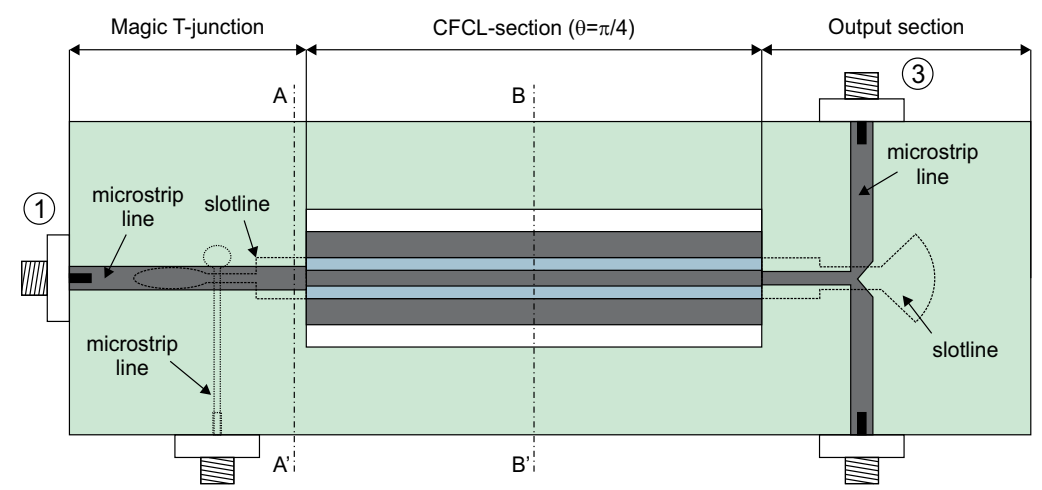

(2)

(4)

AA' cross-section

BB' cross-section

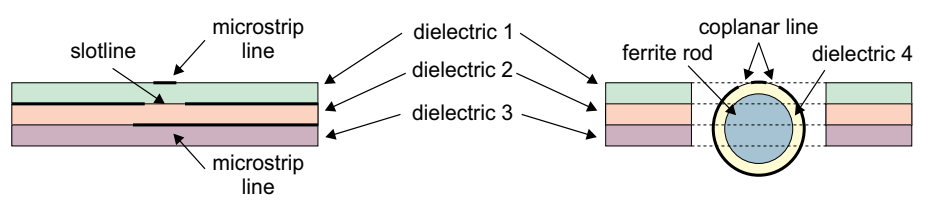

Figure 27. Top and bottom view of four-port circulator utilizing cylindrical ferrite coupled line junction

the cylindrical ferrite coupled line to microstrip lines. In the case of port (1) excitation of this section, the signal is transmitted to port (3). Similarly, when port (2) is excited, the signal is transmitted to port (4). For a complete understanding of the operation of the device, the excitation in ports (1) or (2) can be represented as a superposition of the even and odd signals, which are of the same amplitude and phase equal to 0 or $180^{\circ}$. Then, for even-mode excitation, the signal is divided equally between the output ports. On the other hand, in the case of odd-mode excitation, part of the signal is guided in the bottom slot of the device. Due to the fact that this slotline is shorted with radial stub, the signal is coupled to a microstrip line and divided between the output ports. In order to reduce the isotropic coupling, which 


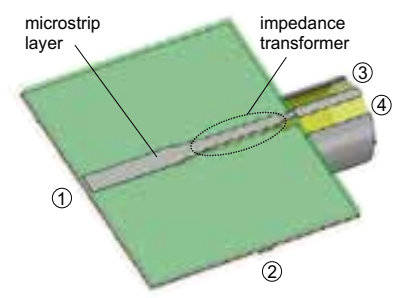

(a)

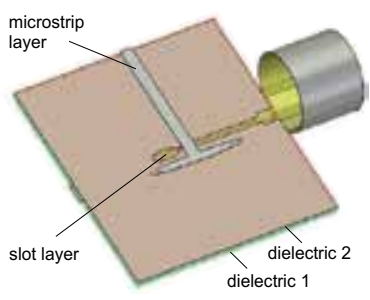

(b)

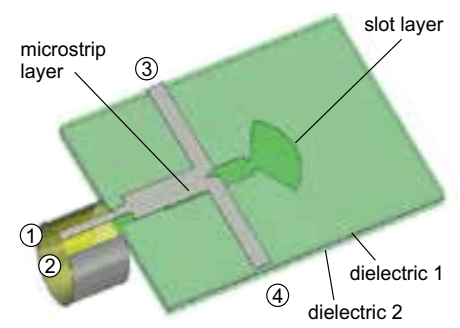

(c)

Figure 28. Feeding circuits of CFCL circulator: (a) magic T, top view; (b) magic T, bottom view; and (c) output transformer from coupled slotlines to microstrip lines

could deteriorate the isolation of the circulator, an additional dielectric layer is used in the considered structure.

The simulation results of the circulator are presented in Fig. 29. It can be seen that the device operates in the frequency range from 7.6 to $8.6 \mathrm{GHz}$. In this frequency range, the device isolation and return losses are better than $18 \mathrm{~dB}$. In the analysis, a lossless section was assumed.

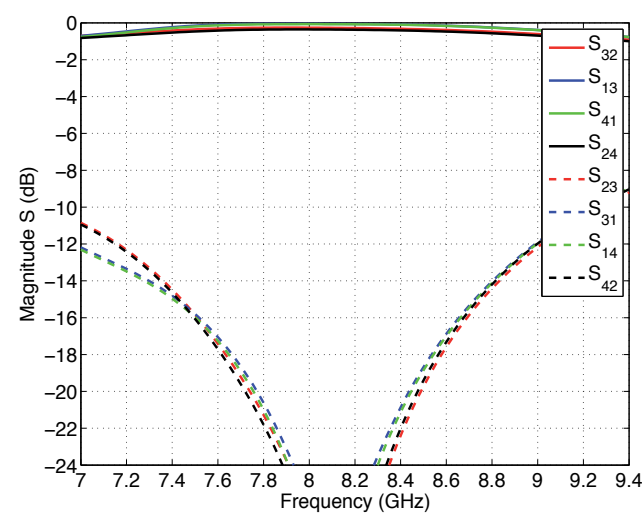

(a)

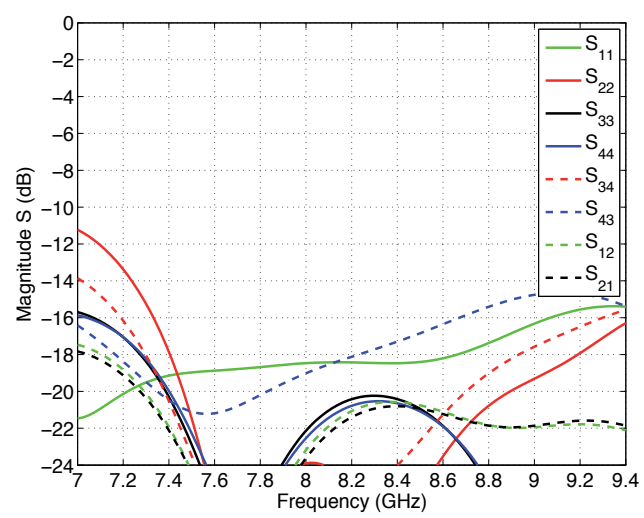

(b)

Figure 29. Simulated scattering parameters of four-port CFCL circulator: (a) transmission with isolation and (b) reflection coefficients with coupling between neighboring ports

The designed device was manufactured (see the photo in Fig. 30) and measured. The obtained scattering parameters characteristics are shown in Fig. 31.

It can be seen that in the frequency range from 7.6 to $8.6 \mathrm{GHz}$, the value of transmission from port (1) to (4) and from port (3) to (1) is about $-1.5 \mathrm{~dB}$, while transmission from port (2) to (3) and port (4) to (2) is about $-3 \mathrm{~dB}$. The isolation between these ports in the considered frequency range is better than $12 \mathrm{~dB}$. Return losses in ports (2), (3), and (4) are better than $17 \mathrm{~dB}$ and in port (1) are better than $8 \mathrm{~dB}$. The isolation between ports (1) and (2) is better than $18 \mathrm{~dB}$ and between ports (3) and (4) better than $8 \mathrm{~dB}$. A higher level of return losses 


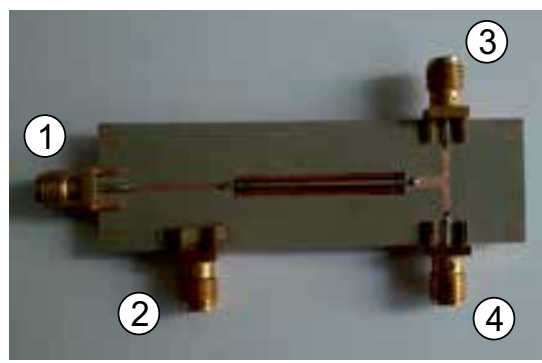

(a)

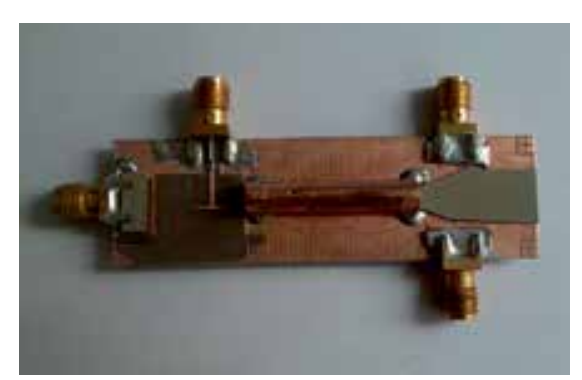

(b)

Figure 30. Photograph of fabricated four-port CFCL circulator: (a) top view and (b) bottom view

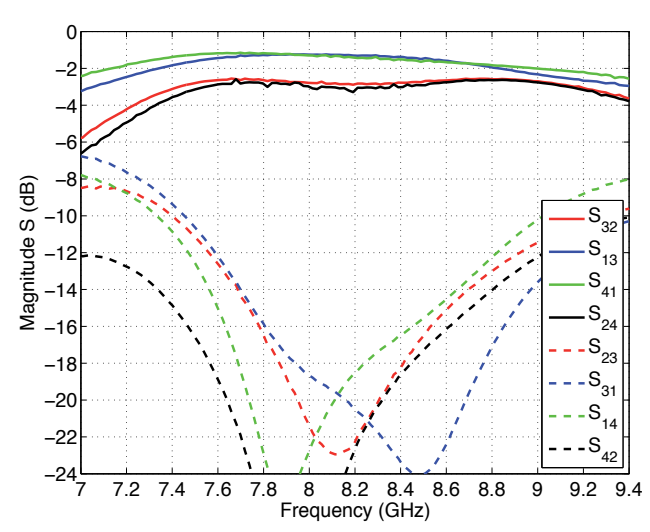

(a)

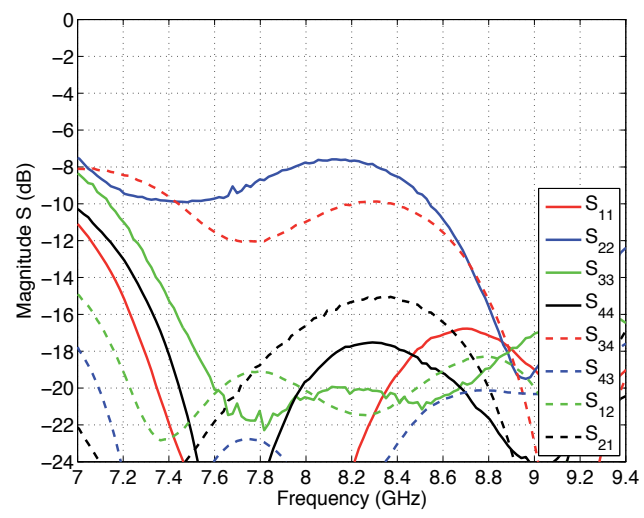

(b)

Figure 31. Measured scattering parameters of four-port CFCL circulator: (a) transmission with isolation and (b) reflection coefficients and coupling between neighboring ports

and a lower level of isolation with respect to simulated results at port (2) result from the inaccuracy of manufacturing process due to technological limitations. The greatest impact on the deterioration of the device parameters had the positioning of the cylindrical ferrite coupled line section with respect to planar structures. Nevertheless, the obtained results of measurements well agree with the results of simulation.

\section{Conclusion}

In this chapter, the research on nonreciprocal devices utilizing longitudinally magnetized ferrite coupled lines junction is presented. The different configurations of FCL junctions were taken into account, comprising planar lines and conformal guides realized as striplines or slotlines placed on cylinder with circular cross section. The description of developed fast and efficient hybrid methods for the analysis of investigated FCL junctions was presented. The numerical results for planar and conformal FCL junctions were calculated and shown. Finally, the numerical and experimental results concerning nonreciprocal devices utilizing proposed planar and conformal FCL junctions were presented and discussed. 


\section{Acknowledgment}

This work was supported from sources of the National Science Center under grant no. DEC-2013/11/B/ST7/04309.

\section{Author details}

Adam Kusiek*, Wojciech Marynowski, Rafal Lech and Jerzy Mazur

*Address all correspondance to: adakus@eti.pg.gda.pl

Gdansk University of Technology, Faculty of Electronics, Telecommunications and Informatics, Poland

\section{References}

[1] Abdalla, M. \& Hu, Z. [2009a]. Compact tuneable single and dual mode ferrite left-handed coplanar waveguide coupled line couplers, Microwaves, Antennas Propagation, IET 3(4): 695-702.

[2] Abdalla, M. \& Hu, Z. [2009b]. Multi-band functional tunable LH impedance transformer, Journal of Electromagnetic Waves and Applications 23(1): 39-47.

[3] Bahri, R., Abdipour, A. \& Moradi, G. [2009]. Analysis and design of new active quasi circulator and circulators, Progress In Electromagnetics Research PIER 96: 377-395.

[4] Cao, M. \& Pietig, R. [2005]. Ferrite coupled-line circulator with reduced length, IEEE Transactions on Microwave Theory and Techniques 53(8): 2572-2579.

[5] Cao, M., Pietig, R., Wu, H. C. \& Gossink, R. G. [2004]. Perturbation theory approach to the ferrite coupled stripline, Microwave Symposium Digest, 2004 IEEE MTT-S International 3: 1903-1906.

[6] Davis, L. E. \& Sillars, D. B. [1986]. Millimetric nonreciprocal coupled slot finline components, IEEE Transactions on Microwave Theory and Techniques 34(7): 804-808.

[7] Fuller, A. J. B. [1986]. Ferrites at Microwave Frequencies, Peter Peregrinus Ltd., London UK.

[8] Itoh, T. [1989]. Numerical Techniques for Microwave and Millimeter-Wave Passive Structures, John Wiley \& Sons, Inc., New York.

[9] Kusiek, A., Marynowski, W. \& Mazur, J. [2007]. Investigations of the circulation effects in the structure using ferrite coupled slot-line section, Microwave and Optical Technology Letters 49(3): 692-696.

[10] Kusiek, A., Marynowski, W. \& Mazur, J. [2011]. Investigations of cylindrical ferrite coupled line junction using hybrid technique, Progress In Electromagnetics Research PIER 120: $143-164$. 
[11] Kusiek, A., Marynowski, W. \& Mazur, J. [2012]. Investigations of nonreciprocal devices employing cylindrical ferrite coupled line junction, Journal of Electromagnetic Waves and Applications JEMWA 26(13): 1685-1693.

[12] Kusiek, A., Marynowski, W. \& Mazur, J. [2013]. Investigations of four-port circulator utilizing cylindrical ferrite coupled line junction, Progress In Electromagnetics Research PIER 134: 379-395.

[13] Marynowski, W., Kusiek, A. \& Mazur, J. [2006]. Microstrip ferrite coupled line isolators, XVI International Microwaves, Radar and Wireless Communications Conference, Vol. 1, Krakow, Poland, pp. 342-345.

[14] Marynowski, W., Kusiek, A. \& Mazur, J. [2008]. Microstrip four-port circulator using a ferrite coupled line section, AEU - International Journal of Electronics and Communications 63(9): 801-808.

[15] Marynowski, W. \& Mazur, J. [2008]. Treatment of the three strip coplanar lines on the ferrite, XVII International Microwaves, Radar and Wireless Communications Conference, Vol. 1, Wroclaw, Poland, pp. 135-138.

[16] Marynowski, W. \& Mazur, J. [2009]. Three-strip ferrite circulator design based on coupled mode method, 2009 International Symposium on Antennas and Propagation (ISAP 2009), Bangkok, Thailand, pp. 205-208.

[17] Marynowski, W. \& Mazur, J. [2010]. Investigations of the double isolator using three-strip ferrite coupled line, 15th Conference on Microwave Techniques, COMITE 2010, Brno, Czech Republic, pp. 73-76.

[18] Marynowski, W. \& Mazur, J. [2011]. Study of nonreciprocal devices using three-strip ferrite coupled line, Progress In Electromagnetics Research PIER 118: 487-504.

[19] Mazur, J., Mazur, M., Michalski, J. \& Sêdek, E. [2002]. Isolator using a ferrite-coupled-lines gyrator, IEE Proceedings - Microwaves, Antennas and Propagation 149(5/6): 291-294.

[20] Mazur, J. \& Mrozowski, M. [1989a]. Nonreciprocal operation of structures comprising a section of coupled ferrite lines with longitudinal magnetization direction, IEEE Transactions on Microwave Theory and Techniques 37(6): 1012-1019.

[21] Mazur, J. \& Mrozowski, M. [1989b]. On the mode coupling in longitudinally magnetized waveguiding structures, IEEE Transactions on Microwave Theory and Techniques 37(1): 159-164.

[22] Mazur, J., Solecka, M., Mazur, M., Poltorak, R. \& Sedek, E. [2005]. Design and measurement of gyrator and isolator using ferrite coupled microstrip lines, IEE Proceedings - Microwaves, Antennas and Propagation 152(1): 43-46.

[23] Mazur, J., Solecka, M., Poltorak, R. \& Mazur, M. [2004]. Theoretical and experimental treatment of a microstrip coupled ferrite line circulator, IEE Proceedings - Microwaves, Antennas and Propagation 151(6): 477-480. 
[24] Queck, C. K. \& Davis, L. E. [2002]. Microstrip and stripline ferrite-coupled-lines (FCL) circulators, IEEE Transactions on Microwave Theory and Techniques 50(12): 2910-2917.

[25] Sajin, G., Simion, S., Craciunoiu, F., Muller, A. \& Bunea, A. [2010]. CRLH CPW antenna on magnetically biased ferrite substrate, Journal of Electromagnetic Waves and Applications 24(5-6): 803-814.

[26] Schmidt, L. \& Itoh, T. [1980]. Spectral domain analysis of dominant and higher order modes in fin-lines, IEEE Transactions on Microwave Theory and Techniques 28(9): 981-985.

[27] Yang, L.-Y. \& Xie, K. [2011]. Design and measurement of nonuniform ferrite coupled line circulator, Journal of Electromagnetic Waves and Applications 25(1): 131-145.

[28] Zahwe, O., Samad, B. A., Sauviac, B., Chatelon, J., Mignon, M. B., Rousseau, J., Berre, M. L. \& Givord, D. [2010]. Yig thin film used to miniaturize a coplanar junction circulator, Journal of Electromagnetic Waves and Applications 24(1): 25-32. 



\title{
Electromagnetic Waves Excitation by Thin Impedance Vibrators and Narrow Slots in Electrodynamic Volumes
}

\author{
Mikhail V. Nesterenko, Sergey L. Berdnik, Victor A. Katrich and \\ Yuriy M. Penkin
}

Additional information is available at the end of the chapter

http://dx.doi.org/10.5772/61188

\begin{abstract}
Linear vibrator and slot radiators, i.e., radiators of electric and magnetic type, respectively, are widely used as separate receiver and transmitter structures, elements of antenna systems, and antenna-feeder devices, including combined vibrator-slot structures. Widespread occurrence of such radiators is an objective prerequisite for theoretical analysis of their electrodynamic characteristics. During the last decades, researchers have published results which make it possible to create a modern theory of thin vibrator and narrow slot radiators. This theory combines the fundamental asymptotic methods for determining the single radiator characteristics, the hybrid analytic-numerical approaches, and the direct numerical techniques for electrodynamic analysis of such radiators. However, the electrodynamics of single linear electric and magnetic radiators is far from been completed. It may be explained by further development of modern antenna techniques and antenna-feeder devices, which can be characterized by such features as multielement structures, integration, and modification of structural units to minimize their mass and dimensions and to ensure electromagnetic compatibility of radio aids, application of metamaterials, formation of required spatial-energy, and spatial-polarization distributions of electromagnetic fields in various nondissipative and dissipative media. To solve these tasks, electric and magnetic radiators, based on various impedance structures with irregular geometric or electrophysical parameters and on combined vibrator-slot structures, should be created. This chapter presents the methodological basis for application of the generalized method of induced EMMF for the analysis of electrodynamic characteristics of the combined vibrator-slot structures. Characteristic feature of the generalization to a new class of approximating functions consists in using them as a function of the current distributions along the impedance vibrator and slot elements; these distributions are derived as the asymptotic solution of integral equations for the current (key problems) by the method of averaging. It should be noted that for simple structures similar to that considered in the model problem, the proposed approach yields an analytic solution of the electrodynamic problem. For more complex structures, the method may be used to design effective numerical-analytical algorithms for their analyses.
\end{abstract}


The demonstrative simulation (the comparative analysis of all electrodynamic characteristics in the operating frequencies range) has confirmed the validity of the proposed generalized method of induced EMMF for analysis of vibrator-slot systems with rather arbitrary structure (within accepted assumptions). Here, as examples, some fragments of this comparative analysis were presented. This method retains all benefits of analytical methods as compared with direct numerical methods and allows to expand significantly the boundaries of numerical and analytical studies of practically important problems, concerning the application of single impedance vibrator, including irregular vibrator, the systems of such vibrators, and narrow slots.

Keywords: Waves excitation, thin impedance vibrators, narrow slots, vibrator-slot structures

\section{Introduction}

At present, linear vibrator and slot radiators, i.e. radiators of electric and magnetic type, respectively, are widely used as separate receiver and transmitter structures, elements of antenna systems, and antenna-feeder devices, including combined vibrator-slot structures [1-4]. Widespread occurrence of such radiators is an objective prerequisite for theoretical analysis of their electrodynamic characteristics. During last decades researchers have published results which make it possible to create a modern theory of thin vibrator and narrow slot radiators. This theory combines the fundamental asymptotic methods for determining the single radiator characteristics [5-7], the hybrid analytic-numerical approaches [8-10], and the direct numerical techniques for electrodynamic analysis of such radiators [11]. However, the electrodynamics of single linear electric and magnetic radiators is far from been completed. It may be explained by further development of modern antenna techniques and antenna-feeder devices which can be characterized by such features as multielement structures, integration and modification of structural units to minimize their mass and dimensions and to ensure electromagnetic compatibility of radio aids, application of metamaterials, formation of required spatial-energy and spatial-polarization distributions of electromagnetic fields in various nondissipative and dissipative media. To solve these tasks electric and magnetic radiators, based on various impedance structures with irregular geometric or electrophysical parameters, and on combined vibrator-slot structures, should be created [12-20].

Mathematical modeling of antenna-feeder devices requires multiparametric optimization of electrodynamic problem solution and, hence, effective computational resources and software. Therefore, in spite of rapid growth of computer potential, there exists a necessity to develop new effective methods of electrodynamic analysis of antenna-feeder systems, being created with linear vibrator and slot structures with arbitrary geometric and electrophysical parameters, satisfying modern versatile requirements, and widening their application in various spheres. Efficiency of mathematical modeling is defined by rigor of corresponding boundary problem definition and solution, by performance of computational algorithm, requiring minimal possible RAM space, and directly depends upon analytical formulation of the models. That is, the weightier is the analytical component of the method the grater is its efficiency. In 
this connection it should be noted that formation of analytical concepts of electrodynamic analysis extending the capabilities of physically correct mathematical models for new classes of boundary problems is always an important problem.

This chapter presents the methodological basis of a new approach to solving the electrodynamic problems associated with combined vibrator-slot structures, defined as a generalized method of induced electro-magneto-motive forces (EMMF). This approach is based on the classical method of induced EMMF, i.e, basis functions, approximating the currents along the vibrator and slot elements, are obtained in advance as analytical solutions of key problems, formulated as integral equations for the currents by the asymptotic averaging method. Bearing this in mind, we present here solutions of two key problems: a single impedance vibrator and slot scatterer in a waveguide, obtained by averaging method, and then solve a problem for the multielement vibrator-slot structures by generalized method of induced EMMF.

\section{Problem formulation and initial integral equations}

Let us formulate the problem of electromagnetic fields excitation (scattering, radiation) by finite-size material bodies in two electrodynamic volumes coupled by holes cut in their common boundary. Suppose that there exists some arbitrary volume $V_{1}$, bounded by a perfectly conducting, impedance, or partially impedance surface $S_{1}$, some parts of which may be infinitely distant. The volume $V_{1}$ is coupled with another arbitrary volume $V_{2}$ through holes $\Sigma_{n}(n=1,2 \ldots N)$, cut in the surface $S_{1}$. The boundary between the volumes $V_{1}$ and $V_{2}$ in the regions around the coupling holes has an infinitely small thickness. Permittivity and permeability of the medium filling volumes $V_{1}$ and $V_{2}$ are $\varepsilon_{1}, \mu_{1}$ and $\varepsilon_{2}, \mu_{2}$, respectively. Material bodies, enclosed in local volumes $V_{m_{1}}\left(m_{1}=1,2, \ldots M_{1}\right)$ and $V_{m_{2}}\left(m_{2}=1,2, \ldots M_{2}\right)$, bounded by smooth closed surfaces $S_{m_{1}}$ and $S_{m_{2}}$ are allocated in the volumes $V_{1}$ and $V_{2}$, respectively. The bodies have homogeneous material parameters: permittivity $\varepsilon_{m_{1}} \varepsilon_{m_{2}}$ permeability $\mu_{m_{1}} \mu_{m_{2}}$, and conductivity $\sigma_{m_{1}} \sigma_{m_{2}}$. The fields of extraneous sources can be specified as the electromagnetic wave fields, incident on the bodies and the holes (scattering problem), or as fields of electromotive forces, applied to the bodies (radiation problem), or as combination of these fields. Without loss of generality, we assume that electromagnetic fields of extraneous sources $\left\{\vec{E}_{0}(\vec{r}), \vec{H}_{0}(\vec{r})\right\}$ exist only in the volume $V_{1}$. The fields $\left\{\vec{E}_{0}(\vec{r}), \vec{H}_{0}(\vec{r})\right\}$ depend on the time $t$ as $e^{i \omega t}$ $(\vec{r}$ is the radius vector of the observation point, $\omega=2 \pi f$ is an circular frequency and $f$ is frequency, measured in Hertz). We seek the electromagnetic fields $\left\{\vec{E}_{V_{1}}(\vec{r}), \vec{H}_{V_{1}}(\vec{r})\right\}$ and $\left\{\vec{E}_{V_{2}}(\vec{r}), \vec{H}_{V_{2}}(\vec{r})\right\}$ in the volumes $V_{1}$ and $V_{2}$, satisfying Maxwell's equations and boundary conditions on the surfaces $S_{m_{1}} S_{m_{2}} \Sigma_{n^{\prime}} S_{1}$ and $S_{2}$ (Figure 1).

To solve the above-mentioned problem we express the electromagnetic fields in volumes $V_{1}$ and $V_{2}$ in terms of the tangential fields components on the surfaces $S_{m_{1}{ }^{\prime}} S_{m_{2}}$ and $\Sigma_{n}$. In the Gaussian CGS system of units, the electromagnetic fields can be represented by the wellknown Kirchhoff-Kotler integral equations [3,4]: 


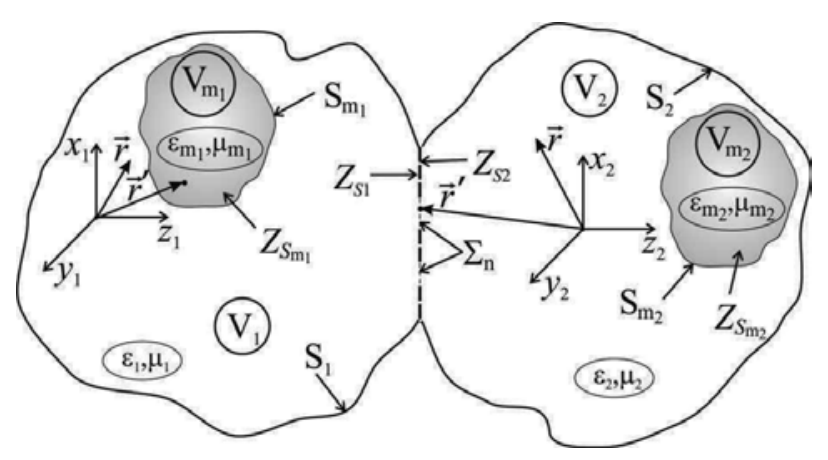

Figure 1. The problem geometry and notations

$$
\begin{aligned}
& \vec{E}_{V_{1}}(\vec{r})=\vec{E}_{0}(\vec{r})+\frac{1}{4 \pi i k \varepsilon_{1}}\left(\operatorname{graddiv}+k_{1}^{2}\right) \sum_{m_{1}=1}^{M_{1}} \int_{S_{m_{1}}} \hat{G}_{V_{1}}^{e}\left(\vec{r}, \vec{r}_{m_{1}}^{\prime}\right)\left[\vec{n}_{m_{1}}, \vec{H}_{V_{1}}\left(\vec{r}_{m_{1}}^{\prime}\right)\right] \mathrm{d} \vec{r}_{m_{1}}^{\prime} \\
& -\frac{1}{4 \pi} \operatorname{rot}\left\{\sum_{m_{1}=1}^{M_{1}} \int_{S_{m_{1}}} \hat{G}_{V_{1}}^{m}\left(\vec{r}, \vec{r}_{m_{1}}^{\prime}\right)\left[\vec{n}_{m_{1}}, \vec{E}_{V_{1}}\left(\vec{r}_{m_{1}}^{\prime}\right)\right] \mathrm{d} \vec{r}_{m_{1}}^{\prime}+\sum_{n=1}^{N} \int_{\Sigma_{n}} \hat{G}_{V_{1}}^{m}\left(\vec{r}, \vec{r}_{n}^{\prime}\right)\left[\vec{n}_{n}, \vec{E}_{V_{1}}\left(\vec{r}_{n}^{\prime}\right)\right] \mathrm{d} \vec{r}_{n}^{\prime}\right\}, \\
& \vec{H}_{V_{1}}(\vec{r})=\vec{H}_{0}(\vec{r})+\frac{1}{4 \pi i k \mu_{1}}\left(\operatorname{graddiv}+k_{1}^{2}\right)\left\{\begin{array}{l}
\sum_{m_{1}=1}^{M_{1}} \int_{S_{m_{1}}} \hat{G}_{V_{1}}^{m}\left(\vec{r}, \vec{r}_{m_{1}}^{\prime}\right)\left[\vec{n}_{m_{1}}, \vec{E}_{V_{1}}\left(\vec{r}_{m_{1}}^{\prime}\right)\right] \mathrm{d} \vec{r}_{m_{1}}^{\prime} \\
+\sum_{n=1}^{N} \int_{\Sigma_{n}} \hat{G}_{V_{1}}^{m}\left(\vec{r}, \vec{r}_{n}^{\prime}\right)\left[\vec{n}_{n^{\prime}}, \vec{E}_{V_{1}}\left(\vec{r}_{n}^{\prime}\right)\right] \mathrm{d} \vec{r}_{n}^{\prime}
\end{array}\right\} \\
& +\frac{1}{4 \pi} \operatorname{rot} \sum_{m_{1}=1}^{M_{1}} \int_{S_{m_{1}}} \hat{G}_{V_{1}}^{e}\left(\vec{r}, \vec{r}_{m_{1}}^{\prime}\right)\left[\vec{n}_{m_{1}}, \vec{H}_{V_{1}}\left(\vec{r}_{m_{1}}^{\prime}\right)\right] \mathrm{d} \vec{r}_{m_{1}}^{\prime} \\
& \vec{E}_{V_{2}}(\vec{r})=\frac{1}{4 \pi i k \varepsilon_{2}}\left(\operatorname{graddiv}+k_{2}^{2}\right) \sum_{m_{2}=1}^{M_{2}} \int_{S_{m_{2}}} \hat{G}_{V_{2}}^{e}\left(\vec{r}, \vec{r}_{m_{2}}^{\prime}\right)\left[\vec{n}_{m_{2}}, \vec{H}_{V_{2}}\left(\vec{r}_{m_{2}}^{\prime}\right)\right] \mathrm{d} \vec{r}_{m_{2}}^{\prime} \\
& -\frac{1}{4 \pi} \operatorname{rot}\left\{\sum_{m_{2}=1}^{M_{2}} \int_{S_{m_{2}}} \hat{G}_{V_{2}}^{m}\left(\vec{r}, \vec{r}_{m_{2}}^{\prime}\right)\left[\vec{n}_{m_{2}}, \vec{E}_{V_{2}}\left(\vec{r}_{m_{2}}^{\prime}\right)\right] \mathrm{d} \vec{r}_{m_{2}}^{\prime}+\sum_{n=1}^{N} \int_{\Sigma_{n}} \hat{G}_{V_{2}}^{m}\left(\vec{r}, \vec{r}_{n}^{\prime}\right)\left[\vec{n}_{n}, \vec{E}_{V_{2}}\left(\vec{r}_{n}^{\prime}\right)\right] \mathrm{d} \vec{r}_{n}^{\prime}\right\}, \\
& \vec{H}_{V_{2}}(\vec{r})=\frac{1}{4 \pi i k \mu_{2}}\left(\text { graddiv }+k_{2}^{2}\right)\left\{\begin{array}{l}
\sum_{m_{2}=1}^{M_{2}} \int_{S_{m_{2}}} \hat{G}_{V_{2}}^{m}\left(\vec{r}, \vec{r}_{m_{2}}^{\prime}\right)\left[\vec{n}_{m_{2}}, \vec{E}_{V_{2}}\left(\vec{r}_{m_{2}}^{\prime}\right)\right] \mathrm{d} \vec{r}_{m_{2}}^{\prime} \\
+\sum_{n=1}^{N} \int_{\Sigma_{n}} \hat{G}_{V_{2}}^{m}\left(\vec{r}, \vec{r}_{n}^{\prime}\right)\left[\vec{n}_{n}, \vec{E}_{V_{2}}\left(\vec{r}_{n}^{\prime}\right)\right] \mathrm{d} \vec{r}_{n}^{\prime}
\end{array}\right\} \\
& +\frac{1}{4 \pi} \operatorname{rot} \sum_{m_{2}=1}^{M_{2}} \int_{S_{m_{2}}} \hat{G}_{V_{2}}^{e}\left(\vec{r}, \vec{r}_{m_{2}}^{\prime}\right)\left[\vec{n}_{m_{2}}, \vec{H}_{V_{2}}\left(\vec{r}_{m_{2}}^{\prime}\right)\right] \vec{r}_{m_{2}}^{\prime}
\end{aligned}
$$


Here $k=2 \pi / \lambda$ is the wave number, $\lambda$ is the free space wavelength, $k_{1}=k \sqrt{\varepsilon_{1} \mu_{1}}$ and $k_{2}=k \sqrt{\varepsilon_{2} \mu_{2}}$ are wave numbers in the media filling the volumes $V_{1}$ and $V_{2}$, respectively; ${ }^{\prime \prime}{ }_{m_{1}, m_{2}, n}$ are radius-vectors of sources allocated at the surfaces $S_{m_{1}} S_{m_{2}}$ and $\Sigma_{n} ; \vec{n}_{m 1, m 2, n}$ are unit vectors of external normals to the surfaces; $\hat{G}_{V_{1}, V_{2}}^{e}\left(\vec{r}, \vec{r}^{\prime}\right)$ and $\hat{G}_{V_{1}, V_{2}}^{m}\left(\vec{r}, \vec{r}^{\prime}\right)$ are the electric and magnetic tensor Green's functions for Hertz's vector potentials in the coupled volumes satisfying the vector Helmholtz equation and the boundary conditions on surfaces $S_{1}$ and $S_{2}$. For the infinitely distant parts of surfaces $S_{1}$ or $S_{2}$ the boundary conditions for the Green's functions are transformed to the Sommerfeld's radiation condition.

Interpretation of the fields in the left-hand side of equations (1) depends upon position of an observation point $\vec{r}$. If the observation point $\vec{r}$ belongs to the surfaces $S_{m_{1}}, S_{m_{2}}$ or to the apertures $\Sigma_{n}$, the fields $\vec{E}(\vec{r})$ and $\vec{H}(\vec{r})$ represent the same fields as in the integrals in the right-hand sides of equations (1). In this case, equations (1) are non-homogeneous linear integral Fredholm equations of the second kind, which are known to have the unique solution. If the observation point lies outside areas $V_{m_{1}}, V_{m_{2}}$ and $\Sigma_{n}$, the equations (1) become the equalities determining the total electromagnetic field by the field of specified extraneous sources. These equalities solve, in general terms, the problem of electromagnetic fields excitation by finite size obstacles if fields on the objects' surfaces are known. Certainly, to find these fields, the Fredholm integral equations should be solved beforehand.

The equations (1) can be also used to solve electrodynamics problems if the fields on the material body surfaces can be defined by additional physical considerations. For example, if induced currents on well-conducting bodies $(\sigma \rightarrow \infty)$ are concentrated near the body surface the skin layer thickness can be neglected and the well-known Leontovich-Shchukin approximate impedance boundary condition becomes applicable [4]

$$
[\vec{n}, \vec{E}(\vec{r})]=\bar{Z}_{S}(\vec{r})[\vec{n},[\vec{n}, \vec{H}(\vec{r})]]
$$

where $\bar{Z}_{S}(\vec{r})=\bar{R}_{S}(\vec{r})+i \bar{X}_{S}(\vec{r})=Z_{S}(\vec{r}) / Z_{0}$ is the distributed complex surface impedance, normalized to the characteristic free space impedance $Z_{0}=120 \pi \mathrm{Ohm}$; the value of $\bar{Z}_{S}(\vec{r})$ may vary over the body surface. It is generally accepted that the boundary condition (2) are physically adequate under condition $\left|\bar{Z}_{S}(\vec{r})\right| \ll 1$. If $\left|\bar{Z}_{S}(\vec{r})\right| \rightarrow 0$, the boundary condition become that for the perfect conductor. In contrast to the limiting case of the perfect conductor, the impedance boundary condition allow to take into account losses in the real material. Since the relative error of (2) is of order $\left|\bar{Z}_{S}(\vec{r})\right|^{3}$, the inequality $0 \leq\left|\bar{Z}_{S}(\vec{r})\right| \leq 0.4$ must hold to obtain valid results by the mathematical model.

Using the impedance boundary condition (2) we can introduce a new unknown, density of surface currents. Let us perform such change of unknown in the equations (1). Without loss of generality, we carry the system of equations (1) the transition to the case when all the material bodies are located in volume $V_{1}$. By placing the observation point on the surface $S_{m}$ (index 1 
is omitted) and using the continuity condition for the tangential components of the magnetic field on the holes $\Sigma_{n}$, we obtain the system of integral equations relative to the density of surface currents: electric $\vec{J}_{m}^{e}\left(\vec{r}_{m}\right)$ at $S_{m}$ and equivalent magnetic $\vec{J}_{n}^{m}\left(\vec{r}_{n}\right)$ at $\Sigma_{n}$. The system can be presented as

$$
\begin{aligned}
& Z_{S q}\left(\vec{r}_{q}\right) \vec{J}_{q}^{e}\left(\vec{r}_{q}\right)+\frac{k}{\omega} \operatorname{rot} \sum_{n=1}^{N} \int_{\Sigma_{n}} \hat{G}_{V_{1}}^{m}\left(\vec{r}_{q}, \vec{r}_{n}^{\prime}\right) \vec{J}_{n}^{m}\left(\vec{r}_{n}^{\prime}\right) \mathrm{d} \vec{r}_{n}^{\prime}= \\
& =\vec{E}_{0}\left(\vec{r}_{q}\right)+\frac{1}{i \omega \varepsilon_{1}}\left(\text { graddiv }+k_{1}^{2}\right) \sum_{m=1}^{M} \int_{S_{m}} \hat{G}_{V_{1}}^{e}\left(\vec{r}_{q}, \vec{r}_{m}^{\prime}\right) \vec{J}_{m}^{e}\left(\vec{r}_{m}^{\prime}\right) \mathrm{d} \vec{r}_{m}^{\prime}+ \\
& +\frac{1}{4 \pi} \operatorname{rot} \sum_{m=1}^{M} \int_{S_{1_{1}}} \hat{G}_{V_{1}}^{m}\left(\vec{r}_{q}, \vec{r}_{m}^{\prime}\right) Z_{S m}\left(\vec{r}_{m}^{\prime}\right)\left[\vec{n}_{m}, \vec{J}_{m}^{e}\left(\vec{r}_{m}^{\prime}\right)\right] \mathrm{d} \vec{r}_{m}^{\prime}, \\
& +\frac{1}{i \omega \mu_{2}}\left(\operatorname{graddiv}+k_{2}^{2}\right) \sum_{n=1}^{N} \int_{\Sigma_{n}} \hat{G}_{V_{2}}^{m}\left(\vec{r}_{p}, \vec{r}_{n}^{\prime}\right) \vec{J}_{n}^{m}\left(\vec{r}_{n}^{\prime}\right) \mathrm{d} \vec{r}_{n}^{\prime} \\
& =\frac{1}{i \omega \mu_{1}}\left(\operatorname{graddiv}+k_{1}^{2}\right) \sum_{n=1}^{N} \int_{\Sigma_{n}} \hat{G}_{V_{1}}^{m}\left(\vec{r}_{p}, \vec{r}_{n}^{\prime}\right) \vec{J}_{n}^{m}\left(\vec{r}_{n}^{\prime}\right) \mathrm{d} \vec{r}_{n}^{\prime} \\
& -\frac{k}{\omega} \operatorname{rot} \sum_{m=1}^{M} \int_{S_{m}} \hat{G}_{V_{1}}^{e}\left(\vec{r}_{p}, \vec{r}_{m}^{\prime}\right) \vec{J}_{m}^{e}\left(\vec{r}_{m}^{\prime}\right) \mathrm{d} \vec{r}_{m}^{\prime}, \\
&
\end{aligned}
$$

where $q=1,2, \ldots, m, \ldots, M, p=1,2, \ldots, n, \ldots, N, \vec{J}_{m}^{e}\left(\vec{r}_{m}\right)=\frac{c}{4 \pi}\left[\vec{n}_{m}, \vec{H}\left(\vec{r}_{m}\right)\right], \vec{J}_{n}^{m}\left(\vec{r}_{n}\right)=\frac{c}{4 \pi}\left[\vec{n}_{n}, \vec{E}\left(\vec{r}_{n}\right)\right], c$ is velocity of light in free space.

Thus, the problem of electromagnetic waves excitation by the impedance bodies of finite dimensions and by the coupling holes between two electrodynamic volumes is formulated as a rigorous boundary value problem of macroscopic electrodynamics, reduced to the system of integral equations for surface currents. Solution of this system is an independent problem, significant in its own right, since it often present considerable mathematical difficulties. If characteristic dimensions of an object are much greater than wavelength (high-frequency region) a solution is usually searched as series expansion in ascending power of inverse wave number. If dimensions of an object are less than wavelength (low-frequency or quasi-static region), representation of the unknown functions as series expansion in wave number powers reduces the problem to a sequence of electrostatic problems. Contrary to asymptotic cases, resonant region, where at least one dimension of an object is comparable with wavelength, is the most complex for analysis, and requires rigorous solution of field equations. It should be noted that, from the practical point of view, the resonant region is of exceptional interest for thin impedance vibrators and narrow slots. 


\section{Integral equations for electric and magnetic currents in thin impedance vibrators and narrow slots}

A straightforward solution of the system (3) for the material objects with irregular surface shape and for holes with arbitrary geometry may often be impossible due to the known mathematical difficulties. However, the solution is sufficiently simplified for thin impedance vibrators and narrow slots, i.e. cylinders, which cross-section perimeter is small as compared to their length and the wavelength in the surrounding media and for holes, which one dimension satisfy the analogous conditions $[19,20]$. The approach used in $[19,20]$ for the analysis of slot-vibrator systems can be generalized for multi-element systems. In addition, the boundary condition (2) can be extended for cylindrical vibrator surfaces with an arbitrary distribution of complex impedance regardless of the exciting field structure and electrophysical characteristics of vibrator material [4].

For thin vibrators made of circular cylindrical wire and narrow straight slots the equation system (3) can be easily simplified using inequalities

$$
\frac{r_{m}}{L_{m}}<<1, \frac{r_{m}}{\lambda_{1,2}}<<1, \frac{d_{n}}{2 L_{n}}<<1, \frac{d_{n}}{\lambda_{1,2}}<<1,
$$

where $r_{m}$ is vibrator radius, $L_{m}$ is vibrator length, $d_{n}$ is slot width, $2 L_{n}$ is slot length, and $\lambda_{1,2}$ is wavelength in the corresponding media. The electric current induced on the vibrator surfaces and equivalent magnetic currents in the slots can be presented using the inequalities (4) as

$$
\vec{J}_{m}^{e}\left(\vec{r}_{m}\right)=\vec{e}_{s_{m}} J_{m}\left(s_{m}\right) \psi_{m}\left(\rho_{m}, \varphi_{m}\right), \quad \vec{J}_{n}^{m}\left(\vec{r}_{n}\right)=\vec{e}_{s_{n}} J_{n}\left(s_{n}\right) \chi_{n}\left(\xi_{n}\right)
$$

where $\vec{e}_{s_{m}}$ and $\vec{e}_{s_{n}}$ are unit vectors directed along the vibrator and slot axis, respectively; $s_{m}$ and $s_{n}$ are local coordinates related to the vibrator and slot axes; $\psi_{m}\left(\rho_{m^{\prime}}, \varphi_{m}\right)$ are functions of transverse $\left(\perp_{m}\right)$ polar coordinates $\rho_{m^{\prime}} \varphi_{m}$ for the vibrators; $\chi_{n}\left(\xi_{n}\right)$ are functions of transverse coordinates $\xi_{n}$ for the slots. The functions $\psi_{m}\left(\rho_{m}, \varphi_{m}\right)$ and $\chi_{n}\left(\xi_{n}\right)$ satisfy the normality conditions

$$
\int_{\perp_{m}} \psi_{m}\left(\rho_{m}, \varphi_{m}\right) \rho_{m} \mathrm{~d} \rho_{m} \mathrm{~d} \varphi_{m}=1, \int_{\xi_{n}} \chi_{n}\left(\xi_{n}\right) \mathrm{d} \xi_{n}=1
$$

and the unknown currents $J_{m}\left(s_{m}\right)$ and $J_{n}\left(s_{n}\right)$ must satisfy the boundary conditions

$$
J_{m}\left( \pm L_{m}\right)=0, \quad J_{n}\left( \pm L_{n}\right)=0,
$$

where upper indexes $e$ and $m$ are omitted. 
Now we take into account that $\left[\vec{n}_{m}, \vec{J}_{m}\left(\vec{r}_{m}\right)\right] \ll 1$ according to inequalities (4) and project the equations (3a) and (3b) on the axes of the vibrators and slots, respectively, and arrive at a system of linear integral equations relative to the currents in the vibrators and slots

$$
\begin{aligned}
& \left(\frac{\mathrm{d}^{2}}{\mathrm{~d} s_{q}^{2}}+k_{1}^{2}\right) \sum_{m=1}^{M} \int_{-L_{m}}^{L_{m}} J_{m}\left(s_{m}^{\prime}\right) G_{s_{m}}^{V_{1}}\left(s_{q}, s_{m}^{\prime}\right) \mathrm{d} s_{m}^{\prime}-i k \vec{e}_{s_{q}} \operatorname{rot} \sum_{n=1}^{N} \int_{-L_{n}}^{L_{n}} J_{n}\left(s_{n}^{\prime}\right) G_{s_{n}}^{V_{1}}\left(s_{q}, s_{n}^{\prime}\right) \mathrm{d} s_{n}^{\prime} \\
& \quad=-i \omega \varepsilon_{1}\left[E_{0 s_{q}}\left(s_{q}\right)-z_{i q}\left(s_{q}\right) J_{q}\left(s_{q}\right)\right], \\
& \frac{1}{\mu_{1}}\left(\frac{\mathrm{d}^{2}}{\mathrm{~d} s_{p}^{2}}+k_{1}^{2}\right) \sum_{n=1}^{N} \int_{-L_{n}}^{L_{n}} J_{n}\left(s_{n}^{\prime}\right) G_{s_{n}}^{V_{1}}\left(s_{p}, s_{n}^{\prime}\right) \mathrm{d} s_{n}^{\prime}+\frac{1}{\mu_{2}}\left(\frac{\mathrm{d}^{2}}{\mathrm{~d} s_{p}^{2}}+k_{2}^{2}\right) \sum_{n=1}^{N} \int_{-L_{n}}^{L_{n}} J_{n}\left(s_{n}^{\prime}\right) G_{s_{n}}^{V_{2}}\left(s_{p}, s_{n}^{\prime}\right) \mathrm{d} s_{n}^{\prime} \\
& \quad+i k \vec{e}_{s_{p}} \operatorname{rot} \sum_{m=1}^{M} \int_{-L_{m}}^{L_{m}} J_{m}\left(s_{m}^{\prime}\right) G_{s_{m}}^{V_{1}}\left(s_{p}, s_{m}^{\prime}\right) \mathrm{d} s_{m}^{\prime}=-i \omega H_{0 s_{p}}\left(s_{p}\right) .
\end{aligned}
$$

Here $z_{i m}\left(s_{m}\right)$ are internal lineal impedance of the vibrators $\left(Z_{S m}\left(\vec{r}_{m}\right)=2 \pi r_{m} z_{i m}\left(\vec{r}_{m}\right)\right)$ measured in $\mathrm{Ohm} / \mathrm{m}, E_{0 s_{m}}\left(s_{m}\right)$ and $H_{0 s_{n}}\left(s_{n}\right)$ are projections of extraneous sources on the vibrators and slots axes, $G_{s_{m}}^{V_{1}}\left(s_{m, n}, s^{\prime}\right)$ and $G_{s_{n}}^{V_{1(2)}}\left(s_{m, n^{\prime}} s^{\prime}{ }_{n}\right)$ are components of the tensor Green's functions in the volumes $V_{1}$ and $V_{2}$.

For solitary vibrator or slot as well as for the absence of electromagnetic interaction between them, the system (8) splits into two independent equations:

$$
\begin{gathered}
\left(\frac{\mathrm{d}^{2}}{\mathrm{~d} s_{v}^{2}}+k_{1}^{2}\right) \int_{-L_{v}}^{L_{v}} J_{v}\left(s_{v}^{\prime}\right) G_{s_{v}}^{V}\left(s_{v}, s_{v}^{\prime}\right) \mathrm{d} s_{v}^{\prime}=-i \omega \varepsilon_{1} E_{0 s_{v}}\left(s_{v}\right)+i \omega \varepsilon_{1} z_{i}\left(s_{v}\right) J_{v}\left(s_{v}\right), \\
\frac{1}{\mu_{1}}\left(\frac{\mathrm{d}^{2}}{\mathrm{~d} s_{s l}^{2}}+k_{1}^{2}\right) \int_{-L_{s l}}^{L_{s l}} J_{s l}\left(s_{s l}^{\prime}\right) G_{s_{s l}}^{V_{1}}\left(s_{s l}, s_{s l}^{\prime}\right) \mathrm{d} s_{s l}^{\prime}+\frac{1}{\mu_{2}}\left(\frac{\mathrm{d}^{2}}{\mathrm{~d} s_{s l}^{2}}+k_{2}^{2}\right) \int_{-L_{s l}}^{L_{s l}} J_{s l}\left(s_{s l}^{\prime}\right) G_{s_{s l}}^{V_{2}}\left(s_{s l}, s_{s l}^{\prime}\right) \mathrm{d} s_{s l}^{\prime}=-i \omega H_{0 s_{s l}}\left(s_{s l}\right) .
\end{gathered}
$$

Here $\vec{e}_{s_{v}^{\prime}}$ and $\vec{e}_{s_{s l}^{\prime}}$ are unit vectors of vibrator and slot axes at the sources, and

$$
\begin{gathered}
G_{s_{v}}^{V}\left(s_{v}, s_{v}^{\prime}\right)=\int_{-\pi}^{\pi} \frac{e^{-i k_{1} \sqrt{\left(s_{v}-s_{v}^{\prime}\right)^{2}+[2 r \sin (\varphi / 2)]^{2}}}}{\sqrt{\left(s_{v}-s_{v}^{\prime}\right)^{2}+[2 r \sin (\varphi / 2)]^{2}}} \psi(r, \varphi) r \mathrm{~d} \varphi, \\
G_{s_{s l}}^{V_{1,2}}\left(s_{s l}, s_{s l}^{\prime}\right)=\int_{-d / 2}^{d / 2} \frac{e^{-i k_{1,2} \sqrt{\left(s_{s l}-s_{s l}^{\prime}\right)^{2}+(\xi)^{2}}}}{\sqrt{\left(s_{s l}-s_{s l}^{\prime}\right)^{2}+(\xi)^{2}}} \chi(\xi) \mathrm{d} \xi .
\end{gathered}
$$


Solution of the integral equation with the exact kernel expressions (11) and (12) may be very difficult, therefore we will use approximate expressions, the so called "quasi-one-dimensional" kernels [5,15]

$$
\begin{gathered}
G_{s_{v}}^{V}\left(s_{v}, s_{v}^{\prime}\right)=\frac{e^{-i k_{1} \sqrt{\left(s_{v}-s_{v}^{\prime}\right)^{2}+r^{2}}}}{\sqrt{\left(s_{v}-s_{v}^{\prime}\right)^{2}+r^{2}}}, \\
G_{s_{s l}}^{V_{1,2}}\left(s_{s l}, s_{s l}^{\prime}\right)=\frac{e^{-i k_{1,2} \sqrt{\left(s_{s l}-s_{s l}^{\prime}\right)^{2}+(d / 4)^{2}}}}{\sqrt{\left(s_{s l}-s_{s l}^{\prime}\right)^{2}+(d / 4)^{2}}}
\end{gathered}
$$

derived with the assumption that source points belong to the geometric axes of the vibrator and slot while observation points belong to vibrator surface and to slot axis, having coordinates $\left\{s_{s l}, \xi / 2\right\}$. In that case the functions $G_{s_{v}}^{V}\left(s_{v}, s_{v}^{\prime}\right)$ and $G_{s_{s l}}^{V_{1,2}}\left(s_{s l}, s_{s l}^{\prime}\right)$ are everywhere continuous and equations for the currents are simplified significantly.

Since the form of the Green's functions was not specified, the equations (8) are valid for any electrodynamic volumes, provided that the Green's functions for any electrodynamic volumes are known or can be constructed. Although the boundary between the volumes $V_{1}$ and $V_{2}$ initially was supposed to be of infinitesimal thickness, its actual thickness can be accounted for by introducing into the equations (8) an effective slot width, defined by the formula given in the Section 5.

\section{Solution of integral equation for current in an impedance vibrator, located in unbounded free space}

Let us use the equation (9) with the approximate kernel (13), being a quasi-one-dimensional analog of the exact integral equation with kernel (11) as starting point for the analysis. Note that impedance $z_{i}(s) \equiv$ const,$\varepsilon_{1}=\mu_{1}=1$, and index $v$ is omitted. Thus, the equation may be written as

$$
\left(\frac{\mathrm{d}^{2}}{\mathrm{~d} s^{2}}+k^{2}\right) \int_{-L}^{L} J\left(s^{\prime}\right) \frac{e^{-i k R\left(s, s^{\prime}\right)}}{R\left(s, s^{\prime}\right)} \mathrm{d} s^{\prime}=-i \omega E_{0 s}(s)+i \omega z_{i} J(s),
$$

where $R\left(s, s^{\prime}\right)=\sqrt{\left(s-s^{\prime}\right)^{2}+r^{2}}$. Let us isolate the logarithmic singularity in the kernel of equation (15) by identical transformation

$$
\int_{-L}^{L} J\left(s^{\prime}\right) \frac{e^{-i k R\left(s, s^{\prime}\right)}}{R\left(s, s^{\prime}\right)} \mathrm{d} s^{\prime}=\Omega(s) J(s)+\int_{-L}^{L} \frac{J\left(s^{\prime}\right) e^{-i k R\left(s, s^{\prime}\right)}-J(s)}{R\left(s, s^{\prime}\right)} \mathrm{d} s^{\prime} .
$$

Here 


$$
\Omega(s)=\int_{-L}^{L} \frac{\mathrm{d} s^{\prime}}{\sqrt{\left(s-s^{\prime}\right)^{2}+r^{2}}}=\Omega+\gamma(s)
$$

$\gamma(s)=\ln \frac{\left[(L+s)+\sqrt{(L+s)^{2}+r^{2}}\right]\left[(L-s)+\sqrt{(L-s)^{2}+r^{2}}\right]}{4 L^{2}}$ is a function, equal to zero at the vibrator center and reaching maximal value at its ends where the current in accordance with boundary condition (7) is equal to zero, $\Omega=2 \ln \frac{2 L}{r}$ is a large parameter. Then, equation (15) in view of (16) is transformed to integral equation with a small parameter

$$
\frac{\mathrm{d}^{2} J(s)}{\mathrm{d} s^{2}}+k^{2} J(s)=\alpha\left\{i \omega E_{0 s}(s)+F[s, J(s)]-i \omega z_{i} J(s)\right\}
$$

Here $\alpha=-\frac{1}{\Omega}=\frac{1}{2 \ln [r /(2 L)]}$ is a natural small parameter of the problem $(|\alpha|<<1)$,

$$
\begin{aligned}
F[s, J(s)]= & -\left.\frac{\mathrm{d} J\left(s^{\prime}\right)}{\mathrm{d} s^{\prime}} \frac{e^{-i k R\left(s, s^{\prime}\right)}}{R\left(s, s^{\prime}\right)}\right|_{-L} ^{L}+\left[\frac{\mathrm{d}^{2} J(s)}{\mathrm{d} s^{2}}+k^{2} J(s)\right] \gamma(s) \\
& +\int_{-L}^{L} \frac{\left[\frac{\mathrm{d}^{2} J\left(s^{\prime}\right)}{\mathrm{d} s^{\prime 2}}+k^{2} J\left(s^{\prime}\right)\right] e^{-i k R\left(s, s^{\prime}\right)}-\left[\frac{\mathrm{d}^{2} J(s)}{\mathrm{d} s^{2}}+k^{2} J(s)\right]}{R\left(s, s^{\prime}\right)} \mathrm{d} s^{\prime}
\end{aligned}
$$

is the vibrator self-field in free space.

To find the approximate analytic solution of equation (18) we will use the asymptotic averaging method. The basic principles of the method are presented in $[3,4]$. To reduce the equation (18) to a standard equation system with a small parameter in compliance with the method of variation of constants we will change variables

$$
\begin{aligned}
& J(s)=A(s) \cos k s+B(s) \sin k s, \\
& \frac{\mathrm{d} J(s)}{\mathrm{d} s}=-A(s) k \sin k s+B(s) k \cos k s, \quad\left(\frac{\mathrm{d} A(s)}{\mathrm{d} s} \cos k s+\frac{\mathrm{d} B(s)}{\mathrm{d} s} \sin k s=0\right), \\
& \frac{\mathrm{d}^{2} J(s)}{\mathrm{d} s^{2}}+k^{2} J(s)=-\frac{\mathrm{d} A(s)}{\mathrm{d} s} \sin k s+\frac{\mathrm{d} B(s)}{\mathrm{d} s} \cos k s,
\end{aligned}
$$

where $A(s)$ and $B(s)$ are new unknown functions. Then the equation (18) reduces to a system of integral equations 


$$
\begin{aligned}
& \frac{\mathrm{d} A(s)}{\mathrm{d} s}=-\frac{\alpha}{k}\left\{i \omega E_{0 s}(s)+F\left[s, A(s), \frac{\mathrm{d} A(s)}{\mathrm{d} s}, B(s), \frac{\mathrm{d} B(s)}{\mathrm{d} s}\right]-i \omega z_{i}[A(s) \cos k s+B(s) \sin k s]\right\} \sin k s, \\
& \frac{\mathrm{d} B(s)}{\mathrm{d} s}=+\frac{\alpha}{k}\left\{i \omega E_{0 s}(s)+F\left[s, A(s), \frac{\mathrm{d} A(s)}{\mathrm{d} s}, B(s), \frac{\mathrm{d} B(s)}{\mathrm{d} s}\right]-i \omega z_{i}[A(s) \cos k s+B(s) \sin k s]\right\} \cos k s .
\end{aligned}
$$

This system is equivalent to the equation (18) and represents the standard equations system unsolvable with respect to derivatives. The right-hand sides of the equations are proportional to small parameter $\alpha$, therefore, the functions $A(s)$ and $B(s)$ in the left-hand sides of the equations system (21) are slowly varying functions and the system can be solved by the asymptotic averaging method. Then, we replace the system (21) by the simplified system wherein assume $\frac{d A(s)}{d s}=0$ and $\frac{d B(s)}{d s}=0$ in rigth-hand members and carry out partial averaging over the explicit variable $s$ to obtain the equations of first approximation. The term partial averaging means that averaging operator acts on all terms, but containing $E_{0 s}(s)$ and it may be done for the system (21). The averaged system can be written as

$$
\begin{aligned}
& \frac{\mathrm{d} \bar{A}(s)}{\mathrm{d} s}=-\alpha\left\{\frac{i \omega}{k} E_{0 s}(s)+\bar{F}[s, \bar{A}(s), \bar{B}(s)]\right\} \sin k s+\chi \bar{B}(s), \\
& \frac{\mathrm{d} \bar{B}(s)}{\mathrm{d} s}=+\alpha\left\{\frac{i \omega}{k} E_{0 s}(s)+\bar{F}[s, \bar{A}(s), \bar{B}(s)]\right\} \cos k s-\chi \bar{A}(s),
\end{aligned}
$$

where $\chi=\alpha \frac{i \omega}{2 k} z_{i}$

$$
\bar{F}[s, \bar{A}(s), \bar{B}(s)]=\left.\left[\bar{A}\left(s^{\prime}\right) \sin k s^{\prime}-\bar{B}\left(s^{\prime}\right) \cos k s^{\prime}\right] \frac{e^{-i k R\left(s, s^{\prime}\right)}}{R\left(s, s^{\prime}\right)}\right|_{-L} ^{L}
$$

is self-field of the vibrator (19), averaged over its length.

We will seek the solution of the equations system (22) in the form

$$
\begin{aligned}
& \bar{A}(s)=C_{1}(s) \cos \chi s+C_{2}(s) \sin \chi s, \\
& \bar{B}(s)=-C_{1}(s) \sin \chi s+C_{2}(s) \cos \chi s .
\end{aligned}
$$

Then, substitution (24) into (22) gives

$$
\begin{aligned}
& \frac{\mathrm{d} C_{1}(s)}{\mathrm{d} s}=-\alpha\left\{\frac{i \omega}{k} E_{0 s}(s)+\bar{F}\left[s, C_{1}, C_{2}\right]\right\} \sin (k+\chi) s, \\
& \frac{\mathrm{d} C_{2}(s)}{\mathrm{d} s}=+\alpha\left\{\frac{i \omega}{k} E_{0 s}(s)+\bar{F}\left[s, C_{1}, C_{2}\right]\right\} \cos (k+\chi) s .
\end{aligned}
$$


Then we find $C_{1}(s)$ and $C_{2}(s)$ by solving system (25), determine $\bar{A}(s)$ и $\bar{B}(s)$ from (24), and substitute them as approximating functions for the current into (20). Thus, the general asymptotic expression in parameter $\alpha$ for the current in a thin impedance vibrator under arbitrary excitation may be presented as

$$
J(s)=\bar{A}(-L) \cos (\tilde{k} s+\chi L)+\bar{B}(-L) \sin (\tilde{k} s+\chi L)+\alpha \int_{-L}^{s}\left\{\frac{i \omega}{k} E_{0 s}\left(s^{\prime}\right)+\bar{F}\left[s^{\prime}, \bar{A}, \bar{B}\right]\right\} \sin \tilde{k}\left(s-s^{\prime}\right) \mathrm{d} s^{\prime}
$$

where $\tilde{k}=k+\chi=k+i(\alpha / r) \bar{Z}_{S}, \bar{Z}_{S}=\bar{R}_{S}+i \bar{X}_{S}$ is the normalized complex surface impedance: $\bar{Z}_{S}=2 \pi r z_{i} / Z_{0}$.

For electrically thin vibrators $\left(\left|(k \sqrt{\varepsilon \mu} r)^{2} \ln \left(k \sqrt{\varepsilon \mu} r_{i}\right)\right|<<1, r_{i}\right.$ is the radius of the inner conductor) with the parameters of material $\varepsilon, \mu, \sigma$, from which they are made, the formulas of the distributed surface impedance $\bar{Z}_{S}$ are presented in Table 1 .

\begin{tabular}{|c|c|c|c|}
\hline № & Design type of vibrator & Breadboard view of vibrator & Formula for impedance \\
\hline 1 & $\begin{array}{l}\text { The solid metallic cylinder of the } \\
r \Delta^{0} \text { radius, } \Delta^{0}=\omega / k \sqrt{2 \pi \sigma \omega \mu} \text { is the } \\
\text { skin-layer thickness }\end{array}$ & & $\bar{Z}_{S}=\frac{1+i}{Z_{0} \sigma \Delta^{0}}$ \\
\hline 2 & $\begin{array}{l}\text { The dielectrical metalized cylinder } \\
\text { with covering, made of the metal of } \\
\text { the } h_{R} \Delta^{0} \text { thickness }\end{array}$ & & $\bar{Z}_{S}=\frac{1}{Z_{0} \sigma h_{R}+i k r(\varepsilon-1) / 2}$ \\
\hline 3 & $\begin{array}{l}\text { The metal-dielectrical cylinder ( } L_{1} \text { is } \\
\text { the thickness of the metal disk, } L_{2} \text { is } \\
\text { the thickness of the dielectric disc) }\end{array}$ & 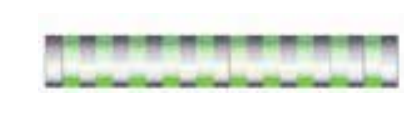 & $\bar{Z}_{S}=-i \frac{L_{2}}{L_{1}+L_{2}} \frac{2}{k r \varepsilon}$ \\
\hline 4 & $\begin{array}{l}\text { The magnetodielectrical metalized } \\
\text { cylinder with the inner conducting } \\
\text { cylinder with the radius } r_{i}\end{array}$ & 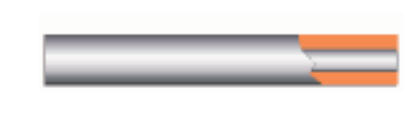 & $\bar{Z}_{S}=\frac{1}{Z_{0} \sigma h_{R}-i / k r \mu \ln \left(r / r_{i}\right)}$ \\
\hline 5 & $\begin{array}{l}\text { The metallic cylinder with covering, } \\
\text { made of magnetodielectric of the } r-r_{i} \\
\text { thickness, or the corrugated } \\
\text { cylinder }\end{array}$ & 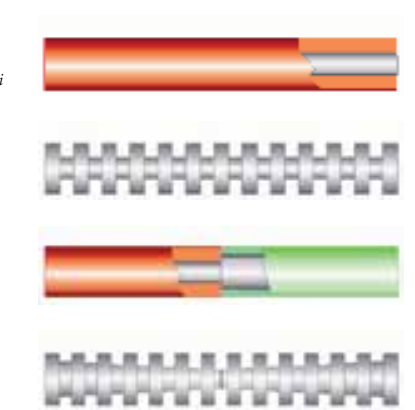 & $\begin{array}{l}\bar{Z}_{S}=i k r \mu \ln \left(r / r_{i}\right) \\
\bar{Z}_{S}(s)=\bar{R}_{S}(s)+i \bar{X}_{S}(s)\end{array}$ \\
\hline
\end{tabular}




\begin{tabular}{llll}
\hline № & Design type of vibrator & Breadboard view of vibrator & Formula for impedance \\
\hline 6 & $\begin{array}{l}\text { The metallic monofilar helix of the } r \\
\text { radius }(k r 1) \text { with the } \psi \text { winding } \\
\text { angle }\end{array}$ & $\bar{Z}_{S}=(i / 2) k r \operatorname{ctg}^{2} \psi$ \\
\hline
\end{tabular}

Table 1. The formulas of the distributed surface impedance $\bar{Z}_{S}$

The formulas have been obtained in the frames of impedance conception [4], and they are just for thin cylinders both of infinite and finite extension, located in free space. It is necessary to introduce the multiplier $\sqrt{\mu_{1} / \varepsilon_{1}}$ in all formulas for the vibrator in the material medium with the $\varepsilon_{1}$ and $\mu_{1}$ parameters. We note, that most of the formulas for impedances include the parameters $\varepsilon$ and $\mu$, smooth change of which (in the case of their dependence from the static electrical and magnetic fields) and the characteristics of radiation of the system, correspondingly, (at its fixed geometrical sizes) can be made, for example, by external field effects.

The constants $\bar{A}( \pm L)$ and $\bar{B}( \pm L)$ can be found employing the boundary conditions (7) and the symmetry conditions [5], unambiguously related to a method of vibrator excitation; if $E_{0 s}(s)=E_{0 s}^{s}(s), J(s)=J(-s)=J^{s}(s)$ and $\bar{A}(-L)=\bar{A}(+L), \quad \bar{B}(-L)=-\bar{B}(+L) \quad$; if $\quad E_{0 s}(s)=E_{0 s}^{a}(s)$, $J(s)=-J(-s)=J^{a}(s)$ and $\bar{A}(-L)=-\bar{A}(+L), \bar{B}(-L)=\bar{B}(+L)$. Then, in terms of symmetric and antisymmetric current components, marked by indexes $s$ and $a$, respectively, for arbitrary vibrator excitation by $E_{0 s}(s)=E_{0 s}^{s}(s)+E_{0 s}^{a}(s)$ it is not difficult to show that

$$
\begin{aligned}
& J(s)=J^{s}(s)+J^{a}(s)=\alpha \frac{i \omega}{k}\left\{\int_{-L}^{s} E_{0 s}\left(s^{\prime}\right) \sin \tilde{k}\left(s-s^{\prime}\right) \mathrm{d} s^{\prime}\right. \\
&-\frac{\sin \tilde{k}(L+s)+\alpha P^{s}[k r, \tilde{k}(L+s)]}{\sin 2 \tilde{k} L+\alpha P^{s}(k r, 2 \tilde{k} L)} \int_{-L}^{L} E_{0 s}^{s}\left(s^{\prime}\right) \sin \tilde{k}\left(L-s^{\prime}\right) \mathrm{d} s^{\prime} \\
&\left.-\frac{\sin \tilde{k}(L+s)+\alpha P^{a}[k r, \tilde{k}(L+s)]}{\sin 2 \tilde{k} L+\alpha P^{a}(k r, 2 \tilde{k} L)} \int_{-L}^{L} E_{0 s}^{a}\left(s^{\prime}\right) \sin \tilde{k}\left(L-s^{\prime}\right) \mathrm{d} s^{\prime}\right\},
\end{aligned}
$$

where $P^{s}$ and $P^{a}$ are the functions of vibrator self-fields equal to

$$
\begin{aligned}
& P^{s}[k r, \tilde{k}(L+s)]=\left.\int_{-L}^{s}\left[\frac{e^{-i k R\left(s^{\prime},-L\right)}}{R\left(s^{\prime},-L\right)}+\frac{e^{-i k R\left(s^{\prime}, L\right)}}{R\left(s^{\prime}, L\right)}\right] \sin \tilde{k}\left(s-s^{\prime}\right) \mathrm{d} s^{\prime}\right|_{s=L}=P^{s}(k r, 2 \tilde{k} L), \\
& P^{a}[k r, \tilde{k}(L+s)]=\left.\int_{-L}^{s}\left[\frac{e^{-i k R\left(s^{\prime},-L\right)}}{R\left(s^{\prime},-L\right)}-\frac{e^{-i k R\left(s^{\prime}, L\right)}}{R\left(s^{\prime}, L\right)}\right] \sin \tilde{k}\left(s-s^{\prime}\right) \mathrm{d} s^{\prime}\right|_{s=L}=P^{a}(k r, 2 \tilde{k} L) .
\end{aligned}
$$

It is evident that if an impedance vibrator is located in restricted volume $V$, the expression for the current coincides with (27), but the functions of vibrator self-field (28) must contain components of electric Green's function for corresponding electrodynamic volume. 
Let us consider a problem of vibrator excitation at its geometric center by a lumped EMF with amplitude $V_{0}$. The mathematical model of excitation is presented as

$$
E_{0 s}(s)=E_{0 s}^{s}(s)=V_{0} \delta(s-0)
$$

where $\delta(s-0)=\delta(s)$ is Dirac delta-function. Then the expression for the current (27) is

$$
J(s)=-\alpha V_{0}\left(\frac{i \omega}{2 \tilde{k}}\right) \frac{\sin \tilde{k}(L-|s|)+\alpha P_{\delta}^{s}(k r, \tilde{k} s)}{\cos \tilde{k} L+\alpha P_{L}^{s}(k r, \tilde{k} L)} .
$$

Here $P_{\delta}^{s}(k r, \tilde{k} s)=P^{s}[k r, \tilde{k}(L+s)]-(\sin \tilde{k} s+\sin \tilde{k}|s|) P_{L}^{s}(k r, \tilde{k} L)$ and $P^{s}[k r, \tilde{k}(L+s)]$ are defined by the formula (28a). Explicit expressions for $P_{\delta}^{s}(k r, \tilde{k} s)$ and $P_{L}^{s}(k r, \tilde{k} L)$ can be expressed explicitly in terms of generalized integral functions [4,5]. Thus, $P_{L}^{s}(k r, \tilde{k} L)$ which will be needed below may be presented as

$$
\begin{gathered}
P_{L}^{s}(k r, \tilde{k} L)=\cos \tilde{k} L\{2 \ln 2-\gamma(L)-(1 / 2)[\operatorname{Cin}(2 \tilde{k} L+2 k L)+\operatorname{Cin}(2 \tilde{k} L-2 k L)] \\
-(i / 2)[\operatorname{Si}(2 \tilde{k} L+2 k L)-\operatorname{Si}(2 \tilde{k} L-2 k L)]\} \\
+\sin \tilde{k} L\{(1 / 2)[\operatorname{Si}(2 \tilde{k} L+2 k L)+\operatorname{Si}(2 \tilde{k} L-2 k L)]-(i / 2)[\operatorname{Cin}(2 \tilde{k} L+2 k L)-\operatorname{Cin}(2 \tilde{k} L-2 k L)]\},
\end{gathered}
$$

where $\operatorname{Si}(x)$ and $\operatorname{Cin}(x)$ are sine and cosine integrals of complex argument.

Since the current distribution (30) is now known we can calculate electrodynamic characteristics of an impedance vibrator. Thus, an input impedance $Z_{i n}=R_{i n}+i X_{i n}$ of vibrator in a feed point is equal

$$
Z_{i n}[\mathrm{Ohm}]=\frac{V_{0}}{J(0)}=\left(\frac{60 i \tilde{k}}{\alpha k}\right) \frac{\cos \tilde{k} L+\alpha P_{L}^{s}(k r, \tilde{k} L)}{\sin \tilde{k} L+\alpha P_{\delta L}(k r, \tilde{k} L)},
$$

where

$$
\begin{aligned}
& P_{\delta L}(k r, \tilde{k} L)=\int_{-L}^{L} \frac{e^{-i k R(s, L)}}{R(s, L)} \sin \tilde{k}|s| \mathrm{d} s \\
& =\sin \tilde{k} L\{-\gamma(L)+(1 / 2)[\operatorname{Cin}(2 \tilde{k} L+2 k L)-\operatorname{Cin}(2 \tilde{k} L-2 k L)]-\operatorname{Cin}(\tilde{k} L+k L)+\operatorname{Cin}(\tilde{k} L-k L) \\
& +(i / 2)[\operatorname{Si}(2 \tilde{k} L+2 k L)-\operatorname{Si}(2 \tilde{k} L-2 k L)]-i[\operatorname{Si}(\tilde{k} L+k L)-\operatorname{Si}(\tilde{k} L-k L)]\} \\
& +\cos \tilde{k} L\{(1 / 2)[\operatorname{Si}(2 \tilde{k} L+2 k L)+\operatorname{Si}(2 \tilde{k} L-2 k L)]-\operatorname{Si}(\tilde{k} L+k L)-\operatorname{Si}(\tilde{k} L-k L) \\
& -(i / 2)[\operatorname{Cin}(2 \tilde{k} L+2 k L)+\operatorname{Cin}(2 \tilde{k} L-2 k L)]+i[\operatorname{Cin}(\tilde{k} L+k L)+\operatorname{Cin}(\tilde{k} L-k L)]\} .
\end{aligned}
$$


Note, that an input admittance $Y_{\text {in }}=G_{i n}+i B_{\text {in }}$ can be calculated as $Y_{\text {in }}=1 / Z_{\text {in }}$.

To confirm the validity of the above analytical formulas we present the results of a comparative analysis of calculated and experimental data available in the literature. Figure 2 and Figure 3 show the graphs of the input admittance for two realizations of surface impedance: 1) metal wire (radius $r_{i}=0.3175 \mathrm{~cm}$ ), covered by dielectric $(\varepsilon=9.0)$ shell (radius $r=0.635 \mathrm{~cm}$ ), the experimental data [21] at Figure 2 and 2$)$ metal wire $\left(r_{i}=0.5175 \mathrm{~cm}\right)$, covered with ferrite $(\mu=4.7)$ shell $(r=0.6 \mathrm{~cm})$, the experimental data from [22] at Figure 3. The plots show that trends of the theoretical curves coincide with that of the experimental curves, especially near the resonance for $B_{i n}=0$, though in absolute values some difference is observed. In our opinion, the discrepancy of theoretical curves, obtained by solving the integral equation for the current by averaging method, and the experimental curves may be caused by evident fact that vibrator self-field (19) was averaged and the current amplitude was determined with some error.
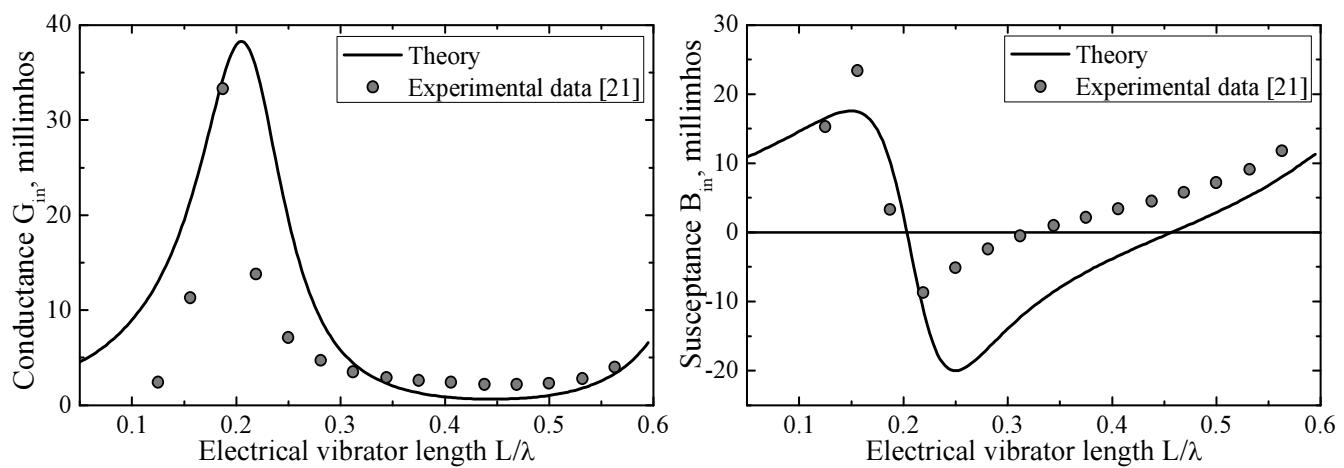

Figure 2. The input admittance of metal wire (radius $r_{i}=0.3175 \mathrm{~cm}$ ), covered by dielectric shell $(\varepsilon=9.0$, radius $r=0.635$ $\mathrm{cm}$ ) versus its electrical length at the frequency $f=600 \mathrm{MHz}$
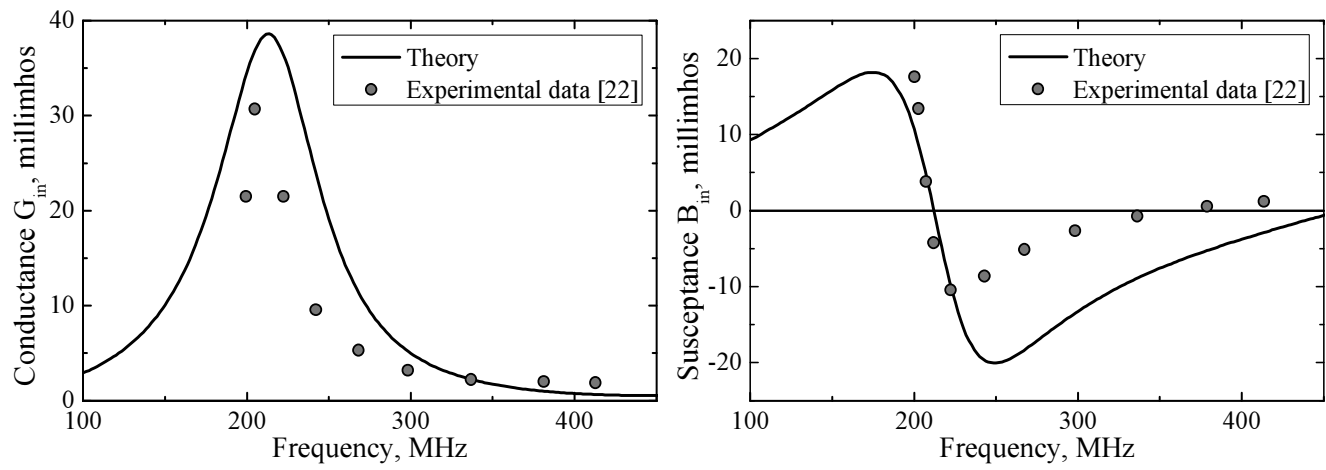

Figure 3. The input admittance of metal wire (radius $\left.r_{i}=5175 \mathrm{~cm}\right)$, covered with ferrite shell $(\mu=4.7, r=0.6 \mathrm{~cm})$ versus frequency for $L=30.0 \mathrm{~cm}$ 


\section{Solution of equation for current in a slot between two semi-infinite rectangular waveguides}

Now let us solve the second key problem. Let a resonant iris is placed in infinite hollow $\left(\varepsilon_{1}=\mu_{1}=\varepsilon_{2}=\mu_{2}=1\right)$ rectangular waveguide so that its slot has arbitrary orientation in the plane of waveguide cross-section and has no contacts with waveguide walls (Figure 4).

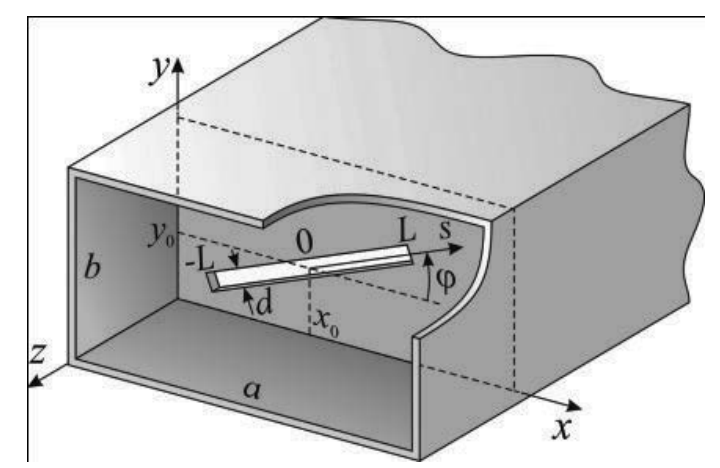

Figure 4. The problem geometry and notations

A starting point for the analysis is equation (10) written as (index sl is omitted)

$$
\left(\frac{\mathrm{d}^{2}}{\mathrm{~d} s^{2}}+k^{2}\right) \int_{-L}^{L} J\left(s^{\prime}\right) 4 \frac{e^{-i k R\left(s, s^{\prime}\right)}}{R\left(s, s^{\prime}\right)} \mathrm{d} s^{\prime}=-i \omega H_{0 s}(s)-\left(\frac{\mathrm{d}^{2}}{\mathrm{~d} s^{2}}+k^{2}\right) \int_{-L}^{L} J\left(s^{\prime}\right)\left[G_{0 s}^{V_{1}}\left(s, s^{\prime}\right)+G_{0 s}^{V_{2}}\left(s, s^{\prime}\right)\right] \mathrm{d} s^{\prime},
$$

where $4 \frac{e^{-i k R\left(s, s^{\prime}\right)}}{R\left(s, s^{\prime}\right)}$ is the Green's function of the slot in infinite perfectly conducting plane, $G_{0 s} V_{1,2}\left(s, s^{\prime}\right)$ are the Green's functions, which takes into account multiple reflection from walls of volumes.

Isolating the logarithmic singularity in the kernel of equation (34) as in (17), we reduce the equation (34) to an integral equation with small parameter

$$
\frac{\mathrm{d}^{2} J(s)}{\mathrm{d} s^{2}}+k^{2} J(s)=\alpha\left\{i \omega H_{0 s}(s)+F[s, J(s)]+F_{0}[s, J(s)]\right\} .
$$

Here $\alpha=1 / 8 \ln \left[d_{e} /(8 L)\right]$ is the natural small parameter of the problem $(|\alpha|<<1), d_{e}=d e^{-\frac{\pi h}{2 d}}$ is equivalent slot width which takes into account a real wall thickness $h(h / \lambda<<1)[3]$, 


$$
\begin{aligned}
F[s, J(s)]= & -4 \frac{\mathrm{d} J\left(s^{\prime}\right)}{\mathrm{d} s^{\prime}} \frac{e^{-i k R\left(s, s^{\prime}\right)}}{R\left(s, s^{\prime}\right)}+4\left[\frac{\mathrm{d}^{2} J(s)}{\mathrm{d} s^{2}}+k^{2} J(s)\right] \gamma(s) \\
& +4 \int_{-L}^{L}\left\{\frac{\left[\frac{\mathrm{d}^{2} J\left(s^{\prime}\right)}{\mathrm{d} s^{\prime 2}}+k^{2} J\left(s^{\prime}\right)\right] e^{-i k R\left(s, s^{\prime}\right)}-\left[\frac{\mathrm{d}^{2} J(s)}{\mathrm{d} s^{2}}+k^{2} J(s)\right]}{R\left(s, s^{\prime}\right)}\right\} d s^{\prime}
\end{aligned}
$$

is self-field of the slot in infinite perfectly conducting plane,

$$
F_{0}[s, J(s)]=-\left.\frac{\mathrm{d} J\left(s^{\prime}\right)}{\mathrm{d} s^{\prime}}\left[G_{0 s}^{V_{1}}\left(s, s^{\prime}\right)+G_{0 s}^{V_{2}}\left(s, s^{\prime}\right)\right]\right|_{-L} ^{L}+\int_{-L}^{L}\left[\frac{\mathrm{d}^{2} J\left(s^{\prime}\right)}{\mathrm{d} s^{\prime 2}}+k^{2} J\left(s^{\prime}\right)\right]\left[G_{0 s}^{V_{1}}\left(s, s^{\prime}\right)+G_{0 s}^{V_{2}}\left(s, s^{\prime}\right)\right] \mathrm{d} s^{\prime}
$$

is self-field of the slot, which takes into account multiple reflection from walls of volumes.

To solve the equation (35) by averaging method we change the variable according to (20) and obtain the standard system of integral equations relative to new unknown functions $A(s)$ and $B(s)$ which is equivalent to initial equation (35)

$$
\begin{aligned}
& \frac{\mathrm{d} A(s)}{\mathrm{d} s}=-\frac{\alpha}{k}\left\{i \omega H_{0 s}(s)+F_{N}\left[s, A(s), \frac{\mathrm{d} A(s)}{\mathrm{d} s}, B(s), \frac{\mathrm{d} B(s)}{\mathrm{d} s}\right]\right\} \sin k s, \\
& \frac{\mathrm{d} B(s)}{\mathrm{d} s}=+\frac{\alpha}{k}\left\{i \omega H_{0 s}(s)+F_{N}\left[s, A(s), \frac{\mathrm{d} A(s)}{\mathrm{d} s}, B(s), \frac{\mathrm{d} B(s)}{\mathrm{d} s}\right]\right\} \cos k s,
\end{aligned}
$$

where $F_{N}=F+F_{0}$ is the total self-field of the slot.

Assuming, as in Section $4, \frac{d A(s)}{d s}=0$ and $\frac{d B(s)}{d s}=0$ in the right-hand members of equations (38) and making partial averaging over the variable $s$, we derive the equations of the first approximation by averaging method

$$
\begin{aligned}
& \frac{\mathrm{d} \bar{A}(s)}{\mathrm{d} s}=-\alpha\left\{\frac{i \omega}{k} H_{0 s}(s)+\bar{F}_{N}[s, \bar{A}, \bar{B}]\right\} \sin k s, \\
& \frac{\mathrm{d} \bar{B}(s)}{\mathrm{d} s}=+\alpha\left\{\frac{i \omega}{k} H_{0 s}(s)+\bar{F}_{N}[s, \bar{A}, \bar{B}]\right\} \cos k s,
\end{aligned}
$$

where 


$$
\begin{aligned}
& \bar{F}_{N}[s, \bar{A}, \bar{B}]=\left.\left[\bar{A}\left(s^{\prime}\right) \sin k s^{\prime}-\bar{B}\left(s^{\prime}\right) \cos k s^{\prime}\right] G_{s}^{\Sigma}\left(s, s^{\prime}\right)\right|_{-L} ^{L}, \\
& G_{s}^{\Sigma}\left(s, s^{\prime}\right)=G_{s}^{V_{1}}\left(s, s^{\prime}\right)+G_{s}^{V_{2}}\left(s, s^{\prime}\right)
\end{aligned}
$$

is the slot total self-field, averaged over the slot length.

Solving the system (39), we obtain the general asymptotic expression for the current in narrow slot, located in arbitrary position relative to the walls of coupling volumes

$$
J(s)=\bar{A}(-L) \cos k s+\bar{B}(-L) \sin k s+\alpha \int_{-L}^{s}\left\{\frac{i \omega}{k} H_{0 s}\left(s^{\prime}\right)+\bar{F}_{N}\left[s^{\prime}, \bar{A}, \bar{B}\right]\right\} \sin k\left(s-s^{\prime}\right) \mathrm{d} s^{\prime}
$$

To determine constants $\bar{A}( \pm L)$ and $\bar{B}( \pm L)$ we will use the boundary conditions (7) and the symmetry conditions, uniquely related both to slot excitation method and its position in waveguide. Then, in terms of symmetric and antisymmetric magnetic current components, marked by indexes $s$ and $a$, respectively, for arbitrary slot excitation by $H_{0 s}(s)=H_{0 s}^{s}(s)+H_{0 s}^{a}(s)$ with an accuracy of order $\alpha^{2}$ we have

$$
\begin{aligned}
& J(s)=J^{s}(s)+J^{a}(s)=\alpha \frac{i \omega}{k}\left\{\int_{-L}^{s} H_{0 s}\left(s^{\prime}\right) \sin k\left(s-s^{\prime}\right) \mathrm{d} s^{\prime}\right. \\
&\left.-\frac{\sin k(L+s) \int_{-L}^{L} H_{0 s}^{s}\left(s^{\prime}\right) \sin k\left(L-s^{\prime}\right) \mathrm{d} s^{\prime}}{\sin 2 k L+\alpha N^{s}\left(k d_{e}, 2 k L\right)}-\frac{\sin k(L+s) \int_{-L}^{L} H_{0 s}^{a}\left(s^{\prime}\right) \sin k\left(L-s^{\prime}\right) \mathrm{d} s^{\prime}}{\sin 2 k L+\alpha N^{a}\left(k d_{e}, 2 k L\right)}\right\},
\end{aligned}
$$

where $N^{s}\left(k d_{e}, 2 k L\right)$ and $N^{a}\left(k d_{e}, 2 k L\right)$ are the functions of self-field which are equal

$$
\begin{aligned}
& N^{s}\left(k d_{e}, 2 k L\right)=\int_{-L}^{L}\left[G_{s}^{\Sigma}(s,-L)+G_{s}^{\Sigma}(s, L)\right] \sin k(L-s) \mathrm{d} s, \\
& N^{a}\left(k d_{e}, 2 k L\right)=\int_{-L}^{L}\left[G_{s}^{\Sigma}(s,-L)-G_{s}^{\Sigma}(s, L)\right] \sin k(L-s) \mathrm{d} s,
\end{aligned}
$$

which are completely defined by the Green's functions of the coupling volumes.

Supposing that dominant wave $H_{10}$ with amplitude $H_{0}$ is propagated from the region $z=-\infty$, we have

$$
H_{0 s}(s)=2 H_{0} \cos \varphi\left[\sin \frac{\pi x_{0}}{a} \cos \frac{\pi(s \cos \varphi)}{a}+\cos \frac{\pi x_{0}}{a} \sin \frac{\pi(s \cos \varphi)}{a}\right] .
$$


The symmetric and antisymmetric components of the slot current, relative to the slot center $s=0$, become equal

$$
\begin{aligned}
& J(s)=J_{0} f(s)=-\alpha 2 H_{0} \cos \varphi \frac{2 i \omega / k^{2}}{\left[1-\left(k_{\varphi} / k\right)^{2}\right]\left[\sin 2 k L+\alpha 2 W_{\varphi}^{s a}\left(k d_{e}, 2 k L\right)\right]} \\
& \times\left\{\sin \frac{\pi x_{0}}{a} \sin k L\left(\cos k s \cos k_{\varphi} L-\cos k L \cos k_{\varphi} s\right)+\cos \frac{\pi x_{0}}{a} \cos k L\left(\sin k \sin k_{\varphi} L-\sin k L \sin k_{\varphi} s\right)\right\},
\end{aligned}
$$

where $J_{0}$ is current amplitude, $f(s)$ is the current distribution function, $k_{\varphi}=\frac{\pi}{a} \cos \varphi$, $W_{\varphi}^{s a}\left(k d_{e}, 2 k L\right)$ is the function of slot self-field, defined by formulas (43).

Reflection and transmission coefficients, $S_{11}$ and $S_{12}$ for the dominant wave in the slot iris are define by the current as

$$
\begin{aligned}
& S_{11}=\left(1+S_{12}\right) e^{2 i \gamma z}, S_{12}=-\alpha \frac{16 \pi k_{g} \cos ^{2} \varphi f\left(k_{\varphi} L\right)}{i a b k^{3}\left[1-\left(k_{\varphi} / k\right)^{2}\right]\left[\cos k L+\alpha 2 W_{\varphi}\left(k d_{e}, k L\right)\right]}, \\
& f\left(k_{\varphi} L\right)=2 \cos k_{\varphi} L \frac{\sin k L \cos k_{\varphi} L-\left(k_{\varphi} / k\right) \cos k L \sin k_{\varphi} L}{1-\left(k_{\varphi} / k\right)^{2}}-\cos k L \frac{\sin 2 k_{\varphi} L+2 k_{\varphi} L}{2\left(k_{\varphi} / k\right)},
\end{aligned}
$$

where $k_{g}=\sqrt{k^{2}-(\pi / a)^{2}}$ is the propagation constant of $H_{10}$ wave.

Figure 5 shows the theoretical and experimental wavelength dependences of power reflection coefficient $\left|S_{11}\right|^{2}$ for the iris, which oriented so that the angle between slot axis $\{0 s\}$ and waveguide axis $\{0 x\}$ are $0^{\circ}$ and $30^{\circ}$.

Note that a comparative analysis of the analytical solution of key problems is not limited only by the examples presented above. Thus, the solution for current in the impedance vibrator, located in free space, was preliminary compared with the known approximate analytical solutions of integral equations. The adequacy of the constructed mathematical models to real physical processes and the reliability of simulation results has been also confirmed by comparative calculations, obtained by the numerical method of moments and other methods, in particular, by the finite element method implemented in the software package Ansoft HFSS.

\section{Combined vibrator-slot structures}

Now let us consider a problem of electromagnetic waves excitation by a narrow straight transverse slot in the broad wall of rectangular waveguide with a two passive impedance vibrators in it. 

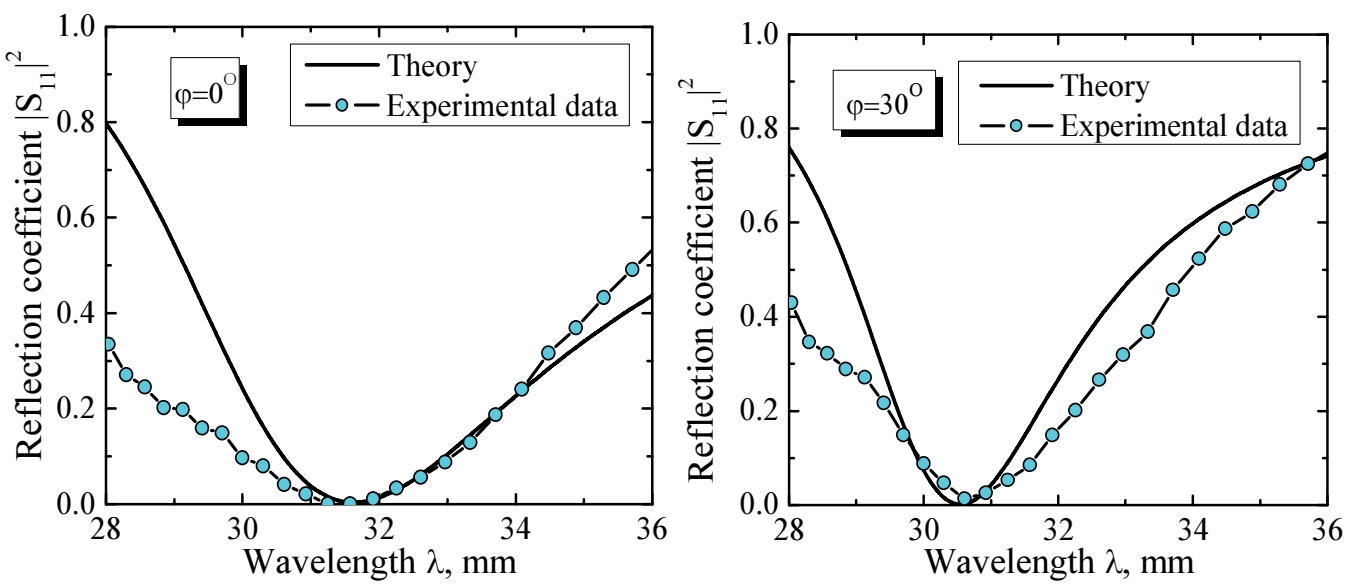

Figure 5. Power reflection coefficient $\left|S_{11}\right|^{2}$ versus wavelength for the iris at $a=23.0 \mathrm{~mm}, b=10.0 \mathrm{~mm}, 2 L=16.0$ $\mathrm{mm}, d=1.5 \mathrm{~mm}, h=2.0 \mathrm{~mm}, x_{0}=a / 2, y_{0}=b / 2$

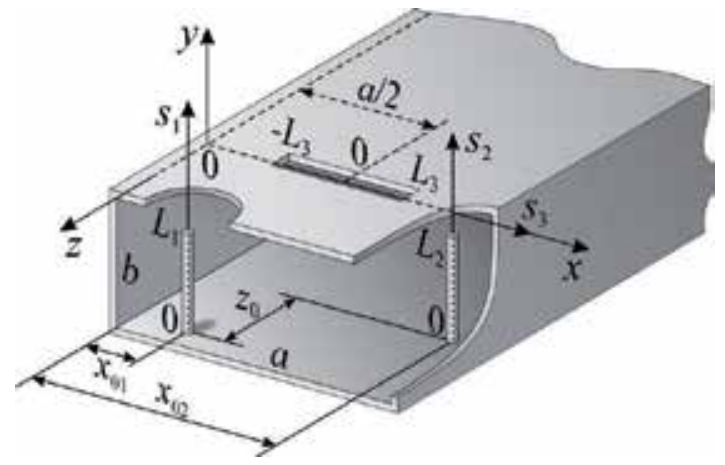

Figure 6. The geometry of three-element vibrator-slot system and notations

Let a fundamental wave $H_{10}$ propagates from the area $z=-\infty$ in a hollow infinite rectangular waveguide, the area index is " $W g^{\prime}$ ". Two thin nonsymmetrical vibrators (monopoles) with variable surface impedance are located in a waveguide with cross-section $\{a \times b\}$. A narrow transverse slot cut in a broad wall of the waveguide symmetrically relative to its longitudinal axis is radiating into free half-space, the area index is " $\mathrm{Hs}^{\prime}$ ". The vibrators radiuses and lengths are $r_{1,2}$ and $L_{1,2}\left(\left(r_{1,2} / L_{1,2}\right)<<1\right)$, the slot width is $d$, the slot length is $2 L_{3}\left(\left(d / L_{3}\right)<<1\right)$ and the waveguide wall thickness is $h$. One vibrator is located in the plane $\{x 0 y\}$ and the second vibrator may be shifted along the axis $\{0 z\}$ at the distance $z_{0}$ (Figure 6).

For this configuration the system of integral equations relative to electrical currents at the vibrators $J_{1,2}\left(s_{1,2}\right)$ and equivalent magnetic current in the slot $J_{3}\left(s_{3}\right)$ in accordance with (8) may be represented as 


$$
\begin{aligned}
& \left(\frac{\mathrm{d}^{2}}{\mathrm{~d} s_{1}^{2}}+k^{2}\right)\left\{\int_{-L_{1}}^{L_{1}} J_{1}\left(s_{1}^{\prime}\right) G_{s_{1}}^{W g}\left(s_{1}, s_{1}^{\prime}\right) \mathrm{d} s_{1}^{\prime}+\int_{-L_{2}}^{L_{2}} J_{2}\left(s_{2}^{\prime}\right) G_{s_{2}}^{W g}\left(s_{1}, s_{2}^{\prime}\right) \mathrm{d} s_{2}^{\prime}\right\}- \\
& -i k \int_{-L_{3}}^{L_{3}} J_{3}\left(s_{3}^{\prime}\right) \tilde{G}_{s_{3}}^{W g}\left(s_{1}, s_{3}^{\prime}\right) \mathrm{d} s_{3}^{\prime} \\
& =-i \omega\left[E_{0 s_{1}}\left(s_{1}\right)-z_{i 1}\left(s_{1}\right) J_{1}\left(s_{1}\right)\right], \\
& \left(\frac{\mathrm{d}^{2}}{\mathrm{~d} s_{2}^{2}}+k^{2}\right)\left\{\int_{-L_{2}}^{L_{2}} J_{2}\left(s_{2}^{\prime}\right) G_{s_{2}}^{W g}\left(s_{2}, s_{2}^{\prime}\right) \mathrm{d} s_{2}^{\prime}+\int_{-L_{1}}^{L_{1}} J_{1}\left(s_{1}^{\prime}\right) G_{s_{1}}^{W g}\left(s_{2}, s_{1}^{\prime}\right) \mathrm{d} s_{1}^{\prime}\right\}= \\
& =-i \omega\left[E_{0 s_{2}}\left(s_{2}\right)-z_{i 2}\left(s_{2}\right) J_{2}\left(s_{2}\right)\right], \\
& \left(\frac{\mathrm{d}^{2}}{\mathrm{~d} s_{3}^{2}}+k_{1}^{2}\right) \int_{-L_{3}}^{L_{3}} J_{3}\left(s_{3}^{\prime}\right)\left[G_{s_{3}}^{W g}\left(s_{3}, s_{3}^{\prime}\right)+G_{s_{3}}^{H s}\left(s_{3}, s_{3}^{\prime}\right)\right] \mathrm{d} s_{3}^{\prime}- \\
& -i k \int_{-L_{1}}^{L_{1}} J_{1}\left(s_{1}^{\prime}\right) \tilde{G}_{s_{1}}^{W g}\left(s_{3}, s_{1}^{\prime}\right) \mathrm{d} s_{1}^{\prime}=-i \omega H_{0 s_{3}}\left(s_{3}\right) .
\end{aligned}
$$

Here $G_{s_{1,2}}^{W g}\left(s_{1,2}, s_{1,2}^{\prime}\right)$ and $G_{s_{3}}^{W g}, H_{s}\left(s_{3}, s^{\prime}\right)$ are components of the Green's functions of the rectangular waveguide and the half-space over the plane $[3,4]$, $\widetilde{G}_{s_{1}}^{W g}\left(s_{3}, s^{\prime}{ }_{1}\right)=\frac{\partial}{\partial z} G_{s_{1}}^{W g}\left[x\left(s_{3}\right), 0, z ; x^{\prime}\left(s_{1}^{\prime}\right), y^{\prime}\left(s_{1}^{\prime}\right), z_{0}\right]$

and $\widetilde{G}_{s_{3}}^{W g}\left(s_{1}, s_{3}^{\prime}\right)=\frac{\partial}{\partial z} G_{s_{3}}^{W g}\left[x\left(s_{1}\right), y\left(s_{1}\right), z ; x^{\prime}\left(s_{3}^{\prime}\right), 0,0\right]$ after substitution $z=0$ into $\widetilde{G}_{s_{1}}^{W g}$ and $z=z_{0}$ into $\widetilde{G}_{s_{3}}^{W g}$ after first derivation, $z_{i 1,2}\left(s_{1,2}\right)$ is the internal impedance per unit length of the vibrators ([Ohm/m]), $E_{0 s_{1,2}}\left(s_{1,2}\right)$ and $H_{0 s_{3}}\left(s_{3}\right)$ are projections of impressed sources fields on the vibrators and the slot axes, $s_{1}=-L_{1}$ and $s_{2}=-L_{2}$ are end coordinates of mirror vibrator images relative to the lower broad wall of the waveguide [4]

We will seek the solution of equations system (47) by a generalized method of induced EMMF $[19,20]$, using functions $J_{1(2)}\left(s_{1(2)}\right)=J_{1(2)}^{0} f_{1(2)}\left(s_{1(2)}\right)$ and $J_{3}\left(s_{3}\right)=J_{3}^{0} f_{3}\left(s_{3}\right)$ as approximating expressions for the currents. Here $J_{1(2)}^{0}$ and $J_{3}^{0}$ are unknown current amplitudes, $f_{1(2)}\left(s_{1(2)}\right)$ and $f_{3}\left(s_{3}\right)$ are predetermined functions of the current distributions. In accordance with (27) and (42) for the vibrator-slot structure excited by the fundamental wave $H_{10}$ we have $f_{1(2)}\left(s_{1(2)}\right)=\cos \tilde{k}_{1(2)} s_{1(2)}-\cos \tilde{k}_{1(2)} L_{1(2)}, \quad f_{3}\left(s_{3}\right)=\cos k s_{3}-\cos k L_{3^{\prime}} \quad \tilde{k}_{1(2)}=k-\frac{i 2 \pi z_{i 1(2)}^{a v}}{Z_{0} \Omega_{1(2)}}$, $z_{i 1(2)}^{a v}=\frac{1}{L_{1(2)}} \int_{0}^{L_{1(2)}} z_{i 1(2)}\left(s_{1(2)}\right) d s_{1(2)}$ are average values [4] of internal impedances, $\Omega_{1(2)}=2 \ln \left(2 L_{1(2)} / r_{1(2)}\right)$.

In accordance with the generalized method of induced EMMF, we multiply equation (47a) by the function $f_{1}\left(s_{1}\right)$, equation (47b) by the function $f_{2}\left(s_{2}\right)$, and the equation $(47 \mathrm{c})$ by the function 
$f_{3}\left(s_{3}\right)$ and integrate the equations (47a) and (47b) over the length of the vibrators, and the equation (47c) over the length of the slot. As a result, we obtain a system of linear algebraic equations relative to the current amplitudes $J_{1,2,3}^{0}$

$$
\begin{aligned}
& J_{1}^{0} Z_{11}^{\Sigma}+J_{2}^{0} Z_{12}+J_{3}^{0} Z_{13}=-\frac{i \omega}{2 k} \int_{-L_{1}}^{L_{1}} f_{1}\left(s_{1}\right) E_{0 s_{1}}\left(s_{1}\right) d s_{1} \\
& J_{2}^{0} Z_{22}^{\Sigma}+J_{1}^{0} Z_{21}=-\frac{i \omega}{2 k} \int_{-L_{2}}^{L_{2}} f_{2}\left(s_{2}\right) E_{0 s_{2}}\left(s_{2}\right) d s_{2} \\
& J_{3}^{0} Z_{33}^{\Sigma}+J_{1}^{0} Z_{31}=-\frac{i \omega}{2 k} \int_{-L_{3}}^{L_{3}} f_{3}\left(s_{3}\right) H_{0 s_{3}}\left(s_{3}\right) d s_{3} .
\end{aligned}
$$

Here

$$
\begin{aligned}
& Z_{11(22)}=\frac{4 \pi}{a b} \sum_{m=1}^{\infty} \sum_{n=0}^{\infty}\left\{\frac{\varepsilon_{n}\left(k^{2}-k_{y}^{2}\right) \tilde{k}_{1(2)}^{2}}{k k_{z}\left(\tilde{k}_{1(2)}^{2}-k_{y}^{2}\right)^{2}} e^{-k_{z} r_{1(2)}} \sin ^{2} k_{x} x_{01(02)}\right. \\
& \left.\times\left[\sin \tilde{k}_{1(2)} L_{1(2)} \cos k_{y} L_{1(2)}-\frac{\tilde{k}_{1(2)}}{k_{y}} \cos \tilde{k}_{1(2)} L_{1(2)} \sin k_{y} L_{1(2)}\right]^{2}\right\}, \\
& F_{1(2)}^{z}=-\frac{i}{r_{1(2)}} \int_{0}^{L_{1(2)}} f_{1(2)}^{2}\left(s_{1(2)}\right) \bar{Z}_{S 1(2)}\left(s_{1(2)}\right) \mathrm{d} s_{1(2)^{\prime}} \\
& Z_{12}=Z_{21}=\frac{4 \pi}{a b} \sum_{m=1}^{\infty} \sum_{n=0}^{\infty}\left\{\frac{\varepsilon_{n}\left(k^{2}-k_{y}^{2}\right) \tilde{k}_{1} \tilde{k}_{2} e^{-k_{z} z_{0}}}{k k_{z}\left(\tilde{k}_{1}^{2}-k_{y}^{2}\right)\left(\tilde{k}_{2}^{2}-k_{y}^{2}\right)} \sin k_{x} x_{01}\right. \\
& \times \sin k_{x} x_{02}\left[\sin \tilde{k}_{1} L_{1} \cos k_{y} L_{1}-\left(\tilde{k}_{1} / k_{y}\right) \cos \tilde{k}_{1} L_{1} \sin k_{y} L_{1}\right] \\
& \left.\times\left[\sin \tilde{k}_{2} L_{2} \cos k_{y} L_{2}-\left(\tilde{k}_{2} / k_{y}\right) \cos \tilde{k}_{2} L_{2} \sin k_{y} L_{2}\right]\right\}, \\
& Z_{33}^{H s}=\operatorname{Si} 4 k L_{3}-i \operatorname{Cin} 4 k L_{3}-2 \cos k L_{3}\left[2\left(\sin k L_{3}-k L_{3} \cos k L_{3}\right)\right. \\
& \left.\times\left(\ln \frac{16 L_{3}}{d_{e}}-\operatorname{Cin} 2 k L_{3}-i \operatorname{Si} 2 k L_{3}\right)+\sin 2 k L_{3} e^{-i k L_{3}}\right] \text {, } \\
& Z_{33}^{W_{g}}=\frac{8 \pi}{a b} \sum_{m=1,3 \ldots n=0,1 . .}^{\infty}\left\{\frac{\varepsilon_{n} k}{k_{z}\left(k^{2}-k_{x}^{2}\right)} e^{-k_{z} \frac{d_{e}}{4}}\left[\sin k L_{3} \cos k_{x} L_{3}-\left(k / k_{x}\right) \cos k L_{3} \sin k_{x} L_{3}\right]^{2}\right\},
\end{aligned}
$$




$$
\begin{aligned}
& Z_{13}=-Z_{31}= \\
& =\frac{4 \pi}{a b} \sum_{m=1}^{\infty} \sum_{n=0}^{\infty}\left\{\frac{\varepsilon_{n} k \tilde{k}_{1} e^{-k_{z} z_{0}}}{i\left(\tilde{k}_{1}^{2}-k_{y}^{2}\right)\left(k^{2}-k_{x}^{2}\right)} \sin k_{x} x_{01} \sin \frac{k_{x} a}{2}\left[\sin \tilde{k}_{1} L_{1} \cos k_{y} L_{1}-\frac{\tilde{k}_{1}}{k_{y}} \cos \tilde{k}_{1} L_{1} \sin k_{y} L_{1}\right]\right. \\
& \left.\times\left[\sin k L_{3} \cos k_{x} L_{3}-\frac{k}{k_{x}} \cos k L_{3} \sin k_{x} L_{3}\right]\right\}, \\
& Z_{11(22)}^{\Sigma}=Z_{11(22)}+F_{1(2)}^{z}, Z_{33}^{\Sigma}=Z_{33}^{H s}+Z_{33}^{W g},
\end{aligned}
$$

where $\varepsilon_{n}=\left\{\begin{array}{ll}1, & n=0 \\ 2, & n \neq 0\end{array}, k_{x(y)}=\frac{m(n) \pi}{a(b)}, k_{z}=\sqrt{k_{x}^{2}+k_{y}^{2}-k^{2}}, m, n\right.$ are integers; Si and Cin are integral sine and cosine.

The energy characteristics of the vibrator-slot system: the reflection and transmission coefficients, $S_{11}$ and $S_{12}$, and power radiation coefficient $\left|S_{\Sigma}\right|^{2}$, are defined by the expressions

$$
\begin{gathered}
S_{11}=\frac{4 \pi i}{a b k k_{g}}\left\{J_{3} \frac{2 k_{g}^{2}}{k^{2}} f\left(k L_{3}\right)-J_{1} \frac{k_{g}}{\tilde{k}_{1}} \sin \left(\frac{\pi x_{01}}{a}\right) f\left(\tilde{k}_{1} L_{1}\right) e^{-i k_{g} z_{0}}-J_{2} \frac{k_{g}}{\tilde{k}_{2}} \sin \left(\frac{\pi x_{02}}{a}\right) f\left(\tilde{k}_{2} L_{2}\right)\right\} e^{2 i k_{g} z}, \\
S_{12}=1+\frac{4 \pi i}{a b k k_{g}}\left\{J_{3} \frac{2 k_{g}^{2}}{k^{2}} f\left(k L_{3}\right)+J_{1} \frac{k_{g}}{\tilde{k}_{1}} \sin \left(\frac{\pi x_{01}}{a}\right) f\left(\tilde{k}_{1} L_{1}\right) e^{i k_{g} z_{0}}+J_{2} \frac{k_{g}}{\tilde{k}_{2}} \sin \left(\frac{\pi x_{02}}{a}\right) f\left(\tilde{k}_{2} L_{2}\right)\right\}, \\
\left|S_{\Sigma}\right|^{2}=1-\left|S_{11}\right|^{2}-\left|S_{12}\right|^{2}
\end{gathered}
$$

In expressions (55)-(57)

$$
\begin{aligned}
& J_{1}=1 /\left(Z_{11}^{\Sigma} Z_{22}^{\Sigma} Z_{33}^{\Sigma}-Z_{21} Z_{12} Z_{33}^{\Sigma}-Z_{31} Z_{13} Z_{22}^{\Sigma}\right) \\
& \times\left[\frac{k^{2}}{k_{g} \tilde{k}_{1}} \sin \frac{\pi x_{01}}{a} f_{1}\left(\tilde{k}_{1} L_{1}\right) e^{-i k_{g} z_{0}} Z_{22}^{\Sigma} Z_{33}^{\Sigma}-\frac{k^{2}}{k_{g} \tilde{k}_{2}} \sin \frac{\pi x_{02}}{a} f_{2}\left(\tilde{k}_{2} L_{2}\right) Z_{12} Z_{33}^{\Sigma}-f_{3}\left(k L_{3}\right) Z_{13} Z_{22}^{\Sigma}\right], \\
& J_{2}=1 /\left(Z_{11}^{\Sigma} Z_{22}^{\Sigma} Z_{33}^{\Sigma}-Z_{21} Z_{12} Z_{33}^{\Sigma}-Z_{31} Z_{13} Z_{22}^{\Sigma}\right) \\
& \times\left[\frac{k^{2}}{k_{g} \tilde{k}_{2}} \sin \frac{\pi x_{02}}{a} f_{2}\left(\tilde{k}_{2} L_{2}\right)\left(Z_{11}^{\Sigma} Z_{33}^{\Sigma}-Z_{31} Z_{13}\right)-\frac{k^{2}}{k_{g} \tilde{k}_{1}} \sin \frac{\pi x_{01}}{a} f_{1}\left(\tilde{k}_{1} L_{1}\right) e^{-i k_{g} z_{0}} Z_{21} Z_{33}^{\Sigma}+f_{3}\left(k L_{3}\right) Z_{13} Z_{21}\right],
\end{aligned}
$$




$$
\begin{gathered}
J_{3}=1 /\left(Z_{11}^{\Sigma} Z_{22}^{\Sigma} Z_{33}^{\Sigma}-Z_{21} Z_{12} Z_{33}^{\Sigma}-Z_{31} Z_{13} Z_{22}^{\Sigma}\right) \\
\times\left[f_{3}\left(k L_{3}\right)\left(Z_{11}^{\Sigma} Z_{22}^{\Sigma}-Z_{21} Z_{12}\right)+\frac{k^{2}}{k_{g} \tilde{k}_{2}} \sin \frac{\pi x_{02}}{a} f_{2}\left(\tilde{k}_{2} L_{2}\right) Z_{12} Z_{31}-\frac{k^{2}}{k_{g} \tilde{k}_{1}} \sin \frac{\pi x_{01}}{a} f_{1}\left(\tilde{k}_{1} L_{1}\right) e^{-i k_{g} z_{0}} Z_{31} Z_{22}^{\Sigma}\right] \\
f_{1(2)}\left(\tilde{k}_{1(2)} L_{1(2)}\right)=\sin \tilde{k}_{1(2)} L_{1(2)}-\tilde{k}_{1(2)} L_{1(2)} \cos \tilde{k}_{1(2)} L_{1(2)^{\prime}} \\
f_{3}\left(k L_{3}\right)=\frac{\sin k L_{3} \cos \left(\pi L_{3} / a\right)-(k a / \pi) \cos k L_{3} \sin \left(\pi L_{3} / a\right)}{1-[\pi /(k a)]^{2}}
\end{gathered}
$$

Let us consider several distribution functions for the surface impedance along the vibrator, namely: 1) $\phi_{0}\left(s_{1(2)}\right)=1$, the constant distribution, 2) $\phi_{1}\left(s_{1(2)}\right)=2\left[1-\left(s_{1(2)} / L_{1(2)}\right)\right]$, the triangular distribution linear decreasing to the vibrator end, and 3) $\phi_{2}\left(s_{1(2)}\right)=2\left(s_{1(2)} / L_{1(2)}\right)$, the triangular linear increasing distribution. All distribution have equal average values $\overline{\phi_{0,1,2}\left(s_{1(2)}\right)}=1$. The expression for $F_{1(2)}^{z 0}$ with the distribution function 1), in accordance with (50), can be presented as

$$
\begin{aligned}
& F_{1(2)}^{z 0}=-\frac{2 i\left(\bar{R}_{S 1(2)}+i \bar{X}_{S 1(2)}\right)}{\tilde{k}_{1(2)}^{2} L_{1(2)} r_{1(2)}}\left[\left(\frac{\tilde{k}_{1(2)} L_{1(2)}}{2}\right)^{2}\left(2+\cos 2 \tilde{k}_{1(2)} L_{1(2)}\right)-\frac{3}{8} \tilde{k}_{1(2)} L_{1(2)} \sin 2 \tilde{k}_{1(2)} L_{1(2)}\right] \\
& =\tilde{F}_{1(2)}^{z}\left(\bar{R}_{S 1(2)}+i \bar{X}_{S 1(2)}\right) \Phi_{1(2)}
\end{aligned}
$$

with the distribution function 2) as

$$
\begin{aligned}
& F_{1(2)}^{z 1}=\tilde{F}_{1(2)}^{z} \\
& \times\left\{\bar{R}_{S 1(2)} \Phi_{1(2)}+i \bar{X}_{S 1(2)}\left[\left(\frac{\tilde{k}_{1(2)} L_{1(2)}}{2}\right)^{2}\left(2+\cos 2 \tilde{k}_{1(2)} L_{1(2)}\right)-\frac{7}{4} \sin ^{2} \tilde{k}_{1(2)} L_{1(2)}-2\left(\cos \tilde{k}_{1(2)} L_{1(2)}-1\right)\right]\right\}
\end{aligned}
$$

and with the distribution function 3) as

$$
\begin{aligned}
& F_{1(2)}^{z 2}=\tilde{F}_{1(2)}^{z}\left\{\bar{R}_{S 1(2)} \Phi_{1(2)}\right. \\
& +i \bar{X}_{S 1(2)}\left[\left(\frac{\tilde{k}_{1(2)} L_{1(2)}}{2}\right)^{2}\left(2+\cos 2 \tilde{k}_{1(2)} L_{1(2)}\right)+\frac{7}{4} \sin ^{2} \tilde{k}_{1(2)} L_{1(2)}\right. \\
& \left.\left.-\frac{3}{4} \tilde{k}_{1(2)} L_{1(2)} \sin 2 \tilde{k}_{1(2)} L_{1(2)}+2\left(\cos \tilde{k}_{1(2)} L_{1(2)}-1\right)\right]\right\}
\end{aligned}
$$


Since the formulas for $F_{1(2)}^{z 0,1,2}$ differ from one another, in spite of equal average values of functions $\phi_{0,1,2}\left(s_{1(2)}\right)$ and identical functional dependences in formulas for currents, the current amplitudes and, hence, energy characteristics will be substantially different.

Figures 7, 8 shows the wavelength dependences of the radiation coefficient, modules of the reflection and transmission coefficients in the wavelength range of the waveguide single-mode regime, obtained using the following common parameters: $a=58.0 \mathrm{~mm}, b=25.0 \mathrm{~mm}, h=0.5 \mathrm{~mm}$, $r_{1,2}=2.0 \mathrm{~mm}, L_{1,2}=15.0 \mathrm{~mm}, \bar{R}_{S 1(2)}=0, x_{01}=a / 8, x_{02}=7 a / 8, d=4.0 \mathrm{~mm}$ and $2 L_{3}=40.0 \mathrm{~mm}$.
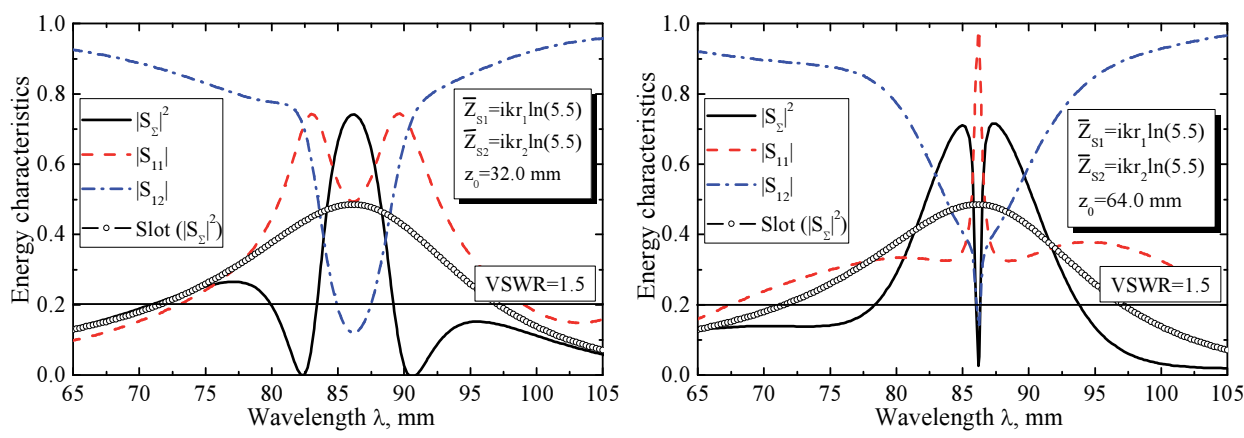

Figure 7. The energy characteristics versus wavelength at $\lambda_{v 1,2}^{r e s}=\lambda_{s l}^{r e s}, \bar{Z}_{S 1}=\bar{Z}_{S 2}$

The choice of slot dimensions was stipulated by its natural resonance at the average wavelength of the waveguide frequency range $\lambda_{3}^{\text {res }}=86.0 \mathrm{~mm}$. The dimensions of the vibrators have been selected so that their resonant wavelength was within the waveguide operating range. Here we present the results only for vibrators with inductive impedances $\left(\bar{X}_{S 1(2)}>0\right)$, known to increase the vibrator electrical length, i.e. to increase $\lambda_{1,2}^{\text {res }}$ as compared to case $\bar{Z}_{S 1(2)}=0$, without decreasing a distance between the vibrators ends and the upper broad wall of the waveguide. This is very important for increasing the breakdown power for waveguide device as a whole.

As might be expected from physical considerations, displacement of the impedance vibrator along the longitudinal axis of the waveguide at a distance $z_{0}$ from the centre of the slot, where the maximum mutual influence between elements of the structure is observed, are multiple of $\lambda_{G} / 4$ (Fig. 7: $z_{0}=\lambda_{G} / 4=32.0 \mathrm{~mm}$ and $z_{0}=\lambda_{G} / 2=64.0 \mathrm{~mm}$ ). Here $\lambda_{G}=2 \pi / \sqrt{\left(2 \pi / \lambda_{\text {sl }}^{\text {res }}\right)^{2}-(\pi / a)^{2}}$ is resonant wavelength of the slot in the waveguide, and $\lambda_{s l}^{\text {res }}$ is the resonant wavelength of the slot in the free half-space over the plane. As seen from Figure 7, an acceptable reflection coefficient $\left|S_{11}\right|$ and high level of radiation could not be achieved if the monopoles have the equal distributed impedances $\bar{Z}_{S 1}=\bar{Z}_{S 2}$. The maximum of radiation coefficient $\left|S_{\Sigma}\right|^{2}$ and almost perfect agreement with the feed line, as well as tuning to other resonant wavelengths can be achieved by changing the distribution functions of impedance along the monopoles axes (Figure 8). Fig. 8 also shows that the results of mathematical modeling are confirmed by the experimental data. Experimental models have been made in the form of corrugated brass rods (see photo in Figure 8). 

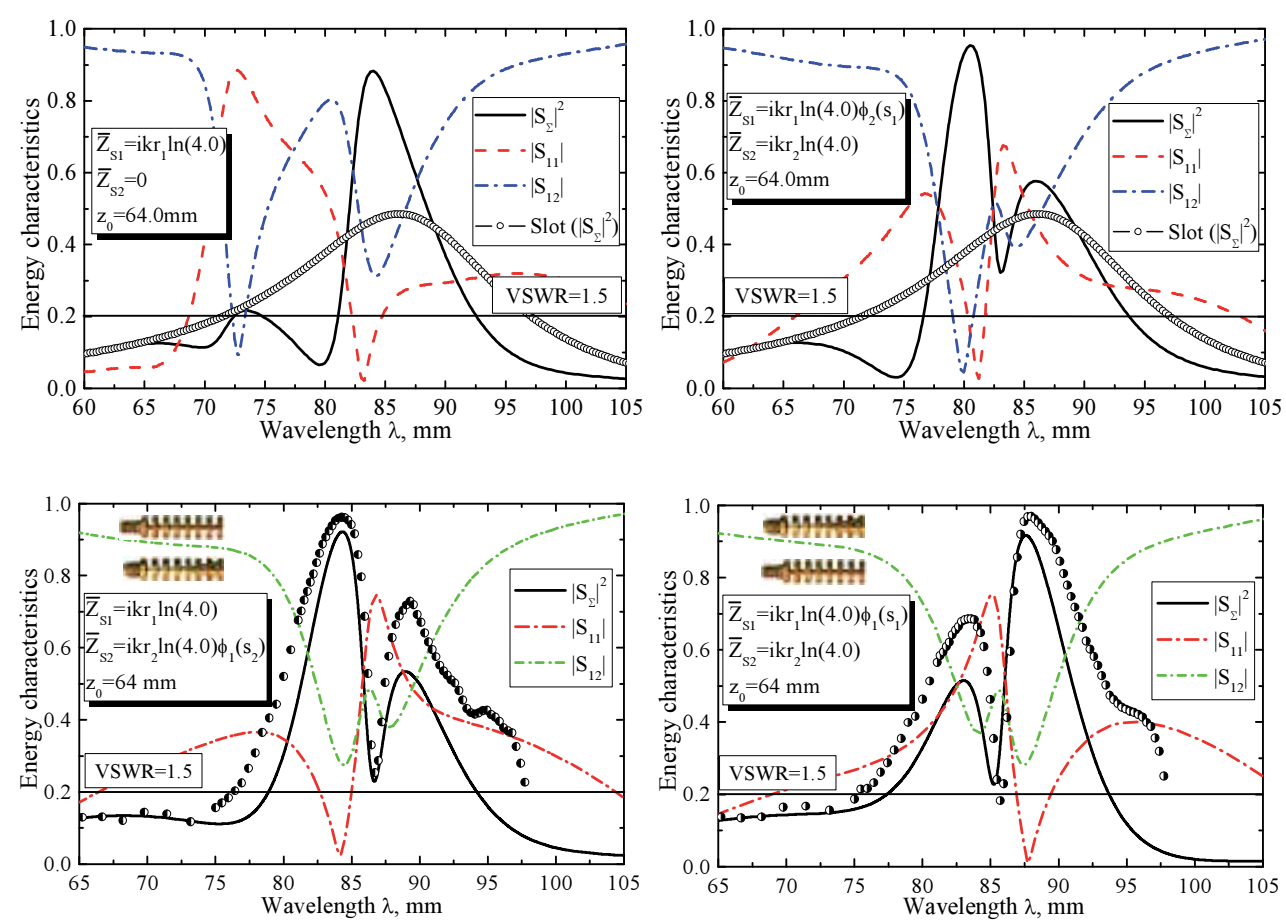

Figure 8. The energy characteristics versus wavelength at $\lambda_{v 1,2}^{r e s} \neq \lambda_{s l}^{r e s}, \bar{Z}_{S 1} \neq \bar{Z}_{S 2}$, experimental data are marked by circles

For the arbitrary vibrator-slot structures and coupled electrodynamic volumes expressions for $f_{v}^{s, a}\left(s_{v}\right)$ and $f_{s l}^{s, a}\left(s_{s l}\right)$ (the subscripts $s, a$ denote the symmetric and antisymmetric components of the currents with respect to the vibrator $\left(s_{v}=0\right)$ and slot $\left(s_{s l}=0\right)$ centers, respectively), in accordance with the results, presented in Sections 4 and 5 (see formulas (27) and (42)), can be obtained from the following relations

$$
\begin{gathered}
f_{v}^{s, a}\left(s_{v}\right) \sim\left\{\begin{array}{l}
\sin \tilde{k}\left(L_{v}-s_{v}\right) \int_{-L_{v}}^{s_{v}} E_{0 s_{v}}^{s, a}\left(s_{v}^{\prime}\right) \sin \tilde{k}\left(L_{v}+s_{v}^{\prime}\right) \mathrm{d} s_{v}^{\prime} \\
+\sin \tilde{k}\left(L_{v}+s_{v}\right) \int_{s_{v}}^{L_{v}} E_{0 s_{v}}^{s, a}\left(s_{v}^{\prime}\right) \sin \tilde{k}\left(L_{v}-s_{v}^{\prime}\right) \mathrm{d} s_{v}^{\prime}
\end{array}\right\}, \\
f_{s l}^{s, a}\left(s_{s l}\right) \sim\left\{\begin{array}{l}
\sin k\left(L_{s l}-s_{s l}\right) \int_{-L_{s l}}^{s_{s l}} H_{0 s_{s l}}^{s, a}\left(s_{s l}^{\prime}\right) \sin k\left(L_{s l}+s_{s l}^{\prime}\right) \mathrm{d} s_{s l}^{\prime} \\
+\sin k\left(L_{s l}+s_{s l}\right) \int_{s_{s l}}^{L_{s l}} H_{0 s_{s l}}^{s, a}\left(s_{s l}^{\prime}\right) \sin k\left(L_{s l}-s_{s l}^{\prime}\right) \mathrm{d} s_{s l}^{\prime}
\end{array}\right\},
\end{gathered}
$$


where $E_{0 s_{v}}^{s, a}\left(s_{v}\right)$ and $H_{0 s_{s l}}^{s, a}\left(s_{s l}\right)$ are projections of symmetric and antisymmetric components of impressed sources on the vibrator and the slot axes. Here the sign $\sim$ means that after integration in expressions (61) only multipliers, depending upon coordinates $s_{v}$ and $s_{s l}$, are left.

Note once more that for arbitrary orientations of the vibrator, or the slot relative to the waveguide walls, or for another impressed field sources, the expressions (61) should be used to determine the distribution functions of electric and magnetic currents in the vibrator and slot. For example, for the longitudinal slot in the broad wall of waveguide, i.e. if axes $\left\{0 s_{s l}\right\}$ and $\{0 z\}$ coincide, we obtain

$$
\begin{aligned}
& f_{s l}^{s}\left(s_{s l}\right)=\cos k s_{s l} \cos k_{g} L_{s l}-\cos k L_{s l} \cos k_{g} s_{s l}, \\
& f_{s l}^{a}\left(s_{s l}\right)=\sin k s_{s l} \sin k_{g} L_{s l}-\sin k L_{s l} \sin k_{g} s_{s l} .
\end{aligned}
$$

If vibrator is excited at its base by voltage $\delta$-generator as in a waveguide-to-coaxial adapter we have

$$
f_{v}\left(s_{v}\right)=\sin \tilde{k}\left(L_{v}-s_{v}\right) .
$$

\section{Conclusion}

This chapter presents the methodological basis for application of the generalized method of induced EMMF for the analysis of electrodynamic characteristics of the combined vibratorslot structures. Characteristic feature of the generalization to a new class of approximating functions consists in using them as a function of the current distributions along the impedance vibrator and slot elements; these distributions are derived as the asymptotic solution of integral equations for the current (key problems) by the method of averaging. Comparison of theoretical and experimental curves indicates that the solution of integral equations for combined vibrator-slot structures by the generalized method of induced EMMF with approximating functions for the currents in the impedance vibrator and the slot, obtained by averaging method is quite legitimate. It should be noted that for simple structures similar to that considered in the model problem, the proposed approach yields an analytic solution of the electrodynamic problem. For more complex structures, the method may be used to design effective numerical-analytical algorithms for their analyses.

The demonstrative simulation (the comparative analysis of all electrodynamic characteristics in the operating frequencies range) has confirmed the validity of the proposed generalized method of induced EMMF for analysis of vibrator-slot systems with rather arbitrary structure (within accepted assumptions). Here, as examples, some fragments of this comparative analysis were presented. This method retains all benefits of analytical methods as compared with direct numerical methods and allows to expand significantly the boundaries of numerical 
and analytical studies of practically important problems, concerning the application of single impedance vibrator, including irregular vibrator, the systems of such vibrators and narrow slots. And this is a natural step in the further development of the general fundamental theory of linear radiators of electric and magnetic types.

\section{Author details}

Mikhail V. Nesterenko*, Sergey L. Berdnik, Victor A. Katrich and Yuriy M. Penkin

*Address all correspondence to: mikhail.v.nesterenko@gmail.com

V.N. Karazin Kharkov National University, Kharkov, Ukraine

\section{References}

[1] Elliott R.S. Antenna Theory and Design. Hoboken: John Wiley \& Sons; 2003.

[2] Weiner M.M. Monopole Antennas. New York: Marcel Dekker; 2003.

[3] Nesterenko M.V., Katrich V.A., Penkin Yu.M, Berdnik S.L. Analytical and Hybrid Methods in Theory of Slot-Hole Coupling of Electrodynamic Volumes. New York: Springer Science + Business Media; 2008.

[4] Nesterenko M.V., Katrich V.A., Penkin Yu.M., Dakhov V.M., Berdnik S.L. Thin Impedance Vibrators. Theory and Applications. New York: Springer Science + Business Media; 2011.

[5] King R.W.P. The Theory of Linear Antennas. Cambr.-Mass.: Harvard University Press; 1956.

[6] Nesterenko M.V., Katrich V.A. The asymptotic solution of an integral equation for magnetic current in a problem of waveguides coupling through narrow slots. Progress In Electromagnetics Research 2006;57 101-129.

[7] Nesterenko M.V. Analytical methods in the theory of thin impedance vibrators. Progress In Electromagnetics Research B 2010;21 299-328.

[8] Nesterenko M.V., Katrich V.A., Penkin Yu.M., Berdnik S.L. Analytical methods in theory of slot-hole coupling of electrodynamics volumes. Progress In Electromagnetics Research 2007;70 79-174.

[9] Nesterenko M.V., Katrich V.A., Dakhov V.M., Berdnik S.L. Impedance vibrator with arbitrary point of excitation. Progress In Electromagnetics Research B 2008;5 275-290. 
[10] Nesterenko M.V., Katrich V.A., Berdnik S.L., Penkin Yu.M., Dakhov V.M. Application of the generalized method of induced EMF for investigation of characteristics of thin impedance vibrators. Progress In Electromagnetics Research B 2010;26 149-178.

[11] Harrington R.F. Field Computation by Moment Methods, New York: IEEE Press ; 1993.

[12] Butler C.M., Umashankar K.R. Electromagnetic excitation of a wire through an aperture-perforated conducting screen. IEEE Trans. Antennas and Propagat. 1976;24 456-462.

[13] Itoh K., Matsumoto T. Theoretical analysis of mutual coupling between slot and unipole antennas. IEICE Trans. Commun. 1978;J61-B 391-397.

[14] Harrington R.F. Resonant behavior of a small aperture backed by a conducting body. IEEE Trans. Antennas and Propagat. 1982;30 205-212.

[15] Naiheng Y., Harrington R.F. Electromagnetic coupling to an infinite wire through a slot in a conducting plane. IEEE Trans. Antennas and Propagat. 1983;31 310-316.

[16] His S.W., Harrington R.F., Mautz J.R. Electromagnetic coupling to a conducting wire behind an aperture of arbitrary size and shape. IEEE Trans. Antennas and Propagat. 1985;33 581-587.

[17] Park S., Hirokawa J., Ando M. Simple analysis of a slot and a reflection-canceling post in a rectangular waveguide using only the axial uniform currents on the post surface. IEICE Trans. Commun. 2003;E86-B 2482-2487.

[18] Kim K.-C., Lim S.M., Kim M.S. Reduction of electromagnetic penetration through narrow slots in conducting screen by two parallel wires. IEICE Trans. Commun. 2005;E88-B 1743-1745.

[19] Nesterenko M.V., Katrich V.A., Penkin Yu.M., Berdnik S.L., Kijko V.I. Combined vibrator-slot structures in electrodynamic volumes. Progress In Electromagnetics Research B 2012;37 237-256.

[20] Nesterenko M.V., Katrich V.A., Penkin D.Yu., Berdnik S.L., Kijko V.I. Electromagnetic waves scattering and radiation by vibrator-slot structure in a rectangular waveguide. Progress In Electromagnetics Research M. 2012;24 69-84.

[21] Lamensdorf D. An experimental investigation of dielectric-coated antennas. EEE Trans. Antennas and Propagat. 1967;15 767-771.

[22] Bretones A.R., Martín R.G., García I.S. Time-domain analysis of magnetic-coated wire antennas. IEEE Trans. Antennas and Propagat. 1995;43 591-596. 

Chapter 7

\title{
Rigorous Approach to Analysis of Two-Dimensional Potential Problems, Wave Propagation and Scattering for Multi-conductor Systems
}

\author{
Galyna Safonova and Elena Vinogradova \\ Additional information is available at the end of the chapter
}

http://dx.doi.org/10.5772/61287

\begin{abstract}
The research described in this chapter analyses two-dimensional potential problems for the multi-body systems, transverse electromagnetic wave propagation along multi-conductor transmission lines and two-dimensional plane wave scattering by various arrays. All conductors may be of arbitrary cross-sections; the only restriction on the system geometry is a smooth parameterization. These problems are mathematically modelled by Dirichlet boundary value problems for either the Laplace or the Helmholtz equation, with the classical integral representation of the solutions in the form of single-layer potential. The analytical-numerical algorithm presented here is based on the method of analytical regularization. The key idea behind this technique is an analytical transformation of the initial ill-posed integral equations to a well-conditioned Fredholm second kind matrix equation. The resulting system of infinite linear algebraic equations is effectively solved using the truncation method: the solution of the truncated system converges to the solution of the infinite system with the guaranteed accuracy that only depends on the truncation number and thus may be pre-specified. The solution obtained is applied to the accurate analysis of 2-D electrostatic- and electrodynamic-field problems for multi-conductor systems with arbitrary profiled conductors. Examples of some conceptual shielded transmission lines incorporating various configurations of conductors and scattering problems for the arrays of thick strips establish the utility of our method and its reliability in various situations
\end{abstract}

Keywords: Scattering, propagation, analytical regularization, Laplace equation, Helmholtz equation 


\section{Introduction}

In electrostatic and electromagnetic studies of highly elongated cylinders, and ensembles or arrays of such cylinders, it is well-known [1] that the most important effects can be treated by replacing the three-dimensional structure by the corresponding cross-sectional two-dimensional (2D) profile. Such cylinders are described by $a<<L$, where $L$ is the length of the cylinder, and $a$ is a parameter characterizing its cross-section. This condition ensures that for real objects such as charged conducting cylinders, the field induced by their ends will have no impact on electric field distribution well away from the ends, or this impact will be vanishingly small as $L \rightarrow \infty$. Such idealization in 2D electrostatics causes nonphysical solutions: the arbitrary profiled charged cylinder, for instance, generates a potential that logarithmically grows instead of vanishing as expected at distant observation points. This means that the potential is unbounded at infinity. The nonphysical nature of the solution is commented by many authors. However, for systems of charged conductors this difficulty can be avoided in one of two ways: by setting the net charge of the system to be equal to zero or by introducing earthed infinite planes or closed earthed shields. This issue is discussed in [2]. In spite of the seeming limitations that the above conditions enforce on 2D electrostatic modelling, they naturally occur in almost all problems arising in practice. In particular, 2D boundary value problems for the Laplace and Helmholtz equation describe many problems of practical interest arising aero- and hydrodynamics (potential fluid flow), electrostatics, electromagnetic scattering studies, acoustics, elasticity theory, etc.

The long-standing interest in the investigation of the electrostatic field in periodic structures continues because of numerous applications. One example is the analysis of the propagation of the transverse electromagnetic (TEM) wave in open and shielded multi-conductor transmission lines [3,4]. When the contour of a conductor coincides with the coordinate surface of one of the coordinate systems in which the Laplace's equation is separable, the Fourier method (method of separation of variables) is used. More generally, a variety of potential problems have been solved by the conformal mapping method. These results are described in many classical handbooks and monographs. The number of such solved problems is highly restricted. Nowadays, the need for simulation of devices used in practice requires development of more universal methods to tackle problems with objects of various finite-width shapes. One of such numerous examples is the capacitance calculation for thick electrodes [5] where a physically reasonable meaning of 'edge capacitance' arises only because an accurate charge distribution over the whole electrode could not be accurately calculated. Though solutions obtained for single objects may adequately describe the real situation, most practical problems deal with a finite number of objects (say, conductors). Even when a conductor is of canonical shape (circular or elliptic cylinder), the solution of an electrostatic multi-conductor problem for an assembly of cylinders of different radii is a very bulky and lengthy procedure. Solving this problem as a classical boundary value problem for Laplace's equation and enforcing the pre-assigned boundary conditions at the surface of each conductor, it is necessary to make multiple re-expansions of the eigen functions of the Laplace's equation in each local coordinate system in terms of that chosen to satisfy the boundary conditions. 
Electromagnetic and acoustic problems described by the Helmholtz equation can also be considered in two dimensions. A great variety of publications consider the problem involving infinite gratings. They are often used in antenna applications as polarizers and filters. In [6], a vector diffraction formulation for the analysis of perfectly conducting gratings of finite width and thickness is presented. The grating is assumed to have a finite number of infinitely long arbitrarily shaped rods, and is illuminated by an arbitrary plane wave. Electric and magnetic field integral equations are used to numerically solve the corresponding TM and TE electromagnetic problems. Periodic structures in the millimetre wave range are considered in [7]: this paper studies single and double periodic devices using a semi-analytical mode-matching technique. Diffraction of the TM-polarized Gaussian beam by $N$ equally spaced slits (finite grating in a planar perfectly conducting thick screen) is investigated in [8]. Numerous publications consider the different types of arrays. A general approach was presented in [9] for solving the 2D scattering of a plane wave by an arbitrary configuration of perfectly conducting circular cylinders in front of a plane surface with general reflection properties. Acoustic scattering by a cluster of small sound-soft obstacles was considered in [10]. The 2D scattering of a Gaussian beam by a periodic array of circular cylinders is studied in [11]. A study of the electromagnetic scattering from multi-layered periodic arrays of parallel circular cylinders is presented in [12]. The electromagnetic scattering by multiple perfectly conducting arbitrary polygonal cylinders is analysed in [13].

It should be noted that the long history of solving the Laplace and Helmholtz equations is marked by the development of many numerical methods which are useful in simulation of practical devices. Such methods include the finite difference technique, extrapolation [14], point-matching method [15], boundary element method [16], spectral-space domain method [17], finite element method [18-20], transverse modal analysis [21] and mode-matching method [22]. A numerical integral equation approach is used in [23] to explore plane-wave scattering from a nonplanar surface with a sinusoidal height profile for the case of the magnetic field parallel to the surface ridges (TM polarization). In spite of effectiveness of these methods in many cases and flexibility in geometrical representation of the structures, modelling of ridges still have some substantial drawbacks. Most of the methods require large resources in terms of computational time and storage. Often the solutions obtained with such purely numerical methods need to be verified through comparison to other results: accuracy generally cannot be guaranteed for a greater number of iterations or larger-scale computations. This problem becomes pronounced in some topologically complex configurations. In electromagnetics, the corresponding class of numerical solutions is applicable in the low to intermediate frequency range. Resonant systems behaviour cannot be reliably analysed with the numerical techniques (see [24]). Analytical-numerical methods such as those based on the method of analytical regularization (MAR) are designed to overcome these drawbacks in the resonant regime. A comparative analysis of the MAR and other methods was conducted in [25], and in [26] the distinctive features of each of the discussed methods are clearly described. The abovementioned methods are mostly suited for analysis of a single or very few conductors. In the case of a significant number of conductors with individual profiles, the effectiveness of such purely numerical methods is highly problematic because of the rapidly growing scale of computations. 
In order to address these difficulties, we present here a semi-analytical approach to the analysis of 2D electrostatic and electrodynamic field problems for multi-conductor systems. The problems to be solved are treated as the classical Dirichlet boundary value problems for the Laplace and Helmholtz equations. It is well-known $[27,28]$ that solutions to the Laplace and Helmholtz equations can be represented as a single-layer potential at points exterior to the body of a single conductor with contour $S$ is given by

$$
U(q)=\int_{S} G(p, q) Z(p) d S_{p}
$$

where $Z(p)$ is related to the linear charge distribution on the contour $S$ in the case of the Laplace equation, and to the linear current density in case of the Helmholtz equation. $G(p, q)=-\frac{1}{2 \pi} \log |p-q|$ is the Green's function for Laplace's equation in 2D space. If the contour $S$ is charged to some prescribed potential value $V_{0}$, then $Z(p)$ may be found by solving the equation:

$$
-\frac{1}{2 \pi} \int_{s} \log |p-q| Z(p) d S_{p}=V_{0}, \quad q \in S_{p}
$$

This equation may be classified as a first kind Fredholm equation with a singular kernel; it is ill-posed [29]. Nevertheless, this problem has been tackled by many authors who used direct numerical schemes for solving its discrete analogue in a form of a first kind algebraic equation. Theoretically, any numerical method applied to solve this equation is unable to guarantee uniform convergence, or pre-determined computational accuracy.

The only way to avoid these shortcomings is to transform the initial equation into a second kind Fredholm equation, discretization of which guarantees uniform convergence and any pre-determined accuracy of the numerical solution depending on truncation number. We employ the MAR, in particular, described in [30,31]. An accurate solution to wave scattering by a single infinitely long cylinder of arbitrary cross-section by the MAR was obtained in [32]. The details of the algorithm for cylinders of closed arbitrary profile are presented in [26, 31]. In this chapter, we generalize the MAR for a multi-conductor potential problem where each body is an arbitrary profiled cylinder.

\section{Regularization of the electrostatic problem: MAR}

\subsection{Problem statement}

Consider (N-1) arbitrary profiled charged perfect electric conductor (PEC) cylinders embedded into a homogeneous dielectric medium with relative permittivity $\varepsilon_{r}$ (Figure 1). The finite dielectric medium is bounded by the infinitesimally thin, grounded cylindrical shell. 


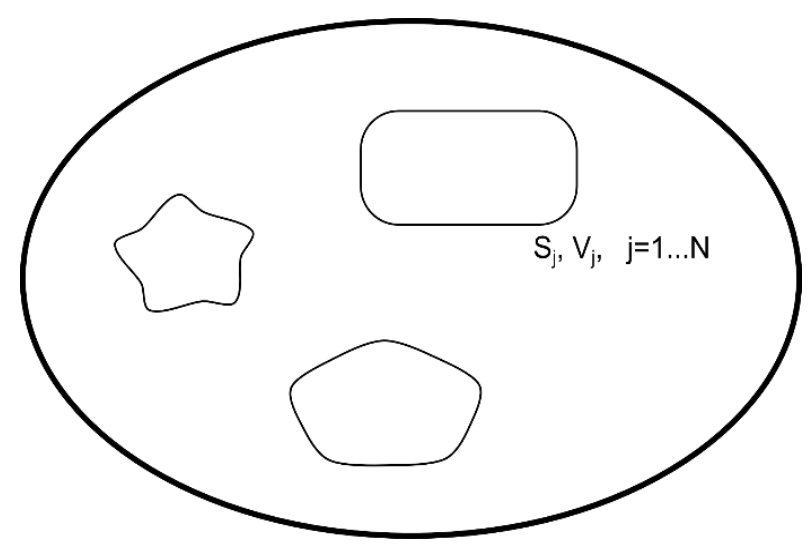

Figure 1. Problem geometry.

The problem is to find electrostatic potential $U$ elsewhere inside the shielded region. This electrostatic problem is fully described by the Dirichlet boundary value problem for the Laplace equation:

$$
\Delta U=0
$$

with boundary conditions of the potentials $V_{n}$ given at the surface of each of $N$ cylinders:

$$
\left.U\right|_{S_{n}}=V_{n}, n=1, \ldots, N-1 ;\left.U\right|_{S_{N}}=V_{N}=0
$$

To employ the regularization procedure, all contours $S_{n}$ must be smooth enough and non-self crossing to provide their continuous parameterization and twice differentiation at each point of $S_{n}$.

\subsection{Problem solution}

The main challenge of this problem is that all the conductors are arbitrary-shaped and the classical separation of variables method is not applicable here. We use a more general approach based on an integral representation. Using the superposition principle, we seek the solution for the total field potential $U$ as the sum of the single-layer potentials contributed by each cylinder:

$$
U(q)=\sum_{j=1}^{N} \int_{S_{j}} G(|p-q|) Z_{j}(p) d S_{p}
$$


where $Z_{j}$ is the unknown line charge density of the $j$ th conductor scaled by $4 \pi / \varepsilon, S_{j}$ is the boundary contour of the $j$ th conductor and points $q$ lie in the area between the contours. The Kernel $G$ of the integral equation (5) is the 2D free space Green's function:

$$
G=-\frac{1}{2 \pi} \log (|p-q|)
$$

where $|p-q|$ is the distance between points $q$ and $p$.

Applying boundary conditions to (5), one can arrive at the coupled system of integral equations for the unknowns $Z_{j}$ :

$$
-\sum_{j=1}^{N} \int_{S_{j}} G_{i j}(|p-q|) Z_{j}(p) d l_{p}=V_{n}, i=1, \ldots, N
$$

Equation (7) represents a system of first kind Fredholm integral equations that is generally illposed.

The contours of the conductors' cross-sections should be smooth. Thus for the analysis of the rectangular and square conductors, corners should be smoothed. The two most common types of parameterization are by angle and by arc length. Here, we use parameterization by angle. After parameterization of the contours $\eta(\theta) \equiv(x(\theta), y(\theta))$ and introducing some new notations:

$$
\begin{aligned}
& z_{j}=l_{j}(\theta) Z_{j}\left(\eta_{j}(\theta)\right), l(\theta)=\left\{\left[x^{\prime}(\theta)\right]^{2}+\left[y^{\prime}(\theta)\right]^{2}\right\}^{1 / 2}, \\
& R_{s j}(\theta, \tau)=|p-q|=\left|\eta_{s}(\theta)-\eta_{j}(\tau)\right|=\left\{\left[x_{s}(\theta)-x_{j}(\tau)\right]^{2}+\left[y_{s}(\theta)-y_{j}(\tau)\right]^{2}\right\}^{1 / 2},
\end{aligned}
$$

we obtain the system of $N$ integral equations:

$$
-\sum_{j=1}^{N} \int_{-\pi}^{\pi} G\left(R_{s j}(\theta, \tau)\right) z_{j}(\tau) d \tau=V_{s}(\theta), s=1,2 \ldots N
$$

The described approach permits us to consider a broader set of possible boundary conditions than simply a constant, though in the application to be described, a constant is deployed on the RHS of (9).

For the kernels $G\left(R_{s j}(\theta, \tau)\right)$ such that $s \neq j$, points corresponding to $\theta$ and $\tau$ belong to different contours and so $R_{s j}(\theta, \tau) \neq 0$ everywhere; hence, the corresponding integral terms do not contain singularities. For $G_{s S}(\theta, \tau)$ the corresponding integral contains a singularity of loga- 
rithmic type at the points $\theta=\tau$. In this case, we analytically separate the Green's function into the singular part and a remainder $L^{s j}$ that does not contain any singularity:

$$
\begin{aligned}
& -2 \pi G\left(R_{s j}(\theta, \tau)\right)=\log \left(R_{s j}(\theta, \tau)\right)=L^{s j}(\theta, \tau), \quad s \neq j, \\
& -2 \pi G\left(R_{s j}(\theta, \tau)\right)=\log \left(R_{s j}(\theta, \tau)\right)=L^{s j}(\theta, \tau)+\log \left(2 \sin \left|\frac{\theta-\tau}{2}\right|\right), \quad s=j .
\end{aligned}
$$

Now we can determine $L{ }^{s j}$ from (10) as follows:

$$
\begin{aligned}
& L^{s j}(\theta, \tau)=\log \left(R_{s j}(\theta, \tau)\right)-\log \left(2 \sin \left|\frac{\theta-\tau}{2}\right|\right), \quad s=j ; \\
& L^{s j}(\theta, \tau)=\log \left(R_{s j}(\theta, \tau)\right), \quad s \neq j .
\end{aligned}
$$

The function $L{ }^{s j}, s=j$ is a regular function, defined everywhere except at points $\theta=\tau$; the function $L^{s j}, s \neq j$ is defined everywhere. It can be shown that for the Laplace's equation this regular function has the same degree of smoothness as the contour parameterization. An exact expression for $L{ }^{s j}, s=j$ at the points of singularity where $\theta=\tau$ was obtained analytically:

$$
L^{s j}(\theta, \tau)=\log (l(\theta))
$$

where $l(\theta)=\sqrt{x(\theta)^{2}+y(\theta)^{2}}$ is an arc length in the point $\theta$.

Now we can redefine function $L^{s j}, s=j$ everywhere by the formula:

$$
L^{s j}=\left\{\begin{array}{l}
\log \left(R_{s j}(\theta, \tau)\right)-\log \left(2 \sin \left|\frac{\theta-\tau}{2}\right|\right), \theta \neq \tau, \\
\log (l(\theta)), \theta=\tau .
\end{array}\right.
$$

Using the well-known Fourier expansion, we can formulate an expression for the singular part of the Green's function:

$$
\log \left(2 \sin \left|\frac{\theta-\tau}{2}\right|\right)=\frac{1}{2} \sum_{n=-\infty}^{\infty} \frac{e^{i n(\theta-\tau)}}{|n|}
$$


As the function $L^{s j}$ is regular, we can expand it into double Fourier series:

$$
L^{s j}(\theta, \tau)=\sum_{n=-\infty}^{\infty} \sum_{m=-\infty}^{\infty} l_{n m}^{s j} e^{i(n \theta+m \tau)}
$$

Also the unknown function $z_{j}$ and the given potential function are represented by their Fourier series:

$$
z_{j}(\tau)=\sum_{-\infty}^{\infty} \xi_{n}^{j} e^{i n \tau}, \quad V_{j}(\theta)=\sum_{-\infty}^{\infty} v_{n}^{j} e^{i n \theta}
$$

After substitution of all expansions into (9), one can arrive at the system of $N$ integral equations:

$$
\sum_{n=-\infty}^{\infty} \frac{\xi_{n}^{s}}{|n|} e^{s n \theta}-2 \sum_{j=1}^{N} \sum_{n=-\infty}^{\infty} e^{i n \theta}\left(\sum_{m=-\infty}^{\infty} l_{n,-m}^{s j} \xi_{m}^{j}\right)=\sum_{n=-\infty}^{\infty} v_{n}^{j} e^{s n \theta}, \theta \in[-\pi, \pi], s=1,2, \ldots, N
$$

Using orthogonal properties and completeness of the functions $\left\{e^{i n \varphi}\right\}_{n=-\infty}^{n=\infty}$ and defining the rescaled unknown Fourier coefficients of charge density function $\xi_{n}^{s}$ as follows: $\xi_{n}^{s}=\frac{\xi_{n}^{s}}{\sigma_{n}}$, $\sigma_{n}=|n|^{1 / 2}$ when $n \neq 0$ and $\sigma_{0}=1$, we obtain the following infinite system of linear algebraic equations:

$$
\tilde{\xi}_{n}^{s}\left(1-\delta_{n 0}\right)+\sum_{j=1}^{N} \sum_{m=-\infty}^{\infty} \sigma_{n} \sigma_{m}^{l s j} n_{n,-m}^{s} \tilde{\xi}_{m}^{j}=\sigma_{n} v_{n}^{s}, n=0, \pm 1, \pm 2 \ldots ; s=1,2, \ldots, N .
$$

Following the steps suggested in [33], it can be shown that coefficient matrix in (18) is square summable:

$$
\sum_{j=1}^{N} \sum_{m=-\infty}^{\infty}\left|\sigma_{n} \sigma_{m} l_{n,-m}^{s j}\right|^{2}<\infty, n=0, \pm 1, \pm 2 \ldots ; s=1,2, \ldots, N
$$

Thus the infinite system (18) is of a second Fredholm kind and can now be effectively solved by a truncation method. The solution of the truncated system monotonically and rapidly converges to the exact solution. The above solution automatically incorporates the reciprocal influence of all charged cylinders, allowing accurate calculation of the line charge densities on the boundaries and then the field potentials at any point of the space between the conductors. Fourier expansions in (18) are calculated numerically as all functions are regular. 


\section{Regularization of the Dirichlet problem for Helmholtz equation: MAR technique for the $\mathrm{N}$-body multiple scattering}

\subsection{Statement of the problem}

In this section, we consider the scattering problem for the structure which consists of $N$ arbitrary profiled perfect electric -conductor cylinders embedded into a homogeneous dielectric medium with relative permittivity $\varepsilon$. The main steps of the solution algorithm are similar to those which were carried out to obtain the solution to the Laplace equation, presented in Section 2.2.

The scattered electromagnetic field $U^{s}$ obeys the Helmholtz equation:

$$
\left(\Delta+k^{2}\right) U^{s}(p)=0
$$

where point $p$ lies exterior to the structure $S, k=2 \pi / \lambda$ is the wave number and $\lambda$ is the corresponding wave length.

Here we consider incident fields in the form of a plane wave. We focus on a transverse magnetic (TM) wave polarization of the incident field $\left(U^{0}\right)$, therefore the scattered field should satisfy the Dirichlet boundary condition on metallic surfaces:

$$
U^{s}(p)=-U^{0}(p), \quad p \in S
$$

The field should also satisfy the Sommerfeld radiation condition:

$$
U^{s}(p)=O\left(|p|^{-1 / 2}\right) ; \frac{\partial U^{s}(p)}{\partial|p|}-i k U^{s}(p)=O\left(|p|^{-1 / 2}\right)
$$

As $|p| \rightarrow \infty$ where $|p|$ is the radial component of the point $p$ in the arbitrary fixed polar coordinate system.

\subsection{Regularized solution}

Solutions to the Laplace equation can be represented as a single-layer potential at points exterior to the body. Using the superposition principle, we seek the solution as the sum of single-layer potentials contributed by each cylinder:

$$
U(q)=\sum_{n=1}^{N} \int_{L_{n}} G\left(\left|p_{n}-q\right|\right) Z_{n}\left(p_{n}\right) d l_{p_{n}}
$$


Here $G\left(\left|p_{n}-q\right|\right)$ is the relevant free space Green's function depending on the distance $\left|p_{n}-q\right|$ between the observation point $q$ and point $p_{n}$ lying on the contour $S_{n}$ : $G(|p-q|)=-\frac{i}{4} H_{0}^{(1)}(k|p-q|)$, and the function $Z(p)$ is related to the linear current density $J(p)$ as $J(p)=i k c Z / 4 \pi$ (where $k$ is a wave number, $c$ the light speed).

Applying boundary conditions to (23), we obtain the system of $N$ integral equations:

$$
\sum_{j=1}^{N} \int_{L_{n}} G\left(\left|p_{n}-q\right|\right) Z_{n}\left(p_{n}\right) d l_{p_{n}}=-U_{m}^{0}(q), \quad q \in S_{m}, m=1, \ldots, N
$$

After parameterization of the contours $\eta_{n}(\theta)=\left(x_{n}(\theta), y_{n}(\theta)\right)$ where $\theta \in[-\pi, \pi]$, we use the definition of a line integral and obtain a functional equation in the form:

$$
\sum_{j=1}^{N} \int_{-\pi}^{\pi} G\left(R_{m n}(\theta, \tau)\right) z_{n}(\tau) d \tau=-U^{0}(\theta)
$$

The following notation is used in (25): $z_{n}(\tau)=l_{n}(\tau) Z_{n}\left(p_{n}(\tau)\right)$, where $l_{n}=\sqrt{\left(x_{n}(\tau)^{\prime}\right)^{2}+\left(y_{n}(\tau)^{\prime}\right)^{2}}, n=1, \ldots, N ; \quad R_{m n}(\theta, \tau)$ is the distance between points $\eta_{m}(\theta)$ and $\eta_{n}(\tau)$ lying on the $m$-th and $n$-th contours, respectively.

The kernel of the integral equation (25) contains a singularity only in the terms $G\left(R_{m n}(\theta, \tau)\right)$. It is of logarithmic type at the points $\theta=\tau$, and we analytically split the Green's function into a singular and a regular part $H^{m n}(\theta, \tau)$ similarly to the solution steps for the Laplace equation in Section 2.2:

$$
G\left(R_{m n}(\theta, \tau)\right)=-\frac{i}{4} H_{0}^{(1)}\left(k R_{m n}(\theta, \tau)\right)= \begin{cases}\frac{1}{2 \pi}\left(\log \left(2 \sin \left|\frac{\theta-\tau}{2}\right|\right)+H^{m m}(\theta, \tau)\right), & m=n \\ \frac{1}{2 \pi} H^{m n}(\theta, \tau), & m \neq n\end{cases}
$$

so that the regular part of Green's function is

$$
H^{m n}(\theta, \tau)= \begin{cases}-\frac{i \pi}{2} H_{0}^{(1)}\left(k R_{m m}\right)-\log \left(2 \sin \left|\frac{\theta-\tau}{2}\right|\right), & m=n \\ -\frac{i \pi}{2} H_{0}^{(1)}\left(k R_{m m}\right), & m \neq n\end{cases}
$$


The function $H^{m n}, m=n$ is a regular function, defined everywhere except at the points $\theta=\tau$; the function $H^{m n}, m \neq n$ is regular everywhere. An exact expression for $H^{m m}$ at the points of singularity is obtained analytically:

$$
H^{m m}(\theta, \tau)_{\mid \theta=\tau}=-\frac{i \pi}{2}+\gamma+\log \frac{k \cdot l_{m}(\theta)}{2},
$$

where $\gamma$ is Euler's constant.

We expand the singular part of Green's functions in the same way as in Section 2.2, and perform the double Fourier series expansion for the regular function $H^{m m}: H^{m n}(\theta, \tau)=\sum_{j, l=-\infty}^{\infty} h_{j l}^{m n} e^{i(j \theta+l \tau)}$. The unknown function $z_{j}$ is also represented by its Fourier series: $z_{n}(\tau)=\sum_{p=-\infty}^{\infty} \xi_{p}^{n} e^{i p \tau}$.

After substitution of all expansions into (25), one can arrive at the system of $N$ integral equations. Following the regularization steps for the Laplace equation from Section 2.3, we obtain an infinite system of linear algebraic equations of the second kind:

$$
\tilde{\xi}_{l}^{m}\left(1-\delta_{n 0}\right)+\sum_{j=n=1}^{N} \sum_{p=-\infty}^{\infty} \sigma_{l} \sigma_{p} h_{l,-p}^{m n} \tilde{\xi}_{p}^{n}=\sigma_{l} g_{l}^{m} \quad l=1,2, \ldots, N ; n=0, \pm 1, \pm 2 \ldots,
$$

where the following notations are used:

$$
\begin{aligned}
& \sigma_{p}= \begin{cases}|p|^{1 / 2} & \text { when } n \neq 0 \\
1 & \text { when } n=0,\end{cases} \\
& \tilde{\xi}_{p}^{n}=\sigma_{p}^{-1} \xi_{p}^{n}, \quad-2 U^{0}=\sum_{m=-\infty}^{\infty} g_{l}^{m} e^{i l \theta} .
\end{aligned}
$$

The infinite systems (31) can be effectively solved by a truncation method. The solution of the truncated system steadily and rapidly converges to the exact solution [34]. There are no limitations on the number of cylinders with arbitrary smooth cross-sections.

\section{Numerical results}

The numerical code was validated by comparing obtained results with known analytical solutions for the coaxial line with a centred inner conductor [35] and the coaxial line with a shifted inner conductor [36]. Results obtained coincide for up to 16 decimal places with the published solutions starting with $N_{t r}=16$ for the centred inner conductor and $N_{t r}=128$ for the 
inner conductor located close to the shield. Our results are also in a good agreement with other semi-analytical and numerical techniques (for example, presented in [37]).

As an illustration of the effectiveness of the obtained solution, we calculate the capacitance matrix for the assembly of arbitrary profiled cylinders located inside the grounded shield. There are no limitations on the number of cylinders with arbitrary smooth cross-sections. The high efficiency of the code is also the result of employment of the discrete Fast Fourier Transform. This makes filling of the matrix very fast routine procedure. For example, the computation time for a problem with the four inner cylinders and truncation number $N_{t r}=256$ does not exceed $4.5 \mathrm{~s}$ on a standard PC.

Efficiency of the developed method is also illustrated by the behaviour of the normalized truncation error versus truncation number (see [30]) calculated in the maximum norm sense as:

$$
e\left(N_{t r}\right)=\frac{\max _{n \leq N_{t r}}\left|x_{n}^{N_{t r}+1}-x_{n}^{N_{t r}}\right|}{\max _{n \leq N_{t r}}\left|x_{n}^{N_{t r}+1}\right|}
$$

where $\left|x_{n}^{N_{t r}+1}\right| N_{n=0}^{N_{t r}}$ and $\left\{x_{n}^{N_{t r}+1}\right\}_{n=0}^{N_{t r}+1}$ denote the solutions to the systems (19) or (29) truncated to $N_{t r}$ and $N_{t r}+1$ equations, respectively. The results of the calculations of truncation error for the infinite system (18) defining the solution to the Laplace equation are shown in the Figure 2. The considered structure is a circular shield of radius 1 with elliptic conductor with major semi-axis $b_{1}=0.5$ and various values of the minor semi-axis $b_{2}$, embedded in the centre of the shield.

Figure 3 shows the condition number behaviour in the same case. The results are quite accurate and stable: for a simple structure like this, the condition number has reached a stable value even for small values of the truncation number.

In these examples, the ellipse is parameterized by the angle as a parameter. Fewer number of points on the sides of a slender ellipse results in decreasing accuracy for smaller $b_{2}$. Arc length parameterization is one way to overcome this drawback. Other parameterizations could be even more effective, but they often require some adjustments for each shape.

Various shapes of conductor will be considered in this chapter. For all system configurations here and below, the inner conductors' potentials are set to be 1 ; the shield is grounded. The profile of each interior conductor is described by the super-ellipse equation (32), where function $\rho(\varphi)$ and its derivative are continuous [38], with

$$
\rho(\varphi)=\left[\left|\frac{1}{a} \cos \left(m \frac{\varphi}{4}\right)\right|^{n_{2}}+\left|\frac{1}{b} \sin \left(m \frac{\varphi}{4}\right)\right|^{n_{3}}\right]^{-1 / n_{1}} .
$$




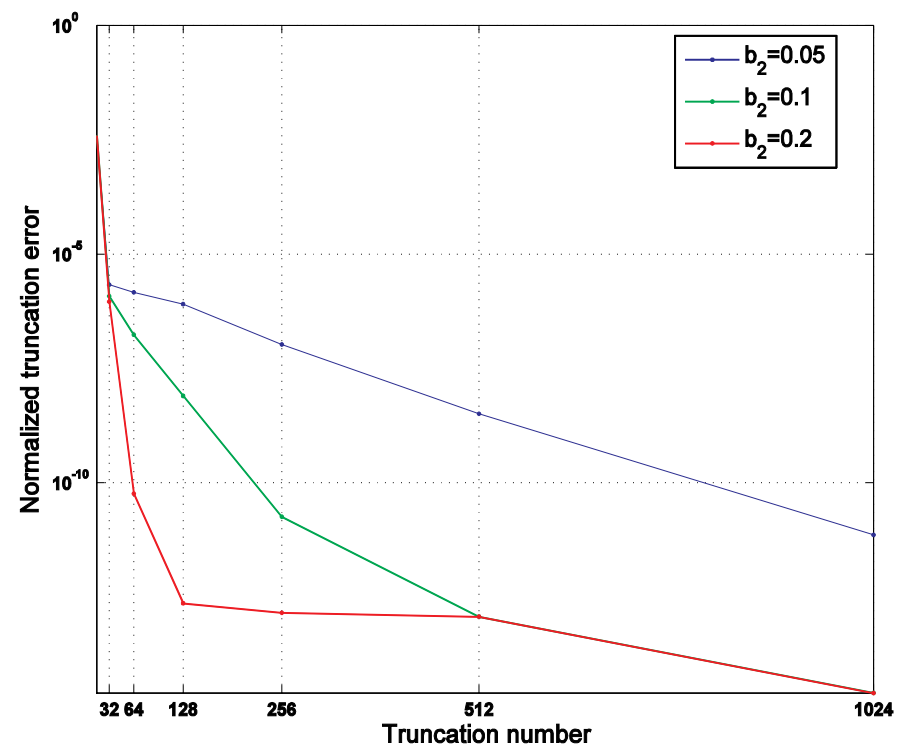

Figure 2. Normalized truncation error versus $N_{t r}$ : major semi-axis of the inner ellipse $b_{1}=0.5$ and various values of the minor semi-axis $b_{2}$.

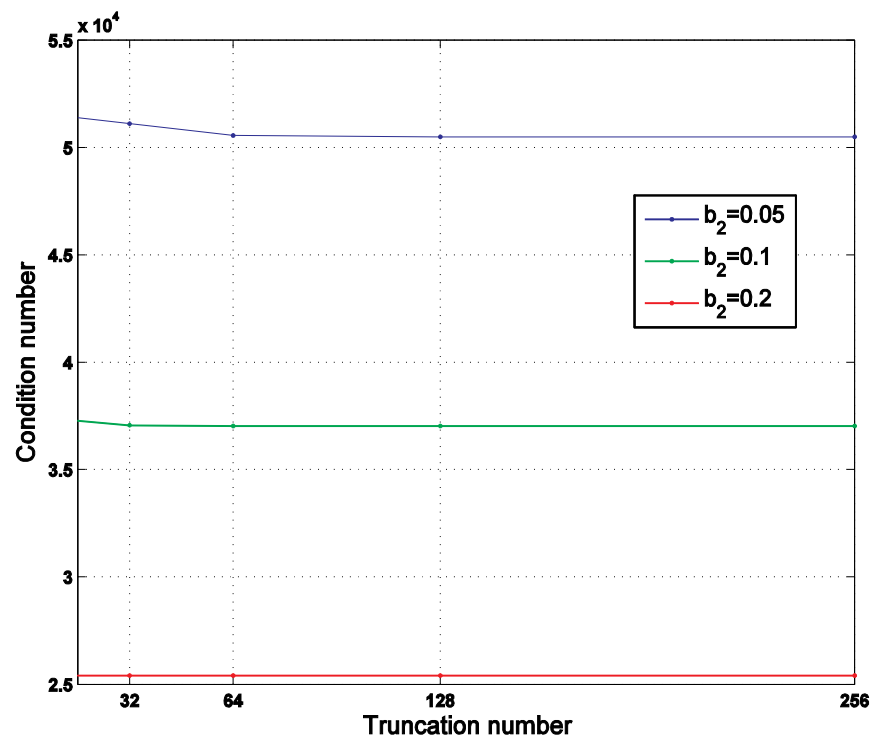

Figure 3. Condition number versus $N_{t r}$. 
In this equation, $a$ and $b$ are the figure size parameters, $n_{1}, n_{2}, n_{3}$ define corner sharpness and $m$ represents the symmetry. This formula allows us to model a great variety of shapes such as an ellipse $\left(n=n_{1}=n_{2}=n_{3}=2, m=4\right)$, a rectangle with rounded-off corners $\left(n=n_{1}=n_{2}=n_{3}>2, m=4\right)$, a star with the smooth rays $\left(n_{1}=2, n_{2}=n_{3}>2, m\right.$ is equal to the number of rays) and many others.

This parameterization is infinitely differentiable which gives us a great advantage in accuracy. To demonstrate this property, comparison of two different parameterizations used in the solution to the Helmholtz equation for a single rectangle with rounded-off corners is presented in Figure 4.

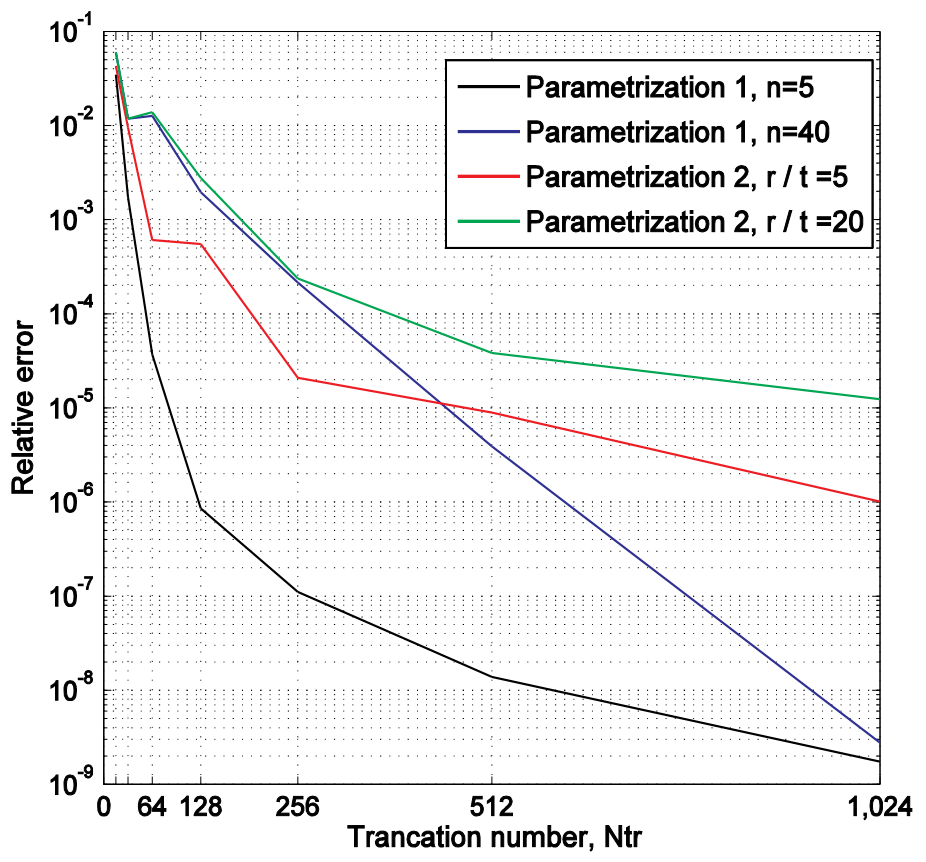

Figure 4. Comparison of super-ellipse and smoothed rectangular parameterizations.

Parameterization 1 stands for a super-ellipse formula; straight lines with a combination of quarter circles are used for the Parameterization 2. The super-ellipse parameterization uses $n=n_{1}=n_{2}=n_{3^{\prime}}$ (see (32)); the greater the $n$ is, the sharper the corners of the rectangle are. Sharper corners require higher truncation number to get the same level of the accuracy due to the parameterization by the angle. In Parameterization 2, $r$ is a radius of curvature used to smoothen the corner, $h$ is rectangle height. In all cases, rectangle height/width ratio is equal to 0.5. Parameterization 2 is not twice differentiable - there is a discontinuity in the second derivative at the joining of the straight line and the quarter circle. This account for slow convergence of the second parameterization as $N_{t r} \rightarrow \infty$. 


\subsection{Electrostatic problems}

\subsubsection{Multi-conductor transmission lines}

Here the power of the method is illustrated by the analysis of multi-conductor transmission lines. Other possible applications of our method for the problems modelled by the Laplace equation include impedance calculations for the transmission lines with adjustable inner conductor, published in [39] and capacitance calculations for the capacitance microscope [40].

The distribution of the electrostatic field for a conceptual configuration of a shielded threeconductor transmission line is shown in Figure 5.
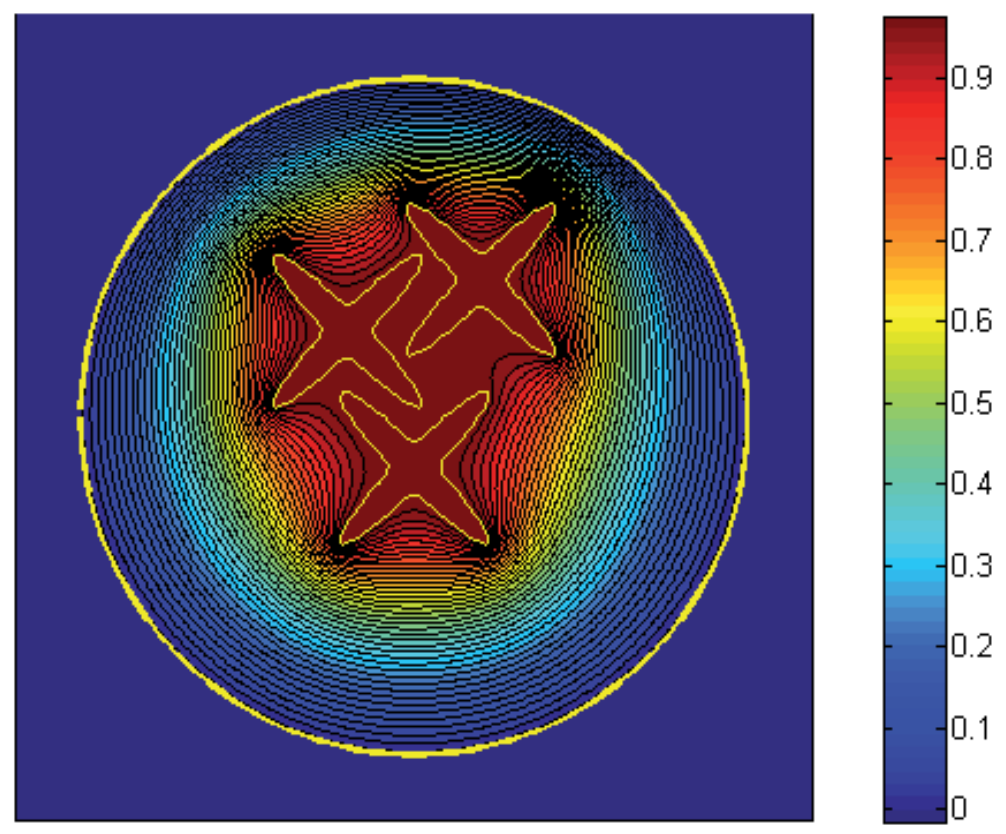

Figure 5. Conceptual circular shielded transmission line with three inner cylinders. $\left(a=b=0.6, m=5, n_{1}=2\right.$, $\left.n_{2}=n_{3}=13\right)$.

It is worth noting that apparently sharp edges are in fact not sharp but have a very small radius of curvature at some points due to parameterization of the contours.

Next we present the calculations for the capacitance matrix (Table 1) calculated by formula $C_{i, j}=\partial Q_{i} / \partial u_{j}$ in the case of the circular shield and conductors of nearly rectangular crosssection (Figure 4). Here $Q_{i}$ is a total charge on the $i$ th cylinder, and $u_{j}$ is the potential of the $j$ th cylinder. 


\begin{tabular}{ccc}
\hline Symmetric alignment: & Non-symmetric alignment: \\
\hline$C=\left(\begin{array}{rrr}3.5583 & -0.4441 & -0.4441 \\
-0.4441 & 3.5580 & -0.4440 \\
-0.4441 & -0.4440 & 3.5580\end{array}\right)$ & $C=\left(\begin{array}{rrr}3.5151 & -0.2713 & -0.4191 \\
-0.2713 & 3.8903 & -1.1851 \\
-0.4191 & -1.1851 & 3.9261\end{array}\right)$ \\
\hline
\end{tabular}

Table 1. Capacitance matrix values for the structure for the square conductors with a circular shield (see Figure 6).

Two configurations are examined - a symmetrical one and another obtained by the translation of one conductor, as indicated in Figure 6. In each case, accuracy was ensured by examining error estimates as a function of truncation number as explained above.

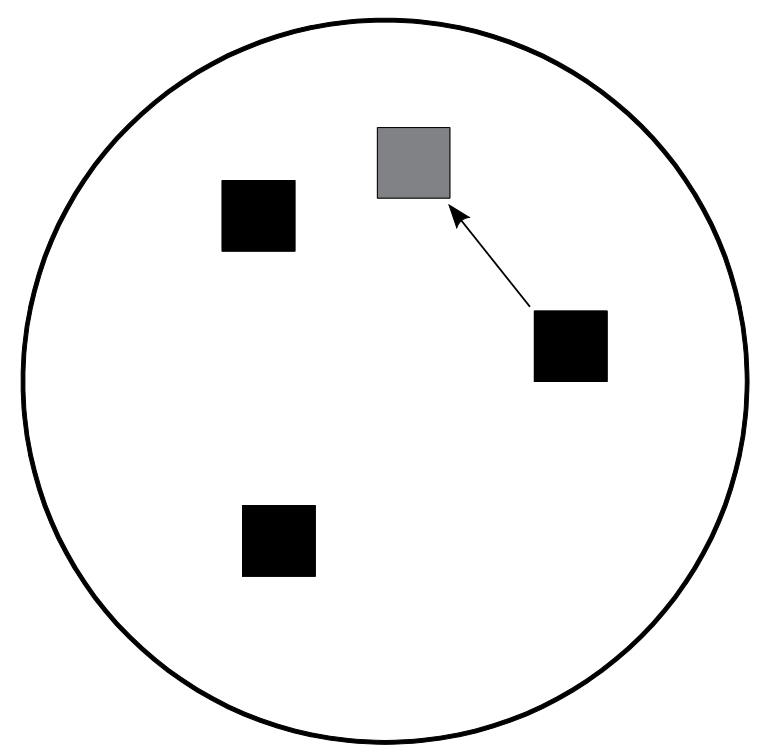

Figure 6. Symmetrical and shifted inner conductors alignment $\left(a=b=0.1, m=4, n_{1}=n_{2}=n_{3}=40\right.$ ).

\subsubsection{Transmission lines with the closely spaced conductors}

Another example demonstrating the effectiveness of the developed algorithm is a study of the closely spaced conductors case (Figure 7). In Table 2, capacitance values for a circular conductor are shown depending on a distance between the inner conductor of a radius 0.1 and a shield of radius 1 . Truncation numbers are chosen to ensure capacitance values and are stable to four decimal places.

The analysis shows that reliable results are obtained when the condition $d \geq \Delta l$ is satisfied, where $d$ is the distance between the conductors, $\Delta l=L / N_{t r}$ is a parameterization step, $L$ is a maximum contour length. 


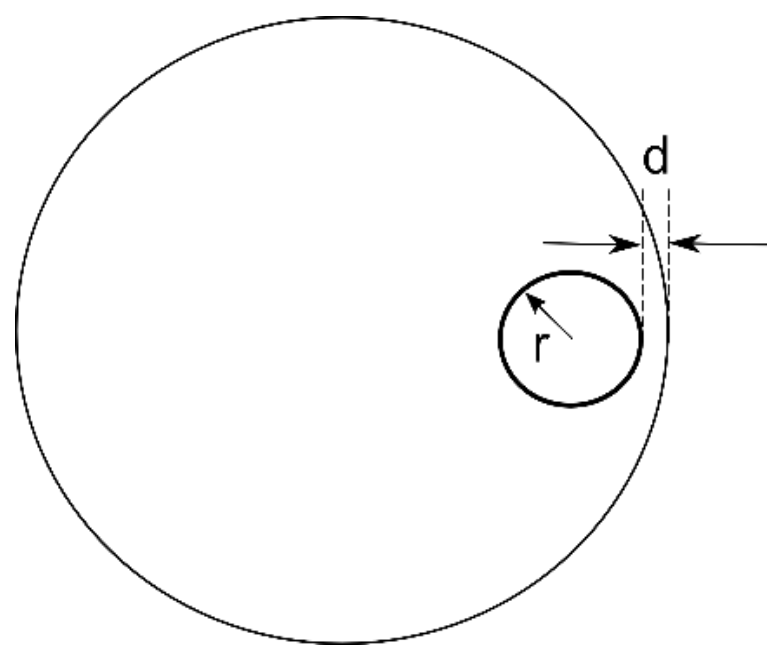

Figure 7. A circular shield with a closely spaced inner conductor.

\begin{tabular}{lll}
\hline $\mathrm{d} / \mathbf{r}$ & $N_{t r}$ & $\mathrm{C}$ \\
\hline 1 & 64 & 5.1270 \\
\hline 0.5 & 128 & 6.9491 \\
\hline 0.1 & 512 & 14.9620 \\
\hline 0.05 & 1024 & 21.0534 \\
\hline
\end{tabular}

Table 2. Capacitance values for the structure with the closely spaced conductors.

\subsection{Scattering of a plane wave by an array of thick strips}

Arrays, which are composed of a finite number of strips, are probably the most common periodic structures. They are employed in various electromagnetic radiating and waveguiding devices. For example, a simple but effective leaky-wave antenna can be designed by placing a microstrip grating above a ground plane, as first proposed by Honey in the 1950s [41] and then studied by different authors with many variations [42]. In addition, periodic structures in the millimetre wave range with high precision requirements must be planar structures, for fabrication reasons [7]. Also, in other applications the strip grating is often used as a circular polarizer [43]. The list of applications can be continued. Nowadays, a lot of attention is paid to more realistic models of the strip gratings: finite gratings, excited by compact directional sources [8]; gratings with thick strips [44, 45]; special elemental positioning $[46,47]$, etc.

In this section, we consider scattering of an obliquely incident E-polarized plane wave by a finite array of metallic thick strips which is relevant to the problems examined in the papers mentioned above. The case of the array of circular cylinders, including resonant effects, was considered in [48]. 


\subsubsection{Linear array of horizontal thick strips}

We consider the scattering of the E-polarized plane wave obliquely incident the linear array composed of the metallic thick strips, as shown in Figure 8. The elemental thick strip is described by its width $w$ and its thickness $t$. The element spacing is characterized by $d$. The incidence angle is $\varphi_{0}$. In our calculations, we set $w=1$, so that $k \equiv k w$ is the relative wave number.

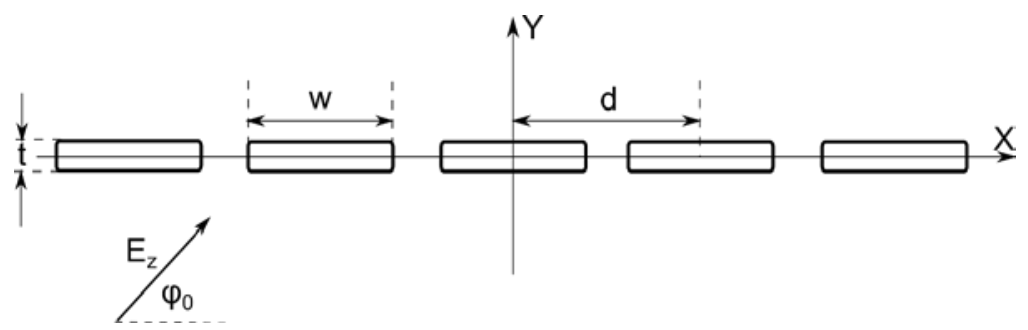

Figure 8. Linear array of horizontal strips.

The radar cross-section (RCS) is determined from the scattered field as $\rho \rightarrow \infty$ in the direction $\varphi=\varphi_{0}+\pi$, where $\varphi_{0}$ is angle of incidence of the incident plane wave, via

$$
R C S=\lim _{\rho \rightarrow \infty} 2 \pi \rho\left|U^{s}\left(\rho, \varphi_{0}+\pi\right)\right|^{2}
$$

The dependence of the RCS ( $R C S\left(\varphi_{0}\right)$, in $\left.\mathrm{dB}\right)$ on the incidence angle, $\varphi_{0}$, for the 3-, 5- and 9elements array $(k=\pi)$ is shown in Figure 9. As the number of elements grows two peaks occurring away from normal incidence begin to dominate over the other minor maxima, due to specular reflection from the elements of the array. The highest peak corresponds to normal incidence $\left(\varphi_{0}=90^{\circ}\right)$; the second peak corresponds to the incidence angle $\varphi_{0}=\cos ^{-1}(1 / 1.5)=48.19^{\circ}$. The calculation of the current density distribution on the contour of each element is of practical interest, especially for the situations when the results obtained for infinite gratings are used for finite gratings. It is reasonable to assume that the near equal current distribution on all elements of the finite grating is a plausible argument to treat such grating as a fragment of the infinite grating. This idea was used, for example, by Kalhor in [49].

In our calculations, we fix the number of the strips $N=5$ in the grating and calculate the current distribution on each element. The results are shown in Figure 10, where we used the parameters: $\varphi_{0}=90, k=\pi, t=0.1, d=1.5$.

Because of the symmetrical (for normal wave incidence) location of the elements in the array, the distribution $\left|J_{z}(\varphi)\right|$ will be identical for the strips, numerated by the indexes $n= \pm 1$ and $n= \pm 2$. Figure 10 demonstrates that current density distributions on all strips are very close to each other, as a front planar surface of each strip is uniformly illuminated by the incidental plane wave. This induces, in particular, a constant current density on most part of the frontal surface of the strip, except in the narrow region near its corners. The current density $\left|J_{z}(\varphi)\right|$ 


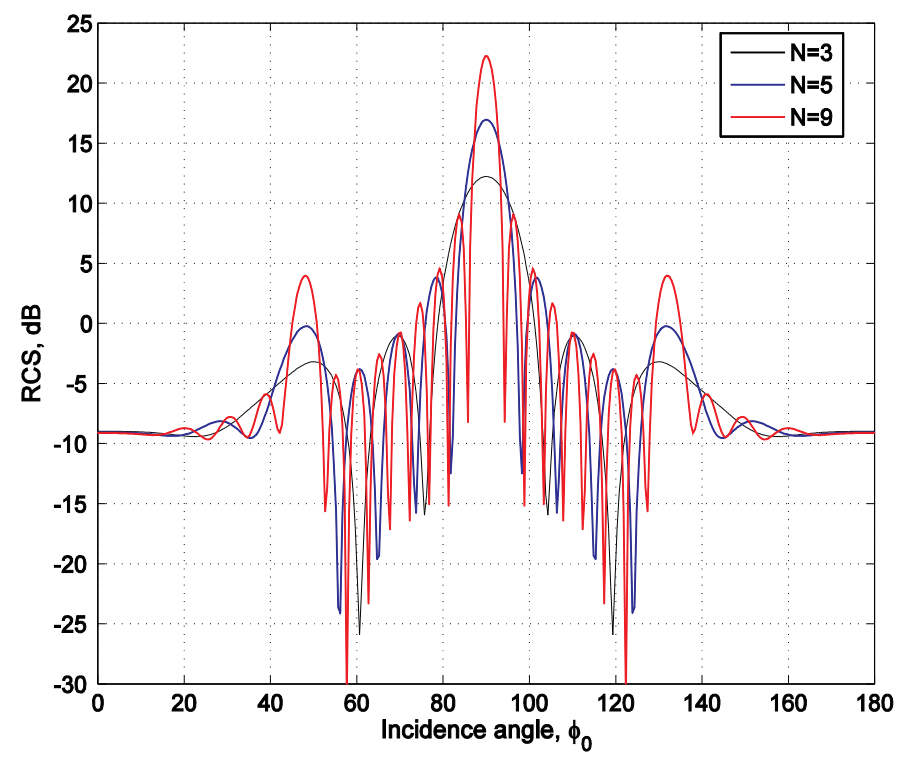

Figure 9. $R C S\left(\varphi_{0}\right),(k=\pi, t / w=0.1, d=1.5)$.

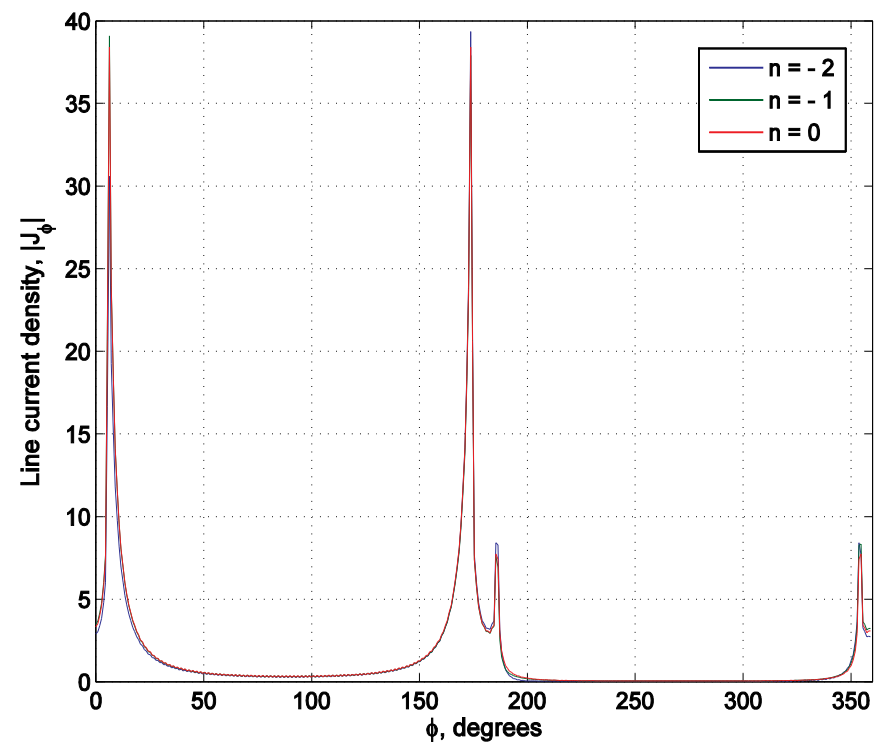

Figure 10. $\left|J_{z}(\varphi)\right|$ at the strips $n=-2,1,0$ in the 5-element array $(k=\pi, t / w=0.1, d=1.5)$. 
has predictable jumps at the angle values, corresponding to the corners of the thick strips. Figure 10 shows four sharp peaks in the distribution of the function $\left|J_{z}(\varphi)\right|$. Two dominant peaks correspond to the directly illuminated corners of the frontal surface of the strip. Two other peaks with significantly smaller magnitude correspond to the corners on the underside of the strip; furthermore, the current density on these parts is relatively small. Due to these peculiarities of the current density distribution on the surface of the thick strip, the dependence of $\left|J_{z}(\varphi)\right|$ on the number of strips in the array is quite weak.

The scattered pattern in the direction $\varphi$ is defined as

$$
S P(\varphi)=\lim _{\rho \rightarrow \infty}\left(\left|U^{s}(\rho, \varphi)\right| / \max _{\varphi \in[-\pi, \pi]}\left|U^{s}(\rho, \varphi)\right|\right) .
$$

The distribution of the scattered field in the far-field zone is shown in Figures 11-13.

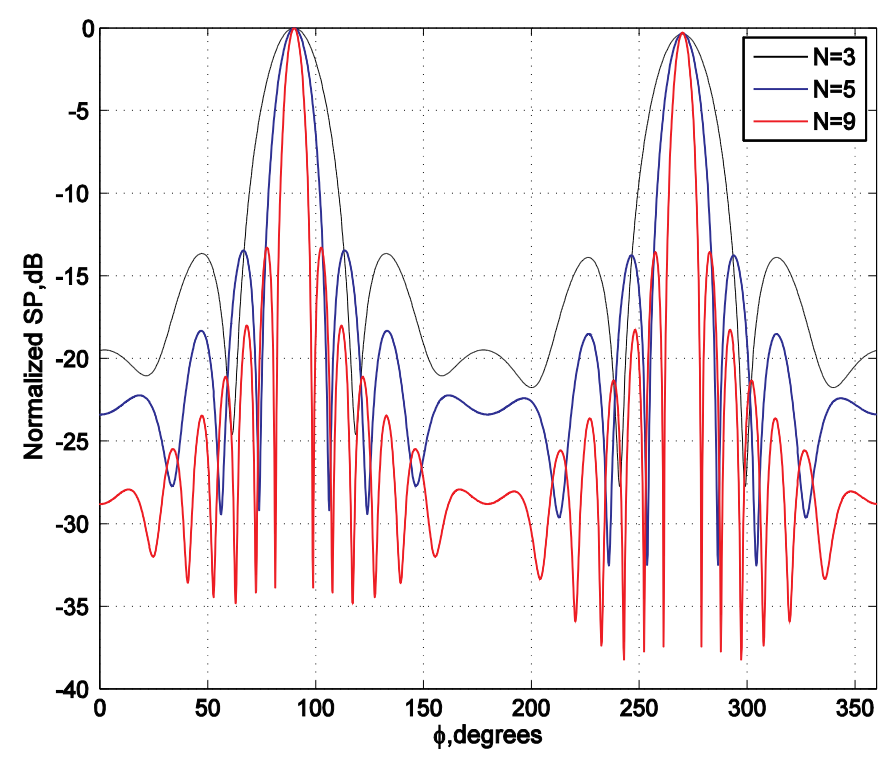

Figure 11. Scattering pattern of the 3-, 5- and 9-strip array $\varphi_{0}=90, k=\pi, t / w=0.1, d=1.5$.

Figure 13 demonstrates the SP for the 3-, 5- and 9-element array with the same geometrical parameters, as in Figure 11, but for a different incidence angle, $\varphi_{0}=30$, and wave number $k=k w=2$. With a small number of strips in the array, the shape of the main beam is not symmetric (the case $N=3$, Figure 12); for formation of a well-focused beam, it is necessary to increase the number of strips in the array. This assertion is confirmed by the substantially improved shape of the main scattering beam when element number increases to $N=9$. 


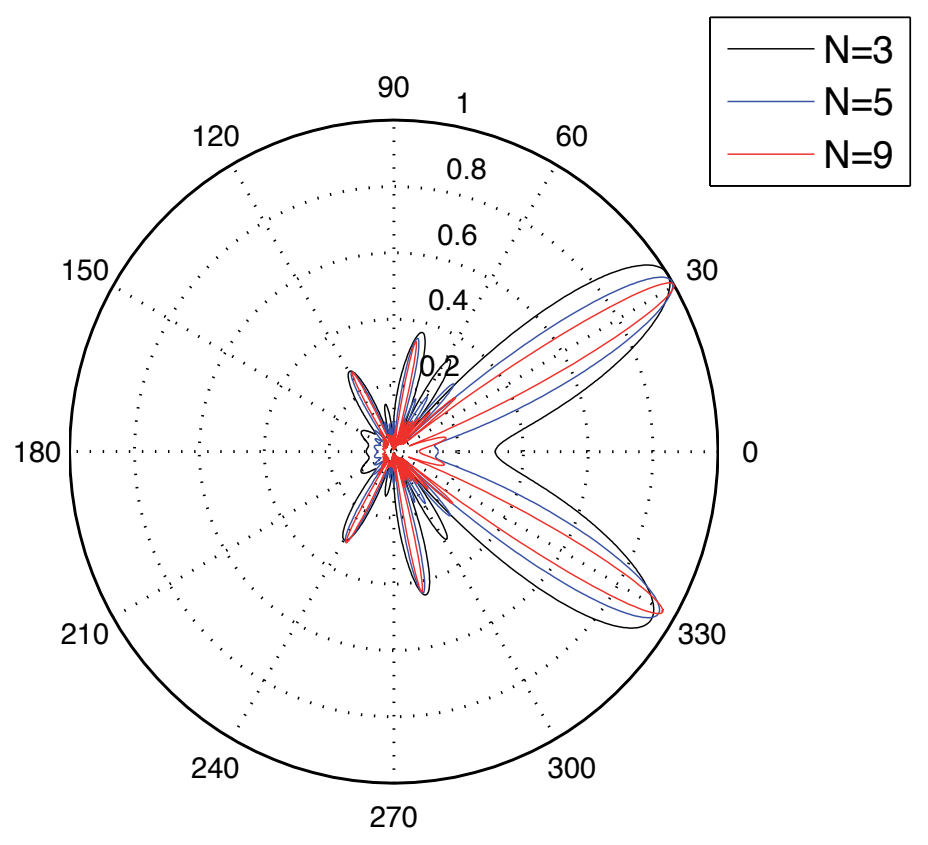

Figure 12. Scattering pattern of the 3-, 5- and 9-strip array $\left(\varphi_{0}=30, k=2 \pi, t / w=0.1, d=1.5\right)$.

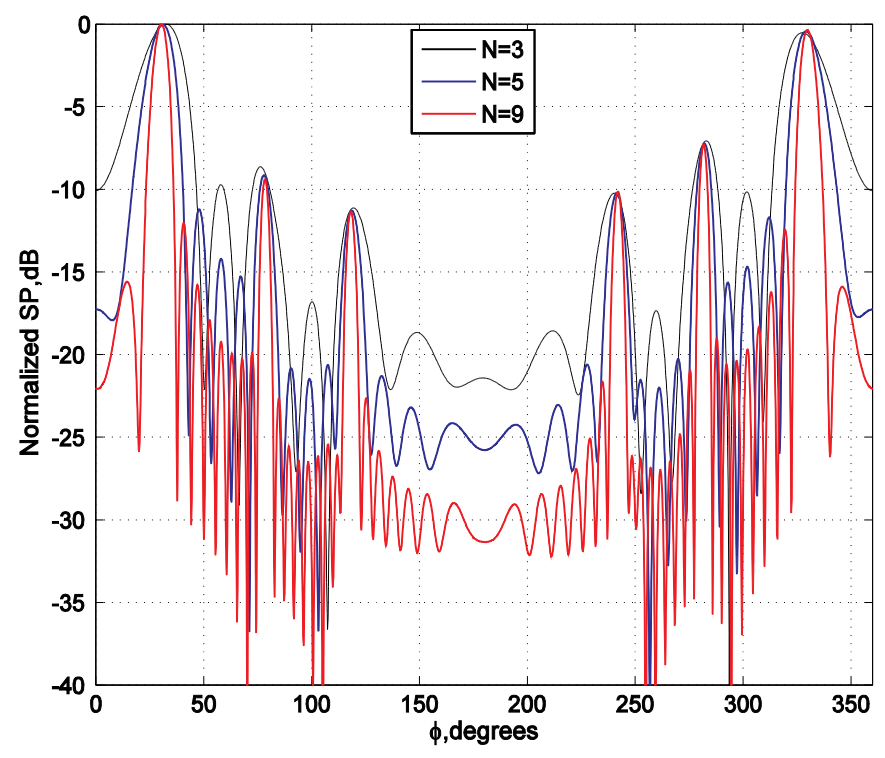

Figure 13. Scattering pattern in $\mathrm{dB}$ of the 3-, 5- and 9-strip array $\left(\varphi_{0}=30, k=2 \pi, t / w=0.1, d=1.5\right)$. 
Next we consider the frequency dependence $R C S(k)$ of the RCS on wavenumber. Setting the array parameters: $N=3,5,9 w=1, t=0.1, d=1.5, \varphi_{0}=90$, we calculate the function $R C S(k)$ in the range $0 \leq k \leq 25$ (see Figure 14).

It is worth noting that extremely high values of the function $\operatorname{RCS}(k)$ are explained by its normalization (the single element width was chosen to be a characteristic parameter, so that $k=k w$. For the 9-element array analysed above the total width $W$ of the array is $W=9 w+8(d-w)=13$ units (all sizes are related to the strip width); hence, $k W=13 k w$. For comparison with the case of a single element case, the RCS should be scaled by the total width of array.

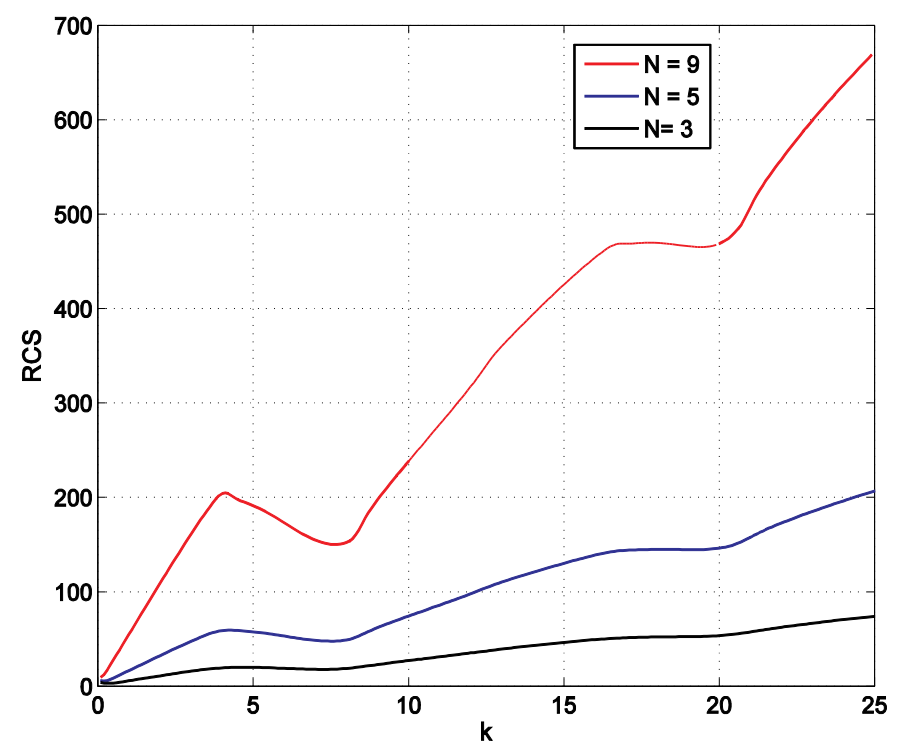

Figure 14. RCS versus relative wave number: $N=3,5,9 ; \varphi_{0}=90, t / w=0.1, d=1.5$.

The next graph in Figure 15 illustrates the effect of perturbing the periodicity of the array on the RCS: the central strip is moved towards to the neighbouring strip by a distance $d=0.4$. At the lower values of the relative wave number $(0 \leq k w \leq 1.8)$, there is no influence of the nonsymmetrical location of the central strip on the value of the RCS. At the value $k w=1.8$, the shift $g=0.9-0.5$ (see Figure 15) becomes slightly greater than the wave size $\lambda / 9$. Hence, we can conclude that the disturbance in the location of the strip in the array is insignificant on the RCS when the shift does not exceed $\lambda / 9$.

Now let us investigate how the thickness of the strips in an array impacts the RCS. In fact, we will consider a more general problem. Usually the term thick strip refers to the strip with the width $w$ greater than its height $h(h / w<1)$; otherwise, it is more reasonable to call such a 


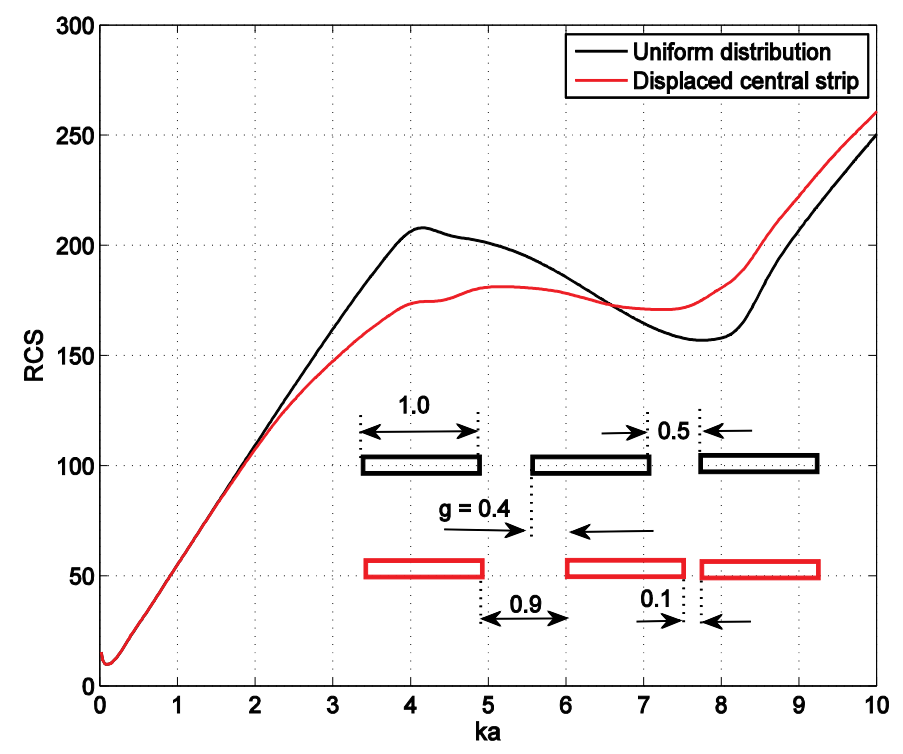

Figure 15. RCS of the 9-element periodic array (black) and array with shifted central strip (red): $N=9 ; \varphi_{0}=90, t / w=0.1, d=1.5$.

structure a rectangular or square bar $(h / w \geq 1)$. The ratio $t=h / w=1$ represents a threshold value; the parameters $w$ and $h$ are better described as height and width as shown in Figure 16.

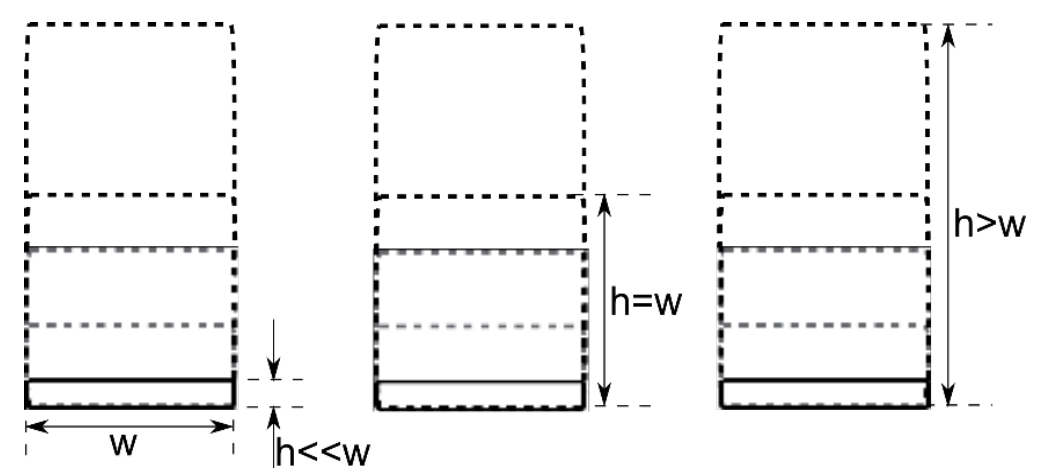

Figure 16. Schematic view of the strip thickening.

Here we consider normal wave incidence $\varphi_{0}=90$ on the array of strips with the fixed parameters: $w=1, d / w=3, k \equiv k w=\pi, 2 \pi$ and $5 \pi$. Starting from the relative strip thickness $t=h / w=0.05$, we consider the dependence of RCS on $t$ for a 3-element array in the range $0.05 \leq t=h / w \leq 3$. The results of these calculations are presented in Figure $17(k=\pi)$, Figure 18 $(k=2 \pi)$ and Figure $19(k=5 \pi)$. 


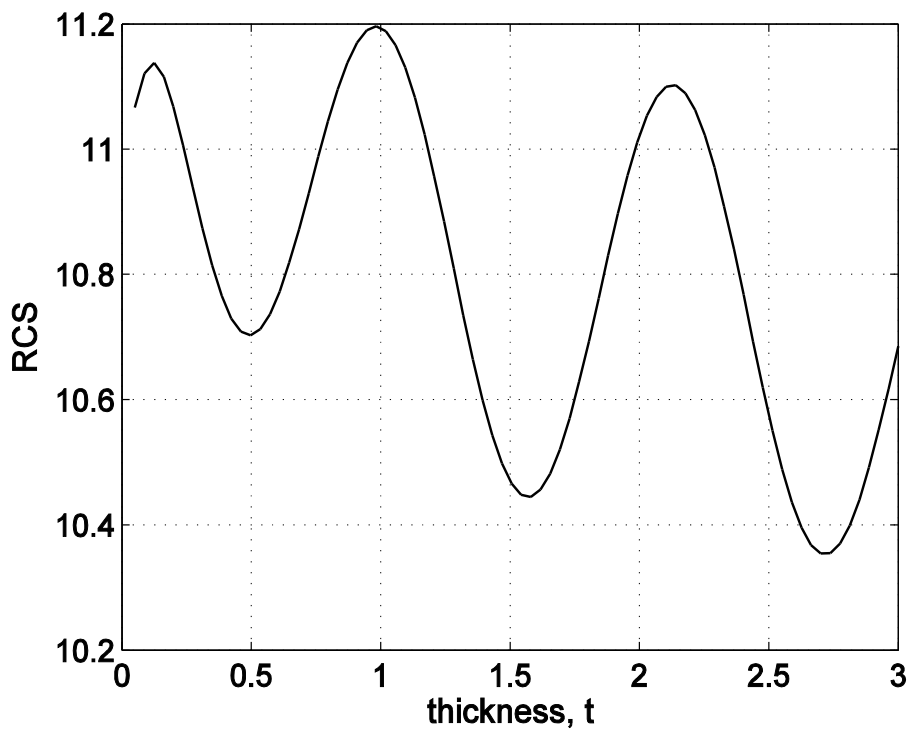

Figure 17. RCS of the 3-element array versus relative strip thickness $t\left(\varphi_{0}=90, k=\pi, w=1, d=3\right)$.

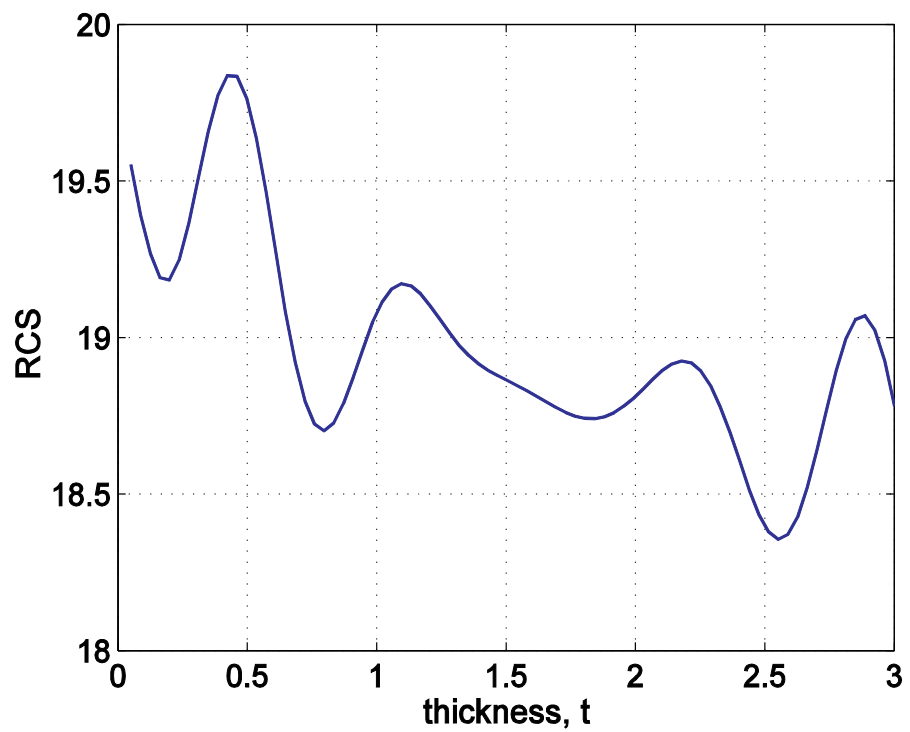

Figure 18. RCS of the 3-element array versus relative strip thickness $t\left(\varphi_{0}=90, k=2 \pi, w=1, d=3\right.$ ). 


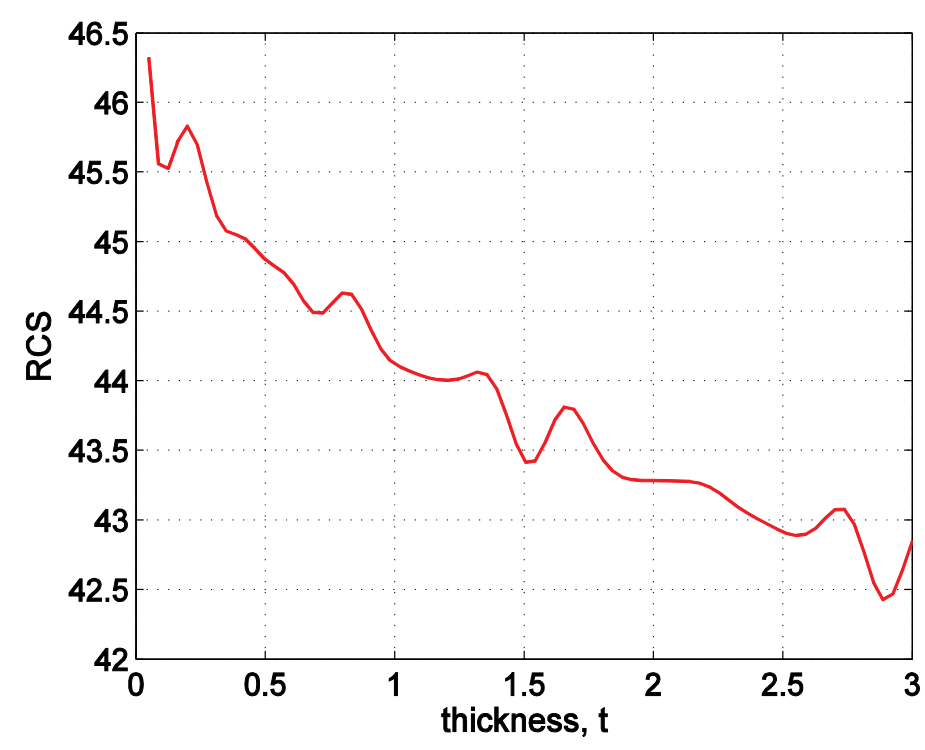

Figure 19. RCS of the 3-element array versus relative strip thickness $t\left(\varphi_{0}=90, k=5 \pi, w=1, d=3\right)$.

Surprisingly the RCS is fairly insensitive to substantial thickening of the original horizontal thin strip $(t=h / w=0.05)$, even at the extreme transformation of the strip into a vertical rectangular cylinder with $t=h / w=3$. The difference between the maximum value $R C S(t)$ and its minimum value for the wavenumbers $k=\pi$ and $k=2 \pi$ does not exceed $7.4 \%$. In the case when $k=5 \pi$, this difference increases to $8.3 \%$.

\subsubsection{Inclined arrays of thick strips}

It was shown previously in this chapter that different positioning of the strips affects the reflection properties. Let us consider another geometry, arranging the strips as a $2 \mathrm{D}$ truncated corner reflector (see Figure 20).

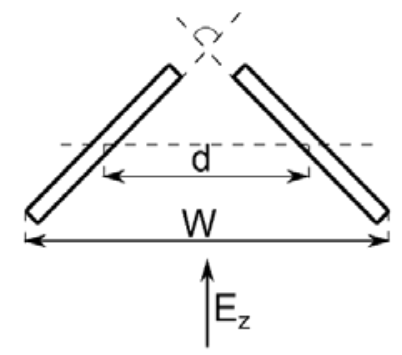

a)

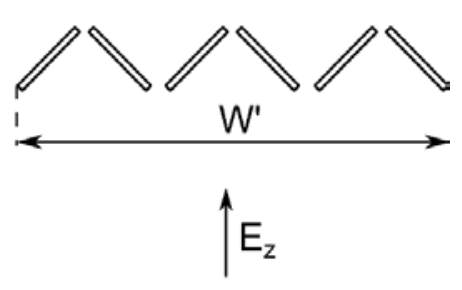

b)

Figure 20. Truncated corner reflector $\left(\varphi_{0}=90\right)$ : (a) isolated reflector and (b) array of three reflectors. 
Here we only consider normal wave incidence, so we normalize the function $R C S(k)$ by the characteristic maximum size of the structure. We define geometric parameters $W$ for the single reflector and $W^{\prime}$ for the array of the corner reflectors (see Figure 20) by $W=d+(w+t) / \sqrt{2}, \quad W^{\prime}=3 W$, where $d$ is the distance between strip centres, $w$ and $t$ are the single strip relative width and thickness, respectively. We calculate the dependence $R C S(k W)$ for the three values $d=0.8,1.2,1.6$. As in previous calculations, we take $w=1, t=0.1$. The Epolarized plane wave excites the truncated corner reflector with incident angle $\varphi_{0}=90^{\circ}$. The frequency dependence of the RCS in the range $0<k W \leq 20$ for the single truncated corner reflector is shown in Figure 21.

The frequency dependence of the RCS in the range for the array of three truncated corner reflectors is shown in Figure 22. The RCS for the single truncated corner reflector is of a regular oscillatory character for each parameter $d=0.8,1.2,1.6$; the average level of the RCS steadily grows along with the relative wave number. Combined as a 3-element array, a moderate enhancement of the RCS is observed. Closer spacing $(d=0.8,1.2)$ of the reflectors preserves the regular oscillatory behaviour of the $R C S(k W)$, in contrast to more separated spacing $(d=1.6)$, where some peaks in the RCS graph (red, Figure 22) become suppressed.

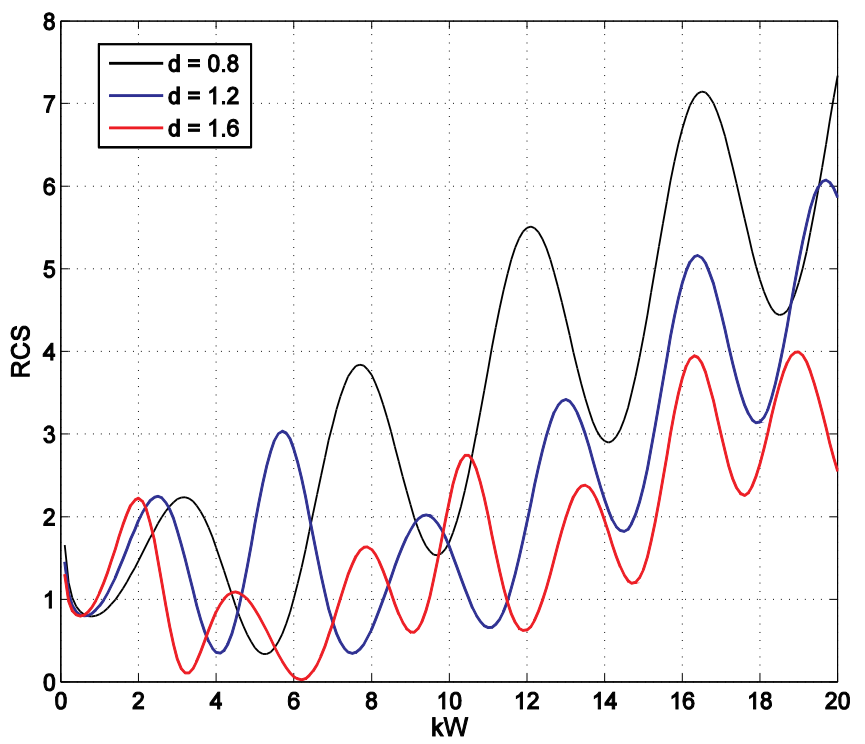

Figure 21. $R C S(k W)$ for the single corner reflector: $\varphi_{0}=90, w=1, t=0.1$. 


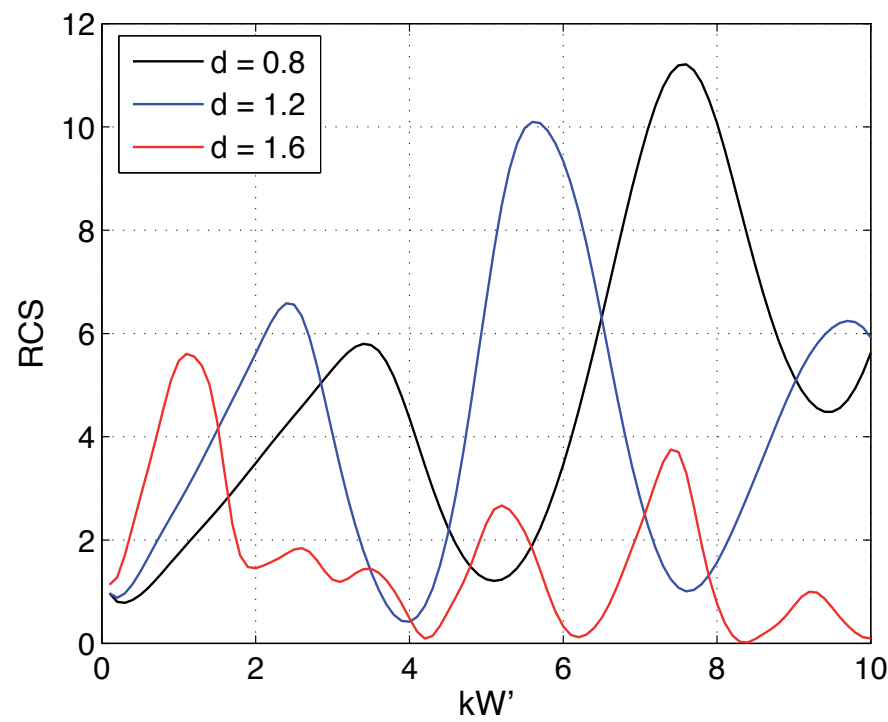

Figure 22. $R C S\left(k W^{\prime}\right)$ for the array of three corner reflectors: $\varphi_{0}=90, w=1, t=0.1$.

\section{Conclusion}

In this chapter, a rigorous approach to the numerical analysis of the multi-conductor problems in electrostatics and multiple wave scattering for metallic cylinders is presented. The problems are treated as a classical Dirichlet boundary value problem for the Laplace and Helmholtz equations. All conductors may be of arbitrary cross-sections; the only restriction on the system geometry is a smooth parameterization of the boundaries.

The 2D multi-conductor problems for the Laplace and Helmholtz equations are rigorously solved by the MAR. The problem is transformed to a numerical analysis of an infinite system of linear algebraic equations of the second kind. This explains its fast convergence and guaranteed computational accuracy, depending only upon truncation number $N_{t r}$. The computation of the matrix elements is based on the discrete FFT, making the matrix filling an accurate and extremely fast procedure. The only limitation imposed on the contour is its smoothness. When the contour incorporates corners, they should be rounded. The developed algorithm is numerically stable and fast, and accuracy of the solution can be pre-specified. The solution obtained is applied to the accurate analysis of 2D electrostatic and electrodynamic field problems for multi-conductor systems with arbitrary profiled conductors. Examples for some conceptual shielded transmission lines incorporating differently profiled conductors and scattering problems for the arrays of thick strips illustrate the efficiency and reliability of the method. 


\section{Author details}

Galyna Safonova* and Elena Vinogradova

*Address all correspondence to: galyna.saf@gmail.com

Department of Mathematics, Macquarie University, Sydney, Australia

\section{References}

[1] Jackson JD. Classical Electrodynamics. New York: Wiley; 1999. p. 808.

[2] Shi F, Ramesh P and Mukherjee S. On the Application of 2D Potential Theory to Electrostatic Simulation. Communications in Numerical Methods in Engineering 1995; 11(8): 691-701.

[3] Paul CR. Analysis of Multiconductor Transmission Lines. New York: Wiley; 1994.

[4] Brando Faria JA. Multiconductor Transmission Lines. Encyclopedia of RF and Microwave Engineering; 2005.

[5] Kamchouchi HE and Zaky AA. A Direct Method for the Calculation of the Edge Capacitance of Thick Electrodes. Journal of Physics D 1975; 8: 1365-1371.

[6] Moreira FJS and Prata A. Vector-Diffraction Analysis of Finite Perfectly Conducting Gratings with Arbitrary Profiles. Journal of Microwaves and Optoelectronics 1999; 1(5): 11-13.

[7] Filtz M and Henke H. Planar Millimeter Wave Structures. Electrical Engineering (Archiv fur Elektrotechnik) 2004; 86(2): 147-155.

[8] Mata-Mendez O, Avendao J and Chavez-Rivas F. Rigorous Theory of the Diffraction of Gaussian Beams by Finite Gratings: TM Polarization. Journal of Optical Society of America A 2006; 23(8): 1889-1896.

[9] Borghi R, Gori F and Santarsiero M. Plane-Wave Scattering by a Set of Perfectly Conducting Circular Cylinders in the Presence of a Plane Surface. Journal of Optical Society of America A 1996; 13(12): 2441-2452.

[10] Argatov II and Sabina FJ. Acoustic Scattering by a Cluster of Small Sound-Soft Obstacles. Wave Motion 2010; 47(8): 537-551.

[11] Yang J, Li LW, Yasumoto K and Liang CH. Two-Dimensional Scattering of a Gaussian Beam by a Periodic Array of Circular Cylinders. IEEE Transactions on Geoscience and Remote Sensing 2005; 43(2): 280-285.

[12] Yasumoto K, Toyama H and Kushta T. Accurate Analysis of Two-Dimensional Electromagnetic Scattering from Multilayered Periodic Arrays of Circular Cylinders Us- 
ing Lattice Sums Technique. IEEE Transactions on Antennas and Propagation 2004; 52(10): 2603-2611.

[13] Lucido M, Panariello G and Schettino F. Electromagnetic Scattering by Multiple Perfectly Conducting Arbitrary Polygon Cylinders. IEEE Transactions on Antennas and Propagation 2008; 56(2): 425-436.

[14] Judd SV, Whiteley I, Clowes RJ and Rickard DC. An Analytical Method for Calculating Microstrip Transmission Line Parameters. IEEE Transactions on Microwave Theory and Techniques 1970; 18(2): 78-87.

[15] Zhu NH, Qiu W, Pun EYB and Chung PS. Quasi-Static Analysis of Shielded Microstrip Transmission Lines with Thick Electrodes. IEEE Transactions on Microwave Theory and Techniques 1997; 45(2): 288-290.

[16] Chang T and Tan C. Analysis of a Shielded Microstrip Line with Finite Metallization Thickness by the Boundary Element Method. IEEE Transactions on Microwave Theory and Techniques 1990; 38(8): 1130-1132.

[17] Gentili GG and Macchiarella G. Quasi-Static Analysis of Shielded Planar Transmission Lines with Finite Metallization Thickness by a Mixed Spectral-Space Domain Method. IEEE Transactions on Microwave Theory and Techniques 1994; 42(2): 249-255.

[18] Khebir A, Kouki AB and Mittra RM. Higher Order Asymptotic Boundary Condition for Finite Element Modeling of Two-Dimensional Transmission Line Structures. IEEE Transactions on Microwave Theory and Techniques 1990; 38(10): 1433-1438.

[19] Slade GW and Webb KJ. Computation of Characteristic Impedance for Multiple Microstrip Transmission Lines Using a Vector Finite Element Method. IEEE Transactions on Microwave Theory and Techniques 1992; 40(1): 34-40.

[20] Alam MS, Hirayama K, Hayashi Y and Koshiba M. Analysis of Shielded Microstrip Lines with Arbitrary Metallization Cross Section Using a Vector Finite Element Method. IEEE Transactions on Microwave Theory and Techniques 1994; 42(11): 2112-2117.

[21] Yee HY and Wu K. Printed Circuit Transmission-Line Characteristic Impedance by Transverse Modal Analysis, IEEE Transactions on Microwave Theory and Techniques 1986; 34(11): 1157-1163.

[22] Hong IP, Yoon N, Park SK and Park HK. Investigation of Metal-Penetrating Depth in Shielded Microstrip Line. Microwave and Optical Technology Letters 1988; 19(6): 396-398.

[23] Zaki KA and Neureuther AR. Scattering from a Perfectly Conducting Surface with a Sinusoidal Height Profile: TM Polarization. IEEE Transactions on Antennas and Propagation 1971; 19(6): 747-751. 
[24] Booker SM and Vinogradov SS. A Two Body Benchmark Problem for Transient and Ultrawideband Scattering. Journal of Electromagnetic Waves and Applications 2002; 16(3): 365-384.

[25] Nosich AI. The Method of Analytical Regularization in Wave-Scattering and Eigenvalue Problems: Foundations and Review of Solutions. IEEE Transactions on Antennas and Propagation 1999; 41(3): 34-48.

[26] Smith PD. Recent Advances in Regularization Techniques for Scattering and Diffraction. Radio Science 2007; 42(6): doi: 10.1029/2007RS003703.

[27] Kellogg O. Foundations of Potential Theory. Berlin-Heidelberg-New York: SpringerVerlag; 1967.

[28] Colton D and Kress R. Integral Equation Methods in Scattering Theory. New York: John Wiley \& Sons; 1983.

[29] Kress R. Linear Integral Equations, ser. Applied Mathematical Sciences. Berlin: SpringerVerlag (Volume 82); 1989.

[30] Vinogradov SS, Smith PD and Vinogradova ED. Canonical Problems in Scattering and Potential Theory, Part 1: Canonical Structures in Potential Theory. Boca Raton: Chapman \& Hall/CRC; 2001.

[31] Vinogradov SS, Vinogradova ED, Wilson C, Sharp I and Tuchkin YA. Scattering of an E-Polarized Plane Wave by Two-Dimensional Airfoils. Electromagnetics 2009; 29(3): 268-282.

[32] Vinogradova ED and Smith PD. Diffraction from 2-D Arbitrary Structures with Cavities or Edges: A Rigorous Approach: Proceedings of the XXVIIth General Assembly of URSI, URSI GASS, 2002, The Netherlands, Maastricht.

[33] Shestopalov VP, Tuchkin YA, Poedinchuk AE and Sirenko YK. New Methods of Solving Direct and Inverse Problems of Diffraction Theory. Kharkiv: Osnova (In Russian); 1997.

[34] Kantorovich LV and Akilov GP. Functional Analysis in Normed Spaces. New York: Pergamon Press; 1974.

[35] Collin RE. Field Theory of Guided Waves. Oxford: Oxford University Press; 1991.

[36] Yakover YM. General Analysis of Shielded Transmission Lines with Cylindrical Geometry Conductors. 27th European Microwave Conference 1997; 2: 955-958.

[37] Malits P, Haridim M and Chattah Y. Characteristic Impedance of a Shielded Transmission Line with Non-Concentric Arc-Shaped Conductors. Electromagnetics 2009; 29: 337-352.

[38] Jielis J. A Generic Geometric Transformation That Unifies a Wide Range of Natural and Abstract Shapes. American Journal of Botany 2003; 90(3): 333-338. 
[39] Safonova G, Vinogradova ED and Tuchkin Y. Accurate Impedance Calculations for Transmission Lines with Adjustable Inner Conductor. Microwave and Optical Technology Letters 2014; 56(9): 2066-2070.

[40] Safonova G and Vinogradova ED. Rigorous Approach to Calculation of Capacitance Images of Metallic Samples for Use in Capacitance Microscopy. Progress in Electromagnetics Research B 2013; 55: 383-399.

[41] Honey R. A Flush-Mounted Leaky-Wave Antenna with Predictable Patterns. IEEE Transactions on Antennas and Propagation 1959; 7(4): 320-329.

[42] Klohn KL, Horn RE, Jacobs H and Freibergs E. Silicon Waveguide Frequency Scanning Linear Array Antenna. IEEE Transactions on Microwave Theory and Techniques 1978; 26(10): 764-773.

[43] Brand GF. The Strip Grating as a Circular Polarizer. American Journal of Physics 2003; 71(5): 452.

[44] Kobayashi K and Miura K. Diffraction of a Plane Wave by a Thick Strip Grating. IEEE Transactions on Antennas and Propagation 1989; 37(4): 459-470.

[45] Peng ST and Shiao CM. Scattering of Plane Waves by Metallic Gratings. Microwave Symposium Digest. IEEE MTT-S International 23-27 May 1994; 2: 879-882, doi: 10.1109/MWSYM.1994.335217.

[46] Filipovic D and Dlabac T. Low Frequency TM Plane-Wave Scattering from a Twolayer Double-strip Grating with Equal Gaps. Series: Electrical Energy 2006; 19(3): http://www.doiserbia.nb.rs/img/doi/0353-3670/2006/0353-36700603379F.pdf (accessed 9 September 2014).

[47] Shimoda M and Itakura T. Scattering of Electromagnetic Plane Waves by an Inclined Parallel Strip Grating. Electronics and Communications in Japan (Part II: Electronics) 1990; 73(8): 56-65.

[48] Safonova G and Vinogradova ED. Accurate Backscattering Studies for Double-Layered Array of Infinitely Long Cylinders. Microwave and Optical Technology Letters 2013; 55: 2127-2131.

[49] Kalhor HA. Plane Metallic Gratings of Finite Number of Strips. IEEE Transactions on Antennas and Propagation 1989; 37(3): 406-407. 

Chapter 8

\title{
Characterization of Magnetic Phases in Nanostructured Ferrites by Electron Spin Resonance
}

\author{
Rebeca Díaz-Pardo and Raúl Valenzuela \\ Additional information is available at the end of the chapter \\ http://dx.doi.org/10.5772/61508
}

\begin{abstract}
This chapter is dedicated to the analysis of the spin resonance response (ESR) of different magnetic phases, in nanoparticles (NPs) of magnetic oxides, or ferrites. Evidence of the correlations between resonance spectrum and magnetic structure has been published, of course, in many works; however, to our knowledge, it is somewhat scattered and not easily accessible. We have chosen to carry out this analysis mainly on ferrite NPs because these magnetic materials exhibit a wide variety of magnetic properties, and as a consequence, a large diversity of classic and novel applications in technological fields ranging from electronics to biomedics.
\end{abstract}

Keywords: EPR, FMR, ESR, Nanoparticles, Ferrites

\section{Introduction}

This chapter is dedicated to the analysis of the spin resonance response (ESR) of different magnetic phases, in nanoparticles (NPs) of magnetic oxides, or ferrites. Evidence of the correlations between resonance spectrum and magnetic structure has been published, of course, in many works; however, to our knowledge, it is somewhat scattered and not easily accessible. We have chosen to carry out this analysis mainly on ferrite NPs because these magnetic materials exhibit a wide variety of magnetic properties, and as a consequence, a large diversity of classic and novel applications in technological fields ranging from electronics to biomedics.

Ferrites can also be easily tailored, and in the nanometric range and at the appropriate temperatures, they exhibit one of the main magnetic structures: ordered ferrimagnetic, superparamagnetic, or paramagnetic. The latter structure is less complex, and electron 
paramagnetic resonance (EPR) has been known for $\sim 50$ years, so in some way, this is a situation both classic and new. Ferrimagnetic and superparamagnetic NPs are more complex, and their resonance response is currently an active research subject.

We first describe the general basis of the classic resonance technique, where a microwave signal of constant frequency is applied on the sample, which is simultaneously subjected to a sweeping magnetic field, in order to achieve the resonance conditions. We tried to establish the main differences between the response of classic and metallic paramagnetic phases and the shapes of magnetically ordered (ferro- or ferrimagnetic) phases in the bulk state.

We then briefly review the novel properties associated with the nanometric size, followed by a short account of crystal structure and magnetic interactions in ferrites. EPR is briefly described, illustrated with some recent results. A basic description of the superparamagnetic phase is then given, with a review of some of the theoretical models proposed, as well as some of the most representative experimental results. The ferrimagnetic phase is then described with an accent on the differences originated by the exchange interactions, magnetocrystalline anisotropy, demagnetization fields, etc., and their effects on the response signal. The general response of magnetic nanoparticles in the ordered state is discussed. In the conclusion, finally, an attempt is made to establish a correlation between these phases and their resonance signals.

\subsection{Nanomagnetism}

In addition to the changes related to the decrease in scale down to the nanometric range, magnetic materials provide another source of novel properties. Many of the critical parameters in magnetism are found in the 1 to 200-nm scale of length, see Table 1 [1]. Two of the most interesting changes in magnetic structure as a consequence of the reduction in size are the change from multidomain to single domain and superparamagnetism.

Bulk samples of ferro- and ferrimagnetic materials are divided into magnetic domains (separated by domain walls) in order to decrease the magnetostatic energy, i.e., the energy associated with the presence of magnetic flux just outside the sample surface. Inside the magnetic domains exchange energy is at a minimum (all spins are parallel coupled) as well as magnetocrystalline energy (all spins are oriented into easy axes), and the magnetization of domains is oriented to provide a continuous magnetic flux inside the sample, thus avoiding any external flux. This is why most magnetic materials do not manifest any attraction or repulsion force (in the absence of an applied magnetic field). Magnetostatic energy is therefore eliminated, except for a small contribution from domain walls, where spins rotate from the orientation of a domain toward the orientation of the neighboring domain. There is also a small contribution to both exchange and anisotropy energy due to domain walls, as spins cannot be strictly parallel, neither oriented into easy directions within the domain wall. These contributions, however, are small as domain wall thickness is in the 10- to 100-nm range. Domain walls represent a very sensitive equilibrium in ferromagnetic materials. They can be displaced by very small applied, fields and their dynamics of propagation though defects (pinning sites) have a fundamental importance for soft magnetic materials [2]. 
As samples become smaller, there is a limit where it becomes more convenient, on an energetic basis, to eliminate the domain walls and form a single domain on the whole sample. This transition becomes feasible because magnetostatic energy is a function of the sample volume, while domain wall energy depends on their total surface. As a single domain, materials become magnetically harder; the coercive field typically exhibits an inverse relationship with the particle size going through a maximum for the transition between single domain and multidomain structure. Single-domain ferromagnets become harder because in the absence of domain wall, the main magnetization process is spin rotation, i.e., the applied magnetic field has to rotate each spin against the anisotropy. This is very different than having domain walls; as they are in a delicate equilibrium, a small field can easily displace them with large variation in oriented domains associated with their passage.

\begin{tabular}{ll}
\hline Property & Typical length range $(\mathrm{nm})$ \\
\hline Exchange interaction & $0.1-1$ \\
\hline RKKY Interaction & $0.1-10$ \\
\hline Single-domain limit & $10-1000$ \\
\hline Superparamagnetic limit & $1-100$ \\
\hline Thickness of domain walls & $10-100$ \\
\hline
\end{tabular}

Table 1. Critical lengths in magnetism. Adapted from Guimaraes [1].

The reduction in scale can also lead to a superparamagnetic (SPM) phase. As discussed with more detail in Section 3, in an SPM phase, the thermal energy dominates over anisotropy, and the magnetization is therefore subjected to random fluctuations. It is interesting to note that in the SPM phase, the exchange interaction maintains the spin coupling up to the Curie temperature, which is higher than the temperature of transition from ordered to SPM phase, also known as blocking temperature. The magnetic properties in the SPM phase are different to both those in the ordered phase and in the paramagnetic phase. SPM properties allow many novel applications, particularly in the field of biomedicine.

\section{Ferrites}

Spinel ferrites are a large family of materials, with the structure of the natural spinel mineral, $\mathrm{MgAl}_{2} \mathrm{O}_{4}$, first determined by Bragg [3]. The spinel is a very stable crystal structure; it is almost enough to satisfy the conditions of neutral electric charge, relatively small cation radii, and a $3 / 4$ cation-to-anion ratio [2]. These conditions allow several cation combinations such as 2,3 (as in $\mathrm{Ni}^{2+} \mathrm{Fe}^{3+}{ }_{2} \mathrm{O}_{4}$ ), 2,4 (as in $\mathrm{Co}_{2} \mathrm{GeO}_{4}$ ), 1,3,4 (as in $\mathrm{LiFeTiO}_{4}$ ), 1,3 (as in $\mathrm{Li}_{0.5} \mathrm{Fe}_{2.5} \mathrm{O}_{4}$ ), 1,2,5 (as in $\mathrm{LiNiVO}_{4}$ ), and 1,6 (as in $\mathrm{Na}_{2} \mathrm{WO}_{4}$ ). Most of ferrites with significant magnetic properties are of the 2,3 type and contain $\mathrm{Fe}^{3+}$. An important ferrite is magnetite $\mathrm{Fe}^{2+} \mathrm{Fe}^{3+}{ }_{2} \mathrm{O}_{4}$, also referred as $\mathrm{Fe}_{3} \mathrm{O}_{4}$, which is the oldest known magnetic material. The name "magnetic" is derived from 
Magnesia, which is the region where magnetite was first discovered in Greece in the 6th century BC. It is interesting that magnetite, the magnetic material first reported in history, is currently an extremely active field in basic and applied research. This is certainly due to its fascinating magnetic and electric properties, associated with the coexistence of ferrous and ferric cations in equivalent crystal sites. A related magnetic material also particularly interesting is maghemite (or $\gamma-\mathrm{Fe}_{2} \mathrm{O}_{3}$ ), which possesses only $\mathrm{Fe}^{3+}$ and the same spinel structure often obtained by oxidation of magnetite. Since the cation to anion ratio is not $3 / 4$, it contains vacancies in a fraction of cation sites; by using the spinel formula, it can be represented as $\square_{1 / 3} \mathrm{Fe}^{3+}{ }_{8 / 3} \mathrm{O}_{4}$. Here, $\square$ stands for vacancies.

The crystal symmetry can be understood by considering a face centered cubic (fcc) lattice of oxygen, leading to two kinds of cation sites: 64 tetrahedral sites and 32 octahedral sites for a unit cell, which is formed by 8 times the basic formula $\mathrm{D}^{2+} \mathrm{T}^{3+}{ }_{2} \mathrm{O}_{4}\left(\mathrm{D}^{2+}\right.$ stands for a divalent cation, and $\mathrm{T}^{3+}$ for a trivalent cation). Only one-eighth of tetrahedral sites is occupied and half of octahedral sites as well. The space group is $\mathrm{Fd} 3 \mathrm{~m}$. This structure is shown in Figure 1.

In the spinel mineral $\mathrm{MgAl}_{2} \mathrm{O}_{4}, \mathrm{Mg}^{2+}$ cations occupy tetrahedral sites and $\mathrm{Al}^{3+}$ cations are found in octahedral sites, which appears as a stable arrangement, as far as divalent cations are surrounded by less anions (four anions in the tetrahedral site) than trivalent cations with higher electric charge and enclosed by 6 anions. This structure is known as the normal spinel, and is indicated by using the system $\left(\mathrm{D}^{2+}\right)\left[\mathrm{T}^{3+}{ }_{2}\right] \mathrm{O}_{4}$. Parenthesis indicates occupancy of tetrahedral sites, also known as " $\mathrm{A}$ " sites, and square brackets show cations on octahedral sites or " $\mathrm{B}$ " sites. A different cations distribution is $\left(\mathrm{T}^{3+}\right)\left[\mathrm{T}^{3+} \mathrm{D}^{2+}\right) \mathrm{O}_{4}$, where the divalent cation goes to an octahedral site and trivalent cations are found both on tetra and octahedral sites. This is the inverse spinel. An intermediate cation distribution has also been observed for some ferrites, $\left(D_{1-\delta} T_{\delta}\right)\left[D_{\delta} T_{2-\delta}\right] O_{4}$, where $\delta$ is the degree of inversion.

The cation distribution in spinels was a problem for some time, but it is now well understood. The involved energies are the elastic energy, associated with the lattice deformation produced by cation radii differences. The electrostatic energy, also known as the Madelung energy, which depends on the overall electric charge distribution; divalent cations on small sites and trivalent cations on larger sites should stabilize the spinel. The crystal field energy has also a large influence on cation site "preference," and it is related mainly to the geometry of $d$-orbitals (or electronic orbitals for nontransition cations). $d^{5}$ orbitals, for instance, can occupy both types of sites as these orbitals (in high-spin state) have spherical geometry (see Table 2).

\begin{tabular}{ccc}
\hline Tetrahedral & Octahedral & Undistinguished \\
\hline $\mathrm{Cd}^{2+}$ & $\mathrm{Co}^{2+}$ & $\mathrm{Fe}^{3+}$ \\
\hline $\mathrm{Zn}^{2+}$ & $\mathrm{Ni}^{2+}$ & $\mathrm{Mn}^{2+}$ \\
\hline & $\mathrm{Cu}^{2+}$ & $\mathrm{Mg}^{2+}$ \\
\hline & $\mathrm{Fe}^{2+}$ & $\mathrm{Mo}^{2+}$ \\
\hline & $\mathrm{Li}^{1+}$ & \\
\hline
\end{tabular}

Table 2. Cation preferences for spinel sites. Adapted from [2]. 

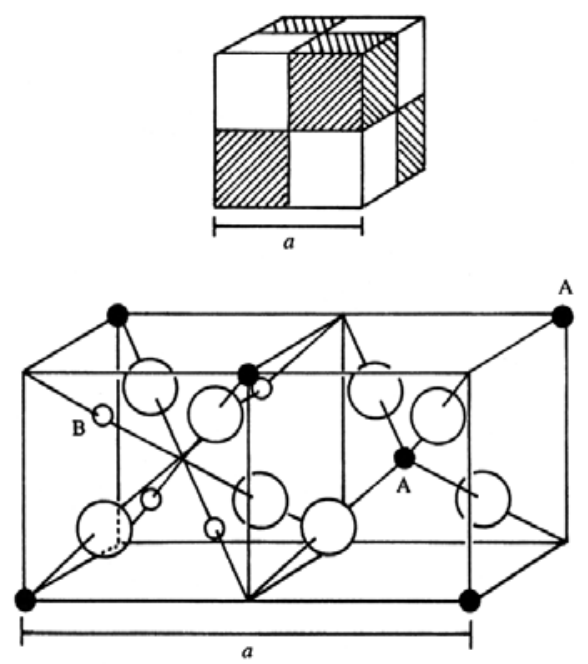

Figure 1. Representation of the spinel structure. The unit cell can be divided into octants (above). The detailed structure of two octants is illustrated below. Large spheres are oxygen, small black spheres indicate cations in tetrahedral sites, and small white spheres represent cations in octahedral sites [2].

An outstanding characteristic of the spinel structure is that it admits an extremely large variety of total solid solutions. The divalent cations in the spinel formula can be formed by a combination of two (or more) cations; $\mathrm{Fe}^{3+}$ can also by partially or totally substituted by another trivalent cation, always maintaining the spinel crystal structure. A very well studied system (or "family") is $\mathrm{Zn}-\mathrm{Ni}$ ferrites with the general formula $\mathrm{Zn}_{x} \mathrm{Ni}_{1-x} \mathrm{Fe}_{2} \mathrm{O}_{4}$, with $0 \leq x \leq 1$. The end compositions are nickel ferrite, $\mathrm{NiFe}_{2} \mathrm{O}_{4}$ (for $x=0$ ), and zinc ferrite, $\mathrm{ZnFe}_{2} \mathrm{O}_{4}(x=1)$. Nickel ferrite is an inverse spinel, while zinc ferrite is a normal one; due to the features of magnetic interactions in spinel ferrites, nickel ferrite is ferrimagnetic with a Curie point about $858 \mathrm{~K}$, while zinc ferrite is antiferromagnetic with a Néel temperature about $9 \mathrm{~K}$. The properties of this family of compounds can be "tailored" between these two extreme behaviors just by varying the chemical composition.

The magnetic structure and interactions in ferrites can be understood on the basis of superexchange interactions between two transition cations separated by an oxygen, as shown in Figure 2. The electron spin up in the $p$-orbital of the oxygen can occupy for a short period of time the empty half of the $d$-orbital in the transition cation at right, if the occupied state in this cation is spin down. As the $p$-orbital of oxygen exhibits, for the same given period of time, an available site for an electron with spin up, the electron of the transition cation on the left side of the oxygen can occupy this place, if its spin is pointing up. This mechanism leads therefore to an antiparallel arrangement of spins on the transition cations. In spinels, there are two sublattices, a tetrahedral one (with one cation per formula) and an octahedral one (with two cations per formula). In most cases, a resultant appears and most ferrites possess a net magnetization. The $\mathrm{Zn}$-Ni ferrite family is a very good example of the richness of magnetic properties and structure of ferrites. 


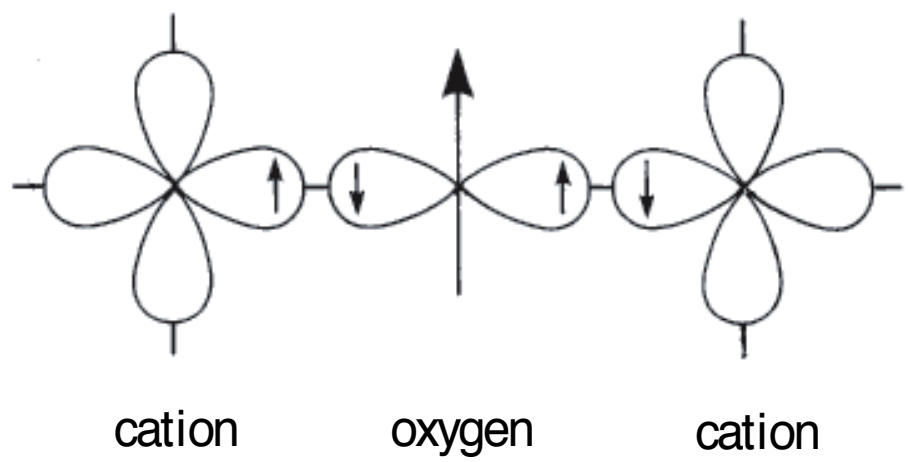

Figure 2. Superexchange interactions in ferrites.

The magnetic interactions in spinel ferrites can be easily understood by considering two B sites and one A site about an oxygen, see Figure 3. The center and right side of this figure, where the large circle represents the oxygen ion, the small circle above it stands for the cation on the A site, and the two small circles below represent $B$ sites. Note that this arrangement is $3 \mathrm{D}$, but for this schematic illustration, it has been simplified on a plane. Nickel ferrite is an inverse spinel, $\mathrm{Ni}^{2+}$ goes to $\mathrm{B}$ sites, one ferric ion occupies the other $\mathrm{B}$ site, and the other $\mathrm{Fe}^{3+}$ is located on the A site. All magnetic interactions between cations are antiparallel (superexchange).
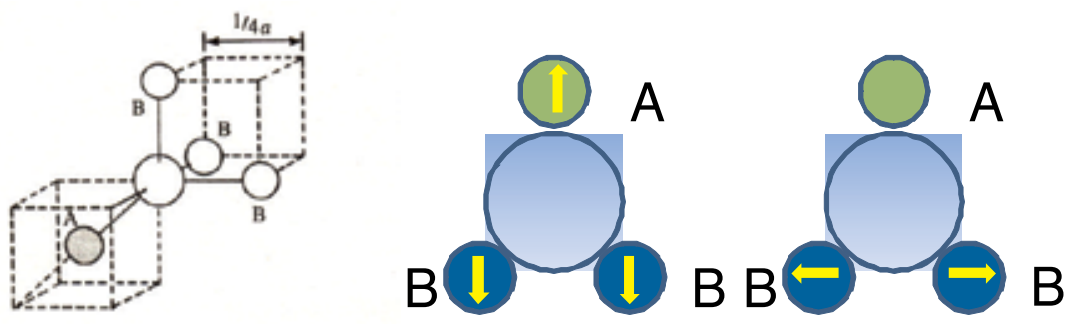

Figure 3. Schematic spin arrangements in spinel ferrites. Left: a section of the crystal structure showing the cation occupancy about an oxygen. Center: the ferrimagnetic spin orientation of the crystal sites for nickel ferrite. Right: the antiferromagnetic structure for the zinc ferrite.

There are A-O-B interactions between the cation on A site and the cations on B sites, and an $\mathrm{B}-\mathrm{O}-\mathrm{B}$ interaction between the cations on the two B sites. However, due to the axial symmetry of oxygen $p$-orbitals, superexchange interaction becomes more efficient when cations and oxygen are in an axial arrangement. This makes a strong difference between interactions; A$\mathrm{O}-\mathrm{B}$ interaction is far more efficient than $\mathrm{B}-\mathrm{O}-\mathrm{B}$ interactions since the latter has a $90^{\circ}$ angle, while the former is closer to $180^{\circ}$. As a result, both spins on B sites are antiparallel to the A spin. The magnetic moment of $\mathrm{Fe}^{3+}$ on $\mathrm{A}$ site cancels with the magnetic moment of $\mathrm{Fe}^{3+}$ on $\mathrm{B}$ site, and the resulting magnetic moment is the spin value for nickel $\mathrm{Ni}^{2+}(2.3 \mathrm{Bohr}$ magnetons), as shown in Figure 3 center. This is a strong interaction; the Curie transition is about $858 \mathrm{~K}$. 
In the case of zinc ferrite, iron ions occupy the B sites and zinc is located on the A site. Since $\mathrm{Zn}^{2+}$ is a $3 d^{10}$ ion, it has no magnetic moment, and the only interaction is $\mathrm{B}-\mathrm{O}-\mathrm{B}$, leading to an antiparallel arrangement of spins (Figure, 3 right). The spins are identical, and the material becomes antiferromagnetic. The Néel temperature is about $9 \mathrm{~K}$, and this clearly illustrates the weakness of this superexchange interaction, as compared with $\mathrm{AOB}$ of the nickel ferrite case. Based on transition temperatures, A-O-B interaction is roughly 95 times stronger than $\mathrm{B}-\mathrm{O}-$ B interaction.

\section{Electron paramagnetic resonance}

Electron paramagnetic resonance (EPR) or electron spin resonance (ESR) is a technique of observing resonance absorption of microwave power by unpaired electron spins aligned with a magnetic field [4]. The electron paramagnetic resonance (EPR) spectroscopy is similar to the nuclear magnetic resonance (NMR) except for the fact that EPR arises from the interaction between unpaired electrons and electromagnetic radiation.

The history of EPR can be told beginning with the Stern-Gerlach experiment in 1921. They observed that a beam of silver atoms split into two lines when it was subjected to a magnetic field. In contrast with the line splitting in optics found by Zeeman in 1896, these phenomena could not be explained by the angular momentum of the electrons. At the time quantum mechanics was still developing and was still an emerging field. Three more years passed before Uhlenbeck and Goudsmit found an explanation for these anomalous Zeeman effect when they postulated the spin $[5,6]$.

The development of the EPR was furthered by the World War II since after the war was over, there was available a great amount of microwave instruments from the radar equipment used in the war. It was in 1945 when a Russian physicist, Zavoisky, observed the first EPR spectrum. $\mathrm{He}$ observed a radiofrequency absorption line from a $\mathrm{CuCl}_{2} \cdot 2 \mathrm{H}_{2} \mathrm{O}$ sample. One year later, the first NMR spectrum was observed and through the first decade both techniques developed simultaneously. Nevertheless, EPR spectroscopy had a few challenges from lack of microwave components and limited microwave power to expensive instrumentation and consequently was left behind by NMR for a couple of decades [4-6].

It was until the 1980s that the instrumentation became cheaper and more manageable and the first pulse EPR was released to the market. A decade later the high field spectrometer was released and since then the interest in EPR as a characterization technique has considerably increased.

The spin angular momentum $S$ gives rise to a magnetic moment $\mu=-\mathrm{g} \mu_{\mathrm{B}} S$, where $g$ is the $\mathrm{g}$ factor; its value for free electrons is $g=2.0023193043617$ and $\mu_{\mathrm{B}}$ is the Bohr magneton. When the electrons are subjected to an external field $H$ (it is customary to place the field along the $z$ axis), the energy levels of the degenerate spin states split depending on their quantum magnetic moment $m_{\mathrm{s}}= \pm 1 / 2$ and the strength of the magnetic field as shown in Figure 4 [4-6]. 


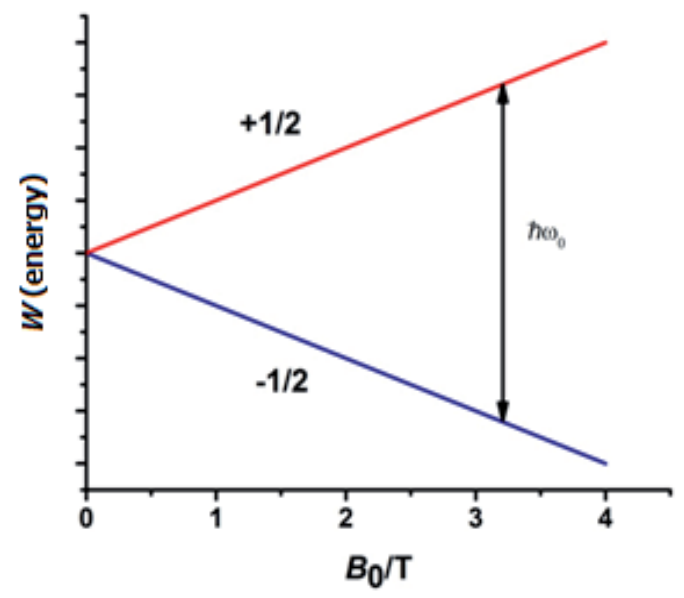

Figure 4. Splitting of the energy levels of an electron spin in a magnetic field at the resonance frequencies.

When the irradiation at a given frequency $\omega_{0}$ is the same as the energy difference $\Delta E$ between both states, a resonant absorption takes place. This frequency is named the Larmor frequency $\omega_{0}=-\gamma H_{0}$ after J. Larmor [6]:

$$
\Delta W=\hbar \omega_{0}=g \mu_{B} H
$$

The detection of that absorption is the most important principle of this spectroscopy.

For temperatures above the Curie temperature, the magnetic moments in an external field obey the Bloch equations. These equations follow the Larmor theorem where the motion of the magnetic moments in a magnetic field originates a torque [6-9]:

$$
\hbar \frac{d \mathbf{M}}{d t}=\mathbf{M} \times \gamma \mathbf{H}
$$

To detect the absorption, there is an oscillating microwave field $\mathbf{H}_{1}=\left(H_{x \prime} H_{y}, 0\right)=\left(H_{1} \cos \left(\omega_{\mathrm{mw}} t\right)\right.$, $\left.H_{1} \sin \left(\omega_{\mathrm{mw}} t\right), 0\right)$ aside from the external magnetic field along the $z$ axis. This magnetic field deviates the magnetization away from its equilibrium position. To describe accurately the motion of the magnetization vector, it is important to take into account the relaxation effects. The relaxation time has two components, the longitudinal relaxation time, which is the average time in which the magnetization vector returns to its thermal equilibrium state, and the transverse relaxation time, which characterizes the loss of coherence in the transverse plane due to spin interactions [5-8].

The evolution of the magnetization as a function of time and its dependence of the magnetic field is given by the equation motion derived by F. Bloch [5-6]: 


$$
\hbar \frac{d \mathbf{M}}{d t}=\mathbf{M}(t) \times \gamma \mathbf{H}(t)-\mathbf{R}\left(\mathbf{M}(t)-\mathbf{M}_{0}\right)
$$

where $\gamma$ is the gyromagnetic ratio, $\mathbf{H}=\left(H_{1} \cos \left(\omega_{\mathrm{mw}} t\right), H_{1} \sin \left(\omega_{\mathrm{mw}} t\right), H_{z}\right)$, and $\mathbf{R}$ is the relaxation tensor given by [6]:

$$
\mathbf{R}=\left(\begin{array}{ccc}
1 / T_{2} & 0 & 0 \\
0 & 1 / T_{2} & 0 \\
0 & 0 & 1 / T_{1}
\end{array}\right)
$$

Relaxation phenomena need to be taken into account to describe the motion of the magnetization vector. $T_{1}$ is the longitudinal relaxation time; this parameter characterizes the process that makes the magnetization vector return to its thermal equilibrium. $T_{2}$ is the transverse relaxation time which describes the loss of coherence in the transverse plane due to spin-spin interactions.

Electron paramagnetic resonance is a very useful technique to study the properties of bulk paramagnetic compounds including their transitions to the magnetic ordered state. Below this temperature, ferromagnetic resonance (FMR) or antiferromagnetic resonance (AFMR) are detected. EPR in paramagnetic samples give information such as the resonance active ion valence and the symmetry of the ligand environment. In the case of nanoparticles, the theory of EPR is not quite the same as in bulk; however, a study of the thermal variations of EPR spectra can be very informative because of the high sensibility of the EPR technique [10].

Let's recall that magnetic resonance is observable only in materials that contain a sufficient number of permanent magnetic dipoles; if the origin of these dipoles is electronic, then the resonance is detectable with a population even fewer than $10^{11}$ dipoles [8].

The first paramagnetic resonance absorption in metals due to the conduction electrons was observed by Feher and Kip [8]. The conduction electrons have an effect on the shape and intensity of the resonance lines. However, until the theoretical study of Dyson, there were not many studies in conduction electron paramagnetic resonance in metals [11].

In a metal, the electrons are assumed to diffuse as free particles and the magnetic moments of each of them can be seen as free-particle moments. When the metal is placed in a radiofrequency electromagnetic field and at the same time in a perpendicular uniform magnetic field, a certain macroscopic magnetization is created as a result of the magnetic moments of the conduction electrons. The penetration of the radio frequency field into the metal is modified by the magnetization. Actually, only the layers near the surface contribute, since the excitation field $H_{1}$ penetrates only a small depth into the metal. This is called the skin effect [11]. The magnetization $M$ shows a resonant behavior and becomes large when the frequency of the field is nearly equal to the resonant frequency. The absorption field observed is a measure of the total energy absorbed in the metal both by eddy currents and by the resistive out of phase component of the magnetization. 
There are four assumptions which need to be taken into account to study the dependence of the line width $[8,11]$ :

1. The electrons which carry the magnetization in a metal are assumed to lie at the top of the Fermi distribution of the conduction electrons and to move with constant velocity.

2. Each electron moves as an independent classical particle with random changes in their direction.

3. The spin of each particle is a quantum independent variable. It is unaffected by collisions and only the local magnetic field has an effect on them.

4. Given any time interval $t$ and $U$, a volume relaxation time there is a probability $\sim \exp (-t / U)$ that the spin state of an electron will not be randomly distributed by collisions during this interval.

These assumptions give as a result a finite line width proportional to $1 / U$ in the resonance signal from nondiffusing electrons. The penetration of the radio frequency field into the metal will be limited by the skin depth, which is given by [11]

$$
\delta=\left(c^{2} / 2 \pi \sigma \omega\right)^{1 / 2}
$$

where $c$ is the speed of light, $\sigma$ is the conductivity of the metal, and $\omega$ is the radio frequency. If the skin depth is larger than the main free path, we are in the domain of the classical skin effects; if it is equal to the main free path, then we are in the anomalous case. In fact, unlike paramagnetic insulators, in which the varying part of the magnetization depends on the magnitude of the applied oscillating field, in metals, there is also a dependence of the local values of the oscillating field on the magnetization because of the skin depth. A typical spectrum (metallic $\mathrm{Na}$ ) is shown in Figure 5.

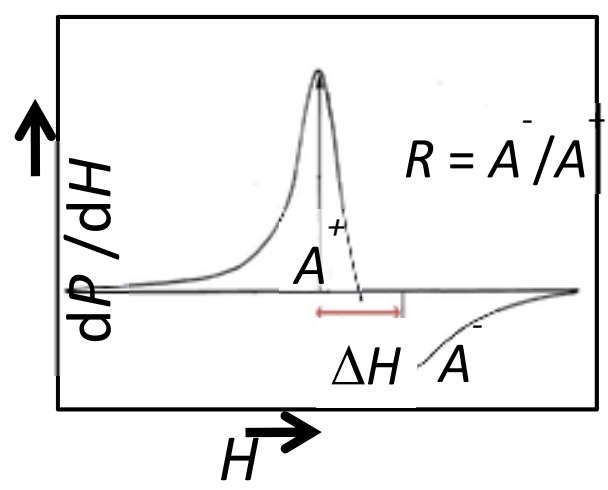

Figure 5. Typical first derivative EPR spectrum observed in colloidal samples of $\mathrm{Na}$, with mean diameter particle small compared to the skin depth. The basic parameters and the definition of $R$ are indicated. Adapted from Feher and Kip [8]. 
The magnetic susceptibility in metals has a diamagnetic component due to the circulation of electrons in the field $H$. This is opposed by the normal paramagnetic component due to unpaired electrons. This is related with another EPR parameter that has been studied in metals: the $g$ factor $[8,12]$. The relation between the resonance values of the constant magnetic field $H_{0}$ and the frequency is determined by the magnitude of the $g$ factor of the conduction electrons. The existence of internal interactions in a metal leads to a shift in the value of the $g$ factor.

Experimentally, the $g$ factor has been measured in different paramagnetic metals giving rise to values very close to the $g$ free electron value $g=2.0023$ as shown in Table 3 . This is a measure of the spin-orbit coupling. It has been possible to study $S$-state ions in metals in order to understand the interaction between the conduction electrons and the inserted ions.

\begin{tabular}{ll}
\hline Metal & Experimental values $\Delta g=g-2.0023$ \\
\hline $\mathrm{Li}$ & $\Delta g<10^{-4}$ \\
\hline $\mathrm{Na}$ & $\Delta g=-(8 \pm 2) \times 10^{-4}$ \\
\hline $\mathrm{Be}$ & $\Delta g=+(9 \pm 2) \times 10^{-4}$ \\
\hline
\end{tabular}

Table 3. Experimental values for the $g$ shift. Adapted from Feher and Kip [8].

EPR spectroscopy has made contributions in understanding the bonding and the electronic structure of molecular species with metal-metal bonds. Some of the information obtained through this technique includes determining whether unpaired electrons reside in metal-based or ligand-based molecular orbitals, giving information of the metal center's total electronic spin, which also provides information of its oxidation state, broadly have information of the distribution of the unpaired electrons between metals and organic ligands [13].

\subsection{Magnetic nanoparticles}

Iron-based oxide nanoparticles materials have been widely studied because of their technological importance. $\gamma-\mathrm{Fe}_{2} \mathrm{O}_{3}$ is a very popular material for magnetic tape purposes. Another popular material in high density recording media is $\mathrm{BaFe}_{12} \mathrm{O}_{19}$ and its substituted derivatives since it can be doped with other cations in order to reduce their magnetocrystalline anisotropy [10].

Bulk $\mathrm{Fe}_{2} \mathrm{O}_{3}$ exists as a ferrimagnetic $\gamma-\mathrm{Fe}_{2} \mathrm{O}_{3}$ (maghemite) or antiferromagnetic $\alpha$ - $\mathrm{Fe}_{2} \mathrm{O}_{3}$ (hematite). Maghemite nanoparticles are a representative model for the experimental study of nanoparticles and for testing theoretical concepts. In contrast, bulk hematite is antiferromagnetic below the Morin temperature and weakly ferromagnetic above this temperature [10].

At room temperature, the EPR of $\gamma-\mathrm{Fe}_{2} \mathrm{O}_{3}$ and $\mathrm{BaFe}_{2} \mathrm{O}_{4}$ and nanoparticles show a tow line pattern as shown in Figure 6 [9], which is typical of superparamagnetic resonance in contrast with the $\mathrm{BaFe}_{12} \mathrm{O}_{19}$ where the narrow line is more pronounced (as shown in Figure 6c). In these sample, the line width $\Delta H \sim 120$ Oe and $g=2.00$; this value of the $g$ factor is typical paramagnetic. 


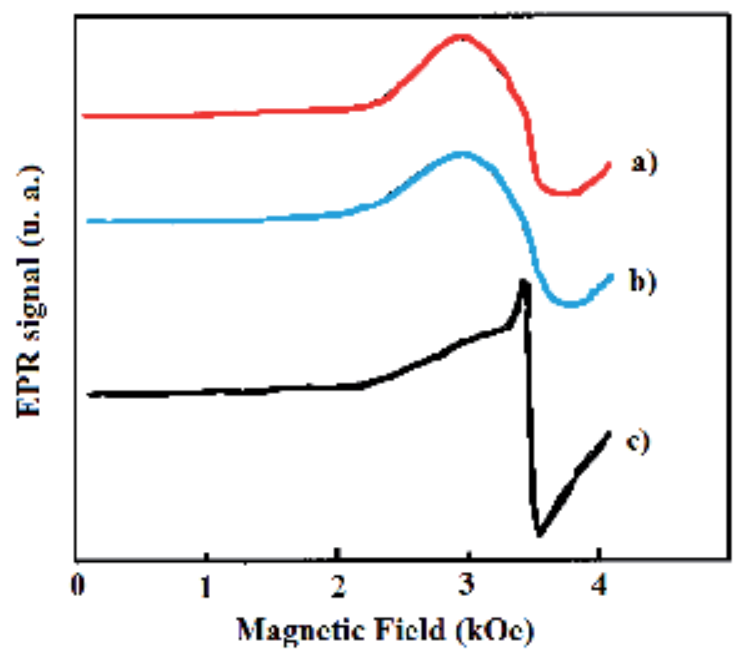

Figure 6. Room temperature EPR spectra of nanoparticles: (a) $\mathrm{Fe}_{2} \mathrm{O}_{3}$, (b) $\mathrm{BaFe}_{2} \mathrm{O}_{4}$, and (c) $\mathrm{BaFe}_{12} \mathrm{O}_{19}$. Adapted from Koksharob et al. [10].

It is shown in Figure 7a that below $100 \mathrm{~K}$, the broad line 1 shows a typical superparamagnetic (SPM) behavior of single-domain particles in the absence of transitions to a magnetic ordered state. SPM behavior will be discussed in the following section. Below $50 \mathrm{~K}$, new resonances appear such as 2 and 3. Point 2 is a typical paramagnetic resonance signal. On the other hand, point 3 is characteristic of a phase transition $\alpha-\mathrm{Fe}_{2} \mathrm{O}_{3}$ of the hematite to antiferromagnetic phase.
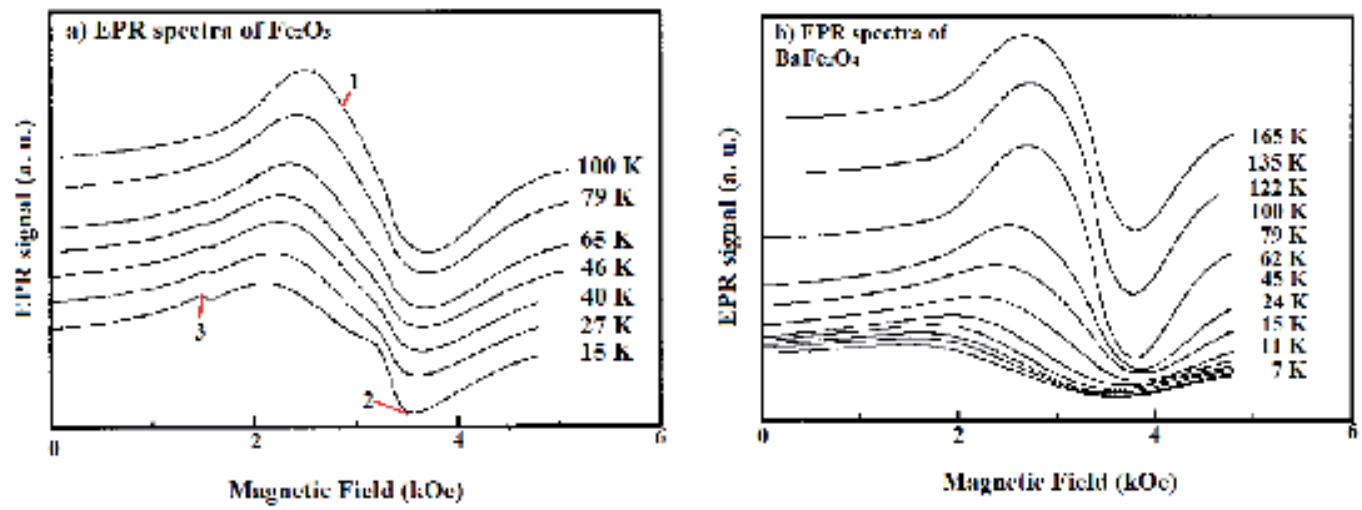

Figure 7. (a) EPR spectra of $\mathrm{Fe}_{2} \mathrm{O}_{3}$ nanoparticles; (b) EPR spectra of $\mathrm{BaFe}_{2} \mathrm{O}_{4}$ nanoparticles. Adapted from Koksharob et al. [10].

The bulk thermodynamics phase diagram [14] shows several interchangeable iron oxide phases. For nanoparticles, the mutual phase transition should be easier. For this reason, it is accurate to expect a multiphase composition in $\mathrm{Fe}_{2} \mathrm{O}_{3}$ nanoparticles. In point 3 , the value $g$ is 4.03 , which is characteristic of a high spin state $\mathrm{Fe}^{3+}$ in the rhombic crystal field. The antifer- 
romagnetic transition is associated with the $\alpha-\mathrm{Fe}_{2} \mathrm{O}_{3}$ phase. In rhombohedral $\alpha-\mathrm{Fe}_{2} \mathrm{O}_{3}$ as well as in $\gamma-\mathrm{Fe}_{2} \mathrm{O}_{3}$, there is a departure from axial symmetry. The paramagnetic signal in point 2 is attributed to the $\gamma-\mathrm{Fe}_{2} \mathrm{O}_{3}$ phase; it has a $g$ value of 2.02, and it is due to the octahedral symmetry sites of $\mathrm{Fe}^{3+}$ in the spinel structure.

Bulk $\mathrm{BaFe}_{2} \mathrm{O}_{4}$ is nonferrimagnetic. On the contrary, $\mathrm{BaFe}_{2} \mathrm{O}_{4}$ demonstrate an EPR anomaly near $125 \mathrm{~K}$ that could indicate the presence of a $\mathrm{Fe}_{2} \mathrm{O}_{3}$ phase. On the other hand, bulk hexaferrite $\mathrm{BaFe}_{12} \mathrm{O}_{19}$ is a representative case of FMR because of its very high uniaxial anisotropy. However, in the nanoparticles, the contribution of the particle's surface is appreciable and can reduce the total anisotropy energy as well as crystalline defects due to stress and strain. The EPR spectra reveal the effect of superparamagnetic fluctuations narrowing the resonance [10] and will be discussed in the next section.

\section{Spin resonance in superparamagnetic phases}

The superparamagnetic (SPM) state is characterized by random fluctuations of the magnetization due to thermal excitation. It appears in ferro- and ferrimagnetic particles, which are sufficiently small and thus single domain, typically in the nanometric range (1-100 nm). It is important to note that in contrast with the paramagnetic state, characterized by random fluctuations of individual, noninteracting spins in the SPM phase, these fluctuations involve the whole magnetization vector, which is the sum of all individual moments of the particle. As SPM occurs at temperatures below the Curie transition, exchange interaction is effective, and spins are therefore coupled. It is by this reason that some authors describe SPM as "macro-spin" fluctuations.

A simple approach to SPM can be obtained by considering the magnetocrystalline anisotropy energy, $E_{\mathrm{K}}=K V$, for a nanoparticle of volume $V$ and anisotropy constant $K$. When this product becomes small and comparable to the thermal energy $E_{\mathrm{T}}=k_{\mathrm{B}} T$ (where $k_{\mathrm{B}}$ is the Boltzmann constant), the magnetization oscillates by thermal excitations and easy directions vanish. A simplified graphical model is shown in Figure 8. A uniaxial nanoparticle has two easy magnetization directions, i.e., two magnetization orientations that lead to a minimum in anisotropy energy. These orientations are separated by an energy barrier $E_{\mathrm{K}}=K V$. Superparamagnetism appears when the thermal energy becomes comparable to anisotropy energy, and therefore the energy barrier is dominated by thermal oscillations.

A nanoparticle of a given composition and constant volume typically shows a ferro/ferrimagnetic behavior at low temperatures and can become superparamagnetic as $T$ increases. At low temperatures, $K$ is large and $T$ is small, and the opposite is true as $T$ increases. The temperature at which SPM occurs is known as the blocking temperature, $T_{\mathrm{B}}$.

The probability of reversal of magnetization is given by the Néel-Arrhenius or Néel-Brown relation $[15,16]$ : 


$$
\tau_{\mathrm{m}}=\tau_{0} \exp \left(K V / k_{\mathrm{B}} T\right)
$$

where $V$ is the volume of the nanoparticle, $K$ is the anisotropy constant, $T$ is the temperature, $\tau_{\mathrm{m}}$ is the average length of time for a magnetization reversal to occur, and $\tau_{0}$ is a characteristic time constant related with the gyromagnetic precession, known also as the attempt time for a given material. Typical values are in the $10^{-9}$ to $10^{-10} \mathrm{~s}$ range. Under an applied magnetic field, the blocking temperature variations can be expressed (for uniaxial materials) as [17]

$$
T_{\mathrm{B}}(H)=\frac{K V}{k_{\mathrm{B}} \ln \left(\tau_{\mathrm{m}} / \tau_{0}\right)}\left[I-\left(\frac{H}{H_{k}}\right)\right]^{2} .
$$

where $H$ is the applied field, $H_{\mathrm{K}}$ is the anisotropy field, and $a$ has a typical value of 1.5 [18].

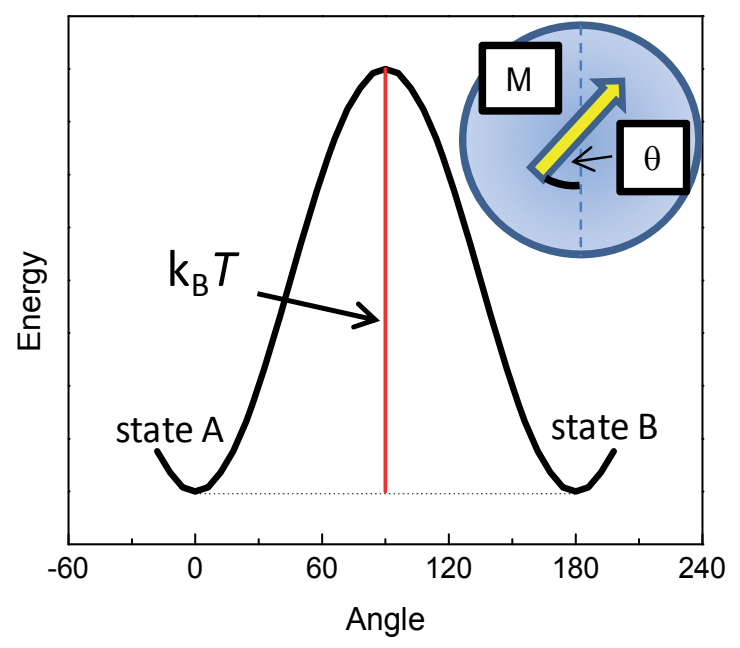

Figure 8. Schematics of the energy in a single-domain particle (with uniaxial anisotropy) as a function of the angle between easy axes and the magnetization direction. For SPM nanoparticles, the energy barrier is comparable to the thermal energy, thus resulting in magnetization random oscillations.

It is interesting to note that the blocking temperature, $T_{\mathrm{B}}$, depends on the time window, $t_{\mathrm{w}}$, of the particular experimental technique. $t_{\mathrm{w}}$ is the effective length of time during the measurement. If $t_{\mathrm{w}} \gg \tau_{\mathrm{m}}$ the magnetization will show several reversals and the state of the particle will we taken as SPM. On the contrary, if $t_{\mathrm{w}} \ll \tau_{\mathrm{m}}$, magnetization will exhibit a stable state, and therefore the material will be considered as in the ordered phase. The time window for $T_{\mathrm{B}}$ determination from magnetization measurements in the ZFC-FC technique (in a SQUID machine) is about $10^{-1} \mathrm{~s}$, while for Mössbauer spectroscopy or spin resonance techniques $t_{\mathrm{w}}$ is about $10^{-6} \mathrm{~s}$. A model to describe the crossover from the SPM state to the blocked phase in magnetic nanoparticles has been recently proposed [19]. It is based on the Stoner-Wohlfarth 
model and also assumes noninteracting nanoparticles with uniaxial anisotropy and uniform random reversal of magnetization.

SPM materials show no hysteresis in $M$ vs. $H$ plots, i.e., absence of both coercive field and remanent magnetization, as illustrated in Figure 9. For an ensemble of noninteracting particles at temperatures low enough $\left(T_{\mathrm{B}}<\mathrm{T}<K V / 10 k_{\mathrm{B}}\right)$, a simple model for the magnetization behavior, based on the paramagnetic theory, is

$$
M(H) \approx n \mu \tanh \left(\frac{\mu_{0} H \mu}{k_{B} T}\right)
$$

At higher temperatures $\left(T>K V / k_{\mathrm{B}}\right)$,

$$
M(H) \approx n \mu L\left(\frac{\mu_{0} H \mu}{k_{B} T}\right)
$$

where $n$ is the density (atoms/volume), $\mu$ is the magnetic moment (the "macrospin"), $M$ is the magnetization, and $L$ is the Langevin function, $L(x)=1 / \tanh (x)-1 / x$, with $x=\mu_{0} H \mu / T k_{\mathrm{B}}$. This behavior is very convenient for many applications, especially in biomedicine, where magnetic nanoparticles manifest a magnetization only when subjected to a magnetic field, thus avoiding interparticle interactions (which could lead to aggregation) at $H=0$. In contrast, SPM is useless for magnetic recording since the absence of a remanent state eliminates any possibility of memory.

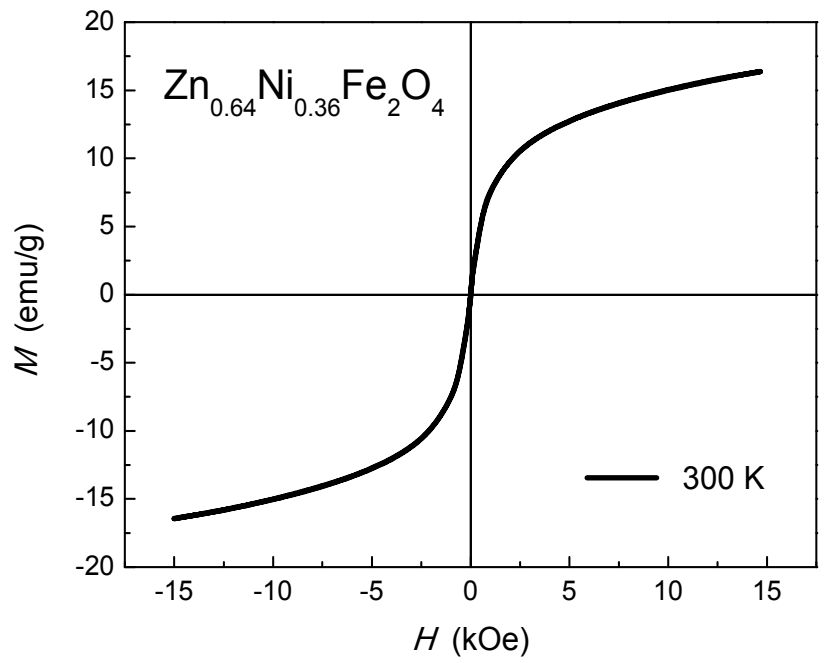

Figure 9. Hysteresis loop of $\mathrm{ZnNi}$ nanoparticles in the superparamagnetic phase [20]. 
The kinetic approach [21,22] is known with this name because it makes use of the FokkerPlanck-type equation used by Brown [16] to analyze viscosity problems. The main criticism for this approach is that it predicts an increase of the line width with temperature, which is opposite to the large majority of experimental results. On some cases, a tendency to show a constant line width has been observed, but to our knowledge, no significant increase in $\Delta H$ has been reported in SPM phases as $T$ increases.

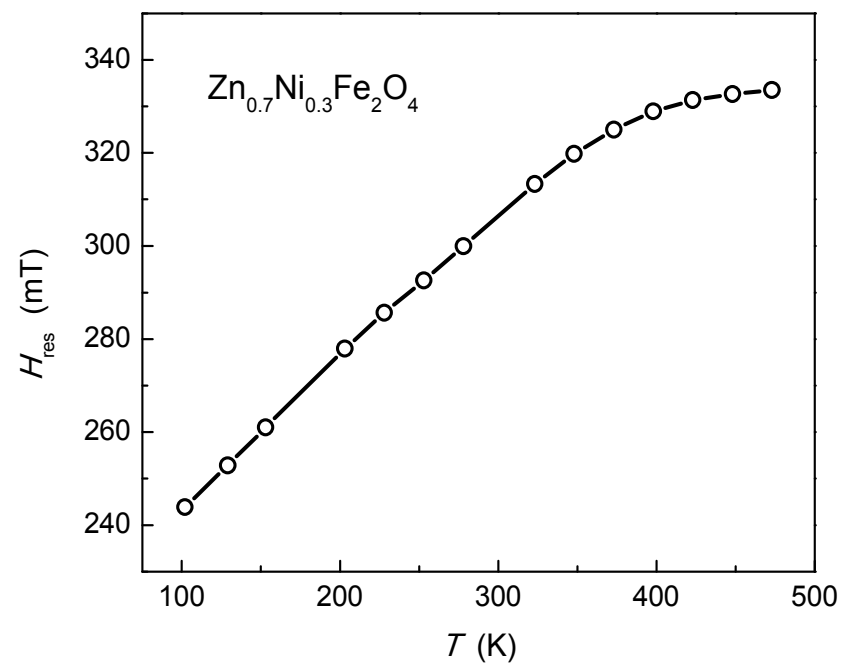

Figure 10. Resonance field as a function of temperature for Zn-Ni ferrite NPs, at $f=9.45 \mathrm{GHz}$ [23].

The SPM state has elicited the proposal of a number of theoretical models to account for its magnetization, and more specifically to understand the features of the resonance spectrum. Some models $[24,25]$ discuss the longitudinal modes, which are more directly associated with magnetization reversal. For spin resonance, however, it is the transverse modes, which are more directly related with magnetization precession and therefore with magnetization resonance. There are two main approaches, the so-called "statistical" approach and the "kinetic" approach.

In the statistical approach [26,27], which has a phenomenological character, the resonance conditions are evaluated by averaging over the equilibrium statistical distribution of all possible directions of particle magnetization moment. Typically, this model assumes that the magnetocrystalline anisotropy is smaller than the interaction energy between the magnetization and the applied field. While modeling can effectively represent some of the experimental results, the averaging of the magnetic anisotropy is not fully clear.

Another approach is also based on the Landau-Lifshitz equation of magnetization dynamics. In this model [26], $\boldsymbol{H}$ is changed by $\boldsymbol{H}_{\mathrm{oc}}$ to take into account both the external field and the effective field of the intrinsic anisotropy of the NPs. The dependence of the line width with temperature is expressed by 


$$
\Delta H=\Delta H_{T} L(X)
$$

where $\Delta H_{\mathrm{T}}$ is the limiting value of the line width for bulk particles and $L(X)$ is a Langevin type $(L(X)=\cot \mathrm{h} X-1 / X)$ of a phenomenological temperature factor $X$ (see Berger et al. [26] for more details).

Experimentally, the behavior of the line width and the resonance field of SPM phases of metallic and oxide NPs is quite clear; as temperature increases, the line width decreases and the resonance field increases, as illustrated by means of some recent results, see Figure 11 [23].

As temperature increases, the resonance field increases from $243 \mathrm{mT}$ for $102 \mathrm{~K}$ to $333 \mathrm{mT}$ for $473 \mathrm{~K}$. This is due to the fact that for temperatures below the Curie transition, there is an internal field formed by all the possible contribution to the ordered state:

$$
H_{\mathrm{int}}=H_{\mathrm{exch}}+H_{\mathrm{anis}}+H_{\mathrm{dem}}+\ldots
$$

where $H_{\text {exch }}$ is the exchange interaction, $H_{\text {anis }}$ is the anisotropy field, and $H_{\text {dem }}$ is the demagnetization field. As a result, the magnetization vector is subjected to a total field composed by the internal field and the applied field,

$$
H_{\text {tot }}=H_{\text {appl }}+H_{\text {int }}
$$
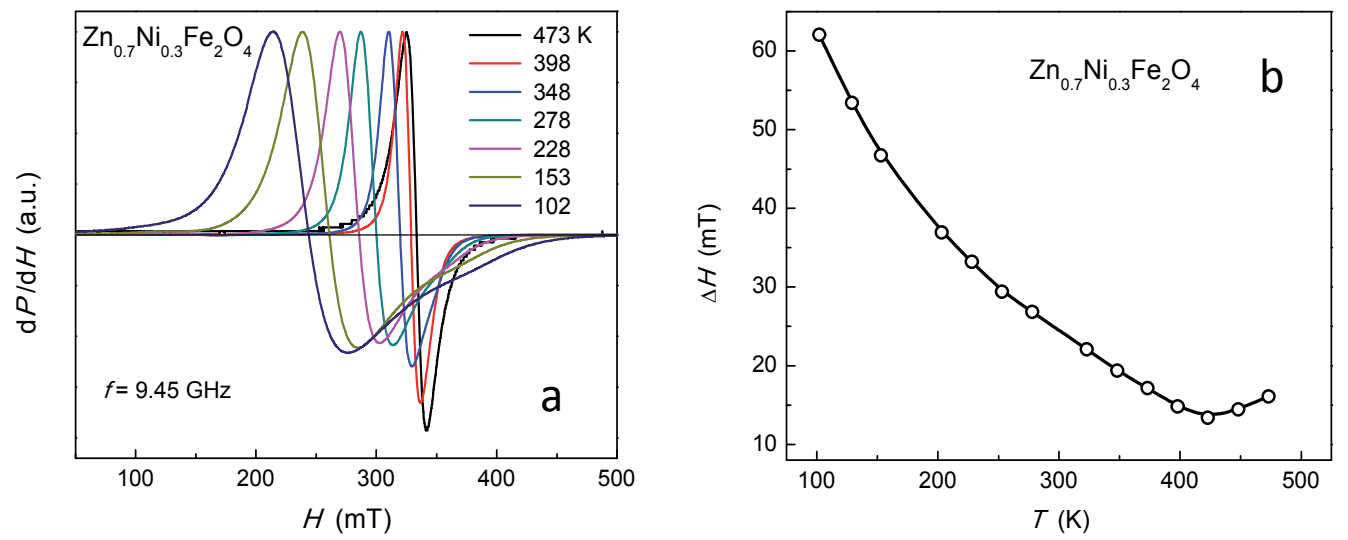

Figure 11. (a) ESR results on $\mathrm{Zn}_{0.7} \mathrm{Ni}_{0.3} \mathrm{Fe}_{2} \mathrm{O}_{4}$ ferrite NPs at a microwave frequency of $9.45 \mathrm{GHz}$, in the 102-473 temperature range [22]. Signals were normalized to have the same value for the maximum (section of the resonance signal for $H<H_{\text {res }}$ ) to facilitate comparisons; (b) temperature behavior of the line width (peak to peak value) [23].

In order to fulfill the resonance conditions (Larmor equation), a smaller external field is required. In the paramagnetic state, thermal energy has overwhelmed the exchange coupling, and the resonance field is identical to the applied field. On the other hand, it is interesting to 
note again that in the SPM state, the exchange interaction is fully effective, and it remains active up to the Curie transition.

In SPM phases, $H_{\text {anis }}$ should be small and decreasing as $T$ increases and thermal energy progressively overwhelms it, but it certainly retains some influence, especially at temperatures close to $T_{\mathrm{B}}$. The transition from the ordered (ferrimagnetic) to the SPM phase is essentially continuous. This is more evident in the $H>H_{\text {res }}$ section of the resonance signal, as this section exhibits a larger broadening and becomes asymmetric. As we will see, this section of the signal is also associated with the magnetocrystalline anisotropy in the case of ferrimagnetic phases.
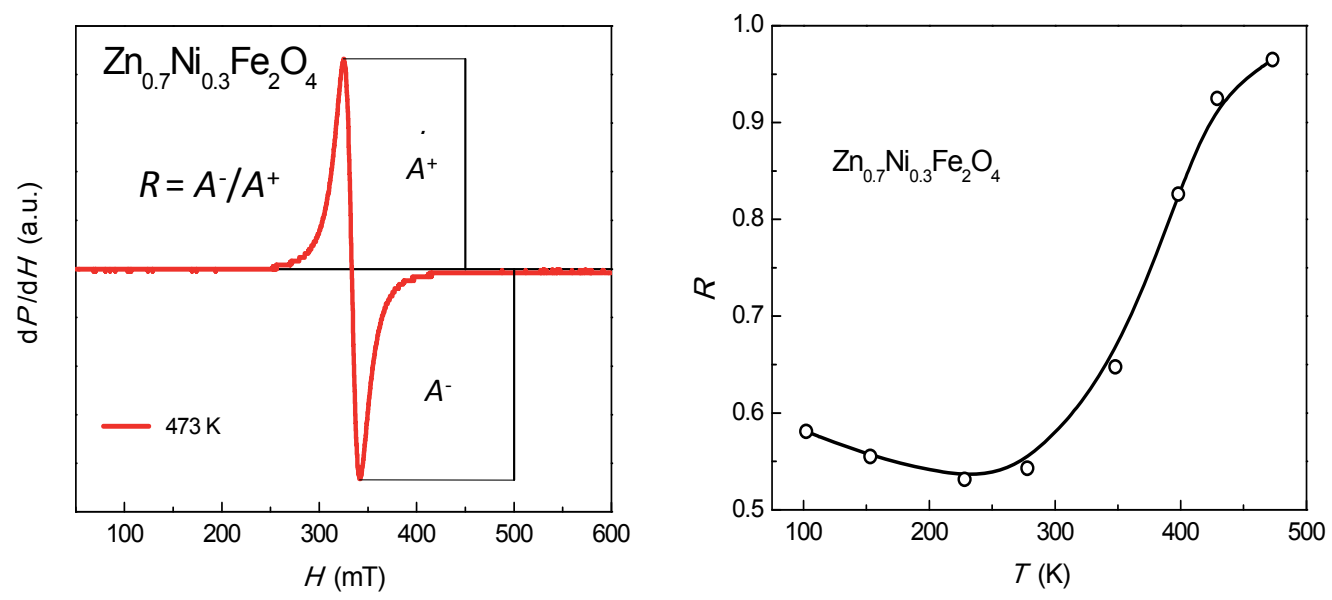

Figure 12. (a) Definition of the $R$ symmetry parameter, as the ratio of resonance signal amplitude $L_{\mathrm{A}}\left(H>H_{\text {res }}\right)$ to $L_{\mathrm{B}}(H$ $<H_{\mathrm{res}}$; (b) behavior of $R$ for the measured temperature range [23].

As an attempt to get more insight into the changes associated with the SPM phase, we have proposed a parameter $R$, which measures the symmetry of the resonance signal. This parameter is defined as the ratio $R=A^{-} / A^{+}$, between the amplitude of the section in the negative part of the spectrum, $A^{-}$, and the amplitude of the positive part, $A^{+}$, as shown in Figure 12. In many ferrite NPs, $R$ has been observed to tend to unity as temperature increases and the ferrite progresses to the paramagnetic phase.

\section{Ferromagnetic resonance}

While the physical principals behind EPR experiments are due to the Zeeman effect, ferromagnetic resonance has a different description because FMR arises from the precessional motion of the whole magnetization $\mathbf{M}$ of a ferromagnetic material in an external magnetic field $\mathbf{H}$ as shown in Figure 13 [27]. 

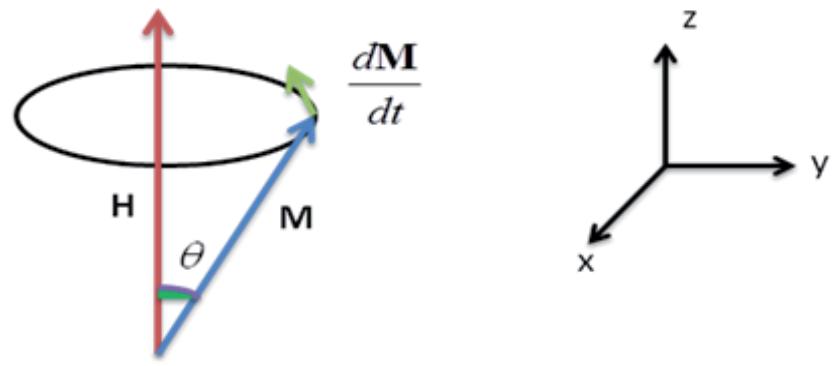

Figure 13. Magnetization vector $M$ precessing around an external field $H$.

Actually ferromagnetic resonance at microwave frequencies is similar in principle to nuclear spin resonance. The total electron magnetic moment of the sample precesses around the direction of the external field and the absorption is due to the energy absorbed from the $r f$ transverse field when its frequency is equal to the precessional frequency $[27,28]$.

The ferromagnetic resonance was unknowingly discovered by V. K. Arkad'yev when he observed the absorption of ultrahigh frequency radiation by ferromagnetic materials. However, it was officially observed by Griffiths in the Clarendon Laboratory in Oxford in 1946 and then confirmed by Yager and Bozorth who found a sharp peak in a supermalloy (Ni 75\%-Fe $20 \%-\mathrm{Mo} 5 \%$ ) sample for a field strength near $5 \mathrm{kOe}$ with a 24,000-Mc/s frequency [28].

Ferromagnetic resonance was theoretically discussed before it was knowingly observed, particularly in the paper written by Landau and Lifshitz in Kharkov in 1935. C. Kittel provided a more complete theoretical formulation in 1951 [27,28]. The resonant absorption in ferromagnetic metals for a given frequency is controlled by an effective field $H_{\text {eff }}$ which is the sum of an external applied field and the contributions of internal magnetization, while in EPR the effective field in which the absorption takes place is the same as the external applied field [27-29].

From the theoretical point of view, the ferromagnetic resonance is described by the FMR equations. These are differential equations that connect time derivatives of the magnetization components with the components of the magnetization, the external field, the static magnetic susceptibility, and the time relaxation $[9,27]$. The FMR equations are different depending on the temperature regime which one is working in. Below the Curie temperature, the FMR equations are called the Landau-Lifshitz equations. Over the Curie temperature, the FMR equations lead to the Bloch-type ferromagnetic-resonance equations [9].

These equations were obtained empirically; however, for a better understanding of the microscopic phenomena, a quantum statistical derivation was developed by O. A. Olkhov and B. N. Provotorov, taking into account a system with spin magnetism placed in a magnetic field composed by a constant field $H_{0}$ (which causes the state of full saturation), and the alternating field $H_{1}[9]$.

The Hamiltonian for this derivation contemplates the potential energy $\mu$ H.S, the exchange interaction term, the dipolar interaction term, the lattice energy, and the spin-lattice interac- 
tion. For the quantum statistical derivation, the relationship between the relaxation time and the correlation time is very important. The correlation time $\omega_{\mathrm{C}}$ is much less than the relaxation time $\omega_{R}$. This means that the equilibrium in the spin system is established by the strong exchange interaction, while the relaxation is conditioned by the weak dipolar interaction, so the equilibrium of the system is established by the strong exchange interaction much more quickly than the relaxation process. This assumption is confirmed by experimental data on the inelastic magnetic scattering of neutrons [9].

The principal result of the theory is that the resonance condition is given by $[9,28]$

$$
\omega_{0}=g \frac{e}{2 m c}\left\{\left[H_{z}+\left(N_{y}-N_{z}\right) M_{z}\right] \times\left[H_{z}+\left(N_{x}-N_{z}\right) M_{z}\right]\right\}^{1 / 2}
$$

Instead of the Larmor condition, where $\omega_{0}$ is the frequency at resonance, $\gamma=g e / 2 m c$, is the magnetomechanical ratio for an electron spin, $H_{z}$ is the static magnetic field, $M_{z}$ is the component of the magnetization along the $z$ axis, and $N_{x, y, z}$ are the components of the demagnetization.

It is important to take into account that the resonance condition is closely related to the demagnetization field, and this depends on the shape of the sample. For this reason, there are some special cases for the resonance frequency, for instance, the plane, the sphere, and a long circular cylinder $[27,28]$.

Another important consideration is that the energy of ferromagnetic crystals depends in part on the anisotropy energy. The anisotropy energy has an effect in the resonance condition. In the case of a single crystal, the value of the magnetic field required for resonance at fixed frequency depends on the direction of the crystal axes relative to the shape axes of the sample.

In a polycrystalline sample, the absorption line will, in general, look broader than in a single crystal sample because the distribution in direction of the crystalline axes causes a distribution in the field strengths for resonance $[27,28]$.

It is convenient to consider the effect of the anisotropy energy as an equivalent magnetic field $H^{\text {s; }}$ this field is defined such that the torque exerted on the sample by this field is equal to the torque exerted by the anisotropy energy,

$$
\frac{\partial f}{\partial \theta}=\mathbf{M}_{S} \times \mathbf{H}^{s}
$$

However, this equivalent magnetic field is not completely determined by equation 2 because the magnitude and the direction are arbitrary. One can express the components of $\mathbf{H}^{\mathrm{S}}$ in terms of an effective demagnetization factor, which will be added to the usual demagnetization factor in the equation for the resonance frequency. The resonance condition depends on the shape of the sample as well as on the orientation of the crystal. 


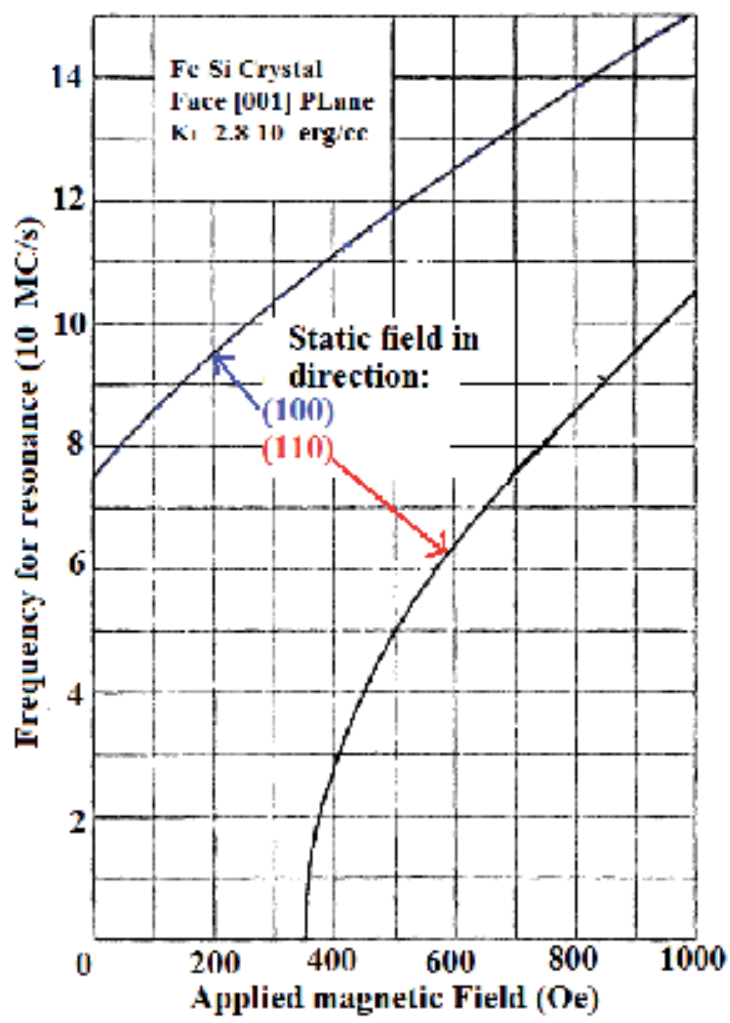

Figure 14. Comparison of the theoretical resonance condition for [100] and [110] directions in Fe-Si single crystal with a (001) plane surface. Adapted from Kittel [28].

A classic example of this is the difference of the magnetic field required for resonance with the (100) crystal face in a crystal with cubic anisotropy when the static field $H_{z}$ is in the [110] direction and when $H_{z}$ is in the [100], see Figure 14. In the first case, $H_{z}$ is greater than in the second. The difference is of the order of $4 \mathrm{~K}_{1} / \mathrm{M}_{\mathrm{s}} \cdot[6]$.

\subsection{Ferrimagnetic nanoparticles}

Nanoparticles of magnetic systems are of particular interest since the reduction to nanosized dimensions of the magnetic lattice gives rise to many interesting and different properties with respect to bulk materials. For instance, the surface spins, which constitute an important fraction of the total spins, undergo decrease in the coordination number and, therefore, a deficiency in exchange interactions. This situation can lead to severe changes in magnetization and anisotropy behavior. The relaxation processes of magnetization are also strongly temperature and size dependent.

In order to make a comparison between the three phases in ferrite NPs, Figure 15, we have selected the spectra obtained at $103 \mathrm{~K}$ (ordered, ferrimagnetic phase), $323 \mathrm{~K}$ (SPM phase), and $448 \mathrm{~K}$ (paramagnetic phases) [23]. The main difference exhibited by the ordered phase (in 
addition to the decrease in the resonance field) is the broadening in the section at $H>H_{\text {res }}$ i.e., $A^{-}$. The origin of this feature should be found in the effect of the internal field, which is the main difference with the other phases, and in particular, in the magnetocrystalline anisotropy field.

We present here two examples to show the complexity of changes driven by the reduction to nanosize dimensions, as illustrated by FMR. The first example involves cobalt-doped zinc ferrite nanoparticles $\left(\mathrm{Co}_{0.73 y} \mathrm{Zn}_{0.73(1-y)} \mathrm{Fe}_{2.18} \square_{0.09} \mathrm{O}_{4}\right)$. After synthesis, they were solubilized in aqueous solution containing $10 \%$ of polyvinyl alcohol [29]. Upon evaporation and during the polymerization process, the samples were subjected to a magnetic field in order to obtain an alignment of anisotropy axes.

The magnetization curves for these samples showed that the coercitivity increases as the percentage of Co increased, as well as the ratio of the remanence magnetization to saturated magnetization $M_{\mathrm{R}} / M_{\mathrm{S}}$. This latter result was interpreted as a change from uniaxial anisotropy for the $\mathrm{Zn}$ ferrite to a cubic anisotropy for the ferrites containing Co. This result was confirmed by the FMR spectrums as shown in Figure 15, associated with a broadening of the line width. This is attributed to the strong cubic magnetocrystalline anisotropy of cobalt ions in octahedral sites. This is somewhat contradictory as in bulk materials, it is well known that $\mathrm{Co}^{2+}$ on octahedral sites leads to change in the anisotropy sign (negative in most ferrites) from negative to positive (hence, uniaxial) [30].

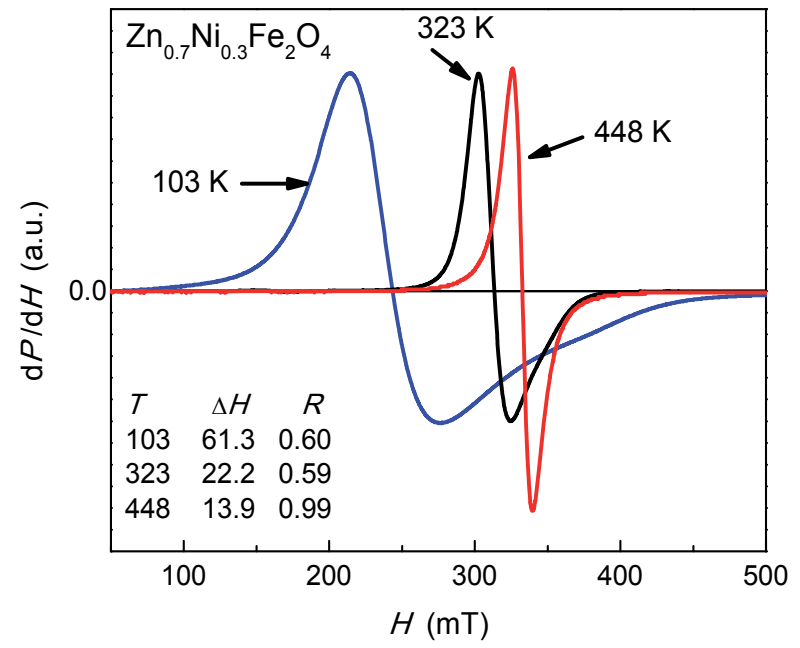

Figure 15. Typical signals associated with the three magnetic phases; paramagnetic at $448 \mathrm{~K}$, ferrimagnetic at $103 \mathrm{~K}$, and in the middle, the SPM phase which shows a progressive behavior from ferrimagnetic to paramagnetic as $T$ increases [23].

The FMR spectra of 3.7-nm nanoparticles show a drastic change when $10 \%$ of cobalt ions were added. A line width broadening and a shift in resonance toward lower fields are observed as 
the temperature decreases, and this behavior is more evident for the nanoparticles containing Co (Figure 16).

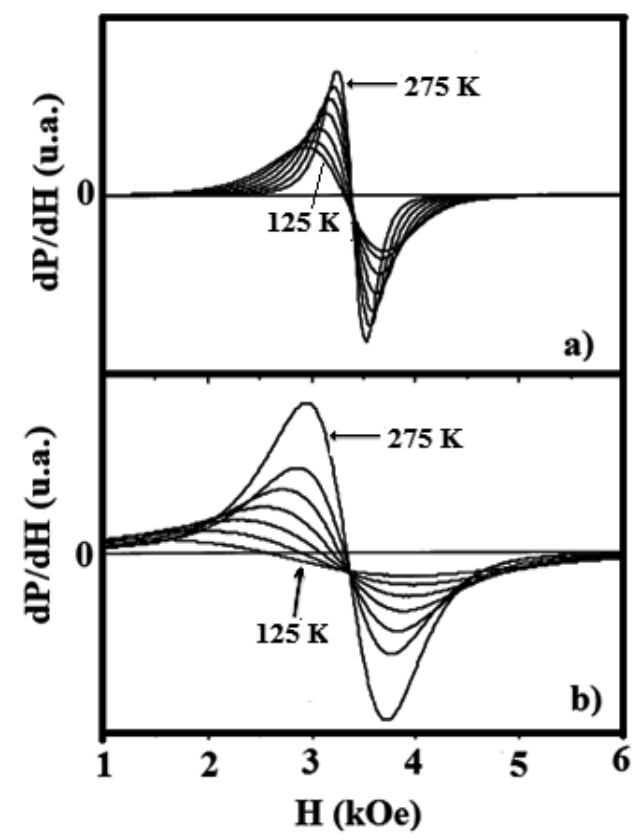

Figure 16. FMR spectra of 3.7-nm diameter ferrite nanoparticles dispersed in PVA, at various temperatures. (a) Zinc ferrite nanoparticles with no cobalt $(y=0)$. (b) Zinc ferrite with $y=10$ of cobalt. Adapted from Gazeau et al. [31].

The next example is a ferrite nanoparticle system of maghemite $\left(\gamma-\mathrm{Fe}_{3} \mathrm{O}_{2}\right)$ [31]. The oxide $\gamma$ $\mathrm{Fe}_{3} \mathrm{O}_{2}$ is an inverse spinel structure with all iron in the trivalent state and ion vacancies in the octahedral sublattice. Anisotropy properties studies have been done for $\gamma-\mathrm{Fe}_{3} \mathrm{O}_{2}$ nanoparticles of diameter from 4.8 to $10 \mathrm{~nm}$ dispersed in glycerol forming a magnetic fluid. This study is done by measuring the samples in a temperature range from $3.5 \mathrm{~K}$ to $300 \mathrm{~K}$ with (i) zero field cooled (ZFC) and thus randomly oriented anisotropy axes and (ii) field cooled (FC) with $H_{\mathrm{fr}}=$ $10 \mathrm{kOe}$. The FMR spectra showed a decrease in the resonance field, and a broadening of the line width, Figure 17. The parameters are shown in Figure 5 [31].

The resonance fields and the line widths of the $\gamma-\mathrm{Fe}_{3} \mathrm{O}_{2}$ samples are represented as a function of temperature, see Figure 18. The ZF experiments are done for $\theta=0^{\circ}$ and for $\theta=90^{\circ}$, where $\theta$ is the angle between the directions of the freezing field and the magnetizing field.

In the field cooled samples, the orientation distribution of the anisotropy axes results from the competition between the magnetic energy, the anisotropy energy and the thermal energy. The distribution in orientation of the anisotropy axes affects the FMR spectrum in angular variation and line width. On the other hand, there is also an effect of the particle size in the anisotropy. This is observed in Figure 19, where $H_{\text {res }}\left(90^{\circ}\right)-H_{\text {res }}\left(0^{\circ}\right)$ and $\Delta H$ are plotted as a function of temperature for different particle sizes. The observed reduction of $H_{\text {res }}\left(90^{\circ}\right)-H_{\text {res }}\left(0^{\circ}\right)$ is related 


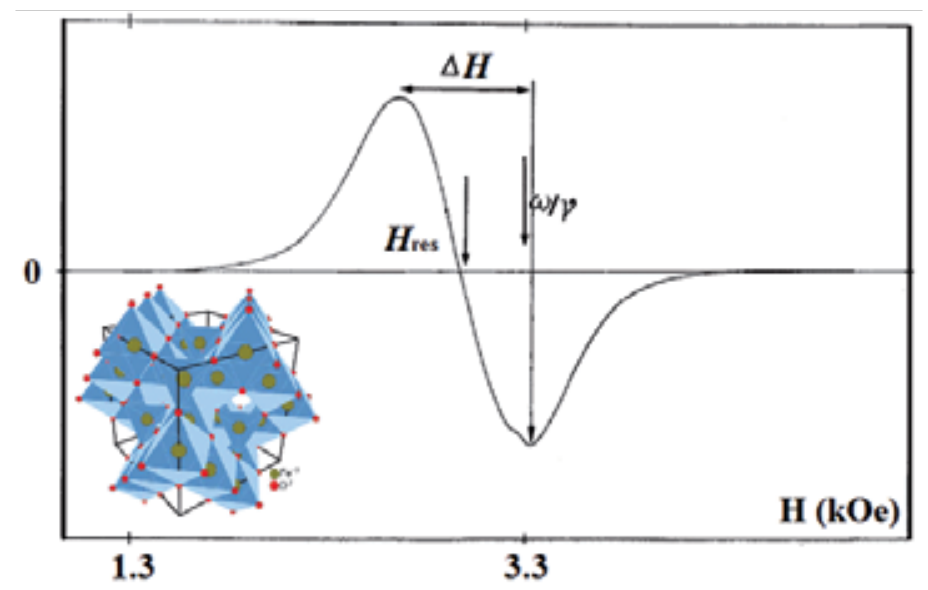

Figure 17. FMR spectrum of maghemite $\left(\gamma-\mathrm{Fe}_{3} \mathrm{O}_{2}\right)$ nanoparticles of $7 \mathrm{~nm}$ of average diameter at room temperature and microwave frequency $f=9.26 \mathrm{GHz}$. The inset shows the spinel structure of maghemite. Adapted from Gazeau et al. [31].
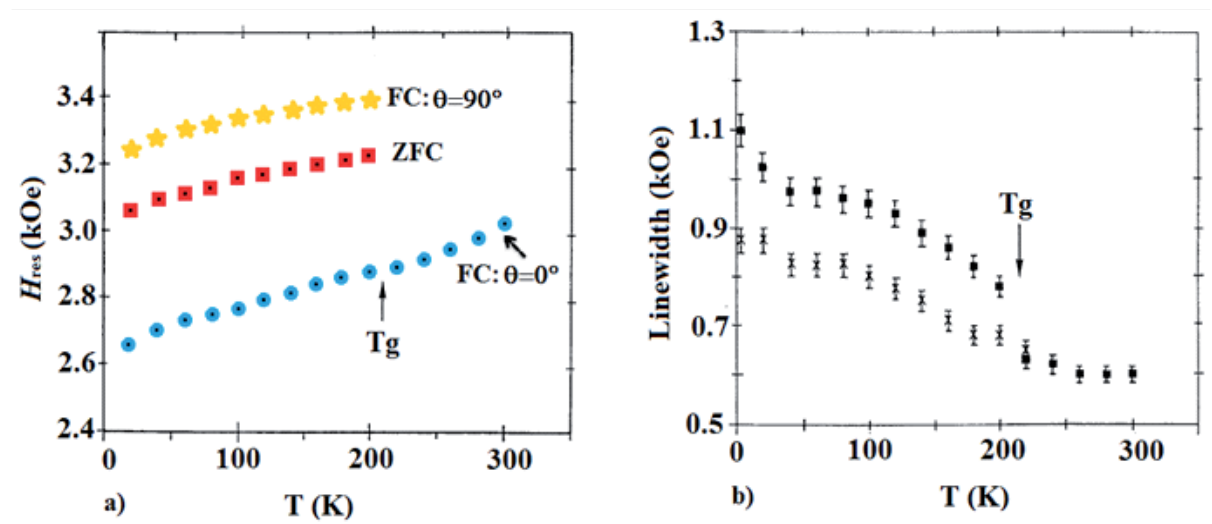

Figure 18. (a) Temperature dependence of the resonance field for 10-nm diameter $\gamma-\mathrm{Fe}_{3} \mathrm{O}_{2}$ nanoparticles cooled under a 10-kOe magnetic field (FC) and cooled without field (ZFC). The arrow indicates the melting temperature of the ferrofluid matrix. (b) Temperature dependence of the peak-to-peak line width for the same samples. Adapted from Gazeau et al. [31].

to the particle size decrease because of the orientation distribution of the anisotropy axes. The FMR study indicates that nanosized particles possess uniaxial anisotropy, even though bulk maghemite has a cubic anisotropy.

By using a theoretical model [32] based on the Landau-Lifshitz-Gilbert dynamics, it is possible to show that for a single domain assembly of magnetic NPs with randomly distributed anisotropy axes, a large broadening of the absorption line is obtained, see Figure 19. In addition to a shift of the resonance frequency, the distribution of anisotropy axes gives rise to a significant change in the shape and symmetry of the line. Figure 20 was calculated at zero temperature; as temperature increases, the shift in resonance field decreases, and due to the typical decrease in anisotropy energy with $T$, the line broadening is also reduced. 

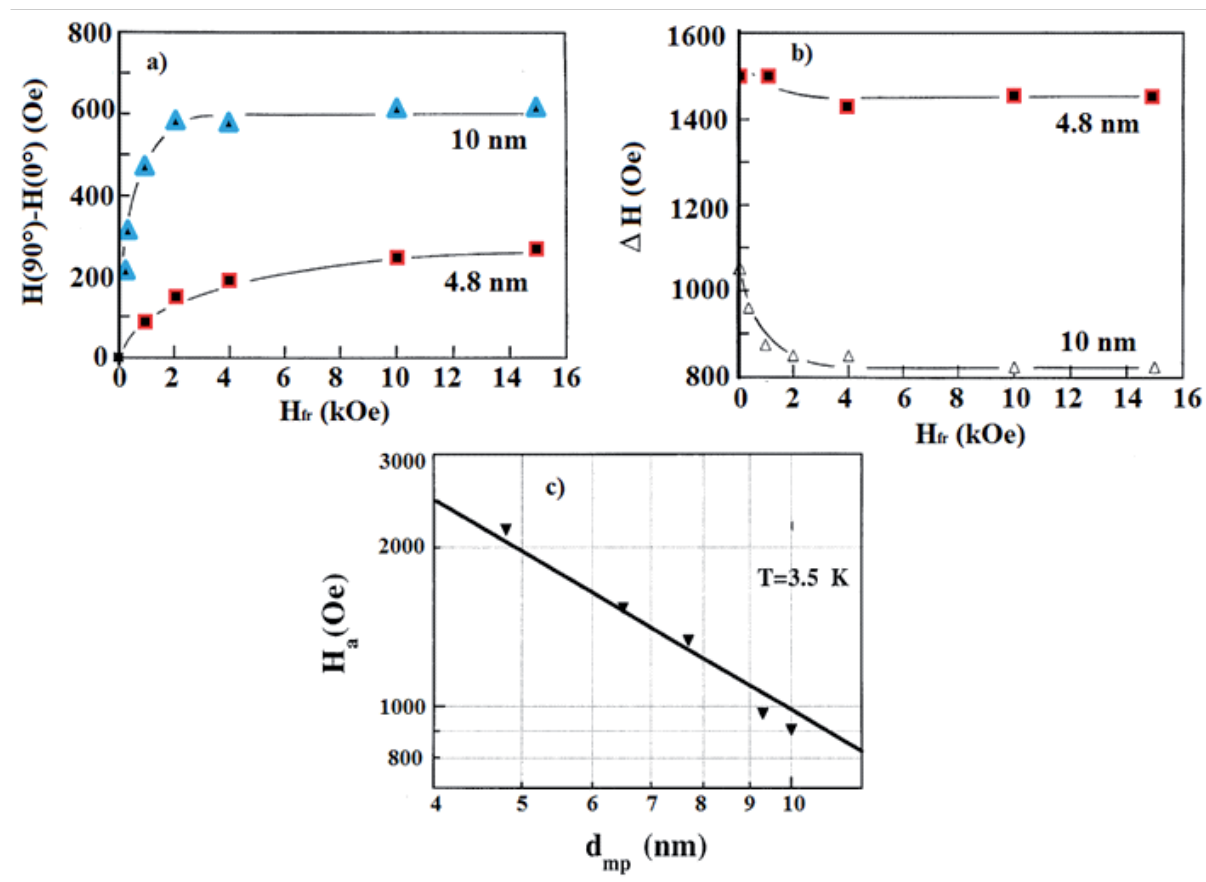

Figure 19. (a) Difference of the resonance fields for anisotropy axis orientated at $90^{\circ}$ and $0^{\circ}$ form the magnetic field. (b) Peak-to-peak line width as a function of microwave field for two different size of $\gamma$ - $\mathrm{Fe}_{3} \mathrm{O}_{2}$ nanoparticles. (c) Anisotropy field deduced for angular variations at $3.5 \mathrm{~K}$ as a function a particle size. Adapted from Sukhov et al. [32].

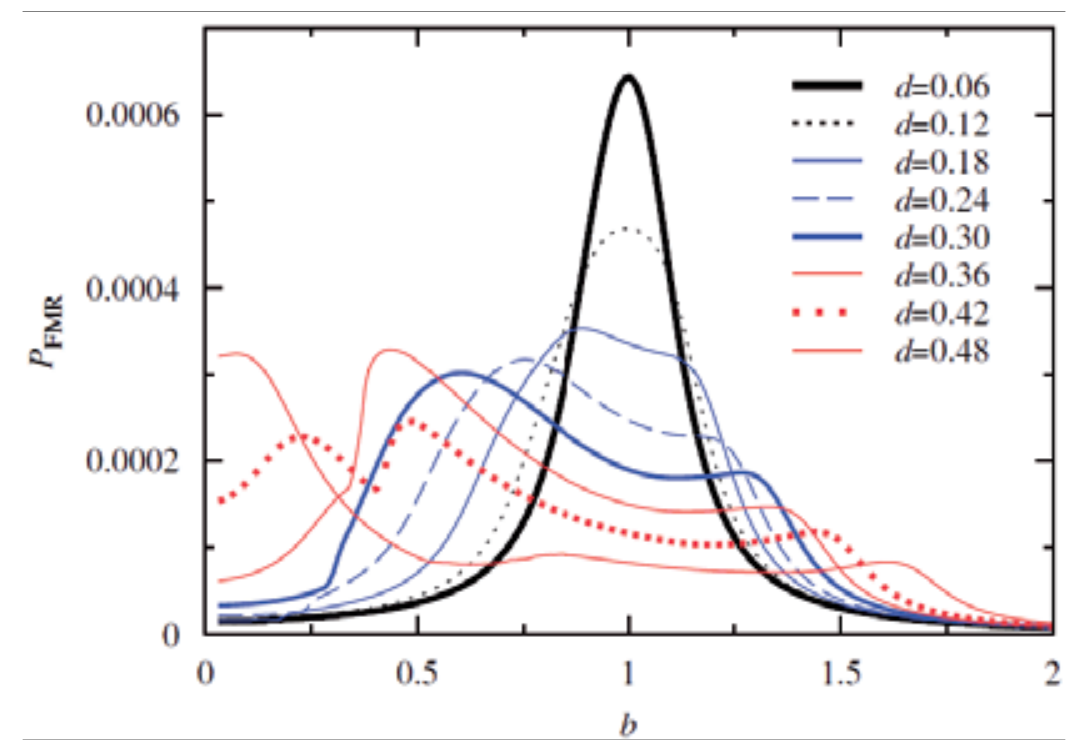

Figure 20. Absorbed FMR power (arbitrary units) vs reduced magnetic field at zero temperature and for different values of the damping parameter in the Landau-Lifshitz-Gilbert dynamics [32]. 


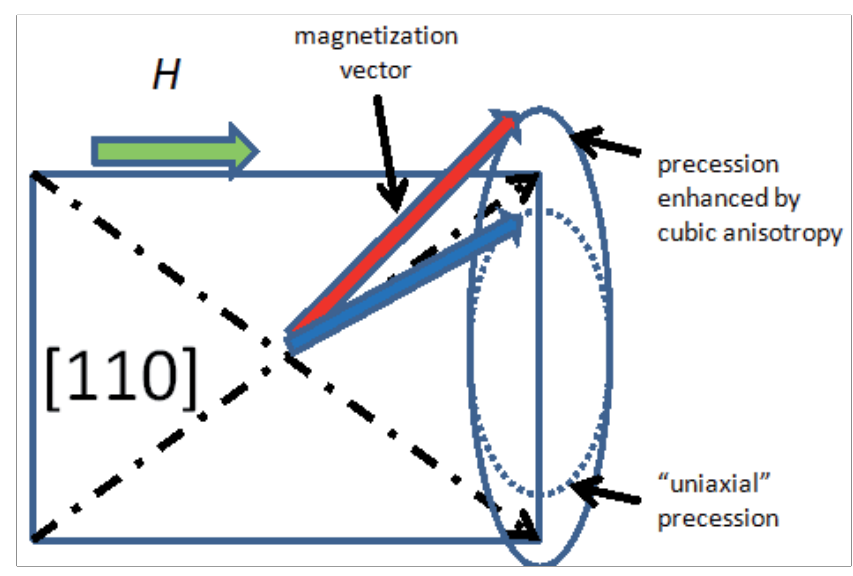

Figure 21. Interpretation of the line broadening in ferrites with cubic anisotropy. On a [110] crystal plane, containing two easy axis of the $\langle 111>$ family, which have an influence on the magnetization dynamics, leading to an increase in the precession amplitude.

These results were obtained for the case of uniaxial materials; in the case of most ferrites with cubic anisotropy axes, it would be expected a larger broadening. In a very cartoon-like model, it is possible to imagine that the magnetization is first oriented along the applied field, which has a value closer to the anisotropy field. Once close to the saturation and once satisfied the resonance conditions, the magnetization is driven by the microwave radiation to precess uniformly, that is in phase. However, magnetocrystaline anisotropy is strong at these temperatures, and it is possible to consider that the magnetization vector can still feel its influence and precesses with a tendency toward the cubic axes. This dynamics leads to a broadening of the line, as far as the main result will be the combination of precession along several anisotropy axes, and has to be averaged over a large NPs population. Figure 21 illustrates this interpretation.

\section{Conclusions}

It can be established that systematic and significant differences occur in the resonance signal of ferrite NPs, associated with the magnetic structure. Paramagnetic phases lead to a high resonance field (typically in the $\sim 330 \mathrm{mT}$ range for the $\mathrm{X}$ band) with a small line width and a tendency to a symmetric signal $(R \sim 1)$ (see signal at $448 \mathrm{~K}$ in Figure 15), in contrast with the ferromagnetic phase, which exhibits a lower resonance field, broad line width and asymmetric shape (signal at $103 \mathrm{~K}$, Figure 15). Between these two different behaviors, there is an SPM phase showing a resonance field closer to the one for the paramagnetic phase, a progressive decrease in the broadening of the minimum section of the line (absorption at $H>H_{\text {res }}$ ), and therefore an increase in the $R$ parameter toward 1.0. The exact transition temperatures are not easy to establish since evolution from one phase to the next is quite continuous. 
As a general conclusion, these reviews confirm that electron spin resonance is a very sensitive experimental method that can be used to characterize magnetic phases in the complex field of magnetic nanoparticles.

\section{Acknowledgements}

Authors acknowledge partial support from DGAPA-UNAM, Mexico, through grant PAPIIT IN 101412.

\section{Author details}

Rebeca Díaz-Pardo and Raúl Valenzuela*

*Address all correspondence to: monjaras@unam.mx

Institute for Materials Research, National Autonomous University of Mexico, Circuito

Exterior, Ciudad Universitaria, Coyoacán, México D.F., México

\section{References}

[1] Guimaraes A.P. Principles of Nanomagnetism. Berlin: Springer-Verlag; 2009.

[2] Valenzuela R. Magnetic Ceramics. Cambridge: Cambridge University Press; 2005.

[3] Bragg, W.H. "The structure of the spinel group of crystals." Philosophical Magazine 1915; 30, 305-315.

[4] Kittel C. Introduction to Solid State Physics. New York: John Wiley \& Sons Inc; 2005.

[5] Abragam A., Bleaney B. Electron Paramagnetic Resonance. Oxford: Oxford University Press; 2012.

[6] Weil John A., Bolton J.R. Electron paramagnetic resonance. New York: John Wiley \& Son; 2007.

[7] Junk M.J.N. Assessing the Functional Structure of Molecular Transporters by EPR Spectroscopy. Berlin: Springer; 2012.

[8] Feher G., Kip A.F. “Electron spin resonance absorption in metals. I. Experimental," Physical Review 1955; 98, 337-348.

[9] Olkhov O.A., Provotorov B.N. “Quantum statistical derivation of ferromagnetic-resonance equations," Physical Review 1965; 140, 1296-1303. 
[10] Koksharob Y.A., Pankratov D.A., Gubin S.P., Kosobudsky I.D., Beltran M., Khodorkovsky Y., Tishin A.M. "Electron paramagnetic resonance of ferrite nanoparticles," Journal of Applied Physics 2001; 89, 2293-2298.

[11] Dyson F.J. "Electron spin absorption in metals. II. Theory of electron diffusion and the skin effect," Physical Review 1955; 98, 349-359.

[12] Khabibullin B.M., Kharakhashýan E.G. "Conduction-electron paramagnetic resonance in metals," Soviet Physics Uspekhi 1974; 16, 806-818.

[13] Dalal N.S., Murillo C.A. “The usefulness of EPR spectroscopy in the study of compounds with metal-metal multiple bonds," Dalton Transactions 2014; 43, 8565-8576.

[14] Lindsay D.L. "Oxide minerals, short course notes," Mineralogical Society of America, Washington, DC, 1976; 3, L66-L71.

[15] Néel L. “Théorie du traînage magnétique des ferromagnétiques en grains fins avec applications aux terres cuites," Annales de Géophysique 1949; 5, 99-136.

[16] Brown W. Jr. "Thermal fluctuations of a single domain particle," Physical Review 1963; 130 (5) 1677-1686.

[17] Wernsdorfer W., Bonet Orozco E., Hasselbach K., Benoit A., Barbara B., Demmoncy N., Loiseau A., Pascard, H., Mailly D. "Experimental evidence of the Néel-Brown model for magnetization reversal," Physical Review Letters 1997; 78, 1791-1795.

[18] Victora R.H. "Predicted time dependence of the switching field for magnetite," Physical Review Letters 1989; 63, 457-459.

[19] de Biasi E., Zysler R., Ramos C.A., Knobel M. "A new model to describe the crossover from superparamagnetic to blocked magnetic nanoparticles." Journal of Magnetism and Magnetic Materials 2008; 320, e312-e315.

[20] de Biasi R.S., Devezas T.C. “Anisotropy field of small magnetic particles as measured by resonance." Journal of Applied Physics 1978; 49, 2466-2469.

[21] Cruz-Franco B., Gaudisson T., Ammar S., Bolarin-Miró A.M., Sánchez de Jesus F., Mazaleyrat F., Nowak S., Vázquez-Victorio G., Ortega-Zempoalteca R., Valenzuela R. "Magnetic properties of nanostructured ferrites," IEEE Transactions on Magnetics 2014; 50(4) 280016-1-280016-5.

[22] Raikher Yu.L., Stepanov V.I., "Magnetization dynamics of single-domain particles by superparamagnetic theory," Journal of Magnetism and Magnetic Materials 2007; 316(2) 417-421.

[23] Raikher Y.L., Stepanov V.I. “Intrinsic magnetic resonance in superparamagnetic systems," Physical Review B 1995; 51 (22) 16428-16431.

[24] Flores-Arias Y., Vázquez-Victorio G., Ortega-Zempoalteca R., Acevedo-Salsa U., Ammar S., Valenzuela R. "Magnetic phase transitions in ferrite nanoparticles character- 
ized by electron spin resonance (ESR)." Journal of Applied Physics 2015; 117(17) 17 A503.

[25] Coffey W.T. Krothers D.S.F., Kalmyvov Yu.P., Massawe E.S., Waldron J.T. Physical Review E 1949; 49, 1869-1872.

[26] Aharoni A. "Susceptibility resonance and magnetic viscosity." Physical Review B 1992; 46, 5434-5441.

[27] Berger R, Bissey J.C., Kliava J., Baubric H., Estournes C. “Temperature dependence of superparamagnetic resonance of iron oxide nanoparticles." Journal of Magnetism and Magnetic Materials (2001) 234, 535-44.

[28] Yulikov M.M., Purtov P.A. "FMR study of superparamagnetic Ni particles with weak and strong anisotropy." Applied Magnetic Resonance 2005; 29, 231-249.

[29] Kittel C. "Ferromagnetic Resonance," Le Journal de Physique et le Radium 1951; 12, 291-302.

[30] Hochepied J.F., Pileni M.P. "Ferromagnetic resonance of nonstoichiometric zinc ferrite and cobalt-doped zinc ferrite nanoparticles." Journal of Magnetism and Magnetic Materials 2001; 231, 45-50.

[31] Bickford L.R., Pappis J., Stull J.L. "Magnetostriction and permeability of magnetite and cobalt-substituted magnetite." Physical Review 1955; 99, 1210-1214.

[32] Gazeau F., Bacri J.C, Gendron F., Perzynski R., Raikher Yu.L., Stepanov V.I., Dubois E. "Magnetic resonance of ferrite nanoparticles: evidence of surface effects." Journal of Magnetism and Magnetic Materials 1998; 186, 175-187.

[33] Sukhov A., Usadel K.D., Nowak U. "Ferromagnetic resonance in an ensemble of nanoparticles with randomly distributed anisotropy axes." Journal of Magnetism and Magnetic Materials 2008; 320, 31-35. 



\title{
Time-Domain Electromagnetic Wave Propagation in Confined Environments
}

\author{
Jorge Avella-Castiblanco, Divitha Seetharamdoo, \\ Marion Berbineau, Michel Ney, Ibrahim Massy and \\ Franois Gallée
}

Additional information is available at the end of the chapter

\begin{abstract}
Confined environments like tunnels are electrically large structures for guided wave propagation. They can have arbitrary cross sections, and the design and optimization of antenna for communication system requires the knowledge of a "full-wave" solution in nearby zones. Current models based on asymptotic approaches do not describe adequately the wave propagation under the above conditions. In addition, a complete "full-wave" analysis of the tunnel propagation performances is not feasible in terms of computer expenditure. After a survey of the most commonly used techniques for propagation in tunnels, some investigation regarding an appropriate approach to find the fields is proposed. It is based on a modal decomposition of the wave propagation that allows an optimization of the coupling with the antenna. To find the mode characteristic for arbitrary cross section, a full-wave method, namely, the transmission-line matrix (TLM), is modified to a so-called 2.5-dimensional TLM algorithm and presented in details. This approach is validated for a canonical structure. Then, it is applied to study the wave propagation in a realistic rectangular tunnel. The concept of surface impedance boundary condition (SIBC) is introduced to reduce the TLM computational domain and model the tunnel walls that can be considered as lossy dielectric. Results show that guided structures with lossy dielectric walls of arbitrary cross section can be studied with this approach.
\end{abstract}

Keywords: wave propagation, wave-guides, Transmission Line Matrix Method, arbitrary cross-section wave-guides, dielectric waveguides, Modes

\section{Introduction}

Railway, roadway and mine tunnels, buildings, and warehouses are some examples of confined environments, in which electromagnetic wave propagation has to be investigated 
for communication channel characterization. In this paper, we shall focus on tunnel environments. The prediction and characterization of the radio-wave propagation is needed to optimize the system performances.

Confined environments are complex real-life electromagnetic (EM) problems. Several techniques and methods are used to study the radio-wave propagation or to design antennas to achieve some good performance. Experimental techniques, analytical methods (exact solutions), numerical methods (approximate solutions), asymptotic methods (approximate high-frequency expansions of Maxwell's equations), and approximate techniques (approximate solutions applicable for certain types of electromagnetic problems) are among the most commonly used techniques in confined structures. Experiments might be expensive and time consuming. Analytical and approximate techniques are limited to some structures. In turn, asymptotic methods are mostly used to study the wave propagation in tunnels. However, in many cases, the antennas employed to provide the communication in these systems are strongly affected by its surrounding environment, affecting the performance of the system. Thus, near-field considerations have to be accounted for, which cannot be considered by these techniques. Finally, the practical utilization of numerical full-wave methods has been hampered by their large computational time compared to asymptotic methods.

With the increasing development of computers, appropriate new models and simplifications are being developed. The formulation of an efficient modal approach stemming from the fact that tunnels can be modeled by an over-sized waveguide and the a priori knowledge of the fields in the axial direction is presented. It allows one to have a better physical insight into wave propagation in confined environments, as well as dealing with electrically large structures like tunnels. The mode parameter determination is carried out by a full-wave time-domain method, namely, the transmission-line matrix (TLM) method.

The calculation volume has to be limited in full-wave volumic methods, and we are interested only in the fields inside the tunnel. Thus, the electromagnetic modeling of the tunnel walls, which can be lossy dielectric, is addressed. Lastly, by assimilating a confined environment to a lossy dielectric waveguides of arbitrary cross section, the mode extraction of these structures is presented. To the best of the authors' knowledge, no such model has been reported.

The chapter is structured as follows: In the first section, an overview of the principal techniques for the description of the EM wave propagation in above structures is briefly presented. In the second section, the formulation of the modal approach is described in detail. In the following section, the implementation for a simple canonical case is shown. Lastly, the numerical analysis of multimodal waveguides representative of confined environments is illustrated in a realistic rectangular tunnel. Finally, discussions and conclusions are developed at the end of the chapter.

\section{Modeling approaches for wave propagation in confined environments}

The correct understanding of wave propagation in confined structures like tunnels has been an important area of research and development. They have been studied in the last 40 years for radio communication system deployment. Unfortunately, as we shall see, current models cannot be generally applied or they do not describe adequately the wave propagation. The 
purpose of this section is to provide an overview of the approaches for the problem of wave propagation in these structures.

\subsection{Analytical models: circular and elliptical dielectric tunnels}

No analytic solution exists for the problem of wave propagation along a dielectric waveguide of arbitrary cross-sectional shape. The circular and elliptical waveguides are the only cases for which analytic solutions for guided waves exist. Field solution in waveguides for analytical and approximate methods is usually expressed in terms of modes. They are classified into different types according to their field configuration: transverse electric modes (TE), with no electric field in the direction of propagation; transverse magnetic modes (TM), with no magnetic field in this direction; transverse electromagnetic modes (TEM), with neither electric nor magnetic field in this direction; and hybrid modes (HE) or (EH), with nonzero electric and magnetic fields in the direction of propagation.

Dielectric circular waveguides can support a family of circularly symmetric $T E_{n m}$ or $T M_{n m}$ modes, with $n=m=0$, and hybrid modes $H E_{n m}$ and $E H_{n m}$. The subscripts $n$ and $m$ denote the number of oscillations with the cylindrical coordinates $\rho$ and $\phi$, respectively. Some discussions considering different excitations, dependence on the constitutive parameters of the tunnel walls, and other interesting topics are treated in [9]. The details of the determination of the fields will not be repeated here. Finally, the wave propagation in elliptical tunnels has not been treated. However, a deformed circular waveguide can be approximated by an elliptical cylinder. Detailed theoretical as well as experimental results can be found in [34].

\subsection{Approximate models: rectangular dielectric tunnels}

An exact analytic solution does not exist for the case of wave propagation in a rectangular tunnel with lossy dielectric walls. An approximate approach, namely, Marcatilli's method, is usually employed to analyze this structure. The a priori assumption of Marcatilli's approach is that most of the energy of the modes propagating in the structure is contained within the core region and very little guided power is contained in the corner regions of the guide [34]. Then, the boundary conditions are only matched along the four sides of the hollow region, and the fields in the corners are ignored. The Helmholtz equation in Cartesian coordinates is solved with separated variables, and approximate solutions for the propagation constant and for the field distributions are found. The modes are classified into $E_{n m}^{x}$ and $E_{n m}^{y}$ with most of its electric field polarized in the horizontal or vertical direction, respectively. This approximation gives the mode parameters with sufficient accuracy as long as the assumptions below are valid:

$$
\begin{gathered}
(n \lambda / 2 w)<<1 \\
(m \lambda / 2 h)<<1 \\
|\bar{\epsilon}-1|^{1 / 2} / \bar{\epsilon}>>(n \lambda / 2 w) \\
|\bar{\epsilon}-1|^{1 / 2} / \bar{\epsilon}>>(m \lambda / 2 h)
\end{gathered}
$$


where $n$ and $m$ are integers describing the $n m$-th mode, $\lambda$ is the free-space wavelength, $w$ and $h$ the waveguide dimensions, and $\bar{\epsilon}$ is the normalized complex permittivity $\bar{\epsilon}=\epsilon_{r}-$ $j \sigma /\left(\omega \epsilon_{0}\right)$, where $\sigma$ is the wall conductivity. Finally, a study of modal propagation in curved rectangular tunnels can be found in [20].

\subsection{Asymptotic models}

Ray models use high-frequency expansions of Maxwell's equations and are based on optical laws to model the fields. They constitute the most commonly used deterministic techniques to analyze confined environments [32]. In ray-based methods, the waves are treated as rays propagating perpendicular to the wave fronts. This approximation is valid at positions sufficiently distant from the source. The resulting spherical waves can be approximated by a locally plane wave on a small portion of the sphere. Two methods are widely used: ray launching and ray tracing $[8,10]$. Curved tunnels, arched cross sections, or bends of a tunnel can be treated by ray methods provided that radii of curvature of the surface be large compared to the wavelength $[22,33]$.

Current tunnel cross sections are getting smaller, and antennas have to operate close to the tunnel walls, such that these interfere with their near-fields. As a result, antenna performances cannot be guaranteed by using asymptotic methods. For this reason, full-wave models accounting for near-field effects become more relevant.

\subsection{Full-wave models}

In electromagnetism, two main families of numerical methods exist: frequency and time-domain methods. Nowadays, time-domain methods are becoming increasingly used $[11,31]$. They are attractive because of their relative simplicity and their ability to account for arbitrary geometries, obstacles like vehicles, nonlinearities, and different materials and to determine transient response and perform wideband characterization $[6,32,35]$. Thus, full-wave methods attract more attention for the purpose of deterministic radio coverage predictions, near-field considerations, simplifications, and practical implementations [13]. However, efficient and adequate models are still needed to describe the wave propagation in tunnel environments. A modal approach is preferred because it allows one to describe the propagation by modes $[1,2,29]$. An efficient time-domain modal approach based on the transmission-line matrix (TLM) method will be introduced in the next section.

\section{The Transmission-Line Matrix (TLM) method for guiding structures}

The transmission-line matrix (TLM) method was developed by P. B. Johns and his co-workers in the 1970s [17]. Its theoretical foundations are based on Huygens's model of wave propagation. The TLM method is very attractive and flexible to analyze electromagnetic field problems and has received increased recognition for full-wave analysis of arbitrary-shaped guiding structures [15]. Through the years, several nodes have been developed in TLM for structured and unstructured meshes in two and three dimensions. We will focus on the symmetrical condensed node (SCN), which is the most widely used for 3D structures. 


\subsection{Description of 3D TLM method}

The symmetrical condensed node ( $\mathrm{SCN}$ ) consists of a network of interconnected multi-port devices where the electromagnetic wave propagation is simulated by the propagation of traveling pulses; a unit cell is presented in (Figure 1). On each arm, two orthogonal ports are employed to account for any polarization.

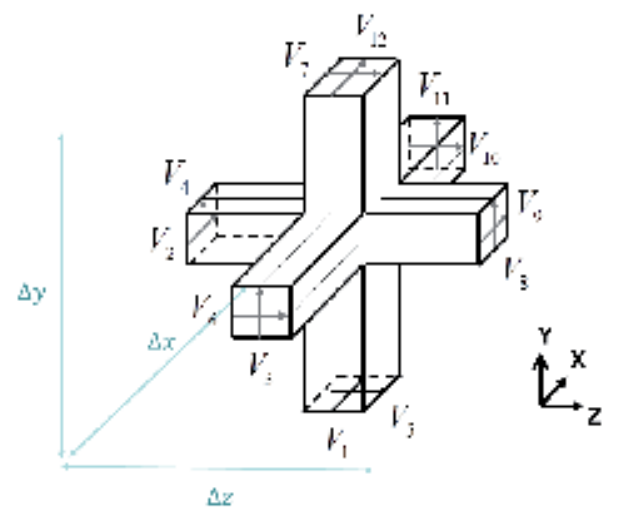

Figure 1. Scheme of the SCN node

To solve an electromagnetic problem, the solution region has to be divided into a number of these elementary nodes.

A rule of thumb consists of taking the maximum cubic cell size as $\Delta l=\lambda / 10$, where $\lambda$ correspond to the medium wavelength of the highest frequency of interest. Then, excitation is imposed. The objective of the source terms is to simulate a desired phenomenon in the structure. For instance, in guiding structures, a given mode can be selectively excited by an adequate field distribution that corresponds to the mode transverse configuration (mode template). Some other problems require to analyze the structure over a wide frequency range. This is achieved by exciting the structure with a wideband time signal such as Dirac, Gaussian, etc. Thus, in general, the excitation corresponds to a space-time distribution. Nodes are interconnected by these virtual transmission lines, and the excitation propagates from the source nodes to the adjacent nodes at each time step.

The method is carried out basically through two processes: scattering and connection. In the scattering process, voltage pulses ${ }_{n} V^{i}$ are incident upon the node from each of the link-lines (halfway between two nodes) at each time step $n \Delta t$. These pulses are then scattered to produce a set of scattered voltages, ${ }_{k} V^{r}$, which become incident on adjacent nodes at the next time step $(n+1) \Delta t$. In the connection process, pulses are simply exchanged among immediate neighbors.

Volumic methods such as TLM full-wave methods require a limitation of the computational domain for open problems in which fields exist at large distances. Thus, somehow the problem has to be bounded. The boundary conditions are the set of conditions which specifies the behavior of fields at the boundaries of the computational domain. 


\subsubsection{Boundary conditions in TLM}

The boundary conditions link the electromagnetic fields through the tangential or normal field values. Since TLM is based on the equivalence between Maxwell's equations and equations for voltages and currents that travel in a mesh of interconnected transmission lines, a relationship of the involved voltages at the boundary can be found. The scattered voltages $V^{r}$ are always known values, and the incident voltages $V^{i}$ are unknown. Any resistive load at a boundary may be simulated by introducing a reflection coefficient $\Gamma$ as shown in (3), [7].

$$
{ }_{k+1} V_{a r m j}^{i}(x, y, z)=\Gamma_{k} V_{a r m j}^{r}(x, y, z)
$$

This formalism allows us to represent a variety of boundary conditions as long as a reflection coefficient $\Gamma$ can be defined. For instance, for a perfectly electric conductor (PEC), boundary is simulated by choosing $\Gamma=-1$; a perfectly magnetic conductor (PMC) is implemented by choosing $\Gamma=1$. The reflection coefficient for lossy boundaries [15], relating the incident and reflected voltages, can be expressed in Laplace domain by the (4).

$$
\Gamma(s)=\frac{V^{r}(s)}{V^{i}(s)}=\frac{\left[\Delta y Z_{s}(s)-Z_{z y} \Delta z\right]}{\left[\Delta y Z_{s}(s)+Z_{z y} \Delta z\right]}
$$

where $\Delta y$ and $\Delta z$ are the cell dimensions; $Z_{z y}$ the impedance of the arms, equal to $Z_{0}=$ $\sqrt{\mu_{0} / \epsilon_{0}}$ for the standard SCN node; and $Z_{S}$ is the surface impedance (SI). In the case of a good conductor, an approximation by a real number for $\Gamma$ is presented in [28]. However, $\Gamma$ is in general complex and would alter the shape of the excitation pulses, which cannot be accounted for in the TLM method [7].

\subsection{Application to confined environments modeled by arbitrary cross-section oversized waveguides: $2.5 \mathrm{D}$ TLM approach}

The analysis of electrically large structures like tunnels involves a high computation time by using full-wave methods like TLM. However, some astutenesses can be considered to efficiently model them, which translates in finding an approach to simplify the analysis of oversized waveguides. From the geometrical point of view, tunnels can have any arbitrary shape, and this constraint has to be considered. From the electromagnetic point of view, waves propagating in tunnels can be classified in modes. This problem is well known in electromagnetic theory. As we shall see, a full-wave model based on a modal decomposition of the waves for the analysis of the radio propagation in tunnels at high frequencies is presented. Besides, the concept is used to further reduce the complexity of this electromagnetic problem.

\subsubsection{The TLM modal approach}

Modal approaches in frequency domain are based on the expansion of fields in terms of modes. In general, total fields can be represented as the sum of modes as shown in (5): 


$$
F(x, y, z)=\sum_{i=1}^{N} A_{i}(x, z) e^{-\left(\alpha_{i}+j \beta_{i}\right) y}
$$

where $N$ is the total number of modes, $A_{i}(x, z)$ the amplitude of the $i$-th mode at the coordinate $(x, z)$ in the plane perpendicular to the propagation direction $y, \alpha_{i}$ its attenuation, and $\beta_{i}$ its phase constant. The mode amplitude $A_{i}(x, z)$ gives the electric or magnetic field distribution of the mode. The phase constant $\beta$ represents the change in phase along the path traveled by the wave. Lastly, the attenuation constant is a measure of losses in the structure.

In the literature, the simplification of uniform guide problems in TLM was first introduced by [16] to obtain the dispersion properties. They proposed the reduction of the calculation region by introducing the field dependence solution in the propagation direction $y$. The longitudinal dependence can be described by exponential terms $e^{-j \beta y}$, where $\beta$ is the phase constant and $y$ the longitudinal distance in the guide. Hence, for a specific mode, two points along the longitudinal distance of the guide have only a phase difference $\beta\left(y_{2}-y_{1}\right)$. Then, it is possible to find a relationship between the reflected voltages of the node at the time $k \Delta t$ and the incident ones at the time $(k+1) \Delta t$. This approach allows one to reduce the computation domain from a 3D mesh of $3 \mathrm{D}$ nodes to a $2 \mathrm{D}$ one, avoiding to calculate the fields over all points along the propagation direction.

The concept of mode will be employed to find a more efficient formulation, to obtain a reduced 2.5-dimensional TLM modal approach for finding fields. The term $2.5 \mathrm{D}$ is used as the $3 \mathrm{D}$ computational domain is reduced to a $2 \mathrm{D}$ mesh in the guide cross section. However, cells are 3D ones that account for all 6 electromagnetic field components. The reduced model can be applied for a uniform (invariant cross section along $y$ ) tunnel of arbitrary cross section.

\subsection{Formulation of the 2.5D TLM node for guiding structures}

To obtain the reduced TLM formulation for guiding structures, the voltages in each arm should be updated at each time step. In doing this, eight relationships between the reflected and incident voltages are needed. As mentioned before, stubs are added to model the background media with constitutive parameters $\varepsilon$ and $\mu$. Then, six additional relationships are needed for these stubs.

In [25], a procedure to find the required updating relationships based on Maxwell's equations is presented. To employ this procedure, six additional relationships are required to update all field components. The process is divided into two steps. The first step consists of finding the relationship between fields at the center of the node and the incident and reflected voltages taken at each arm. Then, the six relationships for the fields at instant $n \Delta t$ are found by using the integral form of Maxwell's equations in the three principal planes $(z, y),(x, z)$, and $(x$, $y$ ). In the next step, field continuity equations at the center are applied by using the integral form of Maxwell's equations for contours passing through the center of the three principal planes. Then, 12 relationships for the voltages are found in terms of the fields at the center. By manipulating these expressions, the required eight relationships to update the voltages are found. Finally, the voltages for the stubs are calculated in the same way as the standard $\mathrm{SCN}$ node. The TLM algorithm is then completed. 


\subsubsection{Starting point: maxwell's equations in integral form}

The starting point is the integral form of Maxwell's equations for an isotropic medium and given by (6) and (7).

$$
\begin{gathered}
\oint_{C} \vec{H} \cdot \overrightarrow{d l}=\iint \hat{\varepsilon} \frac{\partial \vec{E}}{\partial t} \cdot \overrightarrow{d s}+\iint \hat{\sigma_{e}} \vec{E} \cdot \overrightarrow{d s}+\iint \overrightarrow{J_{e}} \cdot \overrightarrow{d s} \\
\oint_{C} \vec{E} \cdot \overrightarrow{d l}=-\iint \hat{\mu} \frac{\partial \vec{H}}{\partial t} \cdot \overrightarrow{d s}-\iint \hat{\sigma_{m}} \vec{E} \cdot \overrightarrow{d s}-\iint \overrightarrow{J_{m}} \cdot \overrightarrow{d s},
\end{gathered}
$$

where $\overrightarrow{J_{e}}$ and $\overrightarrow{J_{m}}$ are the electric and magnetic current density sources, respectively; the tensors $\hat{\sigma_{e}}, \hat{\mu}, \hat{\sigma_{e}}, \hat{\sigma_{m}}$ are given by $\hat{\varepsilon_{e}}=\varepsilon_{0} \operatorname{diag}\left(\varepsilon_{x x}, \varepsilon_{y y}, \varepsilon_{z z}\right), \hat{\mu}_{e}=\mu_{0} \operatorname{diag}\left(\mu_{x x}, \mu_{y y}, \mu_{z z}\right), \hat{\sigma}_{e}=$ $\operatorname{diag}\left(\sigma_{e x}, \sigma_{e y}, \sigma_{e z}\right), \hat{\sigma_{m}}=\operatorname{diag}\left(\sigma_{m x}, \sigma_{m y}, \sigma_{m z}\right)$ with $\varepsilon_{0}$ and $\mu_{0}$ the permittivity and permeability of free space, respectively.

\subsubsection{Field sampling}

Consider the standard 3D SCN node. Using the same notation for the SCN node proposed by Johns, samples for the electric and magnetic fields in the cell are presented in (Figure 2).
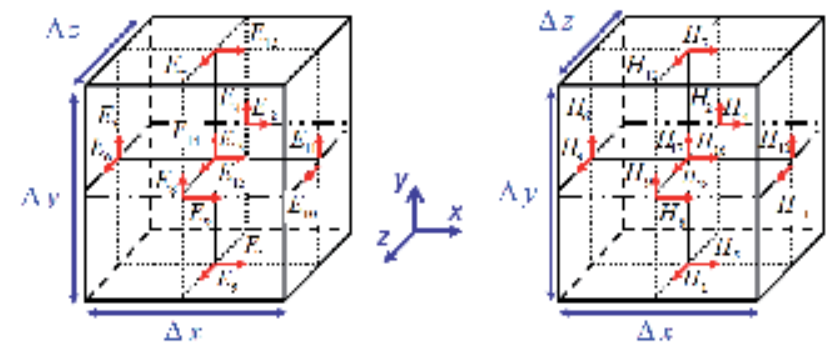

Figure 2. Field sampling in TLM, (a) electric field and (b) magnetic field

$E_{n}$ and $H_{n}$ correspond to field samples at each point of the cell. The incident $V^{i}$ and reflected $V^{r}$ voltages are related with these samples by (8). This equation gives us the relationship between the tangential fields at each face of the node and voltages in TLM network.

$$
V^{i}=\frac{1}{2}\left\{\begin{array}{c}
\Delta x E_{2}^{x}+Z_{z x} \Delta y H_{2}^{y} \\
\Delta y E_{3}^{y}+Z_{x y} \Delta z H_{3}^{z} \\
\Delta y E_{4}^{y}-Z_{z y} \Delta x H_{4}^{x} \\
\Delta z E_{6}^{z}-Z_{x z} \Delta y H_{6}^{y} \\
\Delta y E_{8}^{y}+Z_{z y} \Delta x H_{8}^{x} \\
\Delta x E_{9}^{x}-Z_{z x} \Delta y H_{9}^{y} \\
\Delta z E_{10}^{z}+Z_{x z} \Delta y H_{10}^{y} \\
\Delta y E_{11}^{y}-Z_{x y} \Delta z H_{11}^{z}
\end{array}\right\} V^{r}=\frac{1}{2}\left\{\begin{array}{c}
\Delta x E_{2}^{x}-Z_{z x} \Delta y H_{2}^{y} \\
\Delta y E_{3}^{y}-Z_{x y} \Delta z H_{3}^{z} \\
\Delta y E_{4}^{y}+Z_{z y} \Delta x H_{4}^{x} \\
\Delta z E_{6}^{z}+Z_{x z} \Delta y H_{6}^{y} \\
\Delta y E_{8}^{y}-Z_{z y} \Delta x H_{8}^{x} \\
\Delta x E_{9}^{x}+Z_{z x} \Delta y H_{9}^{y} \\
\Delta z E_{10}^{z}-Z_{x z} \Delta y H_{10}^{y} \\
\Delta y E_{11}^{y}+Z_{x y} \Delta z H_{11}^{z}
\end{array}\right\}
$$


$\vec{E}$ and $\vec{H}$ denote the electric and magnetic fields, respectively; $Z_{i j}$ are the characteristic impedances of each arm. They are equal to $Z_{0}$ for the SCN node. Finally, $\Delta x, \Delta y$, and $\Delta z$ correspond to the cell dimensions. Note that the voltages $V_{1}, V_{5}, V_{7}$, and $V_{12}$ of the SCN node do not appear since they were removed. Six additional voltages (incident and reflected) have to be considered to model materials with a relative permittivity and/or permeability higher than the unity. They can be obtained by using the vector:

$$
\begin{gathered}
\vec{V}_{E}=\frac{1}{2}\left[\Delta x E_{13}^{x} \Delta y E_{14}^{y} \Delta z E_{15}^{z}\right] \\
\vec{V}_{H}=\frac{1}{2} Z_{0}\left[\Delta x H_{16}^{x} \Delta y H_{17}^{y} \Delta z H_{18}^{z}\right]
\end{gathered}
$$

\subsubsection{Determination of the updating equations for the fields}

To find the updating relationships for the electric and magnetic fields, an approximation of Maxwell's equations in the integral form must be done. This can be accomplished by considering the rectangular mesh of Figure 2 and applying (6) and (7) in the main integration planes, $(z, y),(x, z)$, and $(x, y)$.

\subsubsection{Integration in the $(z, y)$-plane}

Figure 3 shows the contour of integration for the magnetic and electric fields in the $(z$, y)-plane. The samples are taken according to Figure 2 and considering the exponential dependence of the fields in the propagation direction.

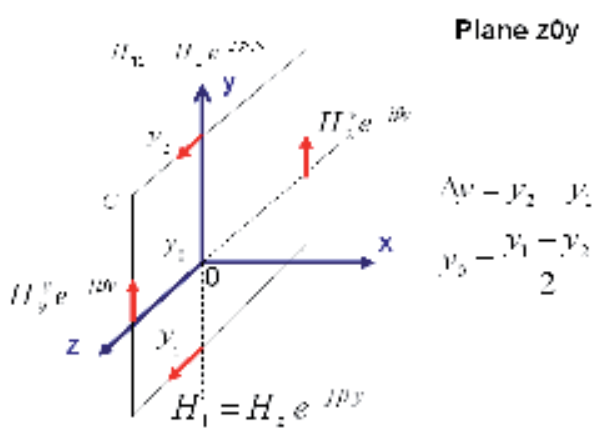

(a)

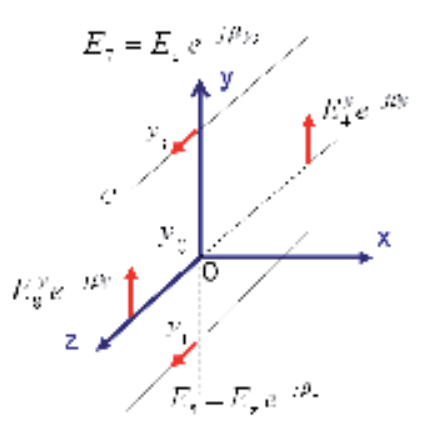

(b)

Figure 3. Integration of Maxwell's equations in the integral form in the plane $(z, y)$. (a) H-field and (b) E-field

Maxwell-Ampère and Maxwell-Faraday equations applied to the contour $\Gamma$ bounding this plane are given by (13) and (14), respectively. Moreover, electric and magnetic laws are given by (15) and (16), respectively. The calculation of these integrals is done by approximating their values as the mean value of the same integral at the time steps $(n+1 / 2) \Delta t$ and $(n-$ $1 / 2) \Delta t$. For the contour integrals, the approximation 


$$
\int_{(n-1 / 2) \Delta t}^{(n+1 / 2) \Delta t} O(t) d t \approx \Delta t\left(\frac{1+T}{2}\right) \widetilde{O},
$$

is used. The term $\widetilde{O}=\oint_{\Gamma} \vec{H} . \overrightarrow{d l}$ or $\widetilde{O}=\oint_{\Gamma} \vec{E} . \overrightarrow{d l}$. Regarding the surface integrals, they can be calculated using

$$
\int_{(n-1 / 2) \Delta t}^{(n+1 / 2) \Delta t} \frac{P(t)}{d t} d t \approx(1-T) P,
$$

where $P(t)=\iint_{S} \vec{D} \cdot \overrightarrow{d S}$ or $P(t)=\iint_{S} \vec{B} \cdot \overrightarrow{d S}$.

The application of the above expressions gives

$$
\begin{gathered}
\oint_{C} \vec{H} \cdot \overrightarrow{d l} \cong\left(\frac{1+T}{2}\right) 2 \sin \left(\frac{\beta \Delta y}{2}\right) e^{-j \beta y_{0}}\left[\frac{1}{\beta}\left(H_{2}^{y}-H_{9}^{y}\right)-j \Delta z H_{z}^{(n)}\right] \\
\oint_{C} \vec{E} \cdot \overrightarrow{d l} \cong\left(\frac{1+T}{2}\right) 2 \sin \left(\frac{\beta \Delta y}{2}\right) e^{-j \beta y_{0}}\left[\frac{1}{\beta}\left(E_{4}^{y}-E_{8}^{y}\right)-j \Delta z E_{z}^{(n)}\right] \\
\varepsilon \iint_{S} \frac{\partial \vec{E}_{x}}{\partial t} \cdot \overrightarrow{d S} \cong(1-T) \frac{\varepsilon_{x} \Delta y \Delta z}{Z_{0} c_{0} \Delta t \beta \Delta x} \Delta x \tilde{E_{x}} e^{-j \beta y_{0}} 2 \sin \left(\frac{\beta \Delta y}{2}\right) \\
-\mu \iint_{S} \frac{\partial \vec{H}_{x}}{\partial t} \cdot \overrightarrow{d S} \cong(T-1) \frac{Z_{0} \mu_{x} \Delta y \Delta z}{c_{0} \Delta t \beta \Delta x} \Delta x \tilde{H}_{x} e^{-j \beta y_{0}} 2 \sin \left(\frac{\beta \Delta y}{2}\right)
\end{gathered}
$$

$T$ corresponds to a delay operator, $Z_{0}$ is the characteristic impedance in free space, and $c_{0}$ the speed of light in vacuum. The terms $\tilde{E}_{x}$ and $\tilde{H}_{x}$ correspond to an approximation of the fields at instant $n \Delta t$. They can be calculated as the mean value of the field components in the plane:

$$
\begin{gathered}
\tilde{E_{x}}=\frac{E_{2}^{x}+E_{9}^{x}+\hat{Y_{s x}} E_{13}^{x}}{\hat{Y_{s x}}+2} \approx E_{x}^{(n)} \\
\tilde{H}_{x}=\frac{H_{4}^{x}+H_{8}^{x}+\hat{Z_{s x}} H_{16}^{x}}{{\hat{Z_{s}}}+2} \approx H_{x}^{(n)}
\end{gathered}
$$

where $\hat{Y_{s x}}$ and $\hat{Z_{s x}}$ are the normalized admittances and impedances, respectively, to model the materials with a relative permeability and/or permittivity higher than unity. Their values are given by

$$
\hat{Y}_{s i}+2=2 \frac{\varepsilon_{i} \Delta j \Delta k}{c_{0} \Delta t \Delta i} \text { for } \mathrm{i} \in(x, z) \hat{Z_{s i}}+2=2 \frac{\mu_{i} \Delta j \Delta k}{c_{0} \Delta t \Delta i} \text { for } \mathrm{i} \in(x, z)
$$


Regarding loss terms, the approximation of the corresponding integrals in (13) is done by taking their value at the center of the cell. This leads to

$$
\begin{gathered}
\iint_{S} \sigma_{e \zeta} \vec{E}_{\zeta} \cdot \overrightarrow{d S} \cong G_{e \zeta}\left[\Delta \zeta \tilde{E}_{\zeta}\right]=\sigma_{e \zeta} \frac{\Delta \chi \Delta \rho}{\Delta \zeta}\left[\Delta \zeta \tilde{E_{\zeta}}\right] \\
\iint_{S} \sigma_{m \zeta} \vec{H}_{\zeta} \cdot \overrightarrow{d S} \cong R_{m \zeta}\left[\Delta \zeta \tilde{H}_{\zeta}\right]=\sigma_{m \zeta} \frac{\Delta \chi \Delta \rho}{\Delta \zeta}\left[\Delta \zeta \tilde{H}_{\zeta}\right],
\end{gathered}
$$

where $\zeta$ denotes the field component $x, y$, or $z$ and $G_{e \zeta}$ and $R_{m \zeta}$ correspond to the electric conductance and magnetic resistance, respectively, for each direction. Finally, the surface integrals of the sources are evaluated by taking their values at the center:

$$
\begin{gathered}
\iint_{S} J_{e \zeta} \cdot \overrightarrow{d S} \cong V_{e \zeta} \\
\iint_{S} J_{m \zeta} \cdot \overrightarrow{d S} \cong V_{m \zeta} \Delta S,
\end{gathered}
$$

where $V_{e \zeta}=J_{e \zeta} \Delta S$ and $V_{m \zeta}=J_{m \zeta} \Delta S$. The infinitesimal area can be calculated, for instance, as $\Delta S=\Delta y \Delta z$ for the $x$-direction $(\zeta=x)$.

By replacing the above expression in Maxwell's equations (6) and (7), the expressions in (24) and (25) can be obtained. Equation (8) was employed to express the fields in terms of reflected and incident voltages.

$$
\begin{aligned}
& V_{2}^{r}+V_{9}^{r}+\hat{Y_{s x}} V_{13}^{r}=V_{2}^{i}+V_{9}^{i}+\hat{Y_{s x}} V_{13}^{i}-\alpha^{\prime} Z_{0} \Delta z H_{z}^{(n)}-\frac{R_{m x}}{Z_{0}} \Delta z H_{x}^{(n)}-\frac{V_{m x}}{Z_{0}} \\
& V_{4}^{r}-V_{8}^{r}+\hat{Z_{s x}} V_{16}^{r}=-V_{4}^{i}+V_{8}^{i}+\hat{Z_{s x}} V_{16}^{i}+\alpha^{\prime} \Delta z E_{z}^{(n)}-\frac{R_{m x}}{Z_{0}} \Delta z H_{x}^{(n)}-\frac{V_{m x}}{Z_{0}},
\end{aligned}
$$

where $\alpha^{\prime}=\beta \Delta y / 2$.

\subsubsection{Integration in the $(x, z)$-plane}

This plane is perpendicular to the propagation direction; thus, any integral is affected by the exponential dependence of the fields. They can be calculated as for the standard SCN node. The contours of integration for the magnetic and electric fields in the $(x, z)$-plane are shown in Figure 4, respectively.

Maxwell-Ampere and Maxwell-Faraday equations applied to these contours are given by

$$
\oint_{C} \vec{H} . \overrightarrow{d l} \cong\left(\frac{1+T}{2}\right)\left[\Delta x\left(H_{4}^{x}-H_{8}^{x}\right)-\Delta z\left(H_{11}^{z}-H_{3}^{z}\right)\right]
$$




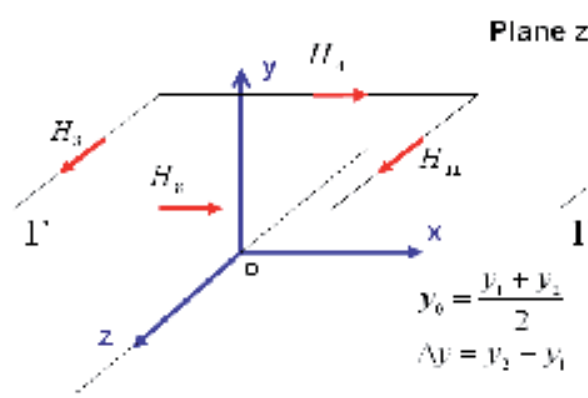

(a)

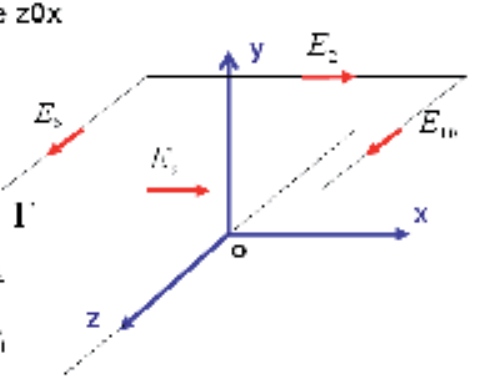

(b)

Figure 4. Integration contour of Maxwell's equations in the integral form in the plane $(x, z)$. (a) H-field and (b) E-field

$$
\oint_{C} \vec{E} \cdot \overrightarrow{d l} \cong\left(\frac{1+T}{2}\right)\left[\Delta x\left(E_{2}^{x}-E_{9}^{x}\right)-\Delta z\left(E_{10}^{z}-E_{6}^{z}\right)\right]
$$

whereas the right-hand side of the Maxwell-Ampere and Maxwell-Faraday equations are given by:

$$
\begin{aligned}
& R H S_{M A} \cong(1-T) \frac{\varepsilon_{y} \Delta x \Delta z}{Z_{0} c_{0} \Delta t \Delta y} \Delta y \tilde{E_{y}}+Z_{0} G_{e y} \Delta y E_{y}^{n}+Z_{0} V_{e y} \\
& R H S_{M F} \cong(T-1) \frac{Z_{0} \mu_{y} \Delta x \Delta z}{c_{0} \Delta t \Delta y} \Delta y \tilde{H}_{y}-\frac{R_{m y}}{Z_{0}} \Delta y H_{y}^{n}-\frac{V_{m y}}{Z_{0}},
\end{aligned}
$$

where $G_{e y}=\sigma_{e y} \Delta z \Delta y / \Delta x$ is the term employed to model the electric losses, $R_{m y}=$ $\sigma_{m y} \Delta z \Delta y / \Delta x$ models the magnetic losses, $T$ and $Z_{0}$ were already defined, $V_{e y}=J_{e y} \Delta z \Delta y$, $V_{m y}=J_{m y} \Delta z \Delta y$, and $c_{0}$ the speed of light in vacuum. $R H S_{M A}$ and $R H S_{M F}$ correspond to the right-hand side of Maxwell's Faraday and Maxwell's (6) and (7), respectively; the terms $\tilde{E_{y}}$ and $\tilde{H}_{y}$ correspond to fields approximations of the fields at instant $n \Delta t$ given by

$$
\begin{gathered}
\tilde{E_{y}}=\frac{E_{4}^{y}+E_{8}^{y}+E_{11}^{y}+E_{3}^{y}+\hat{Y_{s y}} E_{14}^{y}}{\hat{Y_{s y}}+4} \approx E_{y}^{(n)} \\
\tilde{H}_{y}=\frac{H_{2}^{y}+H_{10}^{y}-H_{9}^{y}-H_{6}^{y}+\hat{Z_{s y}} H_{17}^{y}}{\hat{Z_{s y}}+4} \approx H_{y}^{(n)}
\end{gathered}
$$

In this case, the normalized admittances and impedances $\hat{Y_{s y}}$ and $\hat{Z_{s y}}$ are given, respectively, by

$$
\hat{Y}_{s i}+4=2 \frac{\varepsilon_{i} \Delta j \Delta k}{c_{0} \Delta t \Delta i} \text { for } \mathrm{i}=\mathrm{y} \hat{Z_{s i}}+4=2 \frac{\mu_{i} \Delta j \Delta k}{c_{0} \Delta t \Delta i} \text { for } \mathrm{i}=\mathrm{y}
$$


Finally, (26) with (28) and (27) with (29), one obtains (33) and(34), respectively. Again, equation (8) was employed to express the equation in terms of reflected and incident voltages.

$$
\begin{gathered}
V_{3}^{r}+V_{11}^{r}+V_{4}^{r}+V_{8}^{r}+\hat{Y_{s y}} V_{14}^{r}= \\
V_{3}^{i}+V_{11}^{i}+V_{4}^{i}+V_{8}^{i}+\hat{Y_{s y}} V_{14}^{i}-Z_{0} G_{e y} \Delta y E_{y}^{(n)}-Z_{0} V_{e y} \\
-V_{2}^{r}-V_{10}^{r}+V_{9}^{r}+V_{6}^{r}+\hat{Z_{s y}} V_{17}^{r}= \\
V_{2}^{i}+V_{10}^{i}-V_{9}^{i}-V_{6}^{i}+\hat{Z_{s y}} V_{17}^{i}-\frac{R_{m y}}{Z_{0}} \Delta y H_{y}^{(n)}-\frac{V_{m y}}{Z_{0}}
\end{gathered}
$$

\subsubsection{Integration in the $(x, y)$-plane}

This plane is parallel to the propagation direction. Thus, the value of the integrals will depend on the exponential dependence of the fields. The contours of integration for the magnetic and electric fields in the $(x, y)$-plane are shown in Figure 5.

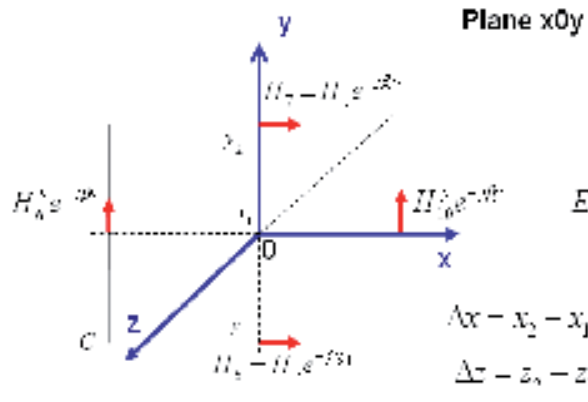

(a)

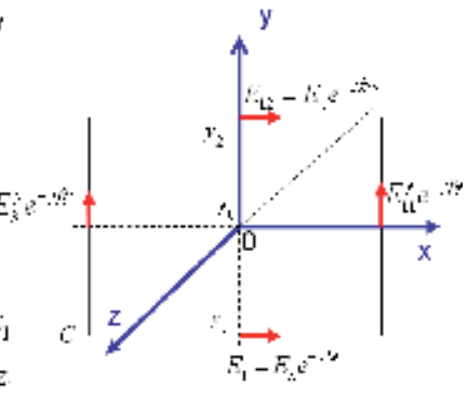

(b)

Figure 5. Integration contour of Maxwell's equations in the integral form in the plane $(x, y)$. (a) H-field and (b) E-field

By using the same procedure as for the other planes, Maxwell-Ampere and Maxwell-Faraday equations and electric and magnetic laws yield, respectively,

$$
\begin{gathered}
\oint_{C} \vec{H} \cdot \overrightarrow{d l} \cong\left(\frac{1+T}{2}\right) 2 \sin \left(\frac{\beta \Delta y}{2}\right) e^{-j \beta y_{0}}\left[\frac{1}{\beta}\left(H_{10}^{y}-H_{6}^{y}\right)-j \Delta x H_{x}\right] \\
\oint_{C} \vec{E} \cdot \overrightarrow{d l} \cong\left(\frac{1+T}{2}\right) 2 \sin \left(\frac{\beta \Delta y}{2}\right) e^{-j \beta y_{0}}\left[\frac{1}{\beta}\left(E_{11}^{y}-E_{3}^{y}\right)-j \Delta x E_{x}\right] \\
R H S_{M A} \cong(1-T) \frac{\varepsilon_{z} \Delta x \Delta y}{Z_{0} c_{0} \Delta t \Delta z} \Delta y \tilde{E}_{z}+Z_{0} G_{e y} \Delta z E_{z}^{n}+Z_{0} V_{e z}
\end{gathered}
$$




$$
R H S_{M F} \cong(T-1) \frac{L_{0} \mu_{z} \Delta x \Delta y}{c_{0} \Delta t \Delta y} \Delta z \tilde{H}_{z}-\frac{\kappa_{m z}}{Z_{0}} \Delta z H_{z}^{n}-\frac{v_{m z}}{Z_{0}}
$$

In the above, $G_{e z}=\sigma_{e z} \Delta x \Delta y / \Delta z$ is the term used to model the electric losses, $R_{m z}=$ $\sigma_{m} \Delta x \Delta y / \Delta z$ models the magnetic losses, $V_{e z}=J_{e z} \Delta x \Delta y, V_{m y}=J_{m y} \Delta x \Delta y$, and the terms $\tilde{E}_{z}$ and $\tilde{H}_{z}$ which correspond to the mean value of all field components in the plane are given, respectively, by

$$
\begin{gathered}
\tilde{E}_{z}=\frac{E_{10}^{z}+E_{6}^{z}+\hat{Y_{s z}} E_{15}^{z}}{\hat{Y_{s z}}+2} \approx E_{z}^{(n)} \\
\tilde{H}_{z}=\frac{H_{11}^{z}+H_{3}^{z}+\hat{Z_{s z}} H_{18}^{z}}{\hat{Z_{s z}}+2} \approx H_{z}^{(n)}
\end{gathered}
$$

The normalized admittances $\hat{Y_{s z}}$ and impedances $\hat{Z_{s z}}$ are given by (19). By following a similar procedure to the other integration planes, the following expressions are obtained:

$$
\begin{gathered}
V_{10}^{r}+V_{6}^{r}+\hat{Y_{s z}} V_{15}^{r}= \\
V_{10}^{i}+V_{6}^{i}+\hat{Y_{s z}} V_{15}^{i}+\alpha Z_{0} \Delta x H_{x}^{(n)}-Z_{0} G_{e z} \Delta z E_{z}^{(n)}-Z_{0} V_{e z} \\
V_{11}^{r}-V_{3}^{r}+\hat{Z_{s x}} V_{18}^{r}= \\
-V_{11}^{i}+V_{3}^{i}+\hat{Z_{s x}} V_{18}^{i}-\alpha Z_{0} \Delta x E_{x}^{(n)}-\frac{R_{m z}}{Z_{0}} \Delta z H_{z}^{(n)}-\frac{V_{m z}}{Z_{0}}
\end{gathered}
$$

The relationships of (24), (25),(33),(34),(41), and (42) allow us to determine $\Delta x E_{x}^{n}, \Delta x H_{x}^{n}$, $\Delta y E_{y}^{(n)}, \Delta y H_{y}^{(n)}, \Delta z E_{z}^{(n)}$, and $\Delta z H_{z}^{(n)}$, from which fields can be computed at any time step. Eight additional relationships are still needed to obtain reflected voltages. By expressing the fields at the center of the cell, these expressions can be found, as detailed in the next subsection.

\subsubsection{Determination of the updating equations for the voltages}

To obtain the reflected voltages at any time step, six new contours have to be defined. Figure 6 shows the integration contours for finding fields at the center of the cell using Maxwell's equations in the integral form in the three planes.

The procedure is the same as presented before. First, Maxwell's equations are approximated for each of these contours. Consider the contour in dashed lines for the plane $(z, y)$; Maxwell-Ampere and the electric flux equations are given, respectively, by

$$
\oint_{C} \vec{H} \cdot \overrightarrow{d l} \cong\left[2 \Delta y H_{y}^{n}-\left(\frac{1+T}{2}\right)\left(H_{2}-H_{9}\right)\right] e^{-j \beta y_{0}} 2 \sin \left(\frac{\beta \Delta y}{2}\right)
$$



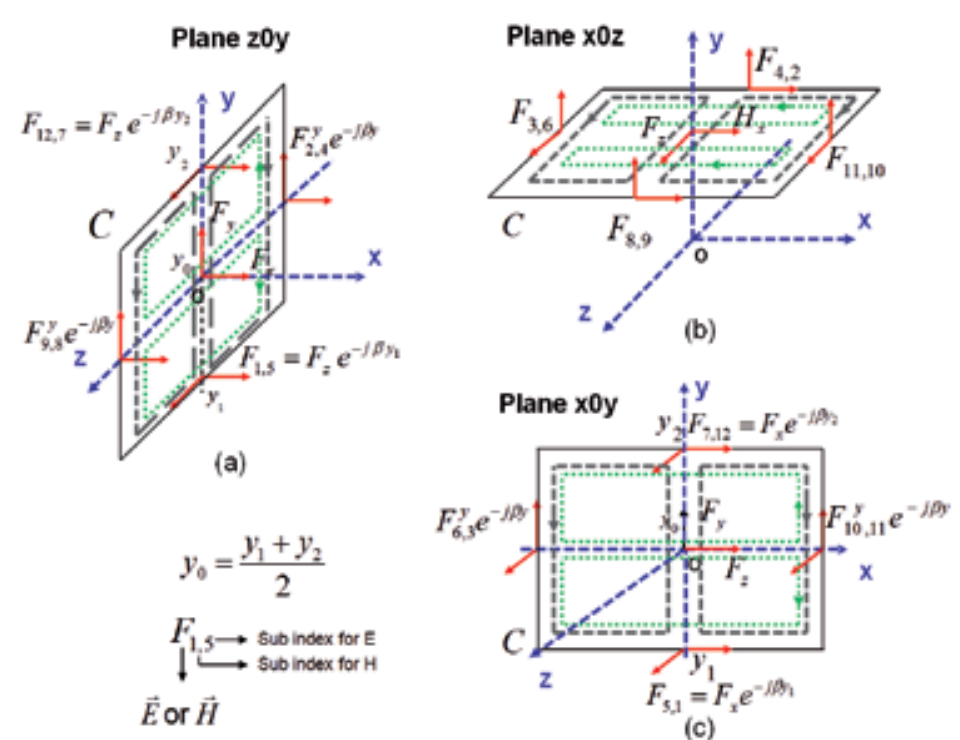

(c)

Figure 6. Integration contours for Maxwell's equations in the integral form for finding fields at the center of the cell: (a) $x 0 z$, (b) $z 0 x$, and (c) $x 0 y$ plane

$$
\varepsilon \iint \frac{\partial \vec{E}_{x}}{\partial t} \cdot \overrightarrow{d S} \cong \frac{1}{2}(1-T) \frac{\varepsilon_{x} \Delta y \Delta z}{Z_{0} c_{0} \Delta t \Delta x} \Delta x\left(E_{9}-E_{2}\right) e^{-j \beta y_{0}} 2 \sin \left(\frac{\beta \Delta y}{2}\right)
$$

Then, the expressions in (43) and (44) are used in combination with (8) to express the result in terms of reflected and incident voltages, yielding (45). By considering the remaining red and blue contours and applying Maxwell's equations in these planes, (46) to (50) can be found.

$$
\begin{aligned}
2 Z_{0} \Delta y H_{y}^{(n)} & =\hat{Y_{z x}}\left(V_{9}^{r}-V_{2}^{r}+V_{9}^{i}-V_{2}^{r}\right) \\
2 \Delta y E_{y}^{(n)} & =\left(V_{8}^{r}+V_{4}^{r}+V_{4}^{i}+V_{8}^{i}\right) \\
2 Z_{0} \Delta y H_{y}^{(n)} & =\hat{Y_{x z}}\left(V_{6}^{r}-V_{10}^{r}+V_{6}^{i}-V_{10}^{r}\right) \\
2 \Delta y E_{y}^{(n)} & =\left(V_{3}^{r}+V_{11}^{r}+V_{3}^{i}+V_{11}^{i}\right) \\
2 Z_{0} \Delta z H_{z}^{(n)} & =\hat{Y_{x y}}\left(V_{11}^{r}-V_{3}^{r}+V_{11}^{i}-V_{3}^{r}\right) \\
2 \Delta y E_{y}^{(n)} & =\left(V_{6}^{r}+V_{10}^{r}+V_{6}^{i}+V_{10}^{i}\right) \\
2 Z_{0} \Delta z H_{z}^{(n)} & =\hat{Y_{y x}}\left(V_{1}^{r}-V_{12}^{r}+V_{1}^{i}-V_{12}^{r}\right) \\
2 \Delta y E_{y}^{(n)} & =\left(V_{5}^{r}+V_{7}^{r}+V_{5}^{i}+V_{7}^{i}\right)
\end{aligned}
$$




$$
\begin{aligned}
2 Z_{0} \Delta x H_{x}^{(n)} & =\hat{Y_{y z}}\left(V_{7}^{r}-V_{5}^{r}+V_{7}^{i}-V_{5}^{r}\right) \\
2 \Delta x E_{x}^{(n)} & =\left(V_{1}^{r}+V_{12}^{r}+V_{1}^{i}+V_{12}^{i}\right) \\
2 Z_{0} \Delta x H_{x}^{(n)} & =\hat{Y_{z y}}\left(V_{4}^{r}-V_{8}^{r}+V_{4}^{i}-V_{8}^{r}\right) \\
2 \Delta x E_{x}^{(n)} & =\left(V_{2}^{r}+V_{9}^{r}+V_{2}^{i}+V_{9}^{i}\right)
\end{aligned}
$$

In summary, 12 expressions at the center were found. These expressions relate fields at instant $n \Delta t$, incident and reflected voltages. They allow us to update the eight voltages in the node. By replacing the above expressions of the fields in (8), one obtains

$$
\left[\begin{array}{c}
V_{9}^{r} \\
V_{11}^{r} \\
V_{8}^{r} \\
V_{10}^{r} \\
V_{4}^{r} \\
V_{2}^{r} \\
V_{6}^{r} \\
V_{3}^{r}
\end{array}\right]=\left[\begin{array}{c}
\Delta x E_{x}^{(n)}-Z_{0} \Delta y H_{y}^{(n)} \\
\Delta y E_{y}^{(n)}-Z_{0} \Delta z H_{z}^{(n)} \\
\Delta y E_{y}^{(n)}+Z_{0} \Delta x H_{x}^{(n)} \\
\Delta z E_{z}^{(n)}+Z_{0} \Delta y H_{y}^{(n)} \\
\Delta y E_{y}^{(n)}-Z_{0} \Delta x H_{x}^{(n)} \\
\Delta x E_{x}^{(n)}+Z_{0} \Delta y H_{y}^{(n)} \\
\Delta z E_{z}^{(n)}-Z_{0} \Delta y H_{y}^{(n)} \\
\Delta y E_{y}^{(n)}+Z_{0} \Delta z H_{z}^{(n)}
\end{array}\right]-\left[\begin{array}{c}
V_{9}^{i} \\
V_{11}^{i} \\
V_{8}^{i} \\
V_{10}^{i} \\
V_{4}^{i} \\
V_{2}^{i} \\
V_{6}^{i} \\
V_{3}^{i}
\end{array}\right]
$$

The calculation of reflected voltages at stubs is done as for standard SCN:

$$
\begin{aligned}
& V_{13}^{r}=\Delta x E_{x}^{(n)}-V_{13}^{i} \\
& V_{14}^{r}=\Delta y E_{y}^{(n)}-V_{14}^{i} \\
& V_{15}^{r}=\Delta z E_{z}^{(n)}-V_{15}^{i} \\
& V_{16}^{r}=\Delta x Z_{0} H_{x}^{(n)}-V_{16}^{i} \\
& V_{17}^{r}=\Delta y Z_{0} H_{y}^{(n)}-V_{17}^{i} \\
& V_{18}^{r}=\Delta z Z_{0} H_{z}^{(n)}-V_{18}^{i}
\end{aligned}
$$

\subsection{Results of the 2.5D modal approach}

To characterize any waveguide field amplitudes, attenuation constant and phase constant must be calculated. Here, we examine how to obtain these parameters by using the $2.5 \mathrm{D}$ TLM approach for guiding structures.

\subsubsection{Phase constant: dispersion diagram $\beta-\omega$}

For each mode, $\beta$ is a function of frequency $\omega$; it is known as the dispersion diagram of the structure. In general, this relationship is not linearly proportional to the frequency for waveguides. The computation of the dispersion diagram with the TLM approach for guiding 
structures is as follows: The structure has to be excited as explained in Section 3.1. Then, the field components at the time step $k \Delta t$ can be found through $(17,18,30,31,39,40)$. After a sufficient number of time-step iterations, the response corresponds to the superposition of the modal fields in the guide. By performing a Fourier transform, the frequency response of the structure can be obtained. The peaks in this spectrum correspond to the modes, and they belong to a specific value of the phase constant $\beta$. Hence, different $\beta$ values have to be imposed to obtain the dispersion diagram.

\subsubsection{Attenuation constant}

The attenuation of the modes can be extracted from the time-domain response at a phase constant $\beta_{l}$ given by

$$
E_{l m}(k \Delta t)=\sum_{k=0}^{M} E^{l m}(0) e^{-\frac{\omega_{l m}}{2 Q_{l m}} k \Delta t} e^{j \omega_{l m} k \Delta t}
$$

where $M$ is the number of modes propagating in the structure, $E^{l m}(0)$ is the initial field magnitude of the $m$-th mode, $Q_{l m}$ its quality factor, and $\omega_{l m}$ the angular resonance frequency of the mode in radians per second for the $l$-th evaluated value of $\beta_{l}$. A similar expression can be found for the magnetic field by replacing $E$ by $H$ in (53). To characterize the attenuation of the modes, the damping parameter has to be estimated. At each frequency $\omega_{l m}$ in the dispersion curve of a given mode, the attenuation constant is related with the quality factor $Q_{l m}$ and the group velocity $V_{g_{l m}}$ by [34]

$$
\alpha_{l m} \approx \frac{\omega_{l m}}{2 Q_{l m} V_{g_{l m}}}=\frac{\omega_{l m}}{2 Q_{l m}} \frac{\partial \beta}{\partial \omega}
$$

The terms $\omega_{l m} / 2 Q_{l m}$ can be extracted from the sampled time-domain response of (53) by using an estimation technique for modal content of a time-varying waveform. For instance, the Matrix pencil method has been a very popular technique for efficient extraction of the modal parameters [14]. Finally, the group velocity in (54), which involves the derivate of $\beta$ with respect to $\omega$, can be approximated by taking the finite differences $\Delta \beta / \Delta \omega$ of the dispersion diagram calculated in the previous section.

\section{Implementation of the 2.5D TLM for guiding structures}

To validate this approach, the study of a simple theoretical "reference solution" for a canonical geometry is considered. A metallic-rectangular waveguide homogeneously filled with a lossy dielectric material is studied in this section.

\subsection{Results for a homogeneously lossy dielectric-filled metallic-rectangular waveguide}

Consider a metallic waveguide with dimensions $a=6 \mathrm{~cm}$ and height $b=4 \mathrm{~cm}$. It was filled with a lossy dielectric with relative permittivity $\epsilon_{r}=2$ and a conductivity $\sigma=0.001 \mathrm{Sm}^{-1}$ and simulated with the 2.5D TLM, as shown in Figure 7 (a). An array of $14 \times 10$ nodes is 
considered, and a perfect electric boundary condition is imposed at the top, bottom, and side walls, as illustrated in Figure 7 (b). To reduce the dispersion error, a mesh size of $\Delta l=\lambda / 15$ at $6 \mathrm{GHz}$ is chosen. The signal is obtained after $N=4000$ time steps and is convolved with a Hanning window.

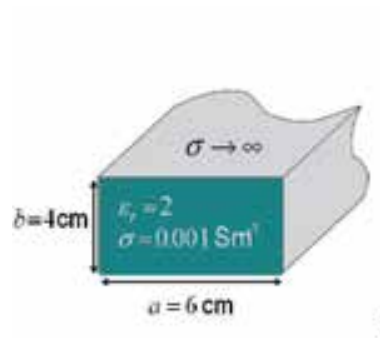

a)

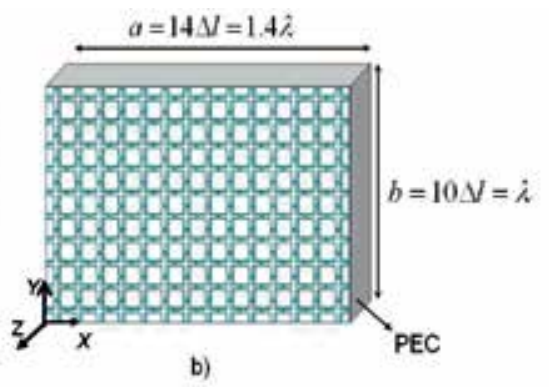

b)

Figure 7. (a) Rectangular-metallic waveguide filled with a lossy dielectric; (b) 2.5D TLM mesh of the rectangular waveguide terminated by a perfect electric boundary condition (PEC). The dimensions are given in terms of the mesh size $\Delta l=\lambda / 15$ at $6 \mathrm{GHz}$

The dispersion diagram is obtained in the frequency range from $1 \mathrm{GHz}$ up to $4 \mathrm{GHz}$ and is compared with the theory. For the case of a rectangular-metallic waveguide, the theoretical expression for the dispersion diagram is given by [26]:

$$
\beta=\sqrt{(\omega \sqrt{\mu \epsilon})^{2}-\left(\frac{m \pi}{a}\right)^{2}-\left(\frac{n \pi}{b}\right)^{2}}
$$

where $\omega$ is the angular frequency; $\mu=\mu_{0} ; \epsilon=\epsilon_{r} \epsilon_{0} ; m, n$ correspond to the order of the modes; and $a$ and $b$ are the waveguide dimensions. The comparison between the numerical dispersion diagram and the theoretical one is shown in Figure 8.

The continuous lines represent the theoretical results, whereas the discrete points are the results obtained by using the 2.5D TLM algorithm. Different $\beta$ values ranging from 0 to $80(\mathrm{rad} / \mathrm{m})$ with 80 samples have been considered. The simulation is carried out for 4000 iterations for each $\beta$ value. Good agreement between theory and the 2.5D TLM for guiding structures was obtained. The dispersion curves of a plane wave propagating in free space and a wave propagating in a medium with the same relative permittivity as the loading material $\left(\epsilon_{r}=2\right)$ are plotted as well. It is worth observing that the dispersion curves of the modes tend to the curve of a plane wave propagating in a medium with relative permittivity $\epsilon_{r}=2$ as the frequency increases. Finally, the theoretical attenuation constant of the metallic waveguide is calculated by [26]:

$$
\alpha_{d}=\frac{k^{2} \tan \delta}{2 \beta}
$$

where $k=\omega \sqrt{\mu \epsilon}$ is the (real) wave number in the absence of losses, $\tan \delta=\left(\omega \epsilon^{\prime \prime}+\sigma\right) / \omega \epsilon^{\prime}$ is the loss tangent with $\epsilon=\epsilon^{\prime}-j \epsilon^{\prime \prime}=\epsilon_{r} \epsilon_{0}-j \sigma / \omega$, and $\beta$ is the phase constant. The results 


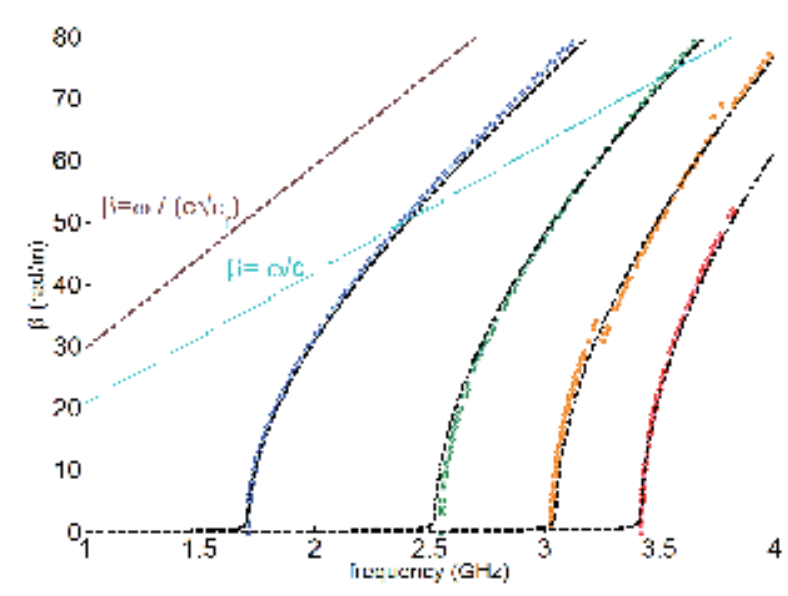

Figure 8. Dispersion diagram of a rectangular-metallic waveguide with dimensions $a=6 \mathrm{~cm}$ and $b=4 \mathrm{~cm}$ : Theory (continuous lines), results using the guiding 2.5D TLM approach (dots)

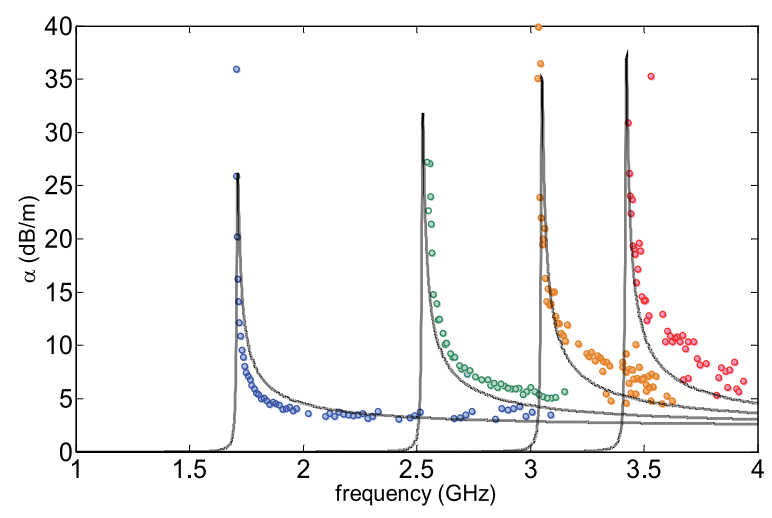

Figure 9. Attenuation constant for a lossy dielectric filled metallic-rectangular waveguide: Theory (continuous lines), simulation (dots)

obtained for the attenuation constants by numerical simulation and theory are shown in Figure 9.

The comparison between both curves validates this approach quite satisfactorily. The discrepancies observed close to the cutoff frequency are explained by the fact that (57) is valid as long as $\alpha<<\beta$.

$$
\alpha \approx \frac{\partial \beta}{\partial \omega} \Delta \omega
$$

Consequently, since $\alpha$ is deduced from $\Delta \beta$ in the numerical approach, an inevitable large calculation error on the attenuation constant $\alpha$ is obtained for a given error on $\Delta \beta$. To 
illustrate this point, let us consider the calculation of the propagated error on $\alpha$. The total differential of a function $z=f\left(x_{1}, x_{2}, \ldots, x_{N}\right)$, where $x_{i}$ is the $i$-th independent variable, is given by

$$
d z=\left(\frac{\partial f}{\partial x_{1}}\right) \Delta x_{1}+\left(\frac{\partial f}{\partial x_{2}}\right) \Delta x_{2}+\ldots+\left(\frac{\partial f}{\partial x_{N}}\right) \Delta x_{N}
$$

the term $d z$ denotes the error in $z$. The uncertainty in the calculation of the attenuation constant for waveguides can be found by considering the differential of the attenuation and phase constants, $\alpha$ and $\beta$, respectively. The differential of the attenuation and phase constants can be computed by

$$
\begin{aligned}
& \Delta \alpha=\left(\frac{\partial \alpha}{\partial \omega}\right) \Delta \omega, \\
& \Delta \beta=\left(\frac{\partial \beta}{\partial \omega}\right) \Delta \omega .
\end{aligned}
$$

Finally, by dividing (59) by (60), the differential of the attenuation constant in terms of the differential of the phase constant is given by

$$
\Delta \alpha=\left(\frac{\partial \alpha}{\partial \omega}\right)\left(\frac{\partial \omega}{\partial \beta}\right) \Delta \beta
$$

is obtained.

As frequency increases, the first partial derivative on the right-hand side of (61) tends to zero and the second to the speed of light in free space, $c_{0}$. Hence, for high frequencies, the error $\Delta \alpha$ is bounded. For low frequencies, the first derivate tends to infinity and the second to a finite value, resulting in a larger error. Indeed, in Figure 9, the highest difference between both approaches occurs at cutoff frequencies of the modes and decreases far from these frequencies. Apart from this relative error on $\alpha$, the comparison between numerical results and theory for both propagation constant $\beta$ and the attenuation constant $\alpha$ confirms the suitability of the 2.5D TLM to characterize modal parameters in waveguiding structures.

\section{Numerical analysis of multimodal waveguide: Model of confined environments}

So far, we have considered the modeling of guiding structures with metallic walls. However, tunnels have dielectric walls and other materials within them (trains, cars, objects, etc.). They also involve boundary conditions which can be time or frequency dependent. The limitation of the computational domain within dielectric materials is usually achieved by using perfectly matched layer (PML) for transmission-line method (TLM), finite-difference time domain (FDTD), or finite element method (FEM) implementations. However, this solution is too expensive in terms of complexity and consequently, computation cost when dealing with very large electrical objects. Since we are interested only in fields within the hollow region of the tunnel, appropriate boundary conditions, namely, the surface 
impedance boundary conditions (SIBCs), constitute the mathematical artifice to limit the computational domain.

\subsection{Assumptions for tunnel wall modeling: SIBC concept}

To apply the SIBC concept, fields in the walls region are assumed to be time exponential decaying, source free, and with no reflections returning to the interface air and tunnels walls. For practical purposes, the wall thickness $t$ should be larger than the skin depth $\delta,(t>>\delta)$. A thickness of $2 \delta$ would ensure an amplitude of $1.8 \%$ of the launched wave [30]. Another important requirement is the smallest radius of curvature on a surface $R_{\min }$, which must be larger than the skin depth $\left(R_{\min }>>\delta\right)$. Consequently, the interface can be considered locally flat and plane-wave concept can be applied. Considering a numerical method like TLM, using the common criteria of $\lambda / 10$ for the maximum mesh size, waves can be treated locally as plane waves. Moreover, at this resolution level, the staircase mesh is a good approximation of curved surfaces.

\subsection{SIBC implementation in time domain for tunnel wall modeling}

The implementation of the SIBC concept was first introduced for good conductors. An overview of the implementations of these techniques in time domain can be found in $[5,23,27]$. Tunnel walls are typically modeled by a lossy dielectric with relative permittivity $\epsilon_{r}=10-12$ and conductivity $\sigma=0.01-0.02 \mathrm{Sm}^{-1}$ [9]. Maloney in [21] first introduced the SIBC for lossy dielectric in FDTD by using an expansion of the impedance model proposed by [5] for good conductors. Finally, [27] proposed a way to improve its implement in FDTD.

In contrast with good conductors, the SIBC for lossy dielectric varies with the angle of incidence and polarization of incident waves. If one considers Figure 10, surface impedances for vertical and horizontal polarizations are given by [24]

$$
\begin{aligned}
& Z_{V}(s)=\frac{E_{x}^{\prime+}(x, 0, s)}{H_{y}^{\prime+}(x, 0, s)}=\eta \frac{\cos \left(\theta_{t}\right)}{s+\sigma / \epsilon} \sqrt{s^{2}+2 \alpha s} \\
& Z_{H}(s)=-\frac{E_{y}^{\prime+}(x, 0, s)}{H_{x}^{\prime+}(x, 0, s)}=\eta \frac{s}{\cos \left(\theta_{t}\right) \sqrt{s^{2}+2 \alpha s}}
\end{aligned}
$$

where

$$
\begin{array}{ccc}
\alpha & =\frac{\sigma}{2 \epsilon \cos ^{2}\left(\theta_{t}\right)}=\frac{\sigma}{2 \epsilon_{0} \epsilon_{r} \cos ^{2}\left(\theta_{t}\right)} \\
\cos \left(\theta_{t}\right) & =\sqrt{1-\frac{v^{2}}{c^{2}} \sin ^{2}\left(\theta_{i}\right)}=\sqrt{1-\frac{\sin ^{2}\left(\theta_{i}\right)}{\mu_{r} \epsilon_{r}}} \\
v & =\quad \frac{1}{\sqrt{\mu \epsilon}}=\frac{1}{\sqrt{\mu_{0} \mu_{r} \epsilon_{0} \epsilon_{r}}}
\end{array}
$$




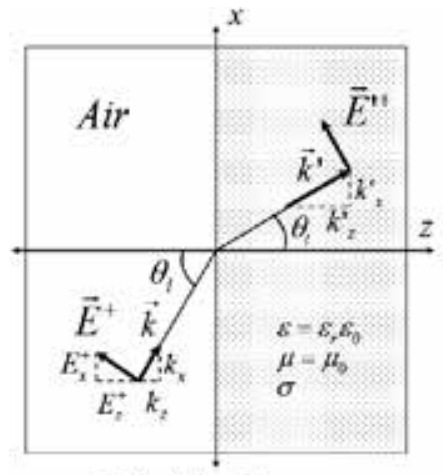

TM-Polarized wave

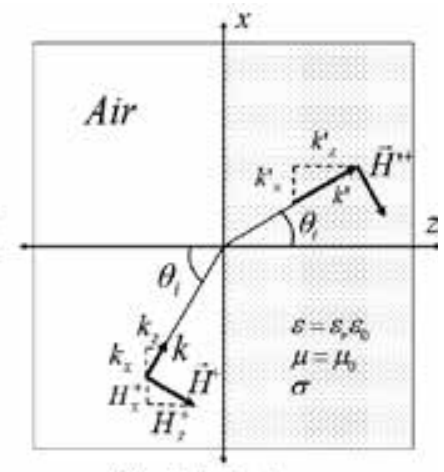

$T E$-Polarized wove

Figure 10. Geometry and coordinate system for a TE and TM polarized waves incident onto a dispersive half space

$Z(s)$ is the surface impedance; $s$ is the complex frequency; $\eta$ is the intrinsic impedance of the lossy dielectric; $\epsilon, \mu$, and $\sigma$ its constitutive parameters; and $\theta_{i}$ and $\theta_{t}$ are the angles of incidence and refraction, respectively. In tunnel environments, the incident angle $\theta_{i}$ for the waves is unknown. Moreover, multiple reflections of waves with different angles of incidence are involved. To the best of the authors' knowledge, no work has been reported for SIBC under these conditions. A procedure to implement it in any time-domain method as well as its validation is presented in [2,4]. Here, a brief explanation of its implementation is described.

\subsection{SIBC formulation for tunnel wall modeling}

The SIBC is inherently a frequency-domain concept. When it is transformed into the time domain for use in methods such as TLM, it is replaced by a convolution integral. Maloney in [21] proposed a recursive formula to overcome the computational difficulties for its calculation. To implement the SIBC in TLM, the reflection coefficient of (4) has to be calculated. This coefficient depends on the polarization, frequency, and angle of incidence. Thus, their influences have to be studied.

For tunnel walls, the relative permittivity $\epsilon_{r}>>1$. Then, for these values in (64), the term $\cos \left(\theta_{t}\right)$ in $($ Eqs.62, 63) tends to one, obtaining the two angle-independent expressions of the surface impedance for both polarizations:

$$
\begin{gathered}
Z_{V}(s)=Z_{\| p o l-T M}=\frac{\eta}{s+\sigma / \epsilon} \sqrt{s^{2}+2 \alpha s} \\
Z_{H}(s)=Z_{\perp p o l-T E}=\eta \frac{s}{\sqrt{s^{2}+2 \alpha s}}
\end{gathered}
$$

By using a rational polynomial approximation [12], the resulting coefficient of (4) can be calculated for both polarizations into the form 


$$
P(s) \approx \tilde{P}(s)=\frac{N(s)}{D(s)}=\Pi_{i=0}^{M}\left[\frac{s-\xi_{i}}{s-\zeta_{i}}\right] \approx \frac{V^{r}(s)}{V^{i}(s)},
$$

where $\xi_{i}$ and $\zeta_{i}$ correspond to the poles and zeros of $\tilde{P}(s)$ and $s_{i}$ are the sample points. Consider discrete values of a function $P(S)$ at points $S_{i}$. One can find two polynomials $N(s)$ and $D(s)$ of order $M$, such that $\tilde{P}(s)=N(s) / D(s)$ best approximates $P(s)$, in the least square sense.

The next step is to obtain (67) in z-domain. The bilinear z-transform can be employed, obtaining the rational polynomial expression

$$
P(z)=\Pi_{i=0}^{M}\left[\frac{z-\xi_{i}^{\prime}}{z-\zeta_{i}^{\prime}}\right]
$$

Finally, the discrete-time state variable representation of (68) can be obtained, which gives us the required relationship between the reflected and incident voltages necessary to implement the boundary in TLM. A canonical representation for the state-space and output equations is given, respectively, by

$$
\begin{gathered}
{\left[\begin{array}{c}
x_{1} \\
\vdots \\
x_{M}
\end{array}\right]=z^{-1}\left[\begin{array}{cccc}
f_{1} & 0 & \cdots & 0 \\
0 & f_{2} & \cdots & 0 \\
\vdots & \vdots & \vdots & \vdots \\
0 & 0 & \cdots & f_{M}
\end{array}\right]\left[\begin{array}{c}
x_{1} \\
x_{2} \\
\vdots \\
x_{M}
\end{array}\right]+z^{-1}\left[\begin{array}{c}
g_{1} \\
g_{2} \\
\vdots \\
g_{M}
\end{array}\right] V^{i}} \\
V^{r}=\left[\begin{array}{llll}
h_{1} & h_{2} & \cdots & h_{M}
\end{array}\right]\left[\begin{array}{c}
x_{1} \\
x_{2} \\
\vdots \\
x_{M}
\end{array}\right]+z^{-1} j_{1} V^{i}
\end{gathered}
$$

This procedure guarantees a good agreement with theory for a relative permittivity $\epsilon_{r}>>1$, i.e., it applies to roadway and railway tunnel modeling.

\subsection{Wave Propagation in a Rectangular Tunnel with Lossy Dielectric Walls Using the SIBC}

The wave propagation in a rectangular tunnel with transverse dimension $w=7 \mathrm{~m} \times h=$ $5 \mathrm{~m}$ was studied. This tunnel was simulated using the TLM for guiding structures and time-domain SIBC implementations presented previously. The tunnel walls were modeled by using a lossy dielectric with $\epsilon_{r}=12$ and $\sigma=0.02 \mathrm{Sm}^{-1}$. The tunnel is shown in Figure 11 .

In a rectangular hollow dielectric waveguide, the modes are classified into two families: the first ones with most of its electric field polarized in the $y$-direction, designed as $E_{n m}^{y}$, and the other ones with most of its electric field polarized in the $x$-direction, designed as $E_{n m}^{x}$. 

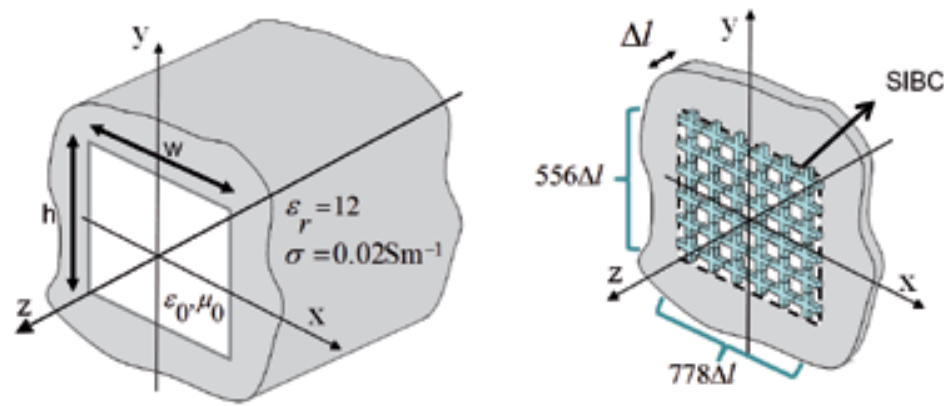

Figure 11. Left: Rectangular tunnel with dimensions $\mathrm{w}=7 \mathrm{~m} \approx 70 \lambda$ and $\mathrm{w}=5 \mathrm{~m} \approx 50 \lambda$. Right: Equivalent 2.5D problem terminated by SIBC (dashed line: TLM staircase section approximation)

Using this notation, the figure on the left of Figure 12 shows a comparison in the range of frequency from $0.4 \mathrm{GHz}$ to $2 \mathrm{GHz}$ of the dispersion curves for the modes $E_{11}^{x}$ and $E_{11}^{y}$ between our procedure and the commonly used Marcatilli's theory for dielectric rectangular waveguides (see Section 2.2). Some very good agreement was obtained for both modes.
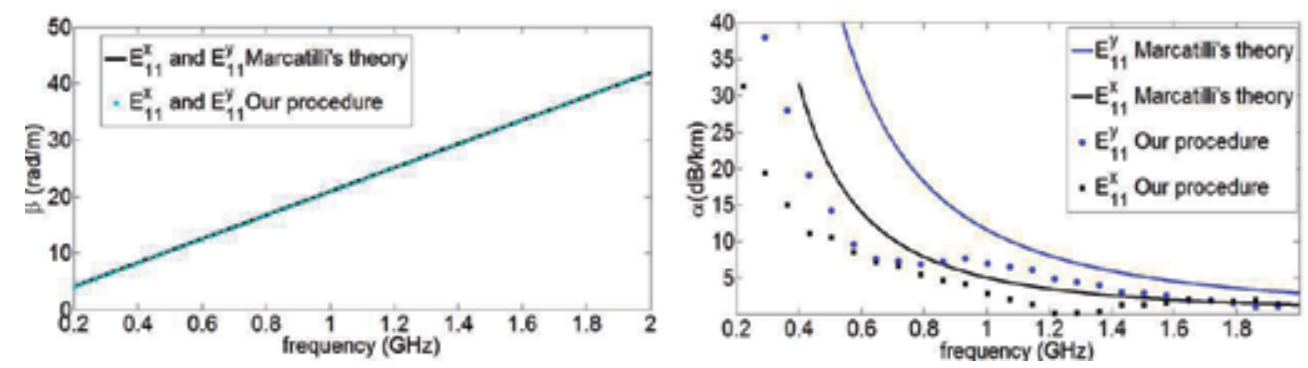

Figure 12. Dispersion curve for: a) $E_{11}^{y}$ mode and b) $E_{11}^{x}$ mode of the rectangular tunnel calculated in the frequency range from $0.4 \mathrm{GHz}$ to $2 \mathrm{GHz}$ with Marcatilli's theory and our procedure, b) dispersion diagram for a rectangular tunnel in the frequency range from $0.2 \mathrm{GHz}$ to $2 \mathrm{GHz}$ using our numerical procedure [4]

As it can be seen, the dispersion curves for the modes tend to the limit $\beta=\omega / c$, and up to $3 \mathrm{GHz}$, they are propagating modes. They propagate with different phase constants and the propagation is strongly multimodal, e.g., around 120 modes at $3 \mathrm{GHz}$. The figure on the right of Figure 12 shows the comparison between both approaches in terms of attenuation constant. One can notice some discrepancies in the low region of the spectrum. One should stress that (54) is valid for $\alpha<<\beta$, and the approximation fails close to the cutoff frequency of the modes (see Section 4.1). Moreover, discrepancies can be explained by the fact that Marcatilli's theory neglects fields at the corners. As the frequency increases, fields are more concentrated in the core region, reducing this error. In Figure 12, the difference between the attenuation constants diminishes as the frequency increases, confirming this fact.

Figure 13 shows the field profiles for the $E_{11}^{y}$ and $E_{11}^{x}$ modes in the waveguide calculated with Marcatilli's theory. Results obtained in Figure 14 were with the 2.5D TLM approach for guiding structures at $1 \mathrm{GHz}$. The structure was excited with a Gaussian pulse and injecting 
the corresponding phase constant of the mode, $\beta \approx 20$ in Figure 12. Good agreement was found for the field profiles of both modes.

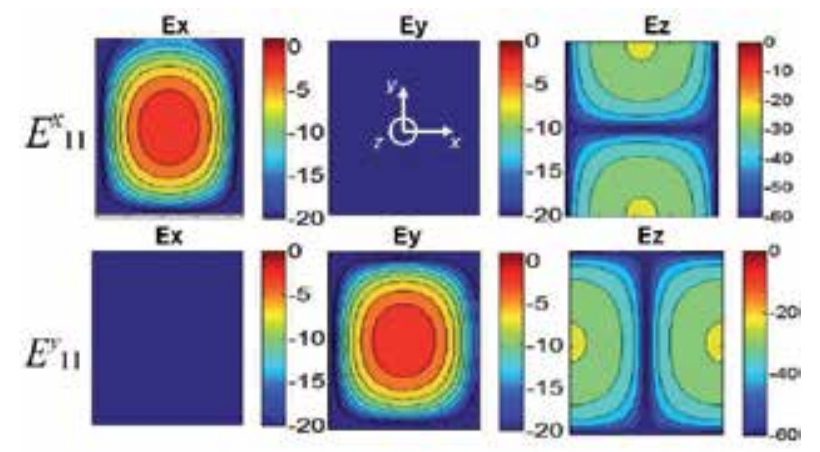

Figure 13. Field configuration in $\mathrm{dBV} / \mathrm{m}$ of the $E_{11}^{y}$ and $E_{11}^{x}$ modes calculated with Marcatilli's theory [18, 19].

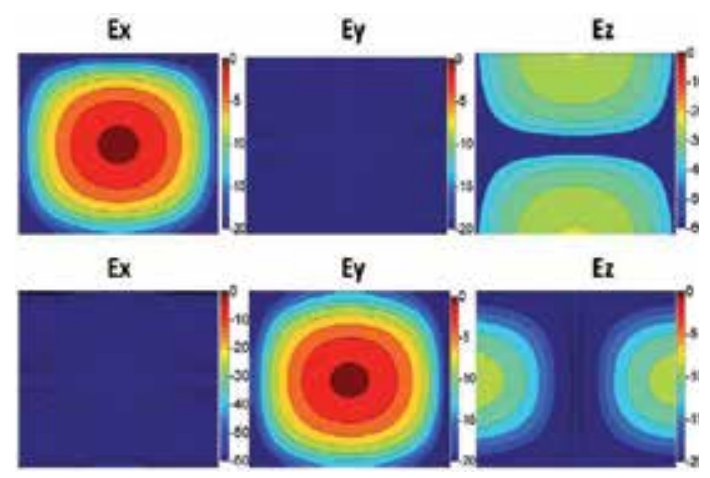

Figure 14. Field configuration in $\mathrm{dBV} / \mathrm{m}$ of the $E_{11}^{y}$ and $E_{11}^{x}$ modes calculated with our procedure [4].

To conclude, it is important to mention that the application of this method can be extended to any uniform waveguiding structure, and also, it can be used to optimally determine antenna field specifications and positioning in confined or waveguide environments as presented in [3].

\section{Conclusion}

The TLM (transmission-line matrix) to characterize modes in guided structures was proposed and described. The 2.5D TLM approach is useful for finding the dispersion diagram, amplitude, and attenuation constant of modes propagating in uniform lossy guiding structures with arbitrary cross section. Its validation in a metallic-rectangular waveguide was presented. This confirms that this approach is well suited for numerical implementation.

Then, the modeling of the wave propagation in lossy waveguides as mine, roadway, or railway tunnels was studied. A procedure to implement the concept of SIBC (surface 
impedance boundary condition) to simulate the lossy dielectric walls was described. This concept allows us to avoid the meshing of the tunnel walls by replacing the wall medium by a frequency-dependent reflection coefficient. Thus, field values beyond the air-wall interface are not required for mode parameter computation. However, the presence of the dielectric beyond the interface is accounted for by the SIBC. The SIBC concept yields satisfactory results as long as $\varepsilon_{r}>>1$, for arbitrary conductivity. The illustration of the capabilities of this method in a rectangular tunnel shows that our procedure is in agreement with theory for the mode amplitudes, attenuation, and phase constants for the $E_{11}^{y}$ and $E_{11}^{x}$ modes when compared with Marcatilli's model.

The 2.5D TLM approach for guiding structures and the SIBC concept might be also applied to study the radio-wave propagation for any cross section. The results in terms of amplitudes and dispersion curves and attenuation constants will allow to study different types of structures and the sensibility of these parameters with the polarization, excitation, shape, size, or frequency.

\section{Acknowledgment}

This work was performed within the French National Project METAPHORT (ANR VTT 2009 Program) and in the frame work of the North of France Regional Cluster CISIT (Campus International Sécurité et Intermodalité des transports). This work was also supported by the French Research Agency, the Nord pas de Calais Region, ITRANS railway cluster, Images et Réseaux cluster of the Brittany region as well as the French ministry of research and the European Commission (FEDER).

\section{Author details}

Jorge Avella-Castiblanco ${ }^{1 *}$, Divitha Seetharamdoo ${ }^{1}$, Marion Berbineau ${ }^{1}$, Michel Ney ${ }^{2}$, Ibrahim Massy ${ }^{2}$, François Gallée ${ }^{2}$

*Address all correspondence to: jeavellac@unal.edu.co

1 Université de Lille Nord de France, French Institute of Science and Technology for Transport (IFSTTAR, LEOST), Villeneuve d'Ascq, France

2 Mines-Télécom Institute, Telecom Bretagne, Brest, France

\section{References}

[1] J. Avella Castiblanco, D. Seetharamdoo, M. Berbineau, M. Ney, and F. Gallee. Determination of antenna specification and positioning for efficient railway communication in tunnels of arbitrary cross section. Intelligent Transport Systems Telecommunications, volume 1, pages 678-683, August 2011.

[2] J. Avella Castiblanco, D. Seetharamdoo, M. Berbineau, M. Ney, and F. Gallee. Surface boundary conditions for lossy dielectrics to model electromagnetic wave propagation in tunnels. In Proceedings of the 5th European Conference on Antennas and Propagation (EUCAP), pages 2331-2335, April 2011. 
[3] J. Avella Castiblanco, D. Seetharamdoo, M. Berbineau, M. Ney, and F. Gallee. Wave Propagation/Book 2, ISBN 980-953-307-493-6, chapter Electromagnetic wave propagation modeling for finding antenna specifications and positions in tunnels of arbitrary cross-section. Intech Europe, Croatia, 2012.

[4] J. Avella Castiblanco, D. Seetharamdoo, M. Berbineau, M. Ney, and F. Gallee. Surface impedance boundary conditions in time domain for guided structures of arbitrary cross section with lossy dielectric walls. IEEE Transaction on Antennas and Propagation, volume 63, no 3, pages 1086-1097, March 2015.

[5] J.H. Beggs, R.J. Luebbers, K.S. Yee, and Karl S. Kunz. Finite-difference time-domain implementation of surface impedance boundary conditions. IEEE Transactions on Antennas and Propagation, 40(1):49-56, January 1992.

[6] Sugiyama K. Chiba, J. Effects of trains on cuttoff frequency and field in rectangular tunnel as waveguide. IEEE Transactions on Microwave Theory and Techniques, 30(5):757-759, May 1982.

[7] C. Christopoulos. The Transmission-Line Modeling (TLM) Method in Electromagnetics. Morgan \& Claypool Publishers, San Rafael, 2006.

[8] D. Didascalou. Ray-Optical Wave Propagation Modelling in Arbitrarily Shaped Tunnels. PhD thesis, Institut fur Hochstfrequenztechnik und Elektronik der Universitat Karlsruhe (TH), 2000.

[9] D.G. Dudley, M. Lienard, S.F. Mahmoud, and P. Degauque. Wireless propagation in tunnels. IEEE Antennas and Propagation Magazine, 49(2):11-26, April 2007.

[10] K. Fujimori and H. Arai. Ray tracing analysis of propagation characteristics in tunnels including transmitting antenna. In Proceedings of AP-S Antennas and Propagation Society International Symposium Digest, volume 2, pages 1222-1225, Baltimore MD, USA, 1996.

[11] K.Y. Fung and Ju Hongbin. Time-domain impedance boundary conditions for computational acoustics and aeroacoustics. International Journal of Computational Fluid Dynamics, 18(6):503-511, August 2004.

[12] P Godfrey. Rational polynomial curve fitting. www.mathworks.com/matlabcentral/, May 2006. fileexchange:11197.

[13] Jean-Marie Gorce, Guillaume Villemaud, and Paul Flipo. On simulating propagation for ofdm/mimo systems with the mr-fdpf model. 2010 Proceedings of the Fourth European Conference on Antennas and Propagation (EuCAP), pages 1-5, Barcelona, April 2010.

[14] Yingbo Hua and Tapan K. Sarkar. Matrix pencil method and its performance. In Proceedings of IEEE International Conference on Acoustics, Speech and Signal Processing (ICASSP), pages 2476-2479, New York, 1988.

[15] T. Itoh. Numerical Techniques for Microwave and Passive Structures. Wiley-Interscience, John Wiley and Sons, Inc., Austin, 1989. 
[16] H. Jin and R. Vahldieck. Full-wave analysis of guiding structures using a 2-d array of 3-d tlm nodes. IEEE Transactions on Microwave Theory and Techniques, 41(3):472-477, March 1993.

[17] P.B. Johns and R.L. Beurle. Numerical solution of 2-dimensional scattering problems using a transmission-line matrix. Proceedings of the Institution of Electrical Engineers, 118(9):1203-1208, September 1971.

[18] H. Krammer. Field configurations and propagation constants of modes in hollow rectangular dielectric waveguides. IEEE Journal of Quantum Electronics, 12(8):505-507, August 1976.

[19] Katherine D. Laakmann and William H. Steier. Waveguides: Characteristic modes of hollow rectangular dielectric waveguides. Applied Optics, 15(5):1334-1340, 1976.

[20] Samir F. Mahmoud and James R. Wait. Guided electromagnetic waves in a curved rectangular mine tunnel. Radio Science, 9(5):567-572, 1974.

[21] J.G. Maloney and G.S. Smith. The use of surface impedance concepts in the finite-difference time-domain method. IEEE Transactions on Antennas and Propagation, 40(1):38-48, January 1992.

[22] E. Masson. Etude de la propagation des ondes electromagnetiques dans les tunnels courbes de section non droite pour des applications metros et ferroviaires. PhD thesis, University of Poitiers, December 2010.

[23] Michel M. Ney. Modeling of highly conducting boundaries with tlm. Electromagnetics, 16(5):521-535, 1996. Highly conducting boundaries; Reflected signals; Transmission line matrix algorithm.

[24] Hsueh-Yuan Pao, Zhaohui Zhu, and S.L. Dvorak. Exact, closed-form representations for the time-domain surface impedances of a homogeneous, lossy half-space. IEEE Transactions on Antennas and Propagation, 52(10):2659-2665, October 2004.

[25] N. Pena Traslavina. Contribution au Developpement de condition aux limites absorbantes pour la metamaterialthode TLM avec applications a l'analyse de circuits hyperfrequences. $\mathrm{PhD}$ thesis, Ecole doctorale de sciences pour l'ingenieur, Universite de Rennes 1, 1997.

[26] D. Pozar. Microwave Engineering. Wiley-Interscience, John Wiley and Sons, Inc., New York, 1998.

[27] J.A. Roden and S.D. Gedney. The efficient implementation of the surface impedance boundary condition in general curvilinear coordinates. IEEE Transactions on Microwave Theory and Techniques, 47(10):1954-1963, October 1999.

[28] Matthew N. O. Sadiku. Numerical Techniques in Electromagnetics, Second Edition. CRC, Austin, 2000. 
[29] D. Seetharamdoo, J. Avella Castiblanco, and M. Berbineau. Metamaterial for trainborne antenna integration and reduction of emi between onboard systems in the railway environment. In 9 th World Congress on Railway Research, pages 678-683, Lille, 2011.

[30] V. Sergey and N. Yuferev. Surface Impedance Boundary Conditions, A Comprehensive Approach. CRC, Boca Raton, 2010.

[31] R. Vahldieck and Wolfang J.R. Hoefer. Encyclopedia of RF and Microwave Engineering, volume 6, chapter Electromagnetic modeling, pages 1232-1248. Wiley-Interscience, John Wiley and Sons, Inc., New Jersey, 2005.

[32] A. Valcarce, G. de la Roche, L. Nagy, J.-F. Wagen, and J.-M. Gorce. A new trend in propagation prediction. IEEE Vehicular Technology Magazine, 6(2):73-81, June 2011.

[33] T. S. Wang and C. F. Yang. Simulations and measurements of wave propagations in curved road tunnels for signals from gsm base stations. IEEE Transactions on Antennas and Propagation, 54(9):2577-2584, 2006.

[34] C. Yeh and F. I. Shimabukuro. The Essence of Dielectric Waveguides. Springer, New York, 2008.

[35] Yan Zhao, Yang Hao, and C. Parini. Fdtd characterization of uwb indoor radio channel including frequency dependent antenna directivities. IEEE Antennas and Wireless Propagation Letters, 6:191-194, 2007. 




\section{Edited by Saad Osman Bashir}

This book endeavors to give the reader a strong base in the advanced theory of electromagnetic waves and its applications, while keeping pace with research in various other disciplines that apply electrostatics/electrodynamics theory. The treatment is highly mathematical, which tends to obscure the principles involved. 